Prepared in cooperation with the Sweetwater Authority

\title{
Water- and Air-Quality and Surficial Bed-Sediment Monitoring of the Sweetwater Reservoir Watershed, San Diego County, California, 2003-09
}

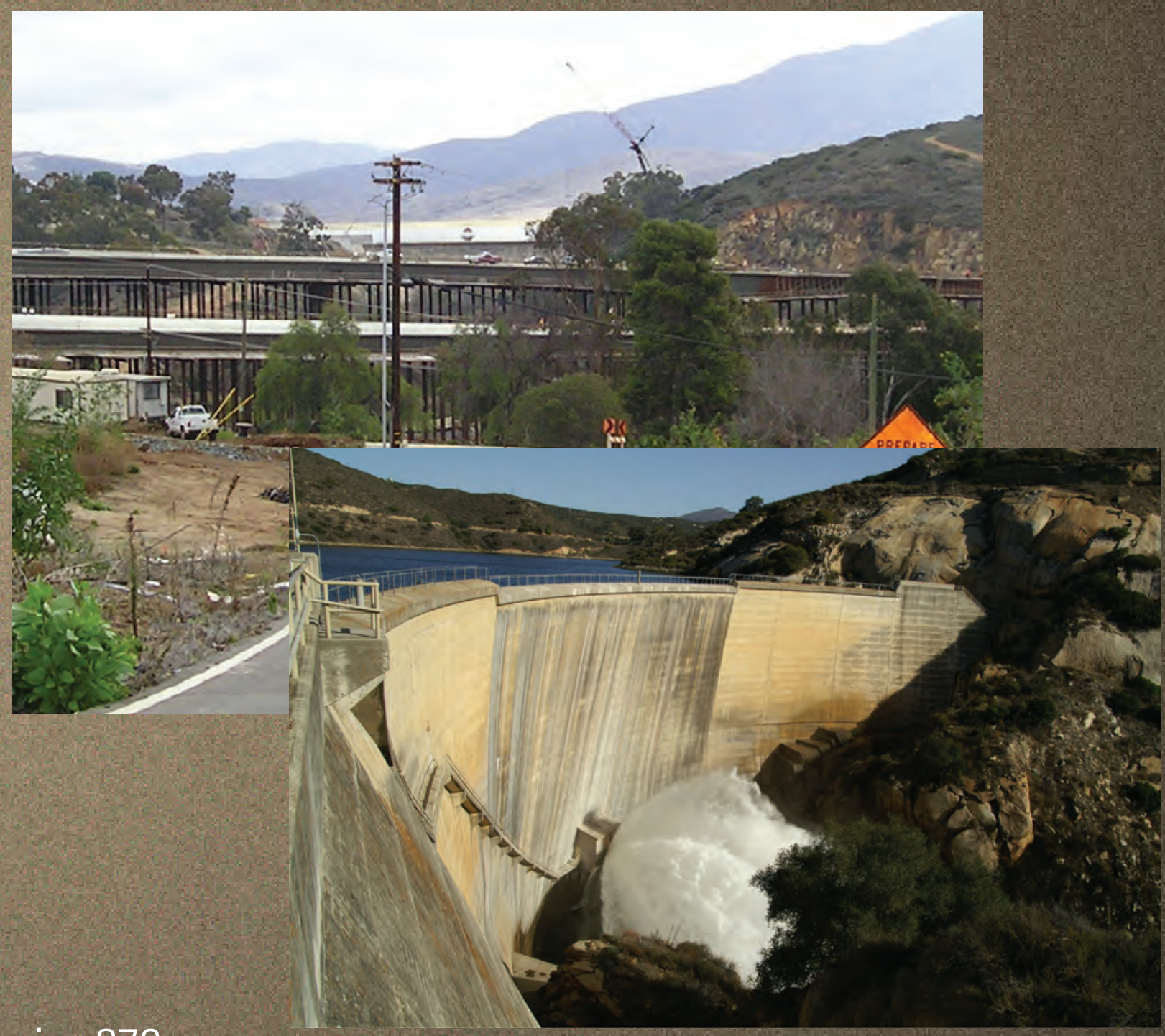

Data Series 879 
Cover. Views of the Sweetwater Reservoir and its surrounding area. Photography by Gregory 0. Mendez, U.S. Geological Survey. 


\section{Water- and Air-Quality and Surficial Bed- Sediment Monitoring of the Sweetwater Reservoir Watershed, San Diego County, California, 2003-09}

By Gregory 0. Mendez, Michael S. Majewski, William T. Foreman, and Andrew Morita

Prepared in cooperation with the Sweetwater Authority

Data Series 879 


\section{U.S. Department of the Interior \\ SALLY JEWELL, Secretary}

\section{U.S. Geological Survey \\ Suzette M. Kimball, Acting Director}

\section{U.S. Geological Survey, Reston, Virginia: 2015}

For more information on the USGS - the Federal source for science about the Earth, its natural and living resources, natural hazards, and the environment, visit http://www.usgs.gov or call 1-888-ASK-USGS.

For an overview of USGS information products, including maps, imagery, and publications, visit http://www.usgs.gov/pubprod

To order this and other USGS information products, visit http://store.usgs.gov

Any use of trade, firm, or product names is for descriptive purposes only and does not imply endorsement by the U.S. Government.

Although this information product, for the most part, is in the public domain, it also may contain copyrighted materials as noted in the text. Permission to reproduce copyrighted items must be secured from the copyright owner.

Suggested citation:

Mendez, G.0., Majewski, M.S., Foreman, W.T., and Morita, Andrew, 2015, Water- and air-quality and surficial bedsediment monitoring of the Sweetwater Reservoir watershed, San Diego County, California, 2003-09: U.S. Geological Survey Data Series 879, 224 p., http://dx.doi.org/10.3133/ds879

ISSN 2327-638X (online) 


\section{Contents}

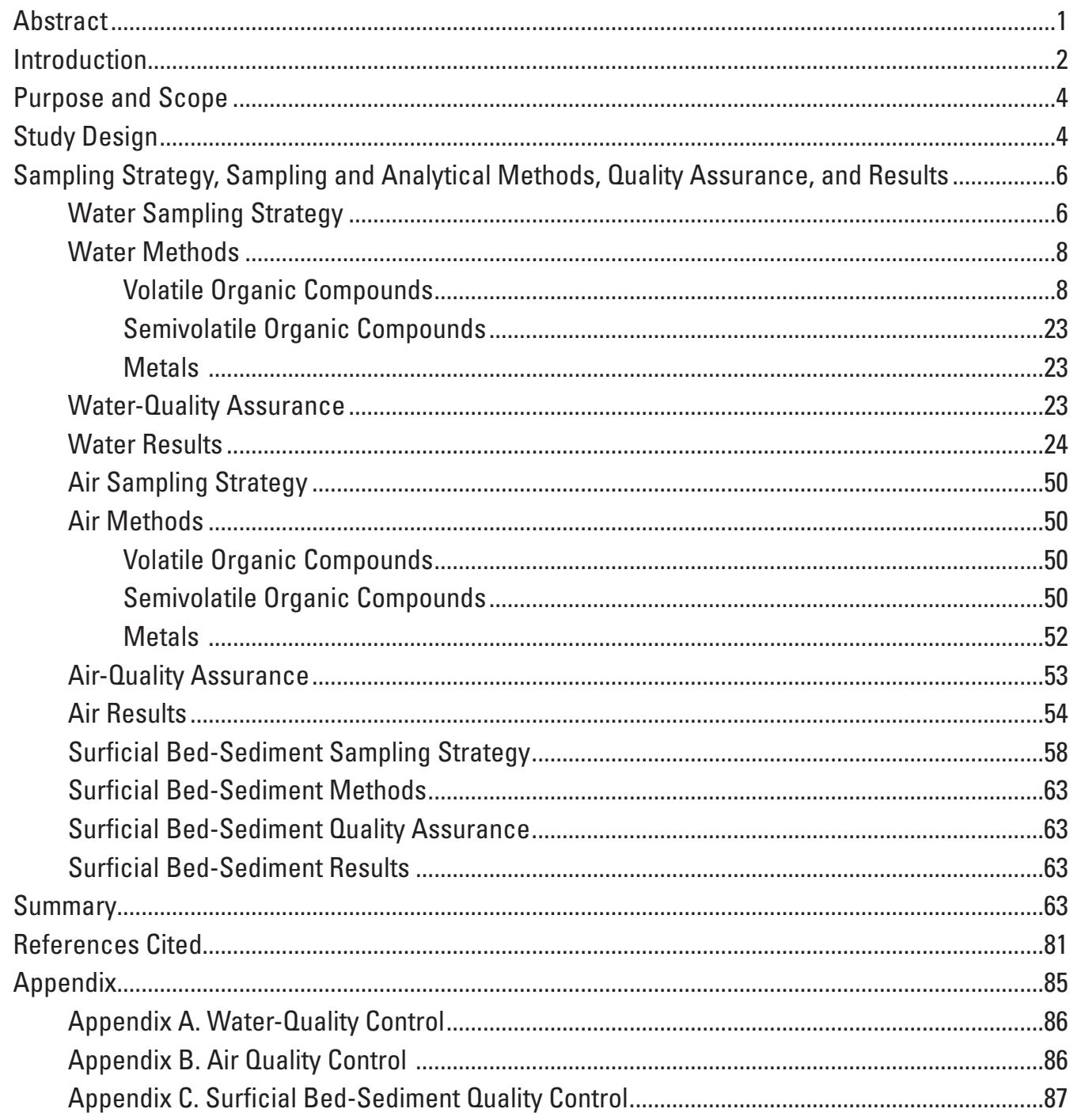




\section{Figures}

1. Map showing the general vicinity of Sweetwater and Loveland Reservoirs and surrounding watershed, San Diego County, California..

2. Map showing water, air, and surficial bed sediment sampling sites and the alignment of State Route 125 near the Sweetwater Reservoir, San Diego County, California...

3. Map showing water and surficial bed sediment sampling sites in Loveland Reservoir, San Diego County, California

4. Figures showing $A$, concentrations in air of methyl tert-butyl ether (MTBE), benzene, and toluene at the Sweetwater Reservoir (SWR) atmospheric sampling site; and concentrations in air and water of $B, \mathrm{MTBE} ; C$, benzene; and $D$, toluene at SWR near the pump tower (site SWR01) and at center of minimum pool (site SWR03), San Diego County, California..

\section{Tables}

1. Site identification numbers, other identifiers, and corresponding site names, Sweetwater and Loveland Reservoirs, San Diego County, California.....

2. Constituent class, medium, sample type, and numbers of constituents analyzed in the environmental and quality-control samples collected from the Sweetwater Reservoir watershed, San Diego County, California.

3. Water-quality depth-profile data for dissolved oxygen, $\mathrm{pH}$, specific conductance, and water temperature for each sampling site and period for the Sweetwater Reservoir watershed, San Diego County, California.

4A. Volatile organic compounds analyzed in whole-water samples, using U.S. Geological Survey National Water Quality Laboratory schedule 2020, for the Sweetwater Reservoir watershed, San Diego County, California

4B. Analytical results for Volatile Organic Compounds detected in whole-water samples, using U.S. Geological Survey National Water Quality Laboratory schedule 2020, from the Sweetwater Reservoir watershed, San Diego County, California (provided as a Microsoft Exce ${ }^{\circledR}$ file).

5A. Base-neutral acid compounds analyzed in whole-water samples, using U.S. Geological Survey National Water Quality Laboratory schedule 1383, for the Sweetwater Reservoir watershed, San Diego County, California

5B. Analytical results for base-neutral acid compounds detected in whole-water samples, using U.S. Geological Survey National Water Quality Laboratory schedule 1383, from the Sweetwater Reservoir watershed, San Diego County, California (provided as a Microsoft Excel ${ }^{\circledR}$ file).

6A. Pesticide compounds analyzed in filtered-water samples, using U.S. Geological Survey National Water Quality Laboratory schedule 2003, for the Sweetwater Reservoir watershed, San Diego County, California

6B. Analytical results for pesticide compounds detected in filtered-water samples, using U.S. Geological Survey National Water Quality Laboratory schedule 2003, from the Sweetwater Reservoir watershed, San Diego County, California.

7A. Pesticide compounds analyzed in filtered-water samples, using U.S. Geological Survey National Water Quality Laboratory schedule 2060, for the Sweetwater Reservoir watershed, San Diego County, California

7B. Analytical results for pesticide compounds detected in filtered-water samples using U.S. Geological Survey National Water Quality Laboratory schedule 2060, from the Sweetwater Reservoir watershed, San Diego County, California 


\section{Tables-Continued}

8A. Anthropogenic organic indicator compounds analyzed in filtered-water samples, using U.S. Geological Survey National Water Quality Laboratory schedule 1433, for the Sweetwater Reservoir watershed, San Diego County, California .

8B. Analytical results for anthropogenic organic indicator compounds detected in filtered-water samples, using U.S. Geological Survey National Water Quality Laboratory schedule 1433, from Sweetwater Reservoir watershed, San Diego County, California.....

9A. Analytical results for metals in whole-water samples, using U.S. Geological Survey National Water Quality Laboratory schedule 1080, from Sweetwater Reservoir watershed, San Diego County, California

9B. Analytical results for metals in filtered-water samples, using U.S. Geological Survey National Water Quality Laboratory schedule 2710, from Sweetwater Reservoir watershed, San Diego County, California.

10A. Analytical results for high-volatility volatile organic compounds (VOCs) processed at the Oregon Graduate Institute for air samples collected from the Sweetwater Reservoir atmospheric site, San Diego County, California.

10B. Analytical results for medium/low-volatility volatile organic compounds (VOCs) processed at the Oregon Graduate Institute for air samples collected from the Sweetwater Reservoir atmospheric site, San Diego County, California (provided as a Microsoft Exce ${ }^{\circledR}$ file).

11A. Polycyclic aromatic hydrocarbons (PAHs) and alkylated-PAH compounds analyzed in air samples, processed at the U.S. Geological Survey National Water Quality Laboratory, from the Sweetwater Reservoir atmospheric site, San Diego County, California

11B. Analyitcal results for Polycyclic Aromatic Hydrocarbon (PAH) and alkylated PAH compounds detected in air samples, processed at the U.S. Geological Survey's National Water Quality Laboratory, from the Sweetwater Reservoir atmospheric site, San Diego County, California (provided as a Microsoft Excel ${ }^{\circledR}$ file)...

12A. Pesticide compounds analyzed in air samples, processed at the U.S. Geological Survey National Water Quality Laboratory, from the Sweetwater Reservoir atmospheric site, San Diego County, California .

12B. Analytical results for pesticide compounds detected in air samples, processed at the U.S. Geological Survey National Water Quality Laboratory, from the Sweetwater Reservoir atmospheric site, San Diego County, California

13. Analytical results for metals in air samples, processed at the U.S. Geological Survey Geologic Division laboratory, collected from the Sweetwater Reservoir atmospheric site, San Diego County, California (provided as a Microsoft Exce ${ }^{\circledR}$ file)...63

14. Analytical results for polycyclic aromatic hydrocarbon (PAHs) compounds in surfical bed sediment, using U.S. Geological Survey National Water Quality Laboratory schedule 2506, from the Sweetwater Reservoir watershed, San Diego County, California

15. Analytical results for organochlorine (OCs) and polychlorinated biphenly (PCBs) compounds in surfical bed sediment, using U.S. Geological Survey National Water Quality Laboratory schedule 5504, from the Sweetwater Reservoir watershed, San Diego County, California.

16. Analytical results for metals in surficial bed-sediment samples, using the U.S. Geological Survey Geologic Division laboratory, collected from the Sweetwater Reservoir watershed, San Diego County, California 


\section{Appendix Tables}

A1A. Quality-control matrix spike results for volatile organic compounds (VOCs) in whole-water samples, using U.S. Geological Survey National Water Quality Laboratory schedule 2020, collected from the Sweetwater Reservoir watershed, San Diego County, California

A1B. Quality-control field blank and field replicate results for volatile organic compound (VOC) concentrations in whole-water samples, using U.S. Geological Survey National Water Quality Laboratory schedule 2020, collected from the Sweetwater Reservoir (SWR) watershed, San Diego County, California

A1C. Quality-control replicate comparison for volatile organic compound (VOC) detected in whole-water samples, using U.S. Geological Survey National Water Quality Laboratory schedule 2020, collected from the Sweetwater Reservoir (SWR) watershed, San Diego County, California

A2A. Quality-control matrix spike results for base-neutral acid (BNA) compounds in whole-water samples, using U.S. Geological Survey National Water Quality Laboratory schedule 1383, collected from the Sweetwater Reservoir watershed, San Diego County, California

A2B. Quality-control replicate results for base-neutral acid (BNAs) compounds in whole-water samples, using U.S. Geological Survey National Water Quality Laboratory schedule 1383, collected from the Sweetwater Reservoir (SWR) watershed, San Diego County, California

A3. Quality-control replicate results of pesticide concentrations in filtered-water samples, using U.S. Geological Survey National Water Quality Laboratory schedule 2003, collected from Sweetwater Reservoir, San Diego County, California

A4. Quality-control replicate results of pesticide concentrations in filteredwater samples, using U.S. Geological Survey National Water Quality Laboratory schedule 2060, collected from the Sweetwater Reservoir watershed, San Diego County, California

A5. Quality-control replicate results for anthropogenic indicator compounds (AICs) analyzed in filtered-water samples, using U.S. Geological Survey National Water Quality Laboratory schedule 1433, collected from the Sweetwater Reservoir watershed, San Diego County, California

A6A. Quality-control spike results for metals in whole-water samples, using U.S. Geological Survey National Water Quality Laboratory schedule 1080, collected from the Sweetwater Reservoir, San Diego County, California.

A6B. Quality-control replicate and blank results for metals in whole-water samples, using U.S. Geological Survey National Water Quality Laboratory schedule 1080, collected from the Sweetwater Reservoir, San Diego County, California

A6C. Quality-control replicate comparison for metals in whole-water samples collected

from the Sweetwater Reservoir watershed, San Diego County, California 133

B1A. Quality-control replicate results for high-volatility volatile organic compounds (VOCs) processed at the Oregon Graduate Institute for air samples collected from the Sweetwater Reservoir atmospheric site, San Diego County, California .........136

B1B. Quality-control replicate results for medium/low-volatility volatile organic compounds (VOCs) processed at the Oregon Graduate Institute for air samples collected from the Sweetwater Reservoir atmospheric site, San Diego County, California 


\section{Appendix Tables-Continued}

B1C. Quality-control replicate comparison for medium/low-volatility volatile organic compounds (VOCs) detected in air samples, processed at the Oregon Graduate Institute, from the Sweetwater Reservoir atmospheric site, San Diego County, California

B2A. Quality-control travel spike results for high-volatility volatile organic compounds (VOCs) processed at the Oregon Graduate Institute for air samples collected from the Sweetwater Reservoir atmospheric site, San Diego County, California .

B2B. Quality-control analytical travel blank results for high-volatility volatile organic compounds (VOCs) processed at the Oregon Graduate Institute for air samples collected from the Sweetwater Reservoir atmospheric site, San Diego County, California

B2C. Quality-control analytical lot blank results for high-volatility volatile organic compounds (VOCs) processed at the Oregon Graduate Institute for air samples collected from the Sweetwater Reservoir atmospheric site, San Diego County, California .

B2D. Quality-control analytical laboratory blank results for high-volatility volatile organic compounds (VOCs) processed at the Oregon Graduate Institute for air samples collected from the Sweetwater Reservoir atmospheric site, San Diego County, California...

B3A. Quality-control travel spike results for medium/low-volatility volatile organic compounds (VOCs) processed at the Oregon Graduate Institute for air samples collected from the Sweetwater Reservoir atmospheric site, San Diego County, California

B3B. Quality-control travel blank detections for medium/low-volatility volatile organic compound (VOCs) processed at the Oregon Graduate Institute for air samples collected from the Sweetwater Reservoir atmospheric site, San Diego County, California...

B3C. Quality-control lot blank detections for medium/low-volatility volatile organic compound (VOCs) processed at the Oregon Graduate Institute for air samples collected from the Sweetwater Reservoir atmospheric site, San Diego County, California

B3D. Quality-control laboratory blank detections for medium/low-volatility volatile organic compounds (VOCs) processed at the Oregon Graduate Institute for air samples collected from the Sweetwater Reservoir atmospheric site, San Diego County, California

B4A. National Water Quality Laboratory quality-control reagent-spike results for polycyclic aromatic hydrocarbon (PAH) compound recoveries for the Sweetwater Reservoir atmospheric site, San Diego County, California

B4B. Quality-control results for polycyclic aromatic hydrocarbon (PAH) compounds detected in National Water Quality laboratory and field reagent-blanks. National Water Quality Laboratory solvent-blank samples for the Sweetwater Reservoir atmospheric site, San Diego County, California

B5A. National Water Quality Laboratory quality-control reagent-spike recoveries for pesticides in air for samples collected from the Sweetwater Reservoir atmospheric site, San Diego County, California . 


\section{Appendix Tables-Continued}

B5B. National Water Quality Laboratory quality-control blank results for pesticide in air samples collected for the Sweetwater Reservoir atmospheric site, San Diego County, California .

B6A. U.S. Geological Survey Geologic Division Laboratory quality-control recovery results for metals from laboratory reagent-spike recoveries for the Sweetwater Reservoir atmospheric site, San Diego County, California

B6B. U.S. Geological Survey Geologic Division Laboratory quality-control replicate results for metals from the Sweetwater Reservoir atmospheric site, San Diego County, California

B6C. U.S. Geological Survey Geologic Division Laboratory quality-control blank replicate comparison for metals from the Sweetwater Reservoir atmospheric site, San Diego County, California

C1. Quality-control replicate results for polycyclic aromatic hydrocarbons (PAHs) in surfical bed sediment, using U.S. Geological Survey National Water Quality Laboratory schedule 2506, from the Sweetwater Reservoir watershed, San Diego County, California .

C2. Quality-control replicate results for organochlorine (OCs) and polychlorinated biphenly (PCBs) compounds in surfical bed sediment, using U.S. Geological Survey National Water Quality Laboratory schedule 5504, from the Sweetwater Reservoir watershed, San Diego County, California

C3A. U.S. Geological Survey Geologic Division Laboratory quality-control matrix-spike results for metals in surficial bed-sediment samples collected in the Sweetwater Reservoir watershed, San Diego County, California

C3B. U.S. Geological Survey Geologic Division Laboratory quality-control replicate results for metals in surficial bed-sediment samples collected in the Sweetwater Reservoir, San Diego County, California

C3C. U.S. Geological Survey Geologic Division Laboratory quality-control replicate comparison for metals in surficial bed-sediment samples collected in the Sweetwater Reservoir, San Diego County, California 


\section{Abbreviations}

$\begin{array}{ll}\text { AOI } & \text { anthropogenic organic indicator } \\ \text { BNAs } & \text { base neutral acids } \\ \text { CCRMP } & \text { Canadian Certified Reference Materials Project } \\ \text { GC/MS } & \text { gas chromatography/mass spectrometry } \\ \text { GFF } & \text { glass-fiber filter } \\ \text { LFDD } & \text { low-flow diversion dam } \\ \text { LLR } & \text { Loveland Reservoir } \\ \text { LRL } & \text { laboratory reporting level } \\ \text { MTBE } & \text { methyl tert-butyl ether } \\ \text { NWIS } & \text { National Water Inventory System } \\ \text { NWOL } & \text { National Water Quality Laboratory } \\ \text { OC } & \text { Organochlorine compound } \\ \text { PAHs } & \text { polycyclic aromatic hydrocarbons } \\ \text { PCBs } & \text { polychlorinated biphenyls } \\ \text { PSE } & \text { pressurized-solvent extraction } \\ \text { PUF } & \text { polyurethane foam } \\ \text { OA } & \text { quality assurance } \\ \text { OFF } & \text { quartz fiber filters } \\ \text { SIM } & \text { selected-ion monitoring } \\ \text { SOCs } & \text { semivolatile organic compounds } \\ \text { SPE } & \text { solid-phase extraction } \\ \text { SR } & \text { State Route } \\ \text { SRM } & \text { standard reference material } \\ \text { SWR } & \text { Sweetwater Reservoir } \\ \text { TSP } & \text { total suspended particle } \\ \text { URDS } & \text { urban runoff diversion system } \\ \text { USEPA } & \text { U.S. Environmental Protection Agency } \\ \text { USGS } & \text { U.S. Geological Survey } \\ \text { OC } & \text { quality control } \\ \text { VOC } & \text { volatile organic compound } \\ & \end{array}$




\section{Conversion Factors}

SI to Inch/Pound

\begin{tabular}{|c|c|c|}
\hline Multiply & By & To obtain \\
\hline \multicolumn{3}{|c|}{ Length } \\
\hline centimeter $(\mathrm{cm})$ & 0.3937 & inch (in.) \\
\hline millimeter (mm) & 0.03937 & inch (in.) \\
\hline meter (m) & 3.281 & foot $(\mathrm{ft})$ \\
\hline kilometer (km) & 0.6214 & mile (mi) \\
\hline meter $(\mathrm{m})$ & 1.094 & yard (yd) \\
\hline \multicolumn{3}{|c|}{ Area } \\
\hline square kilometer $\left(\mathrm{km}^{2}\right)$ & 247.1 & acre \\
\hline square kilometer $\left(\mathrm{km}^{2}\right)$ & 0.3861 & square mile $\left(\mathrm{mi}^{2}\right)$ \\
\hline \multicolumn{3}{|c|}{ Volume } \\
\hline 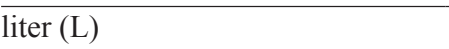 & 0.2642 & gallon (gal) \\
\hline milliliter (mL) & 0.03382 & ounce, fluid (fl. oz) \\
\hline cubic meter $\left(\mathrm{m}^{3}\right)$ & 264.2 & gallon (gal) \\
\hline cubic meter $\left(\mathrm{m}^{3}\right)$ & 0.0008107 & acre-foot (acre-ft) \\
\hline cubic hectometer $\left(\mathrm{hm}^{3}\right)$ & 810.7 & acre-foot (acre-ft) \\
\hline \multicolumn{3}{|c|}{ Mass } \\
\hline gram (g) & 0.03527 & ounce, avoirdupois (oz) \\
\hline milligram (mg) & 0.00003527 & ounce, avoirdupois (oz) \\
\hline nanogram (ng) & 0.00000000003527 & ounce, avoirdupois (oz) \\
\hline \multicolumn{3}{|c|}{ Pressure } \\
\hline kilopascal (kPa) & 0.009869 & atmosphere, standard (atm) \\
\hline \multicolumn{3}{|c|}{ Density } \\
\hline milligram per cubic meter $\left(\mathrm{mg} / \mathrm{m}^{3}\right)$ & 0.00000006242 & pound per cubic foot $\left(\mathrm{lb} / \mathrm{ft}^{3}\right)$ \\
\hline gram per cubic centimeter $\left(\mathrm{g} / \mathrm{cm}^{3}\right)$ & 62.4220 & pound per cubic foot $\left(\mathrm{lb} / \mathrm{ft}^{3}\right)$ \\
\hline
\end{tabular}

Temperature in degrees Celsius $\left({ }^{\circ} \mathrm{C}\right)$ may be converted to degrees Fahrenheit $\left({ }^{\circ} \mathrm{F}\right)$ as follows:

$$
{ }^{\circ} \mathrm{F}=\left(1.8 \mathrm{x}^{\circ} \mathrm{C}\right)+32
$$

Temperature in degrees Celsius $\left({ }^{\circ} \mathrm{C}\right)$ may be converted to the Kelvin (K) scale as follows:

$$
\mathrm{K}={ }^{\circ} \mathrm{C}+273.15
$$

Concentration of a chemical $(y)$ in the atmosphere in units of nanograms per cubic meter $\left(\mathrm{ng} / \mathrm{m}^{3}\right)$ can be converted to the dimensionless volume-fraction units of parts per billion by volume (ppbv) as follow:

Concentration of chemical $y$ in ppbv $=\left(\right.$ concentration in $\left.\mathrm{ng} / \mathrm{m}^{3}\right) \times(R \times T) /\left(p \times M_{y}\right)$ where

$p$ is pressure in Pascal $(\mathrm{Pa})$;

$T$ is temperature in Kelvin (K);

$M_{r}$ is the molecular weight of the chemical $y$ in grams per mole; and

$R$, the gas constant, is $8.314 \mathrm{~Pa}-\mathrm{m}^{3}$ per mole-K.

Vertical coordinate information is referenced to the North American Vertical Datum of 1988 (NAVD 88).

Horizontal coordinate information is referenced to the North American Datum of 1983 (NAD 83).

Altitude, as used in this report, refers to distance above the vertical datum.

Specific conductance is given in microsiemens per centimeter at 25 degrees Celsius ( $\mu \mathrm{S} / \mathrm{cm}$ at $\left.25^{\circ} \mathrm{C}\right)$.

Concentrations of chemical constituents in water are given either in milligrams per liter (mg/L) or micrograms per liter $(\mu \mathrm{g} / \mathrm{L})$. 
This page intentionally left blank. 
This page intentionally left blank. 


\title{
Water- and Air-Quality and Surficial Bed-Sediment Monitoring of the Sweetwater Reservoir Watershed, San Diego County, California, 2003-09
}

\author{
by Gregory 0. Mendez, Michael S. Majewski, William T. Foreman, and Andrew Morita
}

\section{Abstract}

In 1998, the U.S. Geological Survey, in cooperation with the Sweetwater Authority, began a study to assess the overall health of the Sweetwater watershed in San Diego County, California. This study was designed to provide a data set that could be used to evaluate potential effects from the construction and operation of State Route 125 within the broader context of the water quality and air quality in the watershed. The study included regular sampling of water, air, and surficial bed sediment at Sweetwater Reservoir (SWR) for chemical constituents, including volatile organic compounds (VOCs), base-neutral and acid- extractable organic compounds (BNAs) that include polycyclic aromatic hydrocarbons (PAHs), pesticides, and metals. Additionally, water samples were collected for anthropogenic organic indicator compounds in and around SWR. Background water samples were collected at Loveland Reservoir for VOCs, BNAs, pesticides, and metals. Surficial bed-sediment samples were collected for PAHs, organochlorine pesticides, and metals at Sweetwater and Loveland Reservoirs.

To monitor changes in contaminant concentration in water and air at SWR during the construction and operation of State Route 125, this study was divided into three phases. Phase One sampling (September 1998 to September 2004) was designed to establish baseline conditions for target compounds in terms of detection frequency and concentration in air and water. Phase Two (October 2004 to September 2007) continued sampling at selected monitoring sites during construction of State Route 125 to assess any effect from the construction process and the use of heavy equipment to build the roadway. Phase Three (October 2007 to August 2009) continued sampling for 2 years after the opening of State Route 125 to assess the potential changes in water quality related to vehicle emissions from the roadway alignment. Surficial bed-sediment samples were collected three times during the study - at the beginning of the study, at the start of Phase Two, and at the end of the study.
This report describes the study design and the sampling and analytical methods and presents data from water, air, and surficial bed-sediment samples collected from the sixth to eleventh years of the study (October 2003-August 2009), spanning the last year of Phase one and all of Phases Two and Three. Data collected during the first 5 years of sampling have been previously published.

Three types of quality-control samples were used in this study-matrix spikes, blanks, and replicates. Matrix-spike data are considered to be adequate if the recovery concentration is within 30 percent of the matrix concentration. Replicate data are considered to be adequate if the replicate sample concentration is within 30 percent of the environmental sample concentration. Additionally, surrogate compounds were added to most samples to monitor sample-specific performance of the analytical method.

Most VOC matrix-spike recovery data associated with water samples are within acceptable criteria, but three VOCs had recoveries below the acceptable criteria; these compounds may not have been detected in water samples if they were present at low concentrations. Data for blanks associated with water samples for VOCs and metals showed no detections above their laboratory reporting levels. Most replicate data are within acceptable criteria. Quality-control data for VOC air samples resulted in flagging several reported concentrations for acetone, benzene, ethenylbenzene, and naphthalene because they may be biased high. Acetone, benzene, and toluene were detected at low concentrations in almost every VOC air blank. Some PAH and pesticide concentrations in air samples were designated as estimated because of method performance limitations. PAHs in surficial bed sediment had 83 percent of surrogates below the acceptable criteria. No matrix-spike data for metals in surficial bed sediment were outside the acceptable criteria; only beryllium had a replicate comparison outside the acceptable criteria. 
Sampling results show concentrations of the gasoline oxygenate methyl tert-butyl ether in water and air samples declined after it was phased out by the State of California in January 2004. The largest concentrations of gasoline hydrocarbons benzene and toluene in water were detected at or near the surface of the SWR. Isophorone and phenol were the two most frequently detected BNA compounds in water. Diuron, prometon, and simazine were the most frequently detected pesticide compounds in water. Concentrations of benzene and toluene in air samples were highest during the cooler months and had a consistent seasonal pattern over time. Ten PAH compounds were detected frequently in air samples. Twelve pesticide compounds were also detected in air samples. Surficial bed-sediment samples were analyzed for 53 PAHs; 22 of the compounds had one or more detections. Surficial bed-sediment samples were analyzed for 22 organic compounds; only 6 compounds had one or more detections. Surficial bed-sediment samples were analyzed for 37 metals.

\section{Introduction}

The Sweetwater Authority (hereinafter referred to as the "Authority"), under the guidance of its Board of Directors, operates a public drinking-water supply system for over 186,900 residential and commercial customers in Chula Vista and National City, California. The Sweetwater Reservoir (SWR) (fig. 1) is about $15 \mathrm{~km}$ southeast of San Diego, Calif., and has a storage capacity of $34.6 \mathrm{hm}^{3}$. The Authority also stores water at Loveland Reservoir (LLR; fig. 1), which is about $30 \mathrm{~km}$ east of SWR near Alpine, Calif., and has a storage capacity of $31.3 \mathrm{hm}^{3}$. In addition to the two reservoirs, the Authority operates three deep wells in National City and several wells near the Sweetwater River in Chula Vista. The Reynolds Desalination Facility, in Chula Vista, treats brackish groundwater from the wells along the Sweetwater River. Approximately 70 percent of the water provided by the Authority comes from local supplies that include the Sweetwater River, the Sweetwater alluvium, and the San Diego Formation. The remaining water provided is imported from the Colorado River and the Sacramento/San Joaquin River systems through pipelines and aqueducts. Local reservoir and imported water are treated for use as drinking water at the Robert A. Perdue Treatment Plant at the SWR.

The Sweetwater River watershed covers $466 \mathrm{~km}^{2}$ (fig. 1). Although much of the land is undeveloped, the area includes the Sycuan and Viejas Indian Reservations (both having gaming facilities resulting in high vehicle traffic), part of the Cleveland National Forest, agricultural land, rural residential acreage, urban and suburban residential development, mining and industrial land use, commercial recreation, and commercial business development. The watershed also includes three 18-hole golf courses. The Authority started a shore fishing program in 2005 in a restricted area on the south side of the SWR. Shore fishing is allowed at LLR in a restricted area at the east end of the reservoir. The Authority maintains one motorized boat at each reservoir for routine water-quality sampling and regular shore patrols. No other boating is allowed at either reservoir.

The Authority is concerned about the impact that the increasing growth and development in the Sweetwater River watershed will have on the quality of the drinking-water supply. Because of this concern, the Authority has initiated a variety of efforts to protect the watershed, including source assessments, total organic carbon assessments (Fram and others, 2001), watershed stakeholders outreach to identify issues of concern, and the construction and operation of an urban runoff diversion system (URDS; fig. 2). The URDS diverts unwanted water from the river and surrounding urban runoff to a point downstream from the SWR. These programs help the Authority evaluate and manage the overall environmental health of the watershed by monitoring changes that can degrade the quality of the water supply and necessitate additional water treatment as the population increases and land use intensifies. To help improve knowledge of potential watershed issues that will facilitate the Authorities evaluation and management of water resources, the U.S. Geological Survey (USGS), in cooperation with the Authority, began a monitoring study in 1998. Data collected during the first 5 years of sampling are published in Majewski and others (2002) and Mendez and others (2007, 2008).

In addition to the increasing urbanization pressures within the watershed, another Authority concern was the construction and operation of the State Route 125 (SR 125) alignment project. In 1984, the San Diego Association of Governments added SR 125 to the Regional Transportation Plan as part of the future highway system of San Diego. The SR 125 project consists of approximately $18 \mathrm{~km}$ of roadway construction/alignment that extends from SR 54 (northern terminus) to interstate 905 (southern terminus; figs. 1 and 2). The project plans included initial construction of a four-lane toll way, completed in 2007. Two additional lanes may be added for dedicated transit purposes such as high-occupancy vehicle and (or) light-rail operations (California Department of Transportation, 2001). Approximately 200,000 vehicles per day, including a high percentage (more than 10 percent) of heavy diesel trucks from the United States and Mexico, are expected to travel SR 125. The SR 125 alignment is elevated above the reservoir surface and is within about $150 \mathrm{~m}$ of the reservoir at its nearest point. Because the SWR is downwind of the alignment, the Authority was concerned that toxic vehicle emissions, paved road dust, and pesticides used on the roadside might enter the reservoir by atmospheric deposition in concentrations that could affect public health and the cost of treating the drinking-water supply.

In 1996, the Authority commissioned a study (Ogden Environmental and Energy Services, 1997) to model the atmospheric depositional loading to the SWR. The model included a variety of toxic compounds from vehicle emissions and projected risks associated with the SR 125 alignment. The predicted concentrations of select contaminants were 
compared with the standards set by the California Safe Drinking Water Act (California Environmental Protection Agency, 1986) and to California and Federal maximum contaminant levels. The results of the Ogden study showed drinking-water guidance levels for one or more contaminants could be exceeded in the three freeway construction options. Another modeling study done in 1999 resulted in similar conclusions (Byard, 1999). Sources of uncertainty that could affect the interpretation of these modeling studies include the estimates of vehicle emissions and the roles of regional air pollution and land use in the watershed. The Authority and the U.S. Environmental Protection Agency (EPA) Region IX concluded that the findings in the Ogden and Byard reports warranted the implementation of a monitoring program to characterize the potential impact that atmospheric deposition of vehicular emissions from the operation of SR $125 \mathrm{might}$ have on the quality of the drinking water stored in SWR. In 1998, the USGS, in cooperation with the Authority, began a study to assess the overall health of the Sweetwater watershed to provide a data set that could be used to evaluate potential effects from the construction and operation of SR 125, within the broader context of the water quality and air quality in the watershed.

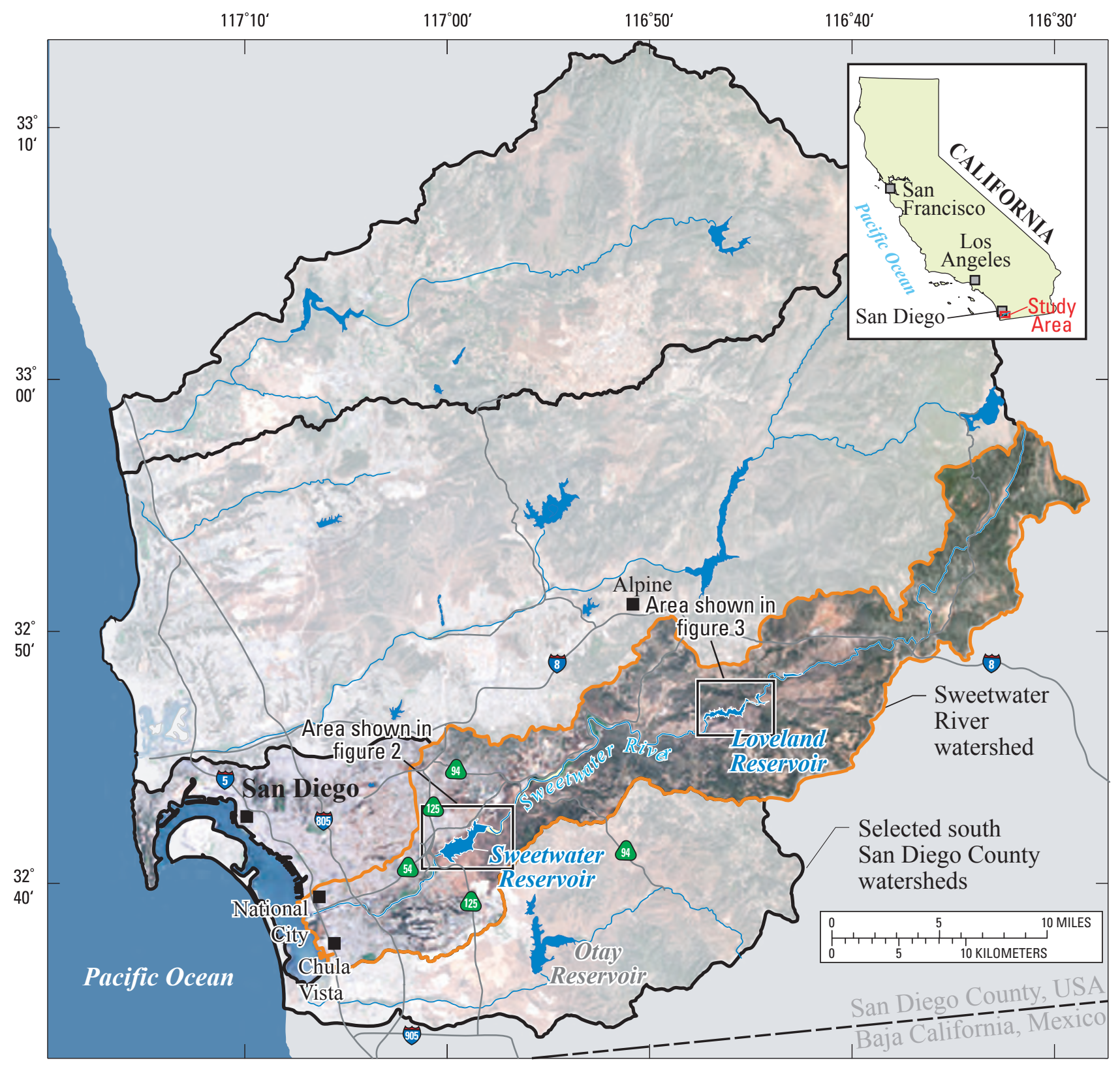

Base from U.S. Geological Survey digital data, 1:100,000, 1981-89; Universal Transverse Mercator Projection (NGVD 29), Zone 11.

Figure 1. The general vicinity of Sweetwater and Loveland Reservoirs and surrounding watershed, San Diego County, California. 


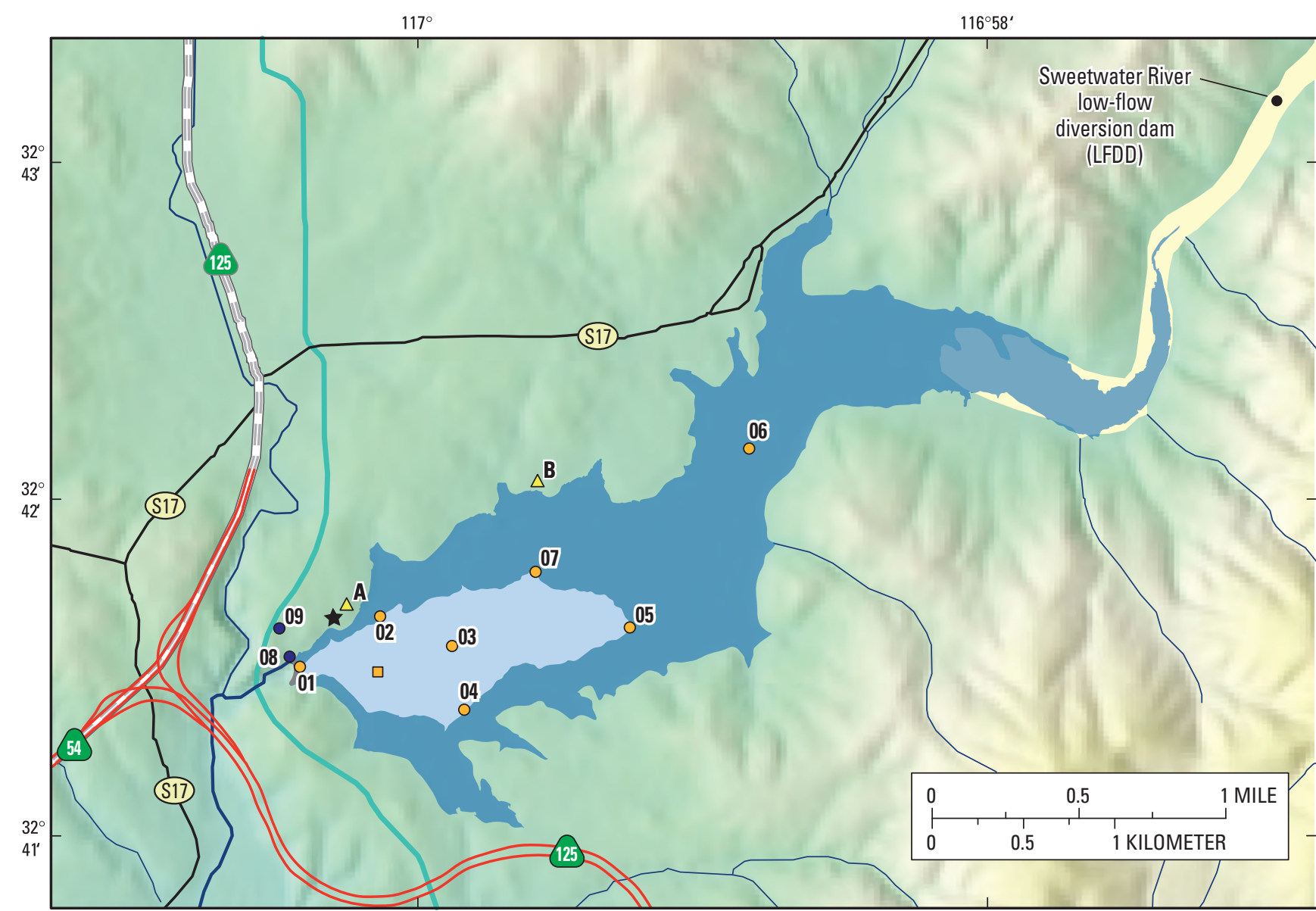

Base from U.S. Geological Survey digital data, 1:100,000, 1981-89; Universal Transverse Mercator Projection (NGVD 29), Zone 11.

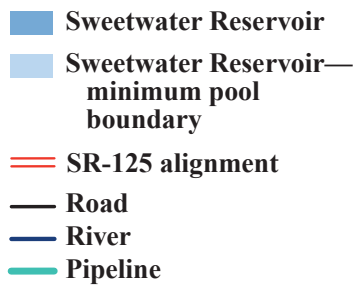

Atmospheric sampling site (AIR01)

$\square$ Sediment core sampling site $\triangle$ Urban Runoff Diversion System pondsA Vista del Lago B Gum Tree Cove

\section{EXPLANATION}

O Water and surficial bedsediment sampling siteSWR01 near pump tower SWR02 near Vista del Lago station SWR03 center of minimum pool SWR04 near recreation area SWR05 minimum pool boundary East SWR06 east-end reservoir-fill boundary SWR07 near Gum Tree Cove Pond
- Water sampling site-

SWR08 Perdue Treatment Plant finished water

SWR09 Perdue Treatment Plant imported raw water

LFDD Sweetwater River at low-flow diversion dam

Figure 2. Water, air, and surficial bed sediment sampling sites and the alignment of State Route 125 near the Sweetwater Reservoir, San Diego County, California.

\section{Purpose and Scope}

This report provides data from water, air, and surficial bed-sediment samples that were collected from October 2003 through August 2009 (water years 2004-09). This report is the fourth in a series of reports that describe the monitoring activities, and presents data that will be used to assess the potential effect on water quality in SWR resulting from land-use changes and development in the watershed.

The scope of this study is to provide analytical results collected over time from three environmental media - water, air, and surficial bed sediment - and determine whether any measured changes in reservoir water quality are the result of atmospheric deposition of organic chemicals and metals originating from the construction and operation of SR 125.

\section{Study Design}

This study was designed to provide a data set that could be used to evaluate potential effects from the construction and operation of SR 125, within the broader context of the water and air quality in the watershed. The study design consisted of three phases: Phase One sampling was performed from October 1998 through September 2004; Phase Two sampling was performed from October 2004 through October 2007; Phase Three sampling was performed from November 2007 through August 2009. The objective of Phase One was to determine the occurrence and concentration of selected organic - volatile organic compounds (VOCs) and polycyclic aromatic hydrocarbons (PAHs) - and inorganic (metal) 
compounds in each of the three environmental media (water, air, and surficial bed sediments) in order to establish baseline conditions for these targeted compounds. The objective of Phase Two was to continue monitoring the targeted compounds during construction of SR 125 and to compare the analytical results to those of Phase One. The objective of Phase Three was to continue monitoring the same targeted compounds during operation of SR 125 and, where applicable, to compare the analytical results to those of Phases One and Two. Pesticides and anthropogenic organic indicator (AOI) compounds were sampled to look at the overall health of the watershed and were not sampled as part of the targeted compounds.

Each of the environmental media in the Sweetwater watershed were sampled and analyzed for the following groups of chemical compounds:

- Surface water-Volatile organic compounds (VOCs), semivolatile organic compounds (SOCs) [base-neutral/acid extractable compounds (BNAs), including polycyclic aromatic hydrocarbons (PAHs), pesticides, and anthropogenic organic indicator (AOI) compounds], and metals.

- Air-VOCs, SOCs (PAHs and pesticides), and metals.

- Surficial bed sediments-PAHs, organochlorine compounds (OCs), total polychlorinated biphenyls (PCBs), and metals.

At the start of Phase One (October 1998), water samples were collected at seven sampling sites at SWR (sites SWR01-07, fig. 2), two sampling sites at the Perdue
Treatment Plant (SWR08 and 09), and two at LLR (fig. 3). Starting in October 1999, only three of the seven sites at SWR (sites SWR01, 03, and 06), both sites at the Perdue Treatment Plant (SWR08 and 09), and one site at LLR were sampled. Phases Two and Three continued the collecting of waterquality samples for the same three sites at SWR and the one site at LLR.

The SWR atmospheric sampling site was located downwind of the proposed SR125 alignment and upwind of SWR (site AIR01, fig. 2) along a transect of the predominant wind direction. The site is wired for AC power (120 volts), equipped with telephone modem access, and secured from the general public by a $2-m$-high chain-link fence. The site includes a fully instrumented meteorological station that records hourly mean values of wind speed and direction, ambient air temperature and relative humidity at two heights, rainfall, and barometric pressure.

At the beginning of Phase One, surficial bed sediments were sampled at 15 sites (Majewski and others, 2002) including sites in the URDS. A one-time coring of the SWR bed sediments was also completed at the start of Phase One (Majewski and others, 2002). During Phase Two (October 2004), surficial bed-sediment samples were collected at four sites in SWR, two sites in LLR, and in the Sweetwater River near the low-flow diversion dam (site LFDD; figs. 2 and 3). Sediment samples were also collected at the SWR atmospheric site (AIR01). At the end of Phase Three (August 2009), surficial bed-sediment samples were collected at four sites in SWR (two samples at site SWR06 because the site moves with the elevation of the reservoir), two sites in LLR, and at site LFDD (figs. 2 and 3).

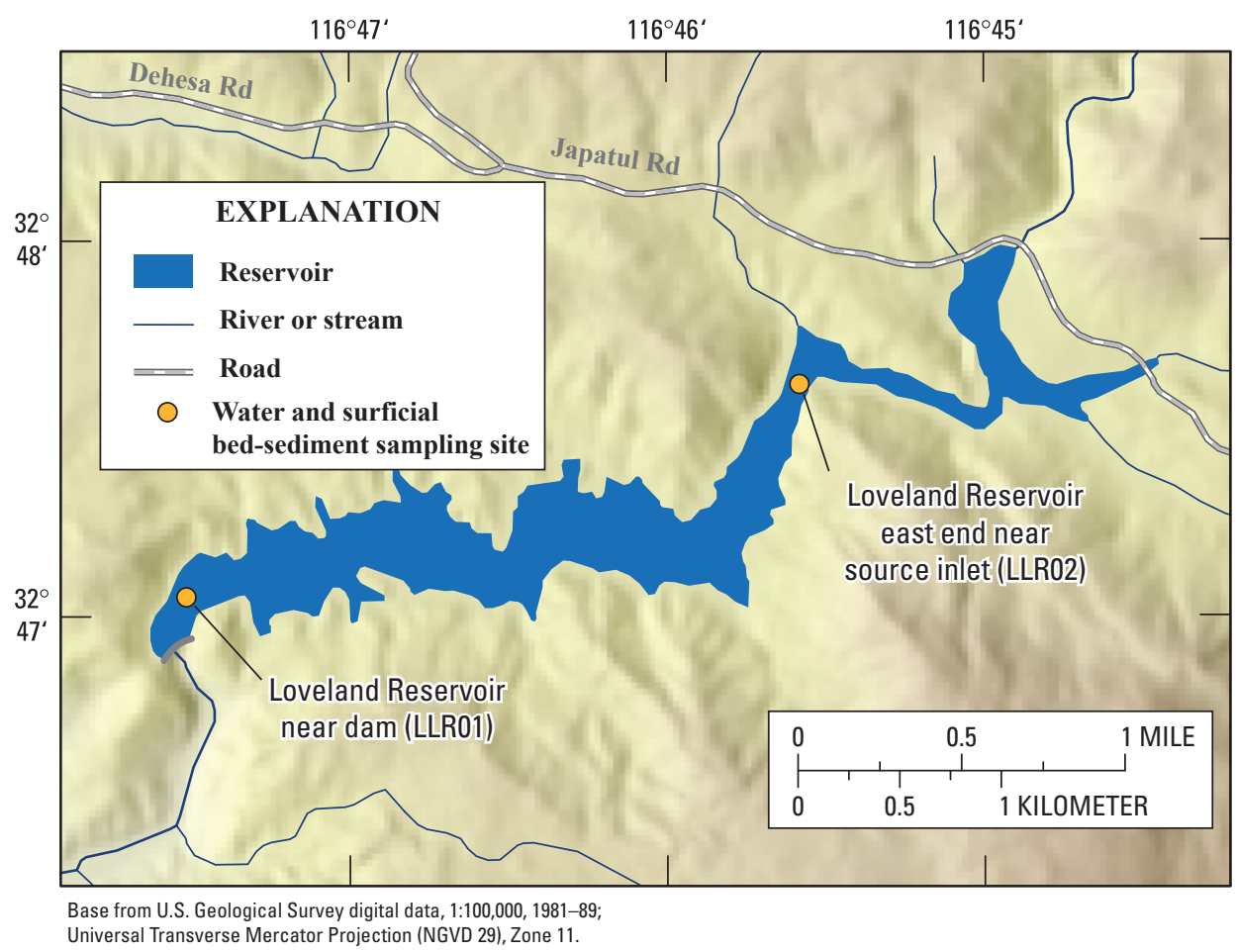

Figure 3. Water and surficial bed sediment sampling sites in Loveland Reservoir, San Diego County, California. 


\section{Sampling Strategy, Sampling and Analytical Methods, Quality Assurance, and Results}

Sampling strategies, methods, quality-assurance steps, and data for water, air, and surficial bed-sediment samples are presented in the following sections. Interpretative analysis of the environmental and quality-control data to address the objectives of the study is not included in this report. All site identification numbers, other identifiers, and sampling site names are listed in table 1 . An inventory of samples collected and their associated quality assurance (QA) is listed in table 2.

\section{Water Sampling Strategy}

Reporting levels for some constituents vary on the basis of differences in sample volumes or the presence of interferences. All water data are available through the USGS National Water Inventory System (NWIS) database web interface (http://nwis.waterdata.usgs.gov/nwis).

Beginning in October 1999, the number of water sampling sites at SWR was reduced from seven to three (fig. 2), and the number of sampling sites at LLR was reduced to one (fig. 3). The locations of the Perdue Treatment Plant and Sweetwater River sampling sites did not change. The number of sampling sites in the SWR was reduced to focus the sampling efforts at "indicator" sites - those sites believed to provide the most relevant information without compromising the scientific integrity of the project. These indicator sites are:
- SWR01-located near the dam and the pump-tower intake for reservoir water to the treatment plant; used to monitor the quality of water entering the treatment plant.

- SWR03-located near the center of the minimum pool; used to monitor the quality of water at the center of the reservoir.

- SWR06 - located near the east end of the reservoir; used to monitor the quality of water entering the reservoir from the Sweetwater River.

- LLR01-located near the dam; used to monitor the quality of the reserve storage water.

Sites SWR01 and SWR03 are within the minimum pool boundary of the reservoir (fig. 2) to ensure that water would always be available for sampling throughout the year. Site SWR06 is in the eastern third of the reservoir in very shallow water. As the water level in the reservoir fell, the water depth at this site decreased, and sometimes the bed sediments were completely exposed. When reservoir water levels fell below $1 \mathrm{~m}$, the sampling site was moved west to where the water depth was over $1 \mathrm{~m}$. Sampling sites at SWR and LLR were marked with stationary buoys anchored to the bottom.

Three additional water-sampling sites (SWR08, SWR09, and LFDD, table 1, fig. 2) were established outside the reservoir boundaries. Site SWR08 monitored the quality of the finished treated water as it left the treatment plant for distribution to customers; this site was sampled because it represents an integrated outflow of lake and imported water. Site SWR09 monitored the quality of the imported raw water

Table 1. Site identification numbers, other identifiers, and corresponding site names, Sweetwater and Loveland Reservoirs, San Diego County, California.

[See figure 2 for site locations near Sweetwater Reservoir. See figure 3 for site locations at Loveland Reservoir. Quality-assurance/quality-control (QA/QC) site is a place holder for the data.]

\begin{tabular}{cll}
\hline $\begin{array}{c}\text { Site-identification } \\
\text { number }\end{array}$ & $\begin{array}{c}\text { Other } \\
\text { identifiers }\end{array}$ & \\
\hline 324130117002501 & SWR01 & Sweetwater Reservoir near pump tower \\
324139117000801 & SWR02 & Sweetwater Reservoir near Vista del Lago station \\
324131117000101 & SWR03 & Sweetwater Reservoir center minimum pool \\
324126116595701 & SWR04 & Sweetwater Reservoir near recreation area \\
324137116592401 & SWR05 & Sweetwater Reservoir minimum pool boundary East \\
324209116585001 & SWR06 & Sweetwater Reservoir east-end reservoir-fill boundary \\
324147116593501 & SWR07 & Sweetwater Reservoir near Gum Tree Cove Pond \\
324132117002701 & SWR08 & Perdue Treatment Plant-finished water at Sweetwater Reservoir \\
324137117002901 & SWR09 & Perdue Treatment Plant-imported raw water at Sweetwater Reservoir \\
324311116565901 & LFDD & Sweetwater River at low-flow diversion dam upstream from Sweetwater Reservoir \\
324703116473101 & LLR01 & Loveland Reservoir near dam \\
324737116453501 & LLR02 & Loveland Reservoir east-end near-source inlet \\
324141117001601 & AIR01 & Sweetwater Reservoir atmospheric site at Sweetwater Dam \\
88888801 & QA/QC & Quality-control site for Sacramento Project Office \\
\hline
\end{tabular}


Table 2. Constituent class, medium, sample type, and numbers of constituents analyzed in the environmental and quality-control samples collected from the Sweetwater Reservoir watershed, San Diego County, California.

\begin{tabular}{|c|c|c|c|c|c|c|c|}
\hline Constituent class & Medium & $\begin{array}{l}\text { Sample } \\
\text { type }\end{array}$ & $\begin{array}{l}\text { Table } \\
\text { number }\end{array}$ & $\begin{array}{c}\text { Number } \\
\text { of } \\
\text { samples }\end{array}$ & Quality-control type & $\begin{array}{l}\text { Quality- } \\
\text { control } \\
\text { table }\end{array}$ & $\begin{array}{c}\text { Number of } \\
\text { quality-control } \\
\text { samples }\end{array}$ \\
\hline Volatile organic compounds & Water & Whole & 4B & 211 & Matrix spike & A1A & 6 \\
\hline Volatile organic compounds & Water & Whole & 4B & 211 & Matrix-spike replicate & A1A & 3 \\
\hline Volatile organic compounds & Water & Whole & 4B & 211 & Equipment blank & A1B & 4 \\
\hline Volatile organic compounds & Water & Whole & 4B & 211 & Field blank & A1B & 3 \\
\hline Volatile organic compounds & Water & Whole & 4B & 211 & Source-solution blank & A1B & 1 \\
\hline Volatile organic compounds & Water & Whole & 4B & 211 & Trip blank & A1B & 1 \\
\hline Volatile organic compounds & Water & Whole & $4 \mathrm{~B}$ & 211 & Replicates & $\mathrm{A} 1 \mathrm{~B}$ and $\mathrm{A} 1 \mathrm{C}$ & 14 \\
\hline Base neutral acid & Water & Whole & $5 \mathrm{~B}$ & 66 & Matrix spike & $\mathrm{A} 2 \mathrm{~A}$ & 1 \\
\hline Base neutral acid & Water & Whole & $5 \mathrm{~B}$ & 66 & Replicates & $\mathrm{A} 2 \mathrm{~B}$ & 3 \\
\hline Pesticides (laboratory schedule 2003) & Water & Filtered & $6 \mathrm{~B}$ & 18 & Replicates & A3 & 1 \\
\hline Pesticides (laboratory schedule 2006) & Water & Filtered & $7 \mathrm{~B}$ & 35 & Replicates & A4 & 3 \\
\hline Anthropogenic indicator compounds & Water & Filtered & $8 \mathrm{~B}$ & 17 & Replicates & A5 & 1 \\
\hline Metals & Water & Whole & $9 \mathrm{~A}$ & 153 & Matrix spike & A6A & 3 \\
\hline Metals & Water & Whole & $9 \mathrm{~A}$ & 153 & Matrix-spike replicate & A6A & 2 \\
\hline Metals & Water & Whole & $9 \mathrm{~A}$ & 153 & Field blank & A6B & 4 \\
\hline Metals & Water & Whole & $9 \mathrm{~A}$ & 153 & Replicates & $\mathrm{A} 6 \mathrm{~B}$ and $\mathrm{A} 6 \mathrm{C}$ & 12 \\
\hline Metals & Water & Filtered & 9B & 18 & None analyzed & & 0 \\
\hline High-volatility volatile organic compounds & Air & Filtered & $10 \mathrm{~A}$ & 166 & Replicates & B1A & 14 \\
\hline High-volatility volatile organic compounds & Air & Filtered & $10 \mathrm{~A}$ & 166 & Travel matrix spike & B2A & 37 \\
\hline High-volatility volatile organic compounds & Air & Filtered & $10 \mathrm{~A}$ & 166 & Travel blank & B2B & 166 \\
\hline High-volatility volatile organic compounds & Air & Filtered & $10 \mathrm{~A}$ & 166 & Lot blank & $\mathrm{B} 2 \mathrm{C}$ & 166 \\
\hline High-volatility volatile organic compounds & Air & Filtered & $10 \mathrm{~A}$ & 166 & Laboratory blank & B2D & 172 \\
\hline $\begin{array}{l}\text { Medium/low-volatility volatile } \\
\text { organic compounds }\end{array}$ & Air & Filtered & $10 \mathrm{~B}$ & 166 & Replicates & $\mathrm{B} 1 \mathrm{~B}$ and $\mathrm{B} 1 \mathrm{C}$ & 11 \\
\hline $\begin{array}{l}\text { Medium/low-volatility volatile } \\
\text { organic compounds }\end{array}$ & Air & Filtered & 10B & 166 & Travel matrix spike & B3A & 36 \\
\hline $\begin{array}{l}\text { Medium/low-volatility volatile } \\
\text { organic compounds }\end{array}$ & Air & Filtered & $10 \mathrm{~B}$ & 166 & Travel blank & B3B & 163 \\
\hline $\begin{array}{l}\text { Medium/low-volatility volatile } \\
\text { organic compounds }\end{array}$ & Air & Filtered & 10B & 166 & Lot blank & $\mathrm{B} 3 \mathrm{C}$ & 166 \\
\hline $\begin{array}{l}\text { Medium/low-volatility volatile } \\
\text { organic compounds }\end{array}$ & Air & Filtered & $10 \mathrm{~B}$ & 166 & Laboratory blank & B3D & 166 \\
\hline Polycyclic aromatic hydrocarbons & Air & Filtered & $11 \mathrm{~B}$ & 144 & Reagent spike & B4A & 14 \\
\hline Polycyclic aromatic hydrocarbons & Air & Filtered & 11B & 144 & Laboratory blank & B4B & 17 \\
\hline Polycyclic aromatic hydrocarbons & Air & Filtered & $11 \mathrm{~B}$ & 144 & Field blank & B4B & 2 \\
\hline Pesticides (Airpest003 method) & Air & Filtered & $12 \mathrm{~B}$ & 23 & Reagent spike & B5A & 6 \\
\hline Pesticides (Airpest003 method) & Air & Filtered & $12 \mathrm{~B}$ & 23 & Blank & B5B & 6 \\
\hline Metals & Air & Filtered & 13 & 142 & Reagent spike & B6A & 10 \\
\hline Metals & Air & Filtered & 13 & 142 & Laboratory blank & B6B & 11 \\
\hline Metals & Air & Filtered & 13 & 142 & Field blank & B6B & 1 \\
\hline Metals & Air & Filtered & 13 & 142 & Field blank replicate & $\mathrm{B} 6 \mathrm{C}$ & 1 \\
\hline Polycyclic aromatic hydrocarbons & Sediment & & 14 & 15 & Replicates & $\mathrm{C} 1$ & 2 \\
\hline $\begin{array}{l}\text { Organochlorine pesticides and } \\
\text { polychlorinated biphenyl compounds }\end{array}$ & Sediment & & 15 & 8 & Replicates & $\mathrm{C} 2$ & 1 \\
\hline Metals & Sediment & & 16 & 16 & Matrix spike & $\mathrm{C} 3 \mathrm{~A}$ & 4 \\
\hline Metals & Sediment & & 16 & 16 & Replicates & $\mathrm{C} 3 \mathrm{~B}$ and $\mathrm{C} 3 \mathrm{C}$ & 2 \\
\hline
\end{tabular}


before it entered the treatment plant or SWR. Site LFDD monitored the quality of the water entering SWR from the watershed (fig. 2). During low flows, the water from site LFDD is diverted into the URDS ponds when water quality parameters are below certain criteria levels. Local urban runoff and the first flush from the Sweetwater River are also diverted into the URDS ponds to prevent contaminated water from entering the SWR.

In most cases, imported raw water was pumped directly from the pipeline into the treatment plant. Occasionally this water was pumped into SWR to augment the local supply. When imported raw water is pumped directly into the reservoir, it increases the water level; volume increases can be on the order of tens of thousands of acre-feet (Peter Baranov, Sweetwater Authority, oral commun., 2002).

Baseline water sampling at SWR and LLR began in September 1998 and continued at 2-month intervals through September 1999. From October 1999 to December 2002, the sampling frequency was reduced to once every third month (quarterly). From December 2002 to April 2004, the sampling frequency was increased to every other month (bi-monthly). Starting July 2004, and thereafter, the sampling frequency was once every third month (quarterly). These sampling frequencies allowed monitoring of various operational modes of the reservoirs, such as recharge or drawdown events that substantially changed the water level in the reservoirs. The data showed the spatial variability in chemical occurrence and concentration in each of the two Sweetwater River reservoirs.

Before any reservoir water was sampled, depth profiles of dissolved oxygen, $\mathrm{pH}$, specific conductance, and water temperature were measured at $1-\mathrm{m}$ intervals from the surface to the bottom at each sampling location (table 3). Because LLR is much deeper than SWR, the depth-profile measurements at LLR below $10 \mathrm{~m}$ were collected every $2 \mathrm{~m}$. If the temperature profile indicated a thermocline (thermal stratification), two sets of water samples were collected at the site - one at midepilimnion and one at midhypolimnion. The epilimnion is defined as the layer in a lake extending from the surface to a depth where photosynthesis no longer occurs and has an abrupt change in the slope of the temperature gradient. The hypolimnion is defined as the poorly illuminated lower region of a stratified lake where denser, colder water occurs and currents are minimal. The temperature of the hypolimnion is nearly uniform, and oxygen is depleted. This stratum of water is characterized by decay rather than by the production of organic matter. If no thermocline was evident, only one sample set was collected at a point midway between the water surface and the reservoir bottom.

Starting in January 2006, two sets of water samples were collected at each reservoir site; one sample was collected just below the water surface, and the other sample was collected either at the midepilimnion, if present, or a point midway between the water surface and the reservoir bottom.

\section{Water Methods}

Procedures for collecting and processing filtered and unfiltered water samples for chemical determinations were based on protocols outlined in the National Field Manual for the collection of water-quality data (U.S. Geological Survey, variously dated). Reservoir water samples for all analyses were collected by using a 1.2 L Teflon Kemmerer thief sampler (Wildco, Saginaw, Michigan) or were obtained as grab samples. The Kemmerer sampler was opened and lowered to the determined sampling depth; a weighted messenger was sent down to close the sampler. The closed sampler was then brought to the surface, and a drain plug at the bottom was opened to fill appropriate sample bottles. Samples that needed filtering were put into $1 \mathrm{~L}$ amber glass bottles and taken back to the Sweetwater Authority laboratory at the Purdue Treatment Plant (Sweetwater laboratory) for processing. These samples were filtered by pulling water from a $1 \mathrm{~L}$ glass bottle through a glass-fiber filter (GFF) by using Teflon tubing and a ceramic piston pump. Surface samples from SWR and LLR and samples from the treatment plant (sites SWR08 and SWR09) and site LFDD were collected by the grab method. Grab samples from the reservoirs or the Sweetwater River were collected by submerging the sample bottle, removing the cap, filling the bottle, and recapping the bottle while still submerged. The samples of finished (treated for drinking use) and imported raw water were collected by filling bottles from their appropriate spigots in the Perdue Treatment Plant. The finished water samples were not treated with dechlorinating reagent at the time of sample collection, and measured concentrations of some compounds in these samples could have been affected by chlorination. The water lines are continually running and were well flushed before the sample bottles were filled. All water samples were shipped on ice to the USGS National Water Quality Laboratory (NWQL), and the container temperature was measured and recorded upon arrival. The multi-parameter probe used to measure depth profiles was calibrated for specific conductance, $\mathrm{pH}$, and dissolved oxygen at the Perdue Treatment Plant before sampling. The water temperature sensor is calibrated yearly at the USGS laboratory in San Diego.

\section{Volatile Organic Compounds}

Unfiltered water samples were analyzed for 87 VOCs on USGS NWQL schedule 2020 by purge and trap capillarycolumn, gas chromatography/mass spectrometry (GC/MS) operated in full-scan mode as described in Connor and others (1998). VOC samples were collected in vials at each site then processed in the Sweetwater laboratory by adding the appropriate amount of hydrochloric acid to lower the $\mathrm{pH}$ of the sample to less than 2 units. 
Table 3. Water-quality depth-profile data for dissolved oxygen, $\mathrm{pH}$, specific conductance, and water temperature for each sampling site and period for the Sweetwater Reservoir watershed, San Diego County, California.

[Time is denoted in 24-hour format. The five-digit parameter code in parentheses below each compound name is used in the U.S. Geological Survey computerized data system (National Water Information System) to uniquely identify a specific constituent or property. Sampling depth is in meters below water surface. Shaded data coincide with reservoir water samples. Abbreviations: hh:mm, hour:minute; m, meter; mg/L, milligram per liter; mm/dd/yyyy, month/day/year; na, not applicable; $\mathrm{PM}$, probe malfunction; $\mu \mathrm{S} / \mathrm{cm}$, microsiemen per centimeter; ${ }^{\circ} \mathrm{C}$, degree Celsius]

\begin{tabular}{|c|c|c|c|c|c|c|}
\hline $\begin{array}{c}\text { Date } \\
\text { (mm/dd/ } \\
\text { yyyy) }\end{array}$ & $\begin{array}{c}\text { Time } \\
\text { (hh:mm) }\end{array}$ & $\begin{array}{c}\text { Sampling } \\
\text { depth } \\
\text { (m) } \\
(00098)\end{array}$ & $\begin{array}{c}\text { Oxygen, } \\
\text { dissolved } \\
(\mathrm{mg} / \mathrm{L}) \\
(00300)\end{array}$ & $\begin{array}{c}\mathrm{pH}, \\
\text { water } \\
\text { whole } \\
\text { field } \\
\text { (standard } \\
\text { units) } \\
(00400)\end{array}$ & $\begin{array}{c}\text { Specific } \\
\text { conduc- } \\
\text { tance } \\
(\mu \mathrm{S} / \mathrm{cm}) \\
(00095)\end{array}$ & $\begin{array}{c}\text { Water } \\
\text { temper- } \\
\text { ature } \\
\left({ }^{\circ} \mathrm{C}\right) \\
(00010)\end{array}$ \\
\hline
\end{tabular}

\begin{tabular}{|c|c|c|c|c|c|c|}
\hline \multicolumn{7}{|c|}{ Sweetwater Reservoir near pump tower (SWR01) } \\
\hline $10 / 14 / 2003$ & $13: 26$ & 0.1 & 9.2 & 7.8 & 1,050 & 24.2 \\
\hline $10 / 14 / 2003$ & $13: 27$ & 1.0 & 8.8 & 8.2 & 1,070 & 23.2 \\
\hline $10 / 14 / 2003$ & $13: 28$ & 2.0 & 7.3 & 8.2 & 1,060 & 22.9 \\
\hline $10 / 14 / 2003$ & $13: 29$ & 3.0 & 6.9 & 8.2 & 1,080 & 22.8 \\
\hline $10 / 14 / 2003$ & $13: 30$ & 4.0 & 4.7 & 8.1 & 1,100 & 22.4 \\
\hline $10 / 14 / 2003$ & $13: 31$ & 5.0 & 4.0 & 8.1 & 1,110 & 22.4 \\
\hline $10 / 14 / 2003$ & $13: 32$ & 6.0 & 3.6 & 8.1 & 1,110 & 22.3 \\
\hline $10 / 14 / 2003$ & $13: 33$ & 7.0 & 3.3 & 8.1 & 1,110 & 22.3 \\
\hline $10 / 14 / 2003$ & $13: 34$ & 7.3 & 1.1 & 8.1 & 1,250 & 22.3 \\
\hline $12 / 16 / 2003$ & $11: 56$ & 0.1 & 10.3 & 6.8 & 983 & 14.7 \\
\hline $12 / 16 / 2003$ & $11: 57$ & 1.0 & 9.4 & 7.5 & 984 & 14.2 \\
\hline $12 / 16 / 2003$ & $11: 58$ & 2.0 & 8.8 & 7.3 & 989 & 13.7 \\
\hline $12 / 16 / 2003$ & $11: 59$ & 3.0 & 8.4 & 7.2 & 987 & 13.7 \\
\hline $12 / 16 / 2003$ & $12: 00$ & 4.0 & 8.2 & 7.9 & 985 & 13.6 \\
\hline $12 / 16 / 2003$ & 12:01 & 5.0 & 8.1 & 7.9 & 986 & 13.6 \\
\hline $12 / 16 / 2003$ & 12:02 & 6.0 & 8.0 & 8.1 & 986 & 13.6 \\
\hline $12 / 16 / 2003$ & $12: 03$ & 7.0 & 8.0 & 8.1 & 985 & 13.6 \\
\hline $12 / 16 / 2003$ & $12: 04$ & 8.0 & 7.8 & 8.2 & 986 & 13.6 \\
\hline $12 / 16 / 2003$ & $12: 05$ & 9.0 & 7.7 & 8.2 & 986 & 13.6 \\
\hline $12 / 16 / 2003$ & $12: 06$ & 9.5 & 2.1 & 7.9 & 1,050 & 13.8 \\
\hline $02 / 17 / 2004$ & $15: 35$ & 0.1 & 6.4 & 8.0 & 1,020 & 14.0 \\
\hline $02 / 17 / 2004$ & $15: 36$ & 1.0 & 6.5 & 8.0 & 1,010 & 13.8 \\
\hline $02 / 17 / 2004$ & $15: 37$ & 2.0 & 6.6 & 8.0 & 1,010 & 13.6 \\
\hline $02 / 17 / 2004$ & $15: 38$ & 3.0 & 6.5 & 8.0 & 1,010 & 13.6 \\
\hline $02 / 17 / 2004$ & $15: 39$ & 4.0 & 6.5 & 8.0 & 1,010 & 13.5 \\
\hline $02 / 17 / 2004$ & $15: 40$ & 5.0 & 6.5 & 8.0 & 1,010 & 13.4 \\
\hline $02 / 17 / 2004$ & $15: 41$ & 6.0 & 6.5 & 8.0 & 1,010 & 13.3 \\
\hline $02 / 17 / 2004$ & $15: 42$ & 7.0 & 6.5 & 8.0 & 1,020 & 13.3 \\
\hline $02 / 17 / 2004$ & $15: 43$ & 8.0 & 6.4 & 8.0 & 1,020 & 13.2 \\
\hline $02 / 17 / 2004$ & $15: 44$ & 9.0 & 6.3 & 8.0 & 1,020 & 13.2 \\
\hline $02 / 17 / 2004$ & $15: 45$ & 10.0 & 5.9 & 8.0 & 1,020 & 13.1 \\
\hline $02 / 17 / 2004$ & $15: 46$ & 11.0 & 5.5 & 8.0 & 1,020 & 13.1 \\
\hline $02 / 17 / 2004$ & $15: 47$ & 11.8 & 1.2 & 7.7 & 1,010 & 13.1 \\
\hline $04 / 13 / 2004$ & $11: 18$ & 0.1 & 11.0 & 8.5 & 988 & 21.7 \\
\hline $04 / 13 / 2004$ & $11: 19$ & 1.0 & 11.4 & 8.5 & 989 & 21.4 \\
\hline $04 / 13 / 2004$ & $11: 20$ & 2.0 & 11.4 & 8.5 & 998 & 21.3 \\
\hline $04 / 13 / 2004$ & $11: 21$ & 3.0 & 11.3 & 8.5 & 988 & 21.1 \\
\hline $04 / 13 / 2004$ & $11: 22$ & 4.0 & 11.1 & 8.5 & 987 & 20.8 \\
\hline $04 / 13 / 2004$ & $11: 23$ & 5.0 & 6.3 & 7.9 & 993 & 18.0 \\
\hline $04 / 13 / 2004$ & $11: 24$ & 6.0 & 2.6 & 7.7 & 990 & 17.3 \\
\hline $04 / 13 / 2004$ & $11: 25$ & 7.0 & 1.5 & 7.5 & 992 & 17.1 \\
\hline $04 / 13 / 2004$ & $11: 26$ & 8.0 & 0.9 & 7.5 & 996 & 16.6 \\
\hline $04 / 13 / 2004$ & $11: 40$ & 9.0 & 0.4 & 7.5 & 1,000 & 16.1 \\
\hline $04 / 13 / 2004$ & $11: 27$ & 10.0 & 0.2 & 7.4 & 1,000 & 15.7 \\
\hline $04 / 13 / 2004$ & $11: 28$ & 11.0 & 0.2 & 7.4 & 1,010 & 15.3 \\
\hline $04 / 13 / 2004$ & $11: 29$ & 12.0 & 0.2 & 7.4 & 1,010 & 15.0 \\
\hline
\end{tabular}

\begin{tabular}{|c|c|c|c|c|c|c|}
\hline $\begin{array}{c}\text { Date } \\
\text { (mm/dd/ } \\
\text { yyyy) }\end{array}$ & $\begin{array}{c}\text { Time } \\
\text { (hh:mm) }\end{array}$ & $\begin{array}{c}\text { Sampling } \\
\text { depth } \\
\text { (m) } \\
(00098)\end{array}$ & $\begin{array}{c}\text { Oxygen, } \\
\text { dissolved } \\
(\mathrm{mg} / \mathrm{L}) \\
(00300)\end{array}$ & $\begin{array}{c}\text { pH, } \\
\text { water } \\
\text { whole } \\
\text { field } \\
\text { (standard } \\
\text { units) } \\
\text { (00400) }\end{array}$ & $\begin{array}{c}\text { Specific } \\
\text { conduc- } \\
\text { tance } \\
(\mu \mathrm{S} / \mathrm{cm}) \\
(00095)\end{array}$ & $\begin{array}{c}\text { Water } \\
\text { temper- } \\
\text { ature } \\
\left({ }^{\circ} \mathrm{C}\right) \\
(00010)\end{array}$ \\
\hline \multicolumn{7}{|c|}{ Sweetwater Reservoir near pump tower (SWR01) —Continued } \\
\hline $04 / 13 / 2004$ & $11: 30$ & 13.0 & 0.2 & 7.7 & 1,120 & 15.1 \\
\hline $07 / 13 / 2004$ & $13: 28$ & 0.1 & 9.3 & 8.5 & 1,050 & 26.8 \\
\hline 07/13/2004 & $13: 29$ & 1.0 & 9.3 & 8.6 & 1,050 & 26.1 \\
\hline $07 / 13 / 2004$ & $13: 30$ & 2.0 & 9.8 & 5.9 & 1,040 & 25.8 \\
\hline $07 / 13 / 2004$ & $13: 31$ & 3.0 & 9.2 & 8.4 & 1,050 & 25.6 \\
\hline 07/13/2004 & $13: 32$ & 4.0 & 9.2 & 8.6 & 1,050 & 23.4 \\
\hline $07 / 13 / 2004$ & $13: 33$ & 5.0 & 2.2 & 8.1 & 1,030 & 23.1 \\
\hline $07 / 13 / 2004$ & $13: 34$ & 6.0 & 0.8 & 7.9 & 1,030 & 22.8 \\
\hline $07 / 13 / 2004$ & $13: 20$ & 7.0 & 0.5 & 7.8 & 1,030 & 22.5 \\
\hline $07 / 13 / 2004$ & $13: 35$ & 8.0 & 0.5 & 7.7 & 1,020 & 22.2 \\
\hline $07 / 13 / 2004$ & $13: 36$ & 9.0 & 0.5 & 7.6 & 1,020 & 21.7 \\
\hline $07 / 13 / 2004$ & $13: 37$ & 10.0 & 0.5 & 7.7 & 1,110 & 19.0 \\
\hline $07 / 13 / 2004$ & $13: 38$ & 10.4 & 0.4 & 7.8 & 1,240 & 19.1 \\
\hline $10 / 12 / 2004$ & $12: 57$ & 0.1 & 10.4 & 7.5 & 1,050 & 23.4 \\
\hline $10 / 12 / 2004$ & $12: 58$ & 1.0 & 8.8 & 7.9 & 1,050 & 22.8 \\
\hline $10 / 12 / 2004$ & $12: 59$ & 2.0 & 7.6 & 8.0 & 1,050 & 22.6 \\
\hline $10 / 12 / 2004$ & $13: 00$ & 3.0 & 7.1 & 8.0 & 1,050 & 22.5 \\
\hline $10 / 12 / 2004$ & $13: 01$ & 4.0 & 6.9 & 8.1 & 1,050 & 22.5 \\
\hline $10 / 12 / 2004$ & $13: 02$ & 5.0 & 6.7 & 8.1 & 1,050 & 22.5 \\
\hline $10 / 12 / 2004$ & $13: 03$ & 6.0 & 6.6 & 8.1 & 1,050 & 22.5 \\
\hline $10 / 12 / 2004$ & 13:04 & 6.6 & 0.8 & 8.1 & 1,170 & 22.1 \\
\hline $01 / 12 / 2005$ & $11: 24$ & 0.1 & 12.6 & 6.4 & 949 & 14.6 \\
\hline $01 / 12 / 2005$ & $11: 25$ & 1.0 & 12.3 & 6.5 & 953 & 14.4 \\
\hline $01 / 12 / 2005$ & $11: 26$ & 2.0 & 11.9 & 6.6 & 978 & 14.0 \\
\hline $01 / 12 / 2005$ & $11: 27$ & 3.0 & 11.1 & 6.7 & 970 & 13.5 \\
\hline $01 / 12 / 2005$ & $11: 28$ & 4.0 & 10.9 & 6.7 & 979 & 13.5 \\
\hline $01 / 12 / 2005$ & $11: 29$ & 5.0 & 10.6 & 6.8 & 985 & 13.4 \\
\hline $01 / 12 / 2005$ & $11: 30$ & 6.0 & 10.6 & 6.8 & 994 & 13.3 \\
\hline $01 / 12 / 2005$ & $11: 31$ & 7.0 & 10.6 & 6.8 & 995 & 13.3 \\
\hline $01 / 12 / 2005$ & $11: 32$ & 8.0 & 10.5 & 6.9 & 995 & 13.2 \\
\hline $01 / 12 / 2005$ & $11: 33$ & 9.0 & 10.4 & 6.9 & 996 & 13.2 \\
\hline $01 / 12 / 2005$ & $11: 34$ & 10.0 & 10.2 & 7.0 & 1,000 & 13.1 \\
\hline $01 / 12 / 2005$ & $11: 35$ & 11.8 & 3.7 & 6.8 & 1,030 & 13.3 \\
\hline $04 / 12 / 2005$ & $13: 07$ & 0.1 & 12.0 & 8.5 & 933 & 20.4 \\
\hline $04 / 12 / 2005$ & 13:08 & 1.0 & 12.2 & 8.3 & 931 & 19.6 \\
\hline $04 / 12 / 2005$ & $13: 09$ & 2.0 & 12.0 & 8.3 & 931 & 19.2 \\
\hline $04 / 12 / 2005$ & $13: 10$ & 3.0 & 10.8 & 8.3 & 932 & 19.1 \\
\hline $04 / 12 / 2005$ & $13: 11$ & 4.0 & 10.4 & 8.2 & 933 & 19.0 \\
\hline $04 / 12 / 2005$ & $13: 12$ & 5.0 & 9.9 & 8.2 & 933 & 18.9 \\
\hline $04 / 12 / 2005$ & $13: 13$ & 6.0 & 5.0 & 8.2 & 934 & 16.6 \\
\hline $04 / 12 / 2005$ & $13: 14$ & 7.0 & 2.0 & 8.1 & 944 & 15.8 \\
\hline $04 / 12 / 2005$ & $13: 15$ & 8.0 & 0.8 & 8.1 & 954 & 15.4 \\
\hline $04 / 12 / 2005$ & $13: 16$ & 9.0 & 0.7 & 8.0 & 958 & 15.2 \\
\hline $04 / 12 / 2005$ & $13: 17$ & 10.0 & 0.6 & 7.9 & 959 & 15.2 \\
\hline $04 / 12 / 2005$ & $13: 18$ & 12.0 & 0.6 & 7.8 & 963 & 15.2 \\
\hline $04 / 12 / 2005$ & $13: 19$ & 14.0 & 0.6 & 7.8 & 968 & 15.0 \\
\hline
\end{tabular}


Table 3. Water-quality depth-profile data for dissolved oxygen, $\mathrm{pH}$, specific conductance, and water temperature for each sampling site and period for the Sweetwater Reservoir watershed, San Diego County, California.-Continued

[Time is denoted in 24-hour format. The five-digit parameter code in parentheses below each compound name is used in the U.S. Geological Survey computerized data system (National Water Information System) to uniquely identify a specific constituent or property. Sampling depth is in meters below water surface. Shaded data coincide with reservoir water samples. Abbreviations: hh:mm, hour:minute; m, meter; mg/L, milligram per liter; mm/dd/yyyy, month/day/year; na, not applicable; PM, probe malfunction; $\mu \mathrm{S} / \mathrm{cm}$, microsiemen per centimeter; ${ }^{\circ} \mathrm{C}$, degree Celsius]

\begin{tabular}{|c|c|c|c|c|c|}
\hline $\begin{array}{c}\text { Date } \\
\text { (mm/dd/ } \\
\text { yyyy) }\end{array}$ & $\begin{array}{c}\text { Time } \\
\text { (hh:mm) }\end{array}$ & $\begin{array}{cc}\begin{array}{c}\text { Sampling } \\
\text { depth }\end{array} & \begin{array}{c}\text { dissolven, } \\
\text { (m) } \\
(\mathrm{mg} / \mathrm{L})\end{array} \\
(00098) & (00300)\end{array}$ & $\begin{array}{c}\text { pH, } \\
\text { water } \\
\text { whole } \\
\text { field } \\
\text { (standard } \\
\text { units) } \\
\text { (00400) }\end{array}$ & $\begin{array}{c}\text { Specific } \\
\text { conduc- } \\
\text { tance } \\
(\mu \mathrm{S} / \mathrm{cm}) \\
(00095)\end{array}$ & $\begin{array}{l}\text { Water } \\
\text { temper- } \\
\text { ature } \\
\left({ }^{\circ} \mathrm{C}\right) \\
(00010)\end{array}$ \\
\hline
\end{tabular}

\begin{tabular}{lrrrrrr}
\hline \multicolumn{5}{c}{ Sweetwater Reservoir near pump tower (SWRO1) Continued } \\
\hline $04 / 12 / 2005$ & $13: 20$ & 15.2 & 0.6 & 7.5 & 1,090 & 14.9 \\
$07 / 12 / 2005$ & $10: 58$ & 0.1 & 8.7 & 8.6 & 1,020 & 25.5 \\
$07 / 12 / 2005$ & $10: 59$ & 1.0 & 9.0 & 8.6 & 1,020 & 25.5 \\
\hline $07 / 12 / 2005$ & $11: 00$ & 2.0 & 9.2 & 8.7 & 1,020 & 25.2 \\
\hline $07 / 12 / 2005$ & $11: 01$ & 3.0 & 9.2 & 8.7 & 1,030 & 25.2 \\
$07 / 12 / 2005$ & $11: 02$ & 4.0 & 9.1 & 8.7 & 1,030 & 25.0 \\
$07 / 12 / 2005$ & $11: 03$ & 5.0 & 2.1 & 8.3 & 991 & 21.6 \\
$07 / 12 / 2005$ & $11: 04$ & 6.0 & 0.7 & 8.1 & 982 & 21.0 \\
$07 / 12 / 2005$ & $11: 05$ & 7.0 & 0.7 & 8.0 & 966 & 19.7 \\
$07 / 12 / 2005$ & $11: 06$ & 8.0 & 0.6 & 7.9 & 961 & 19.1 \\
$07 / 12 / 2005$ & $11: 07$ & 9.0 & 0.5 & 7.8 & 954 & 18.5 \\
$07 / 12 / 2005$ & $11: 08$ & 10.0 & 0.5 & 7.7 & 950 & 17.5 \\
$07 / 12 / 2005$ & $11: 09$ & 11.0 & 0.5 & 7.7 & 949 & 17.2 \\
$07 / 12 / 2005$ & $11: 10$ & 12.0 & 0.4 & 7.7 & 950 & 16.9 \\
$07 / 12 / 2005$ & $11: 11$ & 13.0 & 0.4 & 7.6 & 950 & 16.8 \\
$07 / 12 / 2005$ & $11: 12$ & 14.0 & 0.4 & 7.6 & 987 & 16.7 \\
$10 / 11 / 2005$ & $10: 45$ & 0.1 & 8.2 & 8.8 & 1,080 & 22.4 \\
$10 / 11 / 2005$ & $10: 46$ & 1.0 & 8.2 & 8.6 & 1,080 & 22.1 \\
$10 / 11 / 2005$ & $10: 47$ & 2.0 & 7.9 & 8.5 & 1,080 & 22.0 \\
$10 / 11 / 2005$ & $10: 48$ & 3.0 & 7.6 & 8.4 & 1,080 & 21.9 \\
$10 / 11 / 2005$ & $10: 49$ & 4.0 & 7.4 & 8.4 & 1,080 & 21.9 \\
$10 / 11 / 2005$ & $10: 50$ & 5.0 & 7.2 & 8.4 & 1,080 & 21.8 \\
\hline $10 / 11 / 2005$ & $10: 51$ & 6.0 & 7.1 & 8.3 & 1,080 & 21.8 \\
$10 / 11 / 2005$ & $10: 52$ & 7.0 & 7.0 & 8.3 & 1,080 & 21.8 \\
$10 / 11 / 2005$ & $10: 53$ & 8.0 & 0.9 & 8.0 & 1,050 & 20.6 \\
$10 / 11 / 2005$ & $10: 54$ & 9.0 & 0.7 & 8.0 & 1,030 & 20.1 \\
$10 / 11 / 2005$ & $10: 55$ & 10.0 & 0.6 & 7.8 & 1,020 & 19.5 \\
$10 / 11 / 2005$ & $10: 56$ & 10.6 & 0.6 & 7.7 & 1,260 & 19.0 \\
$01 / 10 / 2006$ & $11: 30$ & 0.1 & 11.6 & 7.4 & 1,130 & 15.7 \\
$01 / 10 / 2006$ & $11: 17$ & 1.0 & 10.6 & 7.3 & 1,140 & 14.4 \\
$01 / 10 / 2006$ & $11: 18$ & 2.0 & 9.6 & 7.3 & 1,140 & 14.3 \\
$01 / 10 / 2006$ & $11: 19$ & 3.0 & 9.5 & 7.3 & 1,140 & 14.2 \\
$01 / 10 / 2006$ & $11: 20$ & 4.0 & 9.3 & 7.3 & 1,140 & 14.2 \\
$01 / 10 / 2006$ & $11: 21$ & 5.0 & 8.8 & 7.3 & 1,140 & 14.2 \\
$01 / 10 / 2006$ & $11: 22$ & 6.0 & 8.2 & 7.3 & 1,140 & 14.1 \\
$01 / 10 / 2006$ & $11: 23$ & 7.0 & 8.0 & 7.3 & 1,130 & 14.1 \\
$01 / 10 / 2006$ & $11: 24$ & 8.0 & 7.3 & 7.3 & 1,130 & 14.0 \\
$01 / 10 / 2006$ & $11: 25$ & 9.0 & 7.1 & 7.2 & 1,130 & 14.0 \\
$01 / 10 / 2006$ & $11: 26$ & 9.5 & 2.9 & 7.1 & 1,220 & 14.4 \\
$04 / 07 / 2006$ & $11: 30$ & 0.1 & 9.7 & 8.1 & 894 & 17.2 \\
\hline $04 / 07 / 2006$ & $11: 17$ & 1.0 & 9.8 & 8.0 & 893 & 17.0 \\
$04 / 07 / 2006$ & $11: 18$ & 2.0 & 8.1 & 8.1 & 893 & 16.9 \\
$04 / 07 / 2006$ & $11: 19$ & 3.0 & 9.7 & 8.0 & 893 & 16.9 \\
$04 / 07 / 2006$ & $11: 20$ & 4.0 & 9.6 & 8.1 & 893 & 16.9 \\
$04 / 07 / 2006$ & $11: 21$ & 5.0 & 9.6 & 8.1 & 893 & 16.8
\end{tabular}

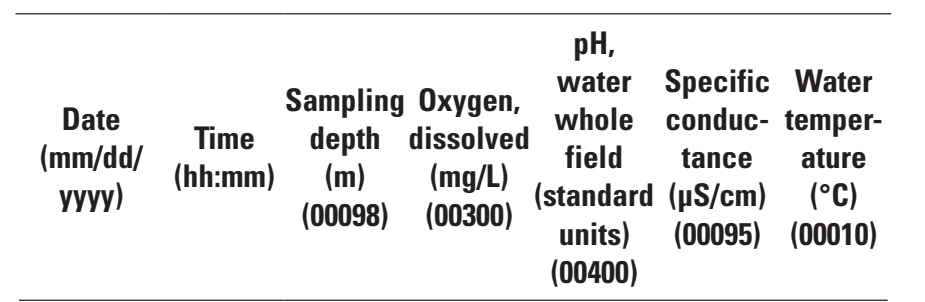

\begin{tabular}{lcrrrrr}
\hline \multicolumn{7}{c}{ Sweetwater Reservoir near } \\
\hline $04 / 07 / 2006$ & $11: 22$ & 6.0 & 9.6 & 8.1 & 893 & 16.8 \\
$04 / 07 / 2006$ & $11: 23$ & 7.0 & 9.3 & 8.1 & 890 & 16.6 \\
$04 / 07 / 2006$ & $11: 24$ & 8.0 & 4.4 & 7.8 & 885 & 14.8 \\
$04 / 07 / 2006$ & $11: 25$ & 9.0 & 2.9 & 7.7 & 886 & 14.8 \\
$04 / 07 / 2006$ & $11: 26$ & 10.0 & 2.6 & 7.6 & 886 & 14.7 \\
$04 / 07 / 2006$ & $11: 27$ & 12.0 & 2.4 & 7.6 & 886 & 14.7 \\
$04 / 07 / 2006$ & $11: 28$ & 14.0 & 2.3 & 7.5 & 887 & 14.6 \\
$04 / 07 / 2006$ & $11: 29$ & 14.7 & 1.3 & 7.5 & 908 & 14.7
\end{tabular}

$\begin{array}{lllllll}07 / 11 / 2006 & 11: 40 & 0.1 & 9.5 & 8.5 & 969 & 28.0 \\ 07 / 11 / 2006 & 11: 29 & 1.0 & 9.1 & 8.6 & 969 & 27.7\end{array}$

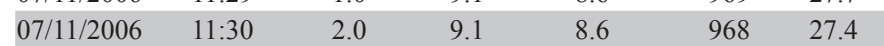

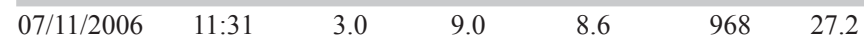

$\begin{array}{lllllll}07 / 11 / 2006 & 11: 32 & 4.0 & 8.7 & 8.6 & 967 & 26.8\end{array}$

$\begin{array}{lllllll}07 / 11 / 2006 & 11: 33 & 5.0 & 3.1 & 7.7 & 943 & 23.0\end{array}$

$\begin{array}{lllllll}07 / 11 / 2006 & 11: 34 & 6.0 & 1.7 & 7.5 & 931 & 21.1\end{array}$

$\begin{array}{lllllll}07 / 11 / 2006 & 11: 35 & 7.0 & 1.6 & 7.5 & 925 & 20.1\end{array}$

$\begin{array}{lllllll}07 / 11 / 2006 & 11: 36 & 8.0 & 1.6 & 7.5 & 919 & 18.6\end{array}$

$\begin{array}{lllllll}07 / 11 / 2006 & 11: 37 & 9.0 & 1.7 & 7.5 & 915 & 18.0\end{array}$

$\begin{array}{lllllll}07 / 11 / 2006 & 11: 38 & 10.0 & 1.7 & 7.5 & 913 & 17.3\end{array}$

$\begin{array}{lllllll}07 / 11 / 2006 & 11: 39 & 11.0 & 1.7 & 7.5 & 913 & 17.0\end{array}$

$\begin{array}{lllllll}07 / 11 / 2006 & 11: 41 & 12.0 & 1.7 & 7.5 & 913 & 16.9\end{array}$

$\begin{array}{lllllll}07 / 11 / 2006 & 11: 42 & 13.1 & 1.7 & 8.0 & 915 & 16.8\end{array}$

$\begin{array}{lllllll}08 / 22 / 2006 & 12: 49 & 0.1 & 10.8 & 8.5 & 955 & 23.4\end{array}$

$\begin{array}{lllllll}08 / 22 / 2006 & 12: 50 & 1.0 & 10.1 & 8.5 & 953 & 26.2\end{array}$

$\begin{array}{lllllll}08 / 22 / 2006 & 12: 51 & 2.0 & 9.6 & 8.5 & 956 & 25.9\end{array}$

$\begin{array}{lllllll}08 / 22 / 2006 & 12: 52 & 3.0 & 9.2 & 8.5 & 956 & 25.8\end{array}$

$\begin{array}{lllllll}08 / 22 / 2006 & 12: 53 & 4.0 & 8.8 & 8.5 & 958 & 25.7\end{array}$

$\begin{array}{lllllll}08 / 22 / 2006 & 12: 54 & 5.0 & 5.1 & 8.1 & 953 & 24.6\end{array}$

$\begin{array}{rrrrrrr}\text { 10/11/2006 } & 11: 00 & 0.1 & \text { PM } & 7.7 & 984 & 22.2 \\ \text { 10/11/2006 } & 11: 01 & 1.0 & \text { PM } & 8.3 & 987 & 21.9 \\ \text { 10/11/2006 } & 11: 02 & 2.0 & \text { PM } & 8.2 & 988 & 21.8 \\ \text { 10/11/2006 } & 11: 03 & 3.0 & \text { PM } & 8.3 & 988 & 21.7 \\ \text { 10/11/2006 } & 11: 20 & 4.0 & \text { PM } & 8.3 & 988 & 21.6 \\ 10 / 11 / 2006 & 11: 04 & 5.0 & \text { PM } & 8.3 & 988 & 21.6 \\ \text { 10/11/2006 } & 11: 05 & 6.0 & \text { PM } & 8.3 & 988 & 21.6 \\ 10 / 11 / 2006 & 11: 06 & 7.0 & \text { PM } & 8.3 & 989 & 21.6 \\ 10 / 11 / 2006 & 11: 07 & 8.0 & \text { PM } & 8.0 & 956 & 19.8 \\ 10 / 11 / 2006 & 11: 08 & 9.0 & \text { PM } & 7.8 & 953 & 19.6 \\ 10 / 11 / 2006 & 11: 09 & 10.0 & \text { PM } & 7.5 & 949 & 19.3 \\ 10 / 11 / 2006 & 11: 10 & 11.0 & \text { PM } & 7.4 & 1,150 & 18.1 \\ 01 / 09 / 2007 & 10: 50 & 0.1 & 15.5 & 6.9 & 993 & 12.9 \\ 01 / 09 / 2007 & 10: 36 & 1.0 & 15.8 & 7.6 & 995 & 12.6 \\ 01 / 09 / 2007 & 10: 37 & 2.0 & 14.1 & 7.9 & 990 & 12.3 \\ 01 / 09 / 2007 & 10: 38 & 3.0 & 12.9 & 7.9 & 990 & 12.2 \\ 01 / 09 / 2007 & 10: 39 & 4.0 & 12.5 & 8.0 & 997 & 12.1\end{array}$


Table 3. Water-quality depth-profile data for dissolved oxygen, $\mathrm{pH}$, specific conductance, and water temperature for each sampling site and period for the Sweetwater Reservoir watershed, San Diego County, California.—Continued

[Time is denoted in 24-hour format. The five-digit parameter code in parentheses below each compound name is used in the U.S. Geological Survey computerized data system (National Water Information System) to uniquely identify a specific constituent or property. Sampling depth is in meters below water surface. Shaded data coincide with reservoir water samples. Abbreviations: hh:mm, hour:minute; m, meter; mg/L, milligram per liter; mm/dd/yyyy, month/day/year; na, not applicable; $\mathrm{PM}$, probe malfunction; $\mu \mathrm{S} / \mathrm{cm}$, microsiemen per centimeter; ${ }^{\circ} \mathrm{C}$, degree Celsius]

\begin{tabular}{|c|c|c|c|c|c|}
\hline $\begin{array}{c}\text { Date } \\
\text { (mm/dd/ } \\
\text { yyyy) }\end{array}$ & $\begin{array}{c}\text { Time } \\
\text { (hh:mm) }\end{array}$ & $\begin{array}{cc}\text { Sampling } & \text { Oxygen, } \\
\text { depth } & \text { dissolved } \\
(\mathrm{m}) & (\mathrm{mg} / \mathrm{L}) \\
(00098) & (00300)\end{array}$ & $\begin{array}{c}\text { pH, } \\
\text { water } \\
\text { whole } \\
\text { field } \\
\text { (standard } \\
\text { units) }\end{array}$ & $\begin{array}{c}\text { Specific } \\
\text { conduc- } \\
\text { tance } \\
(\mu \mathrm{S} / \mathrm{cm}) \\
(\mathbf{0 0 0 9 5 )}\end{array}$ & $\begin{array}{l}\text { Water } \\
\text { temper- } \\
\text { ature } \\
\left({ }^{\circ} \mathrm{C}\right) \\
(00010)\end{array}$ \\
\hline
\end{tabular}

\begin{tabular}{|c|c|c|c|c|c|c|}
\hline \multicolumn{7}{|c|}{ Sweetwater Reservoir near pump tower (SWR01) —Continued } \\
\hline $01 / 09 / 2007$ & $10: 40$ & 5.0 & 11.9 & 8.0 & 998 & 12.1 \\
\hline 01/09/2007 & $10: 41$ & 6.0 & 11.6 & 8.0 & 999 & 12.1 \\
\hline 01/09/2007 & $10: 42$ & 7.0 & 10.2 & 8.0 & 1,000 & 12.1 \\
\hline 01/09/2007 & $10: 43$ & 8.0 & 9.5 & 8.0 & 1,000 & 12.1 \\
\hline 01/09/2007 & $10: 44$ & 9.0 & 8.0 & 8.0 & 1,000 & 12.0 \\
\hline 01/09/2007 & $10: 45$ & 10.0 & 6.5 & 7.9 & 1,000 & 12.2 \\
\hline 01/09/2007 & $10: 46$ & 10.2 & 2.8 & 7.7 & 1,020 & 12.3 \\
\hline $04 / 10 / 2007$ & $11: 30$ & 0.1 & 11.0 & 6.8 & 1,040 & 19.4 \\
\hline $04 / 10 / 2007$ & $11: 38$ & 1.0 & 10.6 & 7.2 & 1,040 & 18.9 \\
\hline $04 / 10 / 2007$ & 11:39 & 2.0 & 10.3 & 7.4 & 1,040 & 18.6 \\
\hline $04 / 10 / 2007$ & $11: 40$ & 3.0 & 10.1 & 7.5 & 1,050 & 18.2 \\
\hline $04 / 10 / 2007$ & $11: 41$ & 4.0 & 10.0 & 7.6 & 1,040 & 18.1 \\
\hline $04 / 10 / 2007$ & $11: 42$ & 5.0 & 9.9 & 7.7 & 1,040 & 18.1 \\
\hline $04 / 10 / 2007$ & $11: 43$ & 6.0 & 5.0 & 7.5 & 1,060 & 17.0 \\
\hline $04 / 10 / 2007$ & $11: 44$ & 7.0 & 3.4 & 7.5 & 1,060 & 16.4 \\
\hline 04/10/2007 & $11: 45$ & 8.0 & 2.4 & 7.4 & 1,060 & 15.8 \\
\hline $04 / 10 / 2007$ & $11: 46$ & 9.0 & 1.5 & 7.4 & 1,060 & 15.5 \\
\hline 04/10/2007 & $11: 47$ & 10.0 & 1.2 & 7.3 & 1,060 & 15.3 \\
\hline $04 / 10 / 2007$ & $11: 48$ & 11.0 & 1.1 & 7.3 & 1,190 & 15.0 \\
\hline 07/10/2007 & $10: 30$ & 0.1 & 6.9 & 7.4 & 1,050 & 25.6 \\
\hline $07 / 10 / 2007$ & $10: 38$ & 1.0 & 7.1 & 7.6 & 1,060 & 25.6 \\
\hline $07 / 10 / 2007$ & $10: 39$ & 2.0 & 7.1 & 7.7 & 1,060 & 25.4 \\
\hline 07/10/2007 & $10: 40$ & 3.0 & 7.2 & 7.7 & 1,060 & 25.4 \\
\hline $07 / 10 / 2007$ & $10: 41$ & 4.0 & 7.2 & 7.8 & 1,060 & 25.3 \\
\hline 07/10/2007 & $10: 42$ & 5.0 & 7.2 & 7.8 & 1,060 & 25.2 \\
\hline 07/10/2007 & $10: 43$ & 6.0 & 1.6 & 7.4 & 1,080 & 22.2 \\
\hline 07/10/2007 & $10: 44$ & 7.0 & 1.6 & 7.4 & 1,080 & 21.9 \\
\hline 07/10/2007 & $10: 45$ & 8.0 & 1.6 & 7.4 & 1,090 & 21.5 \\
\hline 07/10/2007 & $10: 46$ & 9.0 & 1.5 & 7.4 & 1,090 & 21.2 \\
\hline 07/10/2007 & $10: 47$ & 10.0 & 1.6 & 7.3 & 1,100 & 19.4 \\
\hline $07 / 10 / 2007$ & $10: 48$ & 10.8 & 1.5 & 7.4 & 1,160 & 19.1 \\
\hline $10 / 09 / 2007$ & $11: 30$ & 0.1 & 10.1 & 6.6 & 1,100 & 21.6 \\
\hline $10 / 09 / 2007$ & $11: 31$ & 1.0 & 9.0 & 6.8 & 1,100 & 20.8 \\
\hline $10 / 09 / 2007$ & $11: 32$ & 2.0 & 8.4 & 6.9 & 1,100 & 20.6 \\
\hline $10 / 09 / 2007$ & $11: 40$ & 3.0 & 7.7 & 7.0 & 1,100 & 20.6 \\
\hline $10 / 09 / 2007$ & $11: 33$ & 4.0 & 7.3 & 7.1 & 1,100 & 20.5 \\
\hline $10 / 09 / 2007$ & $11: 34$ & 5.0 & 6.8 & 7.1 & 1,100 & 20.5 \\
\hline $10 / 09 / 2007$ & $11: 35$ & 6.0 & 6.5 & 7.2 & 1,100 & 20.5 \\
\hline $10 / 09 / 2007$ & $11: 36$ & 6.5 & 2.2 & 7.0 & 1,220 & 20.6 \\
\hline $10 / 25 / 2007$ & $12: 30$ & 0.1 & 17.4 & 8.5 & 1,100 & 20.2 \\
\hline $01 / 08 / 2008$ & $10: 30$ & 0.1 & 11.3 & 8.2 & 1,150 & 12.6 \\
\hline 01/08/2008 & $10: 31$ & 1.0 & 11.4 & 8.2 & 1,150 & 12.3 \\
\hline 01/08/2008 & $10: 32$ & 2.0 & 10.7 & 8.1 & 1,150 & 12.3 \\
\hline 01/08/2008 & $10: 33$ & 3.0 & 9.8 & 8.1 & 1,150 & 12.2 \\
\hline
\end{tabular}

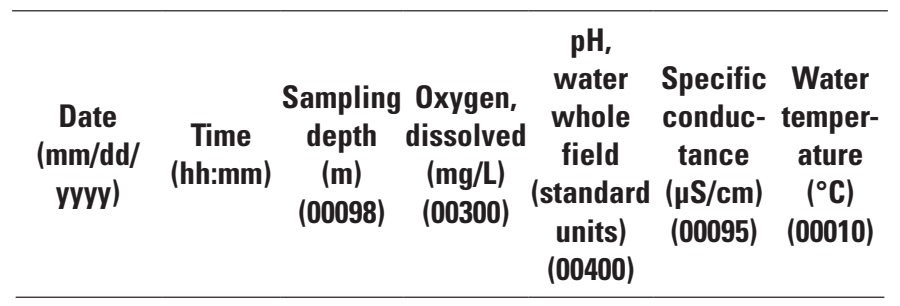

\begin{tabular}{lcccccc}
\hline \multicolumn{7}{c}{ Sweetwater Reservoir near pump tower (SWR01)—Continued } \\
\hline $01 / 08 / 2008$ & $10: 45$ & 4.0 & 9.6 & 8.0 & 1,140 & 12.2 \\
$01 / 08 / 2008$ & $10: 34$ & 5.0 & 9.4 & 7.9 & 1,140 & 12.2 \\
$01 / 08 / 2008$ & $10: 35$ & 6.0 & 8.8 & 8.0 & 1,140 & 12.1 \\
$01 / 08 / 2008$ & $10: 36$ & 7.0 & 8.6 & 7.9 & 1,140 & 12.1 \\
$01 / 08 / 2008$ & $10: 37$ & 7.4 & 5.1 & 7.8 & 1,140 & 12.2
\end{tabular}

$\begin{array}{lllllll}04 / 15 / 2008 & 11: 30 & 0.1 & 12.0 & 8.6 & 856 & 19.9 \\ 04 / 15 / 2008 & 11: 31 & 1.0 & 12.0 & 8.5 & 855 & 19.7 \\ 04 / 15 / 2008 & 11: 32 & 2.0 & 12.0 & 8.5 & 856 & 19.7 \\ 04 / 15 / 2008 & 11: 33 & 3.0 & 11.8 & 8.4 & 853 & 19.4\end{array}$

$\begin{array}{lllllll}04 / 15 / 2008 & 11: 34 & 4.0 & 11.6 & 8.4 & 852 & 19.4\end{array}$

$\begin{array}{lllllll}04 / 15 / 2008 & 11: 35 & 5.0 & 9.3 & 8.3 & 837 & 18.2\end{array}$

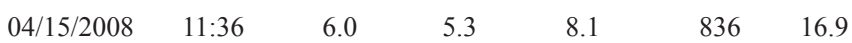

$\begin{array}{lllllll}04 / 15 / 2008 & 11: 37 & 7.0 & 2.8 & 8.0 & 829 & 16.3\end{array}$

$\begin{array}{lllllll}04 / 15 / 2008 & 11: 38 & 8.0 & 1.5 & 8.0 & 825 & 15.6\end{array}$

$\begin{array}{lllllll}04 / 15 / 2008 & 11: 39 & 9.0 & 1.1 & 7.9 & 822 & 15.3\end{array}$

$\begin{array}{lllllll}04 / 15 / 2008 & 11: 40 & 10.0 & 0.9 & 7.9 & 821 & 15.1\end{array}$

$\begin{array}{lllllll}04 / 15 / 2008 & 11: 41 & 11.0 & 0.9 & 7.8 & 819 & 14.9\end{array}$

$\begin{array}{lllllll}04 / 15 / 2008 & 11: 42 & 12.0 & 0.9 & 7.8 & 853 & 14.8\end{array}$

$\begin{array}{lllllll}04 / 15 / 2008 & 11: 43 & 12.2 & 0.8 & 7.8 & 852 & 14.8\end{array}$

$\begin{array}{lllllll}07 / 15 / 2008 & 10: 30 & 0.1 & 8.2 & 8.7 & 1,100 & 26.2\end{array}$

$\begin{array}{lllllll}07 / 15 / 2008 & 10: 31 & 1.0 & 8.7 & 8.2 & 1,100 & 26.0 \\ 07 / 15 / 2008 & 10: 32 & 2.0 & 8.7 & 8.2 & 1,100 & 25.8\end{array}$

$\begin{array}{lllllll}07 / 15 / 2008 & 10: 33 & 3.0 & 8.6 & 8.2 & 1,100 & 25.6\end{array}$

$\begin{array}{lllllll}07 / 15 / 2008 & 10: 34 & 4.0 & 8.4 & 8.1 & 1,100 & 25.5\end{array}$

$\begin{array}{lllllll}07 / 15 / 2008 & 10: 35 & 5.0 & 3.1 & 7.8 & 1,080 & 21.8\end{array}$

$\begin{array}{lllllll}07 / 15 / 2008 & 10: 36 & 6.0 & 1.4 & 7.7 & 1,070 & 20.7\end{array}$

$\begin{array}{lllllll}07 / 15 / 2008 & 10: 37 & 7.0 & 1.3 & 7.6 & 1,070 & 19.7\end{array}$

$\begin{array}{lllllll}07 / 15 / 2008 & 10: 38 & 8.0 & 1.3 & 7.5 & 1,060 & 18.9\end{array}$

$\begin{array}{lllllll}07 / 15 / 2008 & 10: 39 & 9.0 & 1.3 & 7.5 & 1,050 & 17.6\end{array}$

$\begin{array}{lllllll}07 / 15 / 2008 & 10: 40 & 10.0 & 1.3 & 7.4 & 1,050 & 17.3\end{array}$

$\begin{array}{lllllll}07 / 15 / 2008 & 10: 41 & 10.8 & 1.5 & 7.3 & 1,270 & 17.3\end{array}$

$\begin{array}{lllllll}10 / 14 / 2008 & 12: 20 & 0.1 & 8.9 & 7.1 & 1,150 & 22.4 \\ 10 / 14 / 2008 & 12: 21 & 1.0 & 6.5 & 7.0 & 1,150 & 22.1 \\ 10 / 14 / 2008 & 12: 22 & 2.0 & 5.5 & 7.0 & 1,150 & 22.0 \\ 10 / 14 / 2008 & 12: 23 & 3.0 & 5.0 & 7.0 & 1,150 & 21.9 \\ 10 / 14 / 2008 & 12: 24 & 4.0 & 4.1 & 7.0 & 1,150 & 21.9 \\ 10 / 14 / 2008 & 12: 25 & 5.0 & 3.7 & 7.0 & 1,150 & 21.8 \\ 10 / 14 / 2008 & 12: 26 & 5.8 & 1.9 & 7.0 & 1,230 & 21.8 \\ & & & & & & \\ 01 / 13 / 2009 & 11: 10 & 0.1 & 15.8 & 7.2 & 1,080 & 14.8 \\ 01 / 13 / 2009 & 11: 11 & 1.0 & 16.4 & 7.3 & 1,080 & 13.6 \\ 01 / 13 / 2009 & 11: 12 & 2.0 & 15.1 & 7.3 & 1,080 & 13.4 \\ 01 / 13 / 2009 & 11: 13 & 3.0 & 13.3 & 7.3 & 1,070 & 12.8 \\ 01 / 13 / 2009 & 11: 14 & 4.0 & 12.2 & 7.3 & 1,070 & 12.8 \\ 01 / 13 / 2009 & 11: 15 & 5.0 & 5.0 & 7.2 & 1,080 & 12.3\end{array}$


Table 3. Water-quality depth-profile data for dissolved oxygen, $\mathrm{pH}$, specific conductance, and water temperature for each sampling site and period for the Sweetwater Reservoir watershed, San Diego County, California.-Continued

[Time is denoted in 24-hour format. The five-digit parameter code in parentheses below each compound name is used in the U.S. Geological Survey computerized data system (National Water Information System) to uniquely identify a specific constituent or property. Sampling depth is in meters below water surface. Shaded data coincide with reservoir water samples. Abbreviations: hh:mm, hour:minute; m, meter; mg/L, milligram per liter; mm/dd/yyyy, month/day/year; na, not applicable; PM, probe malfunction; $\mu \mathrm{S} / \mathrm{cm}$, microsiemen per centimeter; ${ }^{\circ} \mathrm{C}$, degree Celsius]

\begin{tabular}{|c|c|c|c|c|c|c|}
\hline $\begin{array}{c}\text { Date } \\
\text { (mm/dd/ } \\
\text { yyyy) }\end{array}$ & $\begin{array}{c}\text { Time } \\
\text { (hh:mm) }\end{array}$ & $\begin{array}{l}\text { Sampling } \\
\text { depth } \\
\text { (m) } \\
(00098)\end{array}$ & $\begin{array}{c}\text { Oxygen, } \\
\text { dissolved } \\
\text { (mg/L) } \\
(00300)\end{array}$ & $\begin{array}{c}\text { pH, } \\
\text { water } \\
\text { whole } \\
\text { field } \\
\text { (standard } \\
\text { units) } \\
(00400)\end{array}$ & $\begin{array}{c}\text { Specific } \\
\text { conduc- } \\
\text { tance } \\
(\mu \mathrm{S} / \mathrm{cm}) \\
(00095)\end{array}$ & $\begin{array}{c}\text { Water } \\
\text { temper- } \\
\text { ature } \\
\left({ }^{\circ} \mathrm{C}\right) \\
(00010)\end{array}$ \\
\hline
\end{tabular}

\begin{tabular}{lcccccc}
\hline \multicolumn{5}{c}{ Sweetwater Reservoir near pump tower (SWR01) - Continued } \\
\hline $01 / 13 / 2009$ & $11: 16$ & 6.0 & 4.5 & 7.1 & 1,080 & 12.2 \\
$01 / 13 / 2009$ & $11: 17$ & 7.0 & 4.1 & 7.1 & 1,080 & 12.2 \\
$01 / 13 / 2009$ & $11: 18$ & 8.0 & 4.0 & 7.1 & 1,080 & 12.2 \\
$01 / 13 / 2009$ & $11: 19$ & 9.0 & 3.7 & 7.1 & 1,080 & 12.2 \\
$01 / 13 / 2009$ & $11: 20$ & 9.2 & 2.5 & 7.0 & 1,040 & 12.4 \\
& & & & & & \\
$04 / 14 / 2009$ & $11: 50$ & 0.1 & 9.9 & 8.0 & 1,060 & 18.5 \\
$04 / 14 / 2009$ & $11: 51$ & 1.0 & 9.8 & 8.1 & 1,060 & 18.5 \\
$04 / 14 / 2009$ & $11: 52$ & 2.0 & 8.8 & 8.1 & 1,060 & 18.3 \\
$04 / 14 / 2009$ & $11: 53$ & 3.0 & 8.5 & 8.1 & 1,060 & 18.2 \\
$04 / 14 / 2009$ & $11: 54$ & 4.0 & 8.4 & 8.1 & 1,060 & 18.2 \\
$04 / 14 / 2009$ & $11: 55$ & 5.0 & 8.1 & 8.1 & 1,070 & 18.1 \\
$04 / 14 / 2009$ & $11: 56$ & 6.0 & 6.8 & 8.0 & 1,050 & 17.5 \\
$04 / 14 / 2009$ & $11: 57$ & 7.0 & 5.4 & 7.8 & 1,060 & 16.9 \\
$04 / 14 / 2009$ & $11: 58$ & 8.0 & 4.2 & 7.7 & 1,060 & 16.8 \\
$04 / 14 / 2009$ & $11: 59$ & 9.0 & 3.5 & 7.7 & 1,060 & 16.7 \\
$04 / 14 / 2009$ & $12: 00$ & 10.0 & 3.1 & 7.6 & 1,060 & 16.6 \\
$07 / 07 / 2009$ & $10: 40$ & 0.1 & 10.7 & 7.8 & 1,020 & 24.9 \\
$07 / 07 / 2009$ & $10: 41$ & 1.0 & 10.7 & 8.2 & 1,060 & 24.9 \\
$07 / 07 / 2009$ & $10: 42$ & 2.0 & 11.1 & 8.4 & 1,150 & 24.9 \\
$07 / 07 / 2009$ & $10: 43$ & 3.0 & 11.3 & 8.5 & 1,150 & 24.8 \\
$07 / 07 / 2009$ & $10: 44$ & 4.0 & 11.0 & 8.5 & 1,140 & 24.8 \\
$07 / 07 / 2009$ & $10: 45$ & 5.0 & 10.6 & 8.5 & 1,120 & 24.6 \\
$07 / 07 / 2009$ & $10: 46$ & 6.0 & 2.1 & 7.7 & 1,140 & 22.4 \\
$07 / 07 / 2009$ & $10: 47$ & 7.0 & 1.6 & 7.7 & 1,130 & 21.9 \\
$07 / 07 / 2009$ & $10: 48$ & 8.0 & 1.3 & 7.6 & 1,110 & 21.6 \\
$07 / 07 / 2009$ & $10: 49$ & 9.0 & 1.3 & 7.6 & 1,110 & 21.2 \\
$07 / 07 / 2009$ & $10: 50$ & 10.0 & 1.3 & 7.7 & 1,130 & 20.9 \\
$07 / 07 / 2009$ & $10: 51$ & 10.8 & 1.3 & 7.7 & 1,160 & 20.3 \\
\hline & $S w$ & & & \\
0
\end{tabular}

\begin{tabular}{lllllll}
\hline \multicolumn{6}{c}{ Sweetwater Reservoir center of minimum pool (SWR03) } \\
\hline $10 / 14 / 2003$ & $14: 06$ & 0.1 & 10.6 & 8.8 & 1,080 & 23.8 \\
$10 / 14 / 2003$ & $14: 07$ & 1.0 & 9.7 & 8.8 & 1,090 & 23.6 \\
$10 / 14 / 2003$ & $14: 08$ & 2.0 & 8.1 & 8.7 & 1,100 & 23.0 \\
$10 / 14 / 2003$ & $14: 09$ & 3.0 & 7.5 & 8.6 & 1,100 & 22.8 \\
$10 / 14 / 2003$ & $14: 10$ & 4.0 & 6.9 & 8.6 & 1,100 & 22.6 \\
$10 / 14 / 2003$ & $14: 11$ & 5.0 & 6.5 & 8.5 & 1,110 & 22.5 \\
$10 / 14 / 2003$ & $14: 12$ & 6.0 & 5.1 & 8.5 & 1,110 & 22.4 \\
$10 / 14 / 2003$ & $14: 13$ & 7.0 & 3.5 & 8.4 & 1,110 & 22.3 \\
$10 / 14 / 2003$ & $14: 14$ & 8.0 & 3.1 & 8.3 & 1,110 & 22.3 \\
$10 / 14 / 2003$ & $14: 15$ & 8.5 & 3.1 & 8.3 & 1,120 & 22.3 \\
$12 / 16 / 2003$ & $12: 56$ & 0.1 & 9.5 & 8.4 & 956 & 14.9 \\
$12 / 16 / 2003$ & $12: 57$ & 1.0 & 8.0 & 8.4 & 962 & 14.0 \\
$12 / 16 / 2003$ & $12: 58$ & 2.0 & 7.9 & 8.4 & 967 & 13.7 \\
$12 / 16 / 2003$ & $12: 59$ & 3.0 & 8.0 & 8.4 & 979 & 13.6 \\
$12 / 16 / 2003$ & $13: 00$ & 4.0 & 7.9 & 8.3 & 981 & 13.6 \\
$12 / 16 / 2003$ & $13: 01$ & 5.0 & 8.0 & 8.3 & 981 & 13.5
\end{tabular}

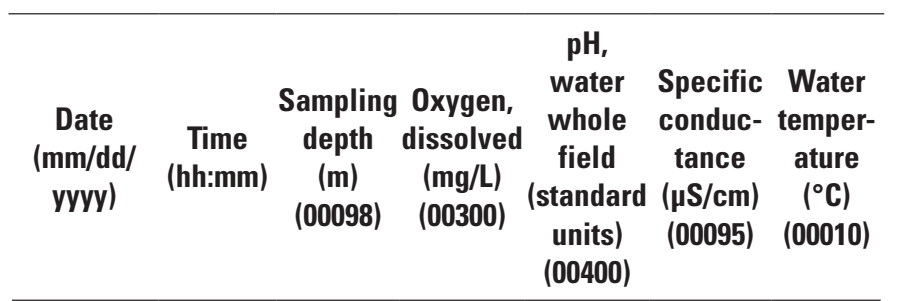

Sweetwater Reservoir center of minimum pool (SWR03)—Continued

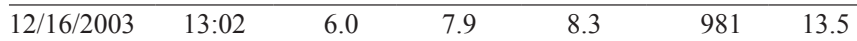

$\begin{array}{lllllll}12 / 16 / 2003 & 13: 03 & 7.0 & 7.9 & 8.3 & 981 & 13.5\end{array}$

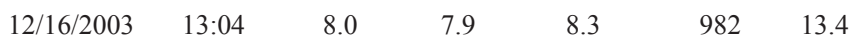

$\begin{array}{lllllll}12 / 16 / 2003 & 13: 05 & 9.0 & 7.7 & 8.3 & 982 & 13.4\end{array}$

$\begin{array}{lllllll}12 / 16 / 2003 & 13: 06 & 9.5 & 7.4 & 8.3 & 983 & 13.4\end{array}$

$\begin{array}{lllllll}02 / 17 / 2004 & 16: 34 & 0.1 & 6.6 & 8.1 & 1,020 & 14.4\end{array}$

$\begin{array}{lllllll}02 / 17 / 2004 & 16: 35 & 1.0 & 6.4 & 8.0 & 1,020 & 14.4\end{array}$

$\begin{array}{lllllll}02 / 17 / 2004 & 16: 36 & 2.0 & 6.3 & 8.0 & 1,020 & 13.8\end{array}$

$\begin{array}{lllllll}02 / 17 / 2004 & 16: 37 & 3.0 & 6.3 & 8.0 & 1,020 & 13.6\end{array}$

$\begin{array}{lllllll}02 / 17 / 2004 & 16: 38 & 4.0 & 6.3 & 8.0 & 1,020 & 13.4\end{array}$

$\begin{array}{lllllll}02 / 17 / 2004 & 16: 39 & 5.0 & 6.3 & 8.0 & 1,020 & 13.3 \\ 02 / 17 / 2004 & 16: 40 & 6.0 & 6.3 & 8.0 & 1,010 & 13.1\end{array}$

$\begin{array}{lllllll}02 / 17 / 2004 & 16: 41 & 7.0 & 5.8 & 8.0 & 1,020 & 12.9\end{array}$

$\begin{array}{lllllll}02 / 17 / 2004 & 16: 42 & 8.0 & 5.5 & 7.9 & 1,020 & 12.9\end{array}$

$\begin{array}{lllllll}02 / 17 / 2004 & 16: 43 & 9.0 & 5.3 & 7.9 & 1,020 & 12.9\end{array}$

$\begin{array}{lllllll}02 / 17 / 2004 & 16: 44 & 10.0 & 5.3 & 7.9 & 1,020 & 12.9\end{array}$

$\begin{array}{lllllll}02 / 17 / 2004 & 16: 45 & 11.0 & 5.0 & 7.9 & 1,020 & 12.9\end{array}$

$\begin{array}{lllllll}02 / 17 / 2004 & 16: 46 & 12.0 & 4.6 & 7.8 & 1,020 & 12.9\end{array}$

$\begin{array}{lllllll}02 / 17 / 2004 & 16: 47 & 12.3 & 0.8 & 7.7 & 1,020 & 12.9\end{array}$

$\begin{array}{lllllll}04 / 13 / 2004 & 12: 08 & 0.1 & 11.2 & 8.6 & 992 & 22.1\end{array}$

$\begin{array}{lllllll}04 / 13 / 2004 & 12: 09 & 1.0 & 11.6 & 8.6 & 992 & 21.6 \\ 04 / 13 / 2004 & 12: 10 & 2.0 & 11.6 & 8.6 & 987 & 21.3\end{array}$

$\begin{array}{lllllll}04 / 13 / 2004 & 12: 11 & 3.0 & 11.3 & 8.6 & 983 & 21.0\end{array}$

$\begin{array}{lllllll}04 / 13 / 2004 & 12: 12 & 4.0 & 11.2 & 8.6 & 987 & 20.8\end{array}$

$\begin{array}{lllllll}04 / 13 / 2004 & 12: 13 & 5.0 & 7.5 & 8.0 & 983 & 18.7\end{array}$

$\begin{array}{lllllll}04 / 13 / 2004 & 12: 14 & 6.0 & 4.8 & 7.8 & 986 & 17.9\end{array}$

$\begin{array}{lllllll}04 / 13 / 2004 & 12: 15 & 7.0 & 2.8 & 7.7 & 992 & 17.2 \\ 04 / 13 / 2004 & 12.20 & 8.0 & 1.0 & 7.5 & 996 & 16.6\end{array}$

$\begin{array}{lllllll}04 / 13 / 2004 & 12: 20 & 8.0 & 1.0 & 7.5 & 996 & 16.6 \\ 04 / 13 / 2004 & 12: 16 & 9.0 & 0.5 & 7.5 & 998 & 16.4\end{array}$

$\begin{array}{lllllll}04 / 13 / 2004 & 12: 17 & 10.0 & 0.3 & 7.5 & 1,010 & 15.5\end{array}$

$\begin{array}{lllllll}04 / 13 / 2004 & 12: 18 & 11.0 & 0.2 & 7.4 & 1,010 & 15.2\end{array}$

$\begin{array}{lllllll}04 / 13 / 2004 & 12: 19 & 12.0 & 0.2 & 7.4 & 1,010 & 15.1\end{array}$

$\begin{array}{lllllll}04 / 13 / 2004 & 12: 22 & 13.0 & 0.2 & 7.4 & 1,010 & 14.9\end{array}$

$\begin{array}{lllllll}04 / 13 / 2004 & 12: 23 & 13.8 & 0.2 & 7.4 & 1,010 & 14.9\end{array}$

$\begin{array}{lllllll}07 / 13 / 2004 & 13: 54 & 0.1 & 10.3 & 8.4 & 1,040 & 26.5\end{array}$

$\begin{array}{lllllll}07 / 13 / 2004 & 13: 55 & 1.0 & 10.2 & 8.5 & 1,040 & 26.5\end{array}$

$\begin{array}{lllllll}07 / 13 / 2004 & 13: 56 & 2.0 & 10.2 & 8.5 & 1,050 & 26.4\end{array}$

$\begin{array}{lllllll}07 / 13 / 2004 & 13.57 & 3.0 & 9.9 & 8.5 & 1,040 & 25.8\end{array}$

$\begin{array}{lllllll}07 / 13 / 2004 & 13: 58 & 4.0 & 6.3 & 8.3 & 1,040 & 24.3\end{array}$

$\begin{array}{lllllll}07 / 13 / 2004 & 13: 59 & 5.0 & 4.0 & 8.1 & 1,040 & 23.7\end{array}$

$\begin{array}{lllllll}07 / 13 / 2004 & 14: 00 & 6.0 & 2.4 & 7.9 & 1,030 & 23.2\end{array}$

$\begin{array}{lllllll}07 / 13 / 2004 & 14: 01 & 7.0 & 0.9 & 7.8 & 1,030 & 22.7\end{array}$

$\begin{array}{lllllll}07 / 13 / 2004 & 14: 02 & 8.0 & 0.7 & 7.7 & 1,020 & 21.6\end{array}$

$\begin{array}{lllllll}07 / 13 / 2004 & 14: 03 & 9.0 & 0.6 & 7.7 & 1,020 & 20.7\end{array}$

$\begin{array}{lllllll}07 / 13 / 2004 & 14: 04 & 10.0 & 0.6 & 7.6 & 1,020 & 19.3\end{array}$ 
Table 3. Water-quality depth-profile data for dissolved oxygen, $\mathrm{pH}$, specific conductance, and water temperature for each sampling site and period for the Sweetwater Reservoir watershed, San Diego County, California.—Continued

[Time is denoted in 24-hour format. The five-digit parameter code in parentheses below each compound name is used in the U.S. Geological Survey computerized data system (National Water Information System) to uniquely identify a specific constituent or property. Sampling depth is in meters below water surface. Shaded data coincide with reservoir water samples. Abbreviations: hh:mm, hour:minute; m, meter; mg/L, milligram per liter; mm/dd/yyyy, month/day/year; na, not applicable; $\mathrm{PM}$, probe malfunction; $\mu \mathrm{S} / \mathrm{cm}$, microsiemen per centimeter; ${ }^{\circ} \mathrm{C}$, degree Celsius]

\begin{tabular}{|c|c|c|c|c|c|c|}
\hline $\begin{array}{c}\text { Date } \\
\text { (mm/dd/ } \\
\text { yyyy) }\end{array}$ & $\begin{array}{c}\text { Time } \\
\text { (hh:mm) }\end{array}$ & $\begin{array}{c}\text { Sampling } \\
\text { depth } \\
\text { (m) } \\
(00098)\end{array}$ & $\begin{array}{c}\text { Oxygen, } \\
\text { dissolved } \\
\text { (mg/L) } \\
(00300)\end{array}$ & $\begin{array}{c}\text { pH, } \\
\text { water } \\
\text { whole } \\
\text { field } \\
\text { (standard } \\
\text { units) } \\
\text { (00400) }\end{array}$ & $\begin{array}{c}\text { Specific } \\
\text { conduc- } \\
\text { tance } \\
(\mu \mathrm{S} / \mathrm{cm}) \\
(00095)\end{array}$ & $\begin{array}{c}\text { Water } \\
\text { temper- } \\
\text { ature } \\
\left({ }^{\circ} \mathrm{C}\right) \\
(00010)\end{array}$ \\
\hline \multicolumn{7}{|c|}{ Sweetwater Reservoir center of minimum pool (SWR03)—Continued } \\
\hline $07 / 13 / 2004$ & $14: 05$ & 11.0 & 0.6 & 7.5 & 1,020 & 18.0 \\
\hline 07/13/2004 & 14:06 & 12.0 & 0.5 & 7.4 & 1,020 & 17.6 \\
\hline 07/13/2004 & $14: 07$ & 12.6 & 0.5 & 7.3 & 1,020 & 17.5 \\
\hline $10 / 12 / 2004$ & $13: 35$ & 0.1 & 9.0 & 8.4 & 1,040 & 23.1 \\
\hline $10 / 12 / 2004$ & $13: 36$ & 1.0 & 8.9 & 8.5 & 1,040 & 23.1 \\
\hline $10 / 12 / 2004$ & $13: 37$ & 2.0 & 8.3 & 8.4 & 1,050 & 23.0 \\
\hline $10 / 12 / 2004$ & $13: 38$ & 3.0 & 6.6 & 8.3 & 1,050 & 22.5 \\
\hline $10 / 12 / 2004$ & $13: 39$ & 4.0 & 6.0 & 8.3 & 1,060 & 22.4 \\
\hline $10 / 12 / 2004$ & $13: 40$ & 5.0 & 5.7 & 8.3 & 1,060 & 22.4 \\
\hline $10 / 12 / 2004$ & $13: 41$ & 6.0 & 5.6 & 8.2 & 1,060 & 22.4 \\
\hline $10 / 12 / 2004$ & $13: 42$ & 7.0 & 5.6 & 8.2 & 1,060 & 22.3 \\
\hline $10 / 12 / 2004$ & $13: 43$ & 8.0 & 5.2 & 8.2 & 1,060 & 22.2 \\
\hline $10 / 12 / 2004$ & $13: 44$ & 9.0 & 4.3 & 8.2 & 1,060 & 22.2 \\
\hline $10 / 12 / 2004$ & $13: 45$ & 9.2 & 1.6 & 7.9 & 1,080 & 22.1 \\
\hline $01 / 12 / 2005$ & $12: 03$ & 0.1 & 13.4 & 7.4 & 884 & 15.6 \\
\hline $01 / 12 / 2005$ & $12: 04$ & 1.0 & 11.2 & 7.5 & 922 & 13.5 \\
\hline $01 / 12 / 2005$ & $12: 05$ & 2.0 & 9.7 & 7.5 & 972 & 13.1 \\
\hline $01 / 12 / 2005$ & $12: 06$ & 3.0 & 9.4 & 7.5 & 986 & 13.1 \\
\hline $01 / 12 / 2005$ & $12: 07$ & 4.0 & 9.3 & 7.5 & 993 & 13.2 \\
\hline $01 / 12 / 2005$ & $12: 08$ & 5.0 & 9.3 & 7.4 & 1,000 & 13.1 \\
\hline $01 / 12 / 2005$ & $12: 09$ & 6.0 & 9.3 & 7.5 & 1,000 & 13.1 \\
\hline $01 / 12 / 2005$ & $12: 10$ & 7.0 & 9.4 & 7.5 & 1,010 & 13.1 \\
\hline $01 / 12 / 2005$ & $12: 11$ & 8.0 & 9.3 & 7.5 & 1,010 & 13.0 \\
\hline $01 / 12 / 2005$ & $12: 12$ & 9.0 & 9.2 & 7.5 & 1,010 & 12.9 \\
\hline $01 / 12 / 2005$ & $12: 13$ & 10.0 & 9.2 & 7.5 & 1,010 & 12.9 \\
\hline $01 / 12 / 2005$ & $12: 14$ & 12.0 & 8.5 & 7.5 & 1,020 & 12.8 \\
\hline $01 / 12 / 2005$ & $12: 15$ & 13.4 & 7.2 & 7.4 & 1,010 & 12.7 \\
\hline $04 / 12 / 2005$ & $13: 32$ & 0.1 & 13.3 & 8.6 & 932 & 20.9 \\
\hline $04 / 12 / 2005$ & $13: 33$ & 1.0 & 13.3 & 8.6 & 933 & 20.7 \\
\hline $04 / 12 / 2005$ & $13: 34$ & 2.0 & 12.5 & 8.6 & 934 & 20.1 \\
\hline $04 / 12 / 2005$ & $13: 35$ & 3.0 & 10.8 & 5.6 & 934 & 19.2 \\
\hline $04 / 12 / 2005$ & $13: 36$ & 4.0 & 9.7 & 8.5 & 932 & 18.8 \\
\hline $04 / 12 / 2005$ & $13: 37$ & 5.0 & 7.1 & 8.5 & 934 & 17.7 \\
\hline $04 / 12 / 2005$ & $13: 38$ & 6.0 & 4.6 & 8.4 & 928 & 17.5 \\
\hline $04 / 12 / 2005$ & $13: 39$ & 7.0 & 1.9 & 8.3 & 924 & 16.3 \\
\hline $04 / 12 / 2005$ & $13: 40$ & 8.0 & 1.0 & 8.3 & 946 & 15.8 \\
\hline $04 / 12 / 2005$ & $13: 41$ & 9.0 & 0.8 & 8.2 & 949 & 15.6 \\
\hline $04 / 12 / 2005$ & $13: 42$ & 10.0 & 0.6 & 8.2 & 952 & 15.5 \\
\hline $04 / 12 / 2005$ & $13: 43$ & 12.0 & 0.6 & 8.2 & 971 & 15.0 \\
\hline $04 / 12 / 2005$ & $13: 44$ & 14.0 & 0.6 & 8.1 & 977 & 14.8 \\
\hline $04 / 12 / 2005$ & $13: 45$ & 16.0 & 0.6 & 8.1 & 972 & 14.8 \\
\hline $07 / 12 / 2005$ & $11: 48$ & 0.1 & 9.2 & 8.8 & 1,030 & 26.1 \\
\hline $07 / 12 / 2005$ & $11: 49$ & 1.0 & 9.3 & 8.8 & 1,030 & 26.1 \\
\hline
\end{tabular}

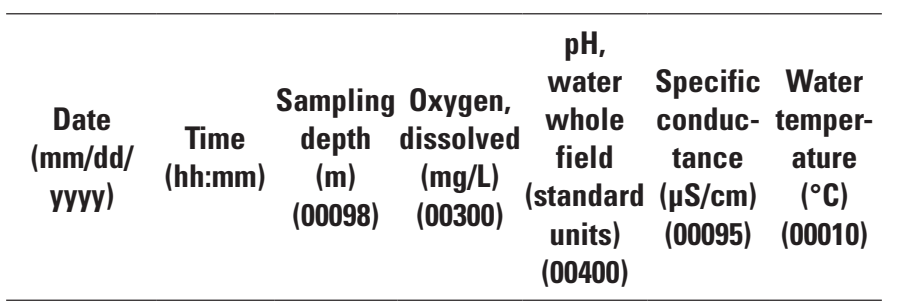

Sweetwater Reservoir center of minimum pool (SWR03)—Continued

\begin{tabular}{|c|c|c|c|c|c|}
\hline $7 / 12 / 2005$ & $11: 50$ & 2.0 & 9.3 & 8.8 & 1,030 \\
\hline $07 / 12 / 2005$ & $11: 51$ & 3.0 & 9.1 & 8.8 & 1,020 \\
\hline
\end{tabular}

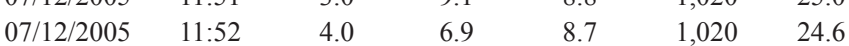

$\begin{array}{lllllll}07 / 12 / 2005 & 11: 53 & 5.0 & 1.9 & 8.4 & 1,000 & 22.6\end{array}$

$\begin{array}{lllllll}07 / 12 / 2005 & 11: 54 & 6.0 & 0.8 & 8.3 & 990 & 21.2\end{array}$

$\begin{array}{lllllll}07 / 12 / 2005 & 11: 55 & 7.0 & 0.6 & 8.1 & 968 & 19.9\end{array}$

$\begin{array}{lllllll}07 / 12 / 2005 & 11: 56 & 8.0 & 0.6 & 8.0 & 962 & 19.2\end{array}$

$\begin{array}{lllllll}07 / 12 / 2005 & 11: 57 & 9.0 & 0.5 & 7.9 & 952 & 18.1\end{array}$

$\begin{array}{lllllll}07 / 12 / 2005 & 11: 58 & 10.0 & 0.5 & 7.8 & 949 & 17.6\end{array}$

$\begin{array}{lllllll}07 / 12 / 2005 & 11: 59 & 11.0 & 0.5 & 7.8 & 948 & 17.4\end{array}$

$\begin{array}{lllllll}07 / 12 / 2005 & 12: 00 & 12.0 & 0.5 & 7.8 & 947 & 17.0\end{array}$

$\begin{array}{lllllll}07 / 12 / 2005 & 12: 01 & 13.0 & 0.4 & 7.7 & 949 & 16.8\end{array}$

$\begin{array}{lllllll}07 / 12 / 2005 & 12: 02 & 14.0 & 0.4 & 7.7 & 949 & 16.7\end{array}$

$\begin{array}{lllllll}07 / 12 / 2005 & 12: 03 & 15.0 & 0.4 & 7.6 & 950 & 16.6\end{array}$

$\begin{array}{lllllll}10 / 11 / 2005 & 11: 24 & 0.1 & 9.0 & 8.4 & 1,080 & 22.6\end{array}$

$\begin{array}{lllllll}10 / 11 / 2005 & 11: 25 & 1.0 & 8.1 & 8.3 & 1,080 & 22.1\end{array}$

$\begin{array}{lllllll}10 / 11 / 2005 & 11: 26 & 2.0 & 7.7 & 8.3 & 1,080 & 21.9\end{array}$

$\begin{array}{lllllll}10 / 11 / 2005 & 11: 27 & 3.0 & 7.4 & 8.3 & 1,080 & 21.9\end{array}$

$\begin{array}{lllllll}10 / 11 / 2005 & 11: 28 & 4.0 & 7.2 & 8.3 & 1,080 & 21.8\end{array}$

$\begin{array}{lllllll}10 / 11 / 2005 & 11: 29 & 5.0 & 7.1 & 8.3 & 1,080 & 21.8\end{array}$

$\begin{array}{lllllll}10 / 11 / 2005 & 11: 30 & 6.0 & 7.0 & 8.3 & 1,080 & 21.7 \\ 10 / 11 / 2005 & 11.31 & 7.0 & 6.2 & 8.3 & 1,080 & 21.4\end{array}$

$\begin{array}{lllllll}10 / 11 / 2005 & 11: 32 & 8.0 & 2.2 & 8.2 & 1,060 & 20.9\end{array}$

$\begin{array}{lllllll}10 / 11 / 2005 & 11: 33 & 9.0 & 1.1 & 8.0 & 1,050 & 20.6\end{array}$

$\begin{array}{lllllll}10 / 11 / 2005 & 11: 34 & 10.0 & 0.7 & 7.9 & 1,010 & 19.3\end{array}$

$\begin{array}{lllllll}10 / 11 / 2005 & 11: 35 & 12.0 & 0.6 & 7.8 & 987 & 18.0\end{array}$

$\begin{array}{lllllll}10 / 11 / 2005 & 11: 36 & 12.5 & 0.5 & 7.6 & 978 & 17.9\end{array}$

$\begin{array}{lllllll}01 / 10 / 2006 & 12: 10 & 0.1 & 13.9 & 8.0 & 1,130 & 14.8 \\ 01 / 10 / 2006 & 11: 56 & 1.0 & 11.5 & 7.9 & 1,130 & 14.3\end{array}$

$\begin{array}{lllllll}01 / 10 / 2006 & 11: 57 & 2.0 & 10.2 & 7.8 & 1,140 & 14.2\end{array}$

$\begin{array}{lllllll}01 / 10 / 2006 & 11: 58 & 3.0 & 9.6 & 7.8 & 1,140 & 14.2\end{array}$

$\begin{array}{lllllll}01 / 10 / 2006 & 11: 59 & 4.0 & 9.2 & 7.8 & 1,140 & 14.2\end{array}$

$\begin{array}{lllllll}01 / 10 / 2006 & 12: 00 & 5.0 & 9.1 & 7.8 & 1,140 & 14.2\end{array}$

$\begin{array}{lllllll}01 / 10 / 2006 & 12: 01 & 6.0 & 8.9 & 7.7 & 1,140 & 14.2\end{array}$

$\begin{array}{lllllll}01 / 10 / 2006 & 12: 02 & 7.0 & 8.5 & 7.8 & 1,140 & 14.2\end{array}$

$\begin{array}{lllllll}01 / 10 / 2006 & 12: 03 & 8.0 & 7.9 & 7.7 & 1,140 & 14.0\end{array}$

$\begin{array}{lllllll}01 / 10 / 2006 & 12: 04 & 9.0 & 7.0 & 7.7 & 1,150 & 14.0\end{array}$

$\begin{array}{lllllll}01 / 10 / 2006 & 12: 05 & 10.0 & 6.4 & 7.5 & 1,150 & 13.9\end{array}$

$\begin{array}{lllllll}01 / 10 / 2006 & 12: 06 & 11.4 & 5.3 & 7.6 & 1,150 & 13.9\end{array}$

$\begin{array}{rrrrrrr}04 / 07 / 2006 & 12: 00 & 0.1 & 9.9 & 8.1 & 893 & 17.2 \\ 04 / 07 / 2006 & 11: 47 & 1.0 & 10.0 & 8.1 & 893 & 17.2 \\ 04 / 07 / 2006 & 11: 48 & 2.0 & 10.0 & 8.1 & 893 & 17.2 \\ 04 / 07 / 2006 & 11: 49 & 3.0 & 9.8 & 8.1 & 893 & 16.9 \\ 04 / 07 / 2006 & 11: 50 & 4.0 & 9.6 & 8.1 & 892 & 16.7\end{array}$


Table 3. Water-quality depth-profile data for dissolved oxygen, $\mathrm{pH}$, specific conductance, and water temperature for each sampling site and period for the Sweetwater Reservoir watershed, San Diego County, California.-Continued

[Time is denoted in 24-hour format. The five-digit parameter code in parentheses below each compound name is used in the U.S. Geological Survey computerized data system (National Water Information System) to uniquely identify a specific constituent or property. Sampling depth is in meters below water surface. Shaded data coincide with reservoir water samples. Abbreviations: hh:mm, hour:minute; m, meter; mg/L, milligram per liter; mm/dd/yyyy, month/day/year; na, not applicable; PM, probe malfunction; $\mu \mathrm{S} / \mathrm{cm}$, microsiemen per centimeter; ${ }^{\circ} \mathrm{C}$, degree Celsius]

\begin{tabular}{|c|c|c|c|c|c|}
\hline $\begin{array}{c}\text { Date } \\
\text { (mm/dd/ } \\
\text { yvyy) }\end{array}$ & $\begin{array}{c}\text { Time } \\
\text { (hh:mm) }\end{array}$ & $\begin{array}{cc}\begin{array}{c}\text { Sampling } \\
\text { depth }\end{array} & \begin{array}{c}\text { dissolven, } \\
\text { dis) } \\
(\mathrm{mg} / \mathrm{L})\end{array} \\
(\mathbf{0 0 0 9 8}) & (00300)\end{array}$ & $\begin{array}{c}\mathrm{pH}, \\
\text { water } \\
\text { whole } \\
\text { field } \\
\text { (standard } \\
\text { units) } \\
\text { (00400) }\end{array}$ & $\begin{array}{c}\text { Specific } \\
\text { conduc- } \\
\text { tance } \\
(\mu \mathrm{S} / \mathrm{cm}) \\
(00095)\end{array}$ & $\begin{array}{c}\text { Water } \\
\text { temper- } \\
\text { ature } \\
\left({ }^{\circ} \mathrm{C}\right) \\
(00010)\end{array}$ \\
\hline
\end{tabular}

\begin{tabular}{|c|c|c|c|c|c|c|}
\hline$\overline{04 / 07 / 2006}$ & $11: 51$ & 5.0 & 9.3 & 8.1 & 891 & 16.6 \\
\hline $04 / 07 / 2006$ & $11: 52$ & 6.0 & 9.2 & 8.1 & 891 & 16.6 \\
\hline $04 / 07 / 2006$ & $11: 53$ & 7.0 & 9.1 & 8.1 & 891 & 16.6 \\
\hline $04 / 07 / 2006$ & $11: 54$ & 8.0 & 6.6 & 8.0 & 886 & 15.4 \\
\hline $04 / 07 / 2006$ & $11: 55$ & 9.0 & 7.8 & 7.9 & 886 & 15.0 \\
\hline $04 / 07 / 2006$ & $11: 56$ & 10.0 & 4.1 & 7.8 & 885 & 15.0 \\
\hline $04 / 07 / 2006$ & $11: 57$ & 12.0 & 3.1 & 7.8 & 886 & 14.7 \\
\hline $04 / 07 / 2006$ & $11: 58$ & 14.0 & 2.2 & 7.7 & 886 & 14.6 \\
\hline $04 / 07 / 2006$ & $11: 59$ & 16.0 & 1.6 & 7.6 & 887 & 14.6 \\
\hline $07 / 11 / 2006$ & $12: 10$ & 0.1 & 9.9 & 8.6 & 958 & 28.2 \\
\hline $07 / 11 / 2006$ & $11: 58$ & 1.0 & 9.2 & 8.6 & 962 & 27.6 \\
\hline $07 / 11 / 2006$ & $11: 59$ & 2.0 & 8.8 & 8.6 & 962 & 27.2 \\
\hline $07 / 11 / 2006$ & $12: 00$ & 3.0 & 8.6 & 8.6 & 961 & 27.0 \\
\hline $07 / 11 / 2006$ & 12:01 & 4.0 & 8.1 & 8.6 & 961 & 26.8 \\
\hline $07 / 11 / 2006$ & $12: 02$ & 5.0 & 1.5 & 7.7 & 940 & 23.7 \\
\hline $07 / 11 / 2006$ & 12:03 & 6.0 & 1.4 & 7.6 & 925 & 20.8 \\
\hline $07 / 11 / 2006$ & 12:04 & 7.0 & 1.4 & 7.5 & 917 & 20.0 \\
\hline $07 / 11 / 2006$ & $12: 05$ & 8.0 & 1.4 & 7.5 & 909 & 18.7 \\
\hline $07 / 11 / 2006$ & $12: 06$ & 9.0 & 1.4 & 7.5 & 903 & 17.9 \\
\hline $07 / 11 / 2006$ & $12: 07$ & 10.0 & 1.4 & 7.5 & 901 & 17.3 \\
\hline $07 / 11 / 2006$ & $12: 08$ & 12.0 & 1.4 & 7.5 & 901 & 16.9 \\
\hline $07 / 11 / 2006$ & 12:09 & 14.0 & 1.4 & 7.5 & 903 & 16.6 \\
\hline $07 / 11 / 2006$ & $12: 11$ & 15.1 & 1.4 & 7.5 & 904 & 16.6 \\
\hline $08 / 23 / 2006$ & 09:49 & 0.1 & 9.6 & 8.5 & 955 & 26.4 \\
\hline $08 / 23 / 2006$ & $09: 50$ & 1.0 & 9.8 & 8.5 & 954 & 26.2 \\
\hline $08 / 23 / 2006$ & 09:51 & 2.0 & 9.1 & 8.5 & 953 & 26.0 \\
\hline $08 / 23 / 2006$ & 09:52 & 3.0 & 9.0 & 8.5 & 954 & 26.0 \\
\hline $08 / 23 / 2006$ & 09:53 & 4.0 & 8.2 & 8.4 & 950 & 25.6 \\
\hline $08 / 23 / 2006$ & 09:54 & 5.0 & 4.2 & 7.9 & 945 & 24.4 \\
\hline $10 / 11 / 2006$ & $11: 30$ & 0.1 & PM & 8.5 & 985 & 21.9 \\
\hline $10 / 11 / 2006$ & $11: 31$ & 1.0 & PM & 8.5 & 985 & 21.9 \\
\hline $10 / 11 / 2006$ & $11: 32$ & 2.0 & PM & 8.4 & 987 & 21.8 \\
\hline $10 / 11 / 2006$ & $11: 33$ & 3.0 & PM & 8.4 & 987 & 21.7 \\
\hline $10 / 11 / 2006$ & $11: 50$ & 4.0 & PM & 8.4 & 987 & 21.6 \\
\hline $10 / 11 / 2006$ & $11: 34$ & 5.0 & PM & 8.4 & 987 & 21.5 \\
\hline $10 / 11 / 2006$ & $11: 35$ & 6.0 & PM & 8.3 & 986 & 21.4 \\
\hline $10 / 11 / 2006$ & $11: 36$ & 7.0 & PM & 8.3 & 986 & 21.4 \\
\hline $10 / 11 / 2006$ & $11: 37$ & 8.0 & PM & 8.1 & 972 & 20.6 \\
\hline $10 / 11 / 2006$ & $11: 38$ & 9.0 & PM & 7.9 & 965 & 20.3 \\
\hline $10 / 11 / 2006$ & $11: 39$ & 10.0 & PM & 7.6 & 938 & 18.6 \\
\hline $10 / 11 / 2006$ & $11: 40$ & 11.0 & PM & 7.5 & 933 & 18.2 \\
\hline $10 / 11 / 2006$ & $11: 41$ & 11.8 & PM & 7.4 & 936 & 17.5 \\
\hline $01 / 09 / 2007$ & $11: 20$ & 0.1 & 14.6 & 8.3 & 1,000 & 12.7 \\
\hline $01 / 09 / 2007$ & $11: 06$ & 1.0 & 13.8 & 8.3 & 1,000 & 12.4 \\
\hline
\end{tabular}

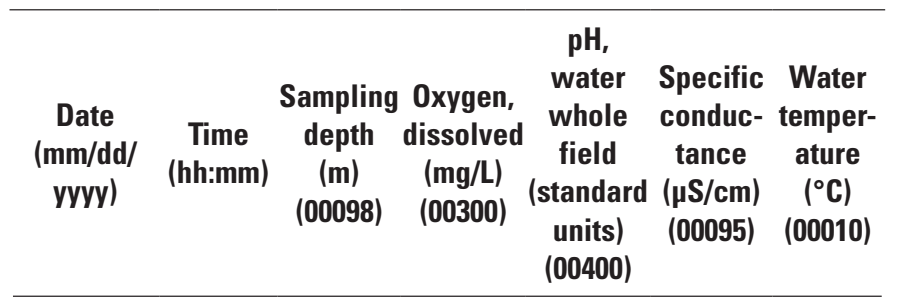

Sweetwater Reservoir center of minimum pool (SWR03)—Continued

\begin{tabular}{lllllll}
\hline $01 / 09 / 2007$ & $11: 07$ & 2.0 & 13.0 & 8.3 & 1,000 & 12.3
\end{tabular}

$\begin{array}{lllllll}01 / 09 / 2007 & 11: 08 & 3.0 & 12.7 & 8.3 & 1,000 & 12.2\end{array}$

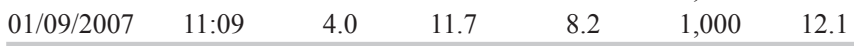

$01 / 09 / 2007 \quad 11: 10 \quad 5.0 \quad 11.3 \quad 8.2 \quad 1,000 \quad 12.1$

$\begin{array}{lllllll}01 / 09 / 2007 & 11: 11 & 6.0 & 11.1 & 8.2 & 1,000 & 12.1\end{array}$

$\begin{array}{lllllll}01 / 09 / 2007 & 11: 12 & 7.0 & 10.7 & 8.2 & 1,000 & 12.1\end{array}$

$\begin{array}{lllllll}01 / 09 / 2007 & 11: 13 & 8.0 & 10.0 & 8.1 & 1,010 & 12.0\end{array}$

$\begin{array}{lllllll}01 / 09 / 2007 & 11: 14 & 9.0 & 9.2 & 8.1 & 1,010 & 12.0\end{array}$

$\begin{array}{lllllll}01 / 09 / 2007 & 11: 15 & 10.0 & 8.9 & 8.0 & 1,000 & 12.0\end{array}$

$\begin{array}{lllllll}01 / 09 / 2007 & 11: 16 & 11.0 & 7.4 & 7.9 & 1,000 & 12.1\end{array}$

$\begin{array}{lllllll}01 / 09 / 2007 & 11: 17 & 11.3 & 5.1 & 7.9 & 1,000 & 12.1\end{array}$

\begin{tabular}{|c|c|c|c|c|c|c|}
\hline $04 / 10 / 2007$ & $12: 00$ & 0.1 & 10.4 & 8.2 & 1,040 & 19.1 \\
\hline $04 / 10 / 2007$ & $12: 08$ & 1.0 & 10.2 & 8.2 & 1,040 & 18.6 \\
\hline $04 / 10 / 2007$ & 12:09 & 2.0 & 10.0 & 8.2 & 1,040 & 18.3 \\
\hline $04 / 10 / 2007$ & $12: 10$ & 3.0 & 9.8 & 8.2 & 1,030 & 18.2 \\
\hline $04 / 10 / 2007$ & $12: 11$ & 4.0 & 9.5 & 8.1 & 1,030 & 18.1 \\
\hline $04 / 10 / 2007$ & $12: 12$ & 5.0 & 9.3 & 8.1 & 1,040 & 18.0 \\
\hline 04/10/2007 & $12: 13$ & 6.0 & 4.5 & 7.8 & 1,050 & 17.2 \\
\hline $04 / 10 / 2007$ & $12: 14$ & 7.0 & 2.8 & 7.7 & 1,050 & 16.4 \\
\hline $04 / 10 / 2007$ & $12: 15$ & 8.0 & 2.1 & 7.6 & 1,050 & 16.2 \\
\hline $04 / 10 / 2007$ & $12: 16$ & 9.0 & 1.3 & 7.5 & 1,050 & 15.7 \\
\hline $04 / 10 / 2007$ & $12: 17$ & 10.0 & 1.1 & 7.5 & 1,060 & 15.1 \\
\hline $04 / 10 / 2007$ & $12: 18$ & 11.0 & 1.0 & 7.5 & 1,060 & 15.1 \\
\hline $04 / 10 / 2007$ & $12: 19$ & 12.0 & 0.9 & 7.5 & 1,060 & 14.9 \\
\hline $04 / 10 / 2007$ & $12: 20$ & 12.2 & 0.9 & 7.4 & 1,060 & 14.9 \\
\hline 07/10/2007 & $11: 10$ & 0.1 & 8.5 & 8.0 & 1,050 & 25.9 \\
\hline $07 / 10 / 2007$ & $11: 18$ & 1.0 & 8.5 & 8.5 & 1,050 & 25.9 \\
\hline 07/10/2007 & $11: 19$ & 2.0 & 8.3 & 8.3 & 1,050 & 25.6 \\
\hline 07/10/2007 & $11: 20$ & 3.0 & 8.1 & 8.1 & 1,050 & 25.5 \\
\hline 07/10/2007 & $11: 21$ & 4.0 & 7.8 & 8.0 & 1,050 & 25.3 \\
\hline $07 / 10 / 2007$ & $11: 22$ & 5.0 & 6.2 & 8.0 & 1,050 & 24.8 \\
\hline $07 / 10 / 2007$ & $11: 23$ & 6.0 & 2.0 & 7.8 & 1,070 & 23.3 \\
\hline $07 / 10 / 2007$ & $11: 24$ & 7.0 & 1.7 & 7.7 & 1,080 & 21.9 \\
\hline $07 / 10 / 2007$ & $11: 25$ & 8.0 & 1.7 & 7.6 & 1,080 & 21.3 \\
\hline $07 / 10 / 2007$ & $11: 26$ & 9.0 & 1.8 & 7.6 & 1,090 & 20.1 \\
\hline $07 / 10 / 2007$ & $11: 27$ & 10.0 & 1.8 & 7.4 & 1,090 & 19.8 \\
\hline $07 / 10 / 2007$ & $11: 28$ & 11.0 & 1.8 & 7.3 & 1,090 & 19.4 \\
\hline $07 / 10 / 2007$ & $11: 29$ & 12.0 & 1.8 & 7.3 & 1,090 & 19.1 \\
\hline 07/10/2007 & $11: 30$ & 13.1 & 1.8 & 7.4 & 1,100 & 18.7 \\
\hline $10 / 09 / 2007$ & $12: 10$ & 0.1 & 10.9 & 7.8 & 1,080 & 21.4 \\
\hline $10 / 09 / 2007$ & $12: 11$ & 1.0 & 10.1 & 7.8 & 1,080 & 21.1 \\
\hline $10 / 09 / 2007$ & $12: 12$ & 2.0 & 8.7 & 7.8 & 1,080 & 20.6 \\
\hline $10 / 09 / 2007$ & $12: 13$ & 3.0 & 8.1 & 7.8 & 1,080 & 20.6 \\
\hline $10 / 09 / 2007$ & $12: 14$ & 4.0 & 7.5 & 7.8 & 1,080 & 20.6 \\
\hline $10 / 09 / 2007$ & $12: 20$ & 5.0 & 7.2 & 7.8 & 1,080 & 20.6 \\
\hline
\end{tabular}


Table 3. Water-quality depth-profile data for dissolved oxygen, $\mathrm{pH}$, specific conductance, and water temperature for each sampling site and period for the Sweetwater Reservoir watershed, San Diego County, California.—Continued

[Time is denoted in 24-hour format. The five-digit parameter code in parentheses below each compound name is used in the U.S. Geological Survey computerized data system (National Water Information System) to uniquely identify a specific constituent or property. Sampling depth is in meters below water surface. Shaded data coincide with reservoir water samples. Abbreviations: hh:mm, hour:minute; m, meter; mg/L, milligram per liter; mm/dd/yyyy, month/day/year; na, not applicable; $\mathrm{PM}$, probe malfunction; $\mu \mathrm{S} / \mathrm{cm}$, microsiemen per centimeter; ${ }^{\circ} \mathrm{C}$, degree Celsius]

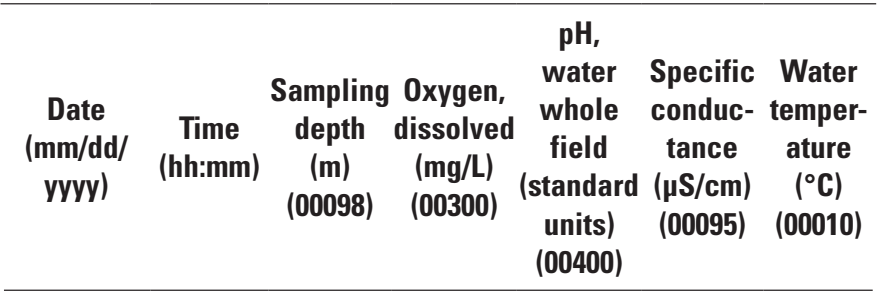

\begin{tabular}{|c|c|c|c|c|c|c|}
\hline \multicolumn{7}{|c|}{ Sweetwater Reservoir center of minimum pool (SWRO3) —Continued } \\
\hline $10 / 09 / 2007$ & $12: 15$ & 6.0 & 6.9 & 7.8 & 1,080 & 20.5 \\
\hline $10 / 09 / 2007$ & $12: 16$ & 7.0 & 6.6 & 7.8 & 1,080 & 20.5 \\
\hline $10 / 09 / 2007$ & $12: 17$ & 8.0 & 6.4 & 7.8 & 1,080 & 20.4 \\
\hline $10 / 09 / 2007$ & $12: 18$ & 9.0 & 6.3 & 7.8 & 1,080 & 20.4 \\
\hline $10 / 09 / 2007$ & $12: 19$ & 9.6 & 3.3 & 7.5 & 1,100 & 20.4 \\
\hline $10 / 25 / 2007$ & $12: 40$ & 0.1 & 15.1 & 8.3 & 1,100 & 20.1 \\
\hline 01/08/2008 & $11: 10$ & 0.1 & 10.9 & 8.0 & 1,150 & 12.9 \\
\hline $01 / 08 / 2008$ & $11: 11$ & 1.0 & 10.1 & 7.9 & 1,150 & 12.3 \\
\hline $01 / 08 / 2008$ & $11: 12$ & 2.0 & 9.1 & 7.9 & 1,150 & 12.2 \\
\hline $01 / 08 / 2008$ & $11: 13$ & 3.0 & 8.9 & 7.9 & 1,150 & 12.2 \\
\hline 01/08/2008 & $11: 14$ & 4.0 & 8.8 & 7.9 & 1,150 & 12.2 \\
\hline $01 / 08 / 2008$ & $11: 20$ & 5.0 & 8.8 & 7.9 & 1,150 & 12.2 \\
\hline $01 / 08 / 2008$ & $11: 15$ & 6.0 & 8.8 & 7.9 & 1,150 & 12.2 \\
\hline 01/08/2008 & $11: 16$ & 7.0 & 8.7 & 8 & 1,150 & 12.2 \\
\hline $01 / 08 / 2008$ & $11: 17$ & 8.0 & 8.7 & 7.9 & 1,150 & 12.2 \\
\hline $01 / 08 / 2008$ & $11: 18$ & 9.0 & 8.4 & 7.9 & 1,150 & 12.2 \\
\hline $01 / 08 / 2008$ & $11: 19$ & 9.3 & 3.7 & 7.8 & 1,150 & 12.2 \\
\hline $04 / 15 / 2008$ & $12: 10$ & 0.1 & 12.3 & 8.4 & 825 & 19.9 \\
\hline $04 / 15 / 2008$ & $12: 11$ & 1.0 & 12.2 & 8.4 & 826 & 19.8 \\
\hline $04 / 15 / 2008$ & $12: 12$ & 2.0 & 12 & 8.4 & 826 & 19.8 \\
\hline $04 / 15 / 2008$ & $12: 13$ & 3.0 & 11.5 & 8.4 & 827 & 19.2 \\
\hline $04 / 15 / 2008$ & $12: 14$ & 4.0 & 11.2 & 8.3 & 826 & 18.9 \\
\hline $04 / 15 / 2008$ & $12: 15$ & 5.0 & 8.9 & 8.2 & 824 & 18.2 \\
\hline $04 / 15 / 2008$ & $12: 16$ & 6.0 & 6.8 & 8.1 & 818 & 12.6 \\
\hline $04 / 15 / 2008$ & $12: 17$ & 7.0 & 3.9 & 8.0 & 808 & 16.3 \\
\hline $04 / 15 / 2008$ & $12: 18$ & 8.0 & 2.2 & 8.0 & 801 & 15.6 \\
\hline $04 / 15 / 2008$ & $12: 19$ & 9.0 & 1.3 & 7.9 & 798 & 15.3 \\
\hline $04 / 15 / 2008$ & $12: 20$ & 10.0 & 1.0 & 7.9 & 797 & 15.1 \\
\hline $04 / 15 / 2008$ & $12: 21$ & 11.0 & 0.9 & 7.8 & 795 & 14.8 \\
\hline $04 / 15 / 2008$ & $12: 22$ & 12.0 & 0.9 & 7.8 & 794 & 14.6 \\
\hline $04 / 15 / 2008$ & $12: 23$ & 13.0 & 0.8 & 7.8 & 793 & 14.5 \\
\hline $04 / 15 / 2008$ & $12: 24$ & 13.6 & 0.8 & 7.8 & 792 & 14.4 \\
\hline $07 / 15 / 2008$ & $11: 10$ & 0.1 & 9.0 & 8.3 & 1,100 & 26.6 \\
\hline $07 / 15 / 2008$ & $11: 11$ & 1.0 & 9.0 & 8.2 & 1,100 & 26.5 \\
\hline $07 / 15 / 2008$ & $11: 12$ & 2.0 & 8.8 & 8.2 & 1,100 & 26.0 \\
\hline $07 / 15 / 2008$ & $11: 13$ & 3.0 & 8.7 & 8.2 & 1,100 & 25.6 \\
\hline 07/15/2008 & $11: 14$ & 4.0 & 6.3 & 8.1 & 1,090 & 24.5 \\
\hline $07 / 15 / 2008$ & $11: 15$ & 5.0 & 1.9 & 7.8 & 1,080 & 23.1 \\
\hline $07 / 15 / 2008$ & $11: 16$ & 6.0 & 1.2 & 7.6 & 1,060 & 20.4 \\
\hline $07 / 15 / 2008$ & $11: 17$ & 7.0 & 1.2 & 7.5 & 1,060 & 19.0 \\
\hline 07/15/2008 & $11: 18$ & 8.0 & 1.2 & 7.5 & 1,050 & 18.4 \\
\hline $07 / 15 / 2008$ & $11: 19$ & 9.0 & 1.2 & 7.4 & 1,050 & 17.9 \\
\hline $07 / 15 / 2008$ & $11: 20$ & 10.0 & 1.2 & 7.4 & 1,050 & 17.5 \\
\hline $07 / 15 / 2008$ & $11: 21$ & 11.0 & 1.2 & 7.3 & 1,050 & 17.3 \\
\hline $07 / 15 / 2008$ & $11: 22$ & 11.9 & 1.3 & 7.2 & 1,060 & 17.2 \\
\hline
\end{tabular}

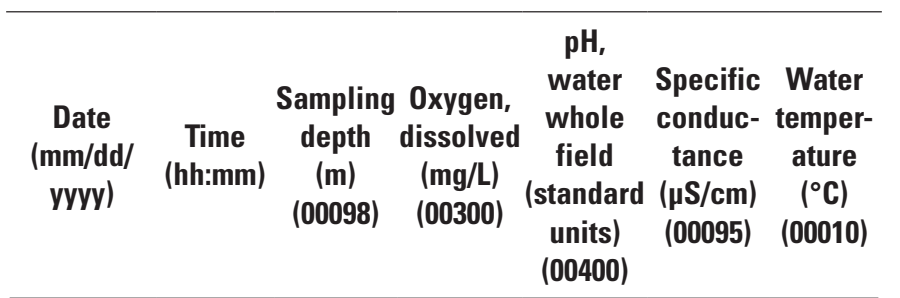

Sweetwater Reservoir center of minimum pool (SWR03)-Continued

\begin{tabular}{lllllll}
\hline $10 / 14 / 2008$ & $13: 00$ & 0.1 & 9.9 & 7.3 & 1,130 & 21.0 \\
$10 / 14 / 2008$ & $13: 01$ & 1.0 & 7.8 & 7.3 & 1,130 & 20.9 \\
$10 / 14 / 2008$ & $13: 02$ & 2.0 & 6.7 & 7.3 & 1,130 & 20.7 \\
$10 / 14 / 2008$ & $13: 03$ & 3.0 & 6.0 & 7.3 & 1,130 & 20.5 \\
$10 / 14 / 2008$ & $13: 04$ & 4.0 & 5.7 & 7.4 & 1,130 & 20.5 \\
$10 / 14 / 2008$ & $13: 05$ & 5.0 & 5.2 & 7.4 & 1,130 & 20.4 \\
$10 / 14 / 2008$ & $13: 06$ & 6.0 & 5.1 & 7.4 & 1,130 & 20.4 \\
$10 / 14 / 2008$ & $13: 07$ & 7.0 & 4.3 & 7.4 & 1,130 & 20.4 \\
$10 / 14 / 2008$ & $13: 08$ & 8.0 & 4.0 & 7.3 & 1,130 & 20.2 \\
$10 / 14 / 2008$ & $13: 09$ & 8.7 & 1.8 & 7.2 & 1,130 & 19.7
\end{tabular}

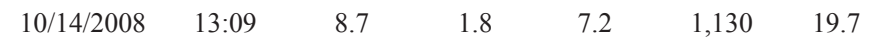

$\begin{array}{rrrrrrr}01 / 13 / 2009 & 11: 50 & 0.1 & 15.8 & 7.6 & 1,080 & 14.4 \\ 01 / 13 / 2009 & 11: 51 & 1.0 & 15.1 & 7.6 & 1,080 & 13.2 \\ 01 / 13 / 2009 & 11: 52 & 2.0 & 13.4 & 7.6 & 1,080 & 13.1 \\ 01 / 13 / 2009 & 11: 53 & 3.0 & 11.7 & 7.6 & 1,080 & 12.8 \\ 01 / 13 / 2009 & 11: 54 & 4.0 & 10.7 & 7.5 & 1,080 & 12.7 \\ 01 / 13 / 2009 & 11: 55 & 5.0 & 9.5 & 7.5 & 1,080 & 12.5 \\ 01 / 13 / 2009 & 11: 56 & 6.0 & 8.7 & 7.5 & 1,080 & 12.4 \\ 01 / 13 / 2009 & 11: 57 & 7.0 & 7.4 & 7.5 & 1,080 & 12.3 \\ 01 / 13 / 2009 & 11: 58 & 8.0 & 6.0 & 7.4 & 1,080 & 12.2 \\ 01 / 13 / 2009 & 11: 59 & 9.0 & 3.9 & 7.3 & 1,080 & 12.2 \\ 01 / 13 / 2009 & 12: 00 & 10.0 & 3.4 & 7.3 & 1,080 & 12.3 \\ 01 / 13 / 2009 & 12: 01 & 10.7 & 1.6 & 7.3 & 1,080 & 12.3\end{array}$

$\begin{array}{rrrrrrr}04 / 14 / 2009 & 12: 20 & 0.1 & 8.1 & 8.2 & 1,060 & 18.4 \\ 04 / 14 / 2009 & 12: 21 & 1.0 & 7.0 & 8.2 & 1,060 & 18.4 \\ 04 / 14 / 2009 & 12: 22 & 2.0 & 7.0 & 8.2 & 1,060 & 18.4 \\ 04 / 14 / 2009 & 12: 23 & 3.0 & 7.0 & 8.2 & 1,060 & 18.3 \\ 04 / 14 / 2009 & 12: 24 & 4.0 & 6.9 & 8.2 & 1,060 & 18.3 \\ 04 / 14 / 2009 & 12: 25 & 5.0 & 6.9 & 8.1 & 1,060 & 17.9 \\ 04 / 14 / 2009 & 12: 26 & 6.0 & 6.8 & 8.1 & 1,060 & 17.8 \\ 04 / 14 / 2009 & 12: 27 & 7.0 & 6.5 & 8 & 1,050 & 17.5 \\ 04 / 14 / 2009 & 12: 28 & 8.0 & 5.6 & 7.9 & 1,050 & 17.3 \\ 04 / 14 / 2009 & 12: 29 & 9.0 & 5.2 & 7.9 & 1,050 & 17.2 \\ 04 / 14 / 2009 & 12: 30 & 10.0 & 4.2 & 7.8 & 1,050 & 16.9 \\ 04 / 14 / 2009 & 12: 31 & 11.0 & 3.1 & 7.6 & 1,050 & 16.4 \\ 04 / 14 / 2009 & 12: 32 & 12.0 & 1.8 & 7.6 & 1,040 & 16.2 \\ 04 / 14 / 2009 & 12: 33 & 12.7 & 1.4 & 7.6 & 1,040 & 16.1 \\ & & & & & & \\ 07 / 07 / 2009 & 11: 30 & 0.01 & 10.9 & 8.5 & 1,040 & 25.6 \\ 07 / 07 / 2009 & 11: 31 & 1.0 & 10.8 & 8.6 & 1,040 & 25.6 \\ 07 / 07 / 2009 & 11: 32 & 2.0 & 10.9 & 8.6 & 1,040 & 25.5 \\ 07 / 07 / 2009 & 11: 33 & 3.0 & 11.0 & 8.6 & 1,040 & 25.0 \\ 07 / 07 / 2009 & 11: 34 & 4.0 & 10.7 & 8.5 & 1,030 & 24.8 \\ 07 / 07 / 2009 & 11: 35 & 5.0 & 8.0 & 8.2 & 1,030 & 23.6 \\ 07 / 07 / 2009 & 11: 36 & 6.0 & 4.2 & 8.0 & 1,030 & 22.9 \\ 07 / 07 / 2009 & 11: 37 & 7.0 & 2.3 & 7.8 & 1,020 & 22.2 \\ 07 / 07 / 2009 & 11: 38 & 8.0 & 1.5 & 7.7 & 1,020 & 21.6\end{array}$


Table 3. Water-quality depth-profile data for dissolved oxygen, $\mathrm{pH}$, specific conductance, and water temperature for each sampling site and period for the Sweetwater Reservoir watershed, San Diego County, California._-Continued

[Time is denoted in 24-hour format. The five-digit parameter code in parentheses below each compound name is used in the U.S. Geological Survey computerized data system (National Water Information System) to uniquely identify a specific constituent or property. Sampling depth is in meters below water surface. Shaded data coincide with reservoir water samples. Abbreviations: hh:mm, hour:minute; m, meter; mg/L, milligram per liter; mm/dd/yyyy, month/day/year; na, not applicable; $\mathrm{PM}$, probe malfunction; $\mu \mathrm{S} / \mathrm{cm}$, microsiemen per centimeter; ${ }^{\circ} \mathrm{C}$, degree Celsius]

\begin{tabular}{|c|c|c|c|c|c|c|}
\hline $\begin{array}{c}\text { Date } \\
\text { (mm/dd/ } \\
\text { yyyy) }\end{array}$ & $\begin{array}{c}\text { Time } \\
\text { (hh:mm) }\end{array}$ & $\begin{array}{c}\text { Sampling } \\
\text { depth } \\
(\mathrm{m}) \\
(00098)\end{array}$ & $\begin{array}{c}\text { Oxygen, } \\
\text { dissolved } \\
(\mathrm{mg} / \mathrm{L}) \\
(00300)\end{array}$ & $\begin{array}{c}\text { pH, } \\
\text { water } \\
\text { whole } \\
\text { field } \\
\text { (standard } \\
\text { units) } \\
\text { (00400) }\end{array}$ & $\begin{array}{c}\text { Specific } \\
\text { conduc- } \\
\text { tance } \\
(\mu \mathrm{S} / \mathrm{cm}) \\
(00095)\end{array}$ & $\begin{array}{c}\text { Water } \\
\text { temper- } \\
\text { ature } \\
\left({ }^{\circ} \mathrm{C}\right) \\
(00010)\end{array}$ \\
\hline
\end{tabular}

\begin{tabular}{lrrrrrr}
\hline \multicolumn{6}{c}{ Sweetwater Reservoir center of minimum pool (SWR03) - Continued } \\
\hline $07 / 07 / 2009$ & $11: 39$ & 9.0 & 1.2 & 7.7 & 1,020 & 21.6 \\
$07 / 07 / 2009$ & $11: 40$ & 10.0 & 1.3 & 7.6 & 1,010 & 20.5 \\
$07 / 07 / 2009$ & $11: 41$ & 11.0 & 1.2 & 7.6 & 1,010 & 20.0 \\
$07 / 07 / 2009$ & $11: 42$ & 12.0 & 1.2 & 7.6 & 1,000 & 19.6 \\
$07 / 07 / 2009$ & $11: 43$ & 12.5 & 1.2 & 7.5 & 1,000 & 19.5 \\
\hline
\end{tabular}

\begin{tabular}{|c|c|c|c|c|c|c|}
\hline \multicolumn{7}{|c|}{ Sweetwater Reservoir east-end reservoir-fill boundary (SWR06) } \\
\hline $10 / 14 / 2003$ & 15:00 & 0.2 & 11.0 & 9.0 & 1,110 & 25.1 \\
\hline $10 / 14 / 2003$ & $15: 01$ & 1.0 & 10.7 & 8.9 & 1,110 & 24.8 \\
\hline $12 / 16 / 2003$ & $13: 30$ & 0.5 & 12.8 & 9.0 & 966 & 14.4 \\
\hline $10 / 14 / 2003$ & $13: 31$ & 1.0 & 12.4 & 8.9 & 989 & 13.5 \\
\hline $02 / 17 / 2004$ & $17: 00$ & 0.2 & 6.7 & 8.0 & 1,020 & 15.6 \\
\hline $10 / 14 / 2003$ & 17:01 & 0.6 & 6.4 & 8.0 & 1,020 & 15.6 \\
\hline $04 / 13 / 2004$ & $12: 39$ & 0.1 & 10.6 & 8.5 & 1,010 & 22.6 \\
\hline $04 / 13 / 2004$ & $12: 40$ & 1.0 & 10.8 & 8.5 & 1,010 & 22.6 \\
\hline $04 / 13 / 2004$ & $12: 41$ & 2.0 & 10.2 & 8.4 & 1,020 & 22.1 \\
\hline $04 / 13 / 2004$ & $12: 42$ & 2.3 & 8.7 & 8.1 & 1,000 & 20.6 \\
\hline $07 / 13 / 2004$ & $14: 30$ & 0.1 & 9.1 & 8.5 & 1,070 & 30.0 \\
\hline $07 / 13 / 2004$ & $14: 31$ & 0.3 & 9.2 & 8.6 & 1,070 & 29.9 \\
\hline $10 / 12 / 2004$ & $14: 00$ & 0.1 & 17.1 & 9.1 & 1,060 & 25.1 \\
\hline $07 / 13 / 2004$ & 14:01 & 0.2 & 17.2 & 9.2 & 1,060 & 25.1 \\
\hline $01 / 12 / 2005$ & $12: 30$ & 0.1 & 9.3 & 7.7 & 552 & 14.8 \\
\hline $07 / 13 / 2004$ & $12: 31$ & 0.9 & 8.3 & 7.6 & 506 & 12.2 \\
\hline $04 / 12 / 2005$ & $14: 28$ & 0.1 & 11.1 & 8.6 & 959 & 20.9 \\
\hline $04 / 12 / 2005$ & $14: 29$ & 1.0 & 11.1 & 8.6 & 960 & 20.9 \\
\hline $04 / 12 / 2005$ & $14: 30$ & 2.0 & 11.0 & 8.6 & 965 & 20.9 \\
\hline $04 / 12 / 2005$ & $14: 31$ & 3.0 & 7.1 & 8.5 & 1,010 & 19.6 \\
\hline $04 / 12 / 2005$ & $14: 32$ & 3.7 & 5.4 & 8.4 & 968 & 18.5 \\
\hline $07 / 12 / 2005$ & $12: 29$ & 0.1 & 9.1 & 8.8 & 1,040 & 26.7 \\
\hline $07 / 12 / 2005$ & $12: 30$ & 1.0 & 8.8 & 8.8 & 1,040 & 26.5 \\
\hline $07 / 12 / 2005$ & $12: 31$ & 2.0 & 6.7 & 8.7 & 1,060 & 25.7 \\
\hline $07 / 12 / 2005$ & $12: 32$ & 2.5 & 5.3 & 8.6 & 1,060 & 25.6 \\
\hline $10 / 11 / 2005$ & $12: 09$ & 0.1 & 8.5 & 8.5 & 1,080 & 22.8 \\
\hline $10 / 11 / 2005$ & $12: 10$ & 1.0 & 8.0 & 8.4 & 1,080 & 22.7 \\
\hline $10 / 11 / 2005$ & $12: 11$ & 1.7 & 5.7 & 8.3 & 1,080 & 22.2 \\
\hline $01 / 10 / 2006$ & $12: 20$ & 0.1 & 11.7 & 8 & 974 & 14.8 \\
\hline $01 / 10 / 2006$ & $12: 21$ & 0.5 & 11.4 & 7.8 & 995 & 14.7 \\
\hline $04 / 07 / 2006$ & $12: 20$ & 0.1 & 9.9 & 8.4 & 907 & 17.8 \\
\hline $04 / 07 / 2006$ & $12: 21$ & 1.0 & 9.9 & 8.3 & 906 & 17.7 \\
\hline $04 / 07 / 2006$ & $12: 22$ & 2.0 & 9.8 & 8.3 & 905 & 17.6 \\
\hline
\end{tabular}

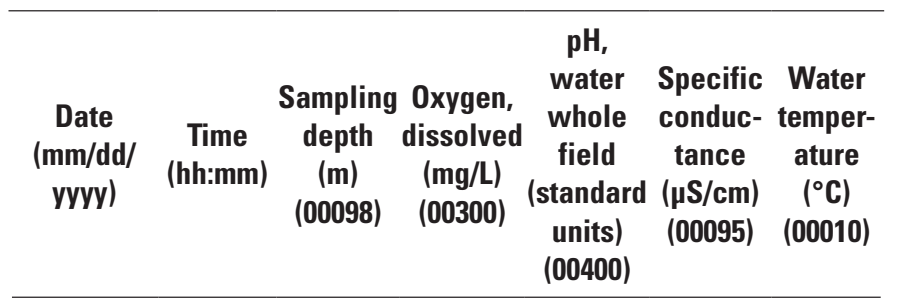

Sweetwater Reservoir east-end reservoir-fill boundary (SWR06)—Continued

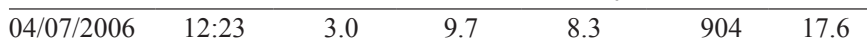

$\begin{array}{lllllll}04 / 07 / 2006 & 12: 24 & 4.0 & 9.6 & 8.3 & 905 & 17.4\end{array}$

$\begin{array}{lllllll}04 / 07 / 2006 & 12: 25 & 5.0 & 8.0 & 8.2 & 895 & 16.4\end{array}$

$\begin{array}{lllllll}07 / 11 / 2006 & 12: 38 & 0.1 & 8.5 & 8.6 & 969 & 28.6\end{array}$

$\begin{array}{lllllll}07 / 11 / 2006 & 12: 39 & 1.0 & 8.2 & 8.6 & 971 & 28.5\end{array}$

$\begin{array}{lllllll}07 / 11 / 2006 & 12: 40 & 2.0 & 8.0 & 8.6 & 971 & 28.4\end{array}$

$\begin{array}{lllllll}07 / 11 / 2006 & 12: 41 & 3.0 & 6.3 & 8.5 & 973 & 27.9\end{array}$

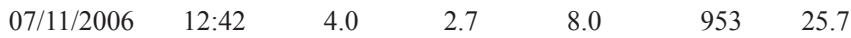

$\begin{array}{lllllll}07 / 11 / 2006 & 12: 43 & 4.3 & 2.7 & 8.0 & 953 & 25.6\end{array}$

$\begin{array}{lllllll}08 / 23 / 2006 & 10: 09 & 0.1 & 8.5 & 8.5 & 964 & 27.1\end{array}$

$\begin{array}{lllllll}08 / 23 / 2006 & 10: 10 & 1.0 & 8.4 & 8.5 & 964 & 26.9\end{array}$

$\begin{array}{lllllll}08 / 23 / 2006 & 10: 11 & 2.0 & 8.2 & 8.5 & 965 & 26.8\end{array}$

$\begin{array}{lllllll}08 / 23 / 2006 & 10: 12 & 2.9 & 4.8 & 8.0 & 962 & 26.0\end{array}$

$\begin{array}{lllllll}10 / 11 / 2006 & 12: 00 & 0.1 & \text { PM } & 8.5 & 992 & 23.1 \\ 10 / 11 / 2006 & 12: 01 & 1.0 & \text { PM } & 8.4 & 990 & 21.8\end{array}$

$\begin{array}{lllllll}10 / 11 / 2006 & 12: 02 & 1.2 & \text { PM } & 8.4 & 990 & 21.8\end{array}$

$\begin{array}{lllllll}01 / 09 / 2007 & 11: 30 & 0.1 & 13.0 & 8.3 & 1,000 & 13.5\end{array}$

$\begin{array}{lllllll}04 / 10 / 2007 & 12: 30 & 0.1 & 14.0 & 8.4 & 1,040 & 20.5\end{array}$

$\begin{array}{lllllll}04 / 10 / 2007 & 12: 31 & 1.0 & 15.4 & 8.5 & 1,040 & 20.5\end{array}$

$\begin{array}{lrrrrrr}07 / 10 / 2007 & 11: 30 & 0.1 & 8.8 & 8.0 & 1,050 & 26.7 \\ 07 / 10 / 2007 & 11: 31 & 1.0 & 8.6 & 8.1 & 1,060 & 26.6 \\ 07 / 10 / 2007 & 11: 32 & 2.0 & 8.5 & 8.2 & 1,060 & 26.4 \\ 10 / 09 / 2007 & 12.30 & 0.1 & 10.4 & 7.8 & 1,070 & 22.0\end{array}$

$\begin{array}{lllllll}10 / 09 / 2007 & 12: 30 & 0.1 & 10.4 & 7.8 & 1,070 & 22.0 \\ 10 / 09 / 2007 & 12: 31 & 0.6 & 10.3 & 7.8 & 1,070 & 21.9\end{array}$

$\begin{array}{lllllll}01 / 08 / 2008 & 11: 50 & 0.1 & 13.2 & 8.2 & 1,130 & 13.5 \\ 01 / 08 / 2008 & 11: 52 & 0.4 & 13.8 & 8.2 & 1,140 & 13.4\end{array}$

\begin{tabular}{lrrrrrr}
$04 / 15 / 2008$ & $12: 30$ & 0.1 & 10.1 & 8.2 & 824 & 21.2 \\
$04 / 15 / 2008$ & $12: 31$ & 1.0 & 10.0 & 8.2 & 826 & 21.1 \\
$04 / 15 / 2008$ & $12: 32$ & 2.0 & 9.9 & 8.2 & 826 & 21.1 \\
$04 / 15 / 2008$ & $12: 33$ & 2.9 & 9.7 & 8.1 & 822 & 21.1 \\
& & & & & & \\
$07 / 15 / 2008$ & $11: 30$ & 0.1 & 10.5 & 8.2 & 1,110 & 27.8 \\
$07 / 15 / 2008$ & $11: 31$ & 1.0 & 9.2 & 8.1 & 1,110 & 27.4 \\
& & & & & & \\
$10 / 14 / 2008$ & $13: 40$ & 0.1 & 12.2 & 8.1 & 1,110 & 22.8 \\
$10 / 14 / 2008$ & $13: 41$ & 0.7 & 17.2 & 8.2 & 1,120 & 23.0 \\
$01 / 13 / 2009$ & $12: 10$ & 0.1 & 12.4 & 7.8 & 1,090 & 15.5 \\
\hline $01 / 13 / 2009$ & $12: 11$ & 0.6 & 13.7 & 7.7 & 1,070 & 14.2 \\
& & & & & & \\
$04 / 14 / 2009$ & $12: 40$ & 0.1 & 7.8 & 8.2 & 1,050 & 19.3 \\
$04 / 14 / 2009$ & $12: 41$ & 1.0 & 7.0 & 8.2 & 1,050 & 19.3 \\
$04 / 14 / 2009$ & $12: 42$ & 2.0 & 7.0 & 8.2 & 1,050 & 19.3
\end{tabular}


Table 3. Water-quality depth-profile data for dissolved oxygen, $\mathrm{pH}$, specific conductance, and water temperature for each sampling site and period for the Sweetwater Reservoir watershed, San Diego County, California.—Continued

[Time is denoted in 24-hour format. The five-digit parameter code in parentheses below each compound name is used in the U.S. Geological Survey computerized data system (National Water Information System) to uniquely identify a specific constituent or property. Sampling depth is in meters below water surface. Shaded data coincide with reservoir water samples. Abbreviations: hh:mm, hour:minute; m, meter; mg/L, milligram per liter; mm/dd/yyyy, month/day/year; na, not applicable; $\mathrm{PM}$, probe malfunction; $\mu \mathrm{S} / \mathrm{cm}$, microsiemen per centimeter; ${ }^{\circ} \mathrm{C}$, degree Celsius]

\begin{tabular}{|c|c|c|c|c|c|c|}
\hline $\begin{array}{c}\text { Date } \\
\text { (mm/dd/ } \\
\text { yyyy) }\end{array}$ & $\begin{array}{c}\text { Time } \\
\text { (hh:mm) }\end{array}$ & $\begin{array}{l}\text { Sampling } \\
\text { depth } \\
\text { (m) } \\
(00098)\end{array}$ & $\begin{array}{c}\text { Oxygen, } \\
\text { dissolved } \\
\text { (mg/L) } \\
(00300)\end{array}$ & $\begin{array}{c}\text { pH, } \\
\text { water } \\
\text { whole } \\
\text { field } \\
\text { (standard } \\
\text { units) }\end{array}$ & $\begin{array}{c}\text { Specific } \\
\text { conduc- } \\
\text { tance } \\
(\mu \mathrm{S} / \mathrm{cm}) \\
(\mathbf{0 0 0 9 5 )}\end{array}$ & $\begin{array}{c}\text { Water } \\
\text { temper- } \\
\text { ature } \\
\left({ }^{\circ} \mathrm{C}\right) \\
(\mathbf{0 0 0 1 0})\end{array}$ \\
\hline
\end{tabular}

$\overline{\text { Sweetwater Reservoir east-end reservoir-fill boundary (SWR06)—Continued }}$

\begin{tabular}{lllllll}
\hline $07 / 07 / 2009$ & $11: 50$ & 0.1 & 9.8 & 8.5 & 962 & 26.6
\end{tabular}

$\begin{array}{lllllll}07 / 07 / 2009 & 11: 51 & 1.0 & 10.0 & 8.5 & 964 & 26.6\end{array}$

\begin{tabular}{lllllll}
$07 / 07 / 2009$ & $11: 52$ & 1.8 & 10.0 & 8.5 & 964 & 26.0 \\
\hline
\end{tabular}

\begin{tabular}{|c|c|c|c|c|c|c|}
\hline Tre & ment & & int & eet & eser & WR08 \\
\hline $10 / 15 / 2003$ & $12: 40$ & na & 10.8 & 8.6 & 789 & 24.9 \\
\hline $12 / 17 / 2003$ & $13: 50$ & na & 10.3 & 9.0 & 976 & 14.2 \\
\hline $02 / 18 / 2004$ & $11: 40$ & na & 10.2 & 7.9 & 1,060 & 13.5 \\
\hline $04 / 14 / 2004$ & 09:30 & na & 10.3 & 7.8 & 1,010 & 20.3 \\
\hline $07 / 13 / 2004$ & $15: 10$ & na & 8.7 & 8.7 & 1,080 & 25.6 \\
\hline $10 / 12 / 2004$ & $15: 00$ & na & 8.8 & 8.2 & 1,040 & 24.7 \\
\hline $01 / 12 / 2005$ & $15: 00$ & na & 11.9 & 7.7 & 846 & 12.9 \\
\hline $04 / 13 / 2005$ & 09:30 & na & 9.8 & 8.3 & 986 & 19.0 \\
\hline $07 / 12 / 2005$ & $14: 10$ & na & 8.1 & 8.6 & 1,060 & 26.1 \\
\hline $10 / 11 / 2005$ & $13: 30$ & na & 9.6 & 8.4 & 1,110 & 21.9 \\
\hline 01/10/2006 & $15: 00$ & na & 12.3 & 7.8 & 1,180 & 16.1 \\
\hline $04 / 06 / 2006$ & $14: 10$ & na & 11.4 & 8.0 & 713 & 16.3 \\
\hline $07 / 11 / 2006$ & $14: 50$ & na & 7.7 & 8.1 & 1,020 & 28.7 \\
\hline $08 / 24 / 2006$ & $14: 30$ & na & 9.0 & 8.2 & 1,020 & 26.5 \\
\hline $10 / 11 / 2006$ & $13: 50$ & na & 10.3 & 8.4 & 1,050 & 23.4 \\
\hline 01/09/2007 & $13: 30$ & na & 12.9 & 8.4 & 735 & 13.7 \\
\hline $04 / 11 / 2007$ & $08: 30$ & na & 11.8 & 8.4 & 777 & 17.7 \\
\hline $07 / 10 / 2007$ & $13: 10$ & na & 9.3 & 8.2 & 885 & 26.6 \\
\hline $10 / 09 / 2007$ & $15: 10$ & na & 8.8 & 7.9 & 1,130 & 21.9 \\
\hline 01/08/2008 & $14: 00$ & na & 13.0 & 8.2 & 839 & 13.0 \\
\hline $04 / 15 / 2008$ & $13: 30$ & na & 12.1 & 8.5 & 686 & 19.4 \\
\hline $07 / 15 / 2008$ & $14: 10$ & na & 10.2 & 8.1 & 1,140 & 27.0 \\
\hline $10 / 15 / 2009$ & $14: 00$ & na & 10.7 & 7.6 & 1,180 & 21.9 \\
\hline $01 / 13 / 2009$ & $14: 00$ & na & 12.0 & 8.0 & 915 & 14.8 \\
\hline $04 / 14 / 2009$ & $14: 30$ & na & 9.2 & 8.1 & 869 & 18.5 \\
\hline $07 / 07 / 2009$ & $14: 00$ & na & 9.7 & 8.6 & 675 & 26.1 \\
\hline
\end{tabular}

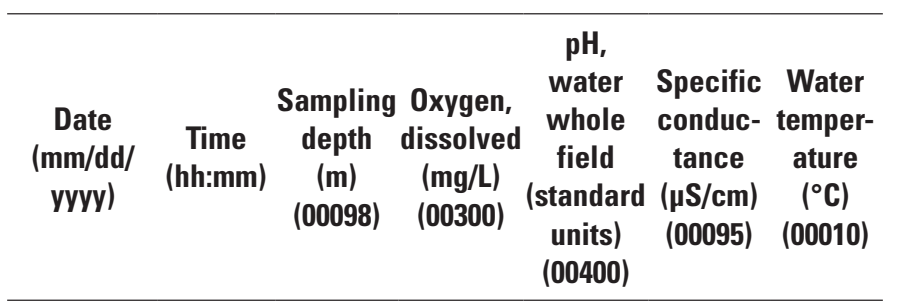

Perdue Treatment Plant-imported raw water at Sweetwater Reservoir (SWR09)

\begin{tabular}{|c|c|c|c|c|c|c|}
\hline$\overline{10 / 15 / 2003}$ & $13: 00$ & na & 8.3 & 8.7 & 778 & 24.9 \\
\hline $12 / 17 / 2003$ & $13: 30$ & na & 10.5 & 8.9 & 834 & 17.5 \\
\hline $02 / 18 / 2004$ & $12: 00$ & na & 10.9 & 8.3 & 442 & 14.7 \\
\hline $04 / 14 / 2004$ & $12: 20$ & na & 9.5 & 8.1 & 774 & 21.1 \\
\hline $10 / 12 / 2004$ & $16: 30$ & na & 8.3 & 8.2 & 775 & 24.0 \\
\hline $01 / 12 / 2005$ & $14: 40$ & na & 11.2 & 7.6 & 821 & 13.9 \\
\hline $04 / 13 / 2005$ & 08:40 & na & 9.3 & 8.6 & 707 & 18.5 \\
\hline $07 / 12 / 2005$ & $14: 00$ & na & 7.4 & 8.8 & 872 & 26.7 \\
\hline $10 / 11 / 2005$ & $13: 50$ & na & 7.8 & 8.4 & 880 & 23.4 \\
\hline 01/10/2006 & $14: 50$ & na & 11.9 & 8.3 & 845 & 15.4 \\
\hline $04 / 06 / 2006$ & $14: 20$ & na & 9.9 & 7.8 & 692 & 15.9 \\
\hline $07 / 11 / 2006$ & $14: 40$ & na & 8.3 & 8.3 & 720 & 28.4 \\
\hline $10 / 11 / 2006$ & $13: 40$ & na & 10.0 & 8.2 & 714 & 22.6 \\
\hline 01/09/2007 & $13: 20$ & na & 14.4 & 8.4 & 693 & 12.9 \\
\hline $04 / 11 / 2007$ & $08: 50$ & na & 12.5 & 8.3 & 747 & 18.5 \\
\hline 07/10/2007 & 13:00 & na & 9.7 & 8.2 & 863 & 27.0 \\
\hline $10 / 09 / 2007$ & $15: 00$ & na & 11.5 & 8.1 & 736 & 20.5 \\
\hline 01/08/2008 & $14: 10$ & na & 11.2 & 8.0 & 806 & 13.1 \\
\hline $04 / 15 / 2008$ & $13: 40$ & na & 11.4 & 8.3 & 672 & 19.9 \\
\hline $07 / 15 / 2008$ & $14: 00$ & na & 12.5 & 8.0 & 895 & 26.4 \\
\hline 01/13/2009 & $14: 10$ & na & 13.6 & 8.0 & 893 & 13.2 \\
\hline 04/14/2009 & $14: 20$ & na & 9.5 & 8.2 & 848 & 18.1 \\
\hline 07/07/2009 & $14: 20$ & na & 9.5 & 8.3 & 662 & 26.0 \\
\hline \multicolumn{7}{|c|}{$\begin{array}{l}\text { Sweetwater River at low-flow diversion dam upstream from } \\
\text { Sweetwater Reservoir (LFDD) }\end{array}$} \\
\hline $10 / 15 / 2003$ & 11:00 & 0.1 & 4.4 & 8.6 & 3,100 & 18.6 \\
\hline $12 / 17 / 2003$ & $13: 00$ & 0.1 & 10.3 & 8.9 & 2,800 & 9.5 \\
\hline $02 / 18 / 2004$ & $15: 10$ & 0.1 & 9.8 & 7.9 & 2,700 & 12.4 \\
\hline
\end{tabular}


Table 3. Water-quality depth-profile data for dissolved oxygen, $\mathrm{pH}$, specific conductance, and water temperature for each sampling site and period for the Sweetwater Reservoir watershed, San Diego County, California._-Continued

[Time is denoted in 24-hour format. The five-digit parameter code in parentheses below each compound name is used in the U.S. Geological Survey computerized data system (National Water Information System) to uniquely identify a specific constituent or property. Sampling depth is in meters below water surface. Shaded data coincide with reservoir water samples. Abbreviations: hh:mm, hour:minute; m, meter; mg/L, milligram per liter; mm/dd/yyyy, month/day/year; na, not applicable; PM, probe malfunction; $\mu \mathrm{S} / \mathrm{cm}$, microsiemen per centimeter; ${ }^{\circ} \mathrm{C}$, degree Celsius]

\begin{tabular}{|c|c|c|c|c|c|}
\hline $\begin{array}{c}\text { Date } \\
\text { (mm/dd/ } \\
\text { yyyy) }\end{array}$ & $\begin{array}{c}\text { Time } \\
\text { (hh:mm) }\end{array}$ & $\begin{array}{c}\begin{array}{c}\text { Sampling } \\
\text { Oxygen, } \\
\text { depth } \\
\text { dissolved } \\
(\mathrm{m})\end{array} \\
\begin{array}{cc}\text { (mg/L) } \\
(00098) & (00300)\end{array}\end{array}$ & $\begin{array}{c}\text { pH, } \\
\text { water } \\
\text { whole } \\
\text { field } \\
\text { (standard } \\
\text { units) } \\
\text { (00400) }\end{array}$ & $\begin{array}{c}\text { Specific } \\
\text { conduc- } \\
\text { tance } \\
(\mu \mathrm{S} / \mathrm{cm}) \\
(00095)\end{array}$ & $\begin{array}{c}\text { Water } \\
\text { temper- } \\
\text { ature } \\
\left({ }^{\circ} \mathrm{C}\right) \\
(00010)\end{array}$ \\
\hline
\end{tabular}

\begin{tabular}{|c|c|c|c|c|c|c|}
\hline \multicolumn{7}{|c|}{$\begin{array}{l}\text { Sweetwater River at low-flow diversion dam upstream from } \\
\text { Sweetwater Reservoir (LFDD)—Continued }\end{array}$} \\
\hline $04 / 14 / 2004$ & $10: 20$ & 0.1 & 7.3 & 7.8 & 2,860 & 16.8 \\
\hline $07 / 14 / 2004$ & $10: 10$ & 0.1 & 3.6 & 7.6 & 3,190 & 21.2 \\
\hline $10 / 13 / 2004$ & $15: 20$ & 0.1 & 5.8 & 7.6 & 3,120 & 18.9 \\
\hline $01 / 13 / 2005$ & 14:00 & 0.1 & 11.7 & 7.8 & 1,020 & 11.5 \\
\hline $04 / 13 / 2005$ & $10: 20$ & 0.1 & 9.7 & 8.1 & 1,830 & 14.9 \\
\hline $07 / 13 / 2005$ & $10: 00$ & 0.1 & 5.2 & 8.3 & 2,440 & 19.5 \\
\hline $10 / 12 / 2005$ & $13: 30$ & 0.1 & 9.5 & 8.1 & 2,580 & 17.2 \\
\hline $01 / 10 / 2006$ & $14: 20$ & 0.1 & 12.2 & 8.0 & 620 & 13.0 \\
\hline $04 / 07 / 2006$ & $13: 20$ & 0.1 & 11.5 & 7.5 & 1,780 & 14.1 \\
\hline $07 / 12 / 2006$ & $12: 30$ & 2.0 & 7.1 & 7.9 & 2,580 & 24.3 \\
\hline $10 / 11 / 2006$ & $13: 10$ & 0.1 & 8.8 & 7.9 & 2,630 & 17.7 \\
\hline 01/10/2007 & $13: 10$ & 0.1 & 13.3 & 7.9 & 2,790 & 10.2 \\
\hline $04 / 11 / 2007$ & $10: 20$ & 0.1 & 8.8 & 7.7 & 2,730 & 15.5 \\
\hline $07 / 11 / 2007$ & $14: 20$ & 0.1 & 10.4 & 7.6 & 2,910 & 22.6 \\
\hline $10 / 09 / 2007$ & $14: 20$ & 0.1 & 10.9 & 7.7 & 2,860 & 15.7 \\
\hline $01 / 09 / 2008$ & $13: 30$ & 0.1 & 13.7 & 7.7 & 2,370 & 12.1 \\
\hline $02 / 01 / 2008$ & 13:00 & 0.1 & 9.6 & 7.9 & 816 & 9.8 \\
\hline $04 / 16 / 2008$ & $10: 10$ & 0.1 & 10.0 & 6.8 & 2,710 & 15.8 \\
\hline $07 / 16 / 2008$ & $13: 20$ & 0.1 & 8.5 & 7.4 & 2,720 & 23.6 \\
\hline $10 / 15 / 2009$ & $13: 30$ & 0.1 & 9.0 & 7.2 & 2,820 & 15.2 \\
\hline $01 / 14 / 2009$ & $12: 40$ & 0.1 & 11.3 & 7.7 & 2,160 & 11.2 \\
\hline $04 / 15 / 2009$ & $10: 20$ & 0.1 & 8.6 & 7.9 & 2,480 & 13.9 \\
\hline $07 / 08 / 2009$ & $11: 00$ & 0.1 & 7.7 & 7.8 & 2,070 & 19.2 \\
\hline \multicolumn{7}{|c|}{ Loveland Reservoir near dam (LLR01) } \\
\hline $12 / 17 / 2003$ & $10: 19$ & 0.1 & 7.9 & 9.1 & 615 & 12.6 \\
\hline $12 / 17 / 2003$ & $10: 20$ & 1.0 & 7.3 & 8.9 & 617 & 12.4 \\
\hline $12 / 17 / 2003$ & $10: 21$ & 2.0 & 7.0 & 8.8 & 617 & 12.4 \\
\hline $12 / 17 / 2003$ & $10: 22$ & 3.0 & 7.0 & 8.8 & 616 & 12.4 \\
\hline $12 / 17 / 2003$ & $10: 23$ & 4.0 & 7.0 & 8.7 & 617 & 12.4 \\
\hline $12 / 17 / 2003$ & $10: 24$ & 5.0 & 7.0 & 8.7 & 617 & 12.3 \\
\hline $12 / 17 / 2003$ & $10: 25$ & 6.0 & 6.9 & 8.7 & 617 & 12.3 \\
\hline $12 / 17 / 2003$ & $10: 26$ & 7.0 & 6.9 & 8.6 & 617 & 12.3 \\
\hline $12 / 17 / 2003$ & $10: 27$ & 8.0 & 6.8 & 8.6 & 618 & 12.3 \\
\hline
\end{tabular}

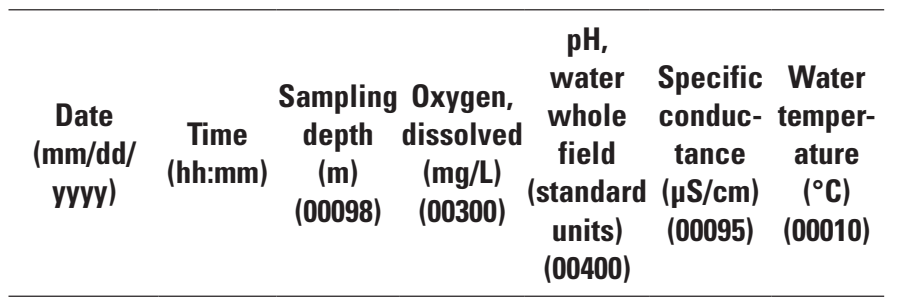

\begin{tabular}{lcrrrrr}
\hline \multicolumn{7}{c}{ Loveland Reservoir near dam (LLR01)—Continued } \\
\hline $12 / 17 / 2003$ & $10: 28$ & 9.0 & 6.8 & 8.6 & 618 & 12.3 \\
$12 / 17 / 2003$ & $10: 29$ & 10.0 & 6.7 & 8.6 & 617 & 12.3 \\
$12 / 17 / 2003$ & $10: 30$ & 12.0 & 6.7 & 8.5 & 618 & 12.3 \\
$12 / 17 / 2003$ & $10: 31$ & 14.0 & 6.6 & 8.5 & 618 & 12.3 \\
$12 / 17 / 2003$ & $10: 32$ & 16.0 & 6.5 & 8.5 & 617 & 12.3 \\
$12 / 17 / 2003$ & $10: 33$ & 18.0 & 6.3 & 8.4 & 617 & 12.3 \\
$12 / 17 / 2003$ & $10: 34$ & 20.0 & 6.2 & 8.4 & 617 & 12.3 \\
$12 / 17 / 2003$ & $10: 35$ & 22.0 & 6.3 & 8.4 & 617 & 12.2 \\
$12 / 17 / 2003$ & $10: 36$ & 24.0 & 6.3 & 8.4 & 617 & 12.2 \\
$12 / 17 / 2003$ & $10: 37$ & 26.0 & 6.2 & 8.4 & 617 & 12.2
\end{tabular}

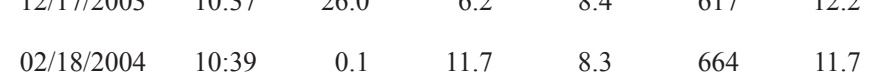

$02 / 18 / 2004 \quad 10: 40 \quad 1.0 \quad 11.7 \quad 8.3 \quad 664 \quad 11.6$

$\begin{array}{lllllll}02 / 18 / 2004 & 10: 41 & 2.0 & 11.6 & 8.3 & 664 & 11.5\end{array}$

$\begin{array}{lllllll}02 / 18 / 2004 & 10: 42 & 3.0 & 11.3 & 8.2 & 664 & 11.5\end{array}$

$\begin{array}{lllllll}02 / 18 / 2004 & 10: 43 & 4.0 & 11.0 & 8.2 & 665 & 11.4\end{array}$

$\begin{array}{lllllll}02 / 18 / 2004 & 10: 44 & 5.0 & 10.9 & 8.2 & 665 & 11.4\end{array}$

$\begin{array}{lllllll}02 / 18 / 2004 & 10: 45 & 6.0 & 10.8 & 8.2 & 665 & 11.4\end{array}$

$\begin{array}{lllllll}02 / 18 / 2004 & 10: 46 & 7.0 & 9.9 & 8.1 & 665 & 11.3\end{array}$

$\begin{array}{lllllll}02 / 18 / 2004 & 10: 47 & 8.0 & 9.7 & 8.1 & 666 & 11.3\end{array}$

$\begin{array}{lllllll}02 / 18 / 2004 & 10: 48 & 9.0 & 9.1 & 8.0 & 666 & 11.2\end{array}$

$\begin{array}{lllllll}02 / 18 / 2004 & 10: 49 & 10.0 & 8.8 & 8.0 & 666 & 11.1 \\ 02 / 18 / 2004 & 10: 50 & 12.0 & 8.6 & 8.0 & 666 & 11.1\end{array}$

$\begin{array}{lllllll}02 / 18 / 2004 & 10: 51 & 14.0 & 8.5 & 8.0 & 666 & 11.1\end{array}$

$\begin{array}{lllllll}02 / 18 / 2004 & 10: 52 & 16.0 & 8.4 & 8.0 & 666 & 11.1\end{array}$

$\begin{array}{lllllll}02 / 18 / 2004 & 10: 53 & 18.0 & 8.4 & 8.0 & 666 & 11.1\end{array}$

$\begin{array}{lllllll}02 / 18 / 2004 & 10: 54 & 20.0 & 8.4 & 8.0 & 666 & 11.1\end{array}$

$\begin{array}{lllllll}02 / 18 / 2004 & 10: 55 & 22.0 & 8.4 & 7.9 & 666 & 11.1\end{array}$

$\begin{array}{lllllll}02 / 18 / 2004 & 10: 56 & 24.0 & 8.3 & 8.0 & 667 & 11.1\end{array}$

$\begin{array}{lllllll}02 / 18 / 2004 & 10: 57 & 26.0 & 1.0 & 7.4 & 667 & 11.2\end{array}$

$\begin{array}{lllllll}07 / 14 / 2004 & 11: 46 & 0.1 & 9.2 & 8.5 & 712 & 26.0\end{array}$

$\begin{array}{lllllll}07 / 14 / 2004 & 11: 47 & 1.0 & 9.3 & 8.5 & 711 & 25.9\end{array}$

$\begin{array}{lllllll}07 / 14 / 2004 & 11: 48 & 2.0 & 9.4 & 8.5 & 711 & 25.9\end{array}$

$\begin{array}{lllllll}07 / 14 / 2004 & 12: 10 & 3.0 & 9.3 & 8.7 & 711 & 25.8\end{array}$

$\begin{array}{lllllll}07 / 14 / 2004 & 11: 49 & 4.0 & 9.3 & 8.5 & 711 & 25.6\end{array}$

$\begin{array}{lllllll}07 / 14 / 2004 & 11: 50 & 5.0 & 8.6 & 8.4 & 710 & 25.2\end{array}$

$\begin{array}{lllllll}07 / 14 / 2004 & 11: 51 & 6.0 & 3.8 & 7.9 & 702 & 27.9\end{array}$

$\begin{array}{lllllll}07 / 14 / 2004 & 11: 52 & 7.0 & 0.9 & 7.6 & 688 & 19.7\end{array}$

$\begin{array}{lllllll}07 / 14 / 2004 & 11: 53 & 8.0 & 0.7 & 7.6 & 683 & 17.2\end{array}$

$\begin{array}{lllllll}07 / 14 / 2004 & 11: 54 & 9.0 & 0.7 & 7.6 & 676 & 15.4\end{array}$

$\begin{array}{lllllll}07 / 14 / 2004 & 11: 55 & 10.0 & 1.0 & 7.6 & 676 & 14.2\end{array}$

$\begin{array}{lllllll}07 / 14 / 2004 & 11: 56 & 12.0 & 0.7 & 7.6 & 673 & 12.6\end{array}$

$\begin{array}{lllllll}07 / 14 / 2004 & 11: 57 & 14.0 & 0.4 & 7.5 & 672 & 11.9\end{array}$

$\begin{array}{lllllll}07 / 14 / 2004 & 11: 58 & 16.0 & 0.4 & 7.5 & 675 & 11.9\end{array}$

$\begin{array}{lllllll}07 / 14 / 2004 & 11: 59 & 18.0 & 0.3 & 7.5 & 675 & 11.8 \\ 07 / 14 / 2004 & 12.00 & 20.0 & 0.3 & 7.5 & 675 & 11.8\end{array}$

\begin{tabular}{lllllll}
$07 / 14 / 2004$ & 12.00 & 20.0 & 0.3 & 7.5 & 675 & 11.8 \\
\hline $07 / 14 / 2004$ & $12: 01$ & 22.0 & 0.3 & 7.5 & 675 & 11.8
\end{tabular} 
Table 3. Water-quality depth-profile data for dissolved oxygen, $\mathrm{pH}$, specific conductance, and water temperature for each sampling site and period for the Sweetwater Reservoir watershed, San Diego County, California.—Continued

[Time is denoted in 24-hour format. The five-digit parameter code in parentheses below each compound name is used in the U.S. Geological Survey computerized data system (National Water Information System) to uniquely identify a specific constituent or property. Sampling depth is in meters below water surface. Shaded data coincide with reservoir water samples. Abbreviations: hh:mm, hour:minute; m, meter; mg/L, milligram per liter; mm/dd/yyyy, month/day/year; na, not applicable; $\mathrm{PM}$, probe malfunction; $\mu \mathrm{S} / \mathrm{cm}$, microsiemen per centimeter; ${ }^{\circ} \mathrm{C}$, degree Celsius]

\begin{tabular}{|c|c|c|c|c|c|c|}
\hline $\begin{array}{c}\text { Date } \\
\text { (mm/dd/ } \\
\text { yyyy) }\end{array}$ & $\begin{array}{c}\text { Time } \\
\text { (hh:mm) }\end{array}$ & $\begin{array}{c}\text { Sampling } \\
\text { depth } \\
\text { (m) } \\
(00098)\end{array}$ & $\begin{array}{c}\text { Oxygen, } \\
\text { dissolved } \\
\text { (mg/L) } \\
(00300)\end{array}$ & $\begin{array}{c}\text { pH, } \\
\text { water } \\
\text { whole } \\
\text { field } \\
\text { (standard } \\
\text { units) } \\
(00400)\end{array}$ & $\begin{array}{c}\text { Specific } \\
\text { conduc- } \\
\text { tance } \\
(\mu \mathrm{S} / \mathrm{cm}) \\
(00095)\end{array}$ & $\begin{array}{c}\text { Water } \\
\text { temper- } \\
\text { ature } \\
\left({ }^{\circ} \mathrm{C}\right) \\
(00010)\end{array}$ \\
\hline \multicolumn{7}{|c|}{ Loveland Reservoir near dam (LLR01)—Continued } \\
\hline $07 / 14 / 2004$ & $12: 02$ & 24.0 & 0.3 & 7.5 & 674 & 11.8 \\
\hline $07 / 14 / 2004$ & $12: 03$ & 26.0 & 0.3 & 7.5 & 675 & 11.7 \\
\hline $07 / 14 / 2004$ & $12: 04$ & 27.0 & 0.3 & 7.3 & 701 & 11.8 \\
\hline $10 / 14 / 2004$ & $10: 47$ & 0.1 & 8.2 & 8.6 & 704 & 21.3 \\
\hline $10 / 14 / 2004$ & $10: 48$ & 1.0 & 8.0 & 8.4 & 704 & 21.3 \\
\hline $10 / 14 / 2004$ & $10: 49$ & 2.0 & 7.9 & 8.4 & 703 & 21.3 \\
\hline $10 / 14 / 2004$ & $10: 50$ & 3.0 & 7.9 & 8.3 & 703 & 21.2 \\
\hline $10 / 14 / 2004$ & $10: 51$ & 4.0 & 7.8 & 8.3 & 703 & 21.2 \\
\hline $10 / 14 / 2004$ & $11: 20$ & 5.0 & 7.8 & 8.3 & 703 & 21.2 \\
\hline $10 / 14 / 2004$ & $10: 52$ & 6.0 & 7.8 & 8.3 & 703 & 21.2 \\
\hline $10 / 14 / 2004$ & $10: 53$ & 7.0 & 7.8 & 8.2 & 703 & 21.2 \\
\hline $10 / 14 / 2004$ & $10: 54$ & 8.0 & 7.8 & 8.2 & 703 & 21.2 \\
\hline $10 / 14 / 2004$ & $10: 55$ & 9.0 & 7.8 & 8.2 & 703 & 21.2 \\
\hline $10 / 14 / 2004$ & $10: 56$ & 10.0 & 7.7 & 8.2 & 703 & 21.1 \\
\hline $10 / 14 / 2004$ & $10: 57$ & 12.0 & 3.0 & 8.1 & 668 & 13.8 \\
\hline $10 / 14 / 2004$ & $10: 58$ & 14.0 & 1.4 & 8.0 & 667 & 12.4 \\
\hline $10 / 14 / 2004$ & $10: 59$ & 16.0 & 1.0 & 7.9 & 668 & 12.2 \\
\hline $10 / 14 / 2004$ & $11: 00$ & 18.0 & 0.8 & 7.8 & 670 & 12.1 \\
\hline $10 / 14 / 2004$ & 11:01 & 20.0 & 0.7 & 7.8 & 668 & 12.1 \\
\hline $10 / 14 / 2004$ & 11:02 & 22.0 & 0.6 & 7.7 & 668 & 12.0 \\
\hline $10 / 14 / 2004$ & $11: 03$ & 24.0 & 0.5 & 7.7 & 668 & 12.0 \\
\hline $10 / 14 / 2004$ & 11:04 & 26.0 & 0.5 & 7.6 & 668 & 12.0 \\
\hline $10 / 14 / 2004$ & $11: 05$ & 27.8 & 0.5 & 7.5 & 671 & 12.0 \\
\hline $01 / 13 / 2005$ & $11: 07$ & 0.1 & 10.4 & 8.2 & 518 & 11.7 \\
\hline $01 / 13 / 2005$ & $11: 08$ & 1.0 & 9.6 & 8.0 & 512 & 11.1 \\
\hline $01 / 13 / 2005$ & 11:09 & 2.0 & 9.3 & 8.0 & 511 & 11.1 \\
\hline $01 / 13 / 2005$ & $11: 10$ & 3.0 & 9.1 & 7.9 & 511 & 11.0 \\
\hline $01 / 13 / 2005$ & $11: 11$ & 4.0 & 9.1 & 7.8 & 515 & 11.0 \\
\hline $01 / 13 / 2005$ & $11: 12$ & 5.0 & 9.0 & 7.8 & 521 & 11.0 \\
\hline $01 / 13 / 2005$ & $11: 13$ & 6.0 & 9.0 & 7.8 & 537 & 11.0 \\
\hline $01 / 13 / 2005$ & $11: 14$ & 7.0 & 8.9 & 7.7 & 542 & 11.0 \\
\hline $01 / 13 / 2005$ & $11: 15$ & 8.0 & 8.9 & 7.7 & 542 & 11.0 \\
\hline $01 / 13 / 2005$ & $11: 16$ & 9.0 & 8.8 & 7.7 & 544 & 11.0 \\
\hline $01 / 13 / 2005$ & $11: 17$ & 10.0 & 8.8 & 7.7 & 546 & 11.0 \\
\hline $01 / 13 / 2005$ & $11: 18$ & 12.0 & 8.8 & 7.7 & 550 & 10.9 \\
\hline $01 / 13 / 2005$ & $11: 19$ & 14.0 & 8.7 & 7.6 & 552 & 10.9 \\
\hline $01 / 13 / 2005$ & $11: 20$ & 16.0 & 8.7 & 7.6 & 556 & 10.9 \\
\hline $01 / 13 / 2005$ & $11: 21$ & 18.0 & 8.4 & 7.6 & 571 & 10.9 \\
\hline $01 / 13 / 2005$ & $11: 22$ & 20.0 & 8.2 & 7.6 & 574 & 10.8 \\
\hline $01 / 13 / 2005$ & $11: 23$ & 22.0 & 8.2 & 7.6 & 561 & 10.7 \\
\hline $01 / 13 / 2005$ & $11: 24$ & 24.0 & 8.4 & 7.6 & 507 & 10.2 \\
\hline $01 / 13 / 2005$ & $11: 25$ & 26.0 & 8.4 & 7.6 & 493 & 10.1 \\
\hline
\end{tabular}

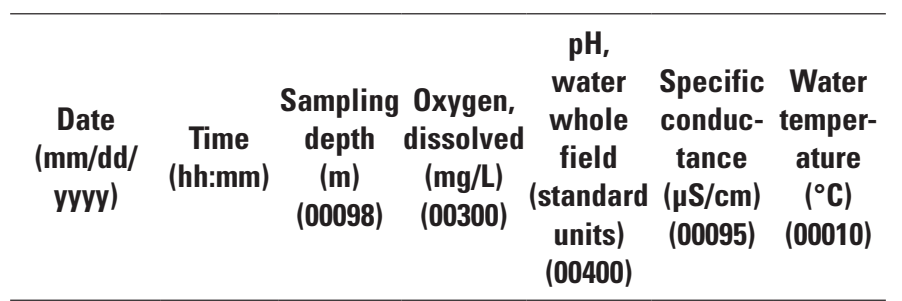

\begin{tabular}{lcccccc}
\hline \multicolumn{7}{c}{ Loveland Reservoir near dam (LLR01)—Continued } \\
\hline $01 / 13 / 2005$ & $11: 26$ & 28.0 & 8.4 & 7.5 & 505 & 10.1 \\
$01 / 13 / 2005$ & $11: 27$ & 30.0 & 6.9 & 7.5 & 531 & 10.3
\end{tabular}

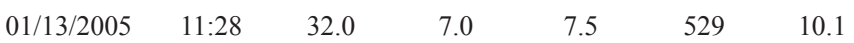

$\begin{array}{lllllll}01 / 13 / 2005 & 11: 29 & 33.4 & 7.4 & 7.4 & 558 & 10.2\end{array}$

$\begin{array}{lllllll}10 / 12 / 2005 & 10: 42 & 0.1 & 8.4 & 8.7 & 512 & 20.5\end{array}$

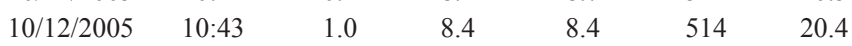

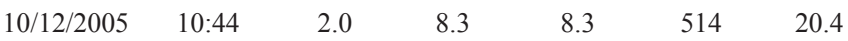

$\begin{array}{lllllll}10 / 12 / 2005 & 10: 45 & 3.0 & 8.3 & 8.2 & 514 & 20.3\end{array}$

$\begin{array}{lllllll}10 / 12 / 2005 & 11: 10 & 4.0 & 8.2 & 8.2 & 514 & 20.3\end{array}$

$\begin{array}{lllllll}10 / 12 / 2005 & 10: 46 & 5.0 & 8.1 & 8.2 & 514 & 20.3\end{array}$

$\begin{array}{lllllll}10 / 12 / 2005 & 10: 47 & 6.0 & 8.1 & 8.2 & 514 & 20.3\end{array}$

$\begin{array}{lllllll}10 / 12 / 2005 & 10: 48 & 7.0 & 8.1 & 8.1 & 514 & 20.2\end{array}$

$\begin{array}{lllllll}10 / 12 / 2005 & 10: 49 & 8.0 & 8.1 & 8.1 & 514 & 20.2\end{array}$

$\begin{array}{lllllll}10 / 12 / 2005 & 10: 50 & 9.0 & 1.7 & 8.0 & 432 & 16.6\end{array}$

$\begin{array}{lllllll}10 / 12 / 2005 & 10: 51 & 10.0 & 0.9 & 7.9 & 427 & 15.1\end{array}$

$\begin{array}{lllllll}10 / 12 / 2005 & 10: 52 & 12.0 & 0.7 & 7.8 & 421 & 13.9\end{array}$

$\begin{array}{lllllll}10 / 12 / 2005 & 10: 53 & 14.0 & 0.6 & 7.7 & 422 & 13.2\end{array}$

$\begin{array}{lllllll}10 / 12 / 2005 & 10: 54 & 16.0 & 0.6 & 7.6 & 436 & 12.4\end{array}$

$\begin{array}{lllllll}10 / 12 / 2005 & 10.55 & 18.0 & 0.5 & 8.0 & 442 & 12.1\end{array}$

$\begin{array}{lllllll}10 / 12 / 2005 & 10: 56 & 20.0 & 0.5 & 7.6 & 444 & 12.0\end{array}$

$\begin{array}{lllllll}10 / 12 / 2005 & 10: 57 & 22.0 & 0.5 & 7.7 & 445 & 11.9\end{array}$

$\begin{array}{lllllll}10 / 12 / 2005 & 10: 58 & 24.0 & 0.5 & 7.5 & 445 & 11.9\end{array}$

$\begin{array}{lllllll}10 / 12 / 2005 & 10: 59 & 26.0 & 0.5 & 7.8 & 445 & 11.8\end{array}$

$\begin{array}{lllllll}10 / 12 / 2005 & 11: 00 & 28.0 & 0.5 & 7.8 & 446 & 11.8 \\ 10 / 12 / 2005 & 11: 01 & 30.0 & 0.4 & 7.6 & 447 & 11.8\end{array}$

$\begin{array}{lllllll}10 / 12 / 2005 & 11: 02 & 32.0 & 0.4 & 7.4 & 447 & 11.8\end{array}$

$\begin{array}{lllllll}10 / 12 / 2005 & 11: 03 & 34.0 & 0.4 & 7.5 & 449 & 11.7\end{array}$

$\begin{array}{lllllll}10 / 12 / 2005 & 11: 04 & 36.0 & 0.4 & 7.6 & 450 & 11.7\end{array}$

$\begin{array}{lllllll}10 / 12 / 2005 & 11: 05 & 38.0 & 0.4 & 7.3 & 450 & 11.7\end{array}$

$\begin{array}{lllllll}10 / 12 / 2005 & 11: 06 & 40.0 & 0.4 & 7.3 & 450 & 11.7\end{array}$

$\begin{array}{lllllll}10 / 12 / 2005 & 11: 07 & 42.0 & 0.4 & 7.3 & 452 & 11.7\end{array}$

$\begin{array}{lllllll}10 / 12 / 2005 & 11: 08 & 44.3 & 0.4 & 7.2 & 489 & 11.7\end{array}$

\begin{tabular}{lllllll}
$04 / 06 / 2006$ & $11: 20$ & 0.1 & 9.7 & 5.2 & 536 & 15.2 \\
\hline $04 / 06 / 2006$ & $11: 06$ & 1.0 & 9.6 & 5.8 & 535 & 14.9 \\
$04 / 06 / 2006$ & $11: 07$ & 2.0 & 9.6 & 6.1 & 535 & 14.7 \\
$04 / 06 / 2006$ & $11: 08$ & 3.0 & 9.5 & 6.2 & 535 & 14.7 \\
$04 / 06 / 2006$ & $11: 09$ & 4.0 & 9.4 & 6.3 & 536 & 14.4 \\
$04 / 06 / 2006$ & $11: 10$ & 5.0 & 9.2 & 6.4 & 536 & 14.4 \\
$04 / 06 / 2006$ & $11: 11$ & 6.0 & 8.9 & 6.5 & 536 & 14.1 \\
$04 / 06 / 2006$ & $11: 12$ & 7.0 & 8.6 & 6.5 & 535 & 13.8 \\
$04 / 06 / 2006$ & $11: 13$ & 8.0 & 8.2 & 6.5 & 535 & 13.4 \\
$04 / 06 / 2006$ & $11: 14$ & 9.0 & 7.3 & 6.5 & 534 & 12.6 \\
$04 / 06 / 2006$ & $11: 15$ & 10.0 & 6.6 & 6.5 & 533 & 12.3
\end{tabular}


Table 3. Water-quality depth-profile data for dissolved oxygen, $\mathrm{pH}$, specific conductance, and water temperature for each sampling site and period for the Sweetwater Reservoir watershed, San Diego County, California.-Continued

[Time is denoted in 24-hour format. The five-digit parameter code in parentheses below each compound name is used in the U.S. Geological Survey computerized data system (National Water Information System) to uniquely identify a specific constituent or property. Sampling depth is in meters below water surface. Shaded data coincide with reservoir water samples. Abbreviations: hh:mm, hour:minute; m, meter; mg/L, milligram per liter; mm/dd/yyyy, month/day/year; na, not applicable; PM, probe malfunction; $\mu \mathrm{S} / \mathrm{cm}$, microsiemen per centimeter; ${ }^{\circ} \mathrm{C}$, degree Celsius]

\begin{tabular}{|c|c|c|c|c|c|c|}
\hline $\begin{array}{c}\text { Date } \\
\text { (mm/dd/ } \\
\text { yyyy) }\end{array}$ & $\begin{array}{c}\text { Time } \\
\text { (hh:mm) }\end{array}$ & $\begin{array}{c}\text { Sampling } \\
\text { depth } \\
\text { (m) } \\
(00098)\end{array}$ & $\begin{array}{c}\text { g Oxygen, } \\
\text { dissolved } \\
(\mathrm{mg} / \mathrm{L}) \\
(00300)\end{array}$ & $\begin{array}{c}\mathrm{pH}, \\
\text { water } \\
\text { whole } \\
\text { field } \\
\text { (standard } \\
\text { units) } \\
(00400)\end{array}$ & $\begin{array}{c}\text { Specific } \\
\text { conduc- } \\
\text { tance } \\
(\mu \mathrm{S} / \mathrm{cm}) \\
(00095)\end{array}$ & $\begin{array}{c}\text { Water } \\
\text { temper- } \\
\text { ature } \\
\left({ }^{\circ} \mathrm{C}\right) \\
(\mathbf{0 0 0 1 0})\end{array}$ \\
\hline
\end{tabular}

\begin{tabular}{lcccccc}
\hline \multicolumn{7}{c}{ Loveland Reservoir near dam (LLR01)-Continued } \\
\hline $04 / 06 / 2006$ & $11: 16$ & 12.0 & 6.1 & 6.4 & 533 & 12.2 \\
$04 / 06 / 2006$ & $11: 17$ & 14.0 & 5.6 & 6.4 & 533 & 12.1 \\
$04 / 06 / 2006$ & $11: 18$ & 16.0 & 5.2 & 6.4 & 533 & 12.1 \\
$04 / 06 / 2006$ & $11: 19$ & 18.0 & 5.1 & 6.4 & 533 & 12.1 \\
$04 / 06 / 2006$ & $11: 21$ & 20.0 & 5.1 & 6.4 & 533 & 12.1 \\
$04 / 06 / 2006$ & $11: 22$ & 22.0 & 5.1 & 6.4 & 533 & 12.1 \\
$04 / 06 / 2006$ & $11: 23$ & 24.0 & 5.2 & 6.4 & 533 & 12.1 \\
$04 / 06 / 2006$ & $11: 24$ & 26.0 & 5.3 & 6.5 & 532 & 12.1 \\
$04 / 06 / 2006$ & $11: 25$ & 28.0 & 5.5 & 6.4 & 532 & 12.0 \\
$04 / 06 / 2006$ & $11: 26$ & 30.0 & 5.4 & 6.5 & 532 & 12.0 \\
$04 / 06 / 2006$ & $11: 27$ & 32.0 & 5.3 & 6.5 & 532 & 12.0 \\
$04 / 06 / 2006$ & $11: 28$ & 34.0 & 5.0 & 6.5 & 532 & 12.0 \\
$07 / 12 / 2006$ & $10: 50$ & 0.1 & 9.0 & 8.7 & 578 & 26.9 \\
\hline $07 / 12 / 2006$ & $10: 28$ & 1.0 & 8.4 & 8.7 & 576 & 26.7 \\
$07 / 12 / 2006$ & $10: 29$ & 2.0 & 8.3 & 8.7 & 577 & 26.7 \\
$07 / 12 / 2006$ & $10: 30$ & 3.0 & 8.3 & 8.7 & 577 & 26.7 \\
$07 / 12 / 2006$ & $10: 31$ & 4.0 & 8.2 & 8.7 & 577 & 26.6 \\
$07 / 12 / 2006$ & $10: 32$ & 5.0 & 8.1 & 8.7 & 577 & 26.5 \\
$07 / 12 / 2006$ & $10: 33$ & 6.0 & 3.0 & 7.7 & 548 & 20.9 \\
$07 / 12 / 2006$ & $10: 34$ & 7.0 & 1.9 & 7.5 & 538 & 17.6 \\
$07 / 12 / 2006$ & $10: 35$ & 8.0 & 1.7 & 7.5 & 535 & 16.2 \\
$07 / 12 / 2006$ & $10: 36$ & 9.0 & 1.4 & 7.4 & 534 & 14.9 \\
$07 / 12 / 2006$ & $10: 37$ & 10.0 & 1.3 & 7.4 & 532 & 14.2 \\
$07 / 12 / 2006$ & $10: 38$ & 12.0 & 1.2 & 7.4 & 528 & 12.8 \\
$07 / 12 / 2006$ & $10: 39$ & 14.0 & 1.2 & 7.4 & 528 & 12.5 \\
$07 / 12 / 2006$ & $10: 40$ & 16.0 & 1.2 & 7.4 & 527 & 12.3 \\
$07 / 12 / 2006$ & $10: 41$ & 18.0 & 1.2 & 7.4 & 528 & 12.3 \\
$07 / 12 / 2006$ & $10: 42$ & 20.0 & 1.2 & 7.4 & 528 & 12.3 \\
$07 / 12 / 2006$ & $10: 43$ & 22.0 & 1.2 & 7.4 & 527 & 12.3 \\
$07 / 12 / 2006$ & $10: 44$ & 24.0 & 1.2 & 7.4 & 529 & 12.2 \\
$07 / 12 / 2006$ & $10: 45$ & 26.0 & 1.2 & 7.4 & 528 & 12.2 \\
$07 / 12 / 2006$ & $10: 46$ & 28.0 & 1.2 & 7.4 & 528 & 12.2 \\
$07 / 12 / 2006$ & $10: 47$ & 30.0 & 1.3 & 7.4 & 528 & 12.2 \\
$07 / 12 / 2006$ & $10: 48$ & 32.0 & 1.3 & 7.4 & 529 & 12.2 \\
$07 / 12 / 2006$ & $10: 49$ & 34.0 & 1.3 & 7.4 & 537 & 12.2 \\
$07 / 12 / 2006$ & $10: 51$ & 35.2 & 1.3 & 7.3 & 575 & 12.2 \\
$08 / 24 / 2006$ & $09: 59$ & 0.1 & 11.4 & 8.6 & 579 & 26.0 \\
$08 / 24 / 2006$ & $10: 00$ & 1.0 & 10.9 & 8.6 & 579 & 25.9 \\
$08 / 24 / 2006$ & $10: 01$ & 2.0 & 10.7 & 8.7 & 579 & 25.8 \\
$08 / 24 / 2006$ & $10: 02$ & 3.0 & 10.5 & 8.6 & 579 & 25.8 \\
$08 / 24 / 2006$ & $10: 03$ & 4.0 & 10.4 & 8.6 & 579 & 25.7 \\
& & & & & &
\end{tabular}

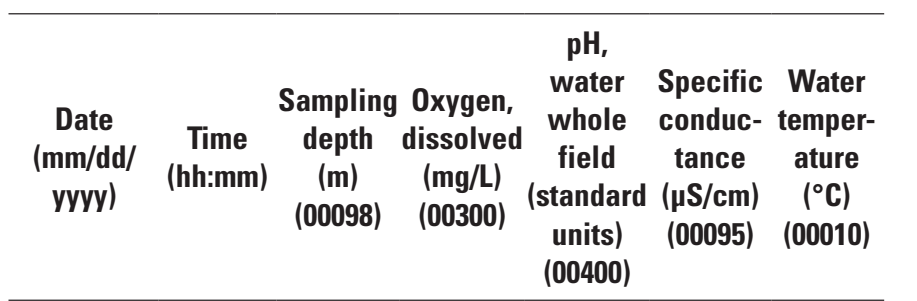

\begin{tabular}{lcrrrrr}
\hline \multicolumn{7}{c}{ Loveland Reservoir near dam (LLR01)—Continued } \\
\hline $08 / 24 / 2006$ & $10: 04$ & 5.0 & 10.2 & 8.7 & 579 & 25.6 \\
$08 / 24 / 2006$ & $10: 05$ & 6.0 & 6.5 & 8.4 & 576 & 24.5
\end{tabular}

$\begin{array}{rrrrrrr}01 / 10 / 2007 & 11: 00 & 0.1 & 11.6 & 8.4 & 570 & 11.5 \\ 01 / 10 / 2007 & 11: 01 & 1.0 & 9.9 & 8.2 & 572 & 11.3 \\ 01 / 10 / 2007 & 11: 02 & 2.0 & 9.5 & 8.1 & 572 & 11.3\end{array}$

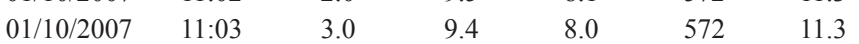

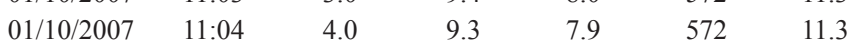

$\begin{array}{lllllll}01 / 10 / 2007 & 11: 05 & 5.0 & 9.2 & 7.8 & 571 & 11.3\end{array}$

$\begin{array}{lllllll}01 / 10 / 2007 & 11: 06 & 6.0 & 9.1 & 7.8 & 571 & 11.3\end{array}$

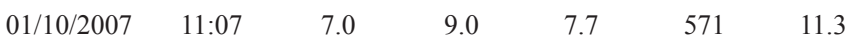

$\begin{array}{lllllll}01 / 10 / 2007 & 11: 08 & 8.0 & 9.0 & 7.7 & 571 & 11.3\end{array}$

$\begin{array}{lllllll}01 / 10 / 2007 & 11: 09 & 9.0 & 8.9 & 7.7 & 571 & 11.3\end{array}$

$\begin{array}{lllllll}01 / 10 / 2007 & 11: 10 & 10.0 & 8.9 & 7.7 & 571 & 11.3\end{array}$

$\begin{array}{lllllll}01 / 10 / 2007 & 11: 11 & 12.0 & 8.9 & 7.7 & 572 & 11.3\end{array}$

$\begin{array}{lllllll}01 / 10 / 2007 & 11: 12 & 14.0 & 8.8 & 7.7 & 572 & 11.3\end{array}$

$\begin{array}{lllllll}01 / 10 / 2007 & 11: 13 & 16.0 & 8.8 & 7.6 & 573 & 11.3\end{array}$

$\begin{array}{lllllll}01 / 10 / 2007 & 11: 14 & 18.0 & 8.8 & 7.6 & 573 & 11.3\end{array}$

$\begin{array}{lllllll}01 / 10 / 2007 & 11: 15 & 20.0 & 8.7 & 7.6 & 573 & 11.3\end{array}$

$\begin{array}{lllllll}01 / 10 / 2007 & 11: 16 & 22.0 & 8.6 & 7.6 & 573 & 11.3\end{array}$

$\begin{array}{lllllll}01 / 10 / 2007 & 11: 17 & 24.0 & 8.5 & 7.6 & 573 & 11.3\end{array}$

$\begin{array}{lllllll}01 / 10 / 2007 & 11: 18 & 26.0 & 8.4 & 7.6 & 573 & 11.2\end{array}$

$\begin{array}{lllllll}01 / 10 / 2007 & 11: 19 & 28.0 & 8.3 & 7.6 & 574 & 11.2\end{array}$

$\begin{array}{lllllll}01 / 10 / 2007 & 11: 20 & 30.0 & 8.3 & 7.6 & 574 & 11.2\end{array}$

$\begin{array}{lllllll}01 / 10 / 2007 & 11: 21 & 32.0 & 8.3 & 7.6 & 574 & 11.2\end{array}$

$\begin{array}{lllllll}01 / 10 / 2007 & 11: 22 & 34.0 & 8.1 & 7.6 & 575 & 11.2\end{array}$

$\begin{array}{lllllll}01 / 10 / 2007 & 11: 23 & 34.8 & 4.4 & 7.5 & 593 & 11.3\end{array}$

\begin{tabular}{lllllll}
$07 / 11 / 2007$ & $11: 10$ & 0.1 & 10.9 & 8.5 & 627 & 25.3 \\
\hline $07 / 11 / 2007$ & $10: 57$ & 1.0 & 10.7 & 8.4 & 626 & 25.2
\end{tabular}

$\begin{array}{lllllll}07 / 11 / 2007 & 10: 57 & 1.0 & 10.7 & 8.4 & 626 & 25.2 \\ 07 / 11 / 2007 & 10: 59 & 2.0 & 10.5 & 8.4 & 625 & 25.2\end{array}$

$\begin{array}{lllllll}07 / 11 / 2007 & 11: 00 & 3.0 & 10.3 & 8.3 & 625 & 25.1\end{array}$

$\begin{array}{lllllll}07 / 11 / 2007 & 11: 01 & 4.0 & 10.2 & 8.3 & 625 & 25.0\end{array}$

$\begin{array}{lllllll}07 / 11 / 2007 & 11: 02 & 5.0 & 10.0 & 8.2 & 625 & 24.9\end{array}$

$\begin{array}{lllllll}07 / 11 / 2007 & 11: 03 & 6.0 & 6.0 & 8.0 & 619 & 21.5\end{array}$

$\begin{array}{lllllll}07 / 11 / 2007 & 11: 04 & 7.0 & 1.9 & 7.5 & 613 & 18.0\end{array}$

$\begin{array}{lllllll}07 / 11 / 2007 & 11: 05 & 8.0 & 1.8 & 7.5 & 610 & 16.0\end{array}$

$\begin{array}{lllllll}07 / 11 / 2007 & 11: 06 & 9.0 & 1.7 & 7.4 & 606 & 14.7\end{array}$

$\begin{array}{lllllll}07 / 11 / 2007 & 11: 07 & 10.0 & 1.6 & 7.3 & 606 & 13.3\end{array}$

$\begin{array}{lllllll}07 / 11 / 2007 & 11: 08 & 12.0 & 1.7 & 7.3 & 602 & 11.7\end{array}$

$\begin{array}{lllllll}07 / 11 / 2007 & 11: 09 & 14.0 & 1.9 & 7.2 & 600 & 11.2\end{array}$

$\begin{array}{lllllll}07 / 11 / 2007 & 11: 11 & 16.0 & 2.2 & 7.2 & 598 & 11.1\end{array}$

$\begin{array}{lllllll}07 / 11 / 2007 & 11: 12 & 18.0 & 2.1 & 7.2 & 598 & 11.0\end{array}$

$\begin{array}{lllllll}07 / 11 / 2007 & 11: 13 & 20.0 & 1.9 & 7.2 & 599 & 11.0\end{array}$ 
Table 3. Water-quality depth-profile data for dissolved oxygen, $\mathrm{pH}$, specific conductance, and water temperature for each sampling site and period for the Sweetwater Reservoir watershed, San Diego County, California.—Continued

[Time is denoted in 24-hour format. The five-digit parameter code in parentheses below each compound name is used in the U.S. Geological Survey computerized data system (National Water Information System) to uniquely identify a specific constituent or property. Sampling depth is in meters below water surface. Shaded data coincide with reservoir water samples. Abbreviations: hh:mm, hour:minute; m, meter; mg/L, milligram per liter; mm/dd/yyyy, month/day/year; na, not applicable; $\mathrm{PM}$, probe malfunction; $\mu \mathrm{S} / \mathrm{cm}$, microsiemen per centimeter; ${ }^{\circ} \mathrm{C}$, degree Celsius]

\begin{tabular}{|c|c|c|c|c|c|c|}
\hline $\begin{array}{c}\text { Date } \\
\text { (mm/dd/ } \\
\text { yyyy) }\end{array}$ & $\begin{array}{c}\text { Time } \\
\text { (hh:mm) }\end{array}$ & $\begin{array}{c}\text { Sampling } \\
\text { depth } \\
\text { (m) } \\
(00098)\end{array}$ & $\begin{array}{c}\text { Oxygen, } \\
\text { dissolved } \\
\text { (mg/L) } \\
(00300)\end{array}$ & $\begin{array}{c}\mathrm{pH}, \\
\text { water } \\
\text { whole } \\
\text { field } \\
\text { (standard } \\
\text { units) } \\
(00400)\end{array}$ & $\begin{array}{c}\text { Specific } \\
\text { conduc- } \\
\text { tance } \\
(\mu \mathrm{S} / \mathrm{cm}) \\
(00095)\end{array}$ & $\begin{array}{c}\text { Water } \\
\text { temper- } \\
\text { ature } \\
\left({ }^{\circ} \mathrm{C}\right) \\
(\mathbf{0 0 0 1 0})\end{array}$ \\
\hline
\end{tabular}

\begin{tabular}{lcccccc}
\hline & Loveland Reservoir near dam (LLR01)-Continued & \\
\hline $07 / 11 / 2007$ & $11: 14$ & 22.0 & 1.9 & 7.1 & 598 & 11.0 \\
$07 / 11 / 2007$ & $11: 15$ & 24.0 & 2.0 & 7.1 & 599 & 11.0 \\
$07 / 11 / 2007$ & $11: 16$ & 26.0 & 2.1 & 7.1 & 599 & 10.9 \\
$07 / 11 / 2007$ & $11: 17$ & 28.0 & 2.2 & 7.1 & 598 & 10.9 \\
$07 / 11 / 2007$ & $11: 18$ & 30.0 & 2.1 & 7.1 & 598 & 10.9 \\
$07 / 11 / 2007$ & $11: 19$ & 32.0 & 1.7 & 7.1 & 598 & 10.9 \\
$07 / 11 / 2007$ & $11: 20$ & 34.0 & 1.6 & 7.1 & 598 & 10.9 \\
$07 / 11 / 2007$ & $11: 21$ & 36.0 & 1.2 & 7.1 & 606 & 10.9 \\
$07 / 11 / 2007$ & $11: 22$ & 38.0 & 1.1 & 7.0 & 640 & 11.0 \\
$07 / 11 / 2007$ & $11: 23$ & 40.0 & 1.1 & 7.0 & 644 & 11.0 \\
$01 / 09 / 2008$ & $10: 50$ & 0.1 & 13.7 & 8.1 & 625 & 11.6 \\
$01 / 09 / 2008$ & $10: 51$ & 1.0 & 11.3 & 8.0 & 625 & 11.3 \\
$01 / 09 / 2008$ & $10: 52$ & 2.0 & 10.3 & 8.0 & 624 & 11.3 \\
$01 / 09 / 2008$ & $10: 53$ & 3.0 & 9.6 & 8.1 & 625 & 11.2 \\
$01 / 09 / 2008$ & $10: 54$ & 4.0 & 9.4 & 7.8 & 625 & 11.2 \\
$01 / 09 / 2008$ & $10: 55$ & 5.0 & 9.3 & 7.9 & 626 & 11.2 \\
$01 / 09 / 2008$ & $10: 56$ & 6.0 & 9.3 & 7.8 & 626 & 11.2 \\
$01 / 09 / 2008$ & $10: 57$ & 7.0 & 9.2 & 7.8 & 625 & 11.2 \\
$01 / 09 / 2008$ & $10: 58$ & 8.0 & 9.2 & 7.8 & 625 & 11.2 \\
$01 / 09 / 2008$ & $10: 59$ & 9.0 & 9.1 & 7.7 & 625 & 11.2 \\
$01 / 09 / 2008$ & $11: 00$ & 10.0 & 9.1 & 7.7 & 625 & 11.2 \\
$01 / 09 / 2008$ & $11: 01$ & 12.0 & 9.1 & 7.7 & 625 & 11.2 \\
$01 / 09 / 2008$ & $11: 02$ & 14.0 & 9.0 & 7.7 & 625 & 11.2 \\
$01 / 09 / 2008$ & $11: 03$ & 16.0 & 8.8 & 7.7 & 626 & 11.1 \\
$01 / 09 / 2008$ & $11: 04$ & 18.0 & 8.7 & 7.7 & 625 & 11.1 \\
$01 / 09 / 2008$ & $11: 05$ & 20.0 & 8.7 & 7.7 & 625 & 11.1 \\
$01 / 09 / 2008$ & $11: 06$ & 22.0 & 8.7 & 7.7 & 626 & 11.1 \\
$01 / 09 / 2008$ & $11: 07$ & 24.0 & 8.7 & 7.7 & 626 & 11.1 \\
$01 / 09 / 2008$ & $11: 08$ & 26.0 & 8.7 & 7.6 & 626 & 11.1 \\
$01 / 09 / 2008$ & $11: 09$ & 28.0 & 8.7 & 7.6 & 626 & 11.1 \\
$01 / 09 / 2008$ & $11: 10$ & 30.0 & 8.7 & 7.6 & 626 & 11.1 \\
$01 / 09 / 2008$ & $11: 11$ & 32.0 & 8.7 & 7.6 & 626 & 11.1 \\
$01 / 09 / 2008$ & $11: 12$ & 34.0 & 8.6 & 7.6 & 627 & 11.1 \\
$01 / 09 / 2008$ & $11: 13$ & 36.0 & 7.9 & 7.6 & 632 & 11.1 \\
$04 / 16 / 2008$ & $11: 30$ & 0.1 & 12.0 & 8.0 & 573 & 17.9 \\
$04 / 16 / 2008$ & $11: 31$ & 1.0 & 11.7 & 8.0 & 569 & 17.7 \\
$04 / 16 / 2008$ & $11: 32$ & 2.0 & 11.5 & 8.0 & 570 & 17.4 \\
$04 / 16 / 2008$ & $11: 33$ & 3.0 & 11.3 & 8.1 & 569 & 17.4 \\
$04 / 16 / 2008$ & $11: 34$ & 4.0 & 11.2 & 8.1 & 569 & 17.2 \\
$04 / 16 / 2008$ & $11: 35$ & 5.0 & 11.2 & 8.1 & 569 & 17.0 \\
$04 / 16 / 2008$ & $11: 36$ & 6.0 & 11.2 & 8.1 & 569 & 16.9 \\
$04 / 16 / 2008$ & $11: 37$ & 7.0 & 10.0 & 8.0 & 572 & 15.9
\end{tabular}

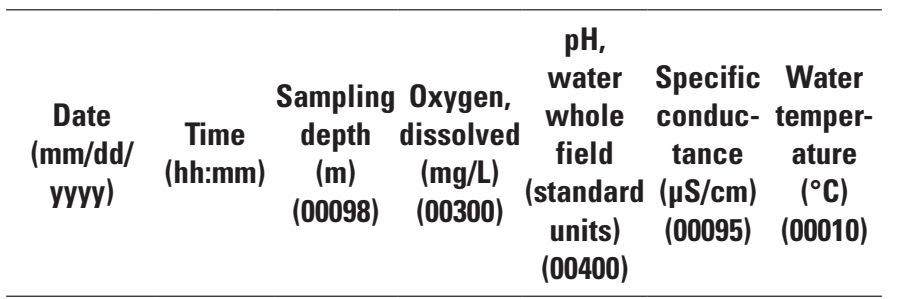

\begin{tabular}{lrrrrrr}
\hline \multicolumn{7}{c}{ Loveland } \\
\hline $04 / 16 / 2008$ & $11: 38$ & 8.0 & 8.4 & 7.8 & 572 & 15.7 \\
$04 / 16 / 2008$ & $11: 39$ & 9.0 & 7.0 & 7.7 & 573 & 13.3 \\
$04 / 16 / 2008$ & $11: 40$ & 10.0 & 5.9 & 7.6 & 576 & 12.2 \\
$04 / 16 / 2008$ & $11: 41$ & 12.0 & 5.1 & 7.6 & 580 & 11.8 \\
$04 / 16 / 2008$ & $11: 42$ & 14.0 & 4.0 & 7.5 & 582 & 11.6 \\
$04 / 16 / 2008$ & $11: 43$ & 16.0 & 3.4 & 7.5 & 583 & 11.5 \\
$04 / 16 / 2008$ & $11: 44$ & 18.0 & 3.0 & 7.5 & 585 & 11.4 \\
$04 / 16 / 2008$ & $11: 45$ & 20.0 & 2.7 & 7.5 & 587 & 11.3 \\
$04 / 16 / 2008$ & $11: 46$ & 22.0 & 2.4 & 7.4 & 589 & 11.3 \\
$04 / 16 / 2008$ & $11: 47$ & 24.0 & 2.1 & 7.4 & 590 & 11.3 \\
$04 / 16 / 2008$ & $11: 48$ & 26.0 & 2.0 & 7.4 & 591 & 11.2 \\
$04 / 16 / 2008$ & $11: 49$ & 28.0 & 2.0 & 7.4 & 593 & 11.2 \\
$04 / 16 / 2008$ & $11: 50$ & 30.0 & 1.8 & 7.4 & 595 & 11.2 \\
$07 / 16 / 2008$ & $11: 10$ & 0.1 & 11.2 & 8.1 & 605 & 26.3
\end{tabular}

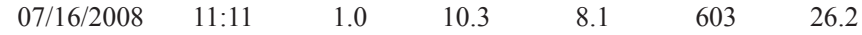

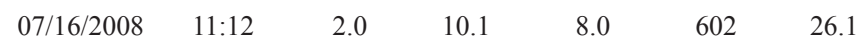

$\begin{array}{lllllll}07 / 16 / 2008 & 11: 13 & 3.0 & 9.9 & 8.0 & 601 & 26.0\end{array}$

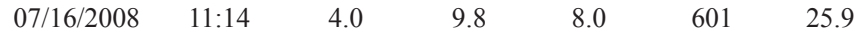

$\begin{array}{lllllll}07 / 16 / 2008 & 11: 15 & 5.0 & 9.8 & 8.0 & 601 & 25.8\end{array}$

$\begin{array}{lllllll}07 / 16 / 2008 & 11: 16 & 6.0 & 7.8 & 7.8 & 590 & 24.2\end{array}$

$\begin{array}{lllllll}07 / 16 / 2008 & 11: 17 & 7.0 & 5.3 & 7.5 & 580 & 19.9\end{array}$

$\begin{array}{lllllll}07 / 16 / 2008 & 11: 18 & 8.0 & 3.3 & 7.2 & 577 & 18.4\end{array}$

$\begin{array}{lllllll}07 / 16 / 2008 & 11: 19 & 9.0 & 2.7 & 7.2 & 575 & 16.5\end{array}$

$\begin{array}{lllllll}07 / 16 / 2008 & 11: 20 & 10.0 & 2.3 & 7.1 & 574 & 15.3\end{array}$

$\begin{array}{lllllll}07 / 16 / 2008 & 11: 21 & 12.0 & 2.1 & 7.1 & 576 & 13.3\end{array}$

$\begin{array}{lllllll}07 / 16 / 2008 & 11: 22 & 14.0 & 2.0 & 7.0 & 576 & 12.3\end{array}$

$\begin{array}{lllllll}07 / 16 / 2008 & 11: 23 & 16.0 & 2.0 & 7.0 & 578 & 11.9\end{array}$

$\begin{array}{lllllll}07 / 16 / 2008 & 11: 24 & 18.0 & 1.9 & 7.0 & 579 & 11.7\end{array}$

$\begin{array}{lllllll}07 / 16 / 2008 & 11: 25 & 20.0 & 1.9 & 6.9 & 581 & 11.7\end{array}$

$\begin{array}{lllllll}07 / 16 / 2008 & 11: 26 & 22.0 & 1.9 & 6.9 & 582 & 11.6\end{array}$

$\begin{array}{lllllll}07 / 16 / 2008 & 11: 27 & 24.0 & 1.9 & 6.9 & 583 & 11.6\end{array}$

$\begin{array}{lllllll}07 / 16 / 2008 & 11: 28 & 26.0 & 1.9 & 6.9 & 584 & 11.6\end{array}$

$\begin{array}{lllllll}07 / 16 / 2008 & 11: 29 & 28.0 & 1.9 & 6.9 & 584 & 11.6\end{array}$

$\begin{array}{lllllll}07 / 16 / 2008 & 11: 30 & 30.0 & 1.8 & 6.9 & 585 & 11.5\end{array}$

$\begin{array}{llllcll}10 / 15 / 2008 & 10: 40 & 0.1 & 8.8 & 8.1 & 599 & 20.1 \\ 10 / 15 / 2008 & 10: 41 & 1.0 & 8.4 & 8 & 605 & 20.0 \\ 10 / 15 / 2008 & 10: 42 & 2.0 & 8.5 & 7.9 & 609 & 19.9 \\ 10 / 15 / 2008 & 10: 43 & 3.0 & 8.4 & 7.8 & 609 & 19.9 \\ 10 / 15 / 2008 & 10: 44 & 4.0 & 8.4 & 7.8 & 611 & 19.9 \\ 10 / 15 / 2008 & 10: 45 & 5.0 & 8.4 & 7.8 & 612 & 19.9 \\ 10 / 15 / 2008 & 10: 46 & 6.0 & 8.4 & 7.8 & 613 & 19.9 \\ 10 / 15 / 2008 & 10: 47 & 7.0 & 8.4 & 7.7 & 613 & 19.9\end{array}$


Table 3. Water-quality depth-profile data for dissolved oxygen, $\mathrm{pH}$, specific conductance, and water temperature for each sampling site and period for the Sweetwater Reservoir watershed, San Diego County, California.-Continued

[Time is denoted in 24-hour format. The five-digit parameter code in parentheses below each compound name is used in the U.S. Geological Survey computerized data system (National Water Information System) to uniquely identify a specific constituent or property. Sampling depth is in meters below water surface. Shaded data coincide with reservoir water samples. Abbreviations: hh:mm, hour:minute; m, meter; mg/L, milligram per liter; mm/dd/yyyy, month/day/year; na, not applicable; PM, probe malfunction; $\mu \mathrm{S} / \mathrm{cm}$, microsiemen per centimeter; ${ }^{\circ} \mathrm{C}$, degree Celsius]

\begin{tabular}{|c|c|c|c|c|c|c|}
\hline $\begin{array}{c}\text { Date } \\
\text { (mm/dd/ } \\
\text { yyyy) }\end{array}$ & $\begin{array}{c}\text { Time } \\
\text { (hh:mm) }\end{array}$ & $\begin{array}{c}\text { Sampling } \\
\text { depth } \\
\text { (m) } \\
(00098)\end{array}$ & $\begin{array}{c}\text { Oxygen, } \\
\text { dissolved } \\
(\mathrm{mg} / \mathrm{L}) \\
(00300)\end{array}$ & $\begin{array}{c}\text { pH, } \\
\text { water } \\
\text { whole } \\
\text { field } \\
\text { (standard } \\
\text { units) } \\
(00400)\end{array}$ & $\begin{array}{c}\text { Specific } \\
\text { conduc- } \\
\text { tance } \\
(\mu \mathrm{S} / \mathrm{cm}) \\
(00095)\end{array}$ & $\begin{array}{c}\text { Water } \\
\text { temper- } \\
\text { ature } \\
\left({ }^{\circ} \mathrm{C}\right) \\
(00010)\end{array}$ \\
\hline \multicolumn{7}{|c|}{ Loveland Reservoir near dam (LLR01) - Continued } \\
\hline $10 / 15 / 2008$ & 10:48 & 8.0 & 8.4 & 7.7 & 614 & 19.9 \\
\hline $10 / 15 / 2008$ & 10:49 & 9.0 & 8.4 & 7.7 & 615 & 19.8 \\
\hline $10 / 15 / 2008$ & 10:50 & 10.0 & 8.0 & 7.7 & 615 & 19.6 \\
\hline $10 / 15 / 2008$ & 10:51 & 12.0 & 2.6 & 7.5 & 586 & 14.1 \\
\hline $10 / 15 / 2008$ & $10: 52$ & 14.0 & 1.4 & 7.4 & 579 & 12.6 \\
\hline $10 / 15 / 2008$ & 10:53 & 16.0 & 1.1 & 7.2 & 581 & 12.0 \\
\hline $10 / 15 / 2008$ & $10: 54$ & 18.0 & 1.1 & 7.2 & 582 & 11.9 \\
\hline $10 / 15 / 2008$ & $10: 55$ & 20.0 & 1.0 & 7.2 & 582 & 11.8 \\
\hline $10 / 15 / 2008$ & $10: 56$ & 22.0 & 1.0 & 7.2 & 583 & 11.8 \\
\hline $10 / 15 / 2008$ & $10: 57$ & 24.0 & 1.0 & 7.1 & 583 & 11.8 \\
\hline $10 / 15 / 2008$ & $10: 58$ & 26.0 & 1.0 & 7.1 & 583 & 11.8 \\
\hline $10 / 15 / 2008$ & 10:59 & 28.0 & 0.9 & 7.1 & 582 & 11.7 \\
\hline $10 / 15 / 2008$ & $11: 00$ & 30.0 & 0.9 & 7.1 & 585 & 11.7 \\
\hline $01 / 14 / 2009$ & $10: 40$ & 0.1 & 9.6 & 7.8 & 605 & 12.4 \\
\hline $01 / 14 / 2009$ & 10:41 & 1.0 & 8.8 & 7.8 & 605 & 11.9 \\
\hline $01 / 14 / 2009$ & $10: 42$ & 2.0 & 8.8 & 7.8 & 604 & 11.6 \\
\hline $01 / 14 / 2009$ & 10:43 & 3.0 & 8.6 & 7.8 & 604 & 11.6 \\
\hline 01/14/2009 & 10:44 & 4.0 & 8.5 & 7.7 & 603 & 11.5 \\
\hline 01/14/2009 & $10: 45$ & 5.0 & 8.5 & 7.7 & 603 & 11.5 \\
\hline 01/14/2009 & $10: 46$ & 6.0 & 8.5 & 7.7 & 603 & 11.5 \\
\hline 01/14/2009 & $10: 47$ & 7.0 & 8.5 & 7.7 & 603 & 11.5 \\
\hline 01/14/2009 & 10:48 & 8.0 & 8.5 & 7.7 & 603 & 11.5 \\
\hline 01/14/2009 & 10:49 & 9.0 & 8.4 & 7.7 & 603 & 11.5 \\
\hline $01 / 14 / 2009$ & $10: 50$ & 10.0 & 8.4 & 7.7 & 603 & 11.5 \\
\hline 01/14/2009 & $10: 51$ & 12.0 & 8.4 & 7.6 & 603 & 11.5 \\
\hline 01/14/2009 & $10: 52$ & 14.0 & 8.1 & 7.6 & 603 & 11.5 \\
\hline $01 / 14 / 2009$ & $10: 53$ & 16.0 & 8.0 & 7.7 & 602 & 11.5 \\
\hline 01/14/2009 & $10: 54$ & 18.0 & 7.9 & 7.7 & 602 & 11.5 \\
\hline 01/14/2009 & 10:55 & 20.0 & 7.9 & 7.7 & 602 & 11.5 \\
\hline 01/14/2009 & $10: 56$ & 22.0 & 8.2 & 7.7 & 602 & 11.4 \\
\hline 01/14/2009 & $10: 57$ & 24.0 & 8.2 & 7.7 & 601 & 11.4 \\
\hline 01/14/2009 & $10: 58$ & 26.0 & 8.2 & 7.7 & 601 & 11.4 \\
\hline 01/14/2009 & $10: 59$ & 28.0 & 8.2 & 7.7 & 601 & 11.4 \\
\hline 01/14/2009 & $11: 00$ & 30.0 & 8.2 & 7.7 & 601 & 11.3 \\
\hline 04/15/2009 & 13:00 & 0.1 & 9.8 & 8.7 & 554 & 15.4 \\
\hline 04/15/2009 & 13:01 & 1.0 & 8.9 & 8.7 & 553 & 15.4 \\
\hline 04/15/2009 & 13:02 & 2.0 & 8.6 & 8.6 & 553 & 15.4 \\
\hline 04/15/2009 & 13:03 & 3.0 & 8.2 & 8.6 & 554 & 15.3 \\
\hline
\end{tabular}

\begin{tabular}{|c|c|c|c|c|c|c|}
\hline $\begin{array}{c}\text { Date } \\
\text { (mm/dd/ } \\
\text { yyyy) }\end{array}$ & $\begin{array}{c}\text { Time } \\
\text { (hh:mm) }\end{array}$ & $\begin{array}{l}\text { Sampling } \\
\text { depth } \\
\text { (m) } \\
(00098)\end{array}$ & $\begin{array}{c}\text { Oxygen, } \\
\text { dissolved } \\
(\mathrm{mg} / \mathrm{L}) \\
(00300)\end{array}$ & $\begin{array}{c}\text { pH, } \\
\text { water } \\
\text { whole } \\
\text { field } \\
\text { (standard } \\
\text { units) } \\
\text { (00400) }\end{array}$ & $\begin{array}{c}\text { Specific } \\
\text { conduc- } \\
\text { tance } \\
(\mu \mathrm{S} / \mathrm{cm}) \\
(00095)\end{array}$ & $\begin{array}{c}\text { Water } \\
\text { temper- } \\
\text { ature } \\
\left({ }^{\circ} \mathrm{C}\right) \\
(00010)\end{array}$ \\
\hline \multicolumn{7}{|c|}{ Loveland Reservoir near dam (LLR01)—Continued } \\
\hline $04 / 15 / 2009$ & 13:04 & 4.0 & 8.0 & 8.5 & 555 & 15.3 \\
\hline 04/15/2009 & 13:05 & 5.0 & 7.8 & 8.5 & 556 & 15.1 \\
\hline $04 / 15 / 2009$ & $13: 06$ & 6.0 & 6.4 & 8.2 & 558 & 14.8 \\
\hline $04 / 15 / 2009$ & 13:07 & 7.0 & 4.8 & 7.9 & 556 & 14.1 \\
\hline $04 / 15 / 2009$ & $13: 08$ & 8.0 & 3.7 & 7.7 & 553 & 13.3 \\
\hline $04 / 15 / 2009$ & 13:09 & 9.0 & 3.1 & 7.7 & 552 & 12.1 \\
\hline $04 / 15 / 2009$ & $13: 10$ & 10.0 & 2.9 & 7.7 & 551 & 11.8 \\
\hline $04 / 15 / 2009$ & $13: 11$ & 12.0 & 2.8 & 7.6 & 550 & 11.5 \\
\hline $04 / 15 / 2009$ & $13: 12$ & 14.0 & 2.8 & 7.6 & 542 & 11.4 \\
\hline $04 / 15 / 2009$ & $13: 13$ & 16.0 & 2.8 & 7.6 & 548 & 11.4 \\
\hline $04 / 15 / 2009$ & $13: 14$ & 18.0 & 2.7 & 7.6 & 547 & 11.4 \\
\hline $04 / 15 / 2009$ & $13: 15$ & 20.0 & 2.4 & 7.6 & 546 & 11.4 \\
\hline $04 / 15 / 2009$ & $13: 16$ & 22.0 & 2.0 & 7.6 & 545 & 11.3 \\
\hline $04 / 15 / 2009$ & $13: 17$ & 24.0 & 1.6 & 7.6 & 545 & 11.3 \\
\hline $04 / 15 / 2009$ & $13: 18$ & 26.0 & 1.3 & 7.6 & 545 & 11.3 \\
\hline $07 / 08 / 2009$ & $13: 00$ & 0.1 & 10.8 & 8.7 & 438 & 25.5 \\
\hline $07 / 08 / 2009$ & $13: 01$ & 1.0 & 10.7 & 8.6 & 442 & 25.3 \\
\hline $07 / 08 / 2009$ & 13:02 & 2.0 & 10.8 & 8.6 & 443 & 24.8 \\
\hline $07 / 08 / 2009$ & 13:03 & 3.0 & 10.7 & 8.6 & 444 & 24.6 \\
\hline $07 / 08 / 2009$ & 13:04 & 4.0 & 10.6 & 8.6 & 446 & 24.5 \\
\hline 07/08/2009 & $13: 05$ & 5.0 & 8.6 & 8.5 & 449 & 24.2 \\
\hline 07/08/2009 & 13:06 & 6.0 & 5.1 & 8.1 & 453 & 19.9 \\
\hline 07/08/2009 & $13: 07$ & 7.0 & 3.1 & 8.0 & 451 & 17.7 \\
\hline 07/08/2009 & 13:08 & 8.0 & 1.7 & 7.9 & 450 & 16.1 \\
\hline $07 / 08 / 2009$ & 13:09 & 9.0 & 1.6 & 7.8 & 450 & 15.1 \\
\hline $07 / 08 / 2009$ & $13: 10$ & 10.0 & 1.5 & 7.8 & 450 & 14.2 \\
\hline 07/08/2009 & $13: 11$ & 12.0 & 1.5 & 7.7 & 452 & 13.0 \\
\hline 07/08/2009 & $13: 12$ & 14.0 & 1.4 & 7.7 & 448 & 11.9 \\
\hline $07 / 08 / 2009$ & $13: 13$ & 16.0 & 1.3 & 7.7 & 448 & 11.7 \\
\hline 07/08/2009 & $13: 14$ & 18.0 & 1.3 & 7.6 & 447 & 11.7 \\
\hline 07/08/2009 & $13: 15$ & 20.0 & 1.3 & 7.6 & 447 & 11.6 \\
\hline $07 / 08 / 2009$ & $13: 16$ & 22.0 & 1.2 & 7.6 & 446 & 11.6 \\
\hline $07 / 08 / 2009$ & $13: 17$ & 24.0 & 1.2 & 7.6 & 446 & 11.6 \\
\hline $07 / 08 / 2009$ & $13: 18$ & 26.0 & 1.2 & 7.6 & 445 & 11.5 \\
\hline $07 / 08 / 2009$ & $13: 19$ & 28.0 & 1.2 & 7.6 & 444 & 11.5 \\
\hline $07 / 08 / 2009$ & $13: 20$ & 29.0 & 1.1 & 7.4 & 451 & 11.5 \\
\hline
\end{tabular}




\section{Semivolatile Organic Compounds}

Unfiltered water samples were analyzed for $57 \mathrm{BNA}$ extractable compounds, including 16 PAHs on NWQL schedule 1383. The analysis included the following modifications to the method originally described in Fishman (1993). Each 1-L water sample was fortified with surrogate compounds (see Quality Control section) and extracted with dichloromethane for 6 hours in a continuous liquid-liquid extractor. The $\mathrm{pH}$ of the sample was made acidic (by addition of $2.0 \mathrm{~mL}$ of 25 percent sulfuric acid) for the first 3 hours and then adjusted to basic (by addition of $7.0 \mathrm{~mL}$ of 37 percent potassium hydroxide) for the remainder of the extraction. The resultant extract was reduced in volume by distillation and then by nitrogen gas evaporation to a final volume of $0.4 \mathrm{~mL}$. The extract was analyzed for 57 BNA compounds by GC/MS operated in the full-scan mode.

Samples collected for methods determining pesticide and AOI compounds were filtered at the Perdue Treatment Plant through 142-mm diameter, $0.7-\mu \mathrm{m}$ nominal pore size GFF into sample bottles (Sandstrom, 1995). Filtered water samples were analyzed for pesticides on NWQL schedules 2003 and 2060. Schedule 2003 includes 65 pesticides and degradates that are a subset of those determined by Zaugg and others (1995), Lindley and others (1996), Sandstrom and others (2001), and Madsen and others (2003). Each water sample was passed through a 0.5 -g octadecylsilyl (C-18) solid-phase extraction (SPE) column to extract the pesticides dissolved in the water. The pesticides were eluted from the columns with $2 \mathrm{~mL}$ of ethyl acetate, and the extracts were reduced in volume, exchanged to toluene to approximately $0.25 \mathrm{~mL}$ final volume, and analyzed by GC/MS operated in the selectedion monitoring (SIM) mode (Sandstrom and others, 2001). Schedule 2060 includes 58 polar pesticides and degradates. Each sample was analyzed by SPE using a graphitized carbon column (Carbopack B, Supelco; Bellefonte, Pennsylvania) and liquid chromatography with mass-spectrometric detection operated in SIM mode as detailed in Furlong and others (2001).

Filtered water samples were analyzed for $63 \mathrm{AOI}$ compounds on NWQL schedule 1433 (Zaugg and others, 2002). Some AOI compounds typically are associated with wastewater effluent, and some are classified as endocrinedisrupting compounds. Approximately $1 \mathrm{~L}$ of a filtered water sample was passed through a 1-g Oasis hydrophilic-lipophilic balance (HLB; Waters Corp., Milford, Massachusetts) SPE column. Eluted analytes were determined by GC/MS operated in full-scan mode.

\section{Metals}

Metals in unfiltered water samples on NWQL schedule 1080 were analyzed by using inductively coupled plasmaoptical emission spectroscopy and inductively coupled plasma-mass spectrometry methods described in Garbarino and Struzeski (1998). Dissolved metals in filtered water samples on NWQL schedule 2710 were analyzed by using inductively coupled plasma-mass spectrometry methods described in Garbarino and Taylor (1996) and Garbarino (1999). Dissolved metal samples were processed through a $0.45-\mu \mathrm{m}$ capsule filter into the appropriate bottle and acidified as needed.

\section{Water-Quality Assurance}

The QA and quality control (QC) procedures used for surface-water sampling followed the protocols described in Mueller and others (1997). The QA plan followed by the USGS NWQL is described in Pirkey and Glodt (1998) and Maloney (2005). Additionally, the USGS Branch of Quality Systems QA program (within the USGS Office of Water Quality) maintains a web page with results from the USGS Inorganic Blind Sample Project (U.S. Geological Survey, variously dated) to document bias and variability for the NWQL through the use of blind QA samples.

Three types of QC samples were used for this studymatrix spikes, blanks, and replicates. Spikes and blanks are used to assess result bias, and replicates are used to assess result variability. Spike recoveries are considered to be acceptable if they are within \pm 30 percent of a known spike value. Replicates are considered to be acceptable if they are within \pm 30 percent of the original value. Additionally, surrogate compounds were added to VOC, BNA, PAH, pesticide, and AOI samples at the laboratory to monitor sample-specific performance of the analytical method. Additional information on water QA methods and QC results are presented in the appendix.

A matrix (replicate environmental sample) spike is prepared by fortifying reagent water with a known concentration of selected analytes and can be used to test the effects of various sampled matrices on the recovery of specific compounds, including matrix interference or matrix-induced analyte degradation. Matrix-spike recoveries are calculated, using the following equation:

$$
\text { Recovery }=100\left(C_{\text {spiked }}-C_{\text {env }} / C_{\text {expected }}\right)
$$

where

$$
\begin{aligned}
& C_{\text {spiked }} \quad \text { is the measured concentration in the spiked } \\
& \text { sample, in micrograms per liter; } \\
& C_{e n v} \text { is the measured concentration in the }
\end{aligned}
$$

Blanks are intended to be free of the analytes of interest and can be prepared in the laboratory or field. Blank samples are analyzed to test for bias that could result from contamination of environmental samples by the analytes of 
interest or by interfering constituents during any stage of sample collection, processing, and analysis. Blanks for water samples were collected by processing USGS laboratorycertified reagent water, known to be free of the analytes of interest, through any part of the sampling and processing stage. A field blank is collected at an environmental sampling location through all field-cleaned sampling equipment. Field blanks are used to show that all stages of sample collection and processing have not introduced contamination. Equipment blanks are collected annually and demonstrate that the equipment used to collect and process the samples is not introducing contamination. Source solution blanks are collected to show that the laboratory-certified reagent water is free of the analytes of interest. Travel (trip) blanks are used to identify contamination that may occur during sample transport and analysis rather than as a result of sample collection and processing. Travel blanks were processed for VOCs from unopened cartridges sent from the lab and processed with the environmental samples.

Replicate samples are collected and processed identically to the environmental sample to measure the variability in the detected analyte concentration during sample processing and analysis. Replicate samples for VOCs and metals (targeted compounds) were evaluated by calculating the percentage difference of detected analytes from the environmental sample.

The quality of analysis was monitored by adding surrogate compounds to each sample before it was processed. These surrogate compounds are added at the NWQL to monitor sample preparation and analysis, and the results are given in their respective data tables. For VOCs, surrogates 1,4-bromofluorobenzene, 1,2-dichloroethane- $d 4$, and toluene- $d 8$ were added to each sample. For BNA, surrogates 2-fluorophenyl, nitrobenzene- $d 5$, phenol- $d 5$, terphenyl, and 2,4,6-tribromophenol were added to each sample. For pesticides on NWQL schedule 2003, diazinon- $d 10$ and $\alpha$-HCH- $d 6$ were added to each sample. For pesticides on NWQL schedule 2060, surrogates barban, caffeine C-13, and 2,4,5-T were added to each sample. For AOI compounds, surrogates bisphenol A- $d 3$, caffeine C-13, decafluorobiphenyl, and fluoroanthene- $d 10$ were added to each sample prior to extraction. Surrogates were not added to the water samples analyzed for metals.

\section{Water Results}

VOCs analyzed in unfiltered water samples (along with their applicable LRLs) are given in table 4A, and the compounds detected are given in table 4B. Of the 85 VOC compounds analyzed, 37 compounds were detected at least once. Four compounds (bromodichloromethane, dibromochloromethane, tribromomethane, and trichloromethane) were detected in 100 percent of the samples at site SWR08. Trichloromethane also was detected in 100 percent of the samples at sites SWR01, SWR08, and SWR09. The highest detections of methyl tert-butyl ether (MTBE), benzene, and toluene in the vertical profile of water samples for each sampling event at sites SWR01 and SWR03 are shown in figures $4 B, 4 C$, and $4 D$, respectively. MTBE was detected in most samples during Phase One until the spring of 2003. MTBE was not detected in Phases Two and Three. Benzene had several detections in Phase One, five detections in Phase Two, and one detection in Phase Three. Toluene was detected many times in Phase One, had the highest concentrations in Phase Two, and was detected five times in Phase Three. To avoid falsely implying trends in figures $4 B-4 D$ because of variations in laboratory reporting limits, all non-detections were plotted at a low concentration $(0.003 \mu \mathrm{g} / \mathrm{L})$ for a normalized appearance. This normalized concentration was chosen because all laboratory reported detections were above this value.

The BNA compounds analyzed in NWQL schedule 1383 (unfiltered water) are given in table 5A, and the compounds detected are given in table 5B. Of the 56 BNA compounds analyzed, 37 were detected at least once. Isophorone and phenol were detected most frequently (45.5 and 40.6 percent, respectively). Pesticide compounds analyzed in NWQL schedules 2003 (filtered water) and 2060 (filtered water) are given in tables $6 \mathrm{~A}$ and $7 \mathrm{~A}$, respectively, and the compound concentrations detected are given in tables $6 \mathrm{~B}$ and $7 \mathrm{~B}$, respectively. Of the 64 pesticide compounds analyzed in schedule 2003, 21 were detected at least once. Prometon and simazine were detected most frequently (94.4 and 83.3 percent, respectively). Of the 59 pesticide compounds analyzed in schedule 2060, 14 were detected at least once. Caffeine and diuron were detected most frequently (54.3 and 91.4 percent, respectively).

A variety of AOI compounds, some that typically are associated with wastewater effluent, were analyzed (table 8A). Concentrations for AOI compounds detected in filtered water samples at sites SWR01, SWR03, SWR08, SWR09, and LFDD (fig. 2) are given in table 8B. Of the 59 AOI compounds analyzed, 17 were detected at least once.

Metals occur naturally but they are also emitted from vehicle exhaust and are found in tire-wear and road dust. The metal analyses were added to the SWR sampling to help understand the role of atmospheric deposition of metals that might originate from the SR 125 alignment and the potential effect of these metals on water quality. Metal concentrations in unfiltered and filtered water samples are given in tables 9A and 9B, respectively. Of the 19 metals analyzed in unfiltered water samples, only silver and thallium were not detected. Of the 22 metals analyzed in filtered water samples, only beryllium, chromium, and thallium were not detected; silver was only detected once. 
Table 4A. Volatile organic compounds analyzed in whole-water samples, using U.S. Geological Survey National Water Quality Laboratory schedule 2020, for the Sweetwater Reservoir watershed, San Diego County, California.

[The parameter code is used in the U.S. Geological Survey computerized data system (National Water Information System) to uniquely identify a specific constituent or property. Laboratory reporting level (LRL) values are reported as micrograms per liter $(\mu \mathrm{g} / \mathrm{L})$, unless noted.]

\begin{tabular}{|c|c|c|c|c|c|}
\hline Compound & $\begin{array}{l}\text { Parameter } \\
\text { code }\end{array}$ & LRL & Compound & $\begin{array}{l}\text { Parameter } \\
\text { code }\end{array}$ & LRL \\
\hline Acetone & 81552 & 6,4 & Hexachlorobutadiene & 39702 & $0.14,0.10,0.06$ \\
\hline Acrylonitrile & 34215 & $1.2,0.8,0.4$ & Hexachloroethane & 34396 & 0.14 \\
\hline Benzene & 34030 & $0.021,0.016$ & 2-Hexanone & 77103 & $0.7,0.4,0.6$ \\
\hline Bromobenzene & 81555 & $0.028,0.02$ & Isopropylbenzene & 77223 & $0.038,0.04$ \\
\hline Bromochloromethane & 77297 & $0.12,0.06$ & 4-Isopropyl-1-methylbenzene & 77356 & $0.08,0.06$ \\
\hline Bromomethane & 34413 & $0.26,0.33,0.4$ & Methyl iodide & 77424 & $0.35,0.50,0.4,0.8$ \\
\hline 2-Butanone & 81595 & $4,2,1.6$ & Methyl methacrylate & 81597 & $0.35,0.20$ \\
\hline$n$-Butylbenzene & 77342 & $0.12,0.14,0.08$ & 4-Methyl-2-pentanone & 78133 & $0.37,0.2,0.4$ \\
\hline sec-Butylbenzene & 77350 & $0.06,0.04,0.02$ & Methyl tert-butyl ether (MTBE) & 78032 & $0.17,0.10$ \\
\hline tert-Butylbenzene & 77353 & $0.06,0.08$ & Naphthalene & 34696 & $0.52,0.4,0.2$ \\
\hline Carbon disulfide & 77041 & $0.038,0.06,0.04$ & tert-Pentyl methyl ether & 50005 & $0.08,0.04,0.06$ \\
\hline 3-Chloropropene & 78109 & $0.5,0.08$ & 1,1,2,2-Tetrachloroethane & 34516 & $0.16,0.08,0.10$ \\
\hline 2-Chlorotoluene & 77275 & $0.04,0.02$ & Tetrachloroethylene & 34475 & $0.06,0.03,0.04$ \\
\hline 4-Chlorotoluene & 77277 & $0.05,0.04,0.02$ & Tetrachloromethane & 32102 & $0.06,0.08$ \\
\hline Dibromochloromethane & 32105 & $0.10,0.12$ & Tetrahydrofuran & 81607 & $2.2,1.0,1.4$ \\
\hline 1,2-Dibromo-3-chloropropane & 82625 & $0.51,0.5,1$ & 1,2,3,4-Tetramethylbenzene & 49999 & $0.14,0.08$ \\
\hline 1,2-Dibromoethane & 77651 & $0.036,0.04$ & 1,2,3,5-Tetramethylbenzene & 50000 & $0.14,0.18,0.12,0.08$ \\
\hline Dibromomethane & 30217 & $0.050,0.04$ & Toluene & 34010 & $0.05,0.02,0.018$ \\
\hline 1,2-Dichlorobenzene & 34536 & $0.048,0.04,0.02$ & Tribromomethane & 32104 & 0.1 \\
\hline 1,3-Dichlorobenzene & 34566 & $0.03,0.04,0.02$ & 1,2,3-Trichlorobenzene & 77613 & $0.27,0.18,0.12$ \\
\hline 1,4-Dichlorobenzene & 34571 & $0.034,0.04,0.02$ & & & $0.08,0.06$ \\
\hline trans-1,4-Dichloro-2-butene & 73547 & $0.70,0.6,0.4$ & 1,2,4-Trichlorobenzene & 34551 & $0.12,0.08,0.04$ \\
\hline 1,3-Dichloropropane & 77173 & 0.06 & 1,2,3-Trimethylbenzene & 77221 & $0.06,0.09,0.08$ \\
\hline 2,2-Dichloropropane & 77170 & $0.05,0.06$ & 1,2,4-Trimethylbenzene & 77222 & $0.056,0.04$ \\
\hline 1,1-Dichloropropene & 77168 & $0.026,0.04$ & $1,3,5$-Trimethylbenzene & 77226 & $0.044,0.04$ \\
\hline cis-1,3-Dichloropropene & 34704 & $0.05,0.06,0.1$ & Vinyl Chloride & 39175 & $0.06,0.08$ \\
\hline trans-1,3-Dichloropropene & 34699 & $0.09,0.10$ & $m$ - and $p$-Xylene & 85795 & $0.06,0.08$ \\
\hline Diethyl ether & 81576 & $0.08,0.12$ & $o$-Xylene & 77135 & $0.038,0.04$ \\
\hline Diisopropyl ether & 81577 & $0.10,0.06$ & & & \\
\hline Ethylbenzene & 34371 & $0.030,0.02,0.04$ & 1,4-Bromofluorobenzene, surrogate & 99834 & percent \\
\hline Ethyl tert-butyl ether & 50004 & $0.05,0.030,0.04$ & 1,2-Dichloroethane- $d 4$, surrogate & 99832 & percent \\
\hline Ethyl methacrylate & 73570 & $0.18,0.14$ & Toluene- $d 8$, surrogate & 99833 & percent \\
\hline
\end{tabular}

$77220 \quad 0.06,0.04,0.02$ 
Table 4B. Analytical results for Volatile Organic Compounds detected in whole-water samples, using U.S. Geological Survey National Water Quality Laboratory schedule 2020, from the Sweetwater Reservoir watershed, San Diego County, California.

(Table 4B is provided separately as a Microsoft Excel ${ }^{\circledR}$ worksheet.)

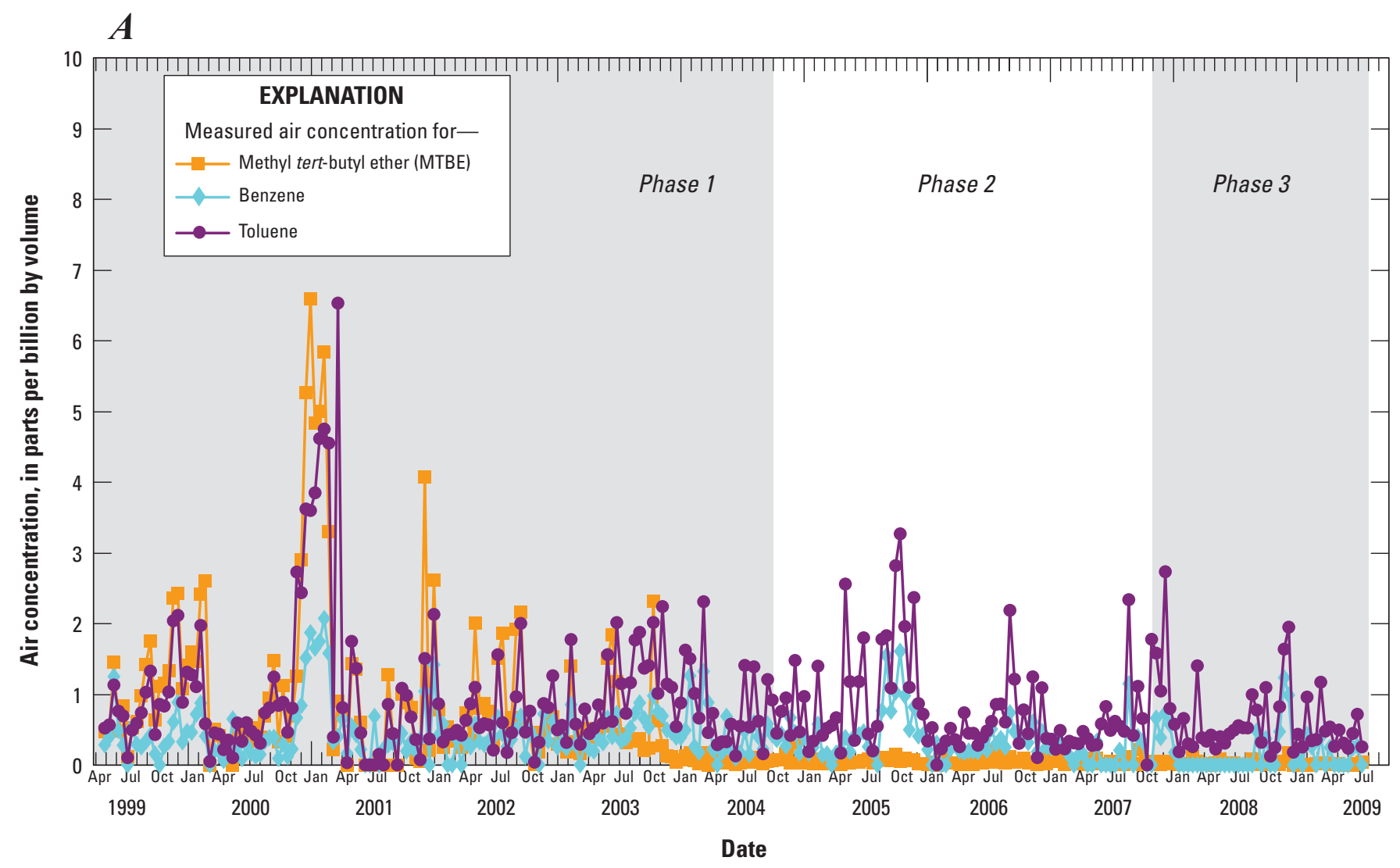

Figure 4. $A$, Concentrations in air of methyl tert-butyl ether (MTBE), benzene, and toluene at the Sweetwater Reservoir (SWR) atmospheric sampling site; and concentrations in air and water of $B, \mathrm{MTBE}$; $C$, benzene; and $D$, toluene at SWR near the pump tower (site SWR01) and at center of minimum pool (site SWR03), San Diego County, California. 
B
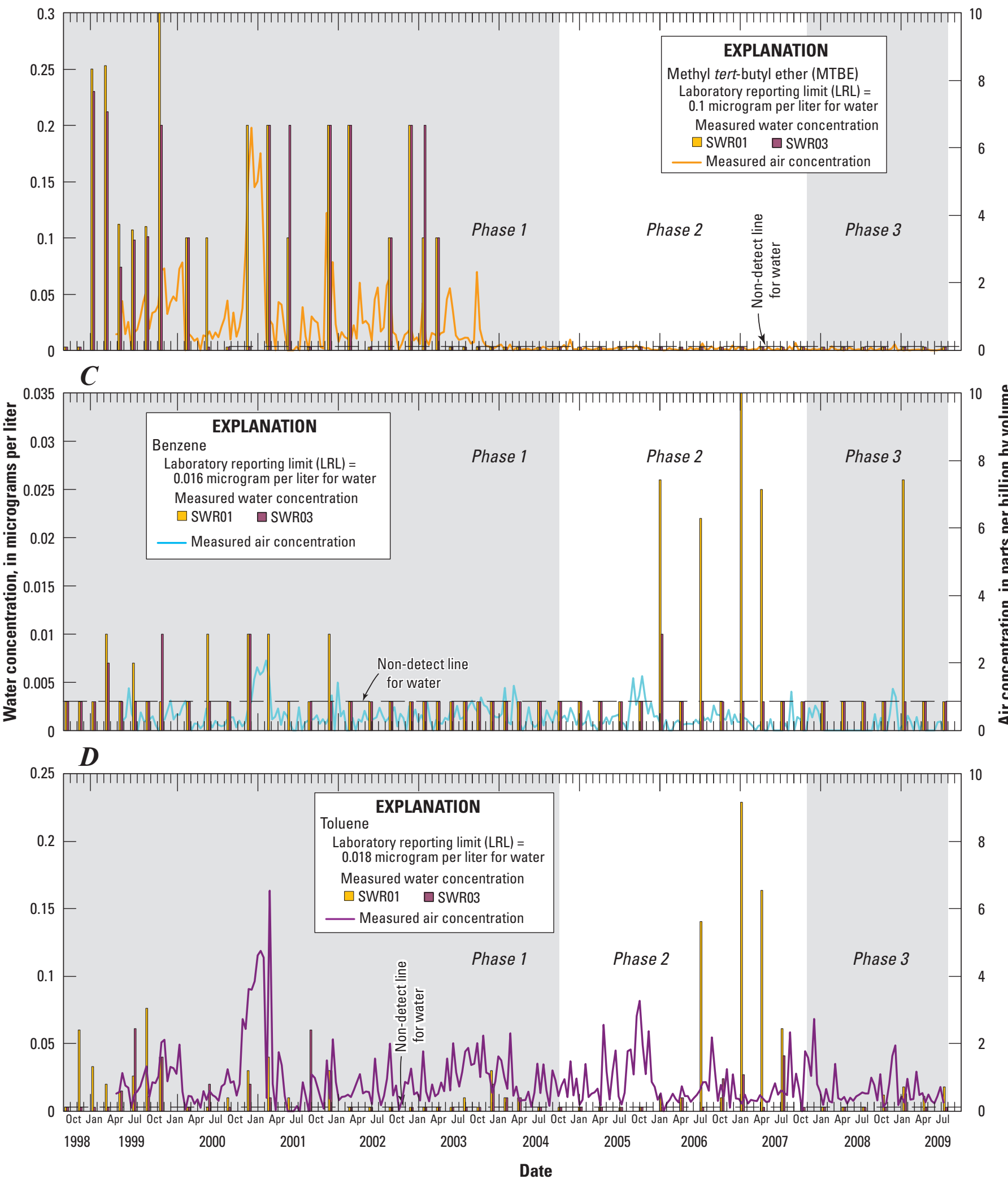

Figure 4. - Continued 
Table 5A. Base-neutral acid compounds analyzed in whole-water samples, using U.S. Geological Survey National Water Quality Laboratory schedule 1383, for the Sweetwater Reservoir watershed, San Diego County, California.

[The parameter code is used in the U.S. Geological Survey computerized data system (National Water Information System) to uniquely identify a specific constituent or property. This schedule is not the preferred method for compounds 1,2-Dichlorobenzene, 1,3-Dichlorobenzene, 1,4-Dichlorobenzene, Hexachlorobutadiene, Hexachloroethane, Naphthalene, and 1,2,4-Trichlorobenzene. Laboratory reporting level (LRL) values are reported as micrograms per liter ( $\mu \mathrm{g} / \mathrm{L}$ ), unless noted. *, denotes polycyclic aromatic hydrocarbons]

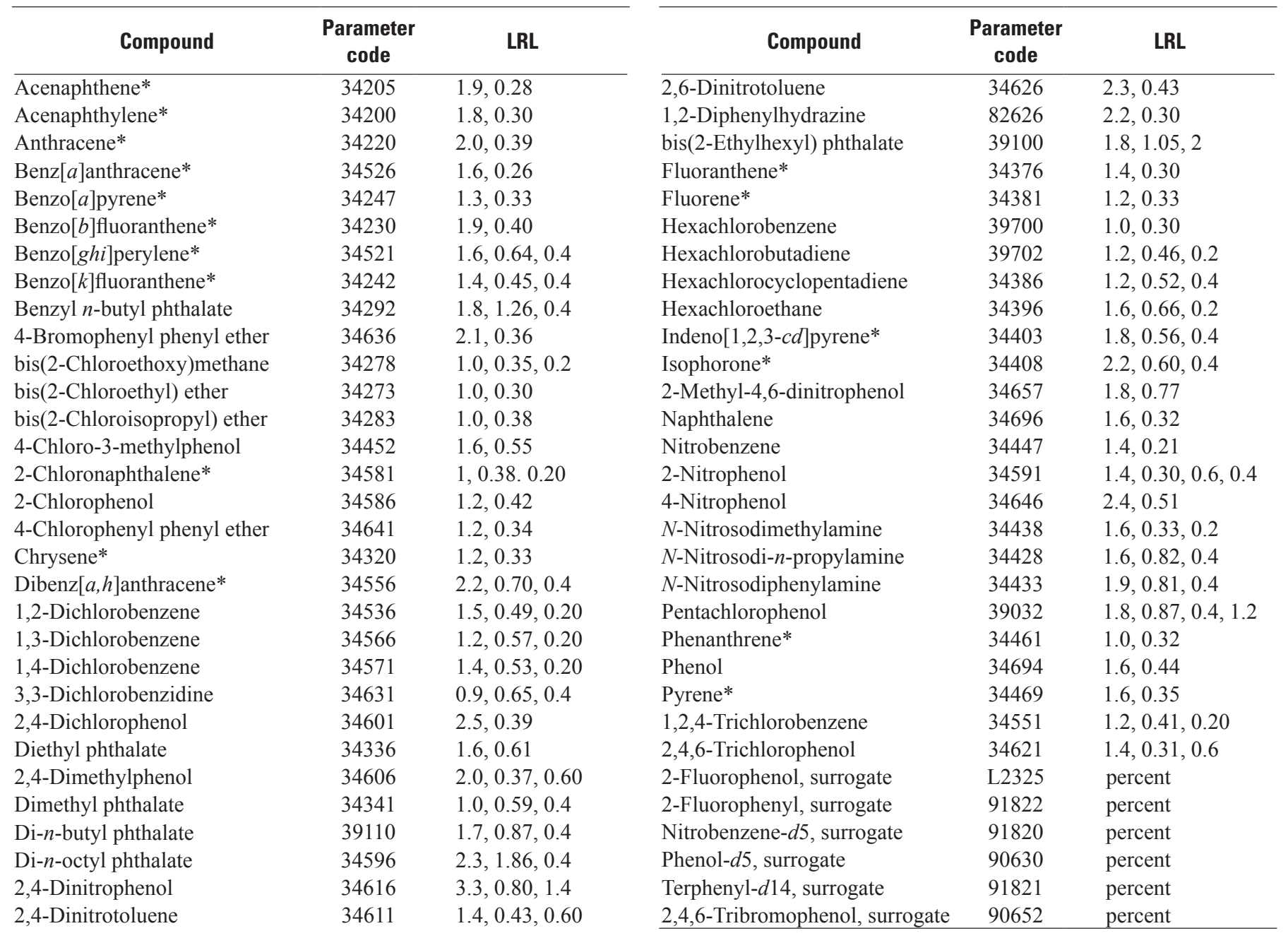

Table 5B. Analytical results for base-neutral acid compounds detected in whole-water samples, using U.S. Geological Survey National Water Quality Laboratory schedule 1383, from the Sweetwater Reservoir watershed, San Diego County, California.

(Table 5B is provided separately as a Microsoft Excel ${ }^{\mathbb{B}}$ worksheet.) 
Table 6A. Pesticide compounds analyzed in filtered-water samples, using U.S. Geological Survey National Water Quality Laboratory schedule 2003, for the Sweetwater Reservoir watershed, San Diego County, California.

[The parameter code is used in the U.S. Geological Survey computerized data system (National Water Information System) to uniquely identify a specific constituent or property. Laboratory reporting level (LRL) values are reported as micrograms per liter $(\mu \mathrm{g} / \mathrm{L})$, unless noted.]

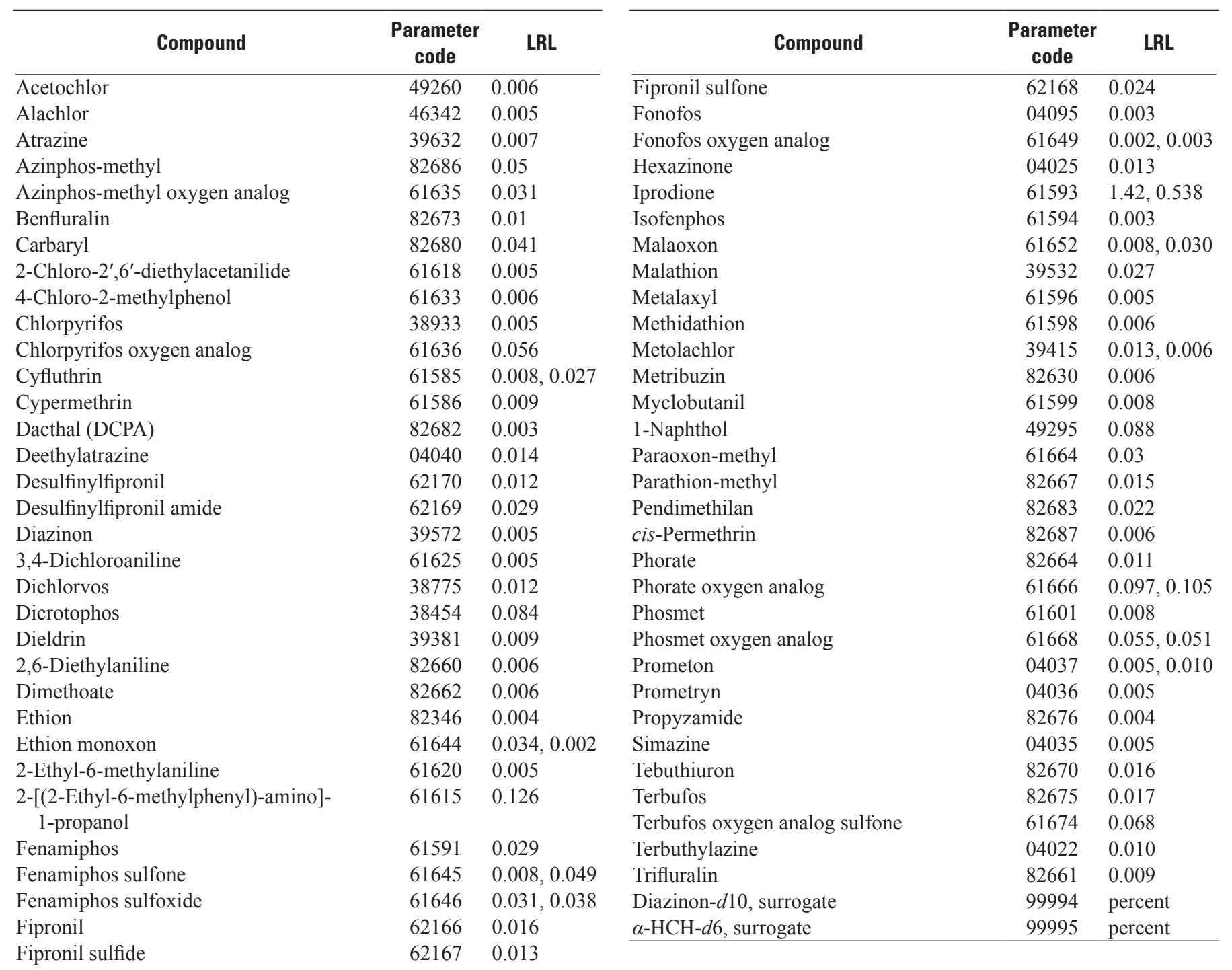



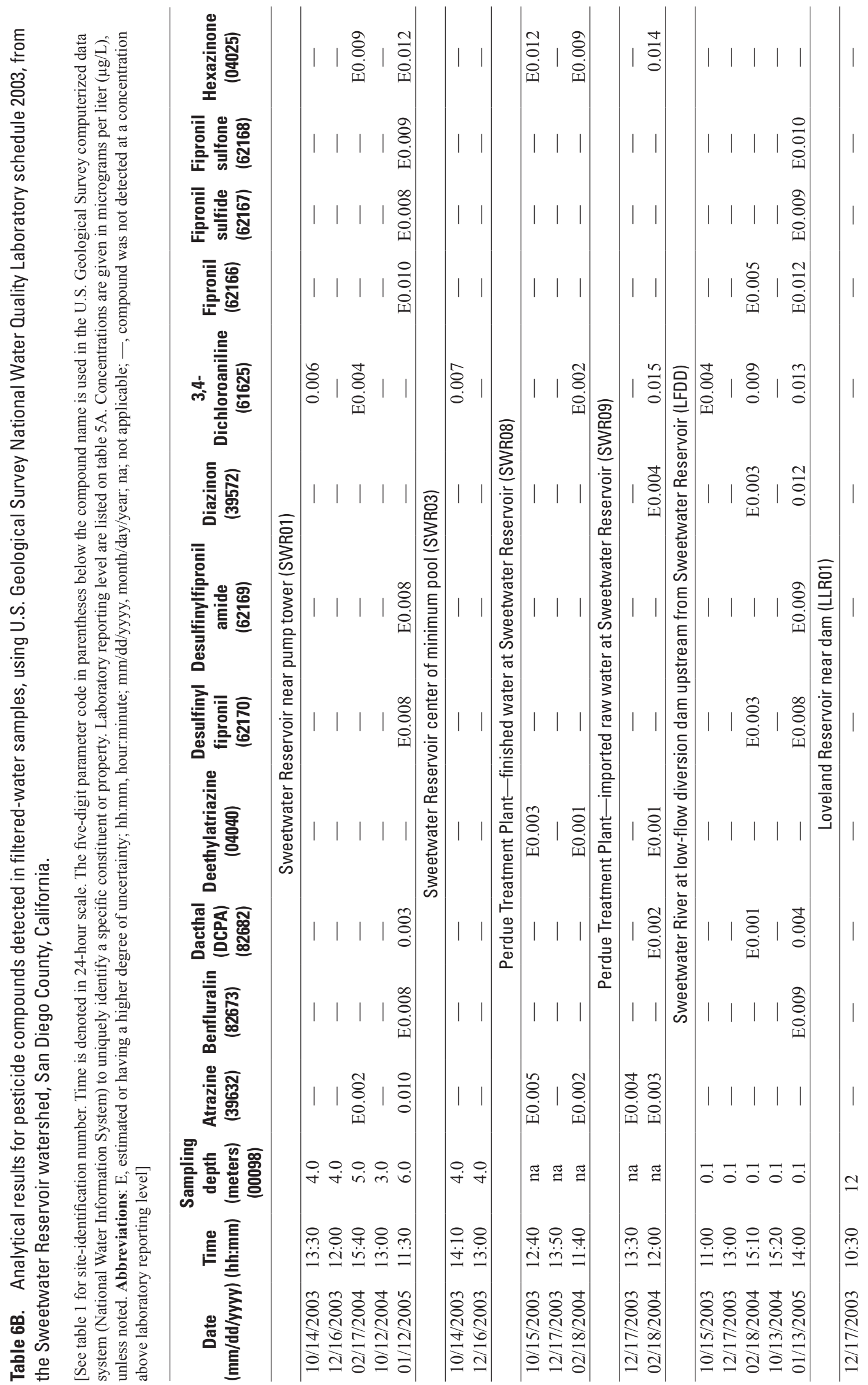


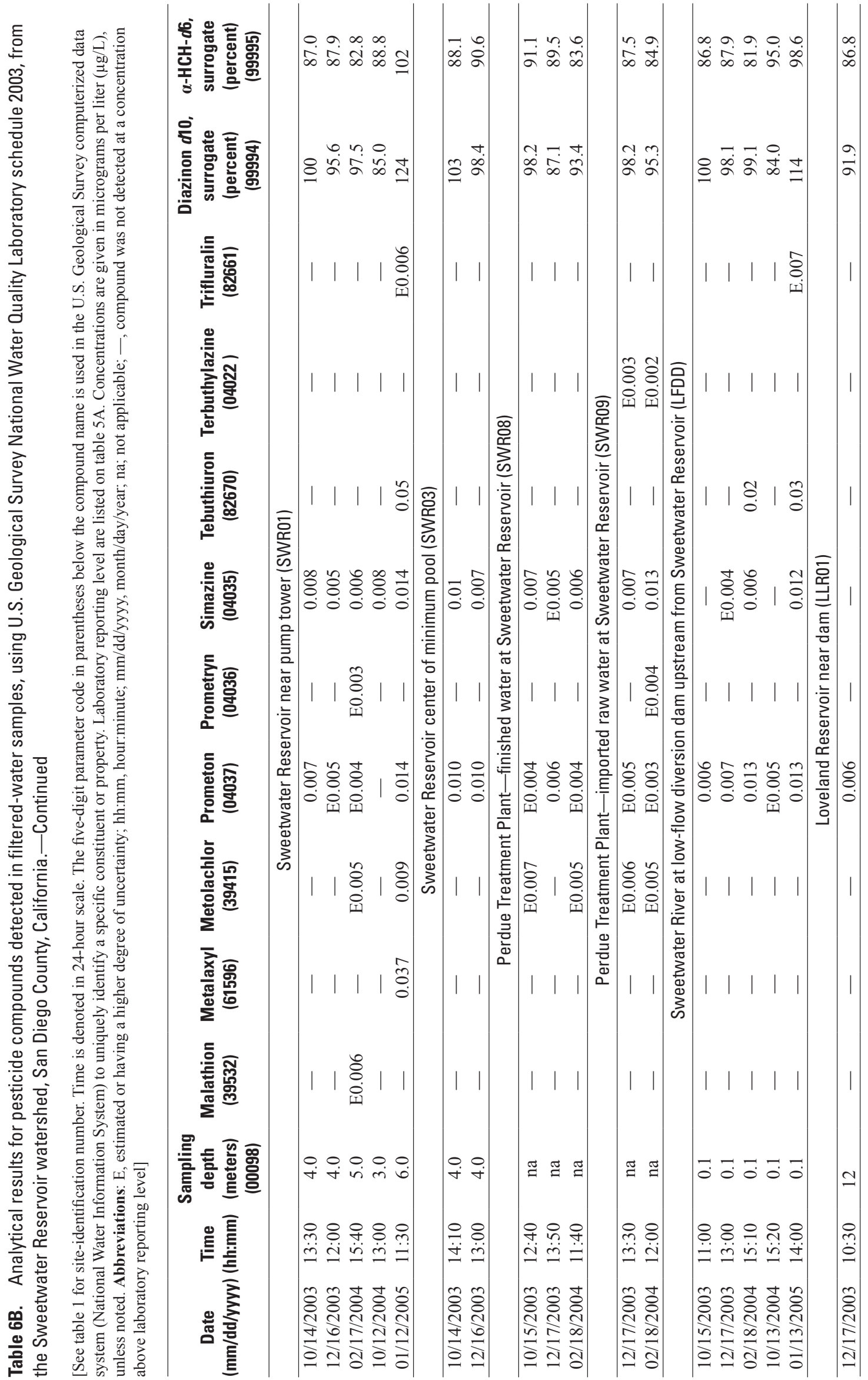


Table 7A. Pesticide compounds analyzed in filtered-water samples, using U.S. Geological Survey National Water Quality Laboratory schedule 2060, for the Sweetwater Reservoir watershed, San Diego County, California.

[The parameter code is used in the U.S. Geological Survey computerized data system (National Water Information System) to uniquely identify a specific constituent or property. Laboratory reporting level (LRL) values are reported as micrograms per liter $(\mu \mathrm{g} / \mathrm{L})$, unless noted. This schedule is not the preferred method for compounds atrazine, 2-chloro-4-isopropylamino-6-amino-s-triazine, caffeine, and tebuthiuron.]

\begin{tabular}{|c|c|c|c|c|c|}
\hline Compound & $\begin{array}{l}\text { Parameter } \\
\text { code }\end{array}$ & LRL & Compound & $\begin{array}{l}\text { Parameter } \\
\text { code }\end{array}$ & LRL \\
\hline Acifluorfen & 49315 & $0.007,0.028$ & Flumetsulam & 61694 & $0.011,0.040$ \\
\hline Aldicarb sulfone & 49313 & $0.02,0.018,0.08$ & Fluometuron & 38811 & $0.031,0.016$ \\
\hline Aldicarb sulfoxide & 49314 & $0.008,0.022$ & 3-Hydroxycarbofuran & 49308 & $0.006,0.008$ \\
\hline Aldicarb & 49312 & 0.04 & 2-Hydroxy-4-isopropylamino-6- & 50355 & $0.008,0.032$ \\
\hline Atrazine & 39632 & $0.009,0.008$ & ethylamino-s-triazine & & \\
\hline Bensulfuron & 61693 & $0.016,0.018$ & Imidacloprid & 61695 & $0.007,0.020$ \\
\hline Bentazon & 38711 & $0.011,0.012$ & Linuron & 38478 & 0.014 \\
\hline Bromacil & 04029 & $0.033,0.018$ & MCPA & 38482 & $0.016,0.030$ \\
\hline Bromoxynil & 49311 & $0.017,0.028$ & МСРB & 38487 & $0.015,0.010$ \\
\hline Caffeine & 50305 & $0.010,0.018$ & Metalaxyl & 50359 & $0.02,0.012$ \\
\hline \multirow{2}{*}{$\begin{array}{l}\text { 2-Chloro-6-ethylamino-4- } \\
\text { amino-s-triazine }\end{array}$} & \multirow[t]{2}{*}{04038} & \multirow[t]{2}{*}{$0.01,0.044,0.04$} & Nicosulfuron & 50364 & $0.013,0.04$ \\
\hline & & & Norflurazon & 49293 & $0.016,0.020$ \\
\hline \multirow{2}{*}{$\begin{array}{l}\text { 2-Chloro-4-isopropylamino-6- } \\
\text { amino-s-triazine (CIAT) }\end{array}$} & \multirow[t]{2}{*}{04040} & \multirow[t]{2}{*}{0.028} & Oryzalin & 49292 & $0.018,0.012$ \\
\hline & & & Oxamyl & 38866 & $0.012,0.030$ \\
\hline$N$-(4-Chlorophenyl)- $N^{\prime}$-methylurea & 61692 & $0.024,0.036$ & Picloram & 49291 & $0.020,0.032$ \\
\hline Clopyralid & 49305 & $0.014,0.024$ & Propham & 49236 & $0.010,0.030$ \\
\hline Cycloate & 04031 & $0.013,0.014$ & Propiconazole & 50471 & $0.021,0.010$ \\
\hline $2,4-\mathrm{D}$ & 39732 & $0.022,0.038$ & Propoxur & 38538 & 0.008 \\
\hline 2,4-DB & 38746 & 0.016 & Siduron & 38548 & $0.017,0.020$ \\
\hline 2,4-D methyl ester & 50470 & 0.009 & Sulfometuron & 50377 & $0.009,0.038$ \\
\hline Dacthal monoacid & 49304 & $0.012,0.028$ & Tebuthiuron & 82670 & $0.006,0.026$ \\
\hline
\end{tabular}


Table 7B. Analytical results for pesticide compounds detected in filtered-water samples using U.S. Geological Survey National Water Quality Laboratory schedule 2060, from the Sweetwater Reservoir watershed, San Diego County, California.

[See table 1 for site-identification number. Time is denoted in 24-hour scale. The five-digit parameter code in parentheses below the compound name is used in the U.S. Geological Survey computerized data system (National Water Information System) to uniquely identify a specific constituent or property. Laboratory reporting levels are listed on table 6A. Concentrations are given in micrograms per liter $(\mu \mathrm{g} / \mathrm{L})$, unless noted. Abbreviations: E, estimated or having a higher degree of uncertainty; hh:mm, hour:minute; mm/dd/yyyy, month/day/year; na, not applicable; —, compound was not detected at a concentration above laboratory reporting level]

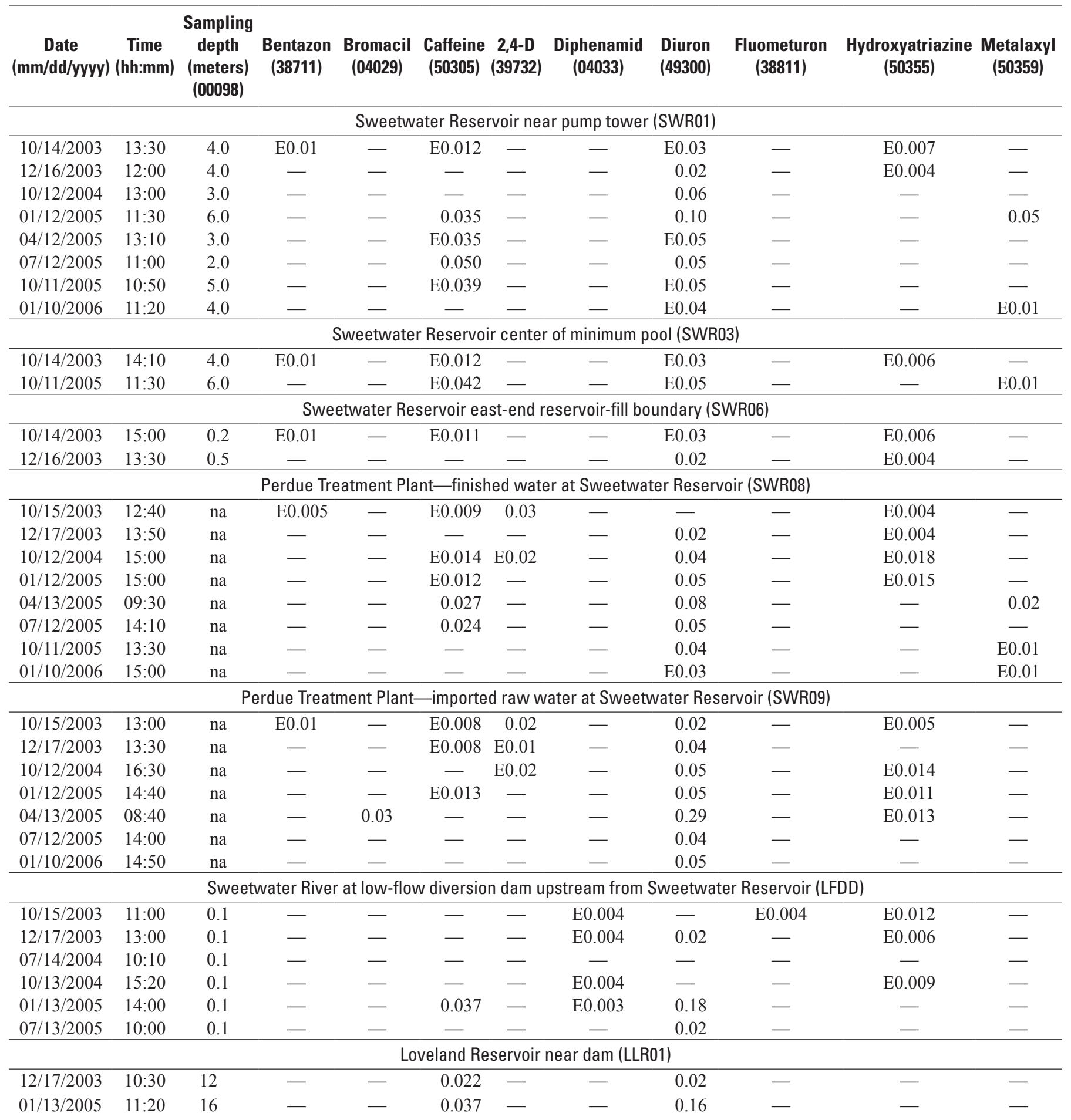


Table 7B. Analytical results for pesticide compounds detected in filtered-water samples using U.S. Geological Survey National Water Quality Laboratory schedule 2060, from the Sweetwater Reservoir watershed, San Diego County, California.-Continued

[See table 1 for site-identification number. Time is denoted in 24-hour scale. The five-digit parameter code in parentheses below the compound name is used in the U.S. Geological Survey computerized data system (National Water Information System) to uniquely identify a specific constituent or property. Laboratory reporting levels are listed on table 6A. Concentrations are given in micrograms per liter $(\mu \mathrm{g} / \mathrm{L})$, unless noted. Abbreviations: E, estimated or having a higher degree of uncertainty; hh:mm, hour:minute; mm/dd/yyyy, month/day/year; na, not applicable; —, compound was not detected at a concentration above laboratory reporting level]

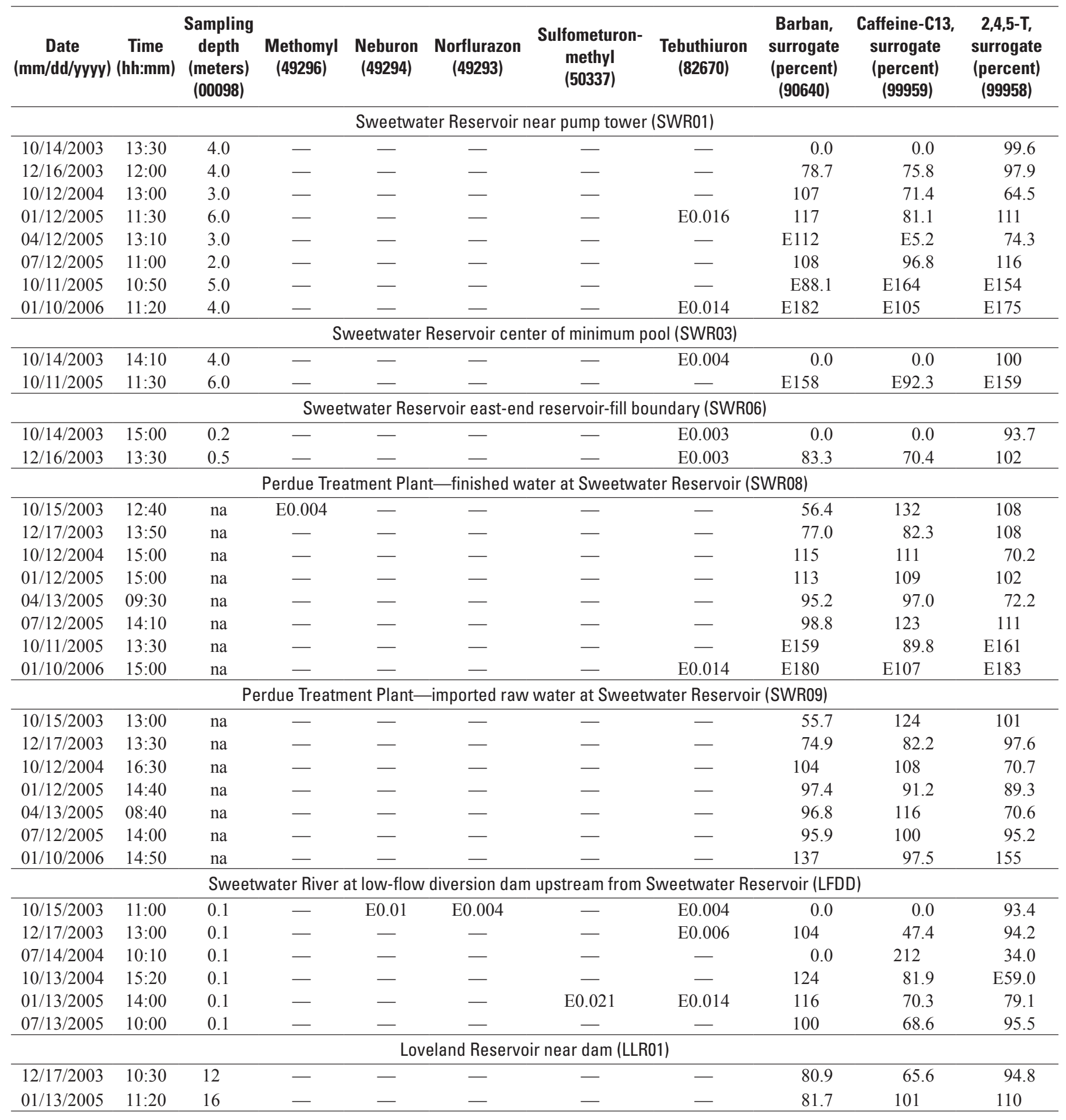


Table 8A. Anthropogenic organic indicator compounds analyzed in filtered-water samples, using U.S. Geological Survey National Water Quality Laboratory schedule 1433, for the Sweetwater Reservoir watershed, San Diego County, California.

[The parameter code is used in the U.S. Geological Survey computerized data system (National Water Information System) to uniquely identify a specific constituent or property. Laboratory reporting level (LRL) values are reported as micrograms per liter $(\mu \mathrm{g} / \mathrm{L})$, unless noted. This schedule is not the preferred method for compounds bromacil, carbaryl, chlorpyrifos, diazinon, metalaxyl, metolachlor, and prometon.]

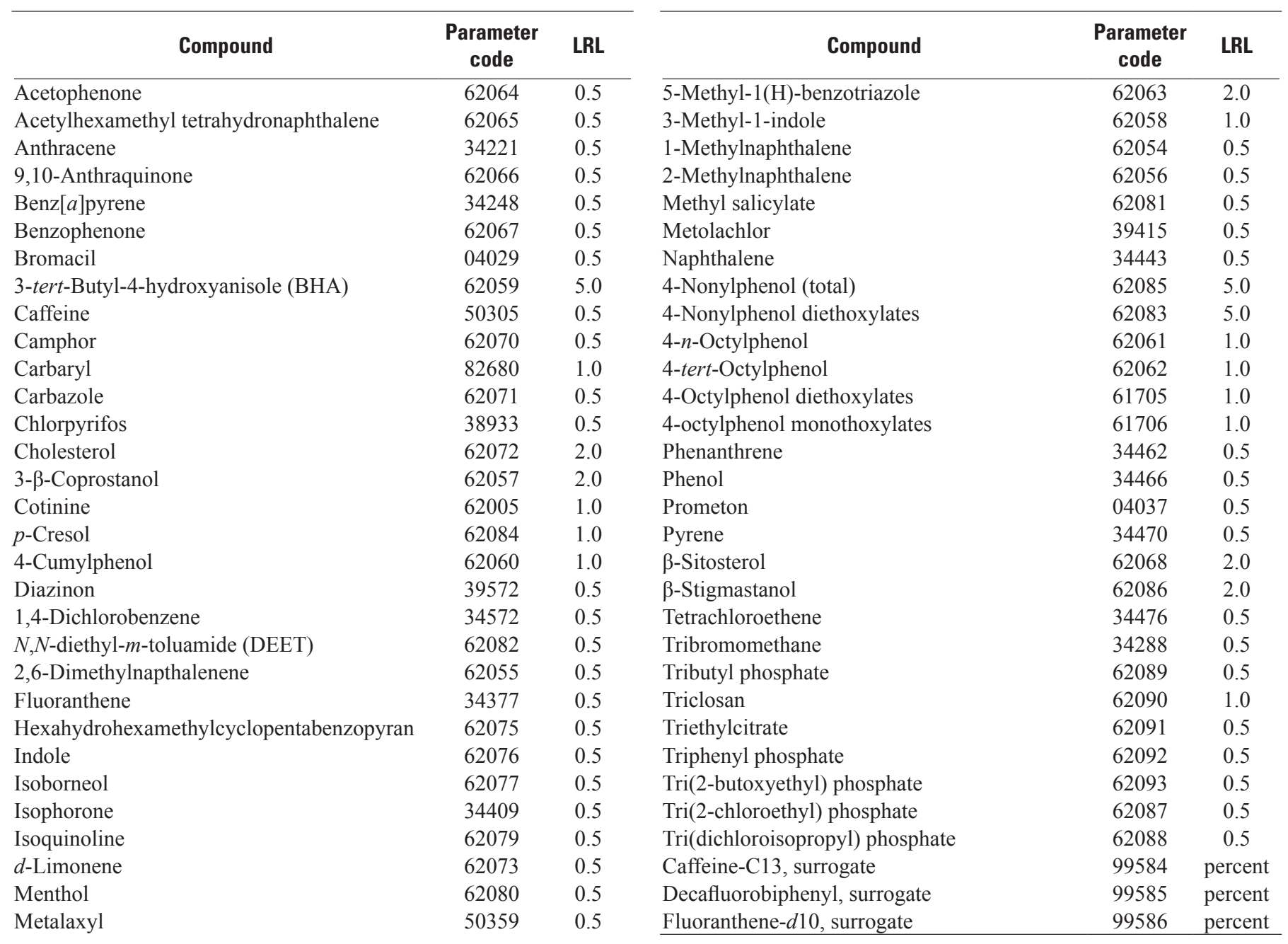




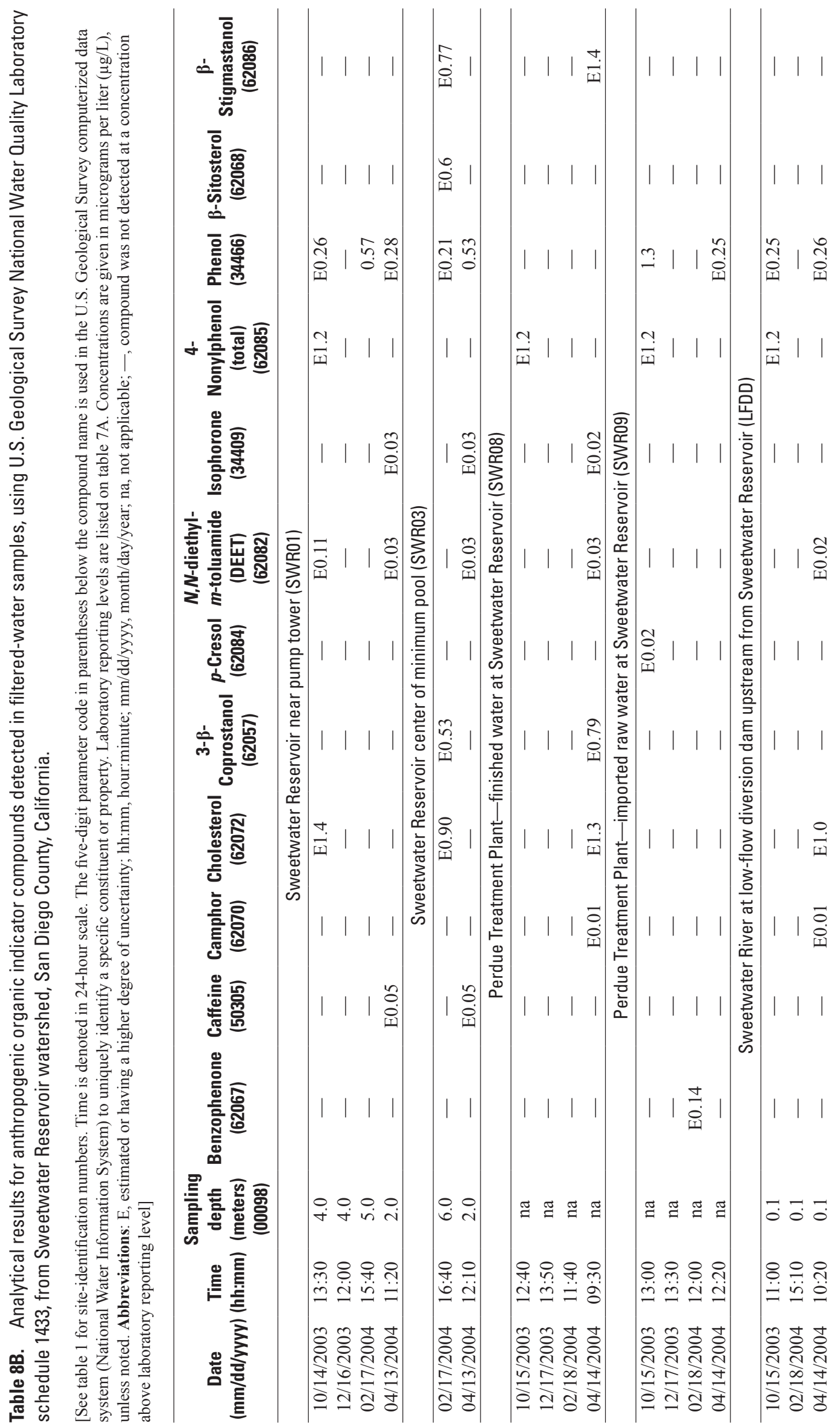



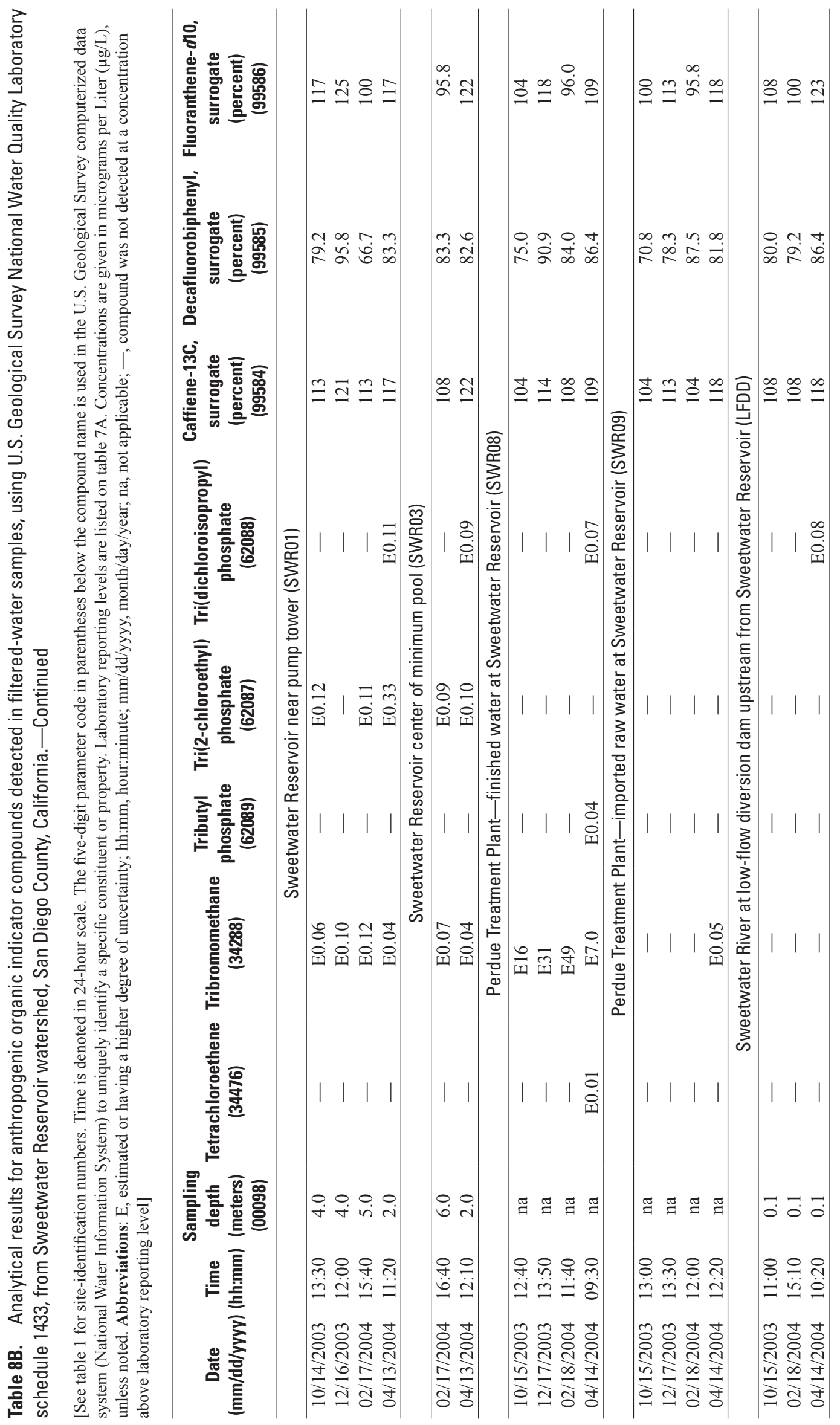


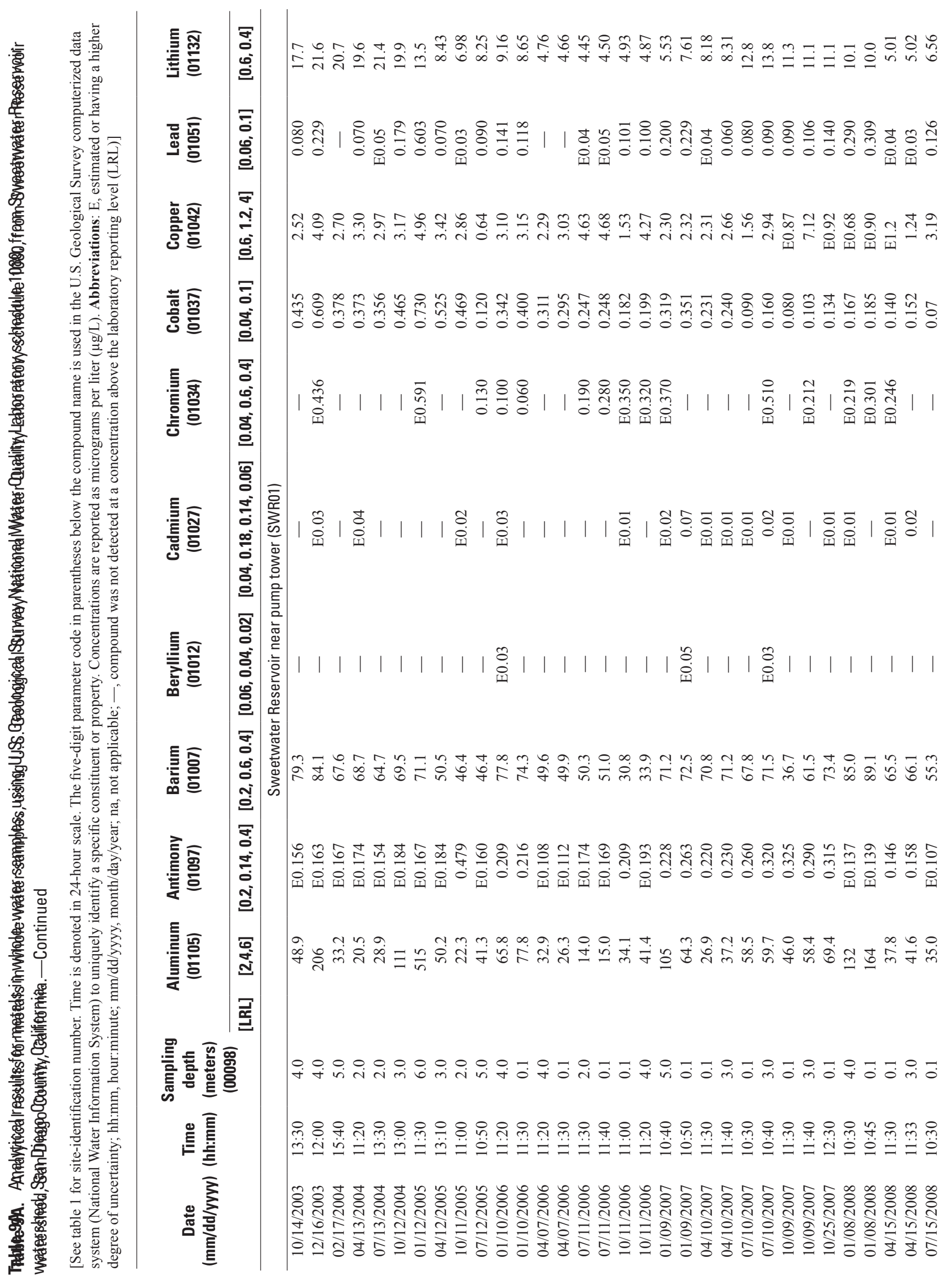




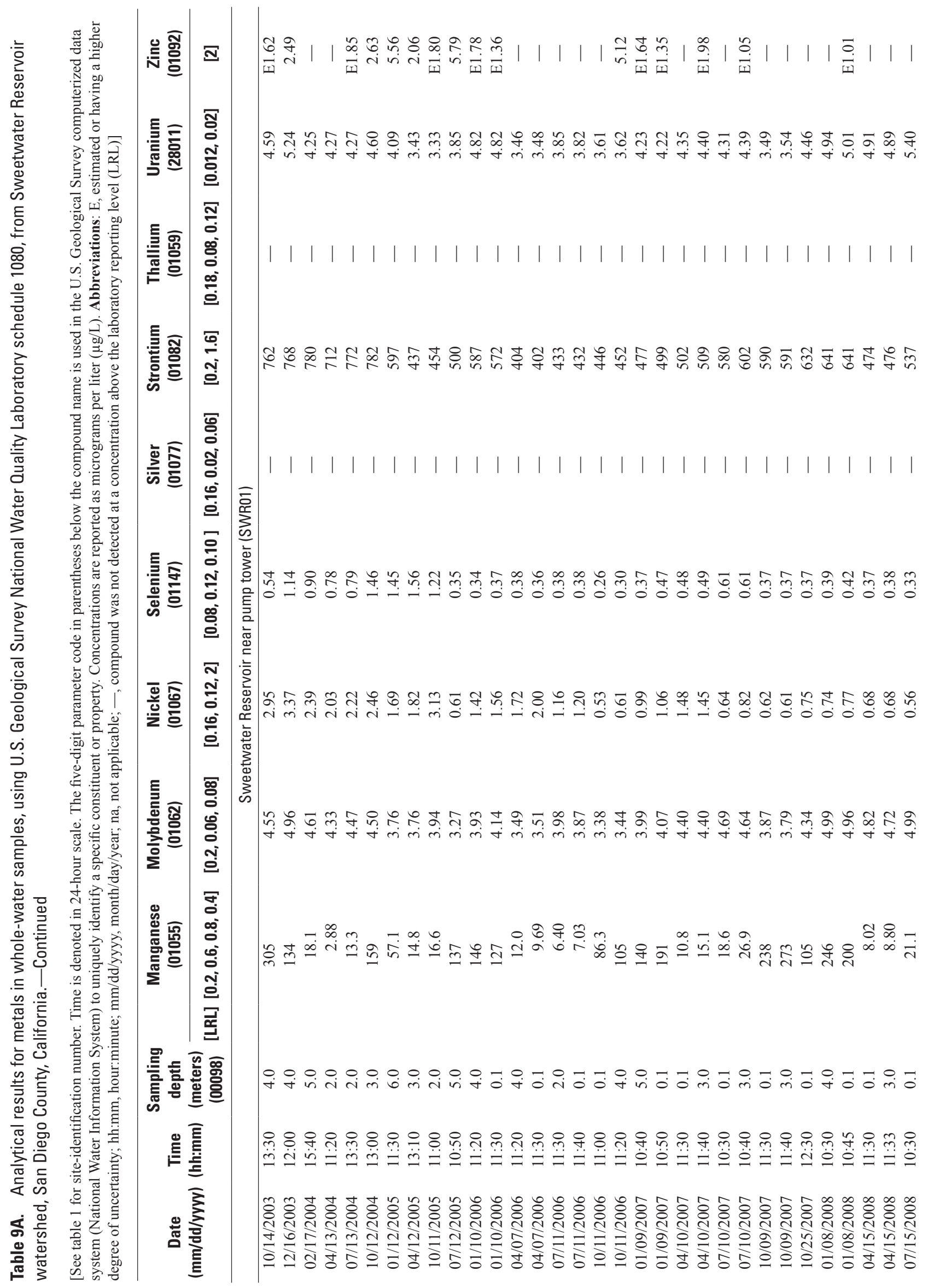




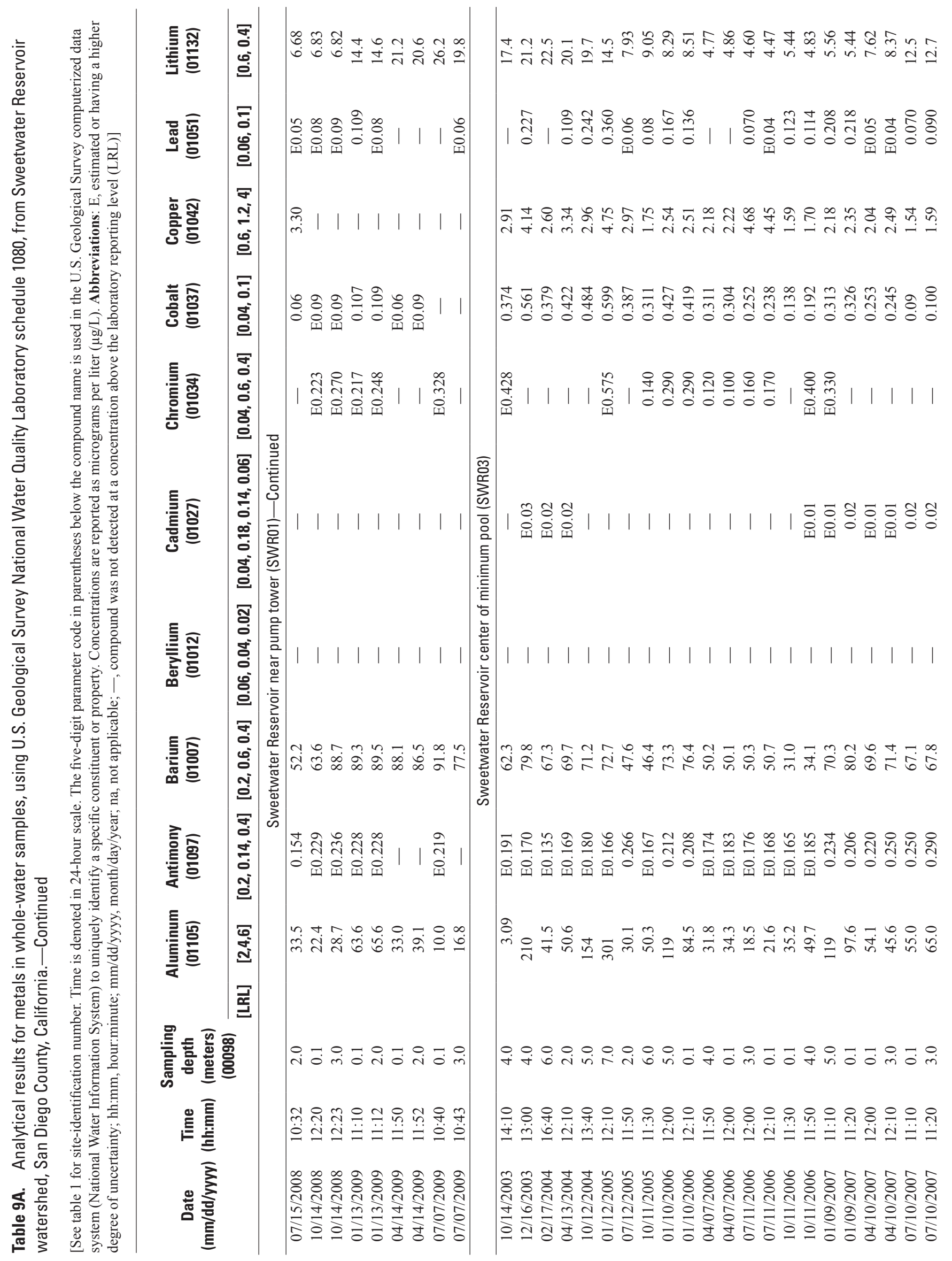




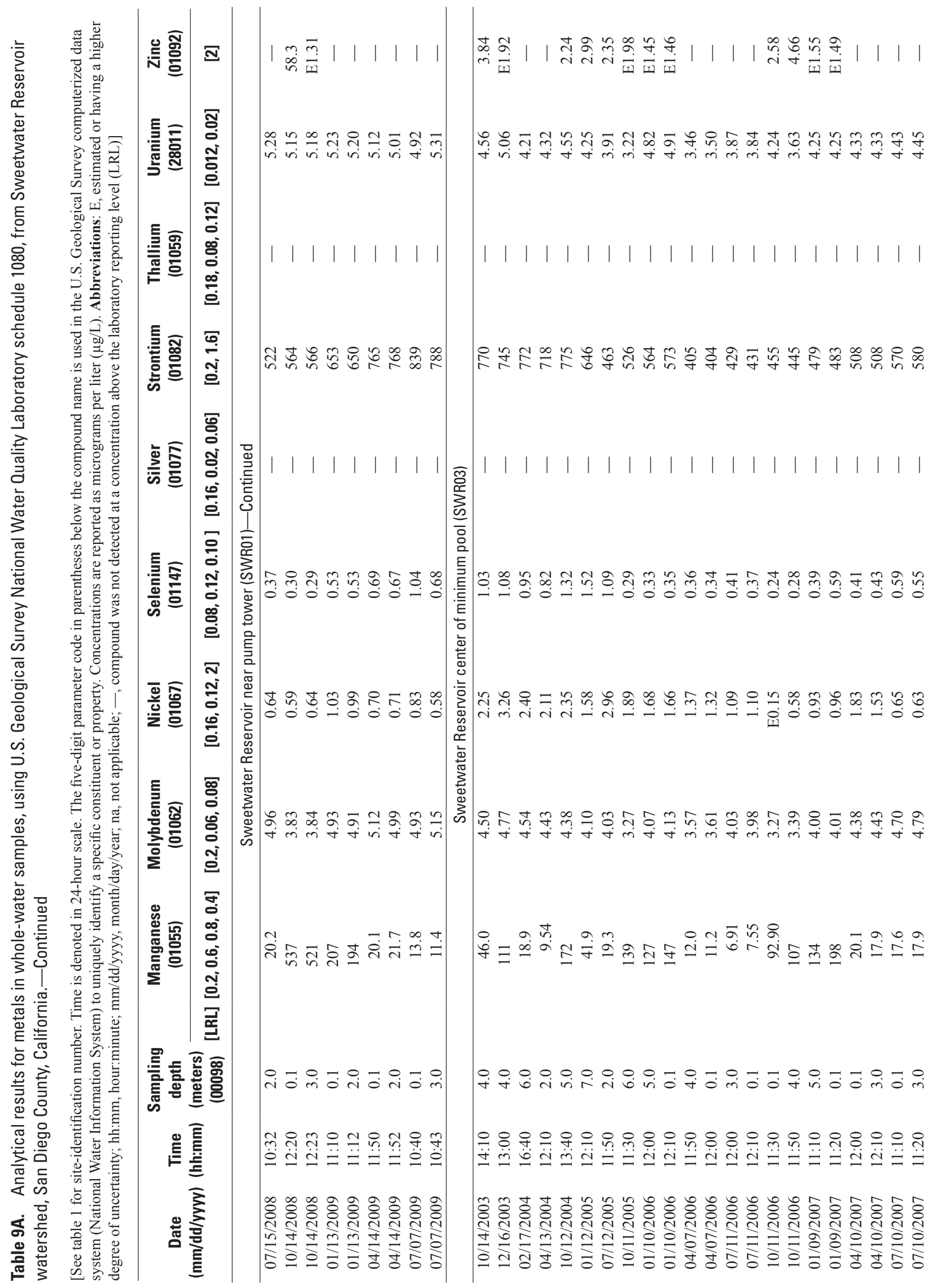




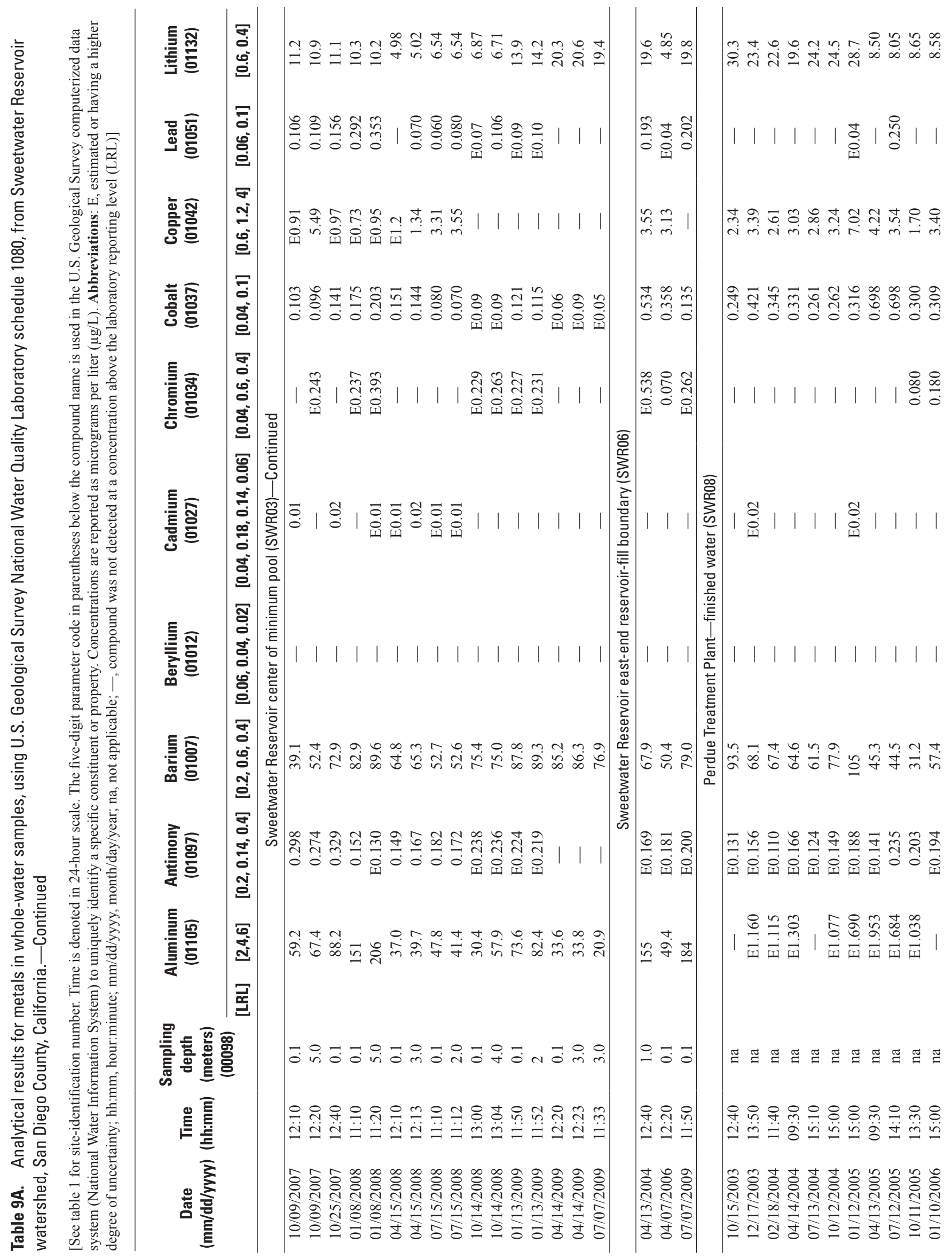




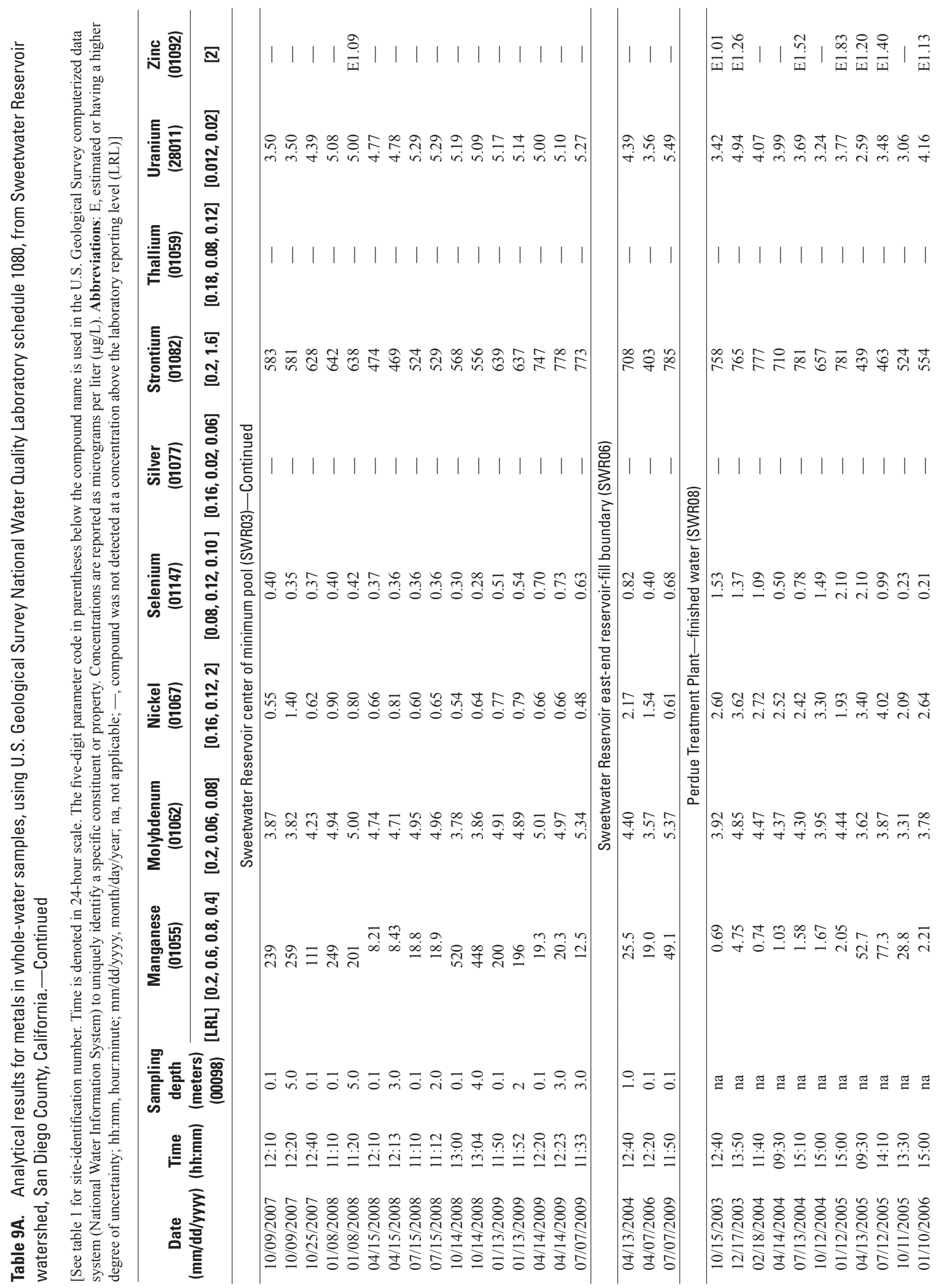




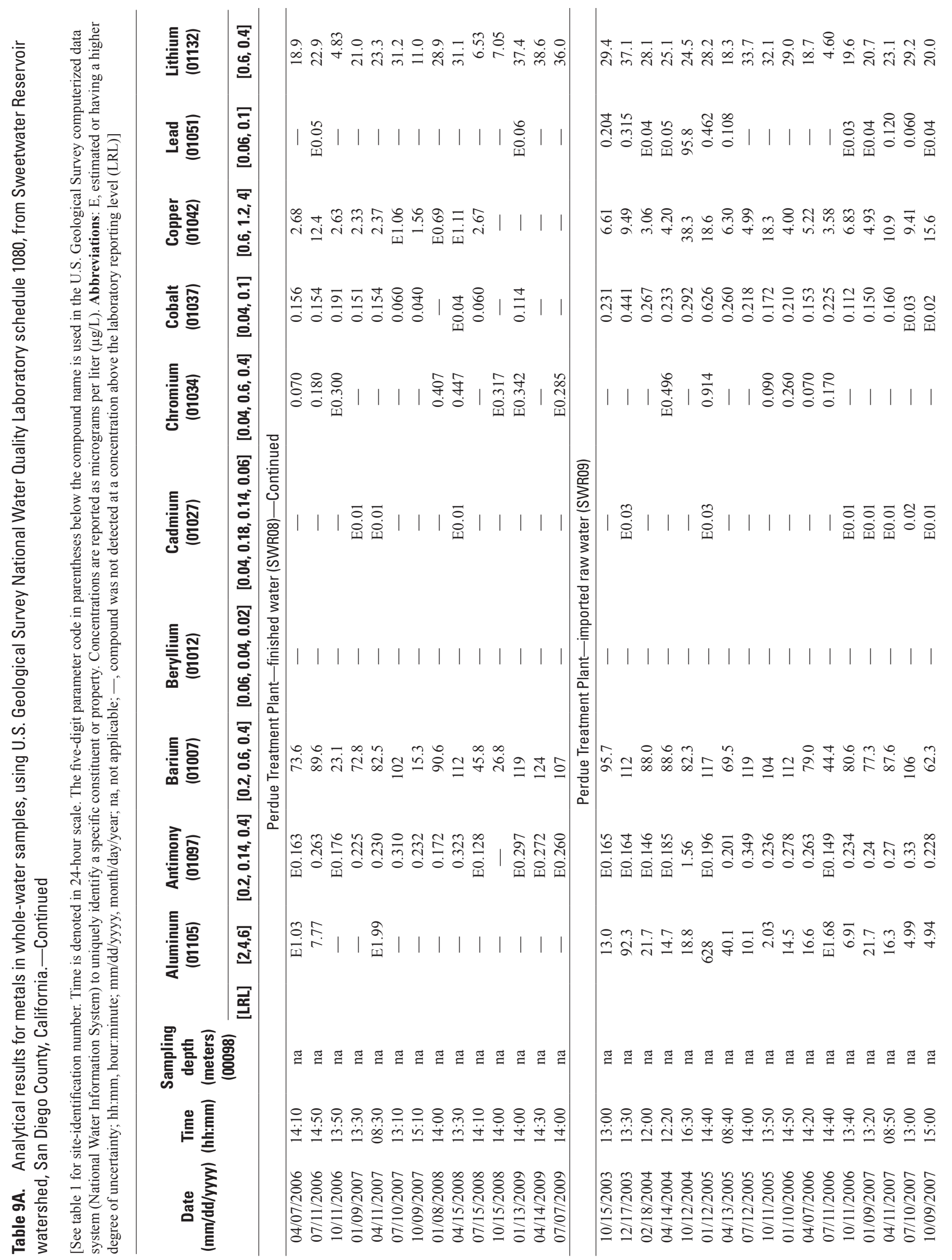




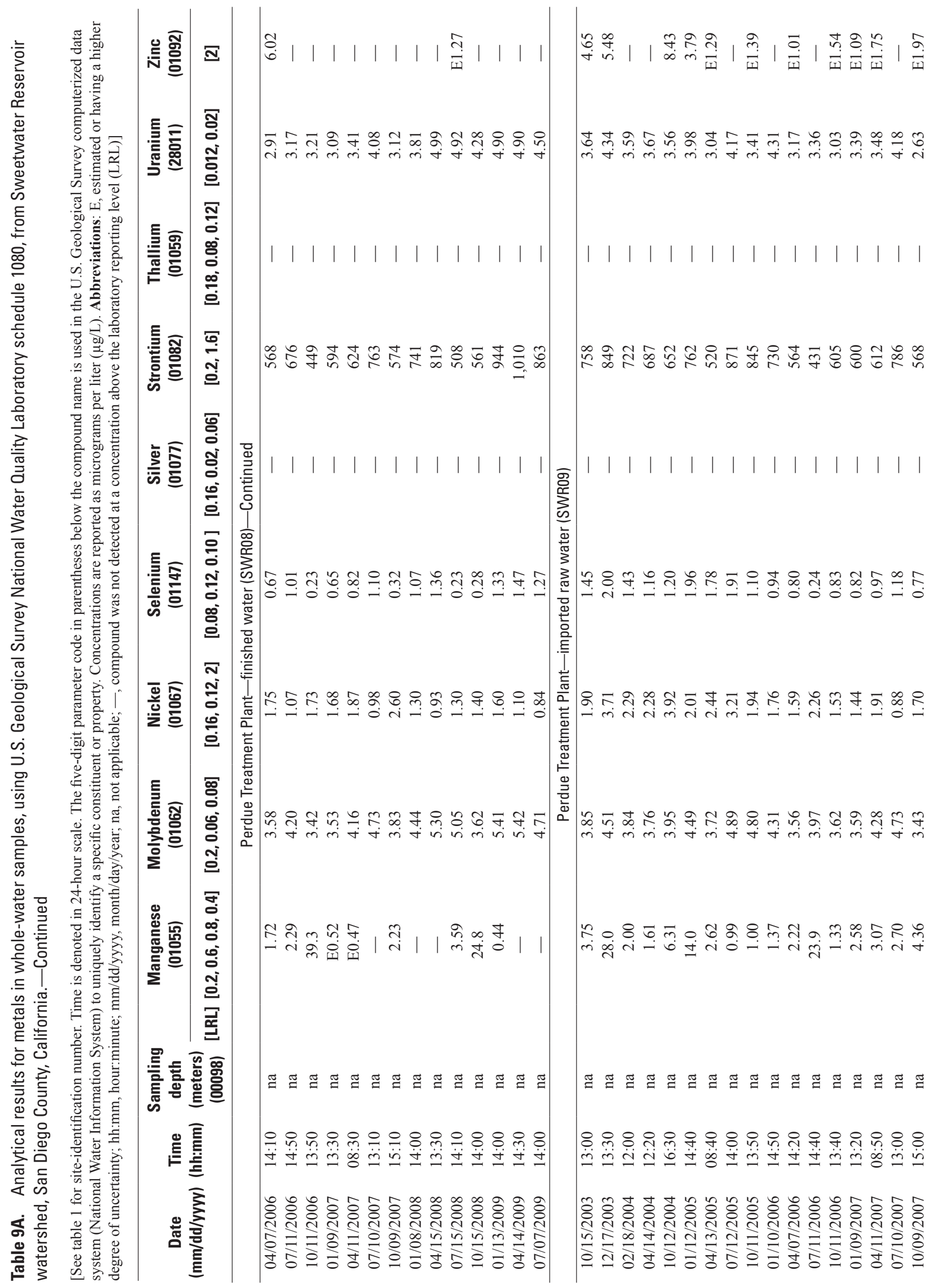




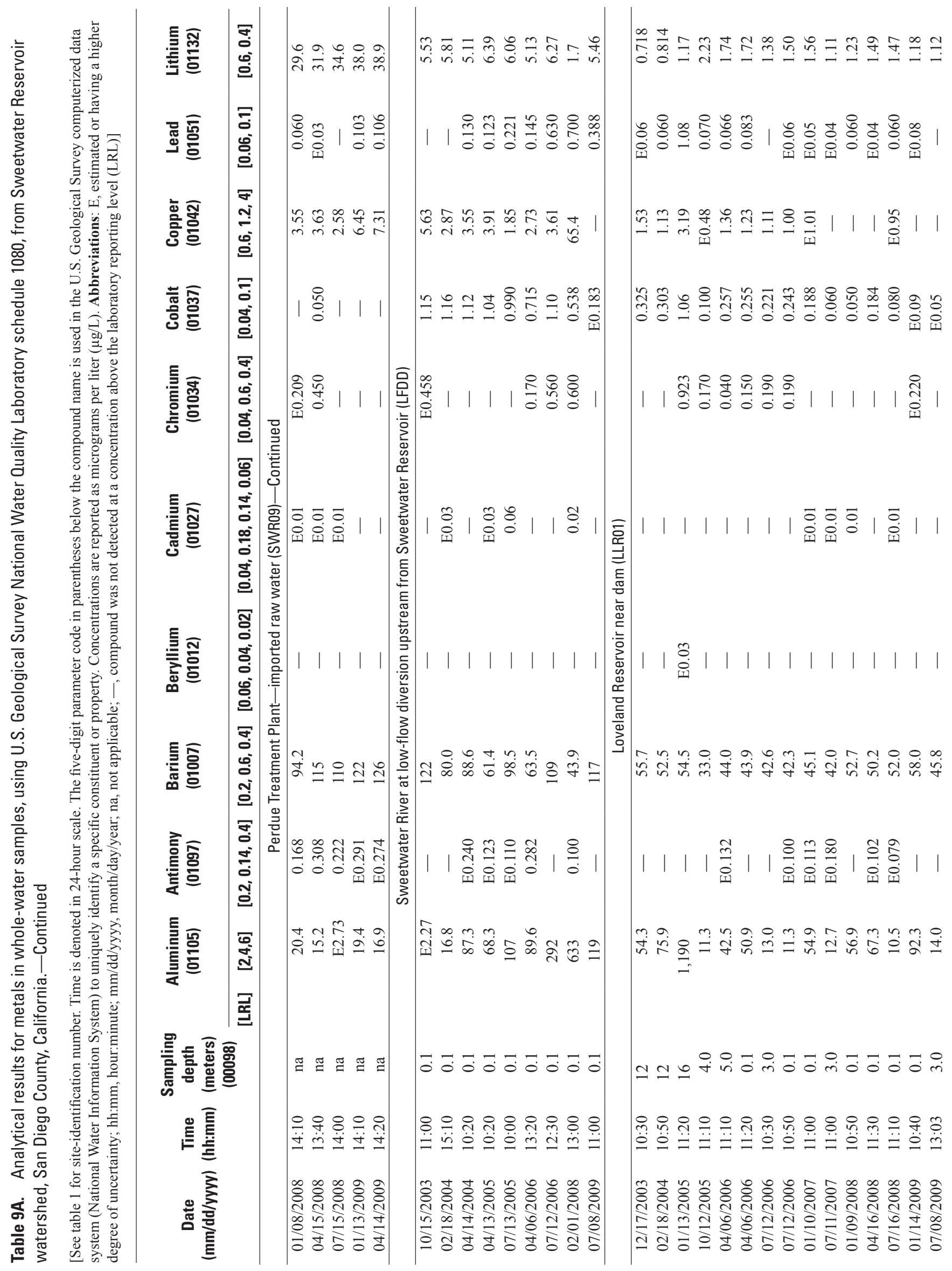




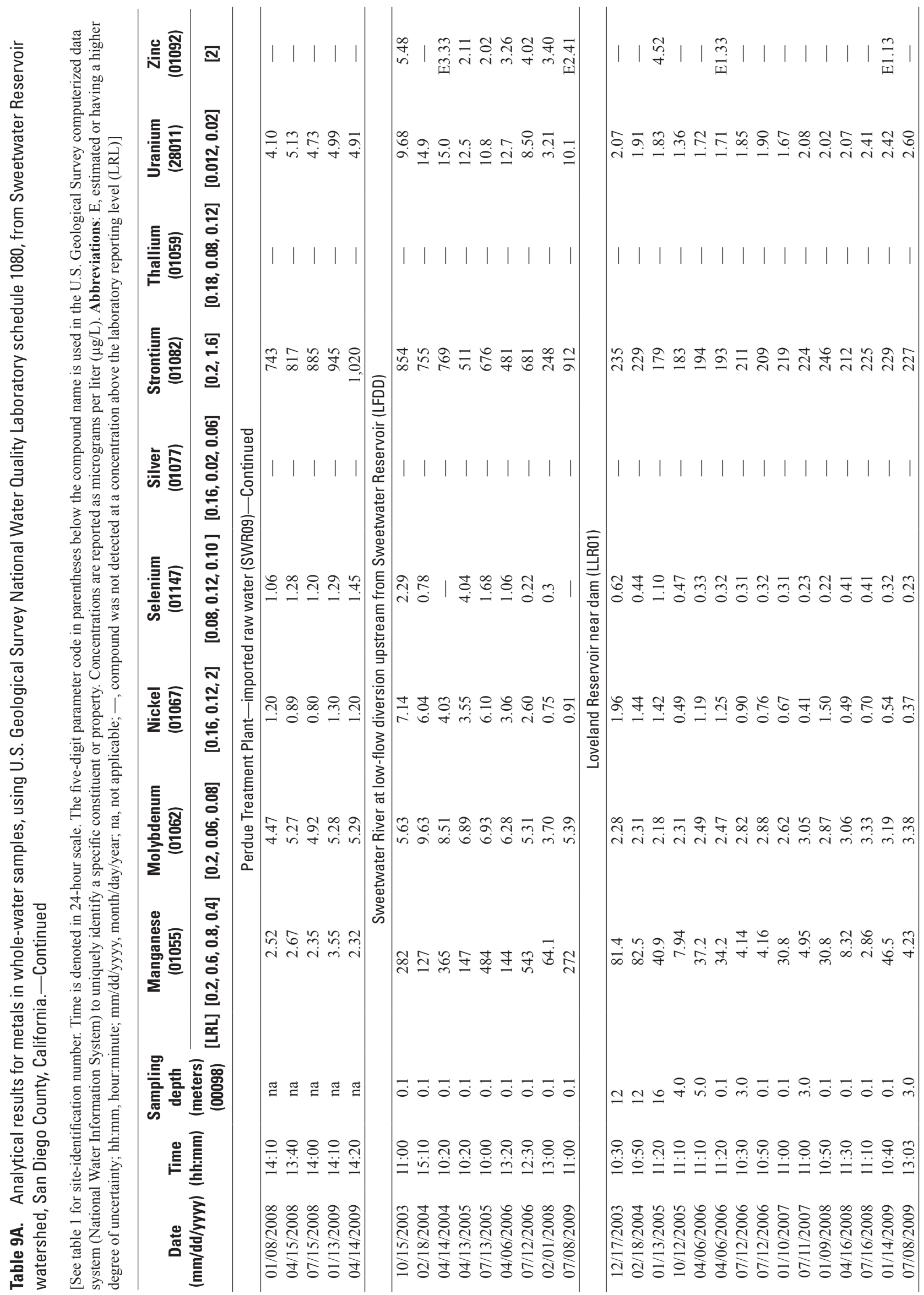




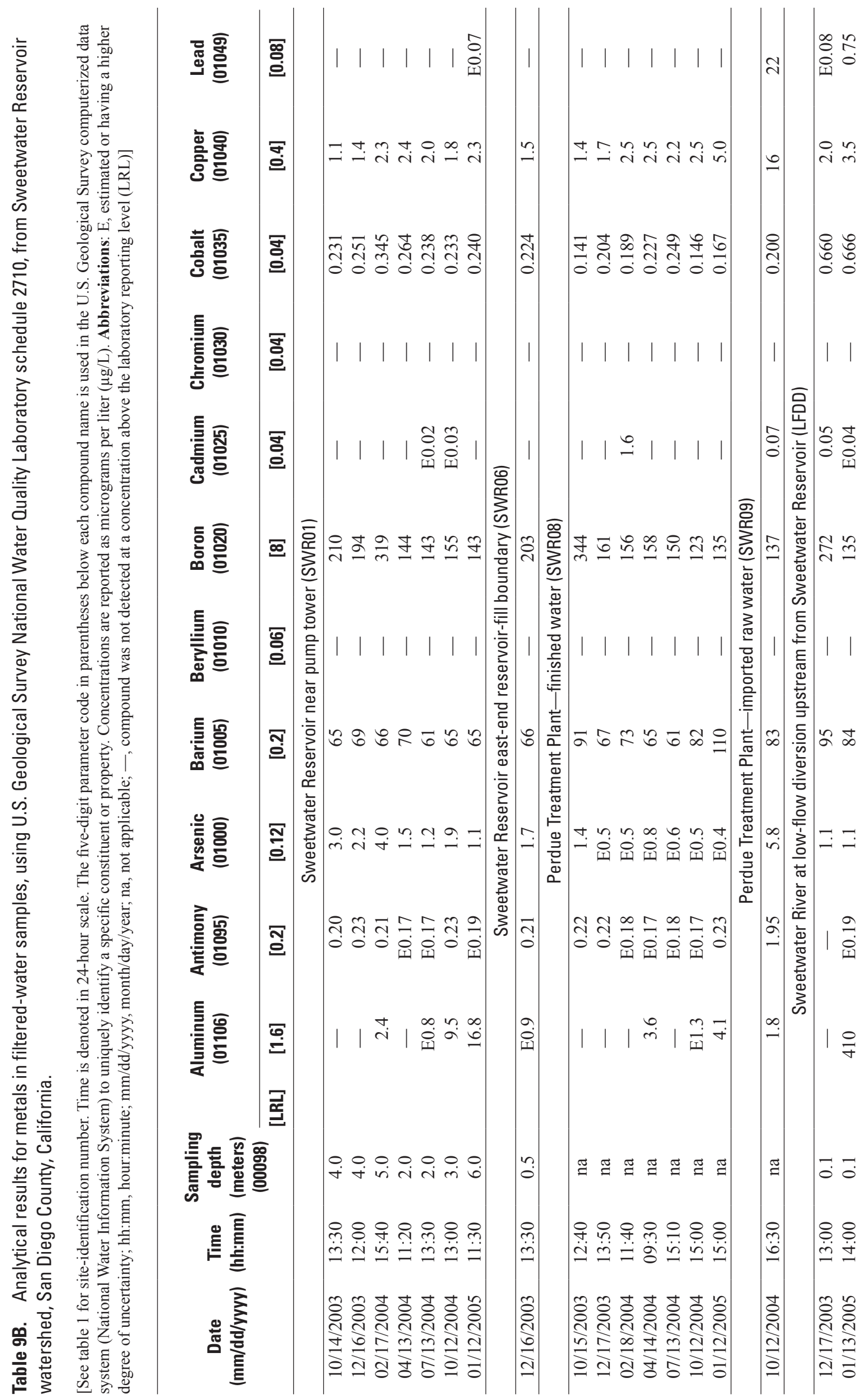




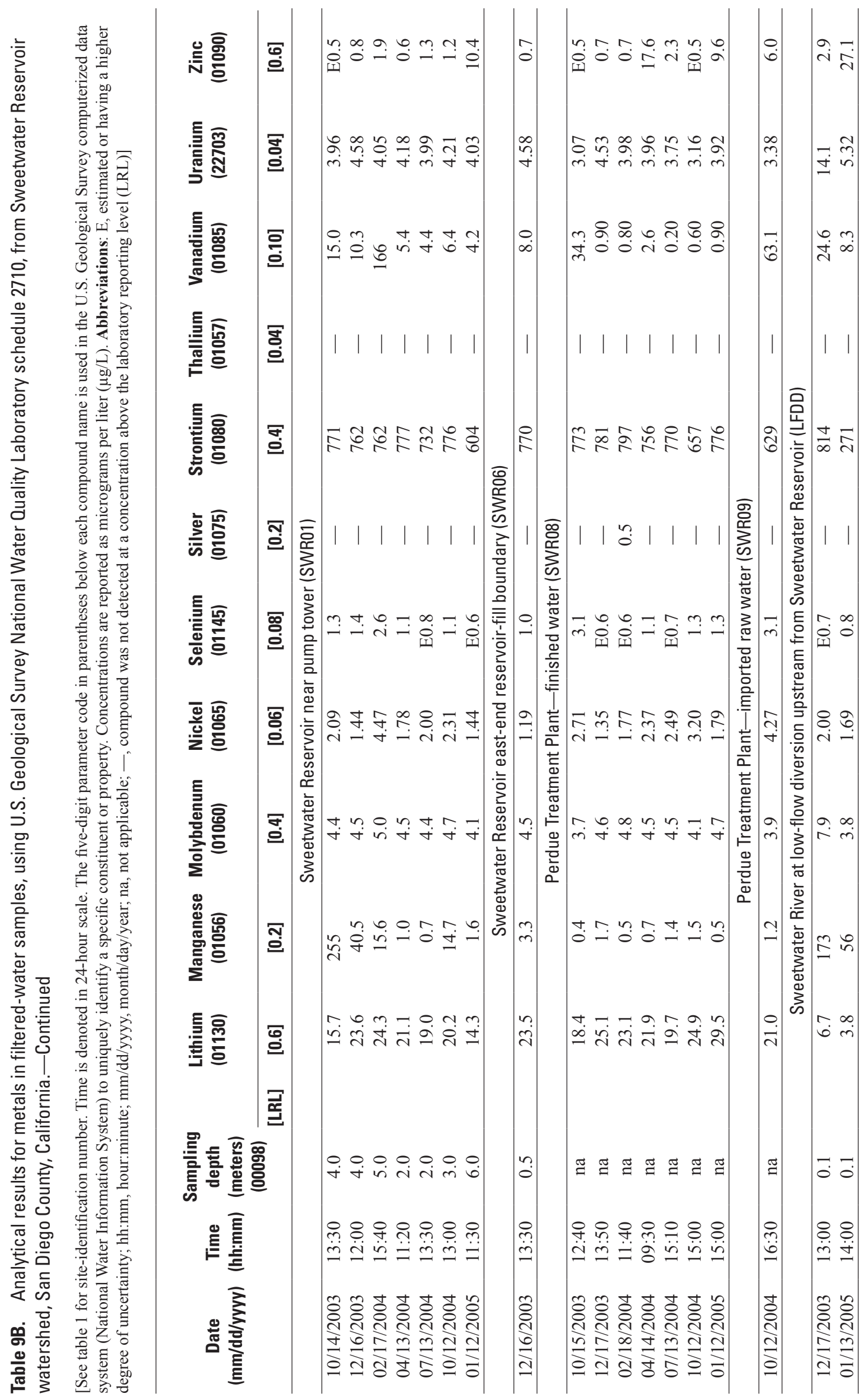




\section{Air Sampling Strategy}

The following section presents the sampling strategy used for the collection of air samples. Air samples were collected for the analysis of VOCs, SOCs, and metals. SOC analysis consisted of PAHs and pesticides (pesticides were sampled through August 2004). Beginning in November 2003, air samples also were collected for analysis of metals. All the air sampling methods are documented in this or the previous SWR project reports or in other publications as noted below. Most air data have not been entered into the USGS NWIS database, except for data from a limited number of samples for the AirPAH method described below.

Air data collected at the SWR atmospheric sampling site may be used to determine the occurrence, temporal patterns, and ambient concentration levels of selected airborne organic compounds (VOCs, PAHs, and pesticides) and metals. Site AIR01 was installed downwind of the SR 125 alignment and upwind of SWR (fig. 2) along the predominant wind direction. The South Coast Air Pollution Control Board (William Brick, oral commun., 1999) helped with the siting and establishment of this atmospheric-sampling station following guidelines outlined by the National Atmospheric Deposition Program (Bigelow, 1984). Air samples were not collected at LLR because its foothill location (30 km east of SWR) was considered sufficiently downwind of SR 125 such that water quality at LLR would be expected to be minimally affected by any airborne contaminants originating from SR 125 .

SOC air samples were collected by using a sampler referred to as the SOC air sampler. Collection times for the SOC air samples were modified several times during this reporting period; the goal was to match the collection periods used for the air samples for VOCs, as well as those used by the California Air Resources Board Air Toxics Program at their sampling station in Chula Vista, Calif. Initially for this reporting period, the SOC air sample was a 24-hour, 7-day weekly composite collected every fourth week, with a 3-week time span between sample collection periods. Beginning November 17, 2003, the collection time was changed to a 24-hour composite collected every 12th day to coincide with the air sample collection period for VOCs. The 24-hour period of active air flow collection was from midnight to midnight; the air sampler was deployed at the sampling site typically between $10 \mathrm{a} . \mathrm{m}$. and $2 \mathrm{p} . \mathrm{m}$. the day before sample collection and was retrieved from the site between 10 a.m. and 2 p.m. the day sample collection ended. Although the air-flow rate was increased to compensate for the change from a weekly to a 24-hour period, air volumes for the SOC air samples were lower (mean volume of $125 \mathrm{~m}^{3}$; range $112-150 \mathrm{~m}^{3}$ ) than for previous sample dates (mean volume of $330 \mathrm{~m}^{3}$; range 220-584 $\mathrm{m}^{3}$ ) and lower than desired. Therefore, beginning March 28, 2004, the collection time was increased to a 48-hour composite ( midnight to midnight), with initiation of sample collection matching the dates of VOC sample collection (mean volume of $382 \mathrm{~m}^{3}$; range $253-412 \mathrm{~m}^{3}$ ).
The SOC collection period returned to a 24-hour composite beginning October 5, 2004, but with a slightly increased air flow that provided a mean volume of $185 \mathrm{~m}^{3}$ (range 159-209 $\mathrm{m}^{3}$ ), and remained at this frequency for the duration of the reporting period. Beginning with the September 2, 2006, SOC sample, wider outlet tubing was installed on the sampler to provide greater sample volumes (mean volume of $437 \mathrm{~m}^{3}$; range $237-522 \mathrm{~m}^{3}$ ) for the 24-hour period. The increased sample volume was desired to help lower method detection levels for the PAHs.

\section{Air Methods}

\section{Volatile Organic Compounds}

The air sampling and analytical methods for VOCs were developed by, and all samples were analyzed by, the research group of Dr. James Pankow at the Oregon Graduate Institute of Oregon Health and Science University. The VOC air sampling and analytical methods are detailed in Pankow and others $(1998,2003)$. All VOC air samples were analyzed by using adsorption/thermal desorption GC/MS. Concentrations of VOCs in air are reported in parts per billion by volume (ppbv). These air methods were also used in several other USGS studies that investigated the occurrence and distribution of VOCs in air (Baehr and others, 1999a, 1999b; Bender and others, 2000). Ambient gas-phase atmospheric concentrations of VOCs were collected by using two programmable lowvolume air sampling pumps (224-PCXR8, SKC ${ }^{\circledR}$ Inc., Eighty Four, $\mathrm{Pa}$.), each attached to a cartridge containing different sorbent materials. One pump pulled approximately $1.5 \mathrm{~L}$ of air through a glass cartridge containing $50 \mathrm{mg}$ of Carbotrap B in series with $280 \mathrm{mg}$ of Carboxen 1000 (Supelco, Bellefonte, $\mathrm{Pa}$.). These samples were analyzed for compounds with the highest volatilities (high-volatility VOCs). The second pump pulled approximately $5 \mathrm{~L}$ of air through a glass cartridge containing $180 \mathrm{mg}$ of Carbotrap B in series with $70 \mathrm{mg}$ of Carboxen 1000 (Supelco, Bellefonte, Pa.). These samples were analyzed for VOCs with lower volatilities (medium/lowvolatility VOCs). Air samples were analyzed for 85 VOCs, which range in volatility from that of chloromethane (vapor pressure of $506 \mathrm{Kilopascal}[\mathrm{kPa}]$ at $20^{\circ} \mathrm{C}$ ) to 1,2,3-trichlorobenzene (subcooled liquid-phase vapor pressure of $58.2 \mathrm{kPa}$ at $25^{\circ} \mathrm{C}$ ) (Mackay and others, 1993).

\section{Semivolatile Organic Compounds}

The air sampling and analytical methods for SOCs were comparable to those described in Foreman and others (2000) for pesticides and to the EPA methods TO-4A for pesticides (U.S. Environmental Protection Agency, 1999a) and TO-13A for PAHs (Foreman and others, 1995; U.S. Environmental Protection Agency, 1999b). The air methods were developed 
to complement those methods for pesticides in water (Zaugg and others, 1995; Sandstrom and others, 2001) and for PAHs/ alkyl PAHs in sediment (Olson and others, 2004) that were used in the SWR monitoring program. The sampling and analytical methods used for the air samples for SOCs have been used in several other specialized studies undertaken by the USGS (Foreman and others, 1997, 2000; Majewski and others, 1998; Mendez and others, 2007). SOC concentrations are reported in nanograms per cubic meter of air and can be converted to parts per billion by volume.

The high-volume air samples for SOCs were collected by drawing air through a $90-\mathrm{mm}$ diameter GFF (type A/E, Pall Corp, East Hills, NewYork) and then through a cartridge containing two polyurethane foam (PUF) plugs. The GFF, containing collected atmospheric particles, was analyzed to provide the operationally defined particulatephase concentration of an SOC. The PUF plugs collected the operationally defined gas-phase concentration of an SOC. Before being used, the GFFs were cleaned by baking them at $450{ }^{\circ} \mathrm{C}$ and allowing them to cool in a desiccator for at least 2 hours. The GFFs were then weighed to the nearest $0.2 \mathrm{mg}$, wrapped in baked aluminum foil, and stored in resealable polyethylene bags. At SWR, a GFF was removed from the foil with clean (methanol-rinsed) stainless-steel forceps and placed in a perfluoralkoxy fluoropolymer (Teflon-PFA) filter holder (series 90, Savillex Corp., Minnetonka, Minnesota) that was modified in the following two ways: (1) the outer closure piece of the filter holder was removed to provide an $80-\mathrm{mm}$ diameter opening that would improve air flow and expose most of the GFF surface, enabling it to better collect atmospheric particles; and (2) the inner closure piece of the filter holder was machined to include a $3.8-\mathrm{cm}$ female National Pipe Thread to allow a direct connection to the $3.8-\mathrm{cm}$ male National PipeThread on the inlet of the PUF cartridge.

PUF plugs were $5 \mathrm{~cm}$ in diameter by $7.6 \mathrm{~cm}$ long and were prepared from open-cell foam that contained no polybrominated diphenyl ether flame retardants. For samples collected through June 15, 2005, the average PUF density was $0.043 \mathrm{~g} / \mathrm{cm}^{3}$ (Netherland Rubber Company, Cincinnati, Ohio). However, the manufacture of this PUF material was discontinued. Beginning with the July 21, 2005, sample, PUF plugs had an average density of $0.022 \mathrm{~g} / \mathrm{cm}^{3}$ (Tisch Environmental, Village of Cleves, Ohio), corresponding to the density prescribed by EPA Method TO-4A. PUF plugs were cleaned by first being rinsed with tap water and then sequentially extracted for at least 12 hours in a "Soxhlet" extractor apparatus with each of the following solvents: acetone, 30-percent ethyl acetate in hexane, and dichloromethane. Beginning July 26, 2004, only acetone was used as the final extraction solvent to clean PUF plugs because it provides a cleaner PUF plug based on the results from a related atmospheric contaminants study (Garrison and others, 2006). Residual solvent was squeezed from the PUF plugs using an acetone-rinsed stainless-steel potato masher, and the plugs were dried in a vacuum oven at $40{ }^{\circ} \mathrm{C}$ for at least 48 hours before being stored in sealed $500-\mathrm{mL}$ wide-mouth jars with Teflon-lined lids.

At the SWR laboratory, primary (top) and secondary (bottom) PUF plugs were positioned in series inside a $24.2-\mathrm{cm}$ long by $3.5-\mathrm{cm}$ internal diameter Teflon-PFA cartridge (Savillex Corporation); the bottom PUF was held in place against a Teflon-PFA screen. The PUF plugs were carefully inserted into the PUF cartridge using methanol-rinsed stainless-steel forceps to ensure that the PUF plugs were wellfitted to the cartridge wall without creases that would allow air to migrate around the plug instead of passing through the foam. At the atmospheric sampling site, the PUF cartridge was connected to the GFF holder, and the GFF-PUF sampling train was positioned inside a high-volume sampler enclosure (Graesby-GMW, Village of Cleves, Ohio) comparable to that described in EPA method TO-4A (U.S. Environmental Protection Agency, 1999a). The outlet of the PUF cartridge was connected by $0.95-\mathrm{cm}$ outer diameter Teflon tubing to a blower motor. Beginning in September 2006, wider (3.9-cm internal diameter) outlet tubing was installed that nearly doubled the flow rate. Air samples were collected at the sampling sites by pulling ambient air through the GFF-PUF sampling train. Sampling periods were controlled by a timer, and sample volume was calculated by multiplying the sample collection time by the air-flow rate measured at the beginning and at the end of the sampling period using a calibrated flow meter. After the samples were collected and returned to the SWR laboratory, the GFF was removed from the holder using clean forceps, returned to the aluminum foil, folded in half (particle-laden side inward), and sealed in the foil and bag. PUF plugs were returned to the jars using forceps; the jars were labeled to identify the top or bottom PUF plug along with other relevant information and were tightly sealed with the lids. The GFF and PUF plugs were stored at $-5^{\circ} \mathrm{C}$ (maximum), if necessary, before being shipped overnight on ice packs to the USGS NWQL.

At the NWQL, the air-sample components were stored at $-5^{\circ} \mathrm{C}$ (maximum) until analyzed. The GFFs were warmed to room temperature then desiccated for 24 hours and weighed to the nearest $0.2 \mathrm{mg}$ to determine particle weight based with comparison with the GFF weight before sample collection. This particle weight was divided by the air volume of the sample to determine the total suspended particle (TSP) concentration in micrograms of particles per cubic meter of air.

Air-sample components were extracted using one of two procedures during this reporting period. Top and bottom PUF plugs were extracted together for all samples from this reporting period except the November 17, 2003, sample, which also is shown as a combined top and bottom PUF result in the data table. The combined PUF extraction was done, in part, to reduce analytical costs, and because results from the separate analysis of PUF plugs from prior samples 
provided sufficient characterization of collection efficiency for the SOCs for the air volumes collected in this study. The GFF and PUF plugs for samples collected from November 2003 through August 19, 2004, were extracted by using pressurized-solvent extraction (PSE) described previously (Mendez and others, 2008). PSE relies primarily on the elevated temperature of a pressurized solvent to achieve rapid extraction (U.S. Environmental Protection Agency, 1999a; Alexandrou and others, 2001). Each GFF was placed into an $11 \mathrm{~mL}$ stainless-steel cell, and top and bottom PUF plugs for a sample were placed into a $100 \mathrm{~mL}$ cell before the compounds were extracted. Surrogate compounds added to the environmental samples were extracted by use of an accelerated solvent extractor 200 or 300 (Dionex Corporation, Salt Lake City, Utah), using two 15-minute static cycles at $100-120^{\circ} \mathrm{C}$ (GFF) or $100^{\circ} \mathrm{C}$ (PUF) under pressurized conditions $(10,342 \mathrm{kPa})$. Although PSE provides excellent extraction efficiency for SOCs, the heated PSE produced greater amounts of coextracted interfering compounds from the PUF plugs compared to traditional solvent extraction procedures previously used in this study (Mendez and others, 2007, 2008). Therefore, PUF plugs for all samples collected after August 19, 2004, as well as many, but not all GFFs, were extracted by traditional solvent extraction. For this approach, top and bottom PUF plugs for a sample were placed in a Soxhlet apparatus. The GFF was placed in a separate $500-\mathrm{mL}$, flat-bottom flask. The GFFs or PUF plugs were fortified with the surrogate compounds (see the QA section) and extracted with $100 \mathrm{~mL}$ (GFF) or $300 \mathrm{~mL}$ (PUF) of 30-percent ethyl acetate in hexane for at least 12 hours. All extracts were dried with sodium sulfate and reduced in volume using KudernaDanish distillation to about 4-6 mL.

For samples collected through August 19, 2004, the extract from each sample was reduced by nitrogen gas evaporation to about $2 \mathrm{~mL}$ and quantitatively (by weight) split in half before column cleanup. One portion (PAH fraction) was introduced to a 0.5 -g C-18 SPE column positioned above a 1-g florisil SPE column (Biotage, Charlottesville, Virginia; the columns were pre-rinsed with acetone, dichloromethane, and ethyl acetate). Analytes were eluted with $6 \mathrm{~mL}$ of ethyl acetate, which was then reduced to about $0.15 \mathrm{~mL}$ by nitrogen gas evaporation, and transferred with a $0.1-\mathrm{mL}$ ethyl acetate rinse to a GC vial containing $500 \mathrm{ng}$ of 1,4-dichlorobenzene and five perdeuterated PAHs (naphthalene- $d 8$, acenaphthene- $d 10$, phenanthrene- $d 10$, chrysene- $d 12$, and perylene- $d 12$ ) in ethyl acetate as injection internal standards. Air extracts for PAHs and alkyl PAHs were analyzed by GC/MS in full-scan mode. Analysis of these compounds used instrumental procedures comparable to those described by Olson and others (2004). All samples except one collected after July 11, 2007, were analyzed in a combined SIM/Scan mode to lower analyte detection levels. The PAH analysis is referred to as the "AirPAH" method in this report. GFF and PUF sample components for samples collected on November 17, 2003, and QC samples processes on November 19, 2003 (sample preparation set 03.32308 ) were analyzed by GC/MS operated in the SIM mode as used for previously reported samples (Mendez and others, 2007, 2008). Concentrations for any detected alkyl PAH were estimated on the basis of the responses from the calibration curves for the corresponding parent PAHs because alkyl PAH standards were not available when calibration standards were prepared (Olson and others, 2004). Numerical results for all these alkyl PAHs were reported as estimated ("E" code), meaning they have a higher degree of uncertainty. Dilution of some extracts from the PUF component was necessary to quantify the results for several PAHs that were greater than the highest calibration standard at typical final extract volumes.

The remaining extract portion (pesticide fraction) for each sample was added to a 0.5 -g graphitized carbon SPE column (CarboPrep 90, Restek Corp; Bellefonte, Pa.; prerinsed with acetone, dichloromethane, and ethyl acetate), and the analytes were eluted with $13 \mathrm{~mL}$ of a 50 -percent dichloromethane in ethyl acetate solution. The eluent was reduced using micro-Kuderna-Danish distillation to about $1 \mathrm{~mL}$. A procedural internal standard solution of three perdeuterated PAHs (acenaphthene- $d 10$, phenanthrene- $d 10$, and chrysene- $d 12$ ) in toluene was added to the extract before final solvent reduction to about $0.15 \mathrm{~mL}$ using nitrogen gas evaporation. The extract was transferred to a $\mathrm{GC}$ vial with a toluene rinse for an approximate $0.25-\mathrm{mL}$ final volume for analysis by GC/MS-SIM (comparable to analytical methodology on NWQL water schedule 2003) for the 66 compounds that are a subset of the high- and moderateuse pesticides and degradates determined using water methods described by Zaugg and others (1995), Sandstrom and others (2001), and Madsen and others (2003). Analysis of pesticide and related degradate compounds is referred to as the "Airpest003" method in this report. Some analytes included in NWQL schedule 2003 were not amenable to the Airpest 003 method because the compounds were inadequately collected by the PUF or recovered during the SPE cleanup step; therefore, these compounds were not reported for the air samples.

Starting with the air sample collected on August 31, 2004, the samples were no longer analyzed for pesticides to reduce analytical costs; therefore, the entire samplecomponent extract was reduced to approximately $1 \mathrm{~mL}$ using nitrogen evaporation then cleaned up and analyzed using the AirPAH procedure as described previously. The final extract volumes ranged from $0.35-0.50 \mathrm{~mL}$ for $\mathrm{GC} / \mathrm{MS}$ analysis. The SOC concentrations are reported in nanograms per cubic meter of air.

\section{Metals}

The Quartz Fiber Filters (QFF) were purchased and handled in the same manner as the GFFs except that nylon forceps were used to handle the filters instead of stainlesssteel forceps. The filters were baked at $400{ }^{\circ} \mathrm{C}$ and stored in a desiccant until cool, then individually weighed and placed in a plastic petri dish. QFFs were installed into the sampling 
cartridge in the same way as the GFFs. At the end of sampling, the QFFs were removed from the sampler, folded in half, and placed back into the petri dish. They did not need to be chilled, so they were stored in the shipping container (a Pelican case) at the SWR laboratory until about 15-20 samples were collected; samples were then shipped to the laboratory where they were reweighed and prepared for analysis.

The environmental samples and blanks for metals in air were analyzed at the USGS Crustal Geophyscis and Geochemistry Science Center (Geologic Division Laboratory) in Denver, Colorado, using inductively coupled plasma massspectroscopy (ICP-MS; Briggs and Meier, 2002; Lamothe and others, 2002). The QFF samples were analyzed in batches consisting of about 15 samples, each including one laboratory blank QFF. The mean laboratory-blank result was subtracted from the result of each field sample to obtain the final mass of analyte per sample. These values were then divided by the total volume of air sampled to obtain the concentration in nanograms per cubic meter of air. The method detection limits (MDLs) were estimated as three times the standard deviation of determined concentrations in three replicate reagent blanks (not QFF blanks) that were prepared along with the QFF samples. Thus, the reported MDL value for each metal is defined here as the minimum concentration of that element that can be measured and reported with 95-percent confidence that the concentration is greater than zero.

\section{Air-Quality Assurance}

The QC air methods used were comparable to the water methods for assessing the analytical method efficiency. Groups of VOC or SOC air samples had one or more associated laboratory-prepared matrix or reagent spike and laboratory blank samples analyzed along with the environmental samples. Additionally, surrogate compounds were added to BNA and pesticide samples at the laboratory to monitor samplespecific performance of the analytical method. No surrogates were added to the VOC air samples. The GFF or PUFs were fortified with surrogate compounds nitrobenzene- $d 5$, 2-fluorobiphenyl, and terphenyl- $d 14$ for the PAH analysis. For the pesticide analysis, surrogate compounds diazinon- $d 10$ and $\alpha-\mathrm{HCH}-d 6$ were added. Additional information on air QA methods and QC results are presented in the appendix.

Air VOC matrix spikes were prepared by fortifying the air sampling media (VOC cartridge) with a known concentration of selected analytes. Matrix spikes also can be used to test the effects of various sampled matrices on the recovery of specific compounds, including matrix interference or matrix-induced analyte degradation. Lot blanks are processed at the lab on a subset of the air VOC cartridges sent for environmental samples; these lot blanks are used to test the matrix that was used during the manufacture of the cartridges. Laboratory blanks are used to assess whether the sample processing steps in the laboratory have introduced contamination. A laboratory blank was processed with every environmental air sample.
Each VOC air sample collected had a travel blank, lot blank, and laboratory blank and about a quarter of the air samples collected had a travel matrix-spike associated with it as described in Pankow and others (1998). Travel matrixspike cartridges were fortified at the laboratory with a known concentration of the VOC analytes, sent to the field, and subsequently analyzed at the laboratory to assess recovery bias and reproducibility at the spike concentration. Travel (trip) blank-sample cartridges were shipped with the field sample cartridges, kept with the field sample cartridges, and shipped back to the laboratory but was not exposed to the air. Travel blanks were shipped with every VOC air sample and were used to identify contamination that may have occurred during sample transport, storage, and analysis rather than as a result of sample collection. Lot blanks consisted of a randomly selected cartridge from a group of simultaneously manufactured (conditioned) air VOC cartridges that were analyzed at the laboratory to test for contaminants derived from the cartridge sorbents or conditioning steps. Similarly, laboratory blanks were randomly selected from conditioned cartridge groups, fortified with the internal standards, and stored at the laboratory until analysis along with associated field samples; the laboratory blanks were used to assess contamination introduced at the laboratory. Blank sample data in this report are presented in concentration units comparable to field sample data by assuming sample volumes of $1.5 \mathrm{~L}$ (high-volatility VOCs) and $5 \mathrm{~L}$ (medium/low-volatility VOCs). Replicate VOC air samples were taken by attaching a Teflon "Y" connector to the pump intake hose, attaching two of the same type sampling cartridges to it, and adjusting the flow through each cartridge to either 1 or $5 \mathrm{Lpm}$ depending on the type of cartridge. Fourteen replicate high-volatility and eleven replicate medium/low-volatility air samples were collected to assess sample reproducibility.

All VOC results for air samples have been

blank-corrected at the laboratory before the data were released. Travel blanks (50-60) from previous and current sets of samples were averaged to obtain the mean blank level plus standard deviation. If the amount in a sample was less than the sum of the mean blank level and three times the standard deviation, the concentration was reported as not detected. If the amount in a sample was larger than the sum of the mean blank level and three times the standard deviation, the mean blank level was subtracted from the amount in the sample and the difference was the reported concentration.

Separate GFF and PUF laboratory blank and laboratory matrix-spike samples were analyzed for each set of prepared SOC environmental samples. They were prepared using one or more of the same lots of GFFs or PUF media used to collect the field samples. Storage conditions for these blank media were comparable to storage conditions at the Sweetwater laboratory. Reported concentrations of PAHs and pesticides in laboratory (and field) blanks were calculated assuming an air volume of $315 \mathrm{~m}^{3}$ to simplify comparison with field sample data. Field sample air data for PAHs and pesticides were not corrected for laboratory blank concentrations. Laboratory 
matrix-spike samples were prepared by fortifying the parent PAH and pesticide compounds (until pesticide analysis was discontinued) directly to the media just prior to extraction. Replicate air samples for PAHs and pesticides were not collected during this study. Each air-sample component (GFF and top and bottom PUF plugs) was fortified with surrogate compounds at the NWQL to monitor performance during sample preparation and analysis.

Two types of field blank samples were collected and analyzed for PAHs during this reporting period. One type, referred to as "Field blank," was used to examine possible contamination resulting from field handling of the GFFPUF sampling components and from post-collection sample processing steps. This Field blank was obtained by loading one clean GFF and two clean PUF plugs into the sampling train at the field laboratory, taking the train out to the site to install and uninstall, returning the train to the field laboratory, and unloading the GFF and PUF plugs for storage and shipping in the same manner as the environmental samples. No air was drawn through the sampling train. This type of blank was collected in October 2004 and June 2009. The second type, referred to as "Exposed field blank," was handled identically to the Field blank, except it also included a 24-hour exposure period when the train was positioned at the sampling site but no air was drawn through the train. The Exposed field blank simulates the approximately 24-hour period of time that the air sampling train was deployed in the field beyond the active air-flow collection period and was used to assess potential passive sampling of PAH by the sampler. An Exposed field blank was collected in January 2007 and June 2009. Ideally, additional field blanks should have been collected during this reporting period to assess SOC contamination potential relative to laboratory blank results. Field blank samples from this reporting period were collected after August 2004 and, thus, were not analyzed for pesticides.

Two types of soil standard reference material (SRM) were used as QC checks of the digestion process and instrument calibration for the analysis of metals in air samples - the National Institute of Standards and Technology (NIST) 2710 (National Institute of Standards and Technology, 1991b) and the Canadian Certified Reference Materials Project (CCRMP) SO-2 and SO-4 standards (Potts and others, 1992). The NIST 2710 material is a certified SRM. The CCRMP standards are not "certified" to the same extent as the NIST standards. They have, however, consensus values from results obtained by about 80 internationally recognized geochemical laboratories and are used in conjunction with the NIST standards to look for systematic biases in the data set. The soil SRM was a solid-phase material that could be processed in the same manner as the QFF substrate used to collect the air samples. If all three percent recovery values of the SRM for a given element were biased by more than 10 percent in the same direction, the data were reviewed and reanalyzed. If the SRM analytical results were less than 10 times the MDL, any lack of agreement was considered statistically insignificant and was ignored. The Potts standards were used in conjunction with the
NIST standards because even though some of the SO-2 and SO-4 results can be more variable than the NIST standards, in some cases no NIST values are available. Including the SO-2 and SO-4 results gives a better indication of the maximum bias that may exist in the data set. Not every major and trace metal in every SRM has values in the certificate of analysis provided by the original suppliers. Therefore, if neither the original supplier of the SRM or the data compilations, such as by Potts and others (1992), provide values for an element, that element is listed as not analyzed.

The percent recovery for metals in the QC samples is the ratio of the analyzed concentration to the expected concentration times 100 . The laboratory reports the total mass of each element measured on the QFF. This enables the easy calculation of airborne concentrations for each element in units of nanograms per cubic meter of air. The QC calculations were performed similarly by calculating a recovery of the total mass of a given element from a QC sample. The laboratory also chose a QC sample weight of $0.1 \mathrm{~g}$. For each element reported in the QC sample, the expected mass recovered after the digestion procedure was 0.1 times the known concentration of that element in the QC sample. Therefore, values from this study have to be multiplied by 10 to be comparable to literature values.

\section{Air Results}

The VOC air method was used to analyze 85 compounds, and the results are presented in tables 10A and 10B.

Chloroethane was the only high-volatility volatile organic compound detected in air samples. Twenty-eight medium/lowvolatility volatile organic compounds had no detections in air samples. Acetone, benzene, ethenylbenzene, and naphthalene had blank detections greater than 30 percent of the adjusted environmental sample concentrations and were flagged as suspect. Because VOC results for air samples were blank corrected (adjusted environmental sample) at the laboratory before the data were released, the true blank value is unknown. The concentration in the current blank associated with each sample was added to the environmental sample, and if the blank was larger than 30 percent of the adjusted environmental sample plus the blank, the compound was considered to be biased high (table 10B). Benzene was detected in the laboratory blank above the acceptable criteria (30 percent of the adjusted environmental sample) in about 30 percent of the environmental samples. Detections of MTBE, benzene, and toluene in air samples at the atmospheric sampling site (fig. 2) during the entire study period are shown in figure $4 A$ for comparison to these compounds in water. These data show a strong correlation between concentrations of these compounds in air throughout the study until MTBE was phased out in January 2004. Concentrations of benzene and toluene continued to have a strong correlation to each other, although toluene was detected at a higher concentration. A detailed analysis of VOCs in air and water is beyond the scope of this report. 
Table 10A. Analytical results for high-volatility volatile organic compounds (VOCs) processed at the Oregon Graduate Institute for air samples collected from the Sweetwater Reservoir atmospheric site, San Diego County, California.

[The site-identification number is 324141117001601. Concentrations are given in part per billion by volume (pbbv). Abbreviations: LRL, laboratory reporting level; $\mathrm{mm}$ of $\mathrm{Hg}$, millimeters of mercury; mm/dd/yyyy, month/day/year; NA, not analyzed; ${ }^{\circ} \mathrm{C}$, degree Celsius; —, compound was not detected at a concentration above the laboratory reporting level]

\begin{tabular}{|c|c|c|c|c|c|c|c|c|}
\hline \multirow{2}{*}{$\begin{array}{c}\text { Date } \\
\text { (mm/dd/yyyy) }\end{array}$} & \multirow{2}{*}{$\begin{array}{l}\text { Pressure } \\
(\mathbf{m m} \text { of } \mathrm{Hg})\end{array}$} & \multirow{2}{*}{$\begin{array}{c}\text { Temperature } \\
\left({ }^{\circ} \mathrm{C}\right)\end{array}$} & \multicolumn{2}{|c|}{ Chloromethane } & \multirow{2}{*}{$\begin{array}{c}\begin{array}{c}\text { Chloroethene } \\
\text { (vinyl chloride) }\end{array} \\
{[0.30]}\end{array}$} & \multirow{2}{*}{$\begin{array}{c}\text { Bromomethane } \\
{[0.50]}\end{array}$} & \multirow{2}{*}{$\begin{array}{c}\text { Chloroethane } \\
{[0.30]}\end{array}$} & \multirow{2}{*}{$\begin{array}{c}\begin{array}{c}\text { Bromoethene } \\
\text { (vinyl bromide) }\end{array} \\
{[0.30]}\end{array}$} \\
\hline & & & [LRL] & {$[0.30]$} & & & & \\
\hline $10 / 12 / 2003$ & 759.7 & 20.7 & & - & - & - & - & - \\
\hline $10 / 24 / 2003$ & 759.0 & 18.2 & & - & - & - & - & - \\
\hline $11 / 05 / 2003$ & 761.7 & 12.7 & & - & - & - & - & - \\
\hline $11 / 17 / 2003$ & 763.0 & 13.3 & & - & - & - & - & - \\
\hline $11 / 29 / 2003$ & 761.9 & 15.0 & & - & - & - & - & - \\
\hline $12 / 11 / 2003$ & 761.6 & 12.0 & & - & - & - & - & - \\
\hline $12 / 23 / 2003$ & 760.7 & 15.4 & & - & - & - & - & - \\
\hline $01 / 04 / 2004$ & 764.9 & 9.5 & & - & - & - & - & - \\
\hline $01 / 14 / 2004$ & 758.0 & 12.9 & & - & - & - & - & - \\
\hline $01 / 28 / 2004$ & 763.5 & 12.3 & & - & - & - & - & - \\
\hline $02 / 09 / 2004$ & 762.5 & 12.4 & & - & - & - & - & - \\
\hline $02 / 21 / 2004$ & 757.9 & 11.7 & & - & - & - & - & - \\
\hline 03/04/2004 & 758.8 & 13.8 & & - & - & - & - & - \\
\hline $03 / 16 / 2004$ & 760.6 & 15.0 & & - & - & - & - & - \\
\hline 03/28/2004 & 760.2 & 20.8 & & - & - & - & - & - \\
\hline 04/09/2004 & 760.3 & 16.6 & & - & - & - & - & - \\
\hline $04 / 21 / 2004$ & 760.8 & 16.0 & & - & - & - & - & - \\
\hline 05/03/2004 & 758.1 & 18.2 & & - & - & - & - & - \\
\hline $05 / 15 / 2004$ & 757.5 & 18.2 & & - & - & - & 1.25 & - \\
\hline 05/27/2004 & 761.2 & 17.5 & & - & - & - & - & - \\
\hline $06 / 08 / 2004$ & 757.3 & 17.1 & & - & - & - & - & - \\
\hline $06 / 20 / 2004$ & 760.7 & 19.2 & & - & - & - & - & - \\
\hline $07 / 02 / 2004$ & 761.7 & 18.9 & & - & - & - & - & - \\
\hline $07 / 14 / 2004$ & 759.7 & 22.9 & & - & - & - & - & - \\
\hline $07 / 26 / 2004$ & 758.9 & 21.2 & & - & - & - & - & - \\
\hline 08/19/2004 & 759.9 & 20.7 & & - & - & - & - & - \\
\hline $08 / 31 / 2004$ & 759.1 & 22.2 & & - & - & - & - & - \\
\hline 09/12/2004 & 757.7 & 22.5 & & - & - & - & - & - \\
\hline 09/23/2004 & 759.2 & 22.1 & & - & - & - & - & - \\
\hline $10 / 06 / 2004$ & 759.0 & 17.9 & & - & - & - & - & - \\
\hline $10 / 18 / 2004$ & 758.9 & 18.7 & & - & - & - & - & - \\
\hline $10 / 30 / 2004$ & 761.7 & 15.0 & & - & - & - & - & - \\
\hline $11 / 23 / 2004$ & 763.3 & 11.2 & & - & - & - & - & - \\
\hline $12 / 05 / 2004$ & 754.7 & 10.5 & & - & - & - & - & - \\
\hline $12 / 28 / 2004$ & 758.4 & 13.4 & & - & - & - & - & - \\
\hline $01 / 10 / 2005$ & 762.2 & 15.3 & & - & - & - & - & - \\
\hline $01 / 22 / 2005$ & 762.3 & 13.6 & & - & - & - & - & - \\
\hline $02 / 03 / 2005$ & 762.4 & 15.7 & & - & - & - & - & - \\
\hline $02 / 15 / 2005$ & 762.4 & 12.8 & & - & - & - & - & - \\
\hline $02 / 27 / 2005$ & 764.0 & 14.0 & & - & - & - & - & - \\
\hline 03/11/2005 & 760.6 & 15.0 & & - & - & - & - & - \\
\hline $03 / 23 / 2005$ & 760.0 & 13.5 & & - & - & - & - & - \\
\hline $04 / 04 / 2005$ & 762.7 & 14.0 & & - & - & - & - & - \\
\hline $04 / 16 / 2005$ & 759.3 & 16.0 & & - & - & - & - & - \\
\hline $04 / 28 / 2005$ & 762.0 & 15.1 & & - & - & - & - & - \\
\hline $05 / 10 / 2005$ & 759.9 & 16.0 & & - & - & - & - & - \\
\hline $06 / 03 / 2005$ & 756.7 & 18.2 & & - & - & - & - & - \\
\hline $06 / 15 / 2005$ & 758.1 & 17.8 & & - & - & - & - & - \\
\hline
\end{tabular}


Table 10A. Analytical results for high-volatility volatile organic compounds (VOCs) processed at the Oregon Graduate Institute for air samples collected from the Sweetwater Reservoir atmospheric site, San Diego County, California.-Continued

[The site-identification number is 324141117001601 . Concentrations are given in part per billion by volume (pbbv). Abbreviations: LRL, laboratory reporting level; mm of Hg, millimeters of mercury; mm/dd/yyyy, month/day/year; NA, not analyzed; ${ }^{\circ} \mathrm{C}$, degree Celsius; —, compound was not detected at a concentration above the laboratory reporting level]

\begin{tabular}{|c|c|c|c|c|c|c|c|c|}
\hline \multirow{2}{*}{$\begin{array}{c}\text { Date } \\
\text { (mm/dd/yyyy) }\end{array}$} & \multirow{2}{*}{$\begin{array}{l}\text { Pressure } \\
(\mathbf{m m} \text { of } \mathrm{Hg})\end{array}$} & \multirow{2}{*}{$\begin{array}{c}\text { Temperature } \\
\left({ }^{\circ} \mathrm{C}\right)\end{array}$} & \multicolumn{2}{|c|}{ Chloromethane } & \multirow{2}{*}{$\begin{array}{c}\begin{array}{c}\text { Chloroethene } \\
\text { (vinyl chloride) }\end{array} \\
{[0.30]}\end{array}$} & \multirow{2}{*}{$\begin{array}{c}\text { Bromomethane } \\
{[0.50]}\end{array}$} & \multirow{2}{*}{$\begin{array}{c}\text { Chloroethane } \\
{[0.30]}\end{array}$} & \multirow{2}{*}{$\begin{array}{c}\begin{array}{c}\text { Bromoethene } \\
\text { (vinyl bromide) }\end{array} \\
{[0.30]}\end{array}$} \\
\hline & & & [LRL] & {$[0.30]$} & & & & \\
\hline $07 / 09 / 2005$ & 759.2 & 18.7 & & - & - & - & - & - \\
\hline $07 / 25 / 2005$ & 757.0 & 25.4 & & - & - & - & - & - \\
\hline $08 / 02 / 2005$ & 758.9 & 20.6 & & - & - & - & - & - \\
\hline $08 / 14 / 2005$ & 757.9 & 19.4 & & - & - & - & - & - \\
\hline $08 / 26 / 2005$ & 759.2 & 25.9 & & - & - & - & - & - \\
\hline 09/07/2005 & 758.9 & 20.3 & & - & - & - & - & - \\
\hline 09/19/2005 & 760.1 & 19.2 & & - & - & - & - & - \\
\hline $10 / 01 / 2005$ & 756.7 & 20.5 & & - & - & - & - & - \\
\hline $10 / 13 / 2005$ & 759.5 & 20.3 & & - & - & - & - & - \\
\hline $10 / 25 / 2005$ & 763.4 & 16.4 & & - & - & - & - & - \\
\hline $11 / 06 / 2005$ & 759.0 & 15.0 & & - & - & - & - & - \\
\hline $11 / 18 / 2005$ & 762.4 & 20.2 & & - & - & - & - & - \\
\hline $12 / 12 / 2005$ & 762.7 & 12.3 & & - & - & - & - & - \\
\hline $12 / 24 / 2005$ & 762.8 & 13.3 & & - & - & - & - & - \\
\hline $01 / 06 / 2006$ & 765.0 & 18.1 & & - & - & - & - & - \\
\hline $01 / 17 / 2006$ & 768.0 & 11.8 & & - & - & - & - & - \\
\hline $01 / 29 / 2006$ & 765.2 & 11.5 & & - & - & - & - & - \\
\hline $02 / 10 / 2006$ & 764.0 & 12.9 & & - & - & - & - & - \\
\hline $02 / 22 / 2006$ & 763.3 & 11.2 & & - & - & - & - & - \\
\hline $03 / 05 / 2006$ & 764.7 & 13.2 & & - & - & - & - & - \\
\hline $03 / 30 / 2006$ & 761.8 & 13.4 & & - & - & - & - & - \\
\hline $04 / 11 / 2006$ & 760.8 & 15.6 & & - & - & - & - & - \\
\hline $04 / 23 / 2006$ & 762.2 & 13.4 & & - & - & - & - & - \\
\hline $05 / 05 / 2006$ & 761.1 & 15.7 & & - & - & - & - & - \\
\hline 06/10/2006 & 757.5 & 17.8 & & - & - & - & - & - \\
\hline $06 / 22 / 2006$ & 760.2 & 19.7 & & - & - & - & - & - \\
\hline $07 / 04 / 2006$ & 760.7 & 21.9 & & NA & NA & NA & NA & NA \\
\hline $07 / 28 / 2006$ & 759.6 & 25.1 & & - & - & - & - & - \\
\hline 08/09/2006 & 758.4 & 23.8 & & - & - & - & - & - \\
\hline $08 / 21 / 2006$ & 758.5 & 21.7 & & - & - & - & - & - \\
\hline 09/02/2006 & 755.5 & 23.2 & & - & - & - & - & - \\
\hline $09 / 14 / 2006$ & 756.1 & 18.8 & & - & - & - & - & - \\
\hline $09 / 26 / 2006$ & 761.0 & 19.3 & & - & - & - & - & - \\
\hline $10 / 08 / 2006$ & 759.4 & 16.5 & & - & - & - & - & - \\
\hline $10 / 20 / 2006$ & 756.8 & 19.4 & & - & - & - & - & - \\
\hline $11 / 01 / 2006$ & 761.6 & 16.5 & & - & - & - & - & - \\
\hline $11 / 13 / 2006$ & 715.9 & 16.9 & & - & - & - & - & - \\
\hline $11 / 25 / 2006$ & 706.9 & 14.5 & & - & - & - & - & - \\
\hline $12 / 07 / 2006$ & 762.7 & 15.7 & & - & - & - & - & - \\
\hline $12 / 31 / 2006$ & 765.7 & 10.1 & & - & - & - & - & - \\
\hline $01 / 12 / 2007$ & 760.5 & 9.4 & & - & - & - & - & - \\
\hline $01 / 24 / 2007$ & 764.2 & 13.4 & & - & - & - & - & - \\
\hline $02 / 05 / 2007$ & 763.2 & 17.3 & & - & - & - & - & - \\
\hline $02 / 17 / 2007$ & 761.7 & 19.1 & & - & - & - & - & - \\
\hline $03 / 01 / 2007$ & 763.3 & 9.9 & & - & - & - & - & - \\
\hline $03 / 13 / 2007$ & 761.4 & 16.2 & & - & - & - & - & - \\
\hline 03/25/2007 & 763.0 & 15.5 & & - & - & - & - & - \\
\hline $04 / 06 / 2007$ & 758.9 & 14.9 & & - & - & - & - & - \\
\hline $04 / 18 / 2007$ & 761.6 & 14.1 & & - & - & - & - & - \\
\hline
\end{tabular}


Table 10A. Analytical results for high-volatility volatile organic compounds (VOCs) processed at the Oregon Graduate Institute for air samples collected from the Sweetwater Reservoir atmospheric site, San Diego County, California.-Continued

[The site-identification number is 324141117001601. Concentrations are given in part per billion by volume (pbbv). Abbreviations: LRL, laboratory reporting level; $\mathrm{mm}$ of $\mathrm{Hg}$, millimeters of mercury; mm/dd/yyyy, month/day/year; NA, not analyzed; ${ }^{\circ} \mathrm{C}$, degree Celsius; - , compound was not detected at a concentration above the laboratory reporting level]

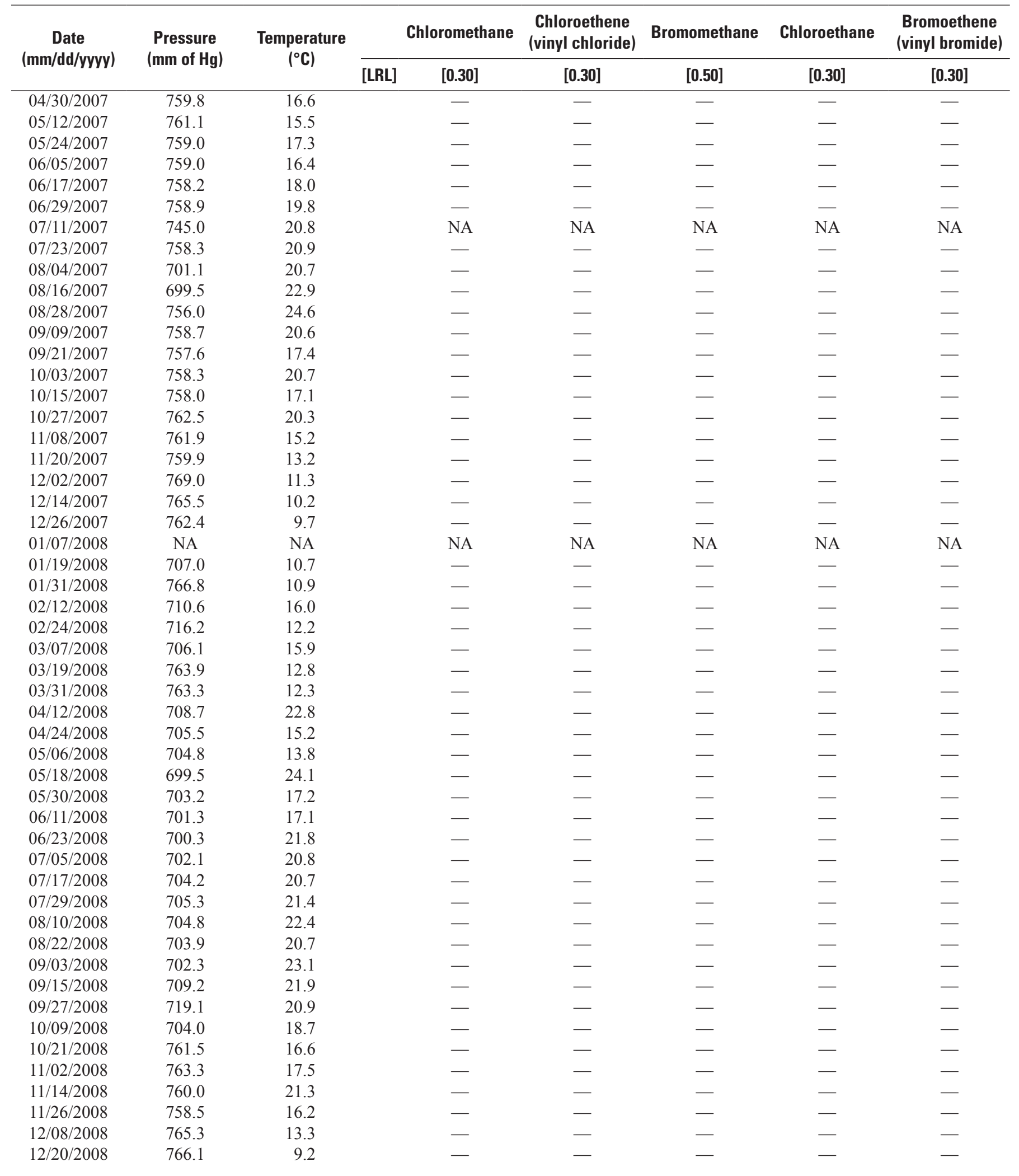


Table 10A. Analytical results for high-volatility volatile organic compounds (VOCs) processed at the Oregon Graduate Institute for air samples collected from the Sweetwater Reservoir atmospheric site, San Diego County, California.-Continued

[The site-identification number is 324141117001601. Concentrations are given in part per billion by volume (pbbv). Abbreviations: LRL, laboratory reporting level; $\mathrm{mm}$ of $\mathrm{Hg}$, millimeters of mercury; mm/dd/yyyy, month/day/year; NA, not analyzed; ${ }^{\circ} \mathrm{C}$, degree Celsius; —, compound was not detected at a concentration above the laboratory reporting level]

\begin{tabular}{|c|c|c|c|c|c|c|c|c|}
\hline \multirow{2}{*}{$\begin{array}{c}\text { Date } \\
\text { (mm/dd/yyyy) }\end{array}$} & \multirow{2}{*}{$\begin{array}{l}\text { Pressure } \\
(\mathrm{mm} \text { of } \mathrm{Hg})\end{array}$} & \multirow{2}{*}{$\begin{array}{l}\text { Temperature } \\
\left({ }^{\circ} \mathrm{C}\right)\end{array}$} & \multicolumn{2}{|c|}{ Chloromethane } & \multirow{2}{*}{$\begin{array}{c}\begin{array}{c}\text { Chloroethene } \\
\text { (vinyl chloride) }\end{array} \\
{[0.30]}\end{array}$} & \multirow{2}{*}{$\begin{array}{c}\text { Bromomethane } \\
{[0.50]}\end{array}$} & \multirow{2}{*}{$\begin{array}{c}\text { Chloroethane } \\
{[0.30]}\end{array}$} & \multirow{2}{*}{$\begin{array}{c}\begin{array}{c}\text { Bromoethene } \\
\text { (vinyl bromide) }\end{array} \\
{[0.30]}\end{array}$} \\
\hline & & & [LRL] & {$[0.30]$} & & & & \\
\hline $01 / 01 / 2009$ & 762.0 & 11.4 & & - & - & - & - & - \\
\hline 01/13/2009 & 765.3 & 17.8 & & - & - & - & - & - \\
\hline $01 / 25 / 2009$ & 762.8 & 11.6 & & - & - & - & - & - \\
\hline $02 / 06 / 2009$ & 761.0 & 13.2 & & - & - & - & - & - \\
\hline $02 / 18 / 2009$ & 766.3 & 11.0 & & - & - & - & - & - \\
\hline 03/02/2009 & 763.0 & 18.3 & & - & - & - & - & - \\
\hline 03/14/2009 & 759.5 & 12.9 & & - & - & - & - & - \\
\hline 03/26/2009 & 759.0 & 12.7 & & - & - & - & - & - \\
\hline 04/07/2009 & 762.5 & 13.7 & & - & - & - & - & - \\
\hline 04/19/2009 & 760.5 & 25.2 & & - & - & - & - & - \\
\hline 05/01/2009 & 761.8 & 16.9 & & - & - & - & - & - \\
\hline 05/13/2009 & 758.3 & 16.6 & & - & - & - & - & - \\
\hline $05 / 25 / 2009$ & 759.7 & 16.0 & & - & - & - & - & - \\
\hline 06/06/2009 & 760.5 & 17.1 & & - & - & - & - & - \\
\hline $06 / 18 / 2009$ & 756.7 & 17.9 & & - & - & - & - & - \\
\hline 06/30/2009 & 757.3 & 20.8 & & - & - & - & - & - \\
\hline $07 / 12 / 2009$ & 760.2 & 24.8 & & - & - & - & - & - \\
\hline 07/24/2009 & 759.0 & 16.7 & & - & - & - & - & - \\
\hline
\end{tabular}

Table 10B. Analytical results for medium/low-volatility volatile organic compounds (VOCs) processed at the Oregon Graduate Institute for air samples collected from the Sweetwater Reservoir atmospheric site, San Diego County, California.

(Table 10B is provided separately as a Microsoft Excel ${ }^{\circledR}$ worksheet.)

Individual PAH compounds and alkylated PAH homologs determined by the AirPAH method, along with estimated reporting levels, are given in table $11 \mathrm{~A}$. Concentrations of $\mathrm{PAH}$ and alkyl PAH detected in air samples are given in table 11B, of which, nineteen compounds were detected. Typically, the PUFs had better recoveries than the GFFs, and most blank detections were an order of magnitude lower than the LRL.

Pesticide and related degradate compounds analyzed in air samples by the Airpest003 method are given in table 12A, and concentrations for the 10 pesticides detected in air samples are given in table 12B. About 10 percent of spike recoveries for Airpest003 were below the acceptable criteria; carbaryl, fenamiphos, phorate, and terbofos had one spike with no recovery. The low spike recoveries may indicate the compound was under-detected and may be reported at lower concentrations than actually occurred.

Concentrations for 32 metals analyzed in air samples are presented in table 13, along with determined TSP concentrations for these samples. Niobium and thallium were the least frequently detected metals in air samples. Ten samples were not collected during this time frame because of mechanical problems or torn filters. Most laboratory-blank metal samples had detections above their LRLs, but the detections vary. Detections near the LRL for these compounds may be caused by the sampling or processing steps.

\section{Surficial Bed-Sediment Sampling Strategy}

Surficial bed-sediment samples were collected for PAHs and metals at the three water sampling sites in SWR (sites SWR01, SWR03, and SWR06), at site LFDD, and at both LLR water sampling sites (sites LLR01 and LLR02) in October 2004 (figs. 2 and 3). Surficial bed-sediment samples were analyzed for PAHs, OC pesticides, and metals at the five water sampling sites in the SWR (sites SWR01, SWR02, SWR03, SWR04, and SWR06), at site LFDD, and at both LLR water sampling sites (sites LLR01 and LLR02) in August 2009 (figs. 2 and 3). All surficial bed sediment data have been entered into the USGS NWIS database, except for metals. Sediment samples for metals also were collected near the atmospheric sampling site (AIR01) in October 2004 (fig. 2). 
Table 11A. Polycyclic aromatic hydrocarbons (PAHs) and alkylated-PAH compounds analyzed in air samples, processed at the U.S. Geological Survey National Water Quality Laboratory, from the Sweetwater Reservoir atmospheric site, San Diego County, California.

[The site-identification number is 324141117001601. The parameter code is used in the U.S. Geological Survey computerized data system (National Water Information System) to uniquely identify a specific constituent or property. Estimated reporting level: for split extract analyzed in full scan mode assuming 315 cubic meters average air volume. Calculated sum of the concentration determined in glass-fiber filter sample component plus the concentration in the combined top and bottom polyurethane foam plug sample components. Estimated analyte reporting levels were 0.05 nanograms per cubic meter (ng/ $\mathrm{m}^{3}$ ) for $\mathrm{GFF}$ and $0.1 \mathrm{ng} / \mathrm{m}^{3}$ for polyurethane foam plug sample components that were analyzed by combined selected ion monitoring and full scan (SIM/Scan) mass spectrometry. Alkylated PAH homolog concentrations are estimated.]

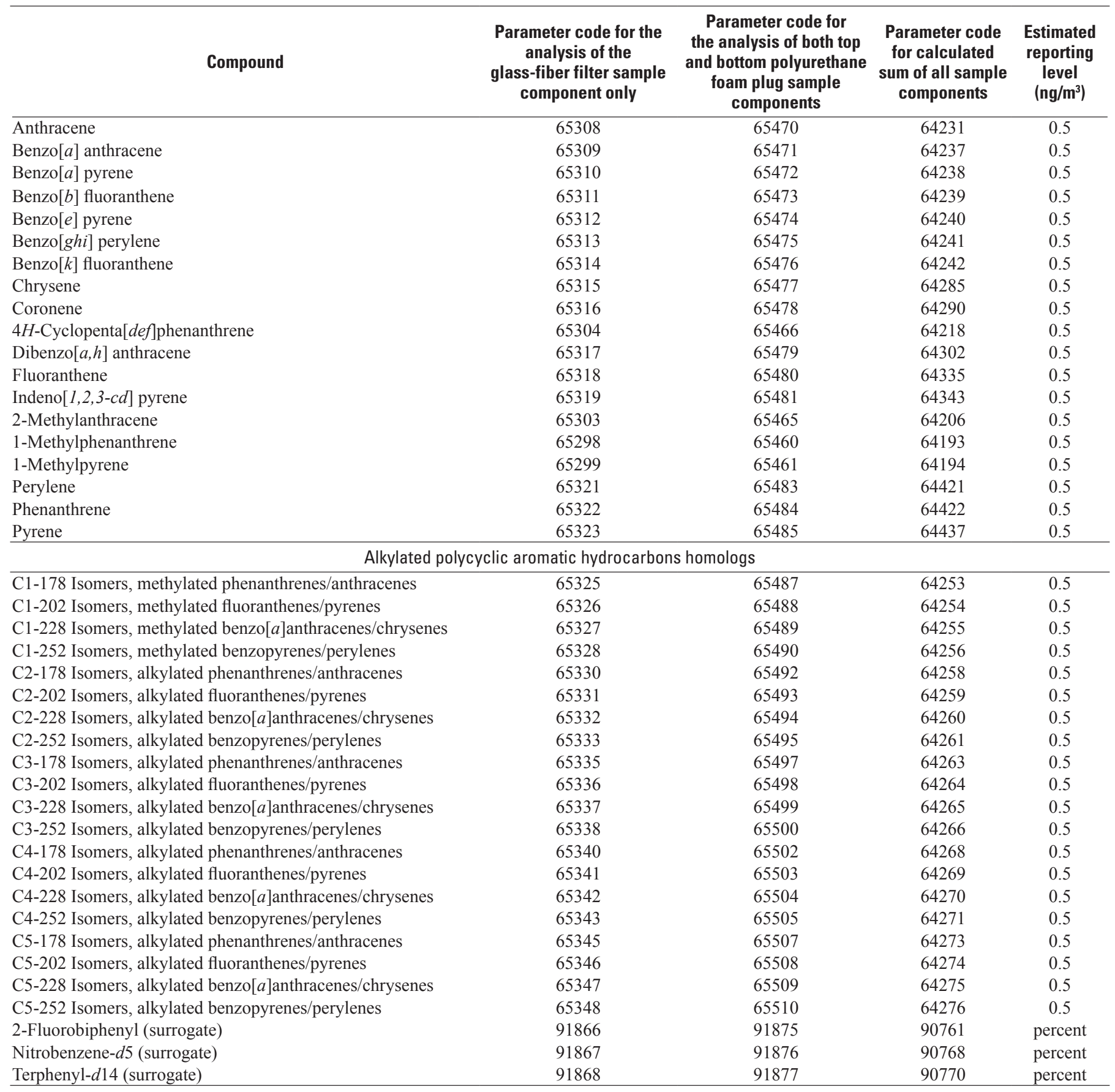

Table 11B. Analyitcal results for Polycyclic Aromatic Hydrocarbon (PAH) and alkylated PAH compounds detected in air samples, processed at the U.S. Geological Survey's National Water Quality Laboratory, from the Sweetwater Reservoir atmospheric site, San Diego County, California.

(Table 11B is provided separately as a Microsoft Excel ${ }^{\circledR}$ worksheet.) 
Table 12A. Pesticide compounds analyzed in air samples, processed at the U.S. Geological Survey National Water Quality Laboratory, from the Sweetwater Reservoir atmospheric site, San Diego County, California.

[The site-identification number is 324141117001601. Estimated reporting level assumes 315 cubic meters as the average air volume. Abbreviation: ng/ $\mathrm{m}^{3}$, nanograms per cubic meter]

\begin{tabular}{|c|c|}
\hline Compound & $\begin{array}{l}\text { Estimated reporting level } \\
\qquad\left(\mathrm{ng} / \mathrm{m}^{3}\right)\end{array}$ \\
\hline Acetochlor & 0.05 \\
\hline Alachlor & 0.05 \\
\hline Atrazine & 0.05 \\
\hline Benfluralin $^{1}$ & 0.30 \\
\hline Carbaryl $^{1}$ & 0.10 \\
\hline 2-Chloro-2,6-diethylacetanilide & 0.08 \\
\hline 2-Chloro-4-isopropylamino-6-amino-s-triazine (CIAT) & 0.11 \\
\hline Chlorpyrifos & 0.06 \\
\hline Dacthal (DCPA) & 0.05 \\
\hline Desulfinyl-fipronil & 0.05 \\
\hline Diazinon & 0.06 \\
\hline Dieldrin & 0.11 \\
\hline Dimethoate & 0.10 \\
\hline Ethion & 0.13 \\
\hline 2-Ethyl-6 methylaniline & 0.11 \\
\hline Fenamiphos $^{1}$ & 0.17 \\
\hline Fenamiphos sulfone $^{1}$ & 0.06 \\
\hline Fenamiphos sulfoxide ${ }^{1}$ & 0.17 \\
\hline Fipronil $^{1}$ & 0.05 \\
\hline Fipronil sulfide & 0.06 \\
\hline Fipronil sulfone & 0.06 \\
\hline Fonofos & 0.06 \\
\hline Hexazinone $^{1}$ & 0.06 \\
\hline Isofenphos & 0.10 \\
\hline Metalaxyl & 0.08 \\
\hline Methidathion & 0.11 \\
\hline Metolachlor & 0.05 \\
\hline Metribuzin & 0.10 \\
\hline Myclobutanil & 0.11 \\
\hline Parathion-methyl & 0.06 \\
\hline Pendi-methilan & 0.06 \\
\hline cis-Permethrin & 0.11 \\
\hline trans-Permethrin & 0.11 \\
\hline Phorate $^{1}$ & 0.13 \\
\hline Phorate oxon ${ }^{1}$ & 0.14 \\
\hline Prometon & 0.05 \\
\hline Prometryn & 0.14 \\
\hline Propyzamide & 0.06 \\
\hline Simazine & 0.06 \\
\hline Terbufos & 0.08 \\
\hline Terbuthylazine & 0.05 \\
\hline Trifluralin $^{1}$ & 0.30 \\
\hline Diazinon- $d 10$ (surrogate) & percent \\
\hline$\alpha-\mathrm{HCH}-d 6$ (surrogate) & percent \\
\hline
\end{tabular}

${ }^{1}$ Concentration estimated. Compound has poor recovery on the basis of laboratory set spikes. 


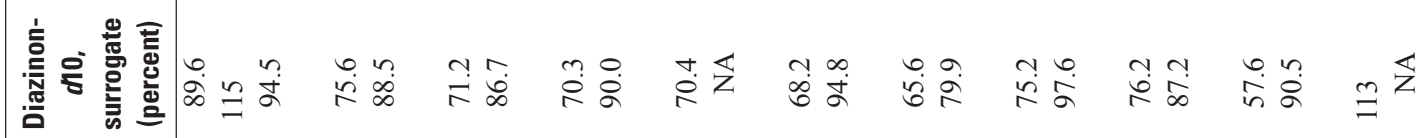

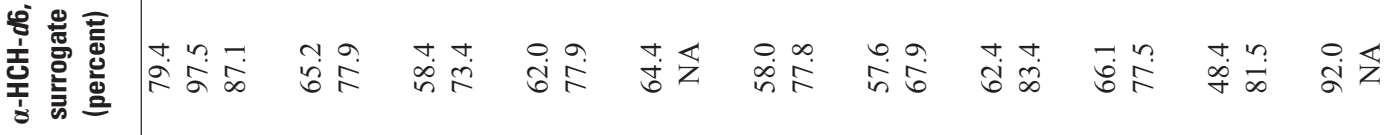

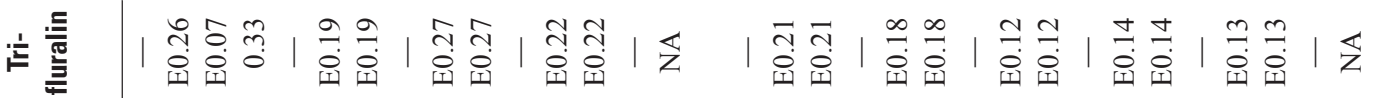
帝 空

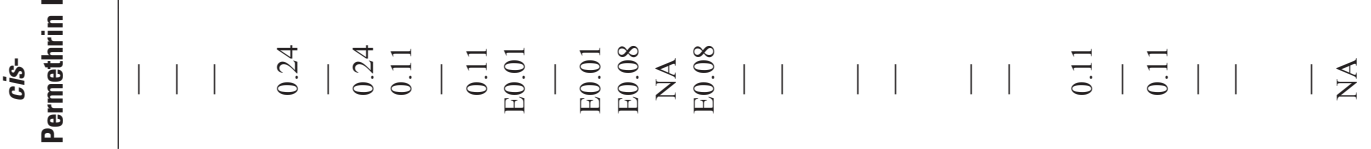

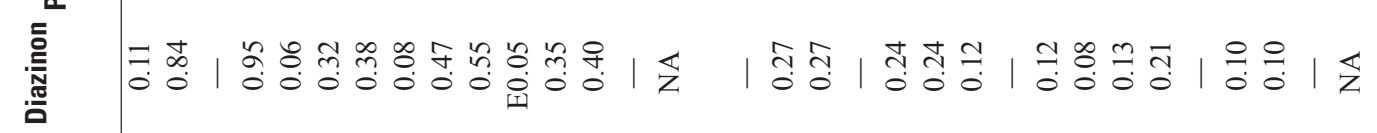
产

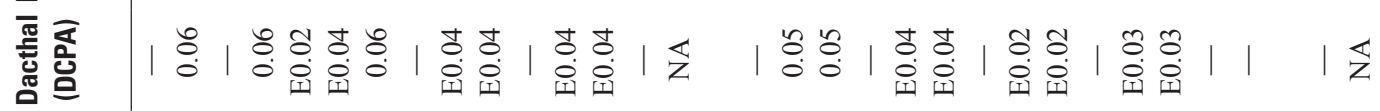

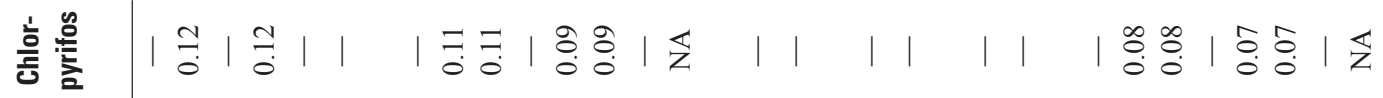

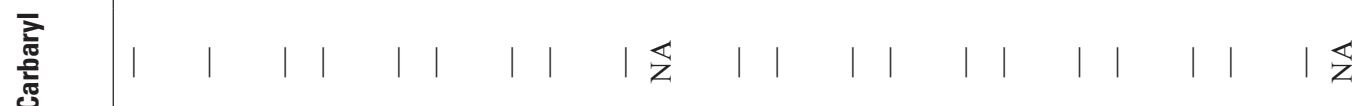

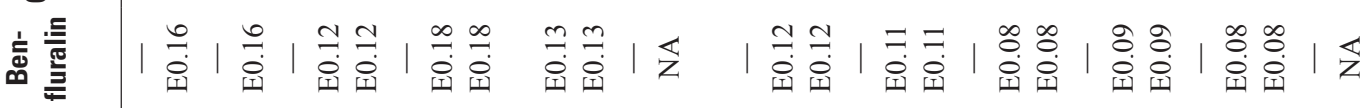

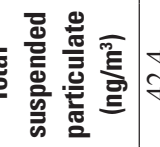
递莺 运富要

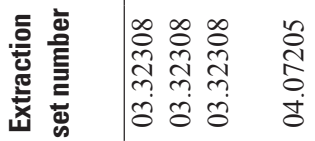

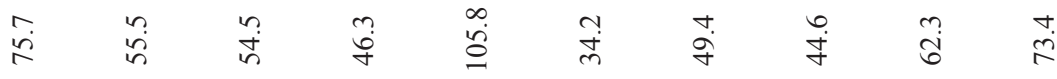

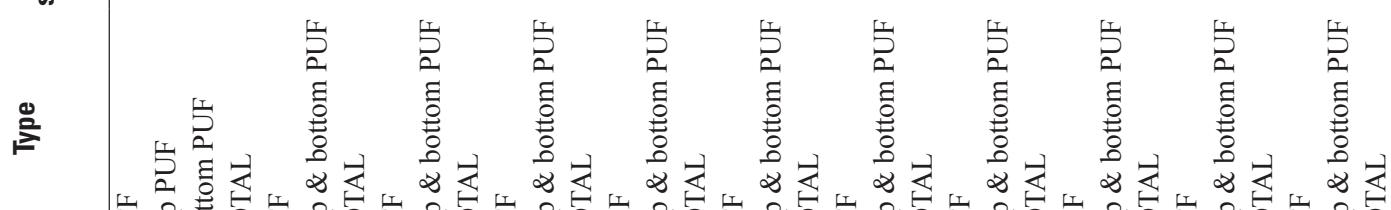

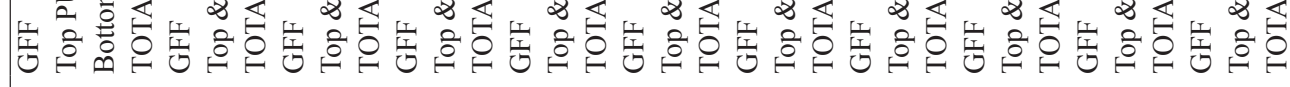

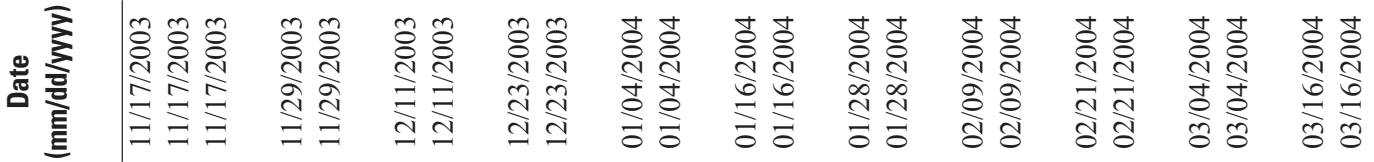




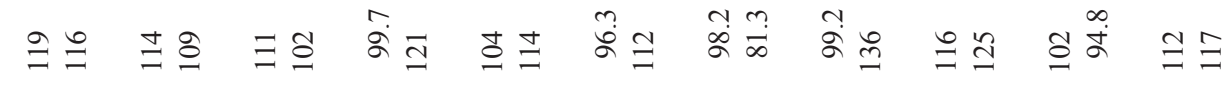

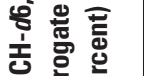

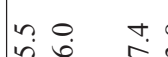

+े 青毫

迹 
Table 13. Analytical results for metals in air samples, processed at the U.S. Geological Survey Geologic Division laboratory, collected from the Sweetwater Reservoir atmospheric site, San Diego County, California.

(Table 13 is provided separately as a Microsoft Excel ${ }^{\circledR}$ worksheet.)

\section{Surficial Bed-Sediment Methods}

Surficial bed-sediment samples were collected by using a stainless steel 15 by 15 by $15 \mathrm{~cm}$ Ekman grab sampler (Wildco, Saginaw, Mich.). The sampler was cleaned with a mild soap solution and rinsed with deionized water prior to sampling and between samples. After the boat was anchored (connected to a buoy at the surface) at each sampling site, the sampler was lowered to the bottom of the reservoir; a messenger was then lowered to close the sampler. The sampler was brought to the surface, and the top $1 \mathrm{~cm}$ was subsampled for analysis. The sample from site LFDD was collected by wading into the river and scooping a sample into the appropriate bottle. Surficial bed-sediment samples were analyzed for 37 metals using methods described in Fishman (1993) and Garbarino and others (2006) at the USGS Crustal Geophyscis and Geochemistry Science Center (Geologic Division Laboratory) in Denver, Colorado. Surficial bed-sediment samples were also analyzed for organic constituents-22 parent PAHs, 10 specific alkyl-PAH isomers, a homologous series of 25 alkyl-PAH isomers, total PCBs, and 16 OC pesticides - using methods described by Noriega and others (2004) and Olson and others (2004).

\section{Surficial Bed-Sediment Quality Assurance}

Two types of QC samples were used for quality assuring bed sediment data - matrix spikes and replicates. Additionally, surrogate compounds were added to PAH and OC samples at the laboratory to monitor sample-specific performance of the analytical method. For PAHs, surrogates 2-fluorobiphenyl, nitrobenzene- $d 5$, and p-terphenyl- $d 14$ were added. For OCs, surrogates a-HCH- $d 6$, isodrin, and nonachlorobiphenyl were added.

Replicate samples were processed for PAHs, OCs, and metals. Two types of soil standard reference material (SRM) were used as QC checks of the digestion process and instrument calibration for the analysis of metals in surficial bed sediment for 2004 samples; they are the NIST 2709 (National Institute of Standards and Technology, 1991a) and the Canadian Certified Reference Materials Project (CCRMP) SO-2 and SO-4 standards (Potts and others, 1992). Only the NIST 2709 soil SRM was used for the 2009 sampling.

\section{Surficial Bed-Sediment Results}

Surficial bed-sediment samples collected for PAHs in October 2004 and August 2009 are given in table 14. Of the 53 compounds analyzed for PAHs, 22 compounds had 1 or more detections, of which, 10 were detected in about
$1 / 2$ of the samples. Surficial bed-sediment samples collected for OCs in August 2009 are given in table 15. Of the 22 compounds analyzed for OCs, only 6 compounds had 1 or more detections. Surficial bed-sediment samples collected for 37 metals in October 2004 and August 2009 are given in table 16 ; silver was the only metal not detected.

\section{Summary}

The primary objective of this study was to monitor changes in composition and concentration of compounds in the air and water before, during, and after the construction of a major thoroughfare (State Route 125) being built upwind of the Sweetwater Reservoir. To accomplish this, the study was divided into three phases. Phase One sampling started in September 1998 and was designed to establish baseline conditions for target compounds (primary sampling) in terms of detection frequency and concentration of select organic and inorganic compounds in air and water. Phase Two sampling began October 2004 and lasted 3 years. Phase Two was designed to monitor the air and water quality in and around the Sweetwater Reservoir during construction of State Route 125. Phase Three sampling began in October 2007, after construction of the roadway was completed, to assess the effect this alignment project may have on the water quality in the Sweetwater Reservoir. Phase Three sampling ended in August 2009. This report provides data from water, air, and surficial bed-sediment samples that were collected from October 2003 through August 2009 (water years 2004-09). This report is the fourth in a series of reports that describe the monitoring activities and present the data. Interpretative analysis of the environmental and quality-control data for targeted compounds to address the objectives of the study is not included in this data report.

Prior to any reservoir water sampling, depth profiles of dissolved oxygen, $\mathrm{pH}$, specific conductance, and water temperature were recorded at each reservoir sampling location. Compounds analyzed in water samples and their results are in tables 4A to 9B. Compounds analyzed in air samples and their results are in tables 10A through 13. Compounds analyzed in surficial bed-sediment samples are given in tables 14 through 16. Quality-control data for all compounds are located in appendixes A-C. Quality-control data for compounds in water samples are in tables A1A to A6C. Quality-control data for compounds in air samples are in tables B1A to B6C. Quality-control data for surficial bedsediment samples are in tables $\mathrm{C} 1$ to $\mathrm{C} 3 \mathrm{C}$. 


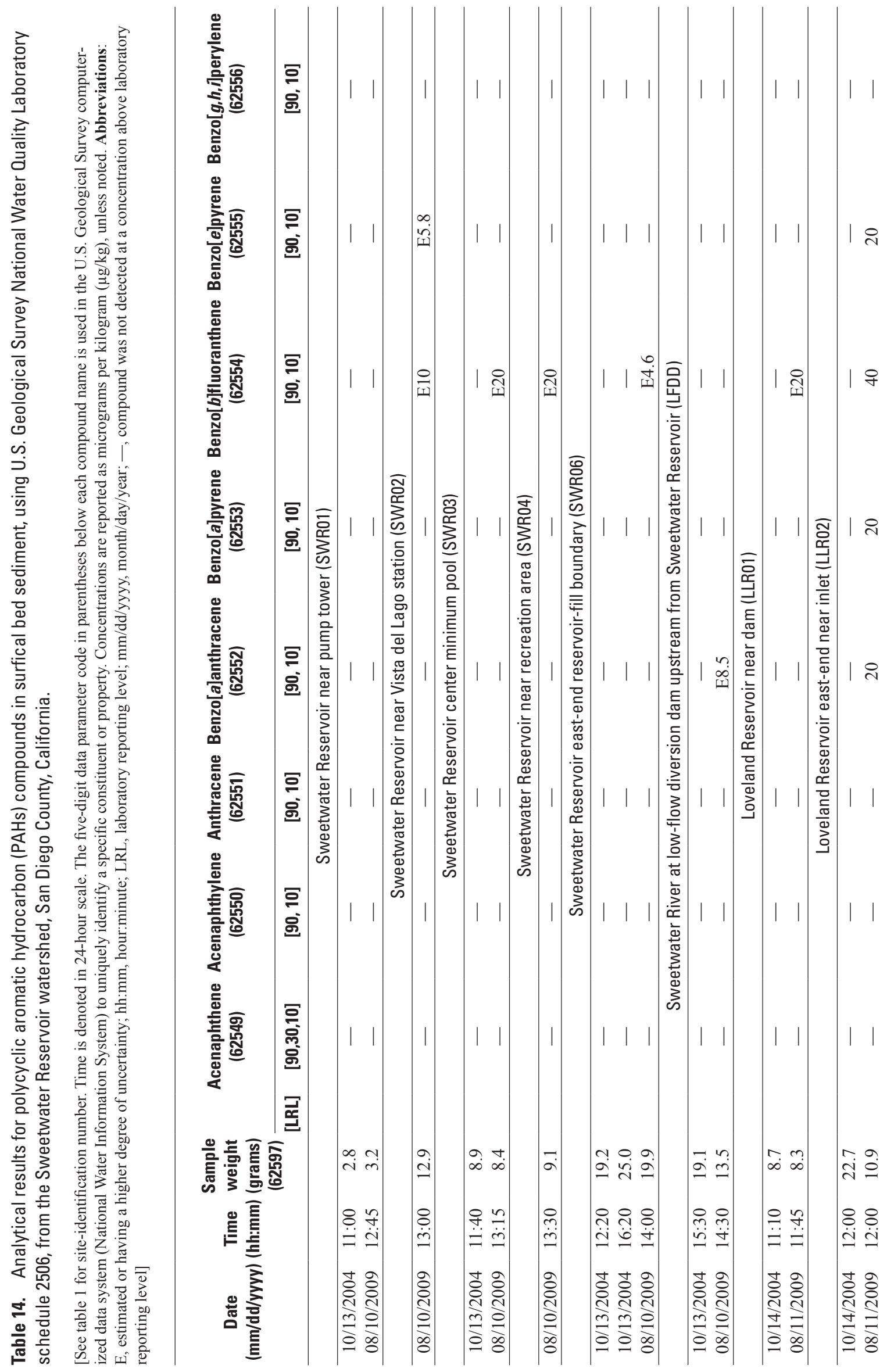




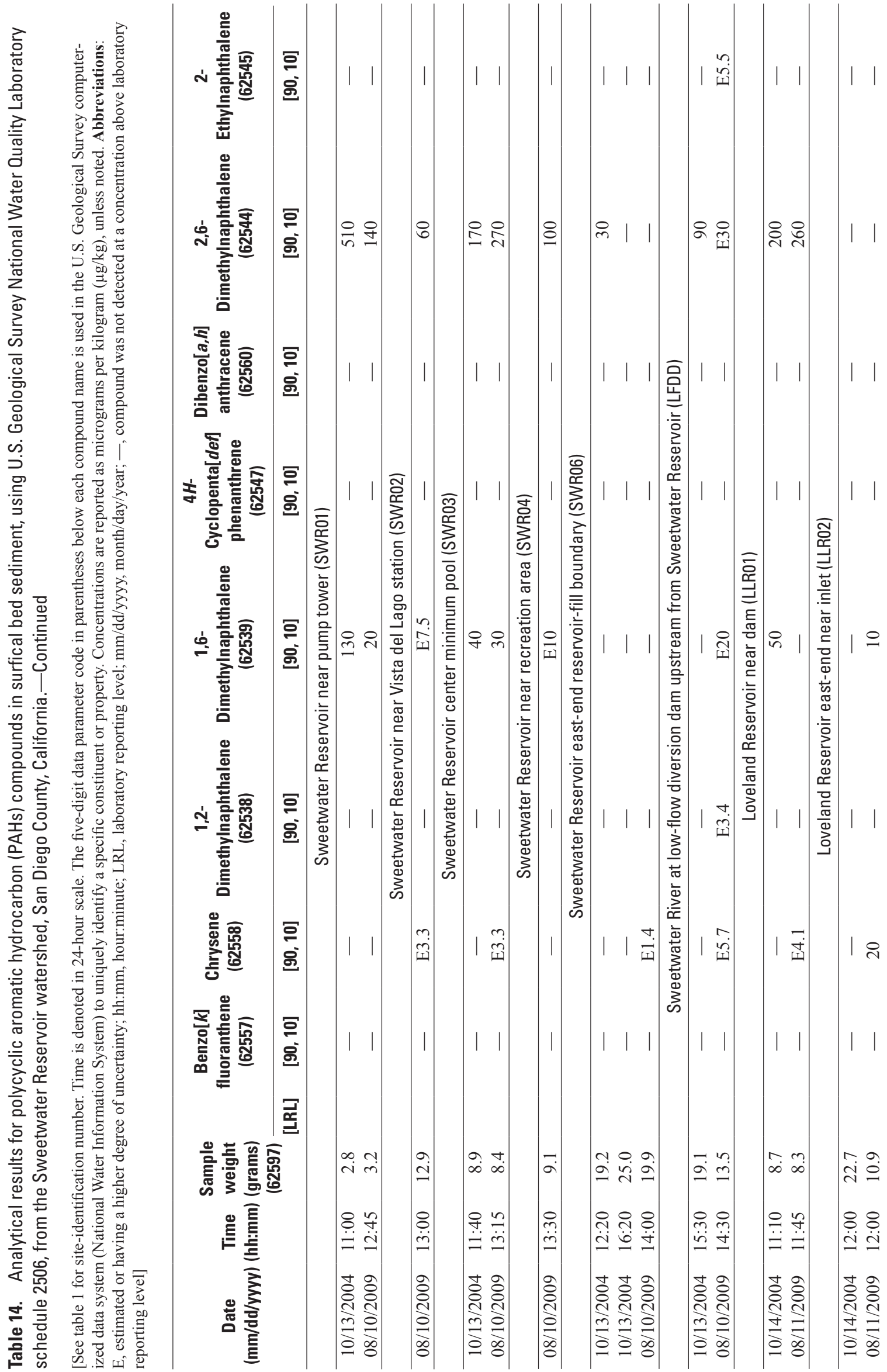




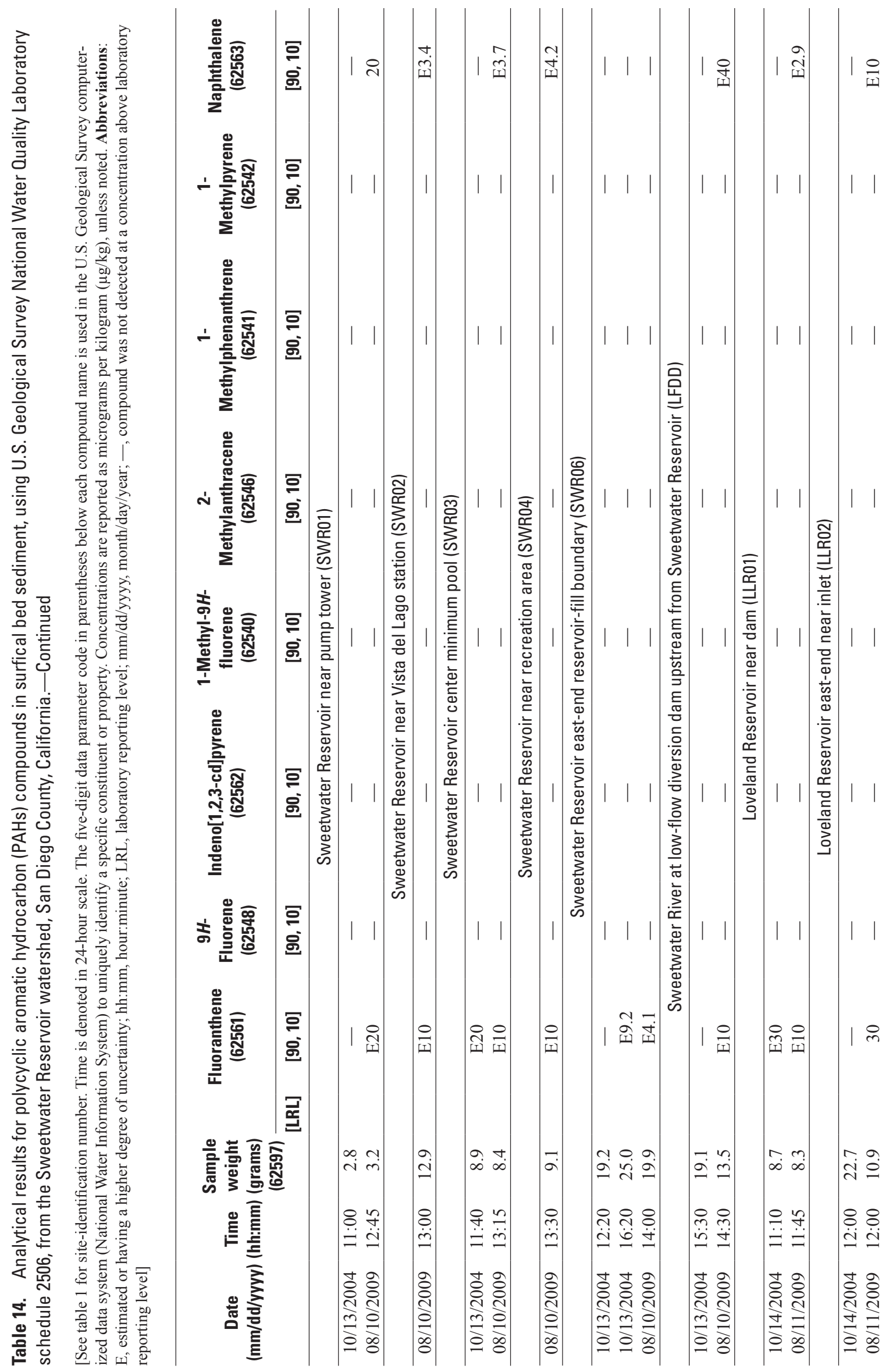




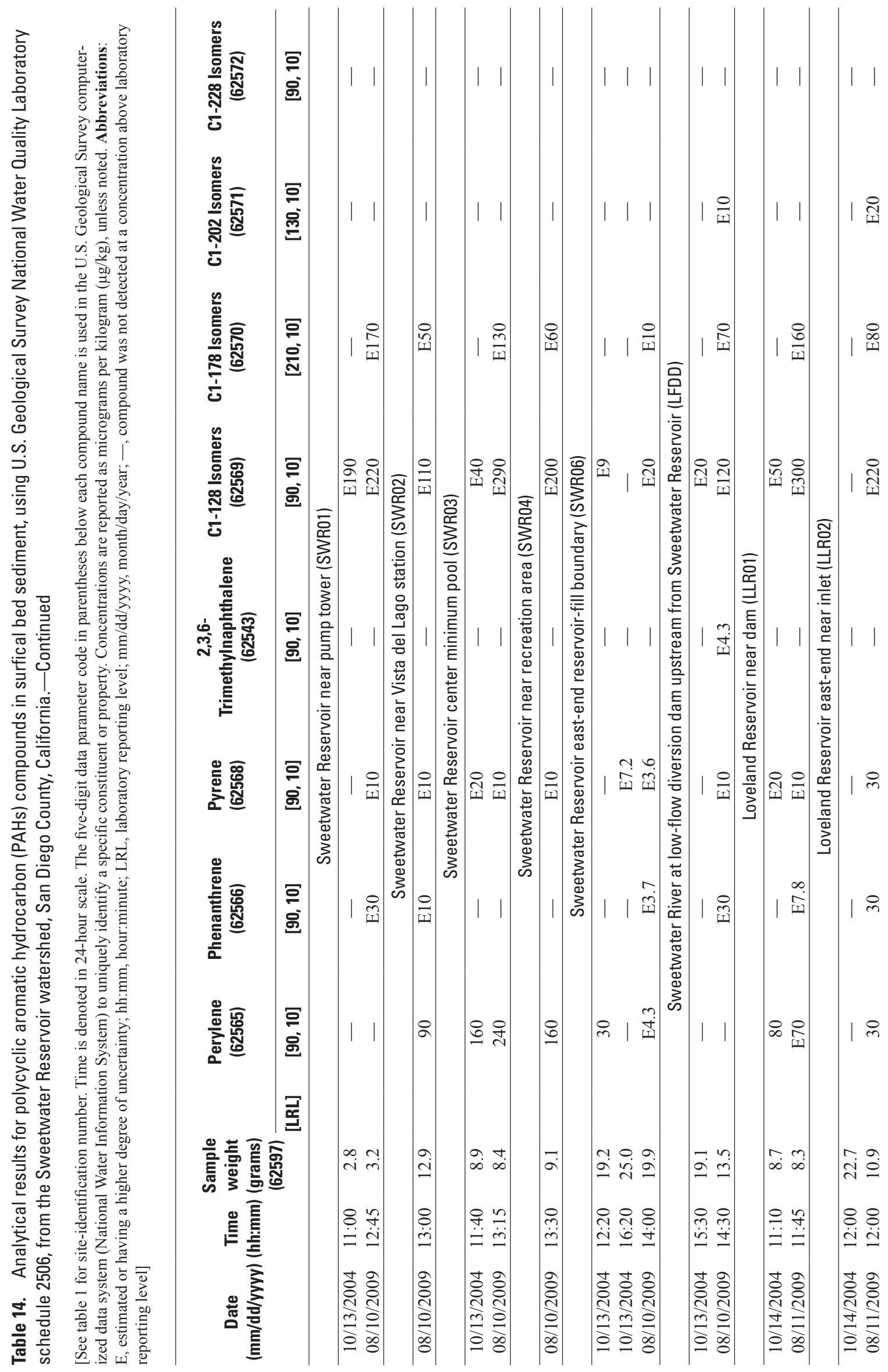




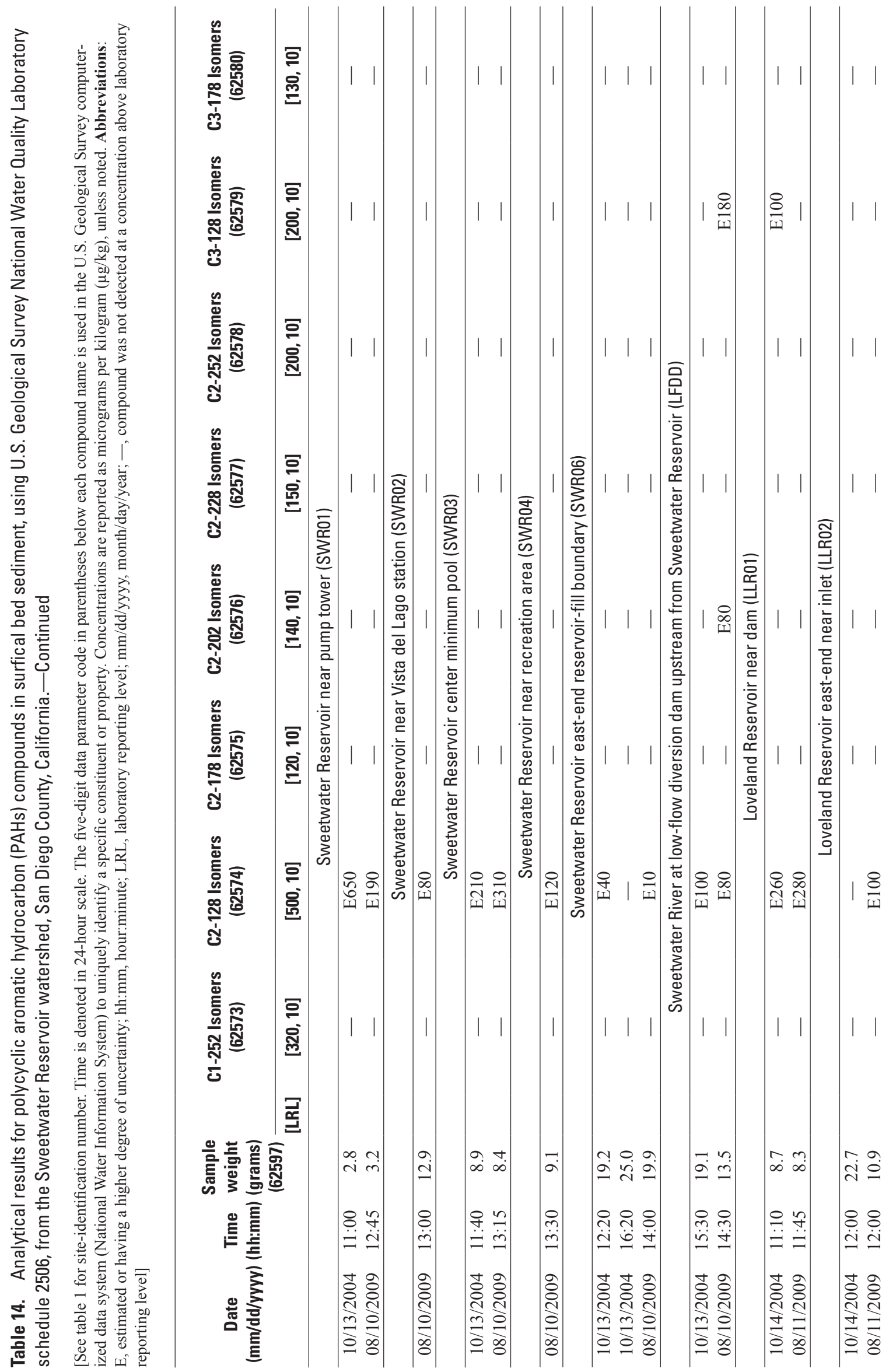




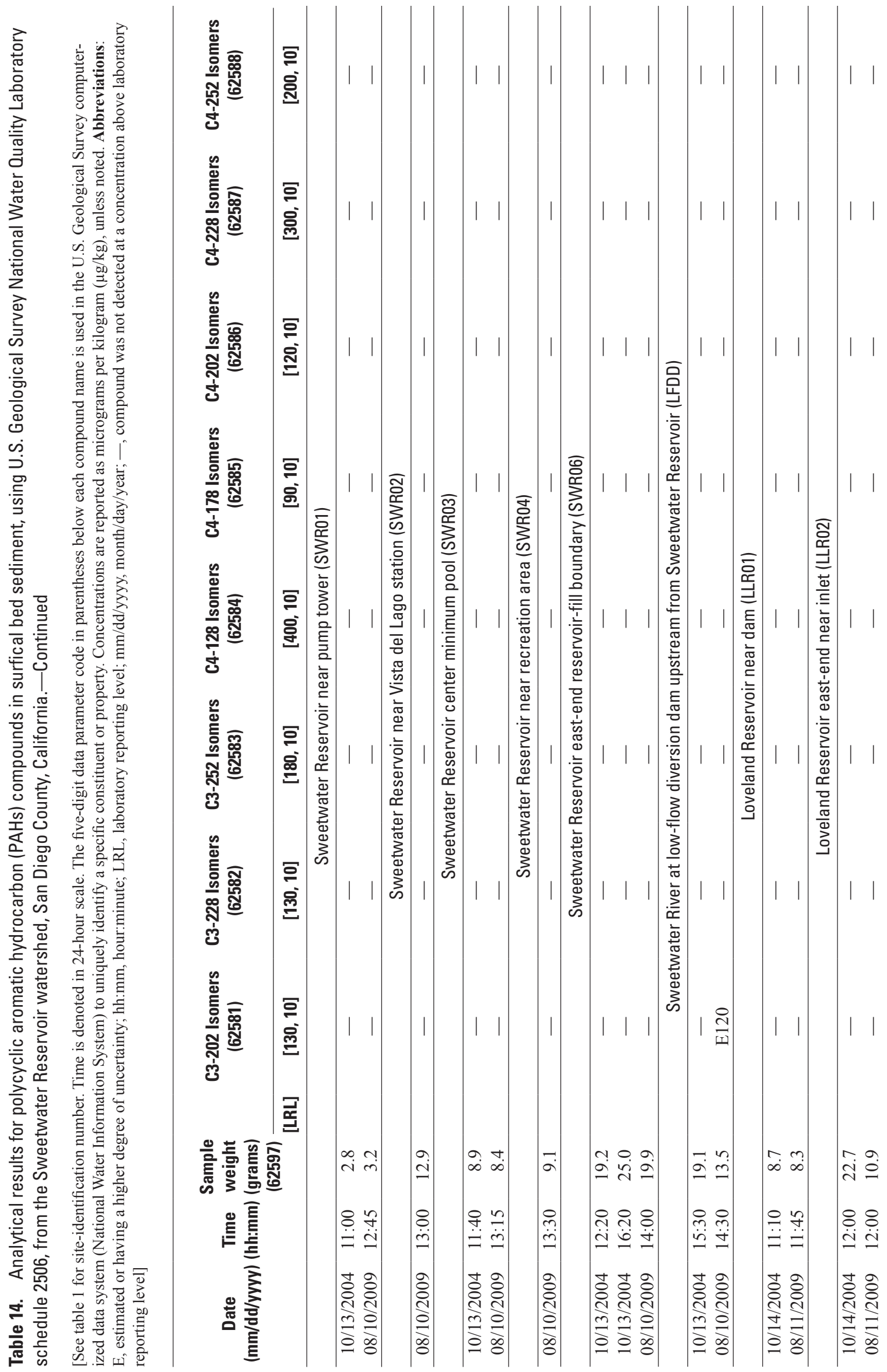




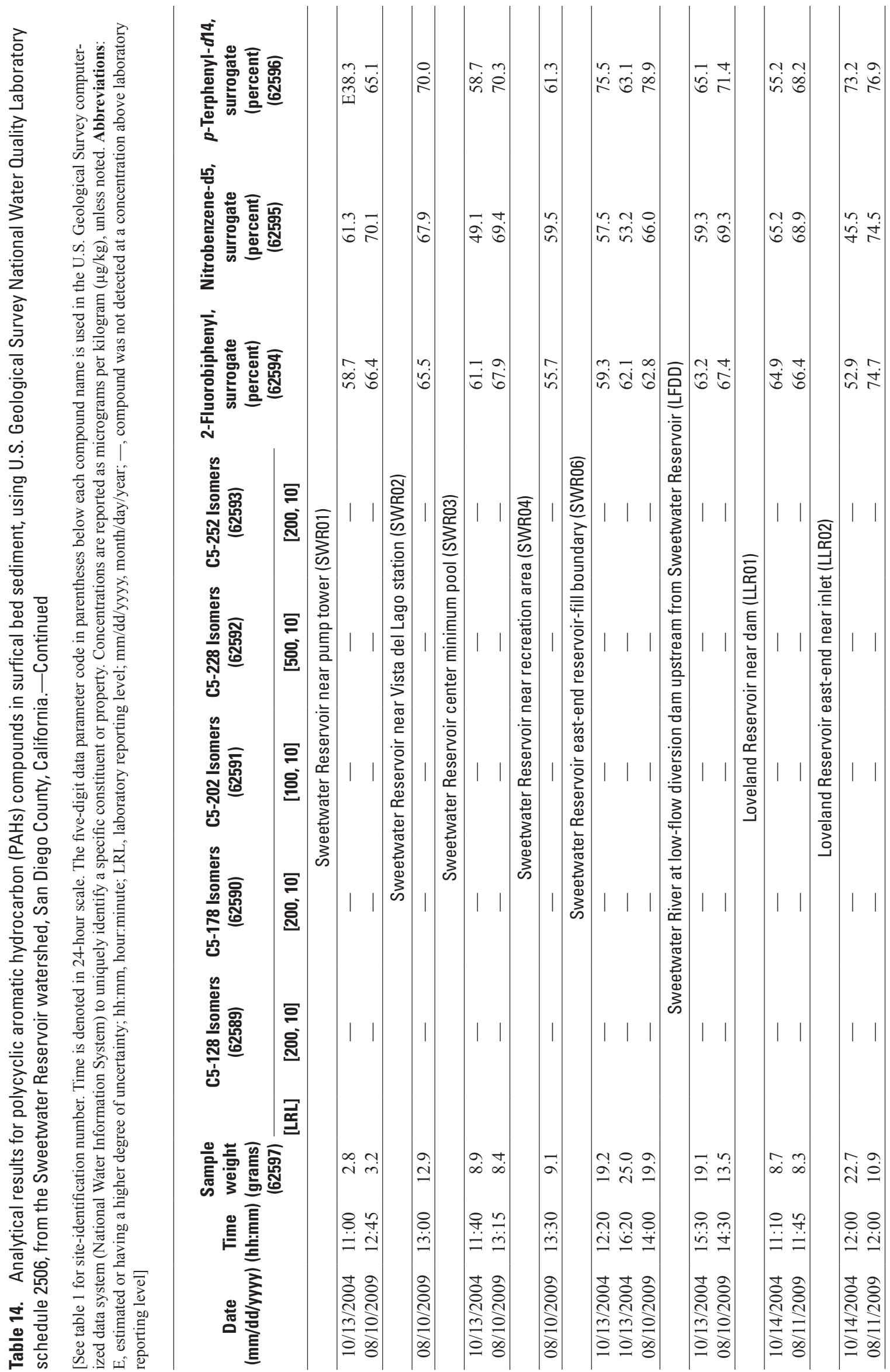




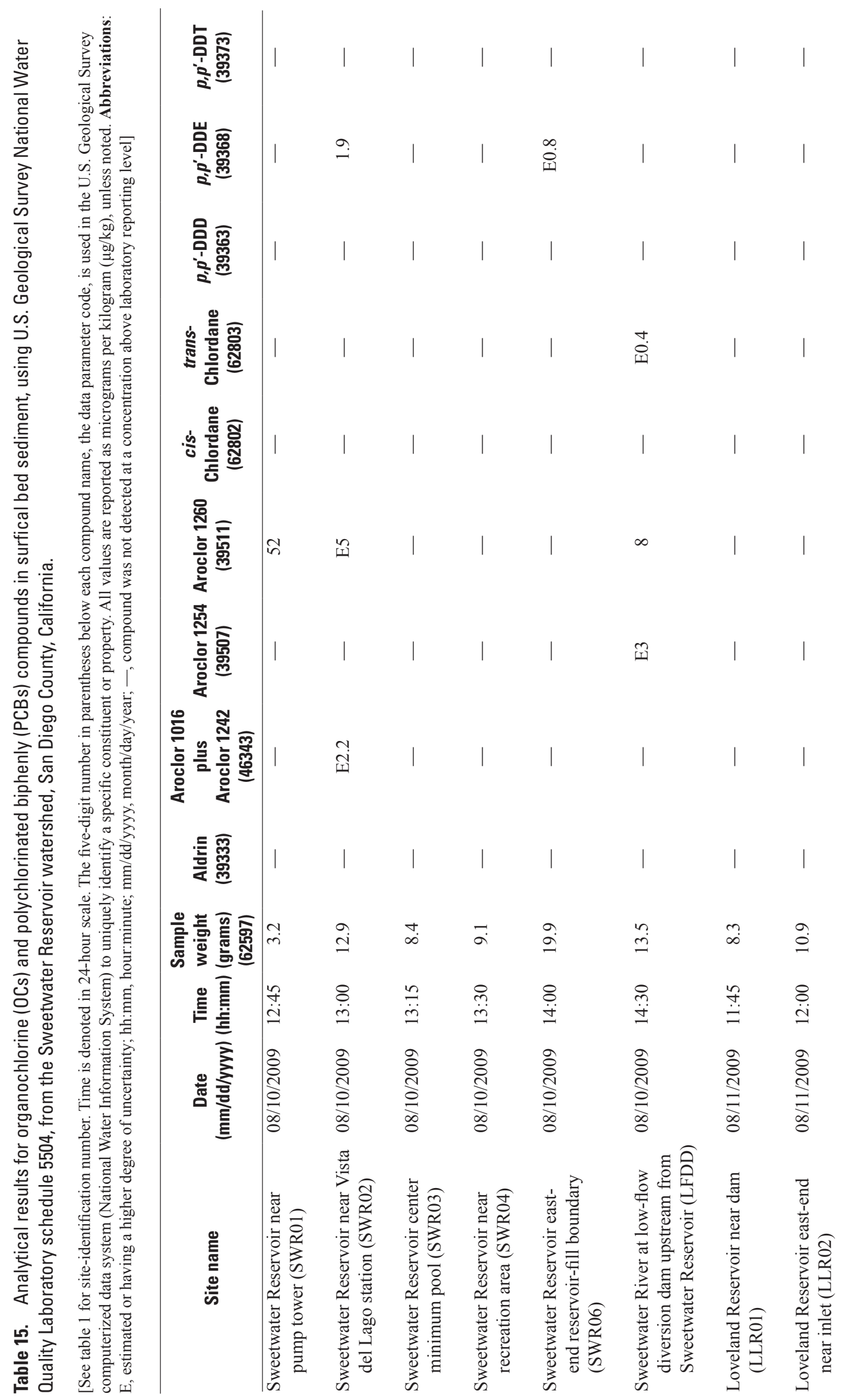




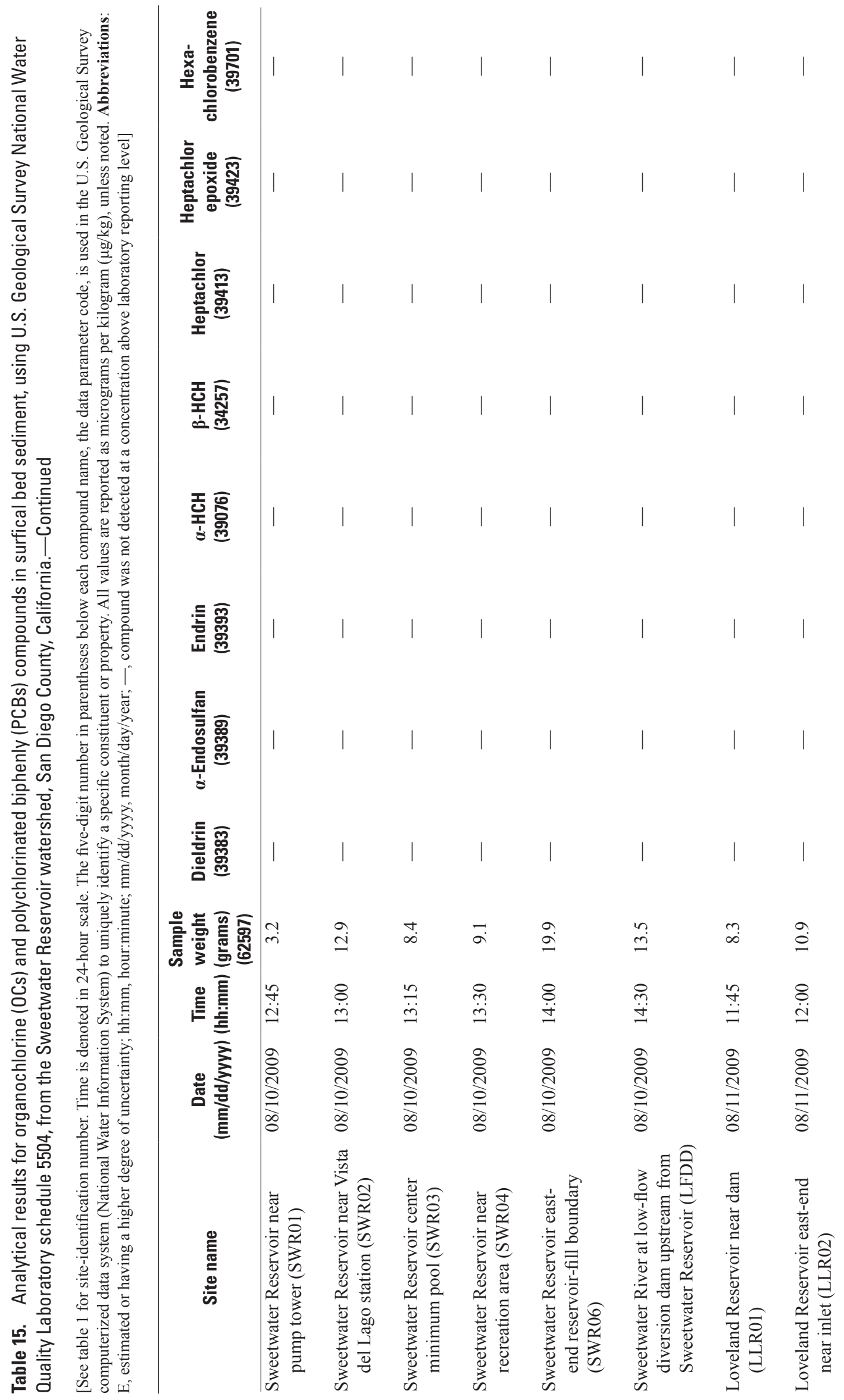




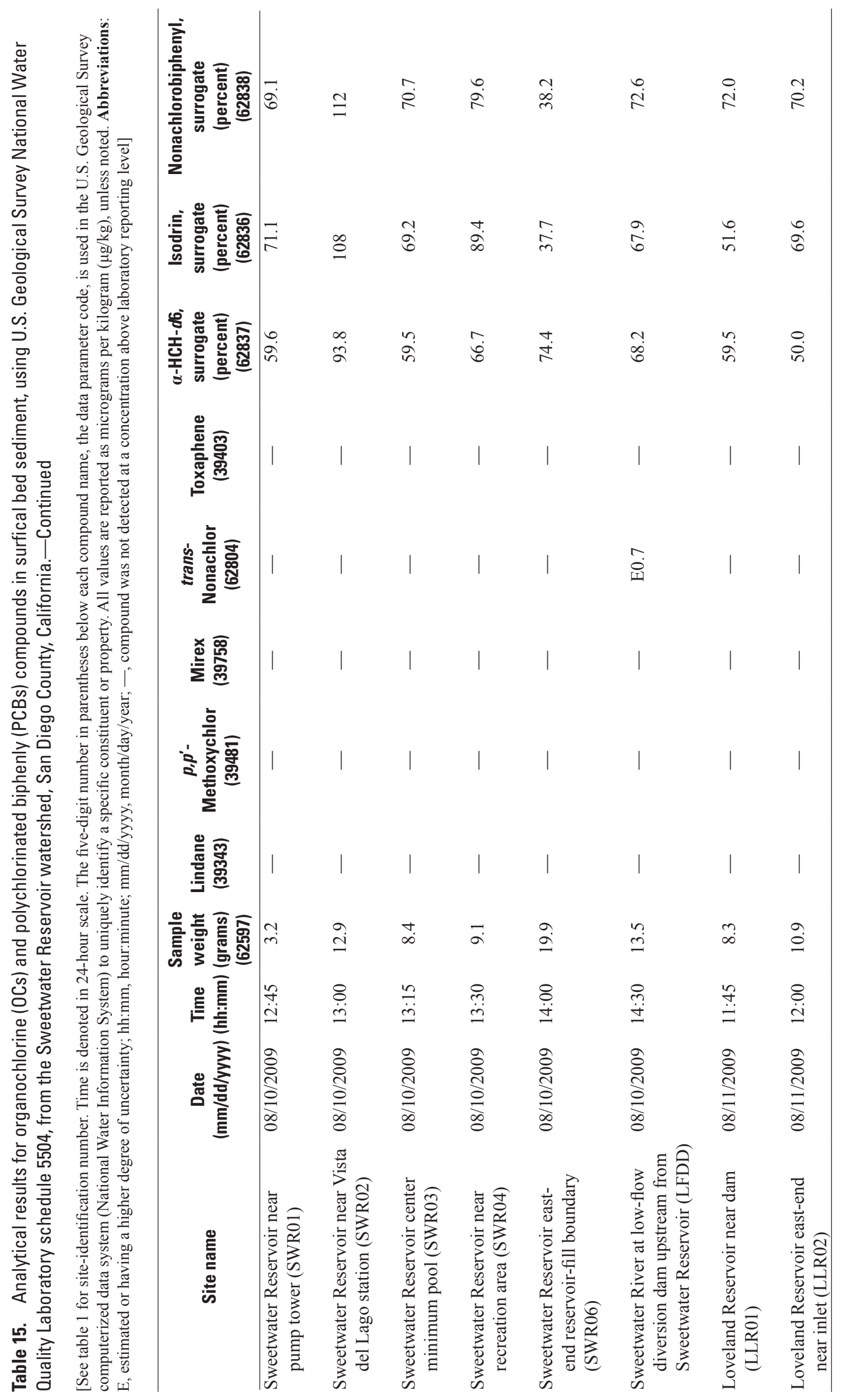




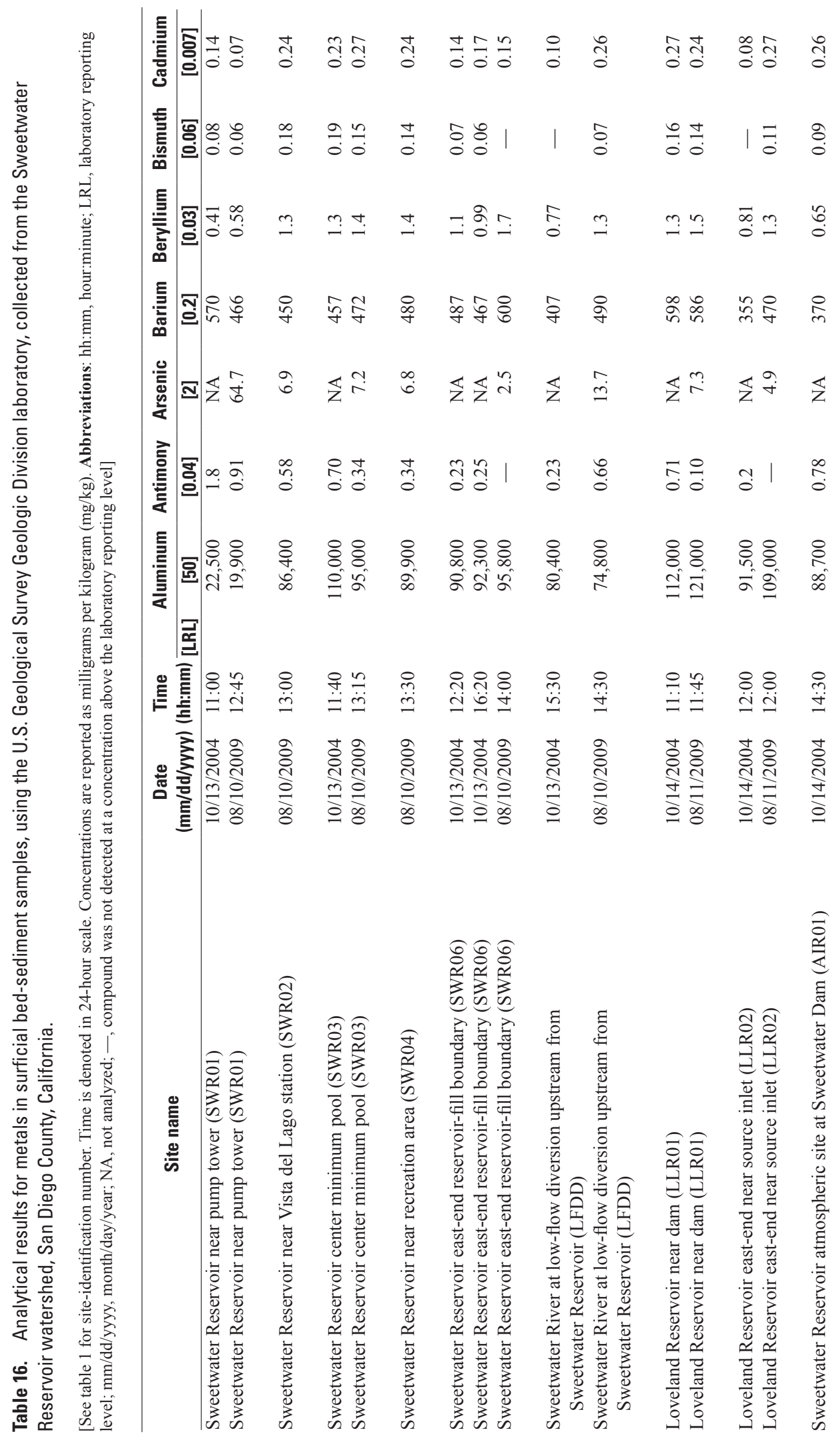




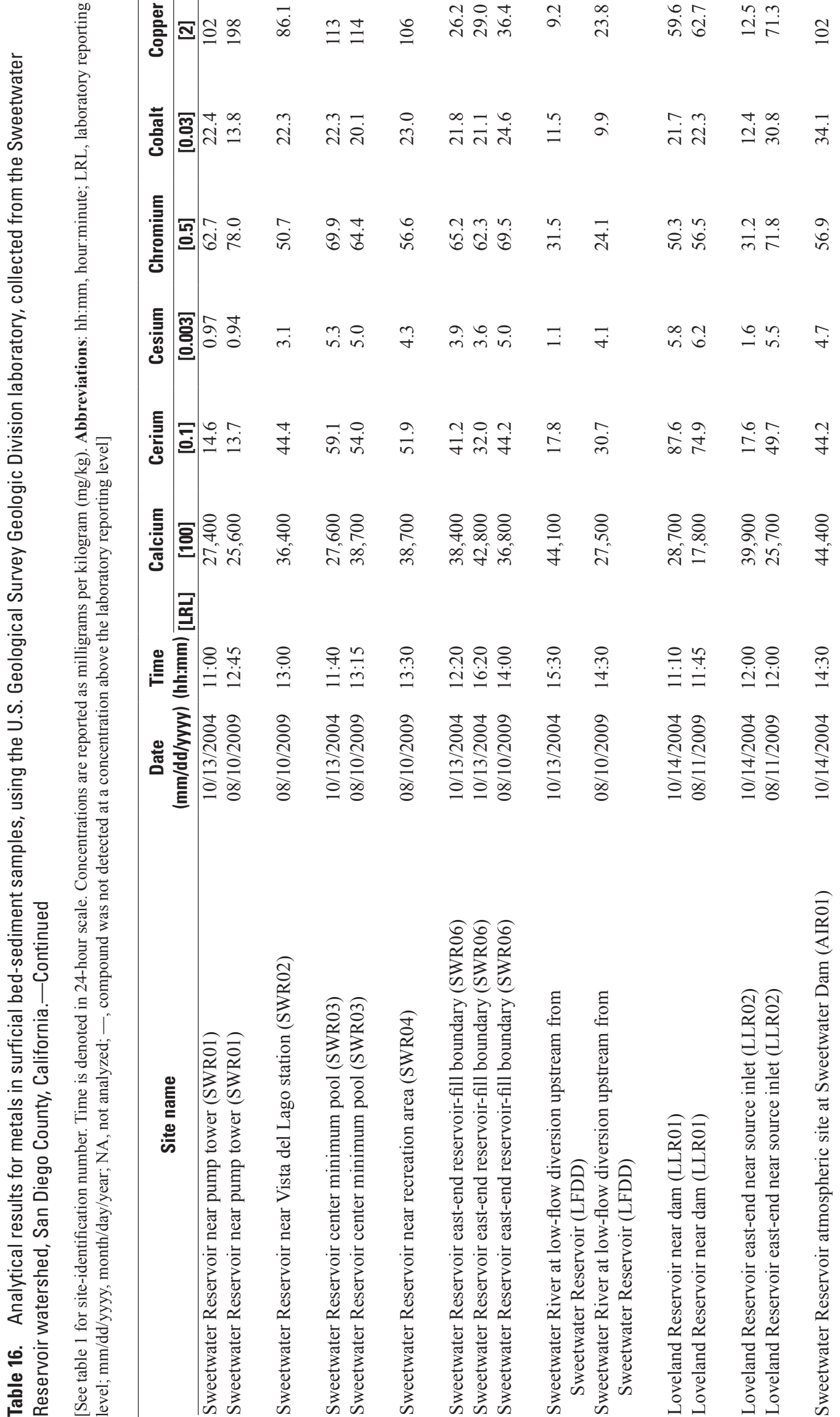




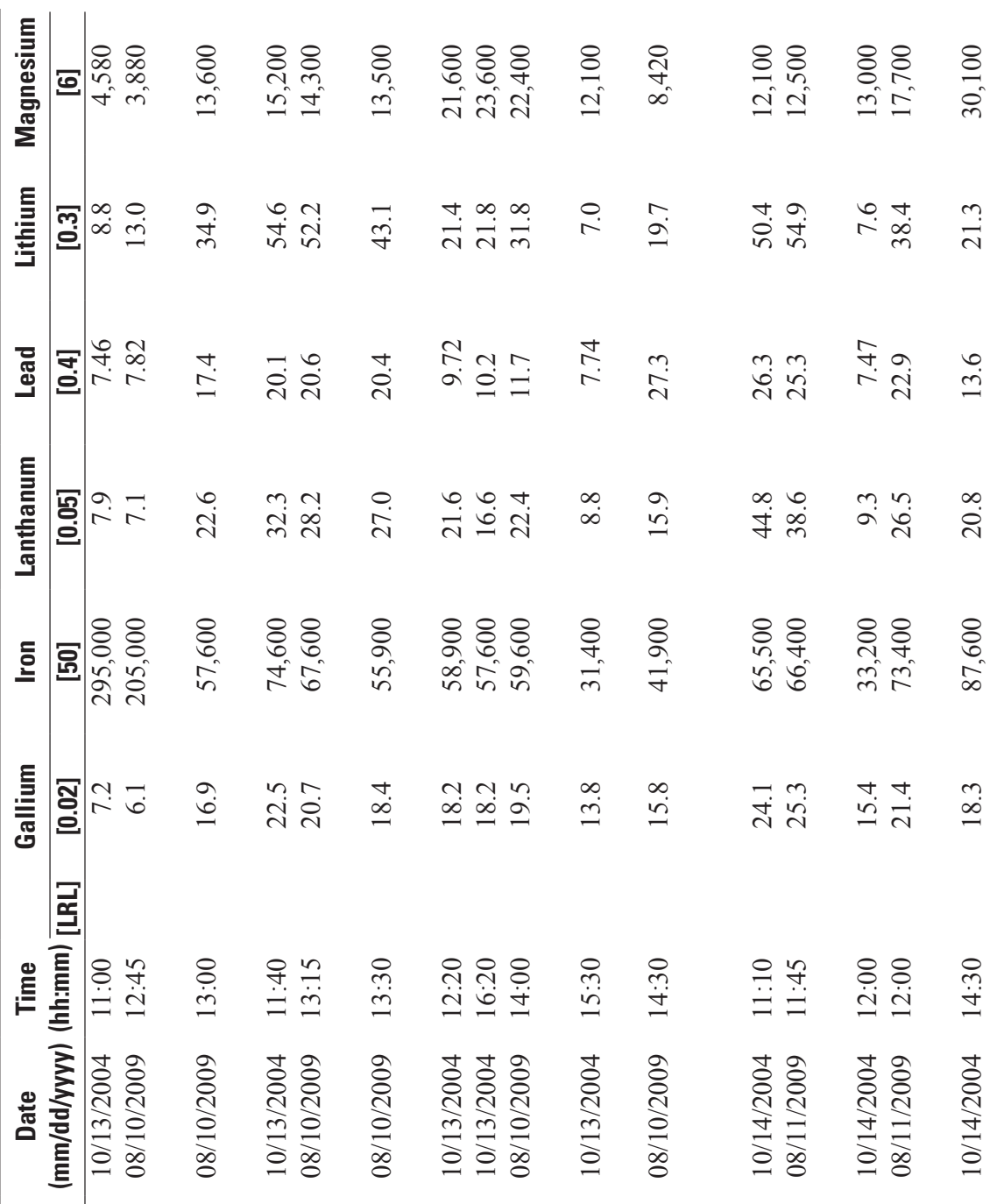




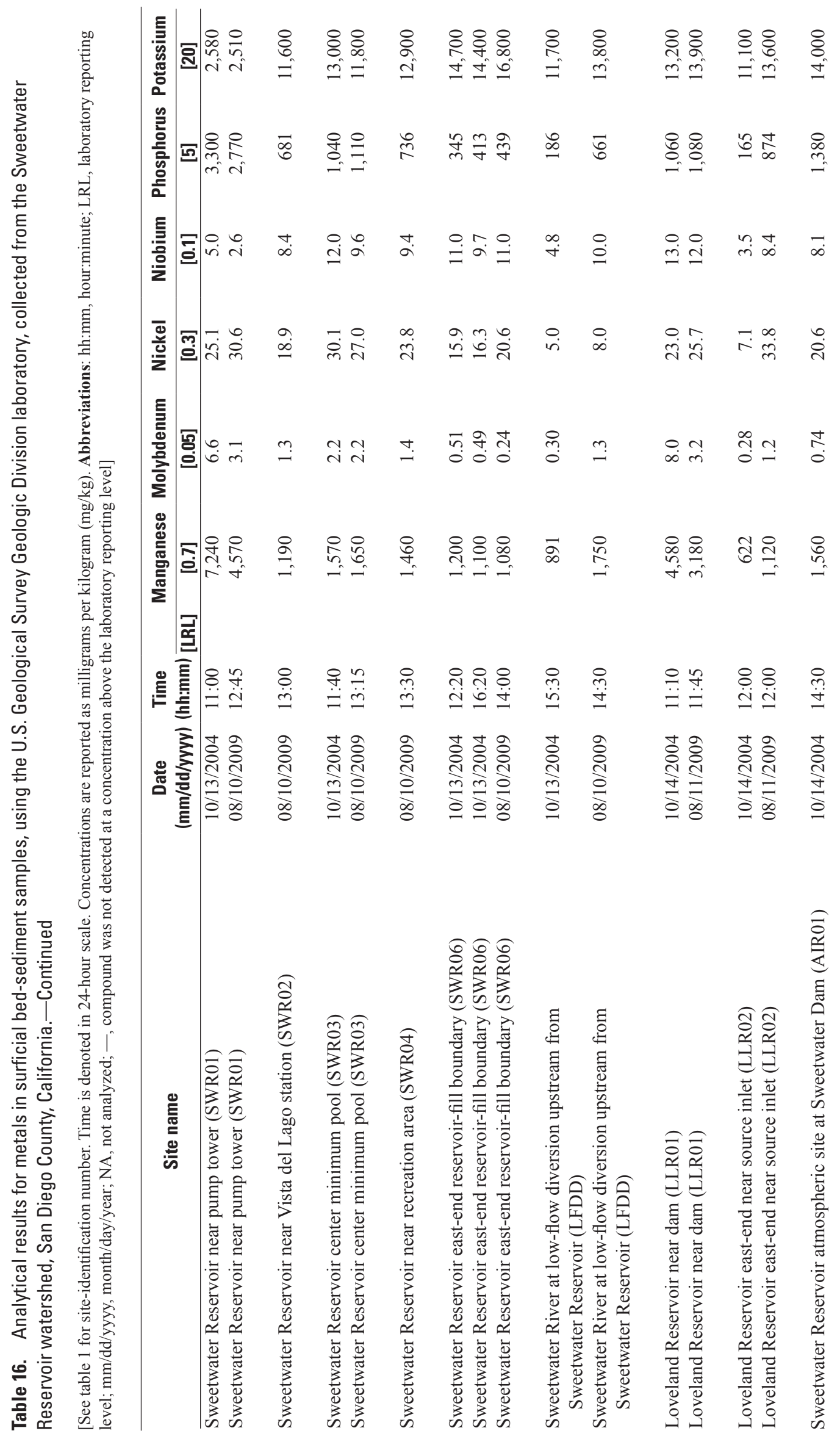




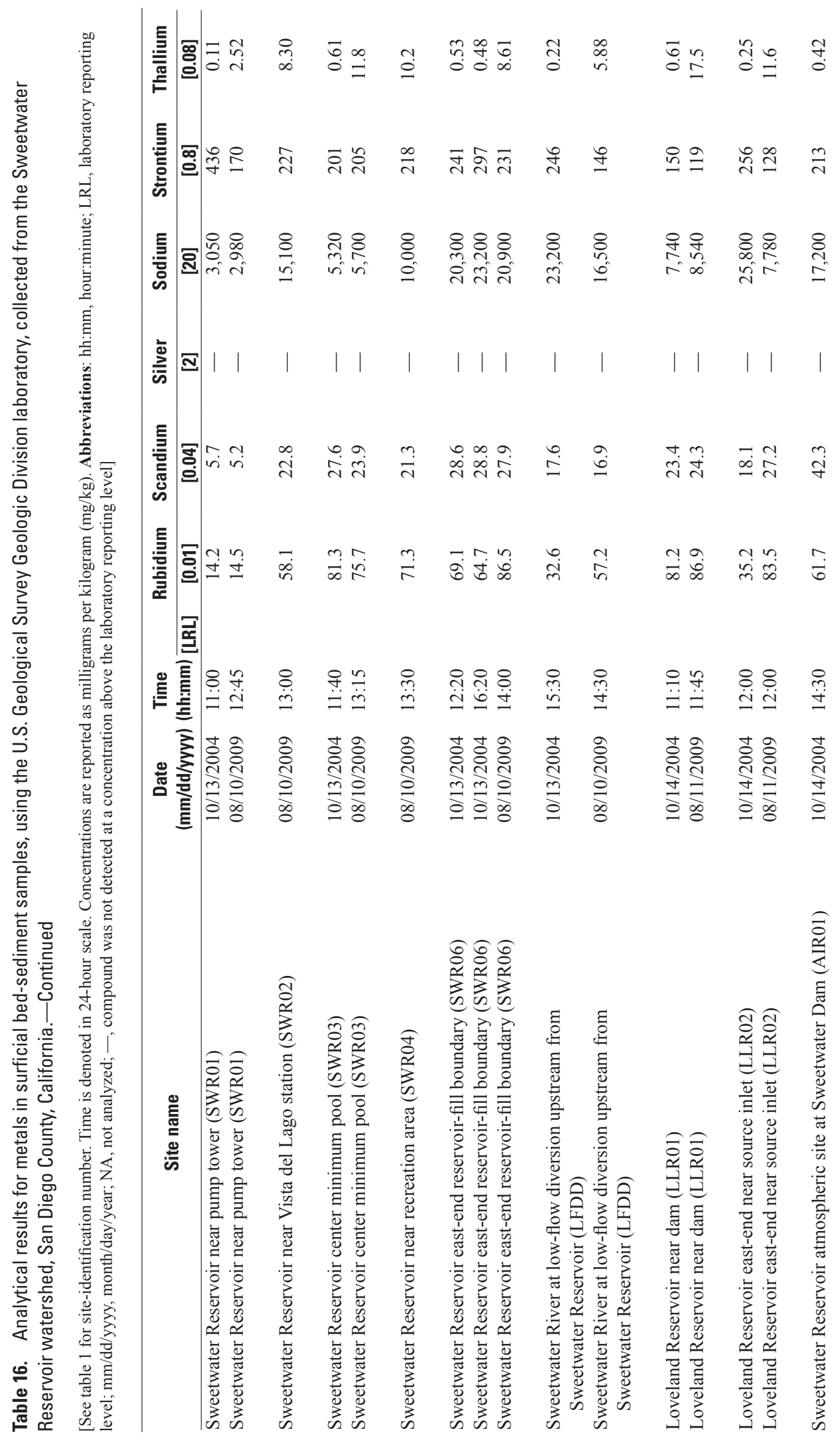




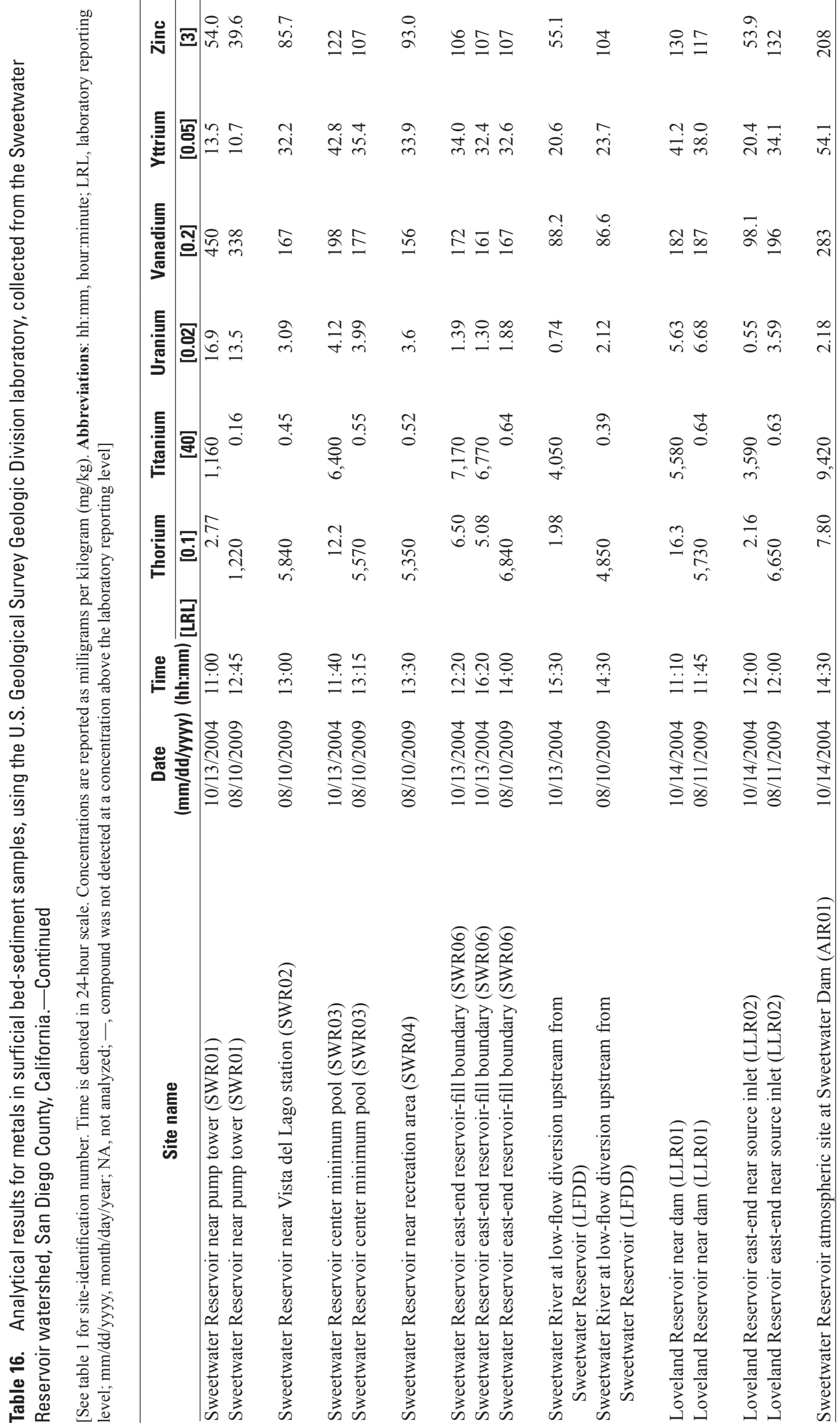


Results showed methyl tert-butyl ether was present in water and air samples until its use was phased out by order of the California state government in January 2004. The highest concentrations of benzene and toluene in water were detected at or near the surface of the reservoir during Phase Two. The concentrations of benzene and toluene in air samples were highest during the cooler months and tended to have the same seasonal pattern over time. Isophorone and phenol were the two most frequently detected BNA compounds in water. Diuron, prometon, and simazine were the most frequently detected pesticide compounds in water. Silver and thallium were not detected in unfiltered water samples. Beryllium, chromium, and thallium had no detections in filtered water samples.

Chloroethane was the only high-volatility volatile organic compound detected in air samples. Twenty-eight medium/low-volatility volatile organic compounds had no detections in air samples. Nineteen polycyclic aromatic hydrocarbon compounds were detected in air samples. Ten pesticide compounds were detected in air samples. Niobium and thallium were the least frequently detected metals in air samples.

Seven polycyclic aromatic hydrocarbons and three isomers were detected in half the surficial bed-sediment samples. Of the 22 organochlorine pesticides analyzed, 6 were detected at least once. Of the 37 metals analyzed in surficial bed-sediment samples, silver was the only metal not detected.

Three types of quality-control samples were used in this study - spikes, blanks, and replicates. Matrix-spike data were considered to be adequate if the recovery was within 30 percent of the expected concentration. Most matrixspike data were within acceptable criteria, but several compounds were below the acceptable criteria. The detected concentrations for these compounds may be under-reported or may not have been detected if present at low concentrations. Some matrix spikes were above the acceptable criteria and concentrations of those compounds may be overestimated. Replicate data are considered to be adequate if within 30 percent of the environmental sample.

Matrix-spike recoveries were within the acceptable criteria for volatile organic compounds in water samples except for carbon disulfide, dichlorodifluoromethane, and trichloromethane, which had recoveries more than 30 percent different from expected in one-third or more of spike samples. High-volatility volatile organic compounds in air samples had only one matrix-spike recovery above the acceptable criteria, and 21 recoveries were below the acceptable criteria, although no recovery was below 59 percent. Medium/low-volatility volatile organic compounds in air samples had 5 matrix-spike recoveries above the acceptable criteria, and 32 recoveries were below the acceptable criteria. The polycyclic aromatic hydrocarbons in the air had no recoveries above the acceptable criteria, but many recoveries were below the acceptable criteria. The recoveries tend to be lower on the glass-fiber filters than on the polyurethane foam. Pesticides in air samples had a similar pattern as the polycyclic aromatic hydrocarbon data for matrix-spike samples; recoveries were lower on the glass-fiber filters than on the polyurethane foam. Eight compounds had one spike recovery above acceptable criteria for Airpest003. About 26 percent of pesticide recoveries were below the acceptable criteria. Three standard references for metals in surficial bed sediment were analyzed for laboratory matrix spikes. All matrix-spike results for reference material NIST 2709 were within the acceptable criteria. All matrixspike results for reference materials SO-2 and SO-4 were within the upper acceptable criteria except niobium (SO-2 recovery of 173 percent). Two matrix-spike recoveries for reference materials SO-2 and SO-4 were below the lower acceptable criteria - antimony and uranium.

Field blank data associated with water samples for volatile organic compounds had detections for acetone and carbon disulfide. Trichloromethane was also detected in a field blank, but at a similar concentration to that in an associated source solution blank. Four metals (molybdenum, silver, thallium, and uranium) were detected in a blank sample at site SWR08, but all detections were below the LRLs. Travel, lot and laboratory blanks were processed with all the volatile organic compound air samples. Acetone, benzene, and toluene were detected at low concentrations in most volatile organic compound air blanks. Two field blanks collected for polycyclic aromatic hydrocarbons in the air had no detections. The 17 laboratory blanks for polycyclic aromatic hydrocarbons in the air had detections, but none were above their laboratory reporting level. No blank data were collected for surficial bed sediments.

The volatile organic compound replicate data for water samples were within acceptable criteria for most samples and compounds. Four replicate data sets collected for pesticide compounds in water indicate most results are within acceptable criteria. Replicate data for metals in water samples indicated that 7 of the 19 compounds analyzed had 5 or fewer replicates outside the acceptable criteria. No highvolatility volatile organic compounds in the air were detected in replicate or associated environmental samples. Twentyseven compounds for medium/low volatility VOCs had at least one replicate value above the acceptable criteria, and twenty-two compounds had at least one replicate value below the acceptable criteria. Replicate air samples for semivolatile organic compounds and metals were not collected during this reporting period. Two replicate data sets were collected for polycyclic aromatic hydrocarbons in surficial bed sediment, and some compounds had replicate pair comparisons outside the acceptable criteria. No detections were found in the surficial bed sediment replicate sample collected for organochlorine and polychlorinated biphenyl compounds. Of the 37 metals analyzed, beryllium had the only replicate outside the acceptable criteria. 


\section{References Cited}

Alexandrou, Nick, Smith, Murray, Park, Richard, Lumb, Kevin, and Brice, Ken, 2001, The extraction of polycyclic aromatic hydrocarbons from atmospheric particulate matter samples by accelerated solvent extraction (ASE): International Journal of Environmental Analytical Chemistry, v. 81 , no. 4, p. 257-280, doi:10.1080/03067310108044248.

Baehr, A.L., Kauffman, L.J., Charles, E.G., Baker, R.J., Stackelberg, P.E., Ayers, M.A., and Zapecza, O.S., 1999a, Sampling throughout the hydrologic cycle to characterize sources of volatile organic compounds in ground water in Morganwalp, D.W., and Buxton, H.T., eds., U.S. Geological Survey toxic substances hydrology program-Proceeding of the technical meeting, Charleston, South Carolina, March 8-12, 1999: U.S. Geological Survey Water Resources Investigations Report 99-4018C, v. 3, p. 21-36.

Baehr, A.L., Stackelberg, P.E., and Baker, R.J., 1999b, Evaluation of the atmosphere as a source of volatile organic compounds in shallow groundwater: Water Resources Research, v. 35, no. 1, p. 127-136, doi:10.1029/1998WR900030.

Bender, D.A., Zogorski, J.S., Luo, Wentai, Pankow, J.F., Majewski, M.S., and Baker, R.J., 2000, Atmosphere-water interaction of chloroform, toluene, and MTBE in small perennial urban streams, in Air \& Waste Management Association, Annual Conference and Exposition, 93 $3^{\text {rd }}$, Salt Lake City, Utah, June 18-22, 2000 [Proceedings], 11 p.

Bigelow, D.S., 1984, Instruction manual-NADP/NTN site selection and installation: Fort Collins, Colo., Colorado State University, Natural Resource Ecology laboratory, variously paged.

Briggs, P.H., and Meier, A.L., 2002, The determination of forty-two elements in geological material by inductively coupled plasma-mass spectrometry, in Taggert, J.E., Jr., ed., Analytical methods for chemical analysis of geologic and other materials: U.S. Geological Survey Open-File Report 02-223, chap. I, p. I-1-I-14.

Byard, J.L., 1999, Impact of SR 125 vehicle emissions on the Sweetwater Reservoir-Executive summary report: Chula Vista, Calif., Sweetwater Authority, 32 p.

California Department of Transportation, 2001, State Route 125 (From I-905 to State Route 54): District 11 Fact Sheet, accessed January 28, 2008, at http://www.dot.ca.gov/dist11/ facts/125spvly.pdf.

California Environmental Protection Agency, 1986, California safe drinking water and toxic enforcement act of 1986 (Proposition 65): Health and Safety Code, chap 6.6, Sections 25249.5 through 25249.13.
Connor, B.F., Rose, D.L., Noriega, M.C., Murtaugh, L.K., and Abney, S.R., 1998, Methods of analysis by the U.S. Geological Survey National Water Quality LaboratoryDetermination of 86 volatile organic compounds in water by gas chromatography/mass spectrometry, including detections less than reporting limits: U.S. Geological Survey Open-File Report 97-829, 78 p.

Fishman, M.J., ed., 1993, Methods of analysis by the U.S. Geological Survey National Water Quality LaboratoryDetermination of inorganic and organic constituents in water and fluvial sediments: U.S. Geological Survey OpenFile Report 93-125, 217 p.

Foreman, W.T., Connor, B.F., Furlong, E.T., Vaught, D.G., and Merten, L.M., 1995, Methods of analysis by the U.S. Geological Survey National Water Quality Laboratory-Determination of organochlorine pesticides and polychlorinated biphenyls in bottom sediment by dual capillary-column gas chromatography with electroncapture detection: U.S. Geological Survey Open-File Report 95-140, $78 \mathrm{p}$.

Foreman, W.T., Goolsby, D.A., Majewski, M.S., Wiebe, F.W., and Battaglin, W.A., 1997, Acetochlor and other herbicides in air and rain in the Midwestern United States [abs.], in Bridging the global environment-Technology, Communication, and Education: Society of Environmental Toxicology and Chemistry Annual Meeting, 18th, San Francisco, Calif., November 16-20, 1997, no. 229, p. 45.

Foreman, W.T., Majewski, M.S., Goolsby, D.A., Wiebe, F.W., and Coupe, R.H., 2000, Pesticides in the atmosphere of the Mississippi River Valley, Part II-Air: Science of the Total Environment, v. 248, no. 2-3, p. 213-226, http://dx.doi. org/10.1016/S0048-9697(99)00544-6.

Fram, M.S., Bergamaschi, B.A., and Fujii, Roger, 2001, Improving water quality in Sweetwater Reservoir, San Diego County, California-Sources and mitigation strategies for Trihalomethane (THM)-forming carbon: U.S. Geological Survey Fact Sheet 112-01, 4 p., accessed January 2007 at $h t t p: / / p u b s . u s g s . g o v / f s / f s-112-01 / p d f /$ framfs.pdf.

Furlong, E.T., Anderson, B.D., Werner, S.L., Soliven, P.P., Coffey, L.J., and Burkhardt, M.R., 2001, Methods of analysis by the U.S. Geological Survey National Water Quality Laboratory-Determination of pesticides in water by graphitized carbon-based solid-phase extraction and high-performance liquid chromatography/mass spectrometry: U.S. Geological Survey Water-Resources Investigations Report 01-4134, 73 p., accessed March 2003 at http://nwql.usgs.gov/Public/pubs/WRIR01-4134.pdf. 
Garbarino, J.R., 1999, Methods of analysis by the U.S. Geological Survey National Water Quality LaboratoryDetermination of dissolved arsenic, boron, lithium, selenium, strontium, thallium, and vanadium using inductively coupled plasma-mass spectrometry: U.S. Geological Survey Open-File Report 99-093, 31 p.

Garbarino, J.R., Kanagy, L.K., and Cree, M.E., 2006, Determination of elements in natural-water, biota, sediment, and soil samples using collision/reaction cell inductively coupled plasma-mass spectrometry: U.S. Geological Survey Techniques and Methods, book 5, sec. B, chap. 1, 88 p.

Garbarino, J.R., and Struzeski, T.M., 1998, Methods of analysis by the U.S. Geological Survey National Water Quality Laboratory-Determination of elements in wholewater digests using inductively coupled plasma-optical emission spectrometry and inductively coupled plasmamass spectrometry: U.S. Geological Survey Open-File Report 98-165, $101 \mathrm{p}$.

Garbarino, J.R., and Taylor, H.E., 1996, Inductively coupled plasma-mass spectrometric method for the determination of dissolved trace elements in natural water: U.S. Geological Survey Open-File Report 94-358, 28 p.

Garrison, V.H., Foreman, W.T., Genualdi, S., Griffin, D.W., Kellogg, C.A., Majewski, M.S., Mohammed, A., Ramsubhag, A., Shinn, E.A., Simonich, S.L., and Smith, G.W., 2006, Saharan dust-A carrier of persistent organic pollutants, metals and microbes to the Caribbean: Revista Biologia Tropical, v. 54 (Suppl. 3), p 9-21.

Lamothe, P.J., Meier, A.L., and Wilson, S.A., 2002, The determination of forty-four elements in aqueous samples by inductively coupled plasma-mass spectrometry, in Taggart, J.E., Jr., ed., Analytical methods for chemical analysis of geologic and other materials: U.S. Geological Survey OpenFile Report 02-223-H, p. H1-H11.

Lindley, C.E., Stewart, J.T., and Sandstrom, M.W., 1996, Determination of low concentrations of acetochlor in water by automated solid-phase extraction and gas chromatography with mass-selective detection: Journal of the Association of Official Analytical Chemists International, v. 79, no. 4, p. 962-966.

Mackay, Donald, Shiu, W.Y., and Ma, K. C., 1993, Illustrated handbook of physical-chemical properties and environmental fate for organic chemicals-Volatile organic chemicals: CRC Press Inc., Boca Raton, Fla., v. 3, 916 p.

Madsen, J.E., Sandstrom, M.W., and Zaugg, S.D., 2003, Methods of analysis by the U.S. Geological Survey National Water Quality Laboratory-A method supplement for the determination of fipronil and degradates in water by gas chromatography/mass spectrometry: U.S. Geological Survey Open-File Report 02-462, 11 p., accessed March 2003 at http://nwql.usgs.gov/Public/pubs/OFR02-462/ OFR02-462.pdf.
Majewski, M.S., Foreman, W.T., Goolsby, D.A., and Nakagaki, Naomi, 1998, Airborne pesticide residues along the Mississippi River: Environmental Science and Technology, v. 32, no. 23, p. 3689-3698.

Majewski, M.S., Sidhu, J.S., and Mendez, G.O., 2002, Waterquality monitoring of Sweetwater and Loveland Reservoirs, San Diego County, California-Phase One results, 19981999: U.S. Geological Survey Open-File Report 02-186, $134 \mathrm{p}$.

Maloney, T.J., ed., 2005, Quality management system, U.S. Geological Survey National Water Quality Laboratory: U.S. Geological Survey Open-File Report 2005-1263, variously paged, accessed November 8, 2010, at http://pubs.usgs.gov/ of $/ 2005 / 1263 /$.

Mendez, G.O., Foreman, W.T., Morita, Andrew, and Majewski, M.S., 2008, Water- and air-quality monitoring of the Sweetwater Reservoir watershed, San Diego County, California-Phase one results continued, 2001-2003: U.S. Geological Survey Data Series Report 347, 158 p.

Mendez, G.O., Foreman, W.T., Sidhu, J.S., and Majewski, M.S., 2007, Water- and air-quality monitoring of the Sweetwater Reservoir watershed, San Diego County, California-Phase One results, continued, 1999-2001: U.S. Geological Survey Data Series Report 233, 270 p.

Mueller, D.K., Martin, J.D., and Lopes, T.J., 1997, Qualitycontrol design for surface-water sampling in the National Water-Quality Assessment Program: U.S. Geological Survey Open-File Report 97-223, 17 p., http://pubs.usgs. gov/of/1997/223/.

National Institute of Standards and Technology, 1991a, Certificate of analysis standard reference material 2709San Joaquin Soil: National Institute of Standards and Technology.

National Institute of Standards and Technology, 1991b, Certificate of analysis standard reference material 2710 - Montana Soil: National Institute of Standards and Technology.

Noriega, M.C., Wydoski, D.S., and Foreman, W.T., 2004, Methods of analysis by the U.S. Geological Survey National Water Quality Laboratory-Determination of organophosphate pesticides and polychlorinated biphenyls in bottom and suspended sediment by gas chromatography with electron-capture detection: U.S. Geological Survey Water-Resources Investigations Report 03-4293, 46 p.

Ogden Environmental and Energy Services, 1997, SR 125 South Route alternatives-Potential air emissions impact on Sweetwater Reservoir: San Diego, Calif. 
Olson, M.C., Iverson, J.L., Furlong, E.T., and Schroeder, M.P., 2004, Methods of analysis by the U.S. Geological Survey National Water Quality Laboratory-Determination of polycyclic aromatic hydrocarbon compounds in sediment by gas chromatography/mass spectrometry: U.S. Geological Survey Water-Resources Investigations Report 03-4318, $45 \mathrm{p}$.

Pankow, J.F., Luo, Wentai, Bender, D.A., Isabelle, L.M., Hollingsworth, J.S., Chen, C., Asher, W.E., and Zogorski, J.S., 2003, Concentrations and co-occurrence correlations of 88 volatile organic compounds (VOCs) in the ambient air of 13 semi-rural to urban locations in the United States: Atmospheric Environment, v. 37, no. 36, p. 5023-5046, http://dx.doi.org/10.1016/j.atmosenv.2003.08.006.

Pankow, J.F., Luo, Wentai, Isabelle, L.M., Bender, D.A., and Baker, R.J., 1998, Determination of a wide range of volatile organic compounds (VOCs) in ambient air using multisorbent adsorption thermal desorption (ATD) and gas chromatography/mass spectrometry (GC/MS): Analytical Chemistry, v. 70, no. 24, p. 5213-5221.

Pirkey, K.D., and Glodt, S.R., 1998, Quality control at the U.S. Geological Survey, National Water Quality Laboratory: U.S. Geological Survey Fact-Sheet 026-98, 4 p., accessed November 12, 2010, at http://pubs.er.usgs.gov/usgspubs/fs/ fs02698.

Potts, P.J., Tindle, A.G., and Webb, Peter, 1992, Geochemical reference material compositions-Rocks, minerals, sediments, soils, carbonates, refractories, and ores used in research and industry: CRC Press Inc., Boca Raton, Fla., $313 \mathrm{p}$.

Sandstrom, M.W., 1995, Filtration of water-sediment samples for the determination of organic compounds: U.S. Geological Survey Water-Resources Investigations Report 95-4105, 13 p., accessed March 2003 at http://nwql.usgs. gov/Public/pubs/WRIR95-4105/WRIR95-4105.pdf.

Sandstrom, M.W., Stroppel, M.E., Foreman, W.T., and Schroeder, M.P., 2001, Methods of analysis by the U.S. Geological Survey National Water Quality LaboratoryDetermination of moderate-use pesticides and selected degradates in water by $\mathrm{C}-18$ solid-phase extraction and gas chromatography/mass spectrometry: U.S. Geological Survey Water-Resources Investigations Report 01-4098, 70 p., accessed March 2003 at http://nwql.usgs.gov/Public/ pubs/WRIR/WRIR-01-4098.pdf.
U.S. Environmental Protection Agency, 1999a, Compendium of methods for the determination of toxic organic compounds in ambient air, second edition, Compendium method TO-4A - Determination of pesticides and polychlorinated biphenyls in ambient air using high volume polyurethane foam (PUF) sampling followed by gas chromatography/multi-detector detection (GC/MD): Center for Environmental Research Information, Office of Research and Development, EPA/625/R-96/010b, 49 p., accessed March 2003 at http://www.epa.gov:80/ttnamtil/ files/ambient/airtox/to-4ar2r.pdf.

U.S. Environmental Protection Agency, 1999b, Compendium of methods for the determination of toxic organic compounds in ambient air, second edition, Compendium method TO-13A - Determination of polycyclic aromatic hydrocarbons (PAHs) in ambient air using gas chromatography/mass spectrometry (GC/MS): Center for Environmental Research Information, Office of Research and Development, EPA/625/R-96/010b, 78 p., accessed March 2003 at http://www.epa.gov/ttn/amtic/files/ambient/ airtox/to-13arr.pdf.

U.S. Geological Survey, variously dated, National field manual for the collection of water-quality data: U.S. Geological Survey Techniques of Water-Resources Investigations, book 9, chaps. A1-A9, accessed February 2, 2011, at http:// water.usgs.gov/owq/FieldManual/.

U.S. Geological Survey, variously dated, USGS Inorganic Blind Sample Project-Data quality assessment summaries, September 2004 to August 2009: Branch of Quality Systems, accessed March 15, 2011, at http://bqs.usgs.gov/ ibsp/qadata.shtml.

Zaugg, S.D., Sandstrom, M.W., Smith, S.G., and Fehlberg, K.M., 1995, Methods of analysis by the U.S. Geological Survey National Water Quality Laboratory-Determination of pesticides in water by $\mathrm{C}-18$ solid-phase extraction and capillary-column gas chromatography/mass spectrometry with selected-ion monitoring: U.S. Geological Survey Open-File Report 95-181, 49 p., http://nwql.usgs.gov/ Public/pubs/OFR95_181.pdf.

Zaugg, S.D., Smith, S.G., Schroeder, M.P., Barber, L.B., and Burkhardt, M.R., 2002, Methods of analysis by the U.S. Geological Survey National Water Quality LaboratoryDetermination of wastewater compounds by polystyrenedivinylbenzene solid-phase extraction and capillary-column gas chromatography/mass spectrometry: U.S. Geological Survey Water-Resources Investigations Reports 01-4186, 37 p., http://nwql.usgs.gov/Public/pubs/WRIR01-4186.html. 
This page intentionally left blank. 


\section{Appendix}

The appendix includes quality-control (QC) tables for the water, air, and surficial bed sediment analyzed as part of the data collected for the Sweetwater Reservoir (SWR) project. The QC associated with the water sampling is listed in tables A1A-A6C. The QC associated with the air sampling is listed in tables B1A-B6C. The QC associated with the bed-sediment sampling is listed in tables $\mathrm{C} 1-\mathrm{C} 3 \mathrm{C}$. Replicate samples for volatile organic compounds (VOCs) and metals (targeted compounds) were evaluated by calculating the percentage difference from the environmental sample. 


\section{Appendix A. Water-Quality Control}

At least one field quality-control (QC) sample type was collected each time the reservoir was sampled. The type of QC sample collected and the sampling location were chosen randomly. The matrix-spike QC data for the volatile organic compounds (VOCs) in water are given in table A1A. The blank and replicate QC data for VOCs are given in table A1B. The replicate data are compared to the environmental samples in table A1C. In six matrix spikes and three matrix-spike recoveries, recoveries were adequate for all the VOCs except the following; carbon disulfide and dichlorodifluoromethane were below the acceptable criteria for about one-third of the samples; and trichloromethane were below the acceptable criteria for more than half of the samples. Of the VOCs detected in the environmental samples, acetone and 2-butanone were detected at estimated concentrations below the laboratory reporting level (LRL) in one of three field blanks; acetone had 35 detections in environmental water samples and 2-butanone had 2 detections. Carbon disulfide was detected in three of the four equipment blanks at site SWR03 and in some environmental water samples. Trichloromethane was detected in one of the three field blanks and in a source solution blank collected the same day as the field blank sample. No compounds were detected in the trip blank at site SWR03. In 14 replicate samples, VOC concentrations were within the acceptable criteria $( \pm 30$ percent of environmental sample) except for the following samples; trichloromethane at site SWR06 in July 2004; six compounds (benzene, ethylbenzene, toluene, 1,2,4-trimethylbenzene, $m$ - and $p$-xylene, and $o$-xylene) at site SWR06 in July 2006; and toluene at site LFDD in October 2006. No replicate comparison was calculated if either the environmental or replicate compound was not detected.

The matrix-spike and replicate QC data for base neutral acid compounds (BNAs) are given in tables A2A and $\mathrm{A} 2 \mathrm{~B}$, respectively. One matrix-spike sample was analyzed for BNAs. Two compounds (di- $n$-butyl phthalate and hexachlorobutadiene) in that sample were not within the acceptable criteria of \pm 30 percent. Di- $n$-butyl phthalate was detected in ten water samples and hexachlorobutadiene was not detected in any water samples. Concentrations of all compounds detected in the three BNA replicate samples were estimated and within the acceptable criteria when a replicate comparison could be calculated, except for phenol in a sample collected from site SWR09 on April 13, 2005. The replicate QC data for pesticides (National Water Quality Laboratory schedules 2003 and 2060) are given in tables A3 and A4, respectively. Most replicate data for pesticides were within the acceptable criteria when a replicate comparison could be calculated, except for caffeine for a sample from site SWR01 on July 12, 2005. No blanks were processed for BNA compounds or pesticides.
No matrix spike or blank samples were processed for anthropogenic organic indicator (AOI) compounds. One replicate sample was collected for AOI compounds (table A5); no AOI compounds were detected in either the environmental or replicate sample.

The matrix-spike QC data for metals in unfiltered water samples are given in table A6A. Two spikes and one spike replicate had recoveries above the acceptable criteria. Metals with recoveries above the acceptable criteria (greater than 30 percent) in three spike and two spike replicates were aluminum, chromium, lithium, selenium, and strontium. Most matrix-spike replicates are within \pm 15 percent of spike data. The replicate and field blank data for metals in unfiltered water samples are given in table A6B. Four metals (molybdenum, silver, thallium, and uranium) were detected in blank samples at site SWR08, but all detections were below the LRLs. Silver was the only metal detected in one additional blank at concentrations just above the LRL. During this reporting period, 12 replicate samples (table A6C) were collected for metals; 7 of the 19 compounds analyzed had 5 or less replicates outside the acceptable criteria.

\section{Appendix B. Air Quality Control}

Replicate volatile organic compound (VOC) air samples collected for high-volatility (14 replicates) and medium/lowvolatility (11 replicates) are presented in tables B1A and B1B, respectively. The VOC replicate air data were compared to the environmental air data in table $\mathrm{B} 1 \mathrm{C}$; no high-volatility VOCs were detected in replicate or associated environmental samples. Twenty-seven compounds for medium/low volatility VOCs had at least one replicate value above the acceptable criteria, and Twenty-two compounds had at least one replicate value below the acceptable criteria. A travel spike was sent with about one-third of the VOC air samples and a travel, lot, and laboratory blank were associated with each VOC air sample collected. For high-volatility VOCs, the travel spike and travel, lot, and laboratory blanks are presented in tables B2A, B2B, B2C, and B2D, respectively. Thirty-seven spikes were analyzed for high-volatility VOCs; chloromethane had one spike recovery above the acceptable criteria and several spike recoveries below the acceptable criteria. Chloroethene and chloroethane also had recoveries below the acceptable criteria, althrough no recovery was below 59 percent. No high-volatility VOCs were detected in travel, lot, or laboratory blanks. For medium/low-volatility VOCs, the travel spike and travel, lot, and laboratory blanks are presented in tables B3A, $\mathrm{B} 3 \mathrm{~B}, \mathrm{~B} 3 \mathrm{C}$, and $\mathrm{B} 3 \mathrm{D}$, respectively. In 36 travel spikes analyzed for 80 medium/low-volatility VOCs, 5 compounds had 1 recovery above the acceptable criteria, and 17 compounds had at least 1 recovery below the acceptable criteria. Of the 80 medium/low-volatility VOCs, 18 were detected in travel and lot blanks, and 17 were detected in laboratory blanks. Acetone, benzene, and toluene were detected in most blank samples of each type. 
The associated laboratory reagent spike and laboratory blanks quality-control (QC) data for the polycyclic aromatic hydrocarbons (PAHs) in air samples are presented in tables B4A and B4B, respectively. Seven sets of PAH spikes were analyzed for the glass-fiber filter (GFF) and polyurethane foam (PUF). Typically, the PUFs had better recoveries than the GFFs. PAHs in the air had no recoveries above the acceptable criteria, but many recoveries were below the acceptable criteria. Of the 17 laboratory PAH samples analyzed, 6 compounds had 2 or more detections, although most blank detections were an order of magnitude lower than the laboratory reporting level (LRL). There were no detections in the two PAH field blanks. The laboratory matrixspike and laboratory blank data for pesticides in air samples (method Airpest003) are presented in tables B5A and B5B, respectively. Eight compounds had one spike recovery above acceptable criteria for Airpest 003 . About 26 percent of spike recoveries for Airpest003 were below the acceptable criteria; four compounds had at least one spike with no recovery. The low spike recoveries may indicate the compound was underdetected and may be reported at lower concentrations than occur. Pesticides were not detected in the six blank samples. Replicate air samples for semivolatile organic compounds (SOCs) were not collected during this reporting period.

Ten laboratory-reagent spike QC samples of standard reference materials for metals in air are presented in tables B6A. Antimony and bismuth were the only compounds with one or more spike recoveries below the acceptable criteria. Five compounds (cadmium, chromium, lead, lithium, and niobium) had one or more recoveries above the acceptable criteria. The spike sample from October 9, 2006, to March 14, 2007, was unusable because of equipment problems. Results from 10 laboratory blank samples and 1 field blank for 32 metals are presented in table B6B. Arsenic, bismuth, thallium, and titanium had no detections in the laboratory blank samples. Caesium had detections in laboratory blanks below the LRL; all other compounds have laboratory blank detections above their LRL. Silver and uranium had a higher detections in the field blank than in environmental samples. One blank replicate sample for metals was analyzed (table B6C), and two compounds are outside the acceptable criteria (cobalt and niobium).

\section{Appendix C. Surficial Bed-Sediment Quality Control}

Replicate data collected for polycyclic aromatic hydrocarbons (PAH's) and organochlorine (OC) and polychlorinated biphenyl compounds in surficial bed-sediment samples are presented in tables $\mathrm{C} 1$ and $\mathrm{C} 2$, respectively. One replicate sample for PAHs was collected during each of the 2004 and 2009 sampling periods. Nine compounds were detected in the $2004 \mathrm{PAH}$ replicate sample; four compounds were detected above 130 percent of the environmental sample. Four compound were detected below 70 percent of the environmental sample. Eleven compounds were detected in the 2009 PAH replicate sample, of which three were detected above 130 percent of the environmental sample. Six compounds were detected below 70 percent of the environmental sample. The one replicate sample collected for OCs during the 2009 sampling event had no detections.

Spike and replicate data collected for metals in surficial bed-sediment samples are presented in tables $\mathrm{C} 3 \mathrm{~A}$ and $\mathrm{C} 3 \mathrm{~B}$, respectively. All matrix-spike results for reference material NIST-2709 were within the acceptable criteria. Matrix-spike results for reference materials SO-2 and SO-4 were mostly within acceptable criteria except niobium (SO-2 recovery of 173 percent), antimony (SO-2 and SO-4 recoveries of 52 and 66 percent, respectively), and uranium (SO-2 recovery of 69 percent). Quality control replicate comparison of metals are listed in table $\mathrm{C} 3 \mathrm{C}$. Of the 36 metals analyzed in a replicate sample in 2004, only antimony (130 percent) had a recovery above the upper acceptable criteria. Of the 36 metals analyzed in the 2009 replicate sample, only beryllium (58.6 percent) had a recovery below the acceptable criteria. 


\section{Appendix A Tables.}




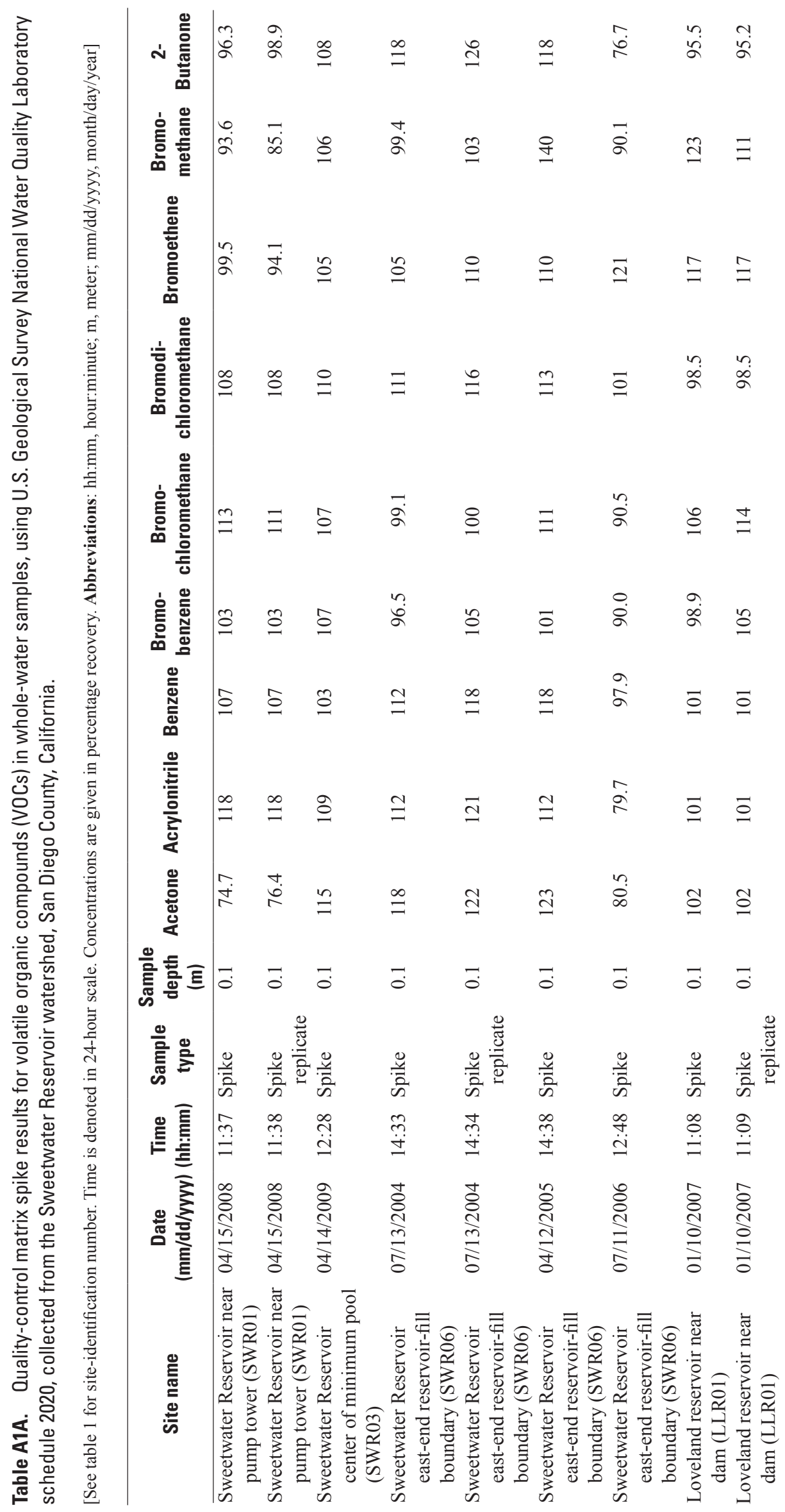




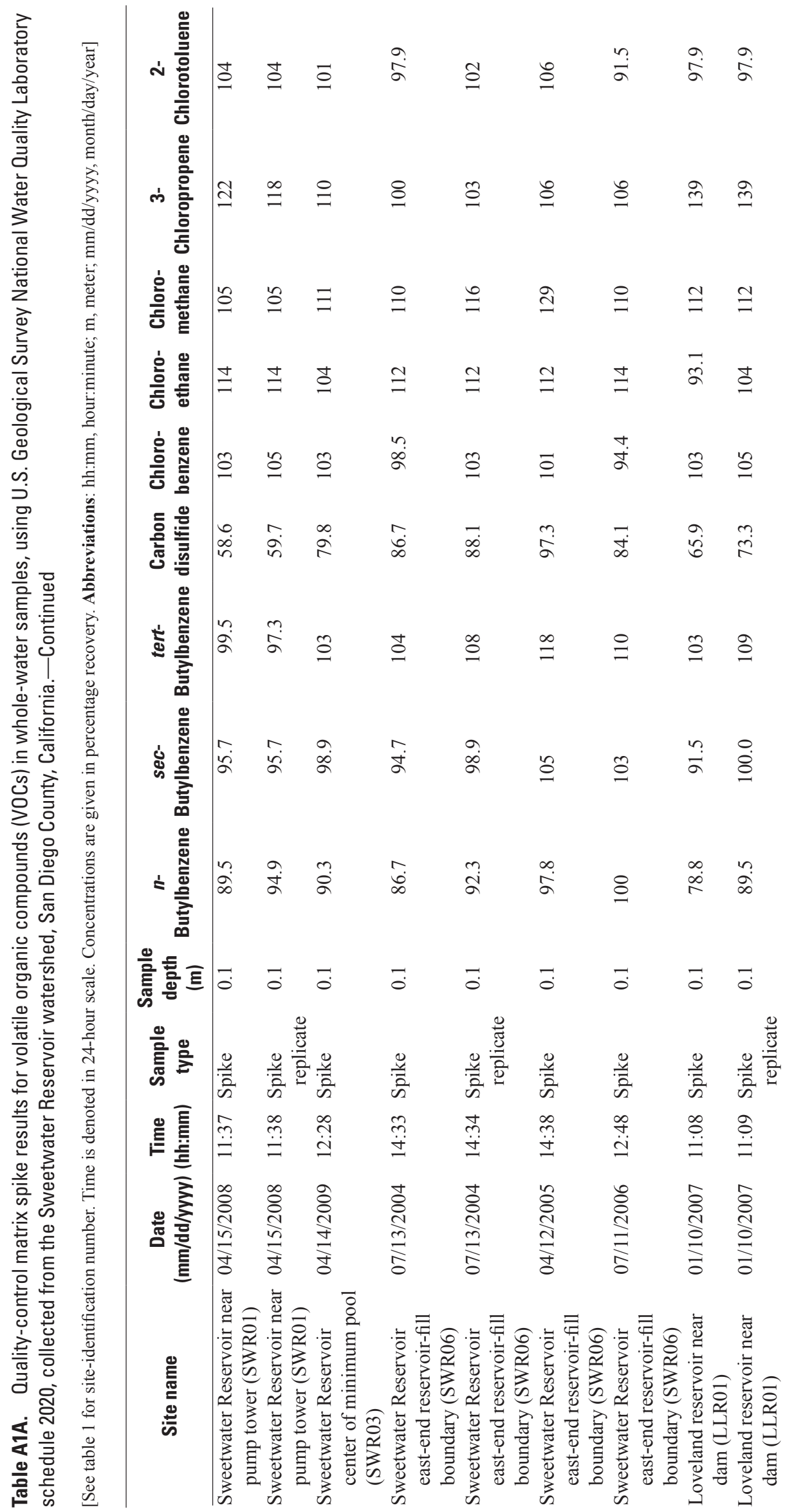




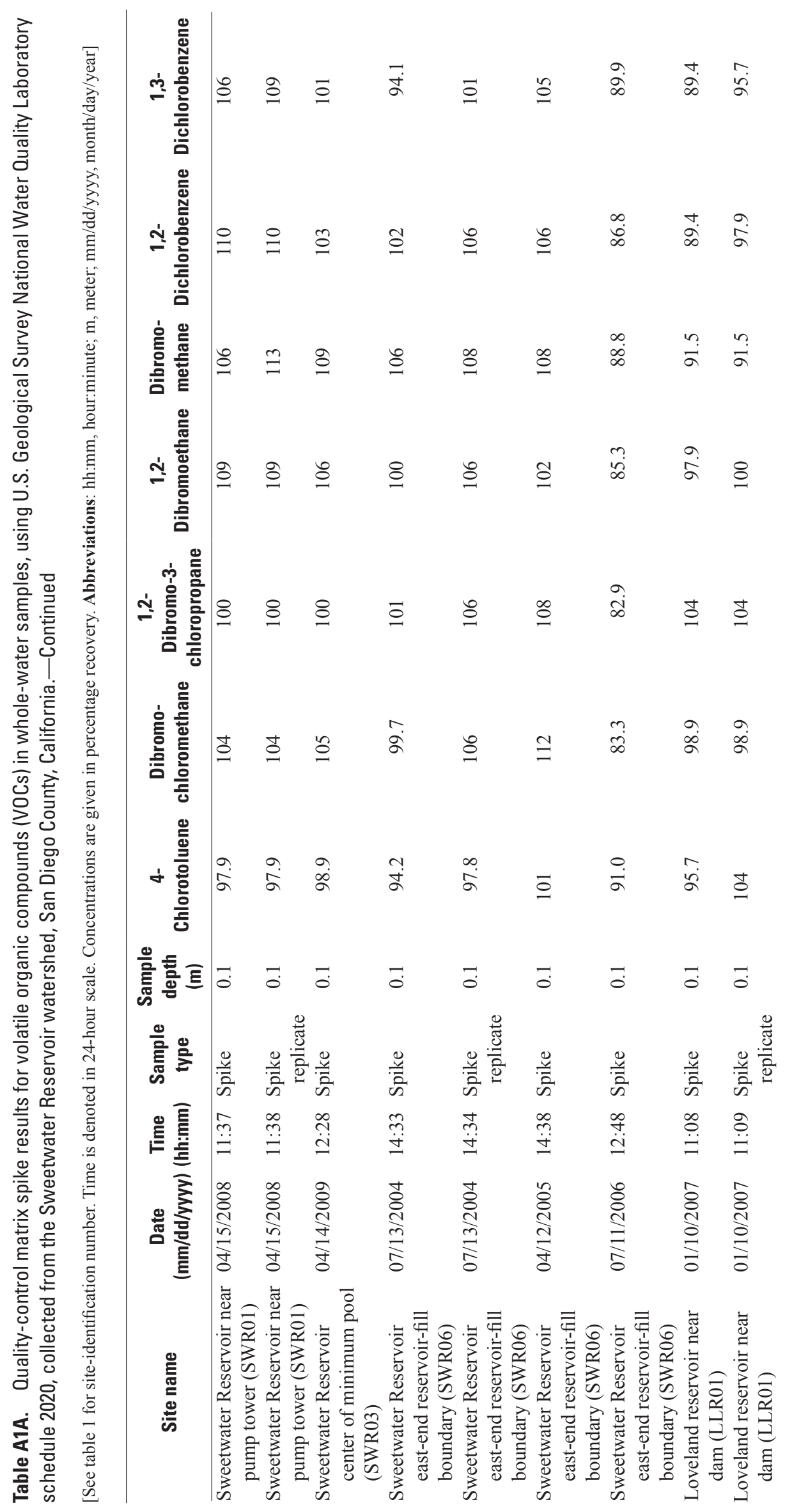




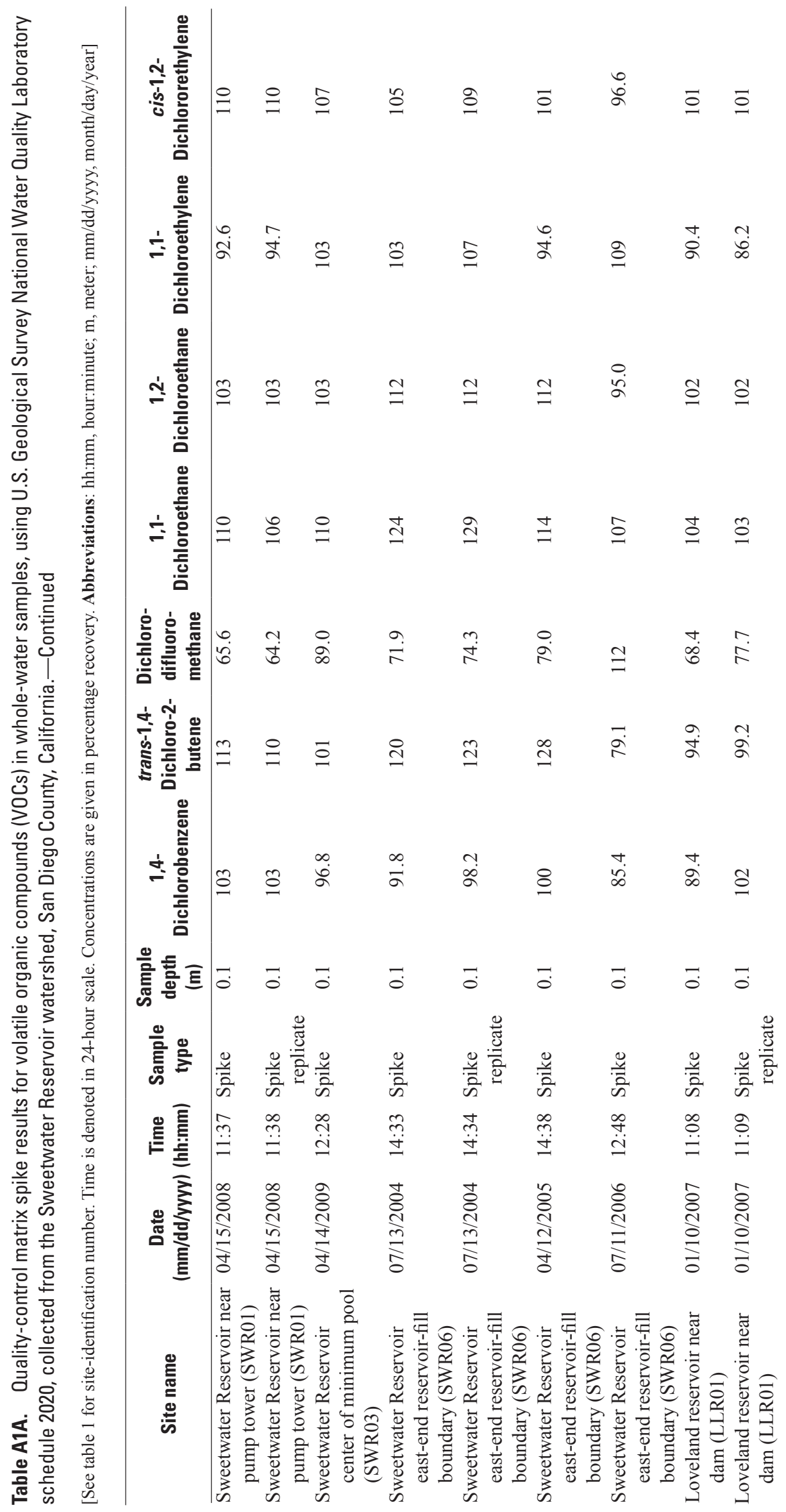




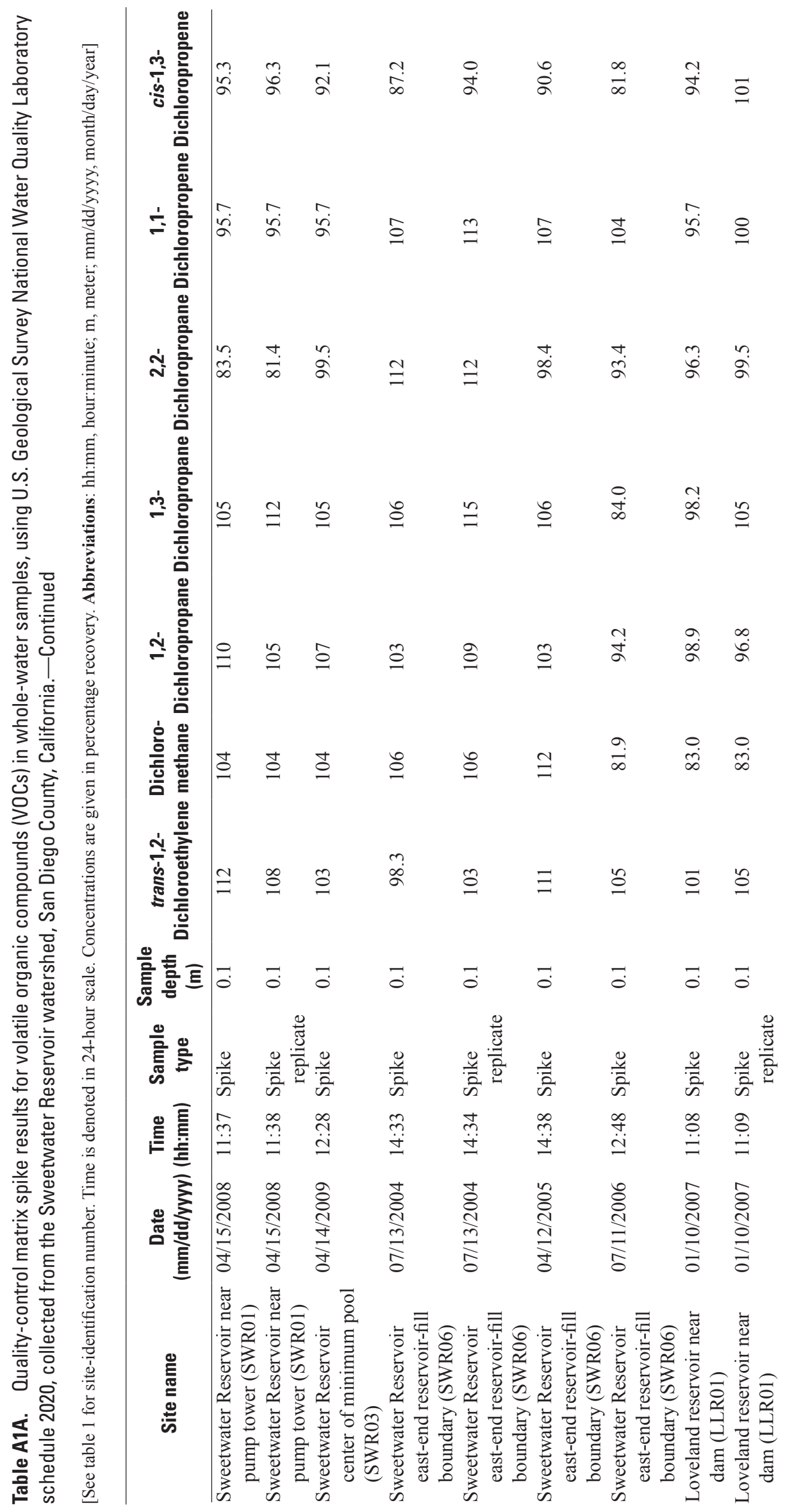




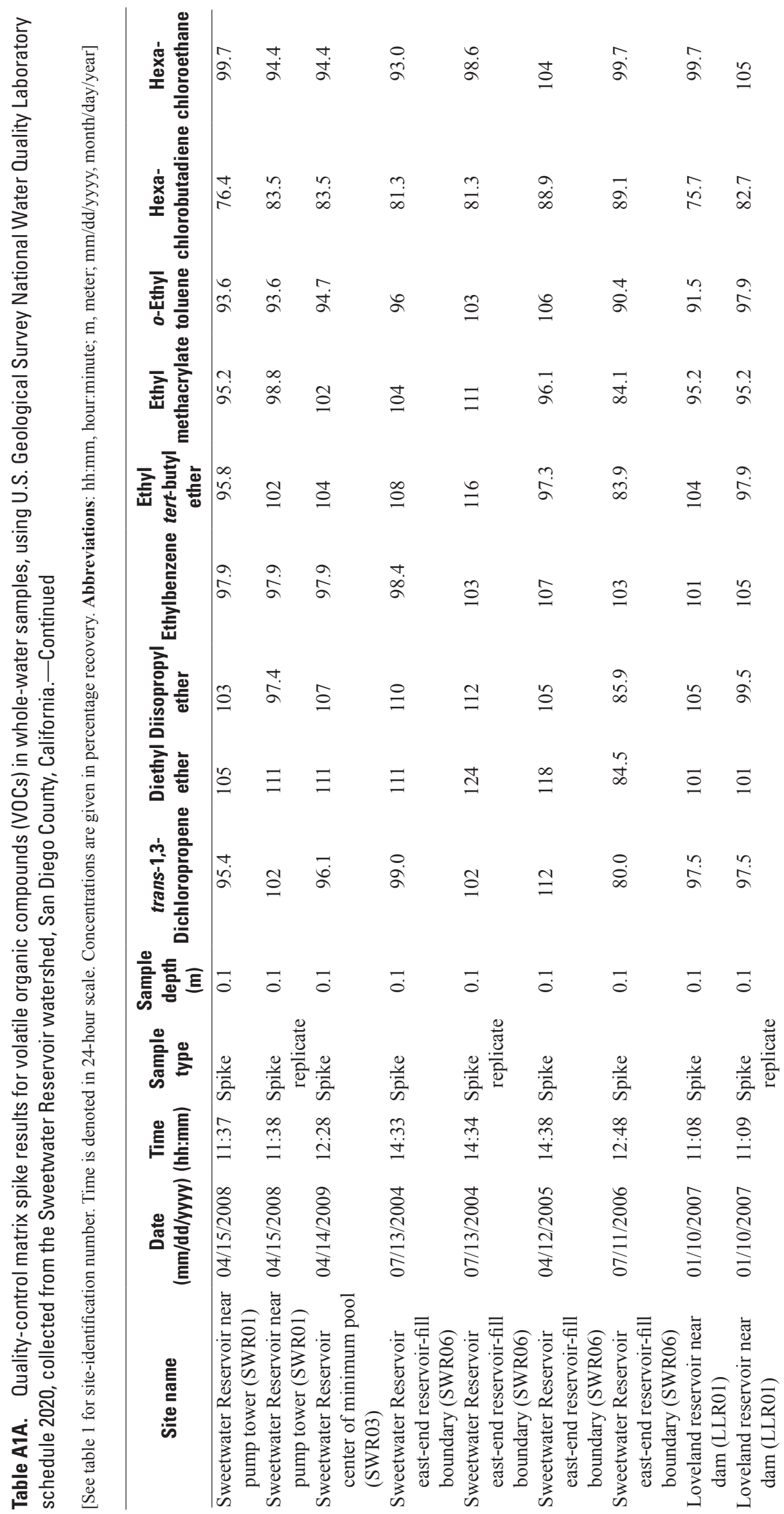




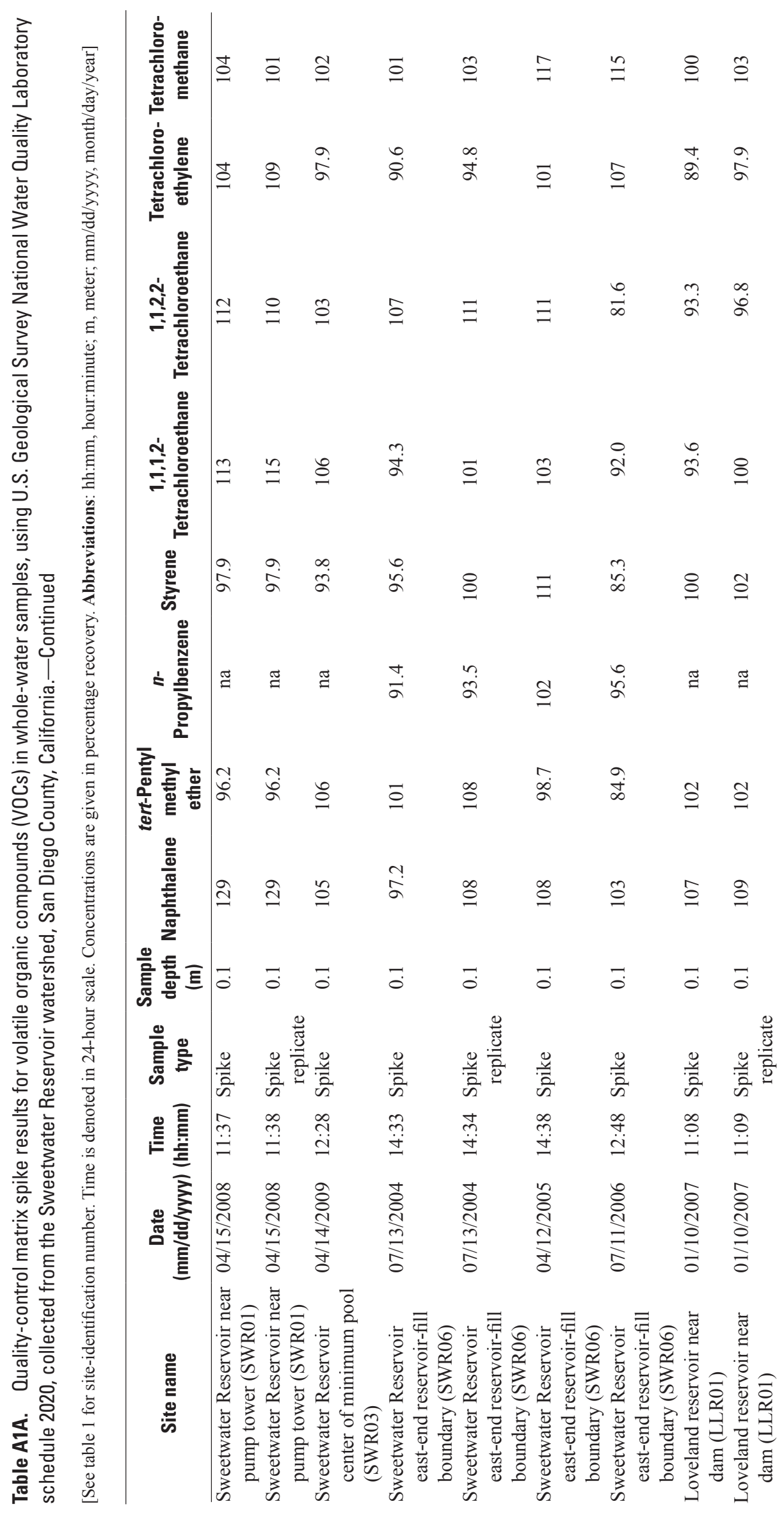




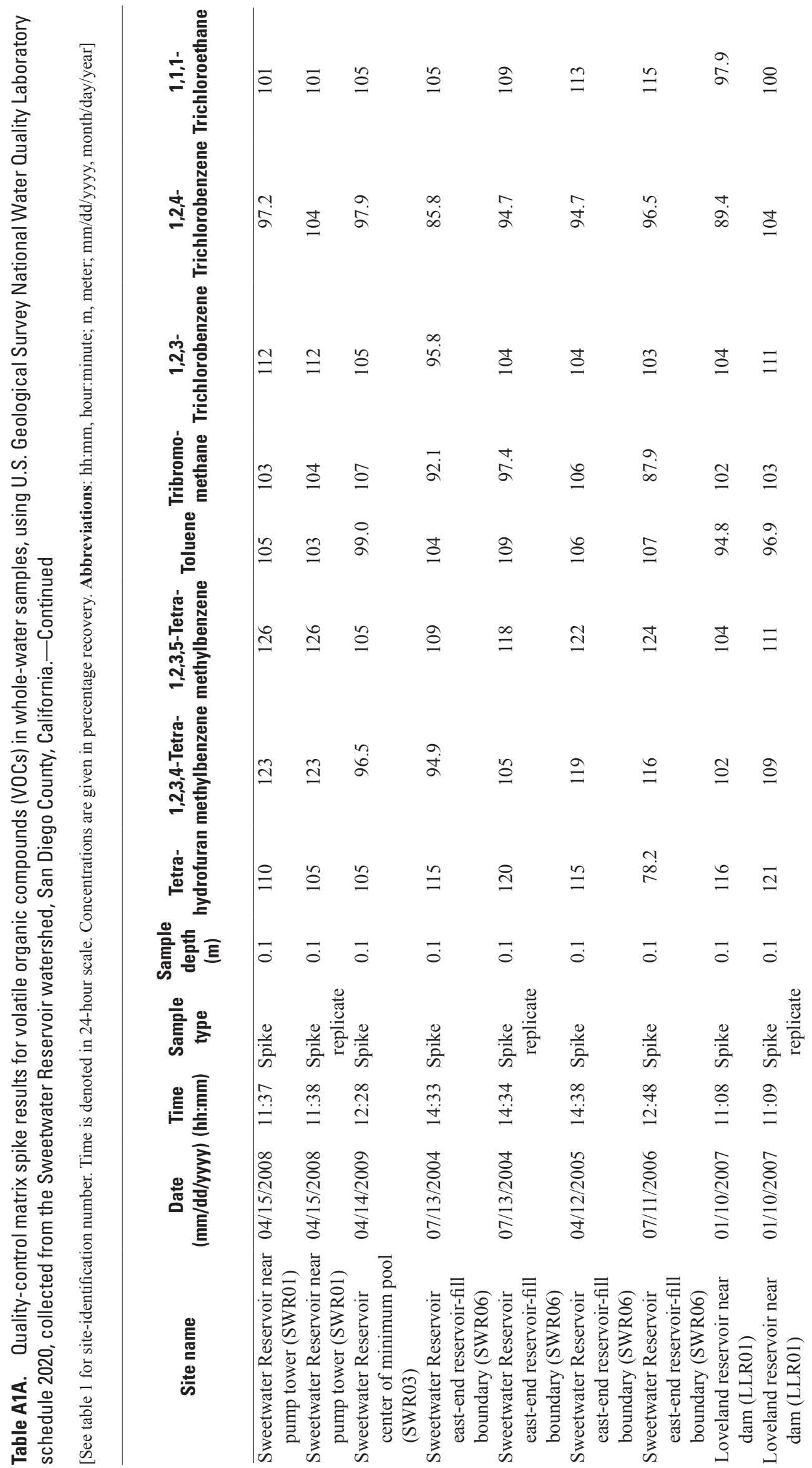




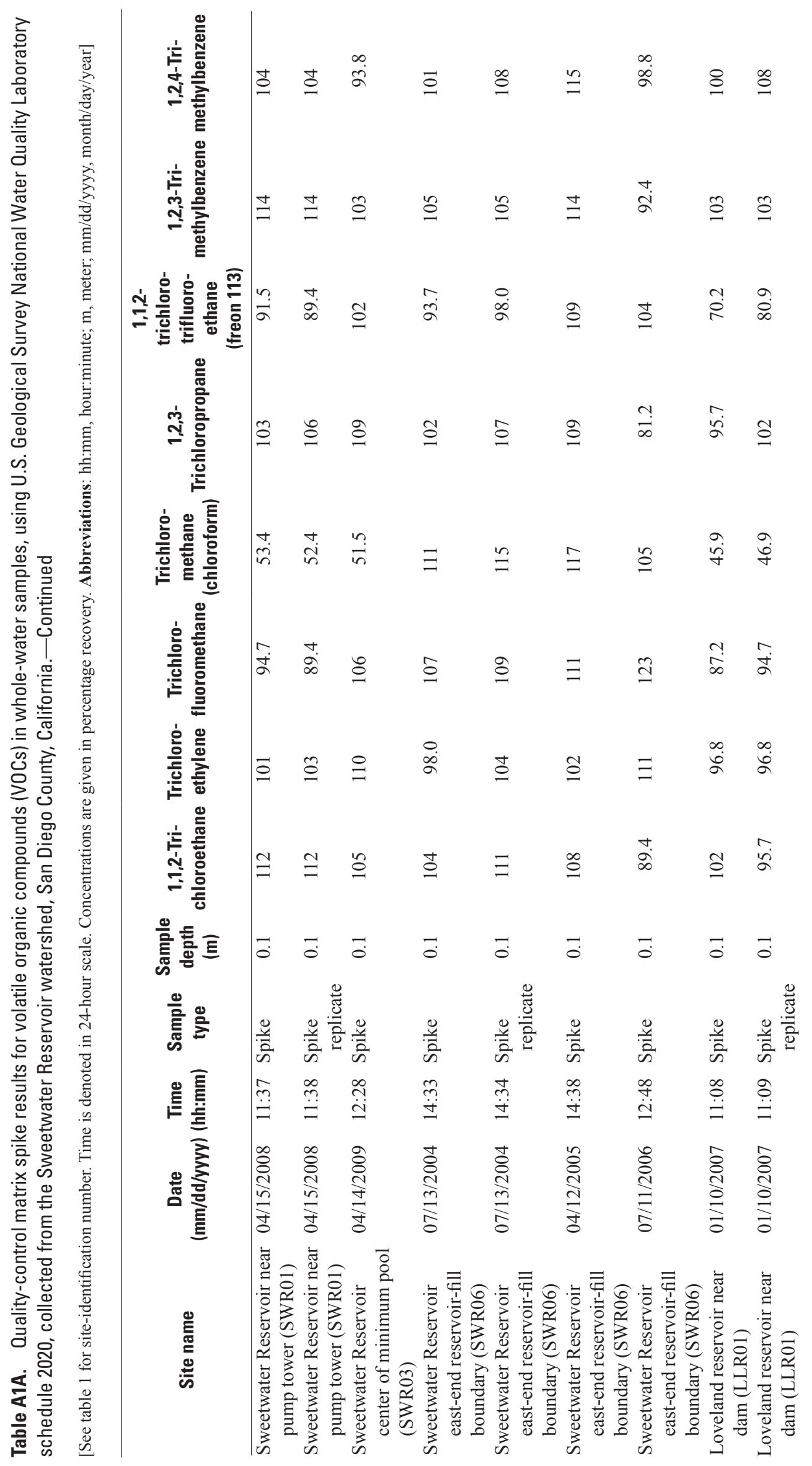




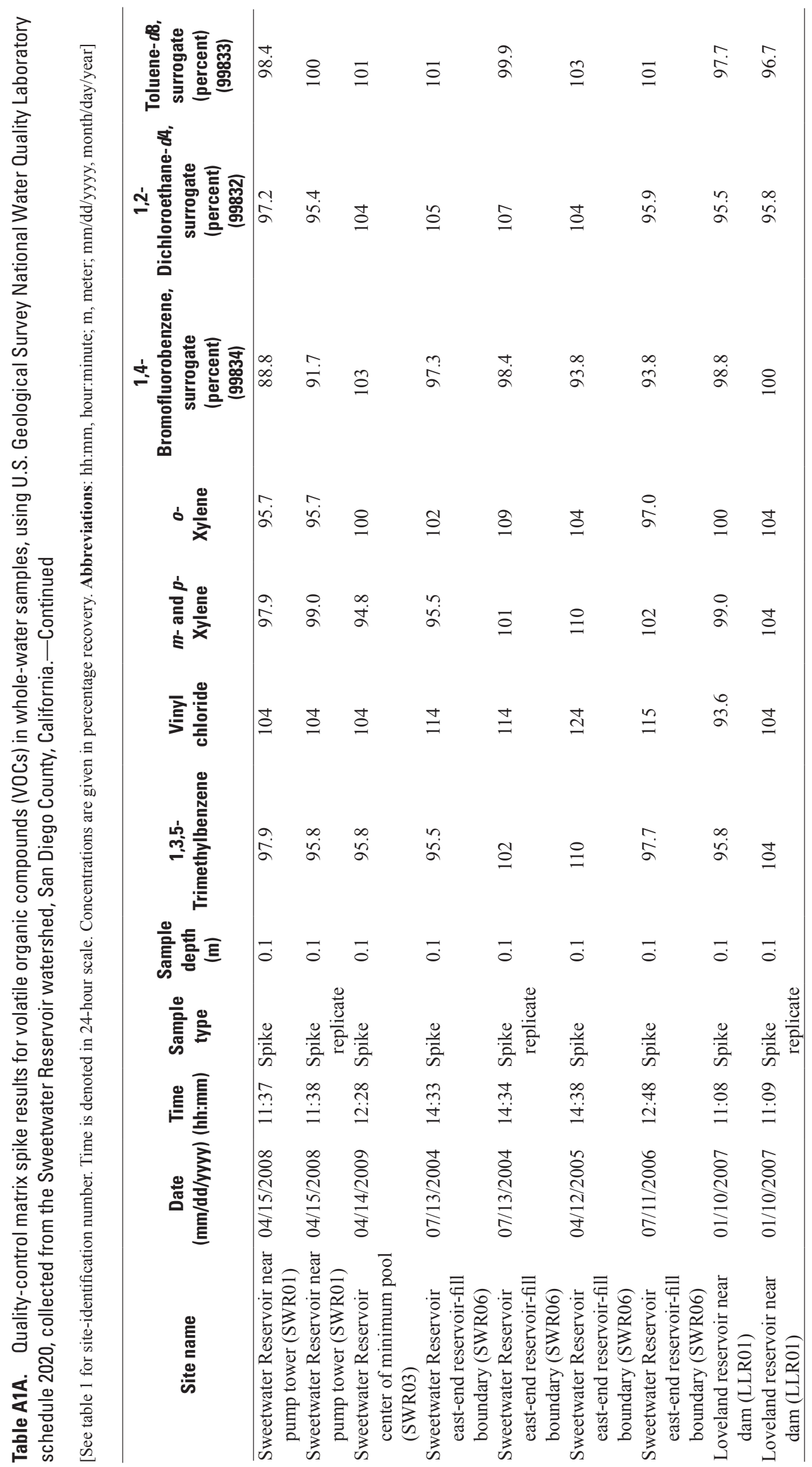




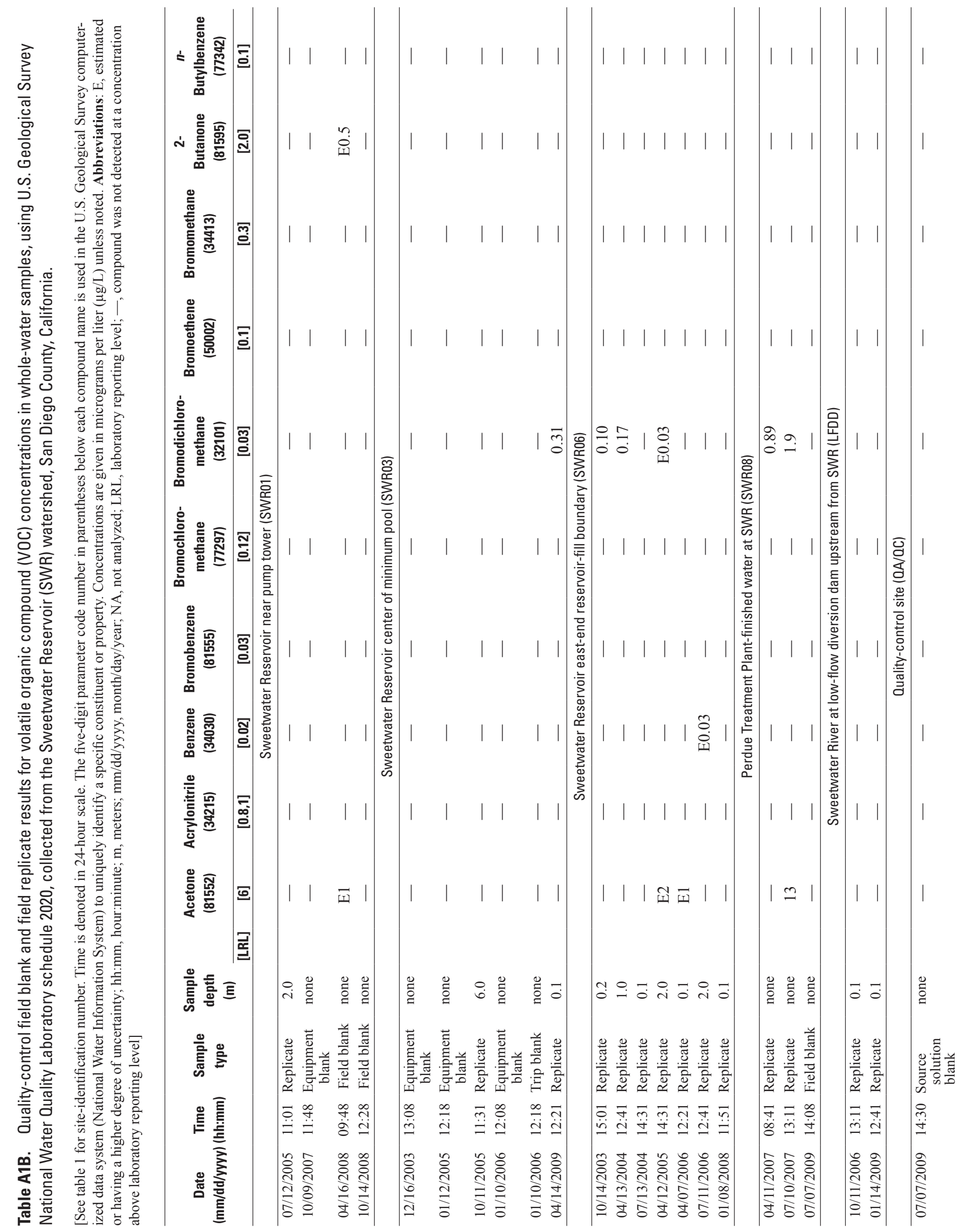




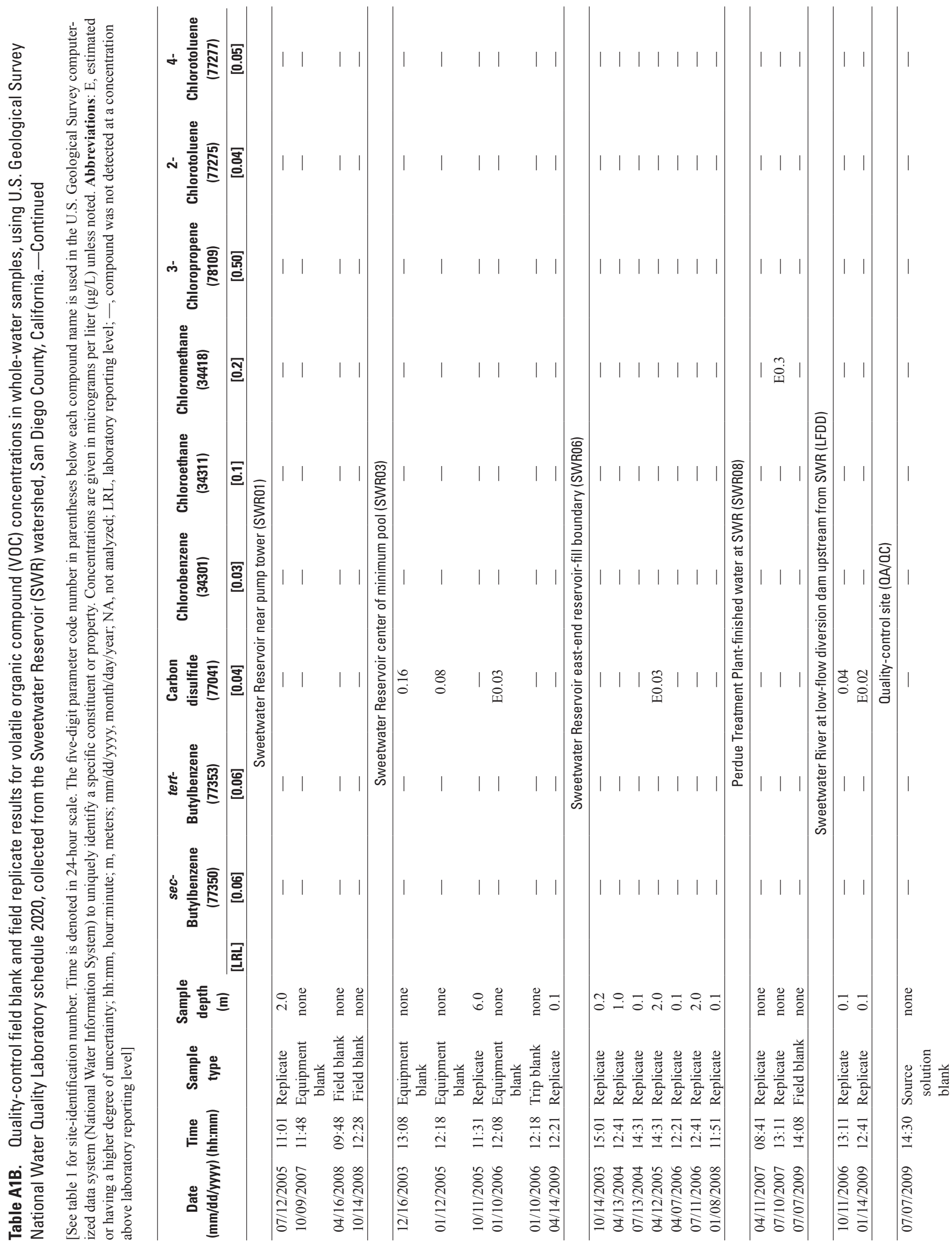




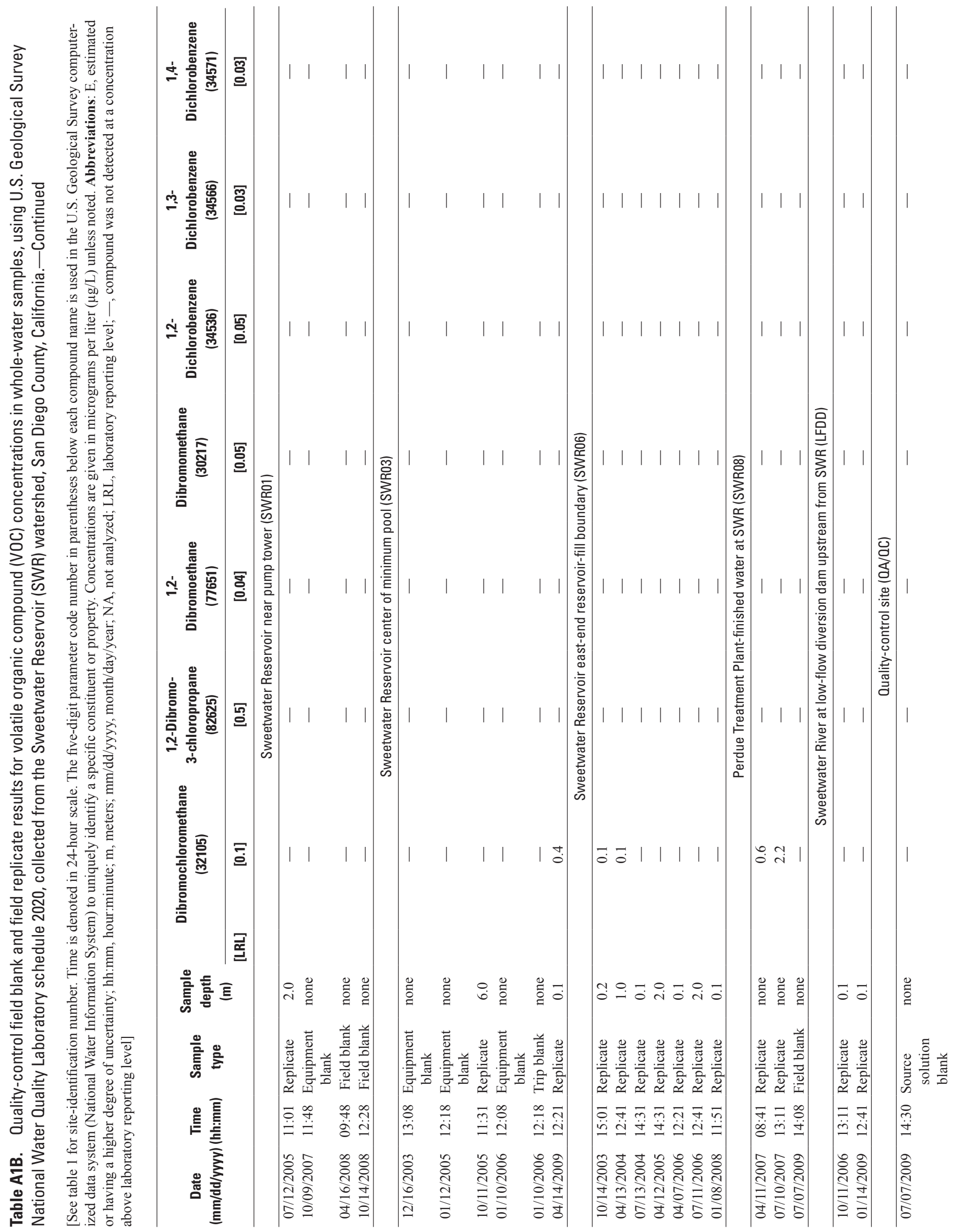




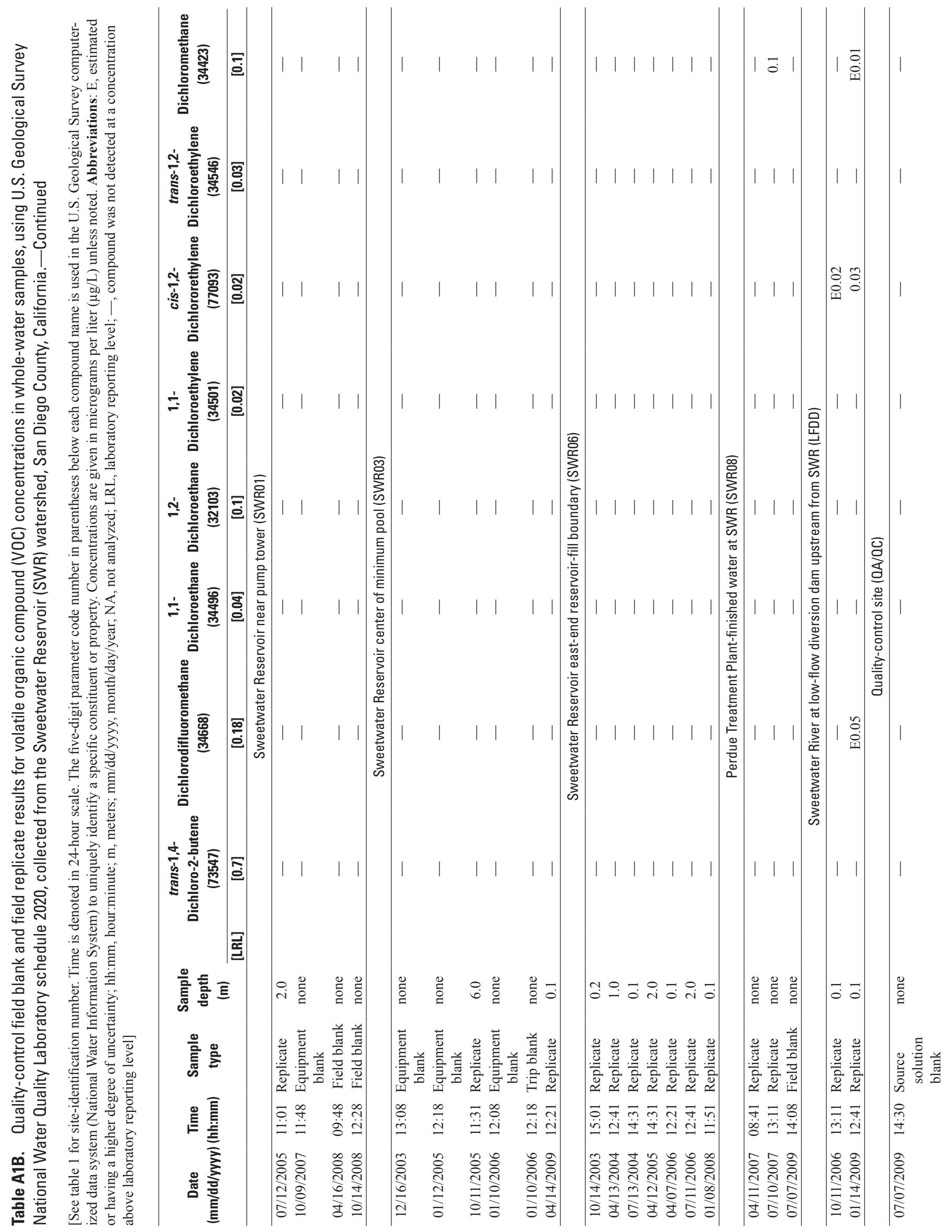




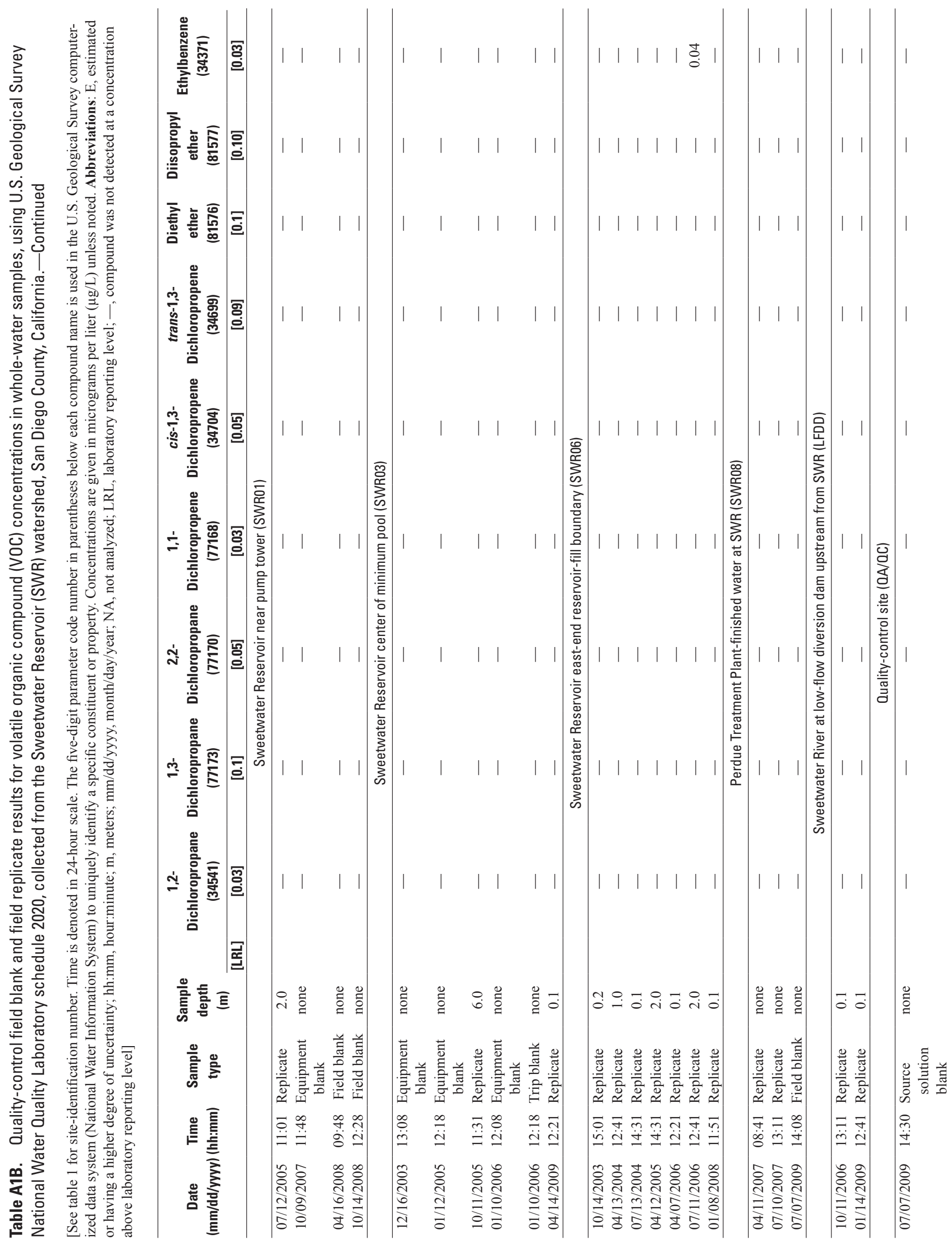




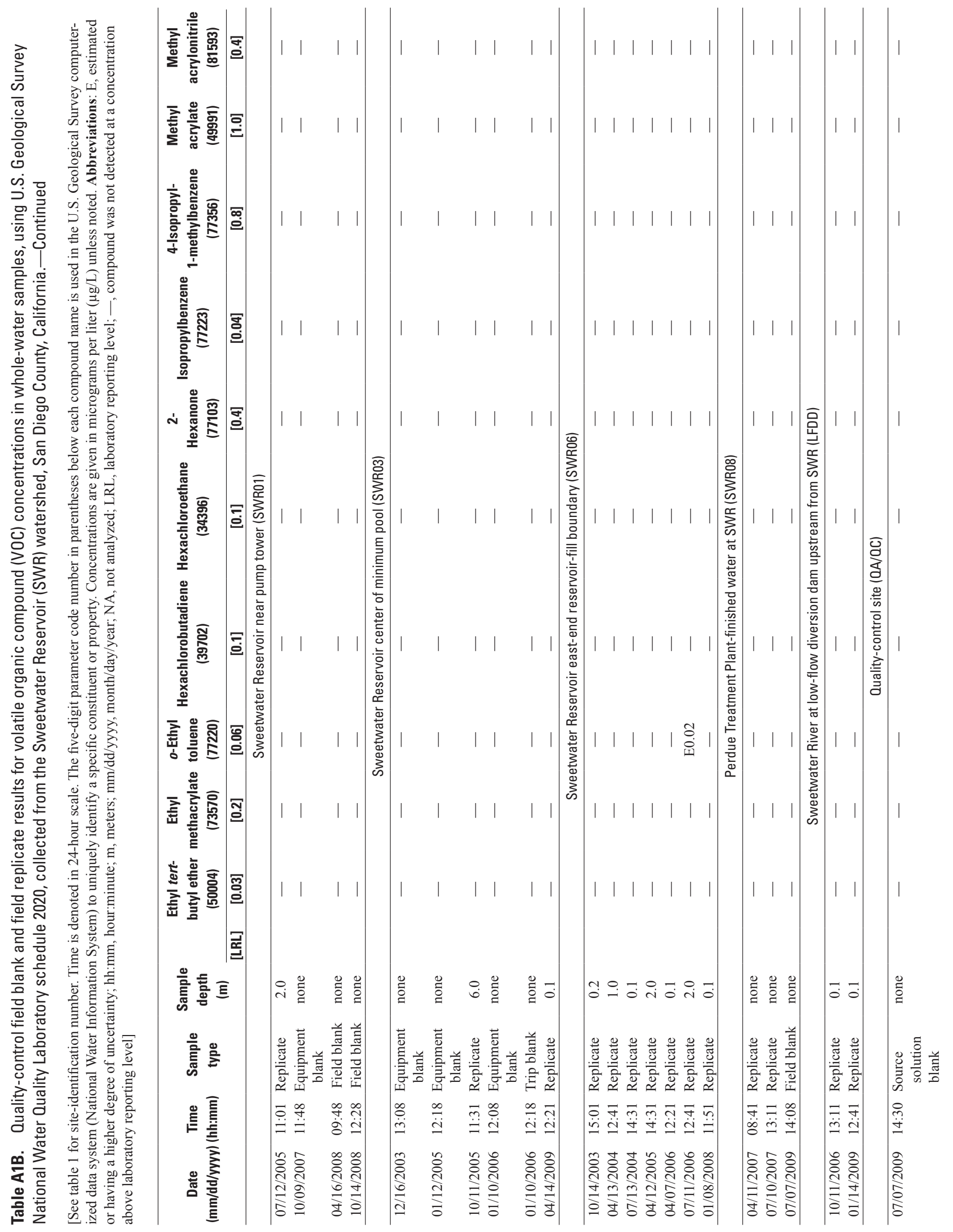




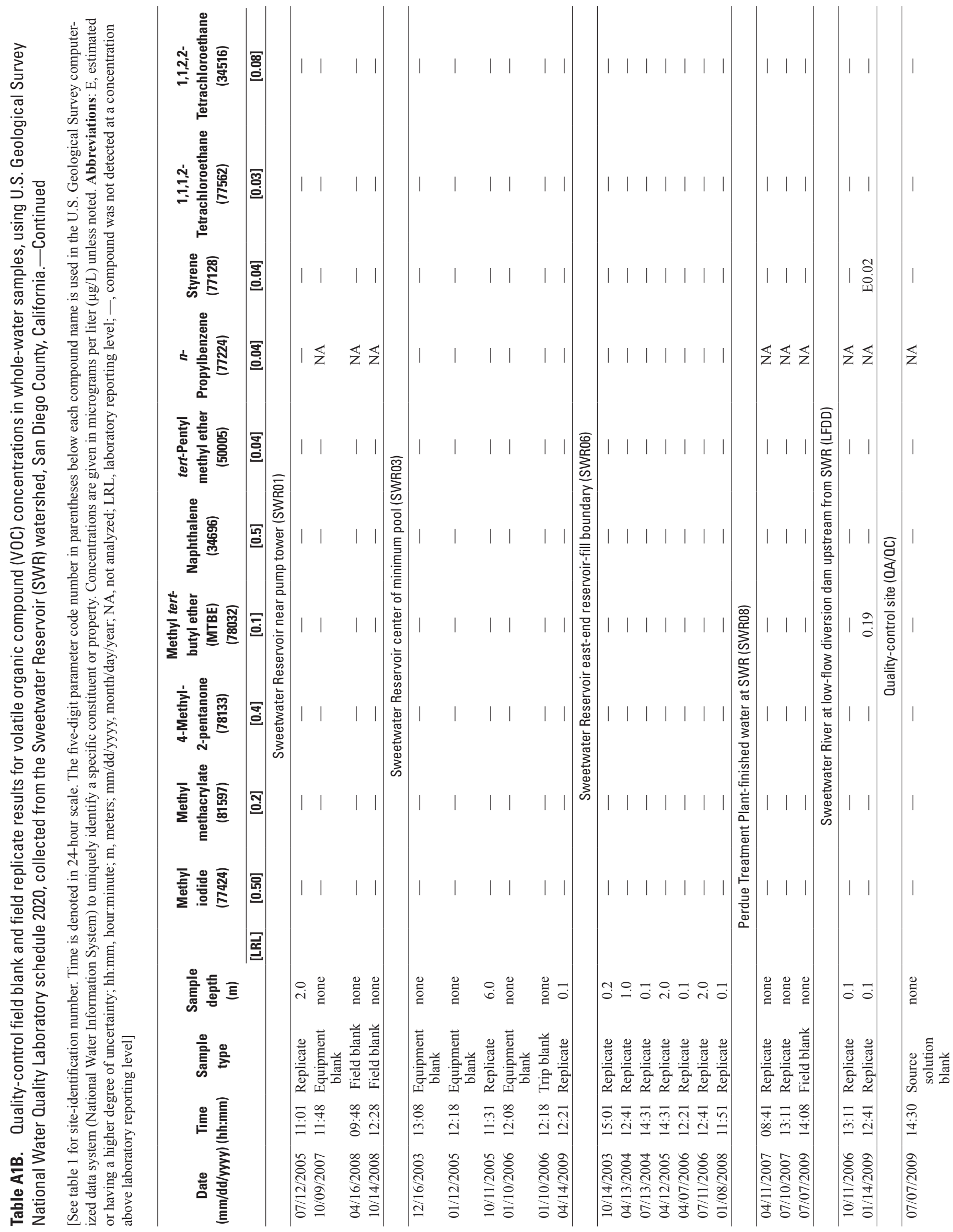




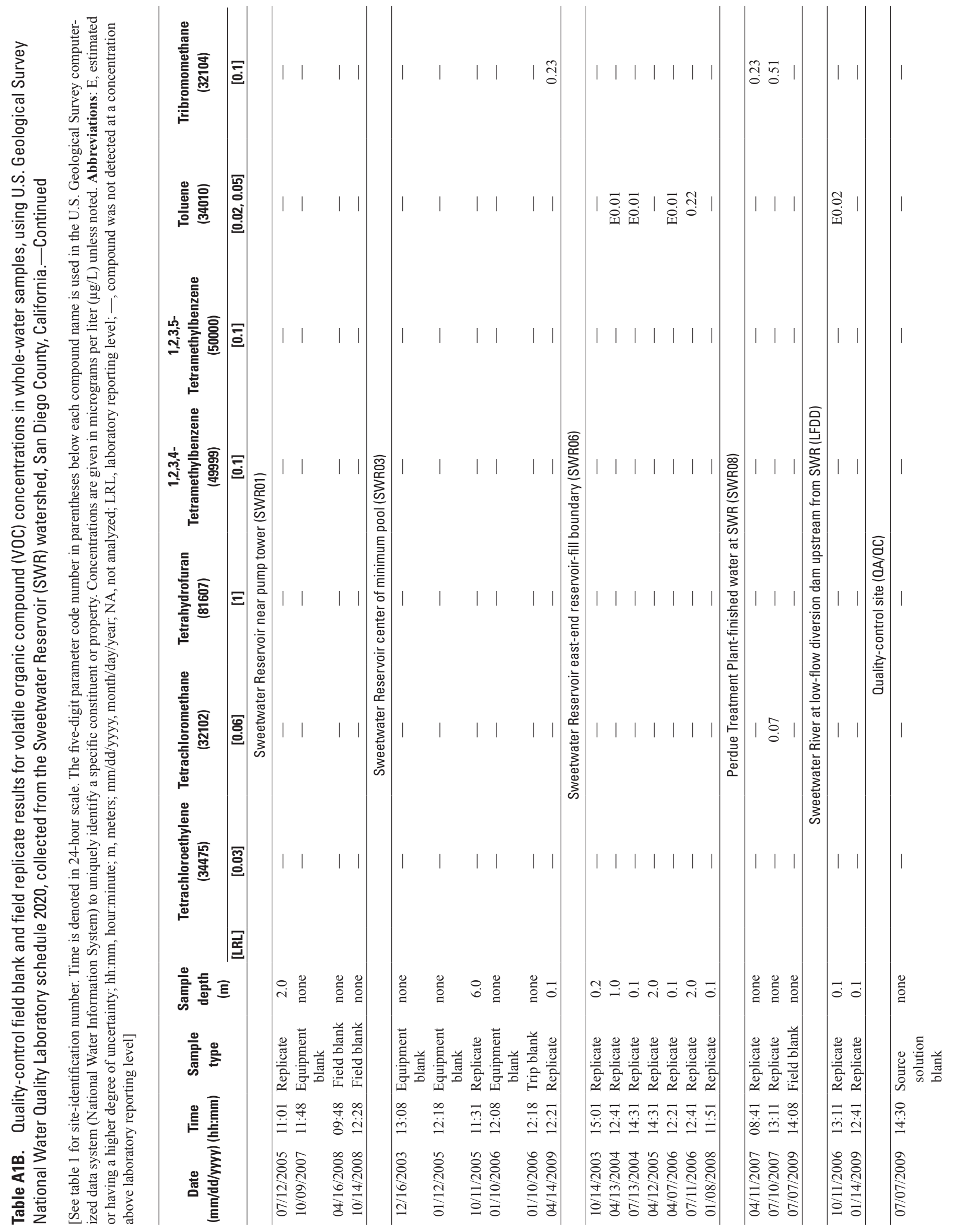




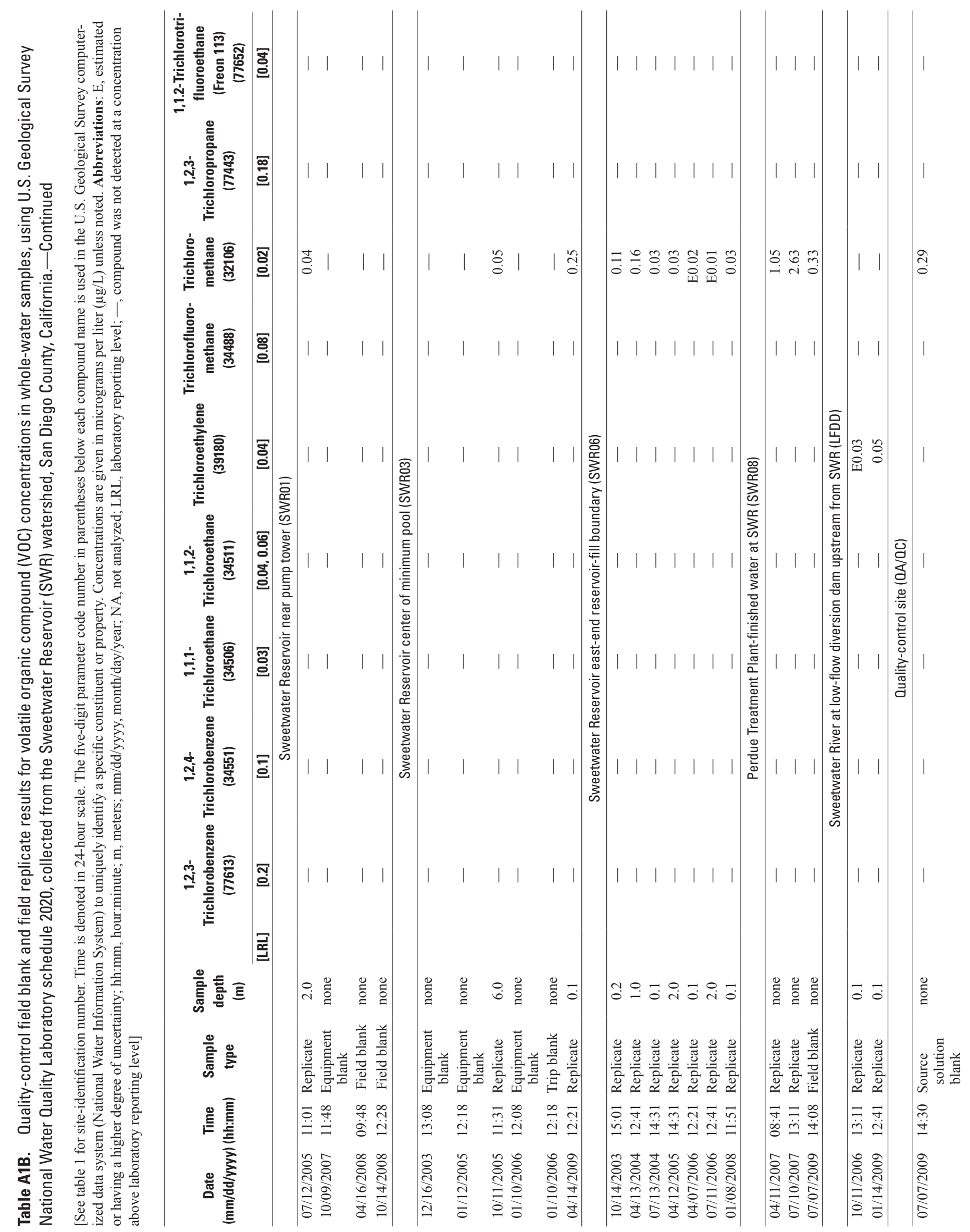




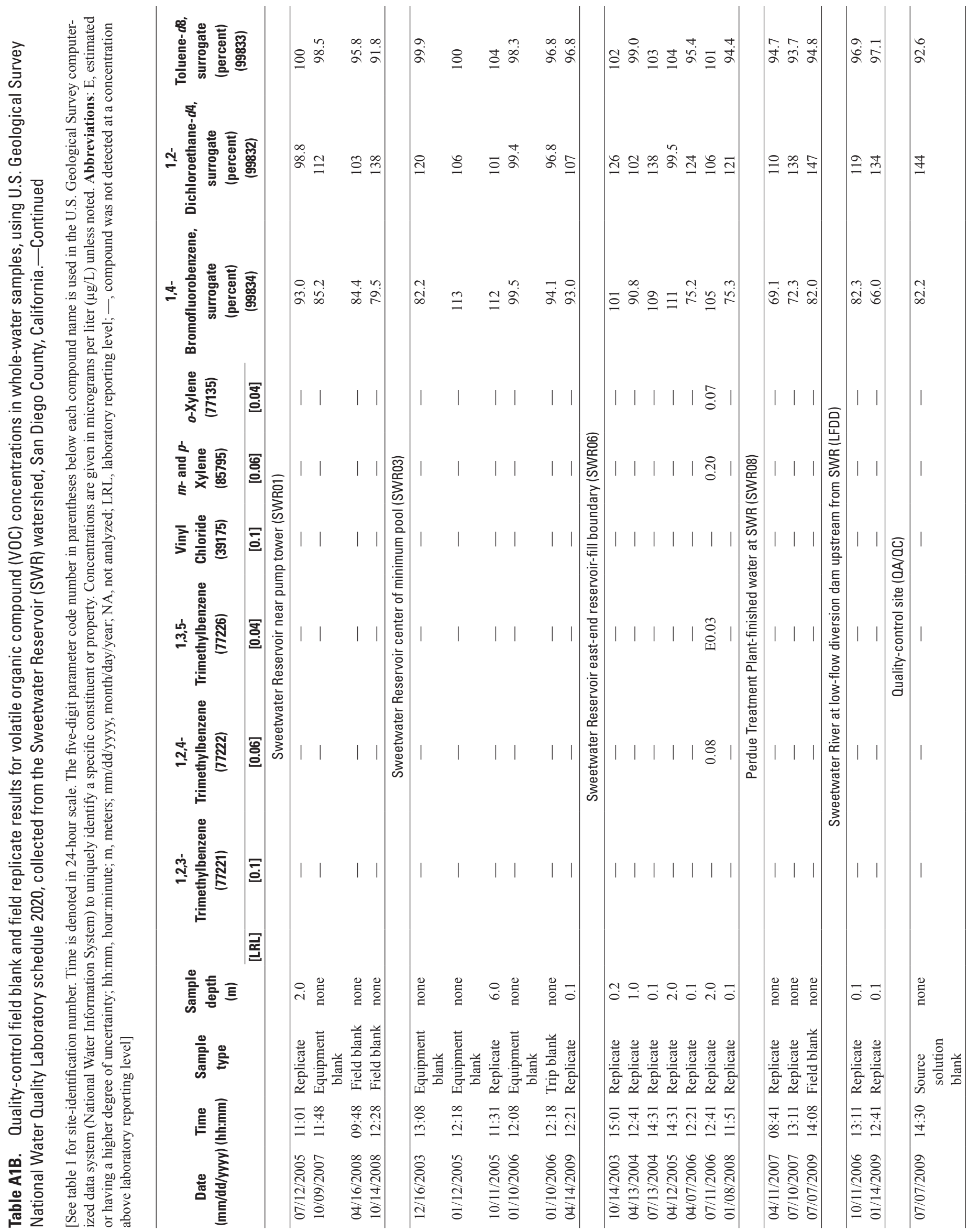




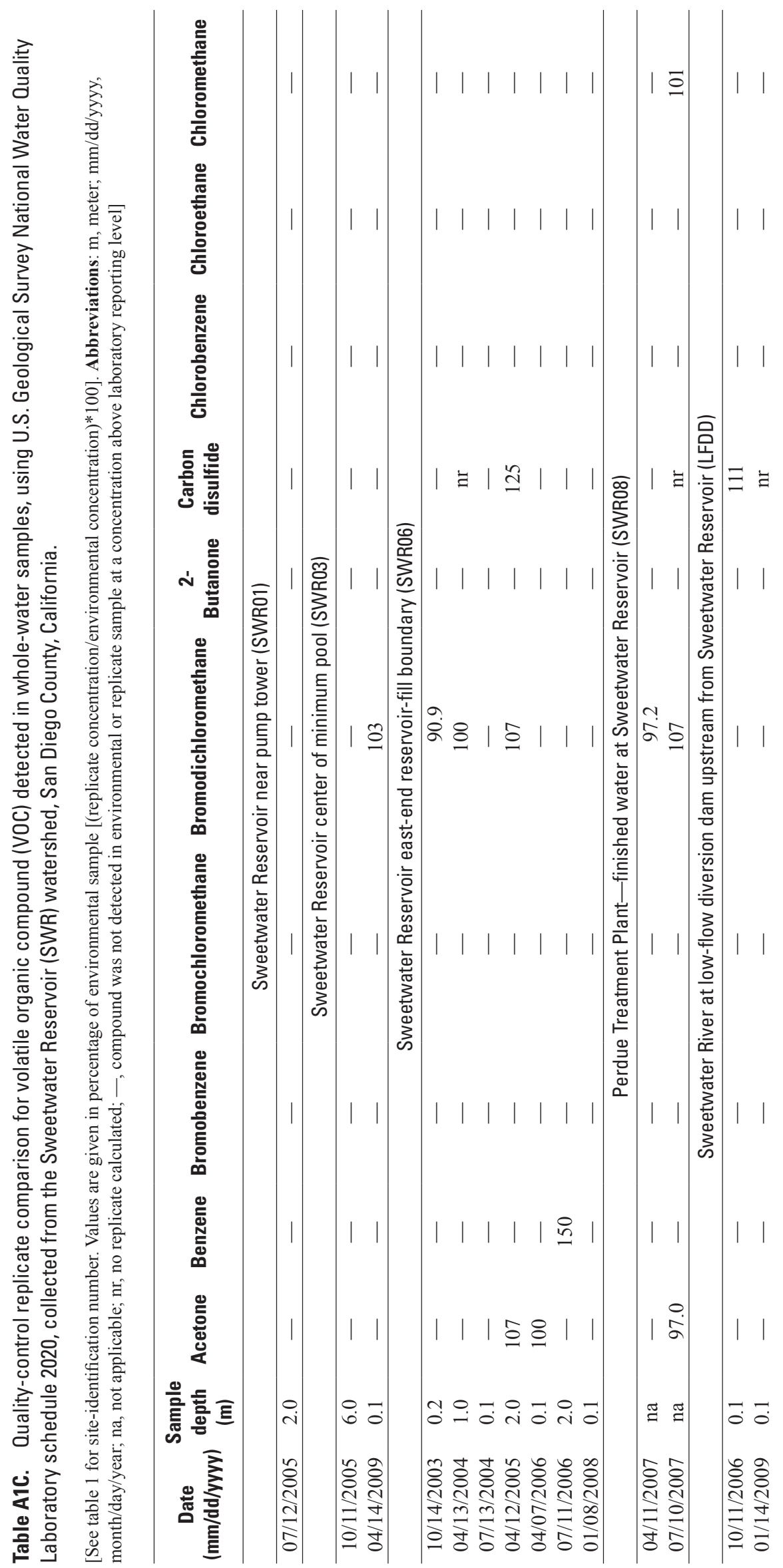




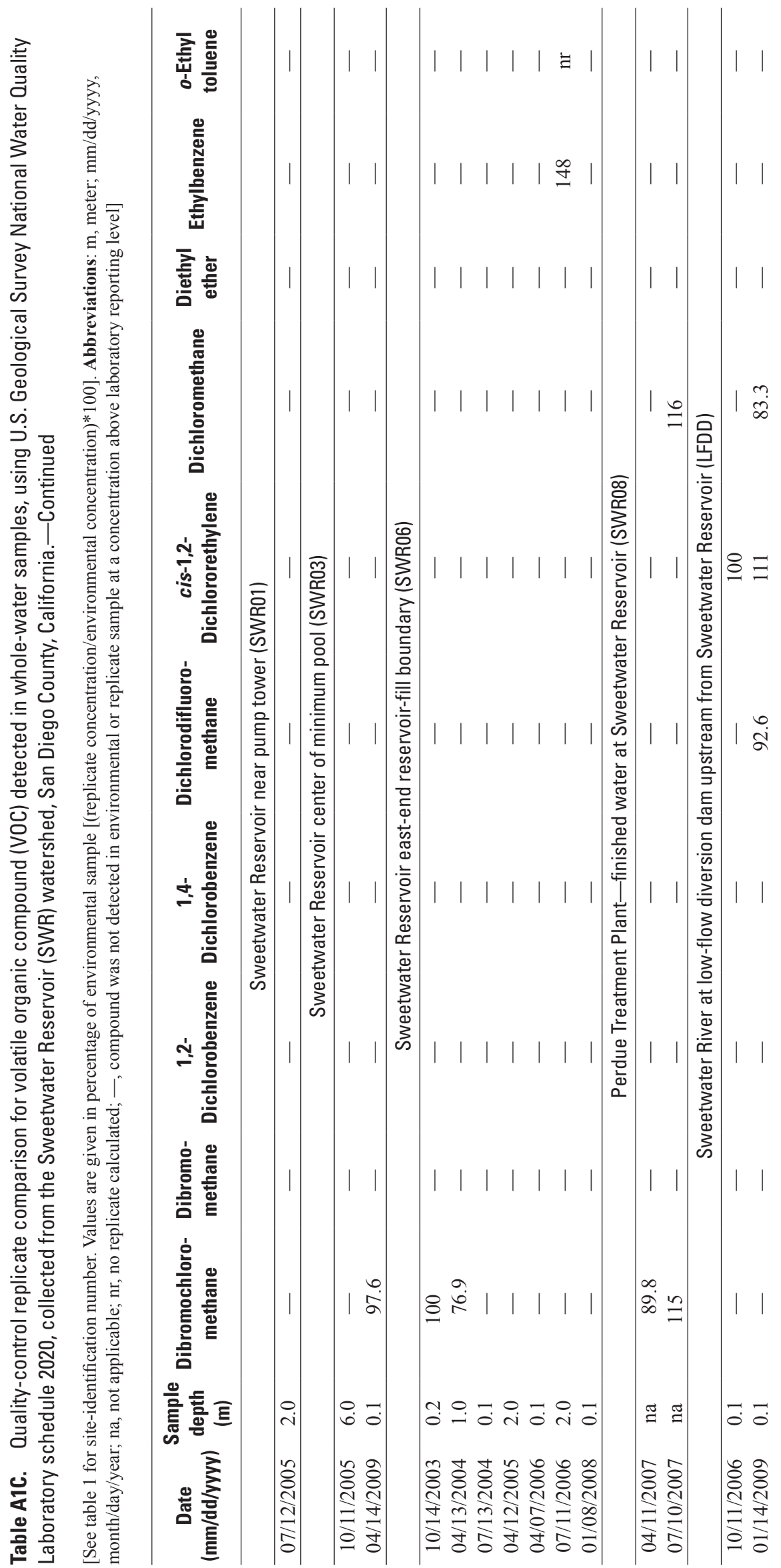




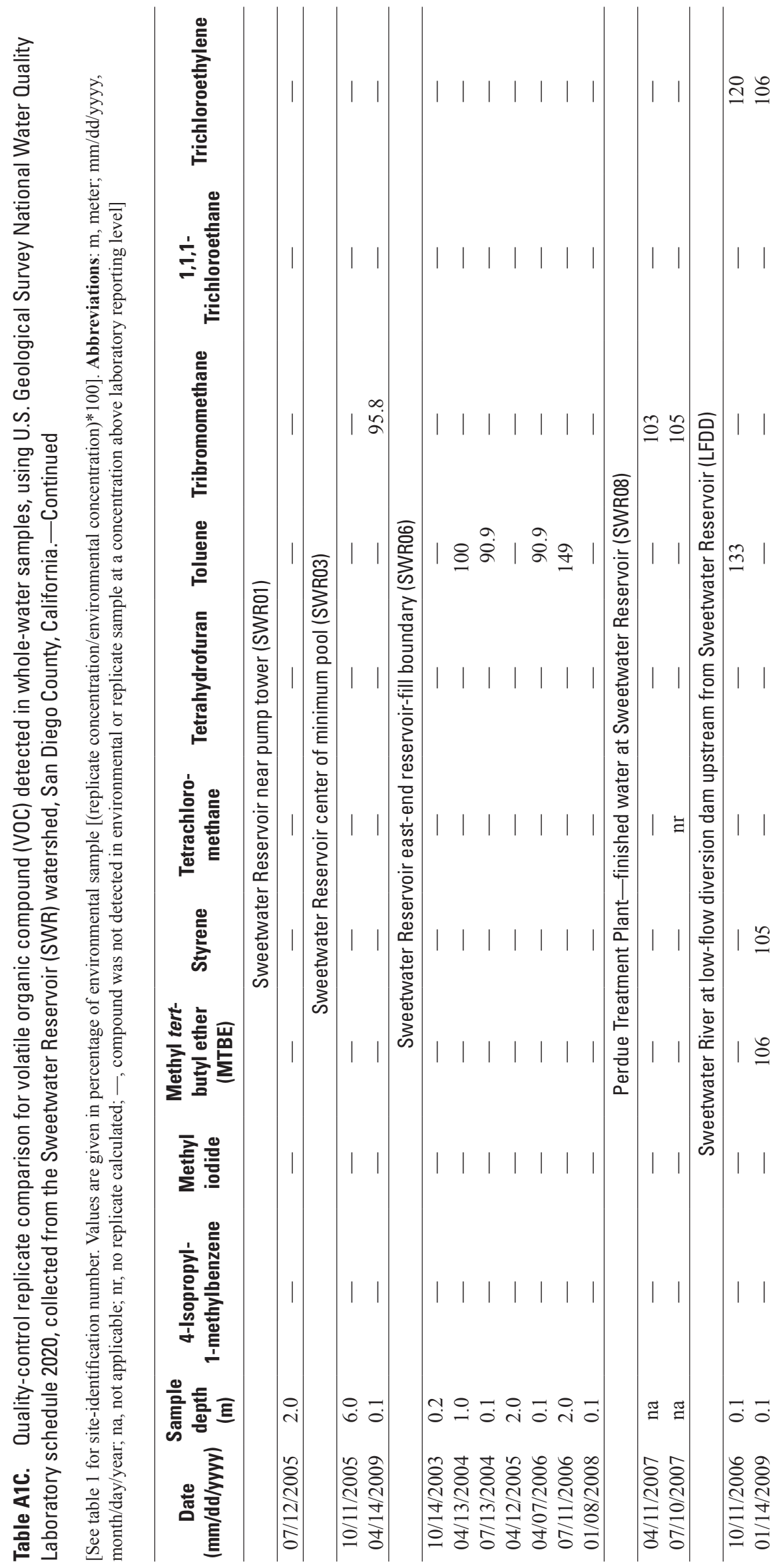




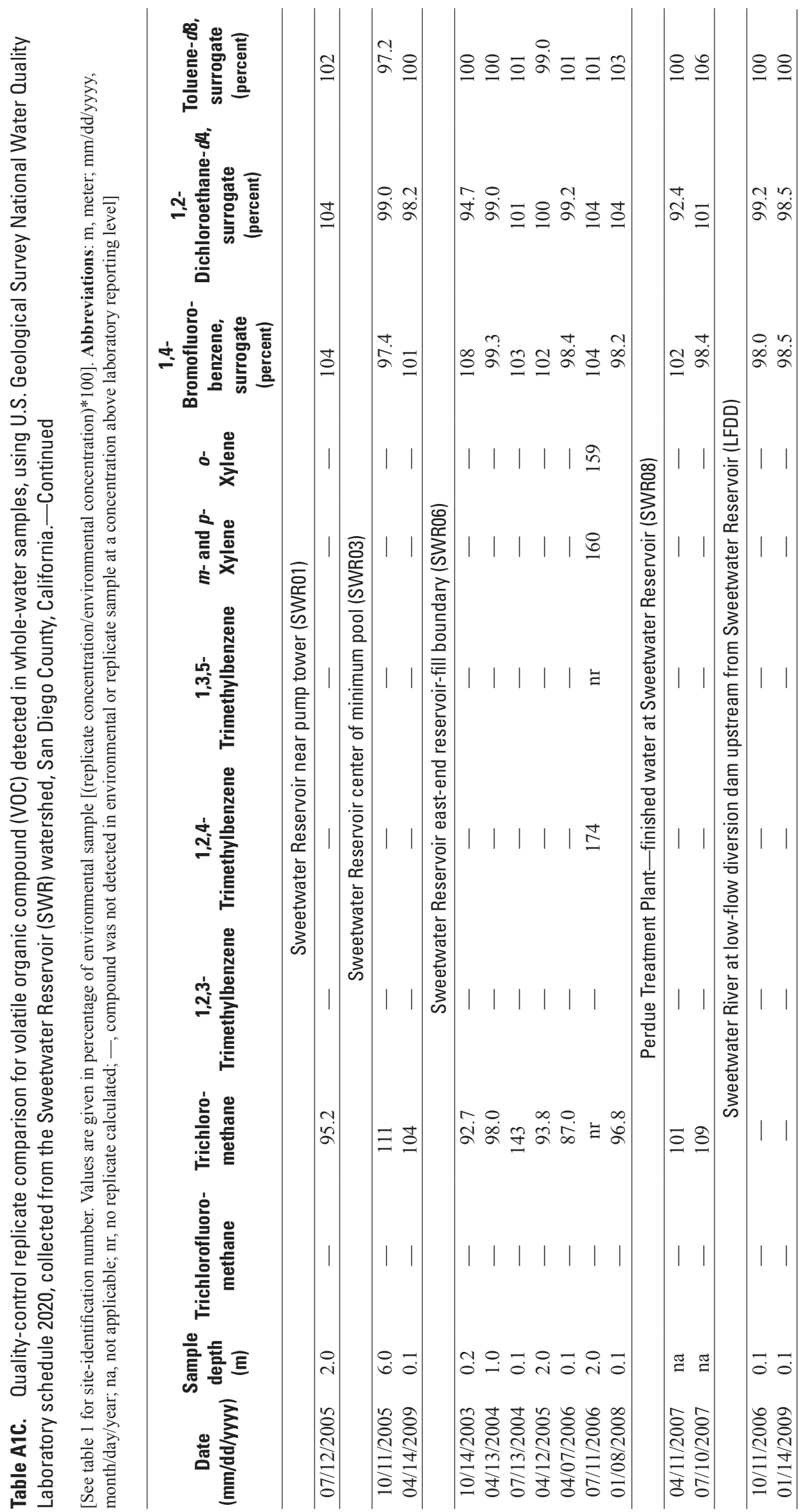



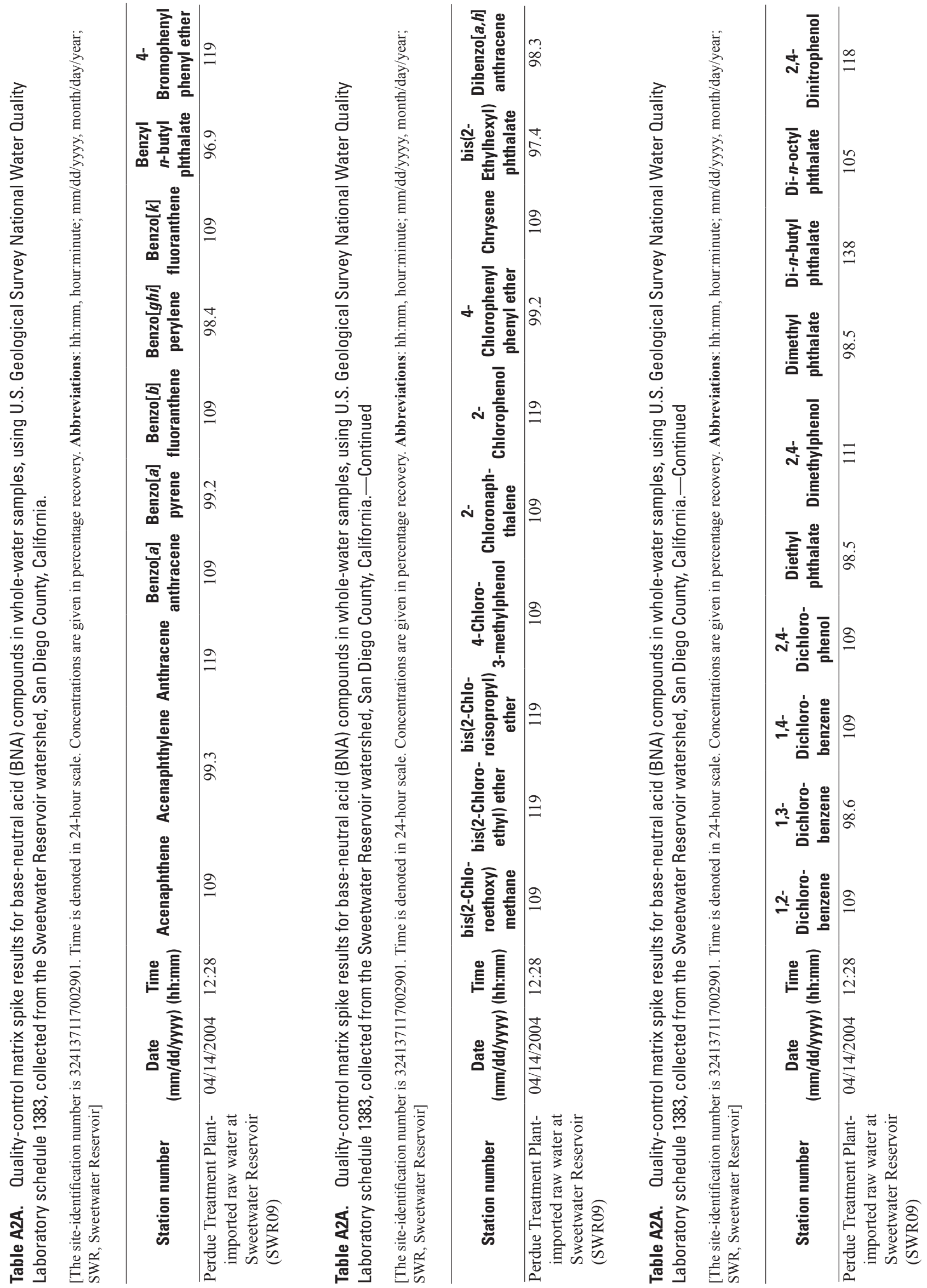

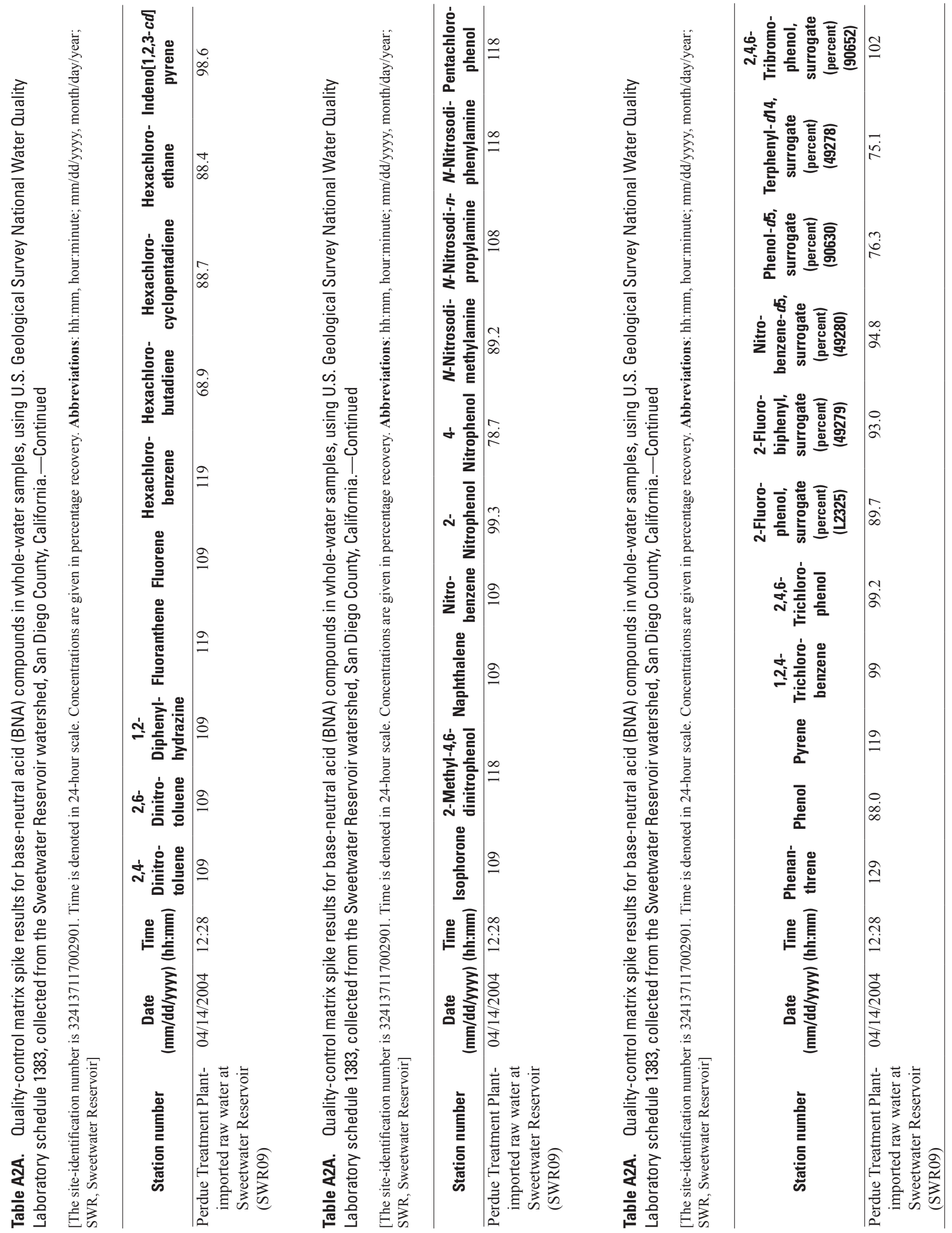

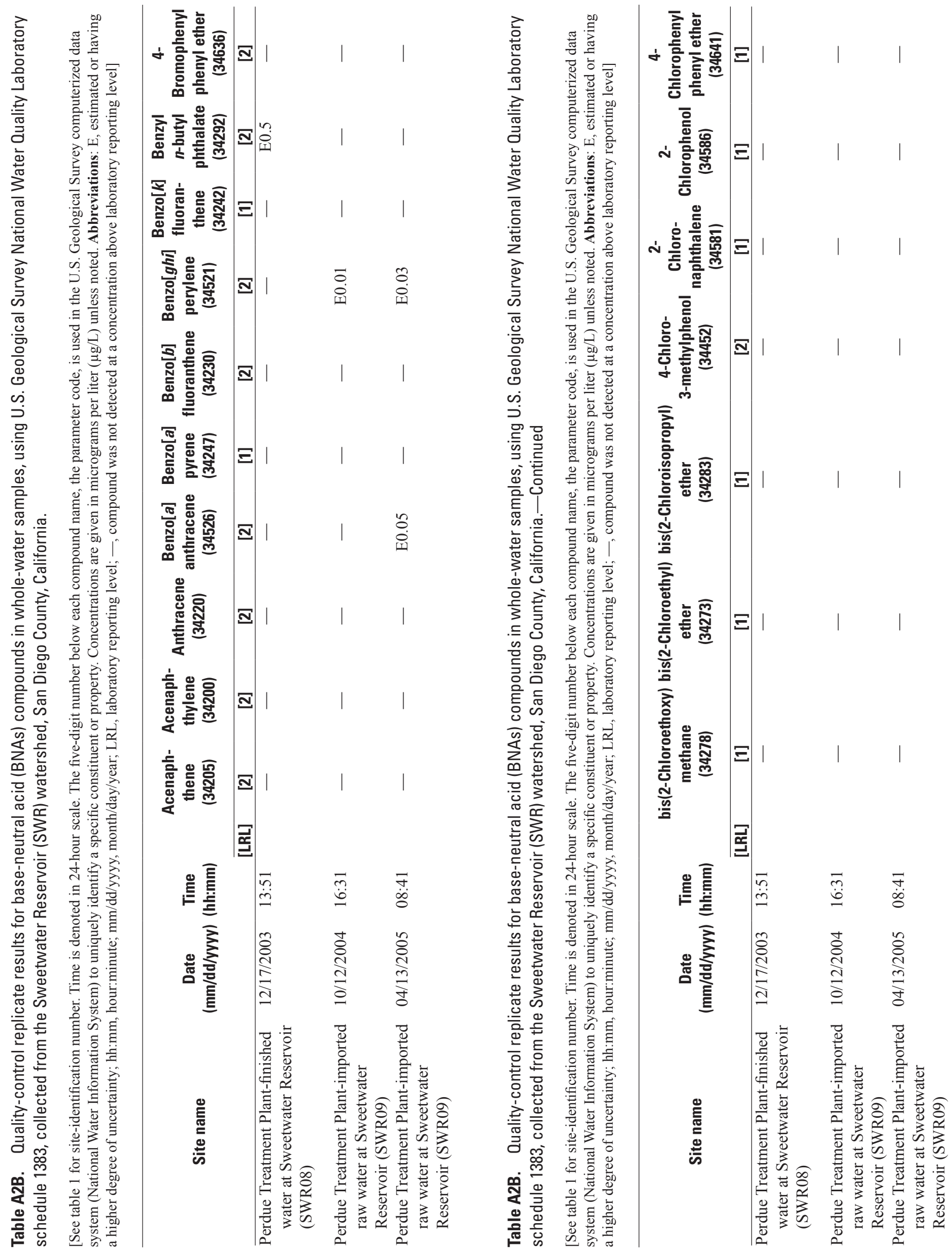

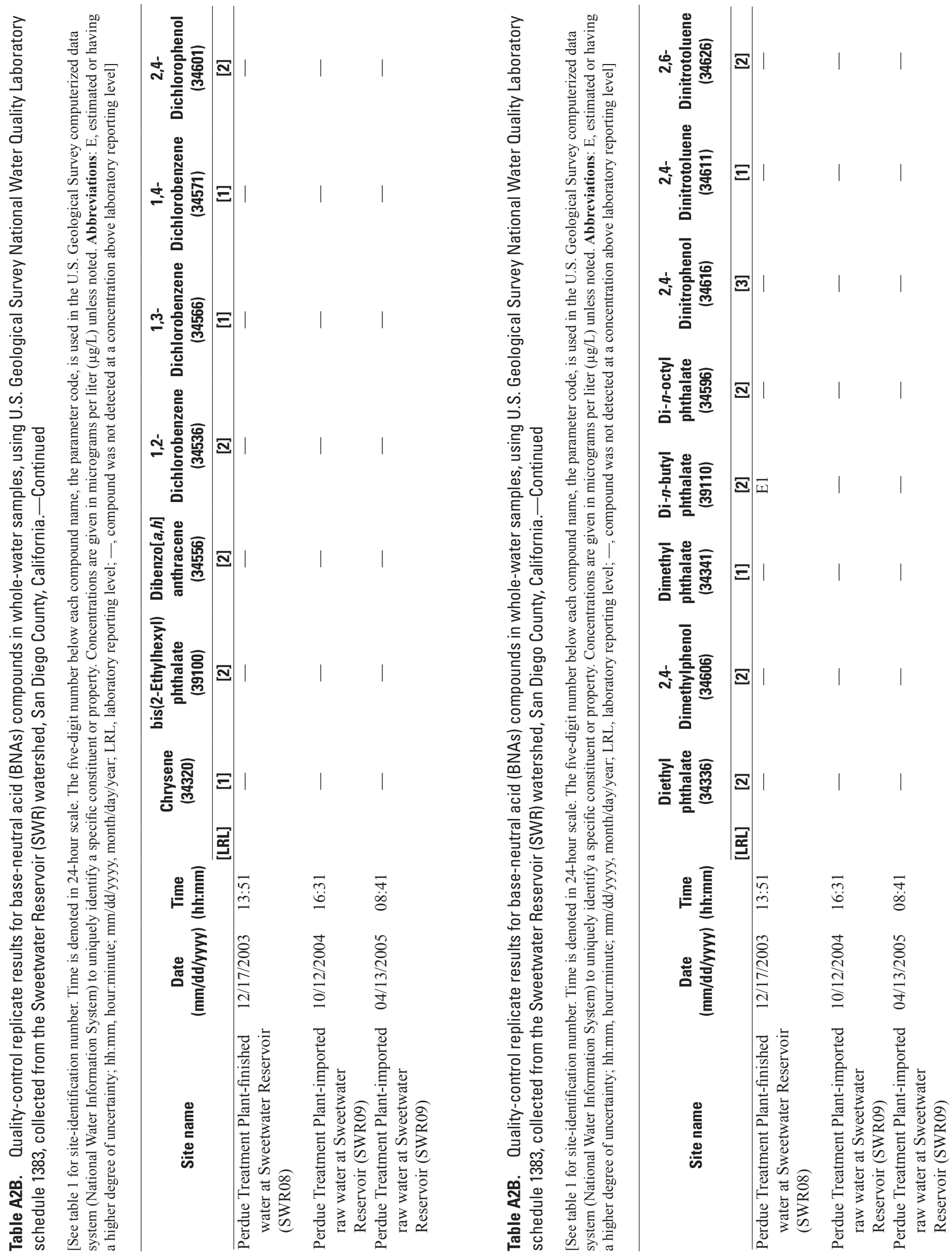

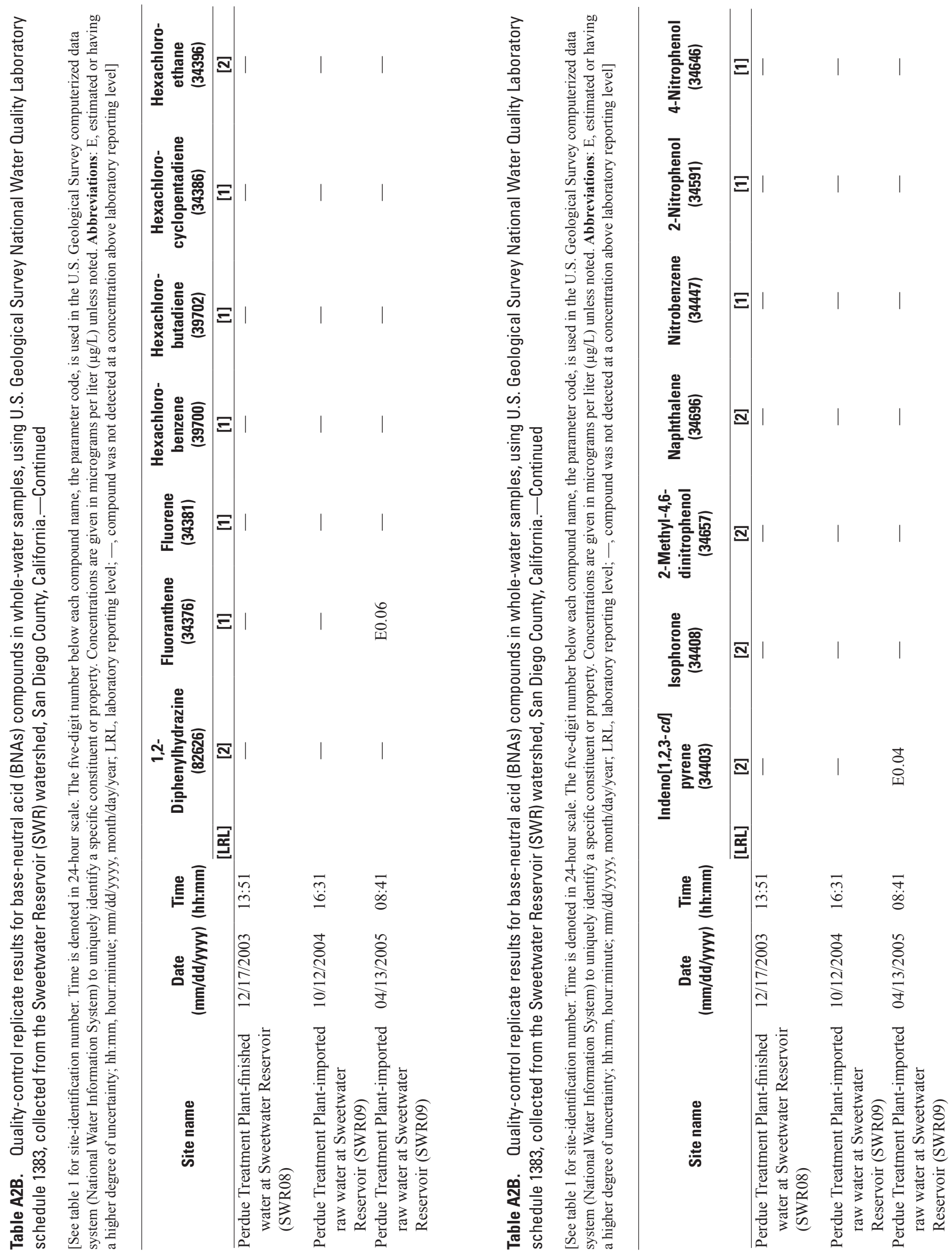

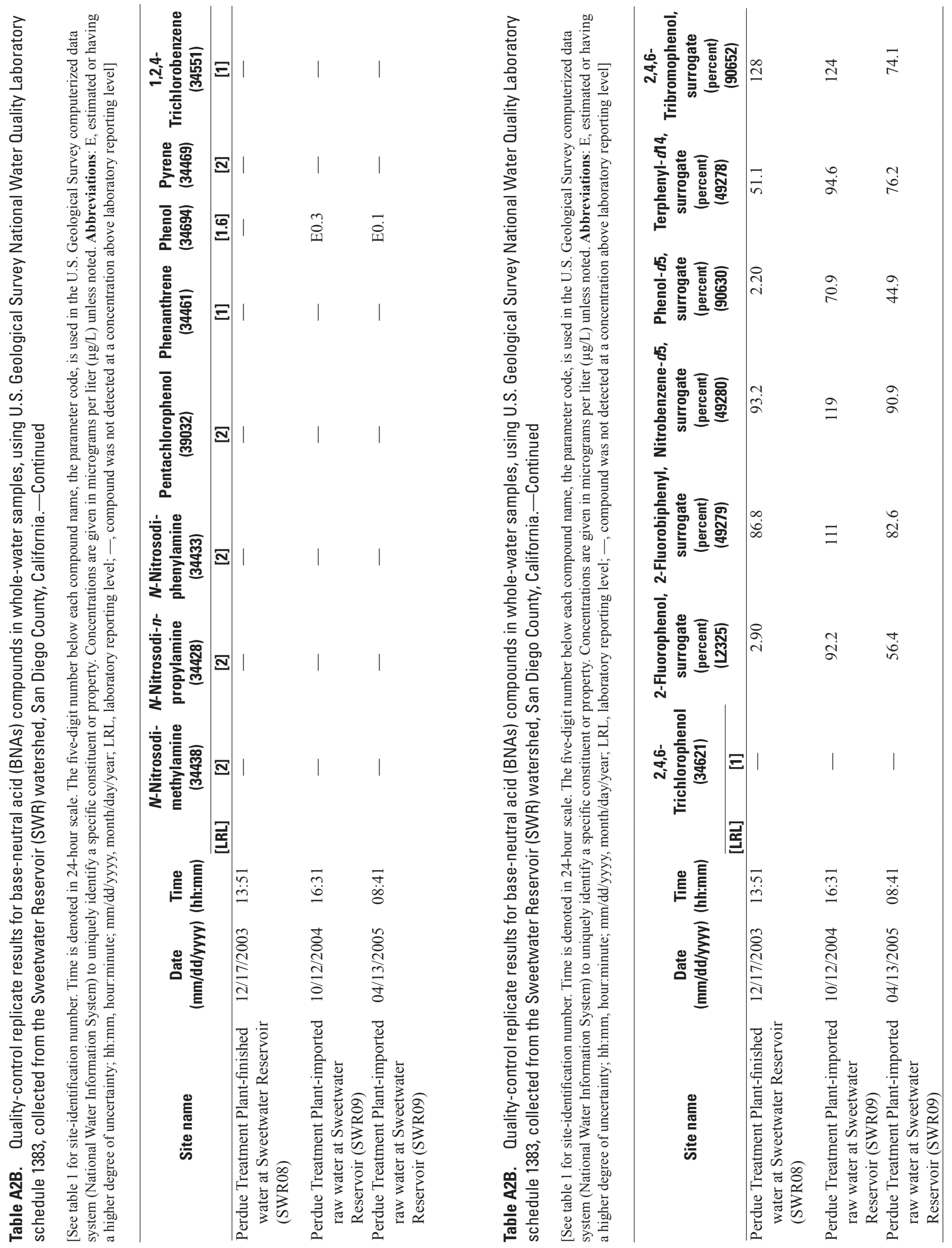

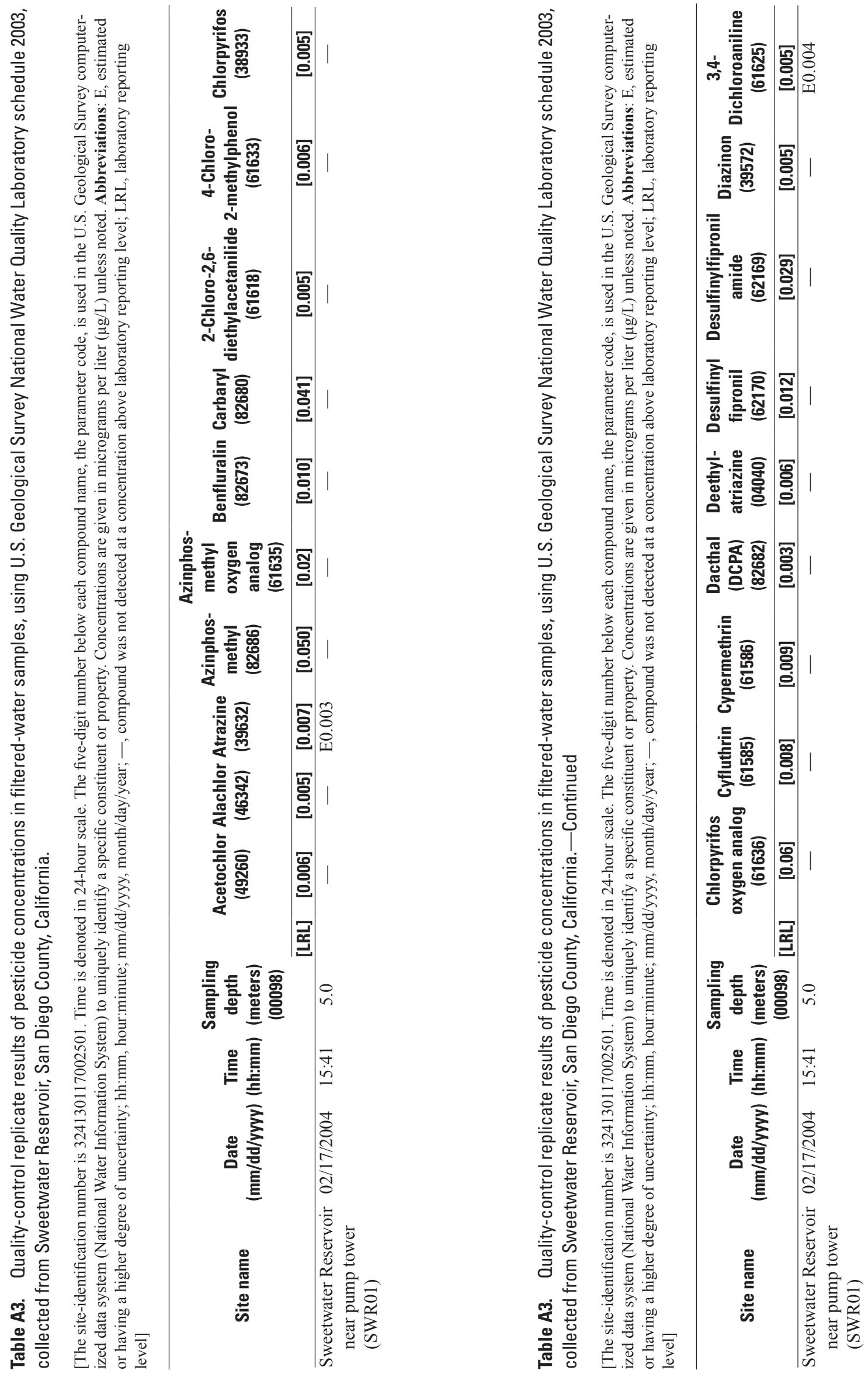

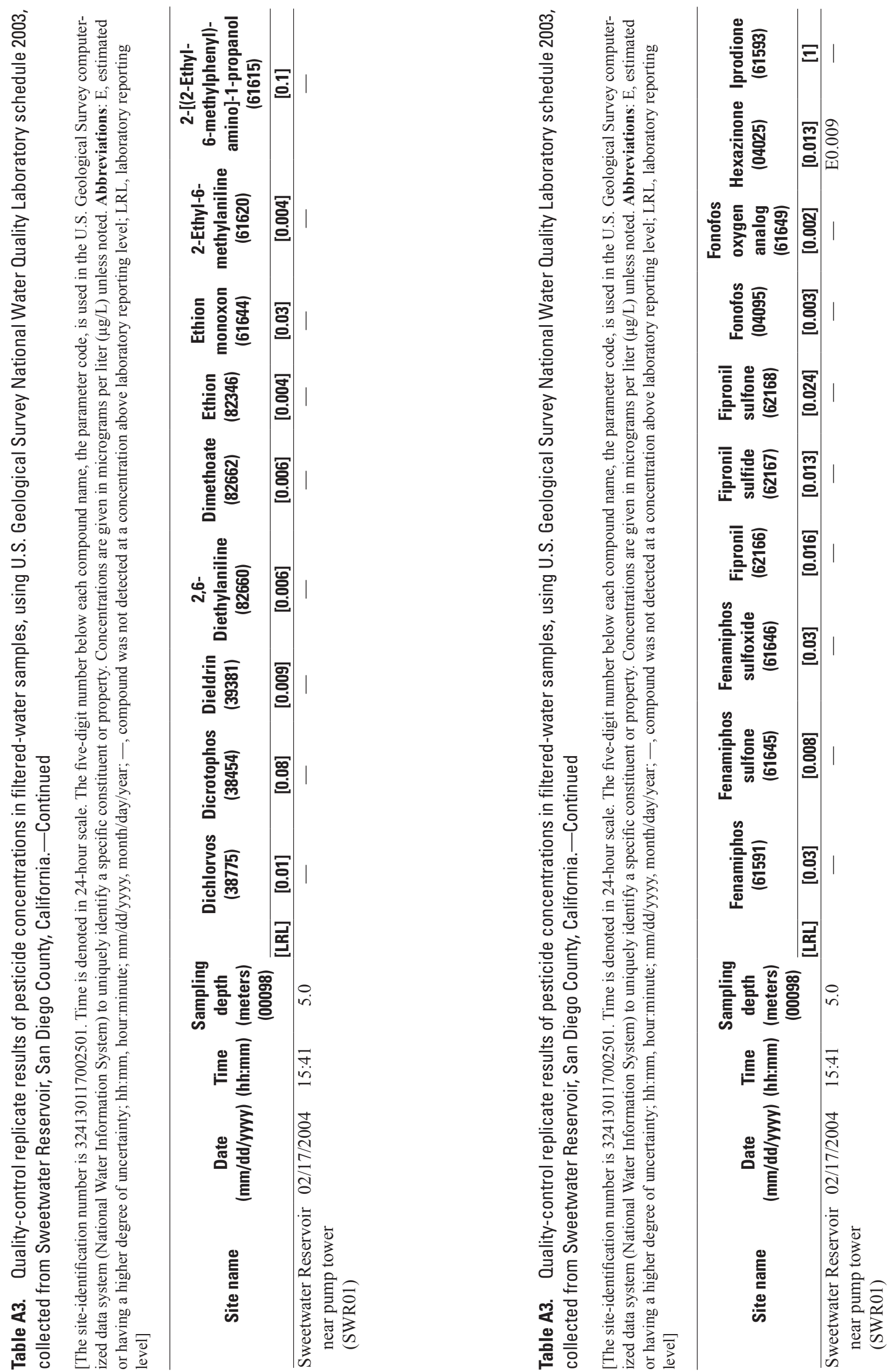

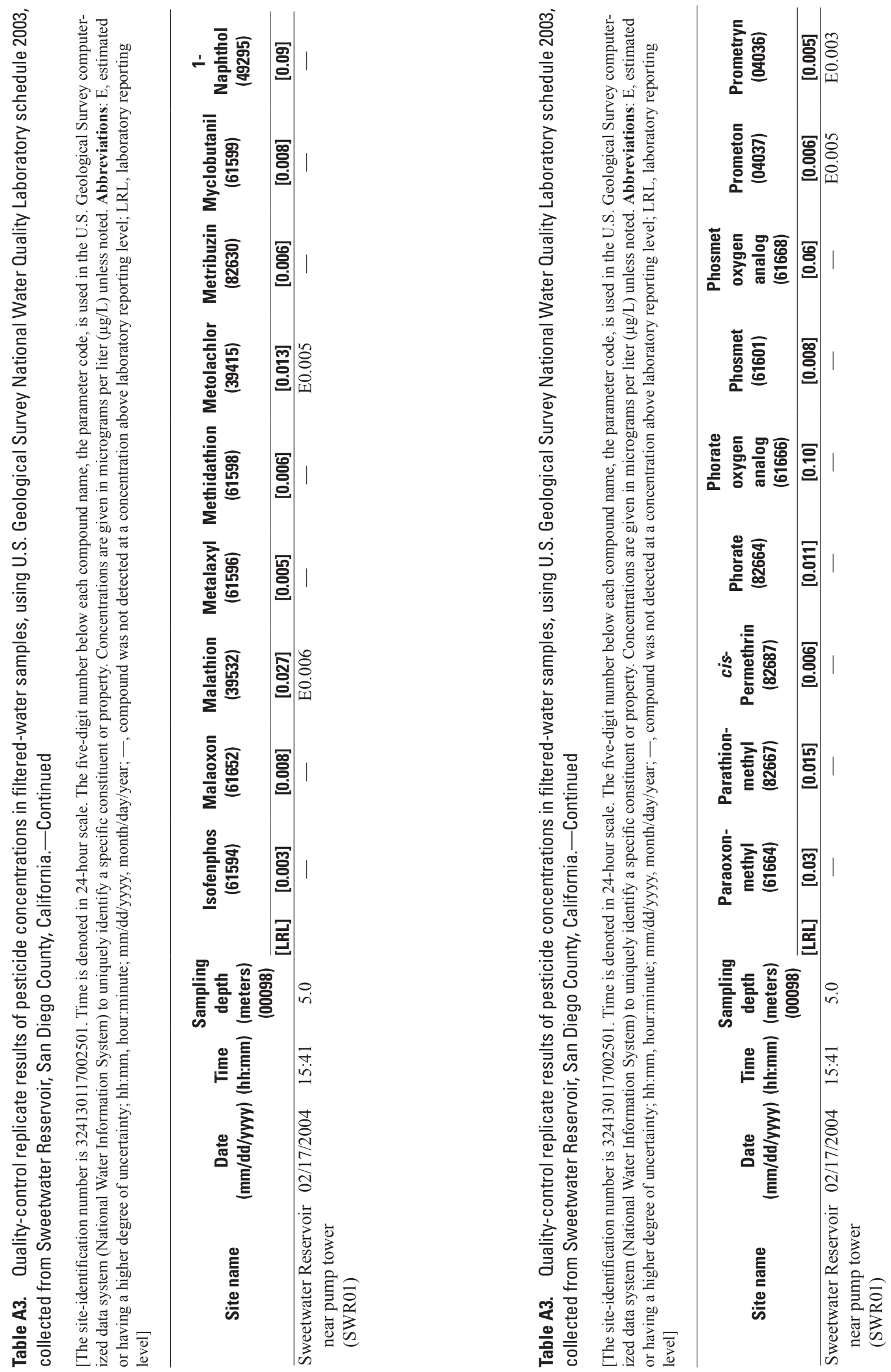


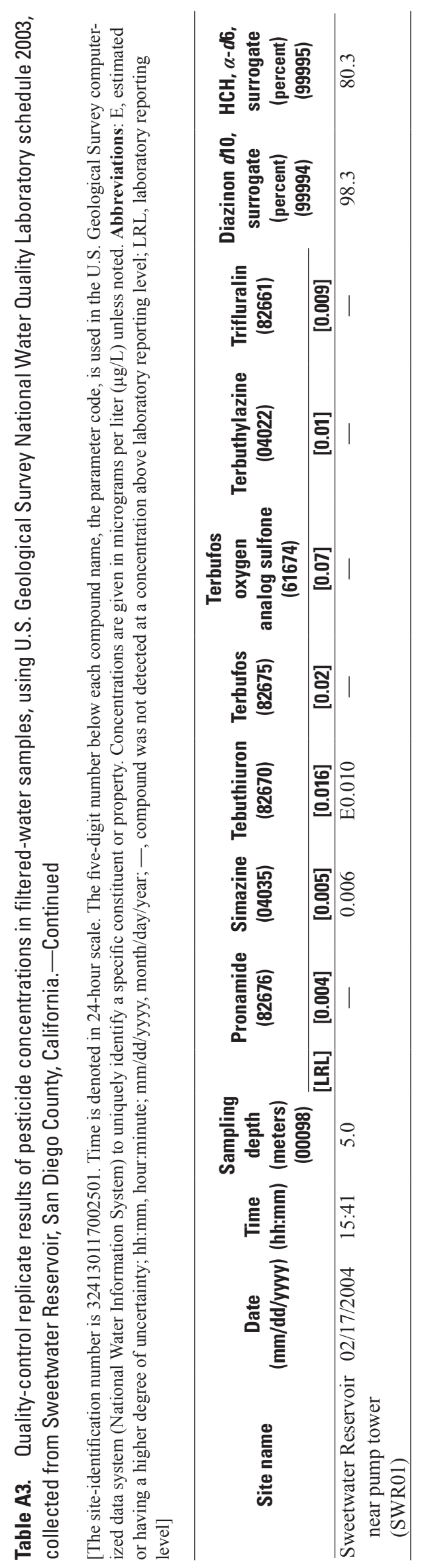



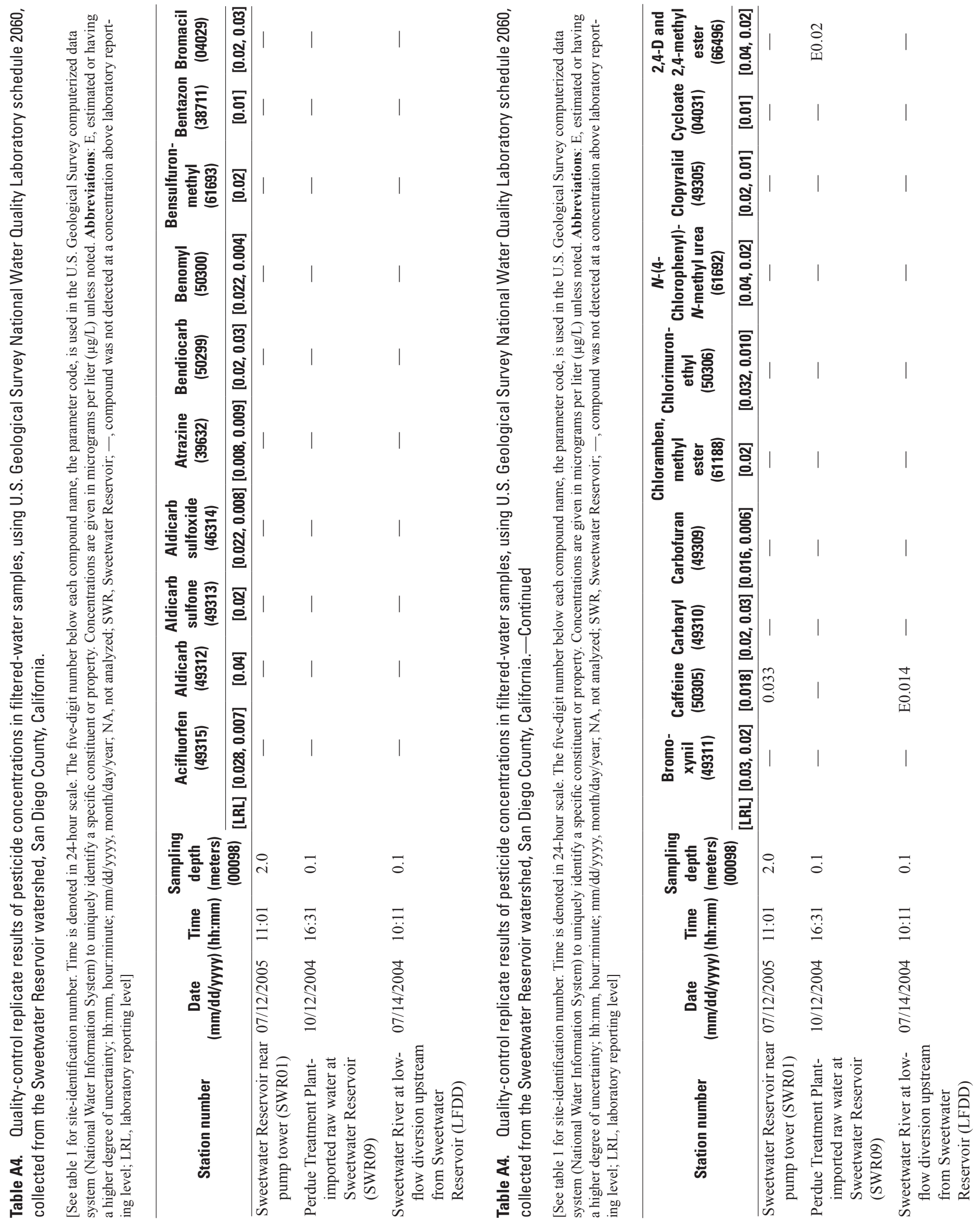

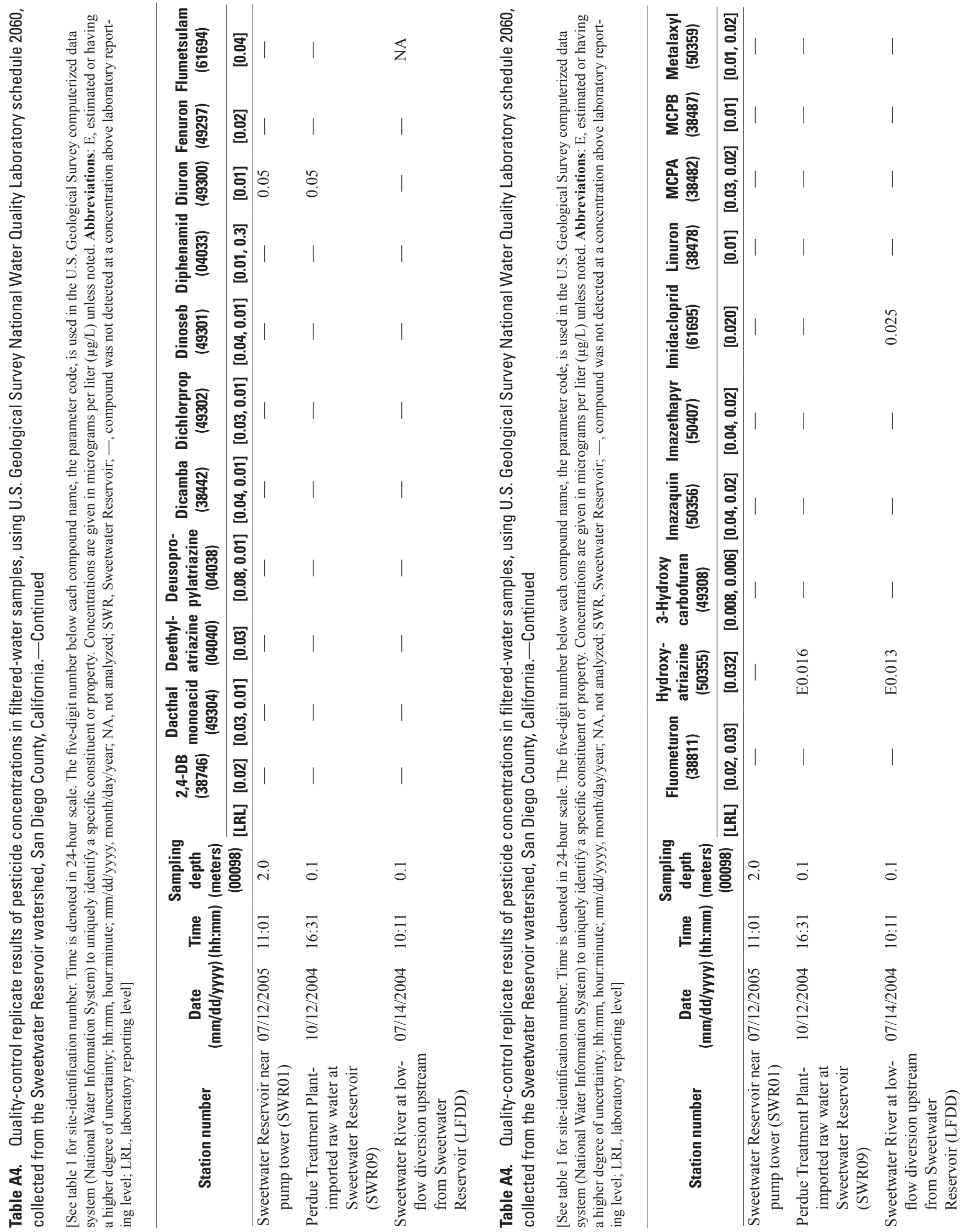

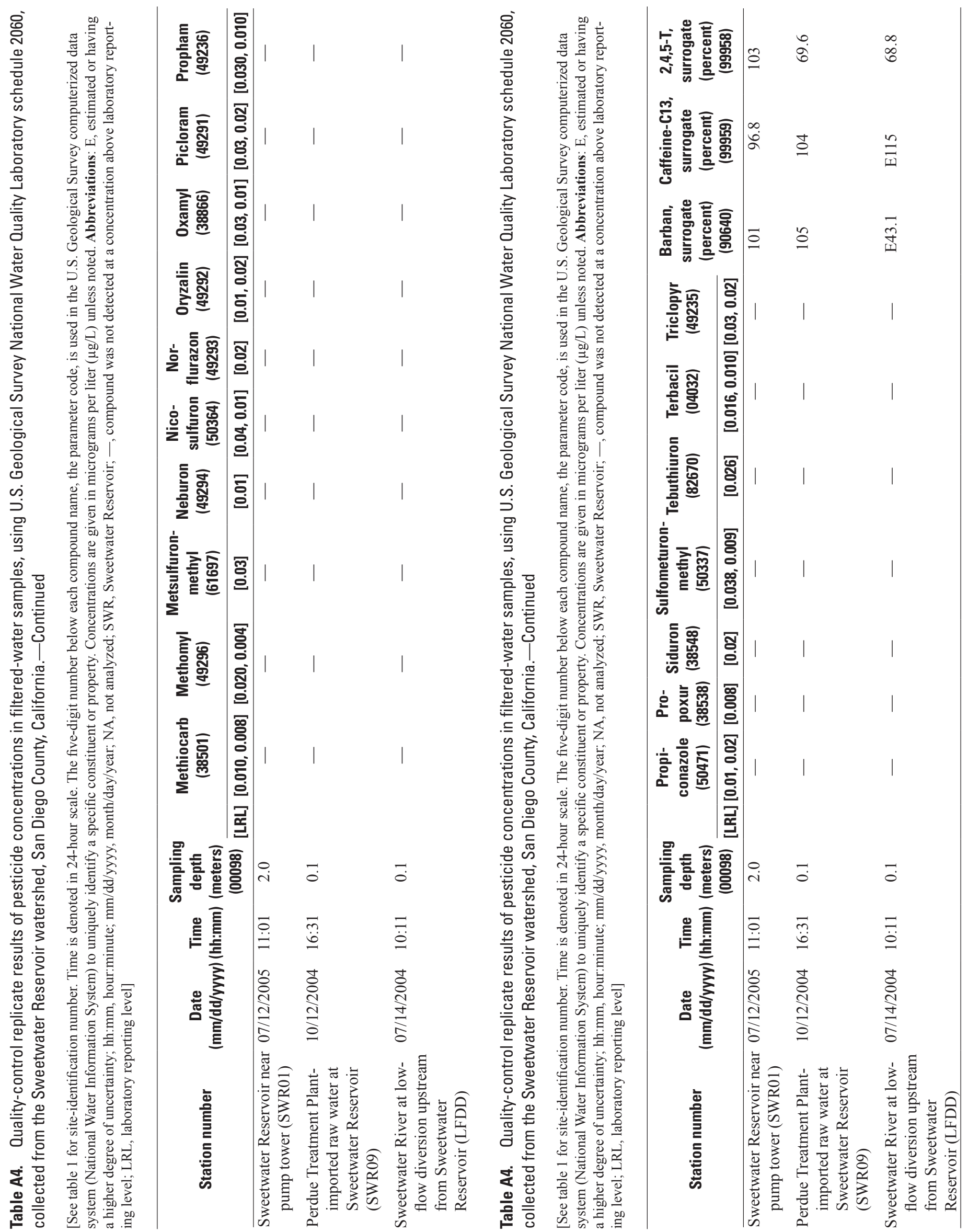


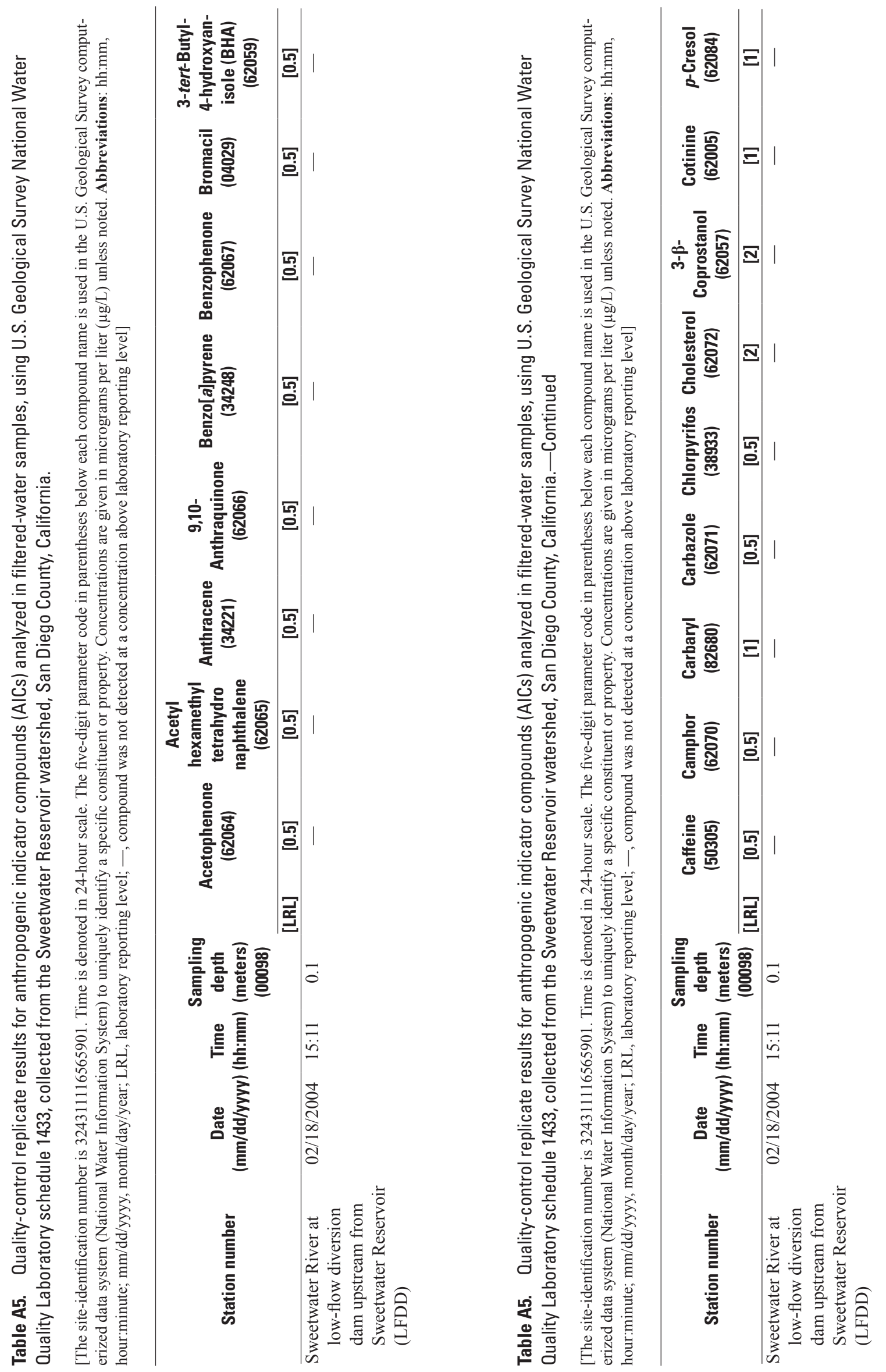



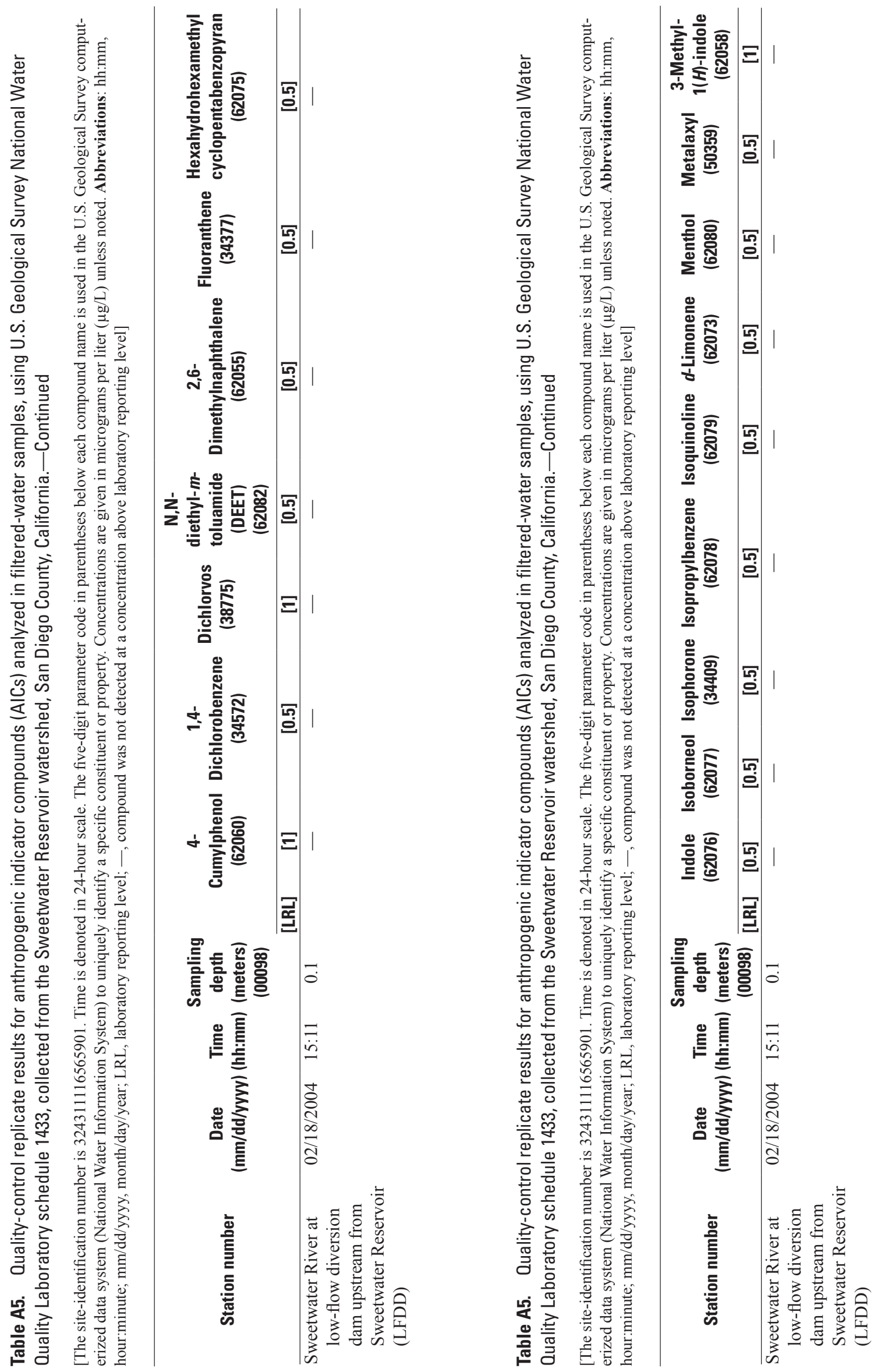

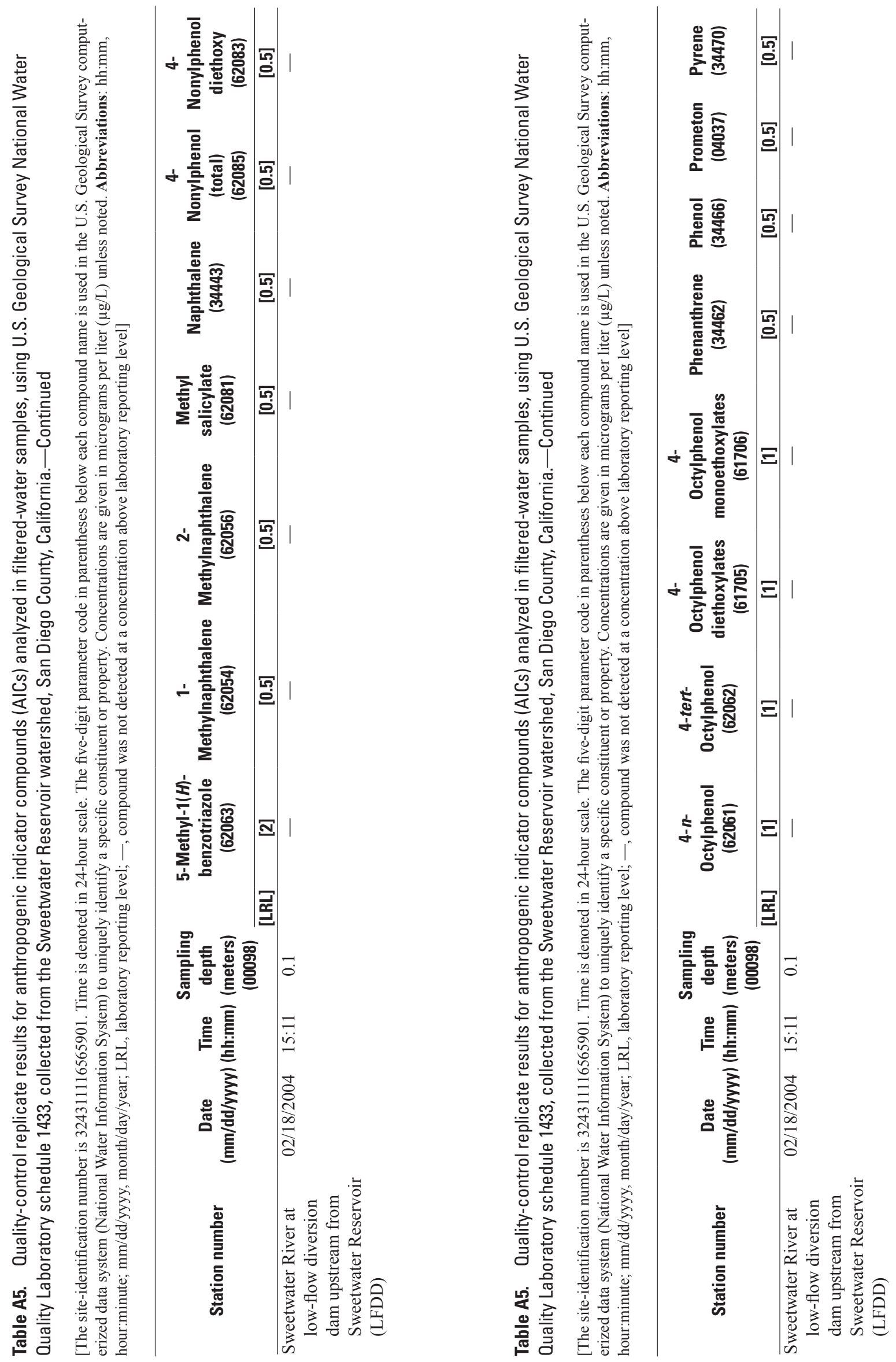

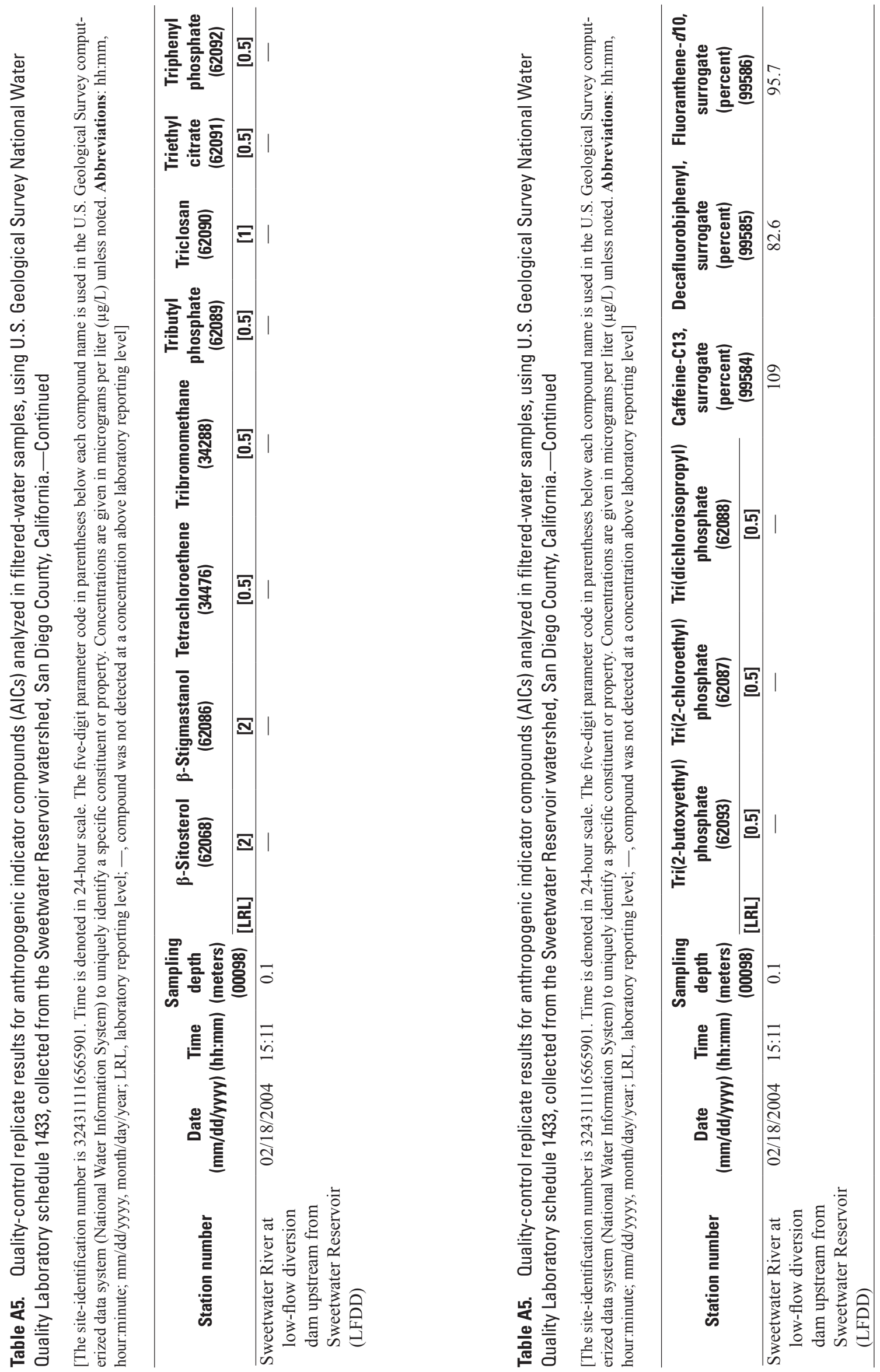

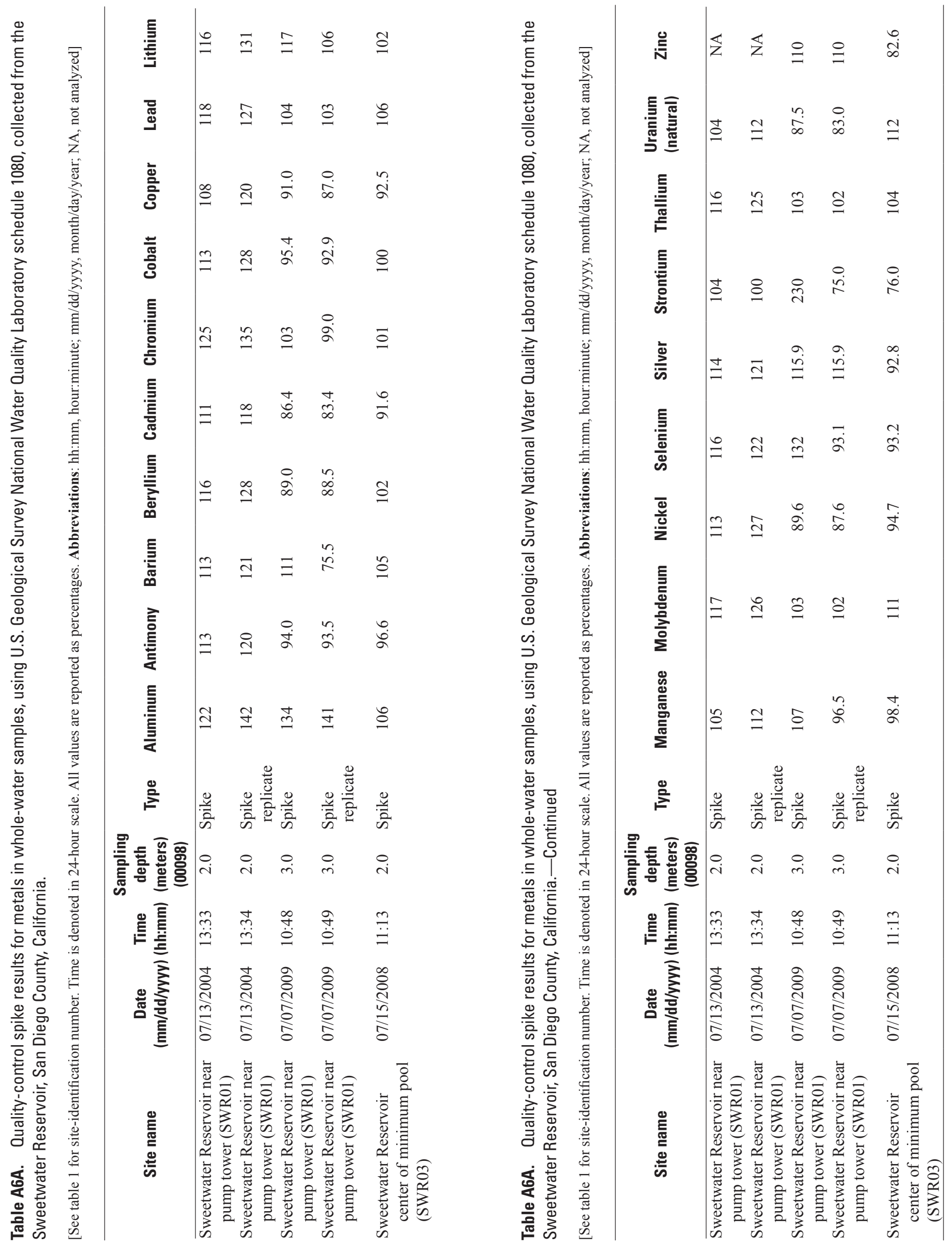


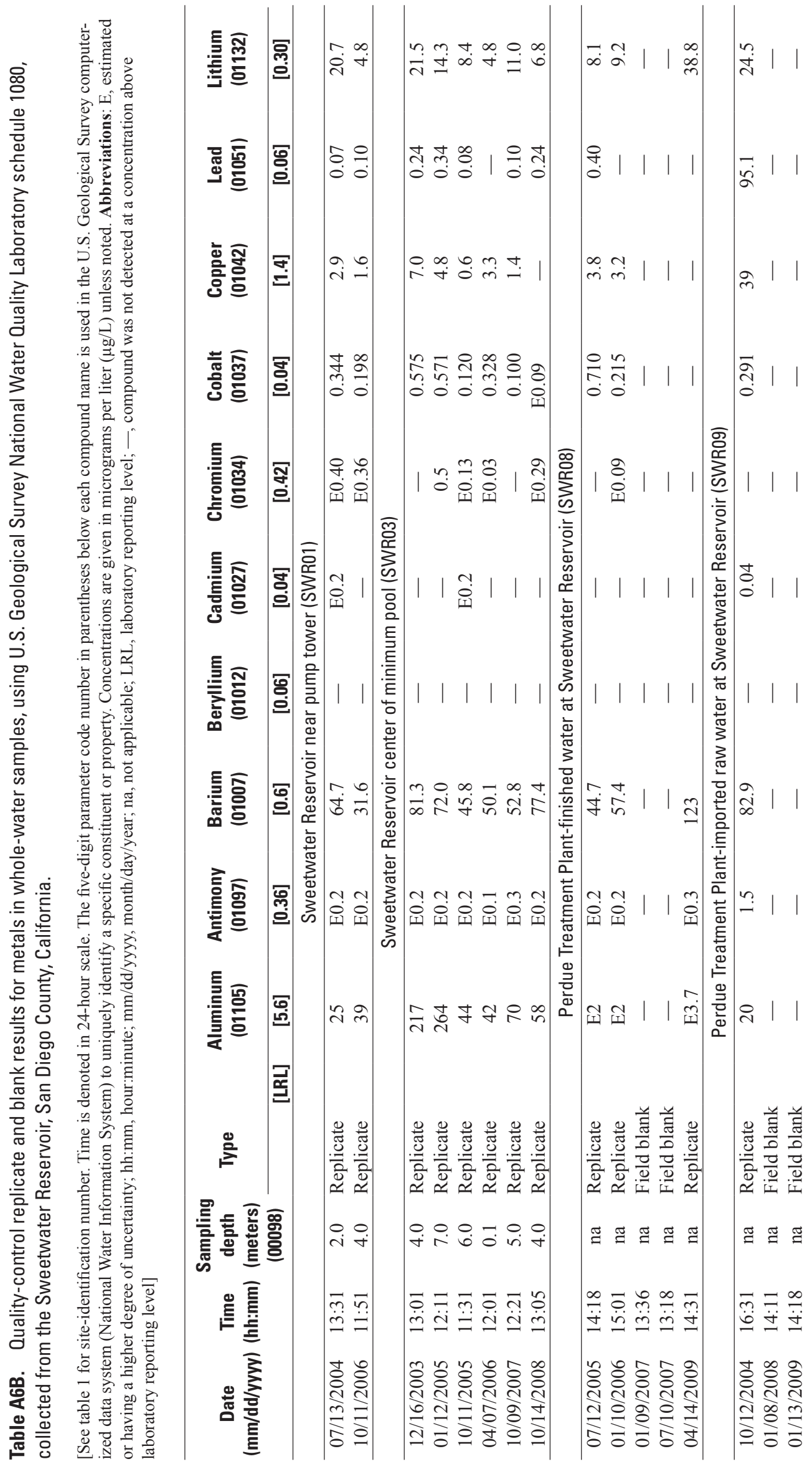




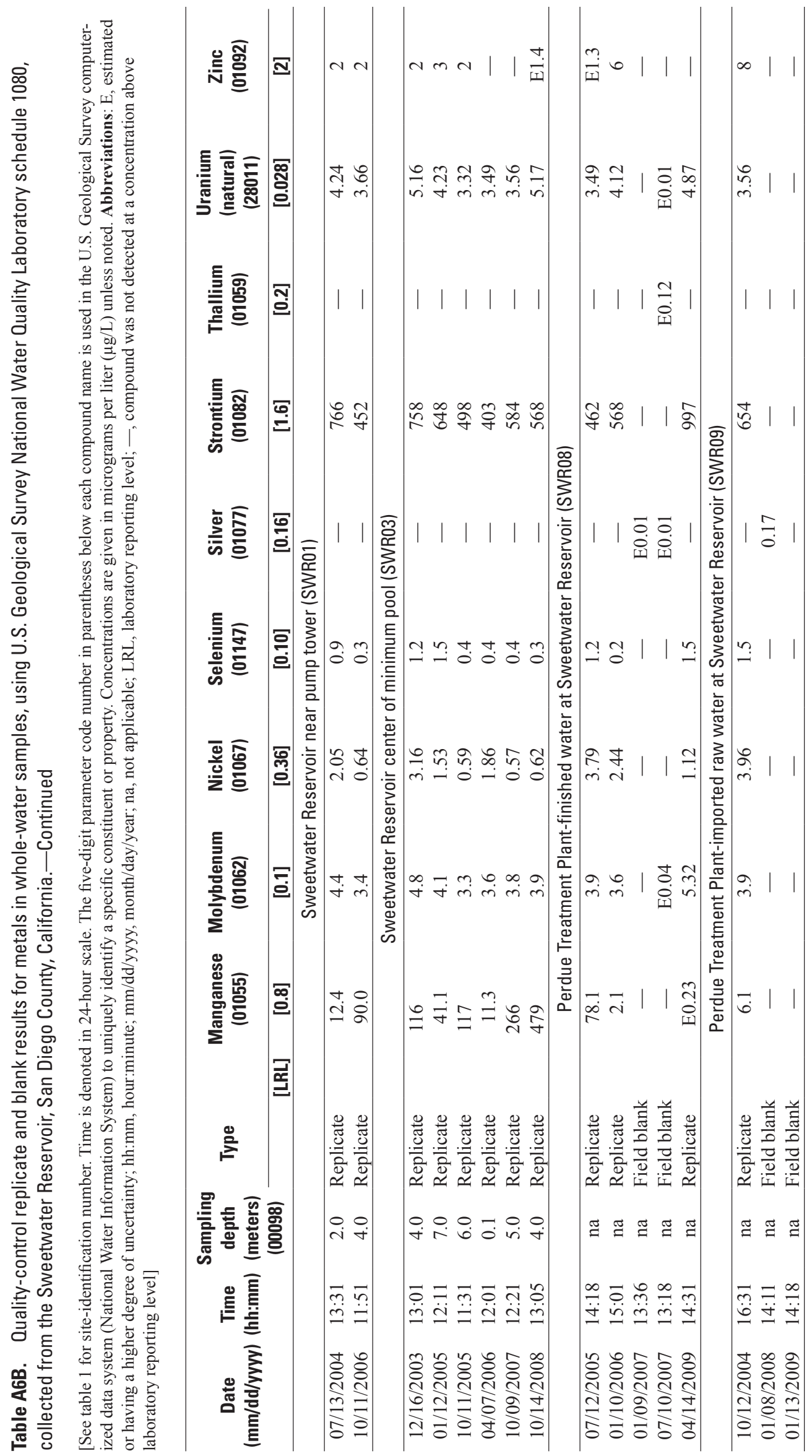




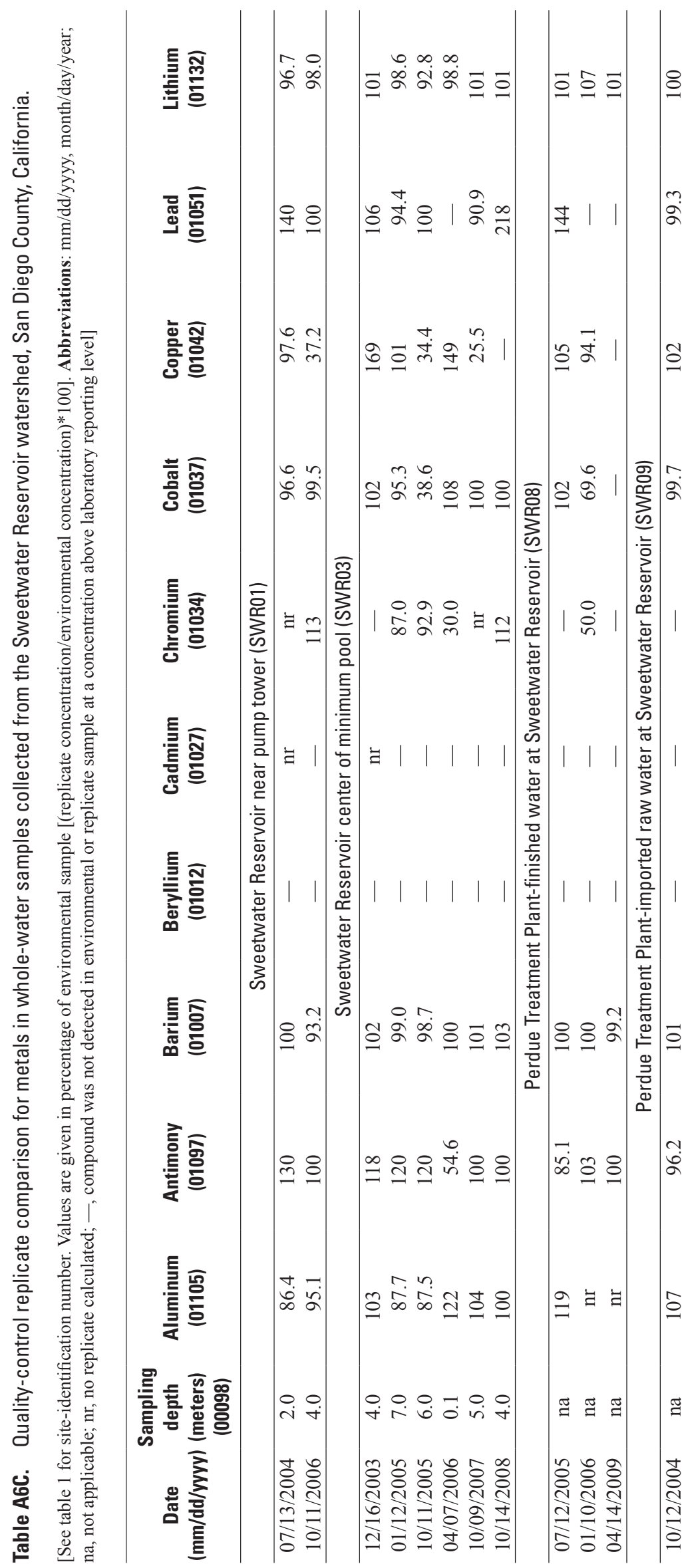




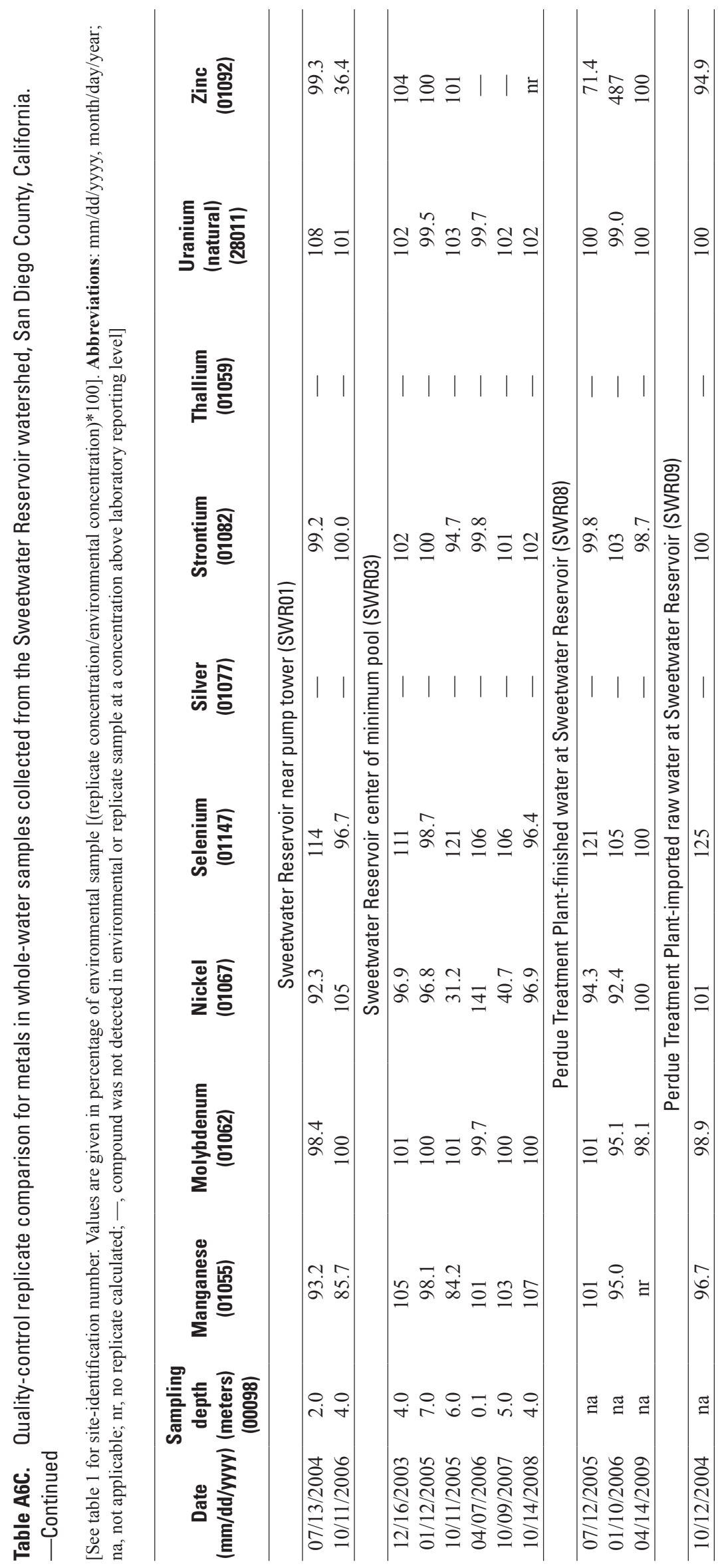


Appendix B Tables. 
Table B1A. Quality-control replicate results for high-volatility volatile organic compounds (VOCs) processed at the Oregon Graduate Institute for air samples collected from the Sweetwater Reservoir atmospheric site, San Diego County, California.

[The site-identification number is 324141117001601. Concentrations are given in part per billion by volume (ppbv). Abbreviations: $\mathrm{mm}$ of $\mathrm{Hg}$, millimeters of mercury; mm/dd/yyyy, month/day/year; LRL, laboratory reporting level; ${ }^{\circ} \mathrm{C}$, degree Celsius; —, compound was not detected at a concentration above the laboratory reporting level]

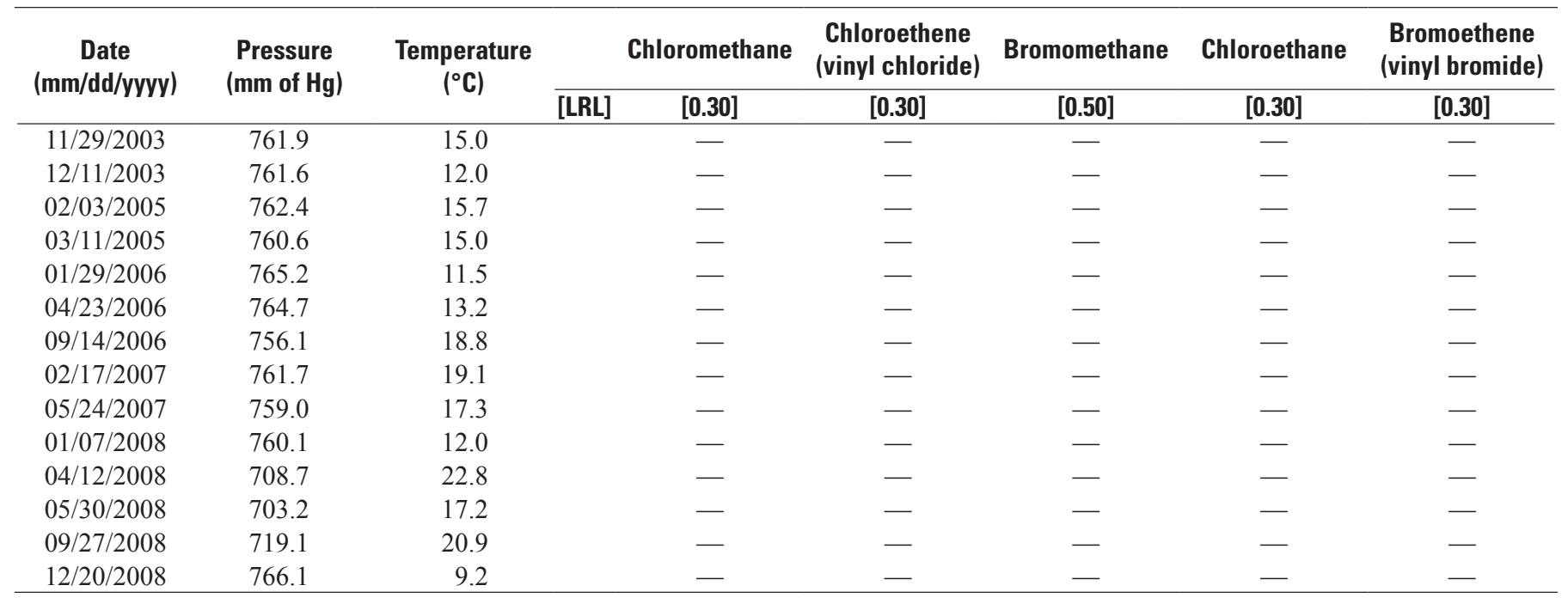



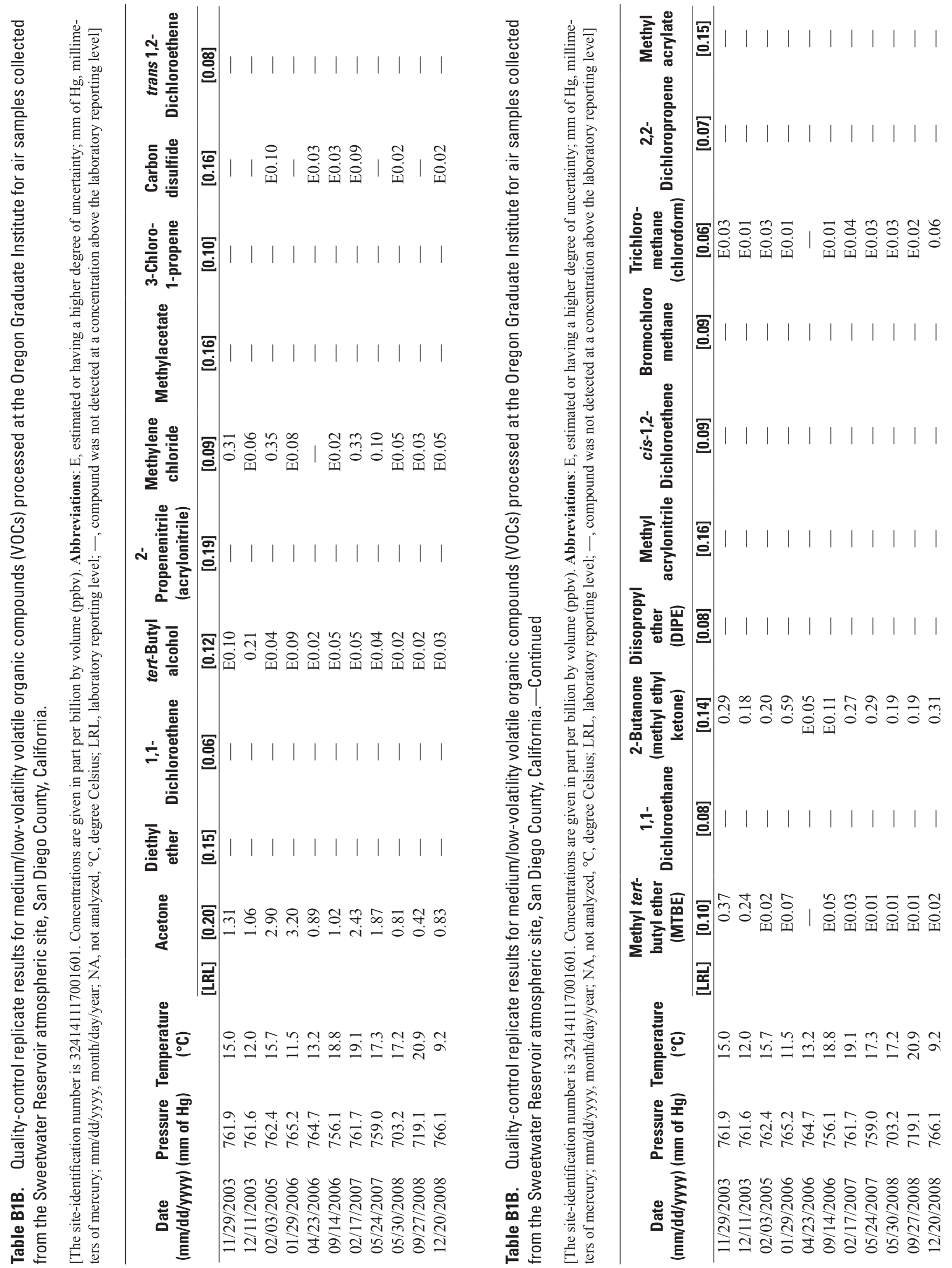

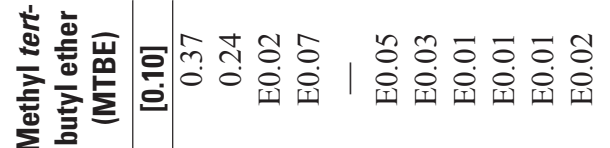

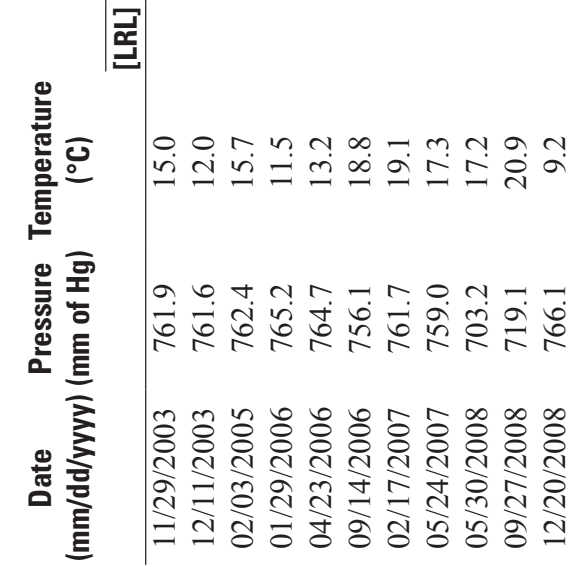



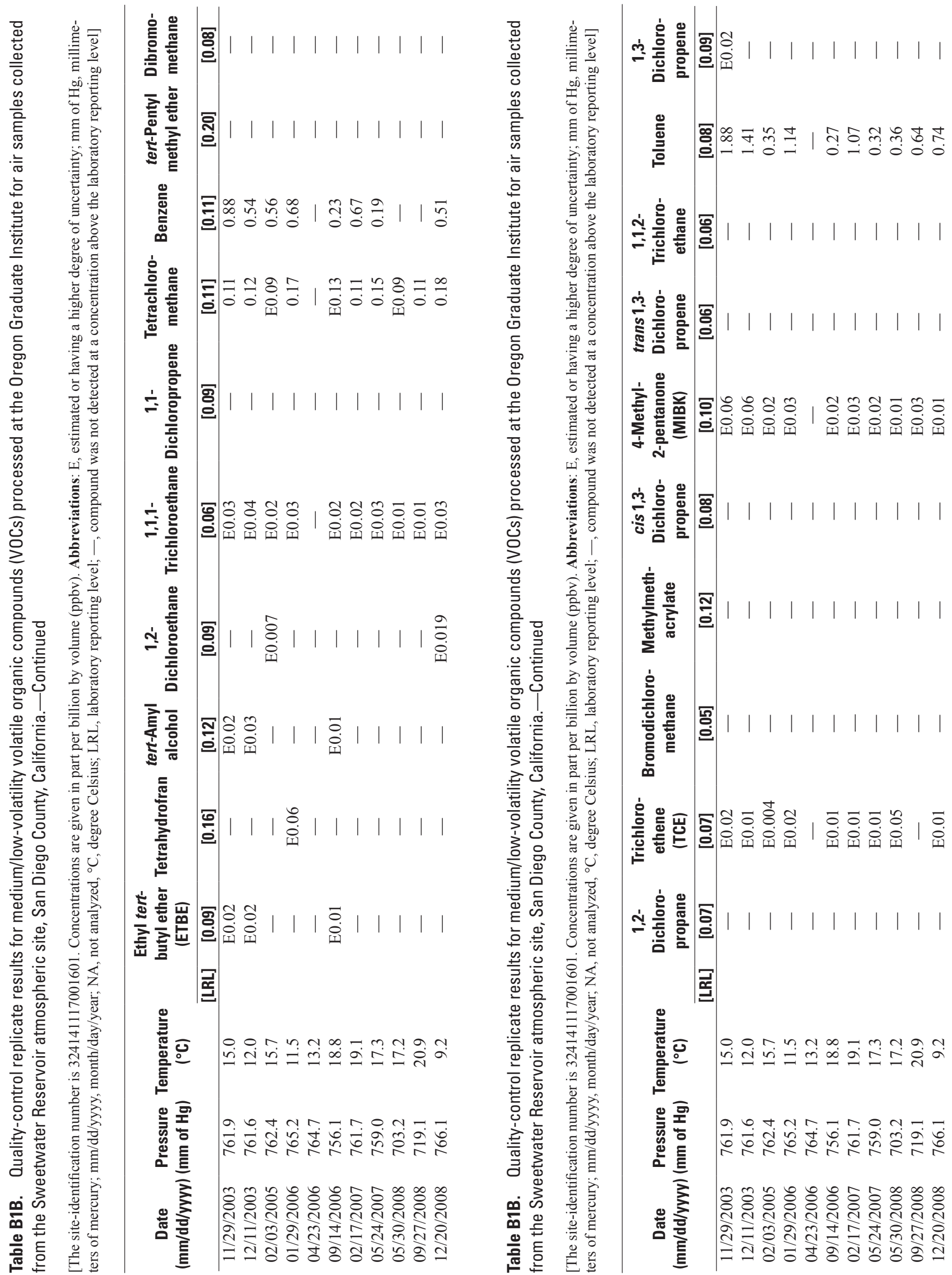

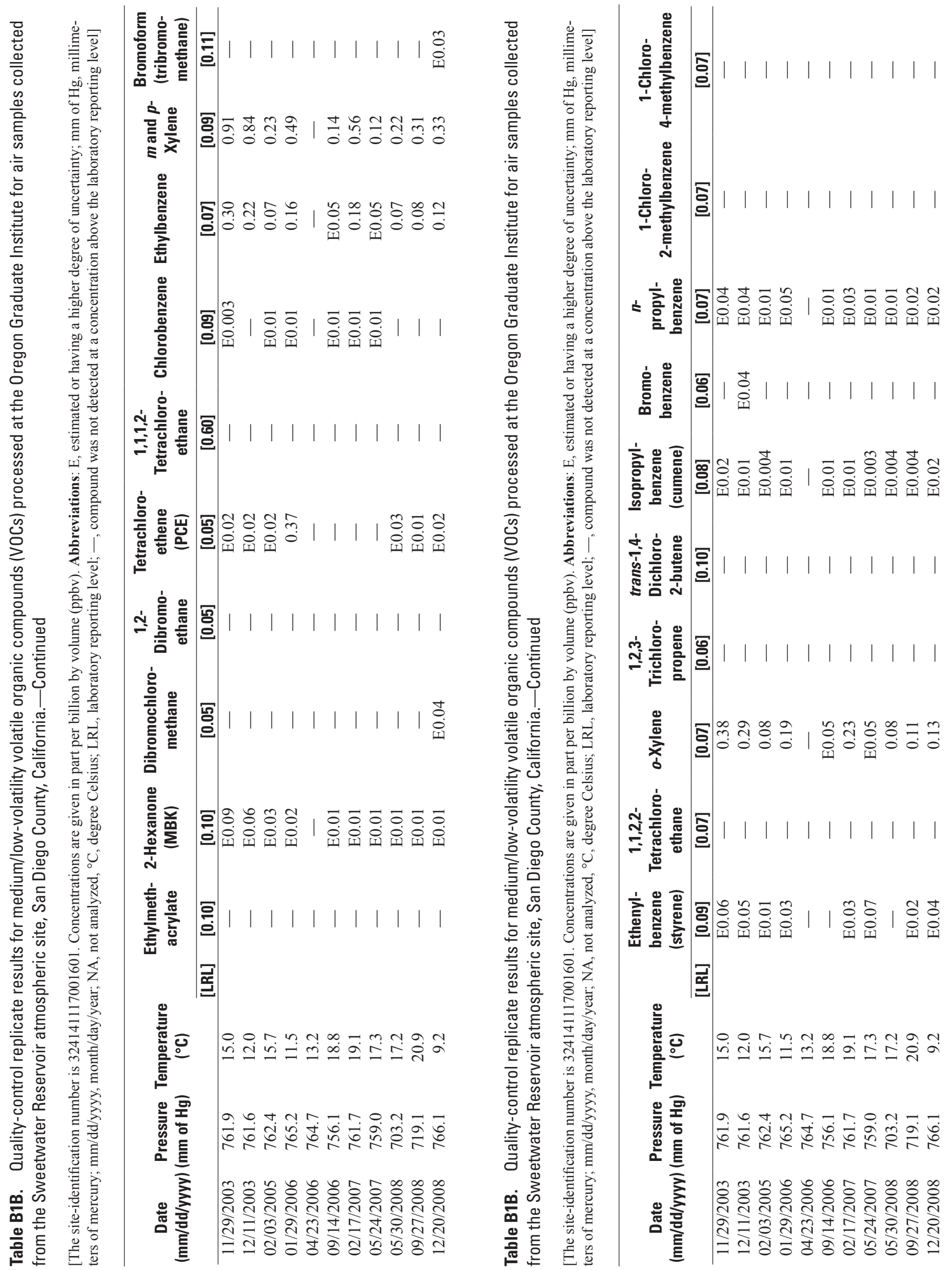

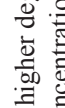

ฮั

ᄂ

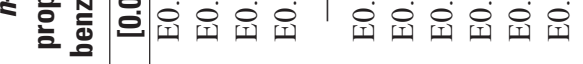

言兽

造

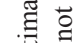

के

它声

言产

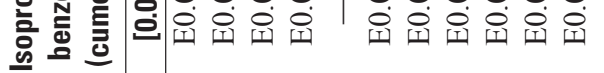

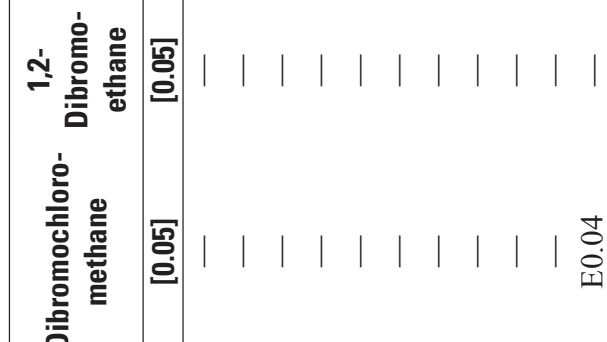

0

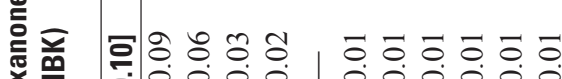

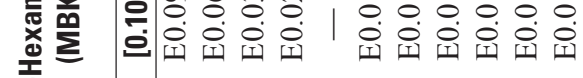

吾

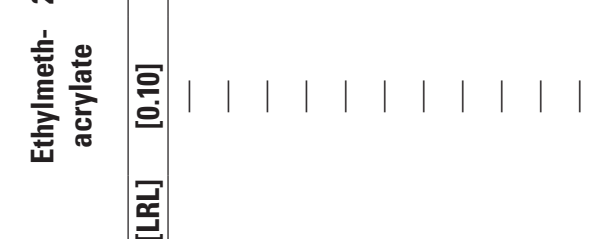

従

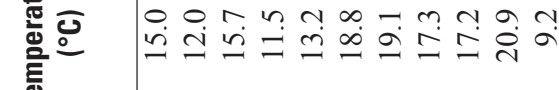

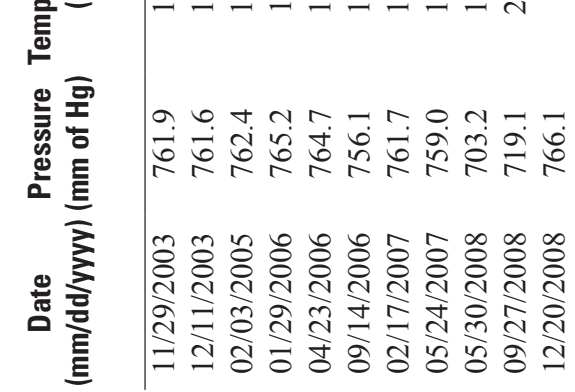

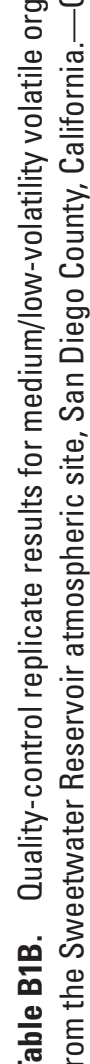

\section{दे}

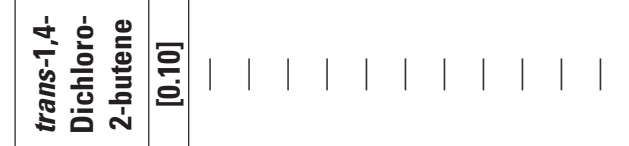

है,

䒠 원

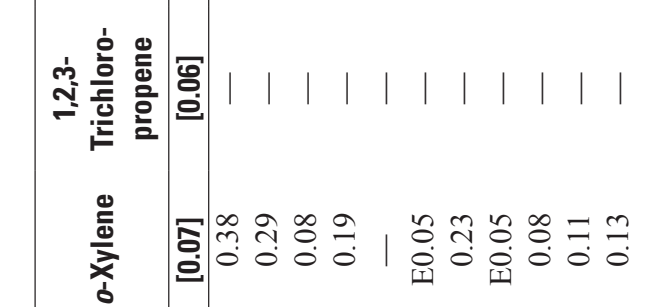

>들

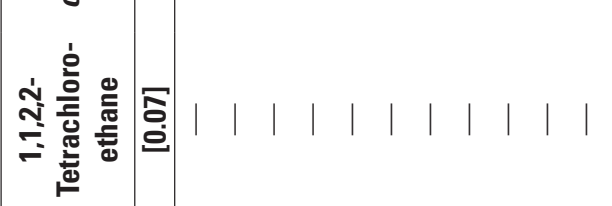

잉

吾 Dी

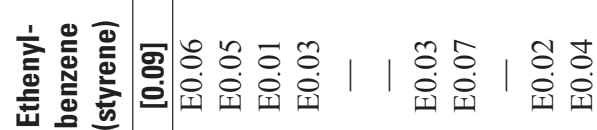

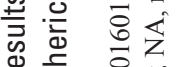

造言

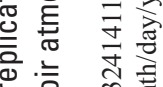

을

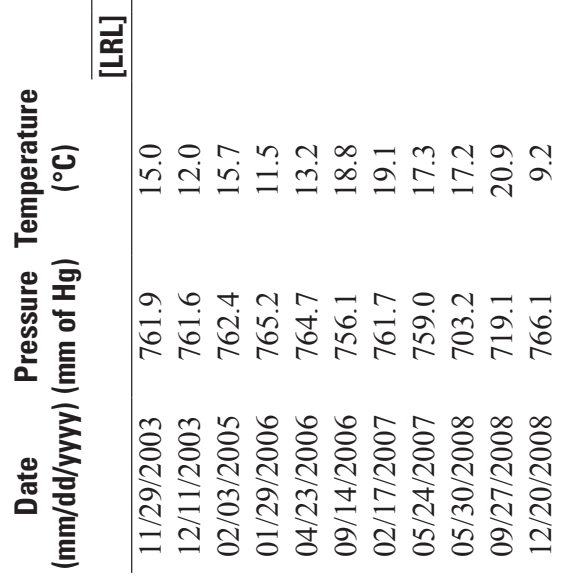



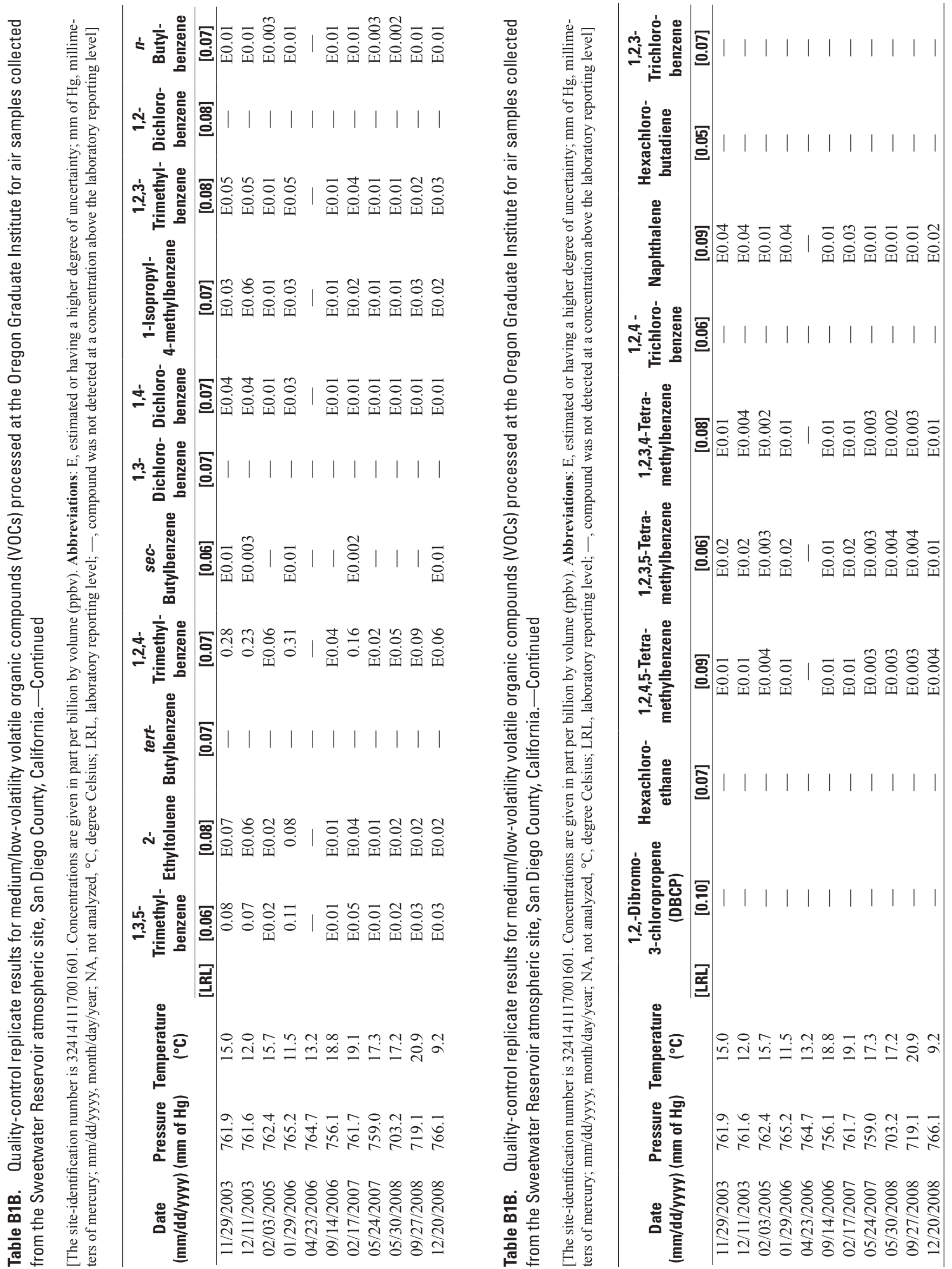

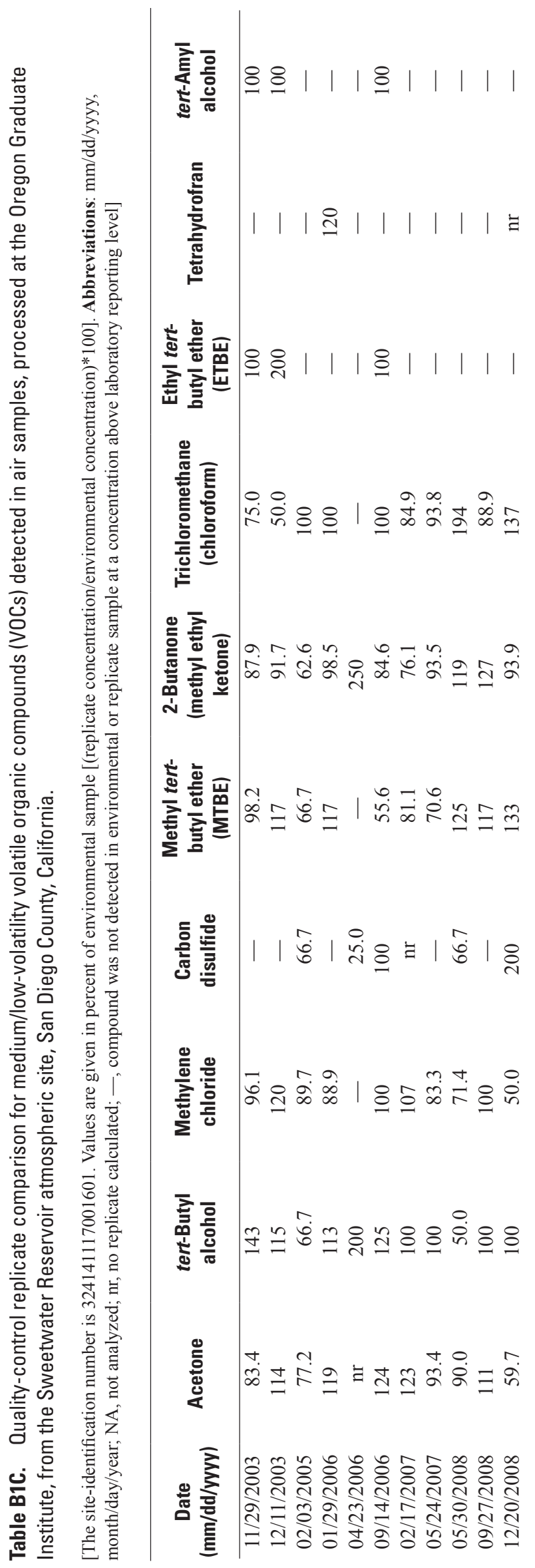

혼

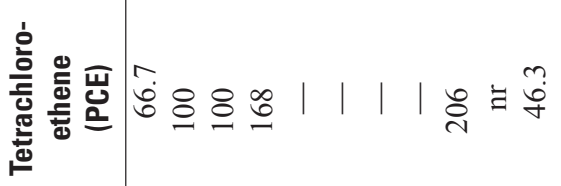
铥 高

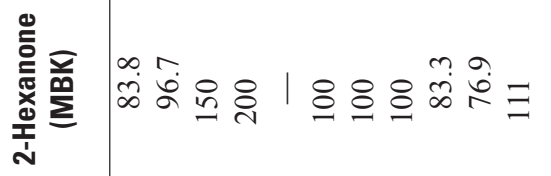

产

辛畜 竞崖 한 


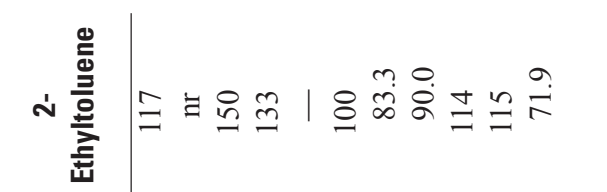

ڤ

定

产

을

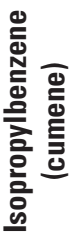

豙

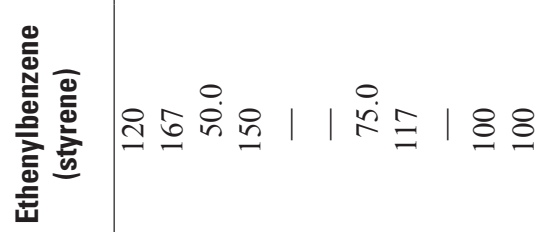

흘

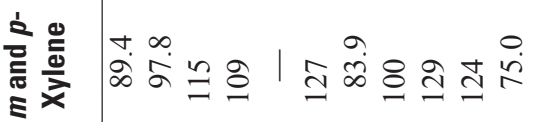

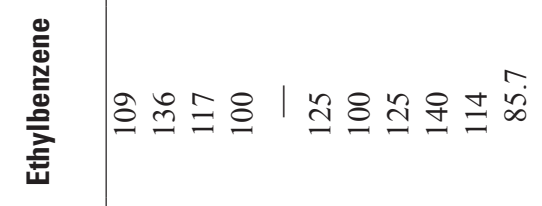

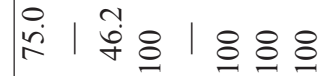

음

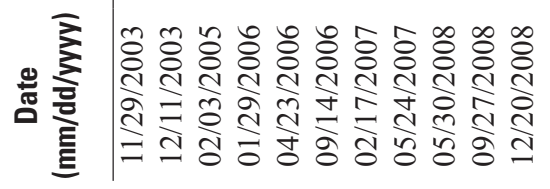

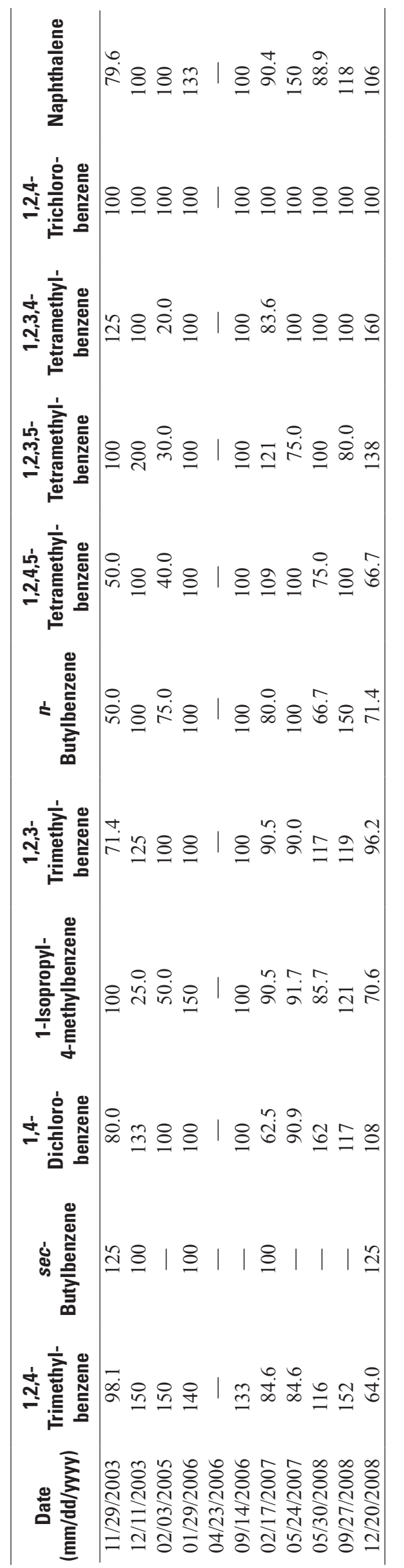


Table B2A. Quality-control travel spike results for high-volatility volatile organic compounds (VOCs) processed at the Oregon Graduate Institute for air samples collected from the Sweetwater Reservoir atmospheric site, San Diego County, California.

[The site-identification number is 324141117001601 . Values are given in percentage recovery. Sample volume for quality-assurance spike is 1.5 liters. Abbreviations: mm/dd/yyyy, month/day/year; NA, not analyzed]

\begin{tabular}{|c|c|c|c|c|c|c|c|}
\hline $\begin{array}{c}\text { Sample } \\
\text { date } \\
\text { (mm/dd/yyyy) }\end{array}$ & $\begin{array}{c}\text { Analyzed } \\
\text { date } \\
\text { (mm/dd/yyyy) }\end{array}$ & $\begin{array}{c}\text { Cartridge } \\
\text { number }\end{array}$ & Chloromethane & $\begin{array}{l}\text { Chloroethene } \\
\text { (vinyl chloride) }\end{array}$ & Bromomethane & Chloroethane & $\begin{array}{l}\text { Bromoethene } \\
\text { (vinyl bromide) }\end{array}$ \\
\hline $11 / 29 / 2003$ & $12 / 18 / 2003$ & Y16058 & 74.3 & 72.3 & NA & 76.2 & 89.4 \\
\hline $12 / 23 / 2003$ & $01 / 29 / 2004$ & Y16119 & 94.3 & 70.5 & NA & 77.6 & 88.8 \\
\hline $01 / 14 / 2004$ & $02 / 06 / 2004$ & Y05951 & 74.9 & 81.5 & NA & 85.9 & 85.6 \\
\hline 04/09/2004 & $04 / 16 / 2004$ & Y16133 & 81.5 & 87.2 & NA & 83.8 & 99.9 \\
\hline $04 / 21 / 2004$ & 05/06/2004 & Y16137 & 113 & 94.1 & NA & 71.4 & 98.9 \\
\hline $07 / 26 / 2004$ & $08 / 25 / 2004$ & Y12889 & 78.0 & 96.5 & NA & 72.2 & 101 \\
\hline $01 / 22 / 2005$ & $02 / 10 / 2005$ & Y15939 & 124 & 72.1 & NA & 77.0 & 97.9 \\
\hline $02 / 15 / 2005$ & $03 / 03 / 2005$ & Y12858 & 76.1 & 75.1 & NA & 78.8 & 82.6 \\
\hline $03 / 11 / 2005$ & $03 / 30 / 2005$ & Y14520 & 90.9 & 95.7 & NA & 69.3 & 98.8 \\
\hline $05 / 10 / 2005$ & $05 / 17 / 2005$ & Y16058 & 86.5 & 85.6 & NA & 74.2 & 86.9 \\
\hline $06 / 03 / 2005$ & $06 / 20 / 2005$ & Y16058 & 78.2 & 75.6 & NA & 65.1 & 90.9 \\
\hline 08/02/2005 & 08/17/2005 & Y16133 & 132 & 68.7 & NA & 71.3 & 83.0 \\
\hline 09/19/2005 & $10 / 05 / 2005$ & Y06586 & 78.4 & 98.4 & NA & 79.1 & 97.1 \\
\hline $10 / 13 / 2005$ & $11 / 02 / 2005$ & Y15161 & 90.2 & 65.1 & NA & 73.0 & 74.3 \\
\hline $01 / 29 / 2006$ & $02 / 15 / 2006$ & Y16096 & 79.3 & 72.7 & NA & 71.4 & 79.0 \\
\hline $02 / 22 / 2006$ & 03/09/2006 & Y12858 & 70.6 & 95.1 & NA & 70.5 & 109 \\
\hline $04 / 23 / 2006$ & $05 / 11 / 2006$ & Y16128 & 90.7 & 105 & NA & 81.6 & 111 \\
\hline 09/14/2006 & $10 / 02 / 2006$ & Y05908 & 83.2 & 93.6 & NA & 72.0 & 108 \\
\hline $10 / 08 / 2006$ & $10 / 25 / 2006$ & Y12870 & 60.2 & 89.3 & NA & 61.8 & 102 \\
\hline $02 / 17 / 2007$ & 03/08/2007 & Y05908 & 63.8 & 74.4 & NA & 78.5 & 83.4 \\
\hline $04 / 30 / 2007$ & $05 / 16 / 2007$ & Y16139 & 69.9 & 76.8 & NA & 65.4 & 83.8 \\
\hline $05 / 24 / 2007$ & $06 / 14 / 2007$ & Y16099 & 81.4 & 78.3 & NA & 71.7 & 85.1 \\
\hline 08/16/2007 & 09/05/2007 & Y16119 & 75.4 & 79.5 & NA & 72.0 & 90.9 \\
\hline $10 / 15 / 2007$ & $10 / 19 / 2007$ & Y05868 & 94.5 & 91.6 & NA & 59.8 & 101 \\
\hline $11 / 20 / 2007$ & $12 / 06 / 2007$ & Y16139 & 96.2 & 87.0 & NA & 78.6 & 99.8 \\
\hline $01 / 07 / 2008$ & $01 / 23 / 2008$ & Y15989 & 81.6 & 71.1 & NA & 92.1 & 94.9 \\
\hline $01 / 31 / 2008$ & $02 / 19 / 2008$ & Y05161 & 83.0 & 91.3 & NA & 61.4 & 109 \\
\hline $02 / 24 / 2008$ & 03/13/2008 & Y05908 & 68.6 & 87.7 & NA & 65.7 & 101 \\
\hline 03/19/2008 & $04 / 03 / 2008$ & Y11977 & 111 & 76.1 & NA & 99.1 & 88.4 \\
\hline $04 / 24 / 2008$ & $04 / 30 / 2008$ & Y12883 & 67.4 & 67.1 & NA & 74.7 & 74.3 \\
\hline $05 / 30 / 2008$ & $06 / 18 / 2008$ & Y05908 & 70.2 & 75.4 & NA & 70.9 & 72.7 \\
\hline 08/10/2008 & 08/28/2008 & Y05161 & 92.4 & 71.6 & NA & 61.8 & 90.5 \\
\hline 09/27/2008 & $10 / 16 / 2008$ & Y16119 & 64.6 & 74.7 & NA & 79.0 & 84.6 \\
\hline $12 / 20 / 2008$ & $12 / 31 / 2008$ & Y12874 & 63.9 & 85.1 & NA & 74.8 & 90.2 \\
\hline 02/18/2009 & 03/04/2009 & Y05951 & 73.0 & 72.1 & NA & 65.4 & 84.9 \\
\hline $04 / 07 / 2009$ & $04 / 23 / 2009$ & Y16052 & 78.4 & 91.6 & NA & 67.6 & 94.7 \\
\hline $05 / 25 / 2009$ & $06 / 11 / 2009$ & Y15939 & 86.4 & 65.8 & NA & 71.4 & 76.7 \\
\hline
\end{tabular}


Table B2B. Quality-control analytical travel blank results for high-volatility volatile organic compounds (VOCs) processed at the Oregon Graduate Institute for air samples collected from the Sweetwater Reservoir atmospheric site, San Diego County, California.

[The site-identification number is 324141117001601. Concentrations are given in part per billion by volume (ppbv). Sample volume for quality-assurance travel blank is 1.5 liters. Pairs of samples analyzed on the same date had been frozen and stored at laboratory before analysis. Abbreviations: E, estimated or having a higher degree of uncertainty; NA, not analyzed; mm/dd/yyyy, month/day/year; mm of Hg, millimeters of mercury; LRL, laboratory reporting level; ${ }^{\circ} \mathrm{C}$, degree Celsius; - , compound was not detected at a concentration above laboratory reporting level]

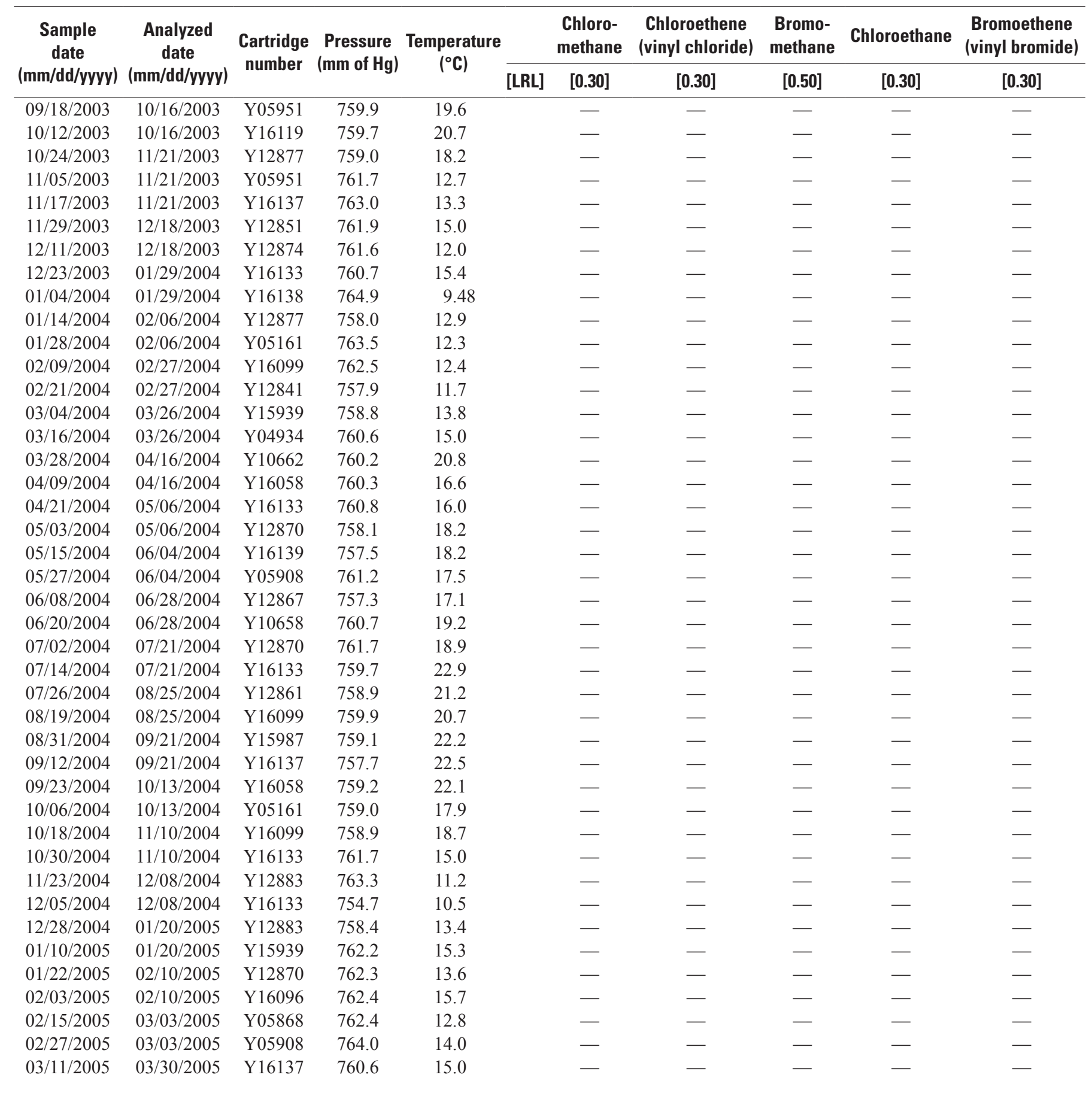


Table B2B. Quality-control analytical travel blank results for high-volatility volatile organic compounds (VOCs) processed at the Oregon Graduate Institute for air samples collected from the Sweetwater Reservoir atmospheric site, San Diego County, California. -Continued

[The site-identification number is 324141117001601 . Concentrations are given in part per billion by volume (ppbv). Sample volume for quality-assurance travel blank is 1.5 liters. Pairs of samples analyzed on the same date had been frozen and stored at laboratory before analysis. Abbreviations: E, estimated or having a higher degree of uncertainty; NA, not analyzed; mm/dd/yyyy, month/day/year; mm of Hg, millimeters of mercury; LRL, laboratory reporting level; ${ }^{\circ} \mathrm{C}$, degree Celsius; - , compound was not detected at a concentration above laboratory reporting level]

\begin{tabular}{|c|c|c|c|c|c|c|c|c|c|c|}
\hline \multirow{2}{*}{$\begin{array}{c}\text { Sample } \\
\text { date } \\
\text { (mm/dd/yyyy) }\end{array}$} & \multirow{2}{*}{$\begin{array}{c}\text { Analyzed } \\
\text { date } \\
\text { (mm/dd/yyyy) }\end{array}$} & \multirow{2}{*}{$\begin{array}{c}\text { Cartridge } \\
\text { number }\end{array}$} & \multirow{2}{*}{$\begin{array}{l}\text { Pressure } \\
(\mathrm{mm} \text { of } \mathrm{Hg})\end{array}$} & \multirow{2}{*}{$\begin{array}{c}\text { Temperature } \\
\left({ }^{\circ} \mathrm{C}\right)\end{array}$} & \multicolumn{2}{|r|}{$\begin{array}{l}\text { Chloro- } \\
\text { methane }\end{array}$} & \multirow{2}{*}{$\begin{array}{c}\begin{array}{c}\text { Chloroethene } \\
\text { (vinyl chloride) }\end{array} \\
{[0.30]}\end{array}$} & \multirow{2}{*}{$\begin{array}{c}\begin{array}{c}\text { Bromo- } \\
\text { methane }\end{array} \\
{[0.50]}\end{array}$} & \multirow{2}{*}{$\begin{array}{c}\text { Chloroethane } \\
{[0.30]}\end{array}$} & \multirow{2}{*}{$\begin{array}{c}\begin{array}{c}\text { Bromoethene } \\
\text { (vinyl bromide) }\end{array} \\
{[0.30]}\end{array}$} \\
\hline & & & & & [LRL] & {$[0.30]$} & & & & \\
\hline $04 / 04 / 2005$ & $04 / 20 / 2005$ & Y12889 & 762.7 & 14.0 & & - & - & - & - & - \\
\hline $04 / 16 / 2005$ & $04 / 20 / 2005$ & Y12874 & 759.3 & 16.0 & & - & - & - & - & - \\
\hline $04 / 28 / 2005$ & $05 / 17 / 2005$ & Y16139 & 762.0 & 15.1 & & - & - & - & - & - \\
\hline $06 / 15 / 2005$ & $06 / 20 / 2005$ & Y15939 & 758.1 & 17.8 & & - & - & - & - & - \\
\hline 07/09/2005 & $07 / 28 / 2005$ & Y16139 & 759.2 & 18.7 & & - & - & - & - & - \\
\hline $07 / 25 / 2005$ & $07 / 28 / 2005$ & Y12889 & 757.0 & 25.4 & & - & - & - & - & - \\
\hline 08/02/2005 & 08/17/2005 & Y15939 & 758.9 & 20.6 & & - & - & - & - & - \\
\hline $08 / 14 / 2005$ & 08/17/2005 & Y16058 & 757.9 & 19.4 & & - & - & - & - & - \\
\hline $10 / 13 / 2005$ & $11 / 02 / 2005$ & Y12883 & 759.5 & 20.3 & & - & - & - & - & - \\
\hline $10 / 25 / 2005$ & $11 / 02 / 2005$ & Y12874 & 763.4 & 16.4 & & - & - & - & - & - \\
\hline $11 / 06 / 2005$ & $11 / 29 / 2005$ & Y12889 & 759.0 & 15.0 & & - & - & - & - & - \\
\hline $11 / 18 / 2005$ & $11 / 29 / 2005$ & Y15939 & 762.4 & 20.2 & & - & - & - & - & - \\
\hline $12 / 12 / 2005$ & $01 / 04 / 2006$ & Y16099 & 762.7 & 12.3 & & - & - & - & - & - \\
\hline $12 / 24 / 2005$ & $01 / 04 / 2006$ & Y15939 & 762.8 & 13.3 & & - & - & - & - & - \\
\hline $01 / 06 / 2006$ & $01 / 24 / 2006$ & Y16137 & 765.0 & 18.1 & & - & - & - & - & - \\
\hline $01 / 17 / 2006$ & $01 / 24 / 2006$ & Y16099 & 768.0 & 11.8 & & - & - & - & - & - \\
\hline 01/29/2006 & $02 / 15 / 2006$ & Y12874 & 765.2 & 11.5 & & - & - & - & - & - \\
\hline $02 / 10 / 2006$ & $02 / 15 / 2006$ & Y16137 & 764.0 & 12.9 & & - & - & - & - & - \\
\hline $02 / 22 / 2006$ & 03/09/2006 & Y12861 & 763.3 & 11.2 & & NA & NA & NA & NA & NA \\
\hline 07/04/2006 & $07 / 12 / 2006$ & Y12841 & 760.7 & 21.9 & & - & - & - & - & - \\
\hline $07 / 28 / 2006$ & 08/14/2006 & Y16096 & 759.6 & 25.1 & & - & - & - & - & - \\
\hline 08/09/2006 & 08/14/2006 & Y12841 & 758.4 & 23.8 & & - & - & - & - & - \\
\hline $08 / 21 / 2006$ & 09/08/2006 & Y12874 & 758.5 & 21.7 & & - & - & - & - & - \\
\hline 09/02/2006 & 09/08/2006 & Y16119 & 755.5 & 23.2 & & - & - & - & - & - \\
\hline 09/14/2006 & $10 / 02 / 2006$ & Y16139 & 756.1 & 18.8 & & - & - & - & - & - \\
\hline $09 / 26 / 2006$ & $10 / 02 / 2006$ & Y12874 & 761.0 & 19.3 & & - & - & - & - & - \\
\hline $10 / 08 / 2006$ & $10 / 25 / 2006$ & Y06313 & 759.4 & 16.5 & & - & - & - & - & - \\
\hline $10 / 20 / 2006$ & $10 / 25 / 2006$ & Y16119 & 756.8 & 19.4 & & - & - & - & - & - \\
\hline
\end{tabular}


Table B2B. Quality-control analytical travel blank results for high-volatility volatile organic compounds (VOCs) processed at the Oregon Graduate Institute for air samples collected from the Sweetwater Reservoir atmospheric site, San Diego County, California. -Continued

[The site-identification number is 324141117001601. Concentrations are given in part per billion by volume (ppbv). Sample volume for quality-assurance travel blank is 1.5 liters. Pairs of samples analyzed on the same date had been frozen and stored at laboratory before analysis. Abbreviations: E, estimated or having a higher degree of uncertainty; NA, not analyzed; mm/dd/yyyy, month/day/year; mm of Hg, millimeters of mercury; LRL, laboratory reporting level; ${ }^{\circ} \mathrm{C}$, degree Celsius; - , compound was not detected at a concentration above laboratory reporting level]

\begin{tabular}{|c|c|c|c|c|c|c|c|c|c|c|}
\hline \multirow{2}{*}{$\begin{array}{c}\text { Sample } \\
\text { date } \\
\text { (mm/dd/yyyy) }\end{array}$} & \multirow{2}{*}{$\begin{array}{c}\text { Analyzed } \\
\text { date } \\
\text { (mm/dd/yyyy) }\end{array}$} & \multirow{2}{*}{$\begin{array}{c}\text { Cartridge } \\
\text { number }\end{array}$} & \multirow{2}{*}{$\begin{array}{l}\text { Pressure } \\
(\mathrm{mm} \text { of } \mathrm{Hg})\end{array}$} & \multirow{2}{*}{$\begin{array}{c}\text { Temperature } \\
\left({ }^{\circ} \mathrm{C}\right)\end{array}$} & \multicolumn{2}{|r|}{$\begin{array}{l}\text { Chloro- } \\
\text { methane }\end{array}$} & \multirow{2}{*}{$\begin{array}{c}\begin{array}{c}\text { Chloroethene } \\
\text { (vinyl chloride) }\end{array} \\
{[0.30]}\end{array}$} & \multirow{2}{*}{$\begin{array}{c}\begin{array}{c}\text { Bromo- } \\
\text { methane }\end{array} \\
{[0.50]}\end{array}$} & \multirow{2}{*}{$\begin{array}{c}\text { Chloroethane } \\
{[0.30]}\end{array}$} & \multirow{2}{*}{$\begin{array}{c}\begin{array}{c}\text { Bromoethene } \\
\text { (vinyl bromide) }\end{array} \\
{[0.30]}\end{array}$} \\
\hline & & & & & $\overline{\text { [LRL] }}$ & {$[0.30]$} & & & & \\
\hline $11 / 13 / 2006$ & $11 / 17 / 2006$ & Y16096 & 715.9 & 16.9 & & - & - & - & - & - \\
\hline $11 / 25 / 2006$ & $12 / 15 / 2006$ & Y06011 & 706.9 & 14.5 & & - & - & - & - & - \\
\hline $12 / 07 / 2006$ & $12 / 15 / 2006$ & Y14520 & 762.7 & 15.7 & & - & - & - & - & - \\
\hline $01 / 24 / 2007$ & $02 / 07 / 2007$ & Y15939 & 764.2 & 13.4 & & - & - & - & - & - \\
\hline $02 / 05 / 2007$ & $02 / 07 / 2007$ & Y12867 & 763.2 & 17.3 & & - & - & - & - & - \\
\hline $02 / 17 / 2007$ & $03 / 08 / 2007$ & Y16058 & 761.7 & 19.1 & & - & - & - & - & - \\
\hline 03/01/2007 & 03/08/2007 & Y12870 & 763.3 & 9.9 & & - & - & - & - & - \\
\hline 03/13/2007 & 03/29/2007 & Y16119 & 761.4 & 16.2 & & - & - & - & - & - \\
\hline $05 / 12 / 2007$ & $05 / 16 / 2007$ & Y15939 & 761.1 & 15.5 & & - & - & - & - & - \\
\hline $05 / 24 / 2007$ & $06 / 14 / 2007$ & Y05161 & 759.0 & 17.3 & & - & - & - & - & - \\
\hline $06 / 05 / 2007$ & $06 / 14 / 2007$ & Y16119 & 759.0 & 16.4 & & - & - & - & - & - \\
\hline $06 / 17 / 2007$ & $07 / 11 / 2007$ & Y12870 & 758.2 & 18.0 & & - & - & - & - & - \\
\hline 06/29/2007 & $07 / 11 / 2007$ & Y12874 & 758.9 & 19.8 & & - & - & - & - & - \\
\hline $07 / 11 / 2007$ & $07 / 13 / 2007$ & Y16052 & 745.0 & 20.8 & & - & - & - & - & - \\
\hline $07 / 23 / 2007$ & 08/09/2007 & Y16133 & 758.3 & 20.9 & & - & - & - & - & - \\
\hline 08/04/2007 & $08 / 09 / 2007$ & Y16058 & 701.1 & 20.7 & & - & - & - & - & - \\
\hline 08/16/2007 & $09 / 05 / 2007$ & Y05161 & 699.5 & 22.9 & & - & - & - & - & - \\
\hline 08/28/2007 & $09 / 05 / 2007$ & Y16099 & 756.0 & 24.6 & & - & - & - & - & - \\
\hline 09/09/2007 & $10 / 02 / 2007$ & Y14520 & 758.7 & 20.6 & & - & - & - & - & - \\
\hline $12 / 14 / 2007$ & $01 / 03 / 2008$ & Y16134 & 765.5 & 10.2 & & - & - & - & - & - \\
\hline $12 / 26 / 2007$ & $01 / 03 / 2008$ & Y16052 & 762.4 & 9.71 & & - & - & - & - & - \\
\hline 01/07/2008 & $01 / 23 / 2008$ & Y12841 & 760.1 & 12.0 & & - & - & - & - & - \\
\hline 01/19/2008 & $01 / 23 / 2008$ & Y16119 & 707.0 & 10.7 & & - & - & - & - & - \\
\hline $01 / 31 / 2008$ & $02 / 19 / 2008$ & Y16134 & 766.8 & 10.9 & & - & - & - & - & - \\
\hline $02 / 12 / 2008$ & $02 / 19 / 2008$ & Y12841 & 710.6 & 16.0 & & - & - & - & - & - \\
\hline $02 / 24 / 2008$ & $03 / 13 / 2008$ & Y12883 & 716.2 & 12.2 & & - & - & - & - & - \\
\hline 03/07/2008 & $03 / 13 / 2008$ & Y05161 & 706.1 & 15.9 & & - & - & - & - & - \\
\hline $03 / 19 / 2008$ & $04 / 03 / 2008$ & Y12867 & 763.9 & 12.8 & & - & - & - & - & - \\
\hline
\end{tabular}


Table B2B. Quality-control analytical travel blank results for high-volatility volatile organic compounds (VOCs) processed at the Oregon Graduate Institute for air samples collected from the Sweetwater Reservoir atmospheric site, San Diego County, California. -Continued

[The site-identification number is 324141117001601 . Concentrations are given in part per billion by volume (ppbv). Sample volume for quality-assurance travel blank is 1.5 liters. Pairs of samples analyzed on the same date had been frozen and stored at laboratory before analysis. Abbreviations: E, estimated or having a higher degree of uncertainty; NA, not analyzed; mm/dd/yyyy, month/day/year; mm of Hg, millimeters of mercury; LRL, laboratory reporting level; ${ }^{\circ} \mathrm{C}$, degree Celsius; - , compound was not detected at a concentration above laboratory reporting level]

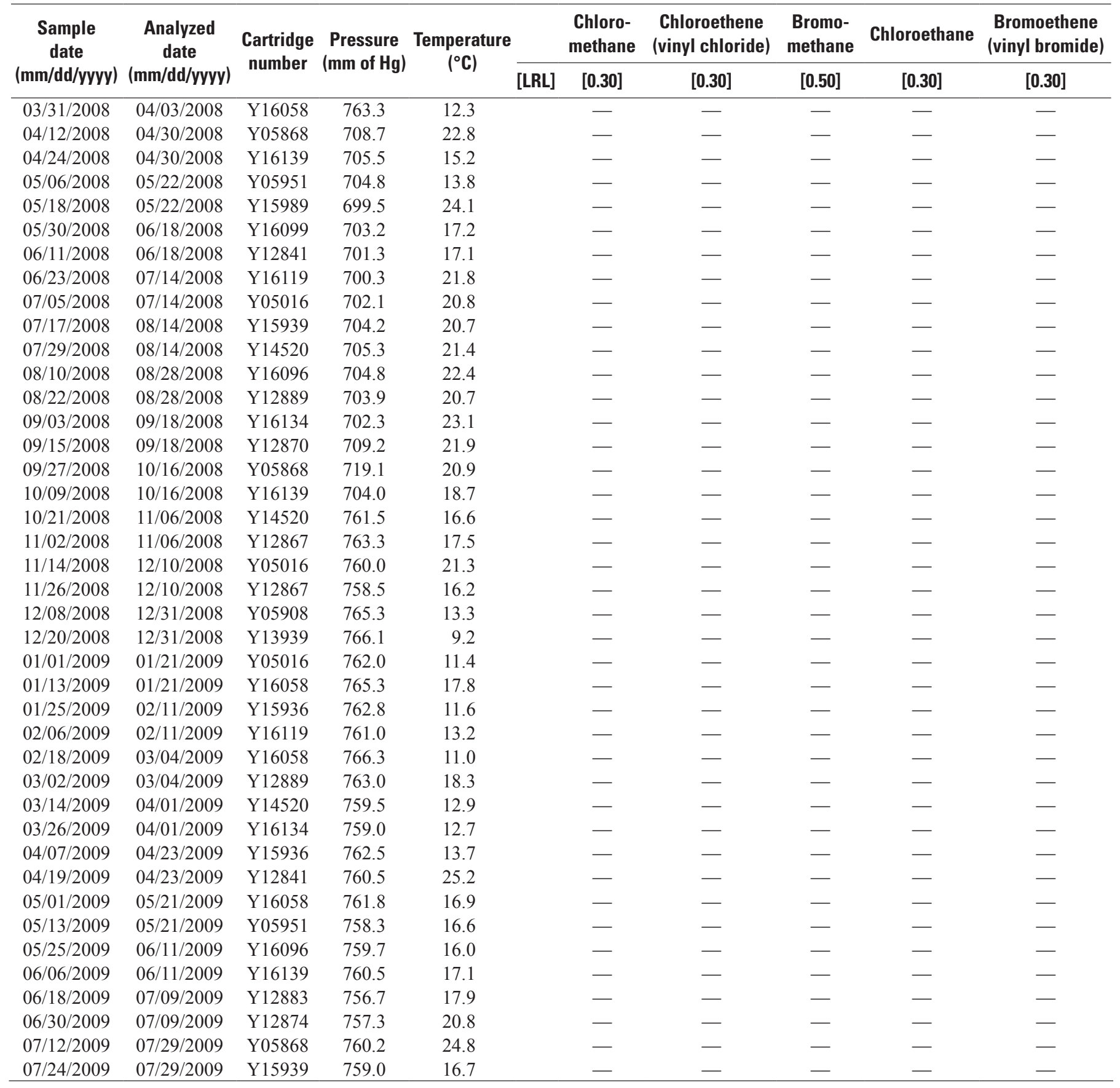


Table B2C. Quality-control analytical lot blank results for high-volatility volatile organic compounds (VOCs) processed at the Oregon Graduate Institute for air samples collected from the Sweetwater Reservoir atmospheric site, San Diego County, California.

[The site-identification number is 324141117001601 . Concentrations are given in part per billion by volume (ppbv). Sample volume for quality-assurance lot-blank is 1.5 liters. Pairs of samples analyzed on the same date had been frozen and stored at laboratory before analysis. Samples processed at 20.0 degrees Celsius and 760 millimeters of mercury. Abbreviations: E, estimated or having a higher degree of uncertainty; mm/dd/yyyy, month/day/year; —, compound was not detected at a concentration above laboratory reporting level; LRL, laboratory reporting level]

\begin{tabular}{|c|c|c|c|c|c|c|c|}
\hline $\begin{array}{c}\text { Sample } \\
\text { date }\end{array}$ & $\begin{array}{l}\text { Analyzed } \\
\text { date }\end{array}$ & Cartridge & Chloromethane & $\begin{array}{l}\text { Chloroethene } \\
\text { (vinyl chloride) }\end{array}$ & Bromomethane & Chloroethane & $\begin{array}{l}\text { Bromoethene } \\
\text { (vinyl bromide) }\end{array}$ \\
\hline (mm/dd/yyyy) & & number [LRL] & {$[0.30]$} & {$[0.30]$} & [0.50] & {$[0.30]$} & {$[0.30]$} \\
\hline $10 / 12 / 2003$ & $10 / 16 / 2003$ & Y12883 & - & - & - & - & - \\
\hline $10 / 24 / 2003$ & $11 / 21 / 2003$ & Y12861 & - & - & - & - & - \\
\hline $11 / 05 / 2003$ & $11 / 21 / 2003$ & Y16138 & - & - & - & - & - \\
\hline $11 / 17 / 2003$ & $11 / 21 / 2003$ & Y12851 & - & - & - & - & - \\
\hline $11 / 29 / 2003$ & $12 / 18 / 2003$ & Y16138 & - & - & - & - & - \\
\hline $12 / 11 / 2003$ & $12 / 18 / 2003$ & Y16096 & - & - & - & - & - \\
\hline $12 / 23 / 2003$ & $01 / 29 / 2004$ & Y12883 & - & - & - & - & - \\
\hline $01 / 04 / 2004$ & $01 / 29 / 2004$ & Y16128 & - & - & - & - & - \\
\hline $01 / 14 / 2004$ & $02 / 06 / 2004$ & Y12874 & - & - & - & - & - \\
\hline $01 / 28 / 2004$ & $02 / 06 / 2004$ & Y12867 & - & - & - & - & - \\
\hline 02/09/2004 & $02 / 27 / 2004$ & Y06012 & - & - & - & - & - \\
\hline $02 / 21 / 2004$ & $02 / 27 / 2004$ & Y12870 & - & - & - & - & - \\
\hline 03/04/2004 & $03 / 26 / 2004$ & Y12858 & - & - & - & - & - \\
\hline $03 / 16 / 2004$ & $03 / 26 / 2004$ & Y16119 & - & - & - & - & - \\
\hline $03 / 28 / 2004$ & $04 / 16 / 2004$ & Y16128 & - & - & - & - & - \\
\hline $04 / 09 / 2004$ & $04 / 16 / 2004$ & Y12858 & - & - & - & - & - \\
\hline $04 / 21 / 2004$ & $05 / 06 / 2004$ & Y15939 & - & - & - & - & - \\
\hline $05 / 03 / 2004$ & 05/06/2004 & Y12874 & - & - & - & - & - \\
\hline $05 / 15 / 2004$ & $06 / 04 / 2004$ & Y16113 & - & - & - & - & - \\
\hline $05 / 27 / 2004$ & $06 / 04 / 2004$ & Y05951 & - & - & - & - & - \\
\hline 06/08/2004 & $06 / 28 / 2004$ & Y10662 & - & - & - & - & - \\
\hline $06 / 20 / 2004$ & $06 / 28 / 2004$ & Y12858 & - & - & - & - & - \\
\hline $07 / 02 / 2004$ & $07 / 21 / 2004$ & Y12861 & - & - & - & - & - \\
\hline $07 / 14 / 2004$ & $07 / 21 / 2004$ & Y12858 & - & - & - & - & - \\
\hline $07 / 26 / 2004$ & $08 / 25 / 2004$ & Y05951 & - & - & - & - & - \\
\hline 08/19/2004 & $08 / 25 / 2004$ & Y16128 & - & - & - & - & - \\
\hline $08 / 31 / 2004$ & $09 / 21 / 2004$ & Y16133 & - & - & - & - & - \\
\hline $09 / 12 / 2004$ & $09 / 21 / 2004$ & Y16139 & - & - & - & - & - \\
\hline $09 / 23 / 2004$ & $10 / 13 / 2004$ & Y12874 & - & - & - & - & - \\
\hline $10 / 06 / 2004$ & $10 / 13 / 2004$ & Y15936 & - & - & - & - & - \\
\hline $10 / 18 / 2004$ & $11 / 10 / 2004$ & Y16119 & - & - & - & - & - \\
\hline $10 / 30 / 2004$ & $11 / 10 / 2004$ & Y06012 & - & - & - & - & - \\
\hline $11 / 23 / 2004$ & $12 / 08 / 2004$ & Y05161 & - & - & - & - & - \\
\hline $12 / 05 / 2004$ & $12 / 08 / 2004$ & Y12867 & - & - & - & - & - \\
\hline $12 / 28 / 2004$ & $01 / 20 / 2005$ & Y12889 & - & - & - & - & - \\
\hline $01 / 10 / 2005$ & $01 / 20 / 2005$ & Y16119 & - & - & - & - & - \\
\hline $01 / 22 / 2005$ & $02 / 10 / 2005$ & Y12877 & - & - & - & - & - \\
\hline $02 / 03 / 2005$ & $02 / 10 / 2005$ & Y05908 & - & - & - & - & - \\
\hline $02 / 15 / 2005$ & $03 / 03 / 2005$ & Y05161 & - & - & - & - & - \\
\hline $02 / 27 / 2005$ & $03 / 03 / 2005$ & Y16133 & - & - & - & - & - \\
\hline $03 / 11 / 2005$ & $03 / 30 / 2005$ & Y15936 & - & - & - & - & - \\
\hline $03 / 23 / 2005$ & $03 / 30 / 2005$ & Y16058 & - & - & - & - & - \\
\hline
\end{tabular}


Table B2C. Quality-control analytical lot blank results for high-volatility volatile organic compounds (VOCs) processed at the Oregon Graduate Institute for air samples collected from the Sweetwater Reservoir atmospheric site, San Diego County, California.—Continued

[The site-identification number is 324141117001601 . Concentrations are given in part per billion by volume (ppbv). Sample volume for quality-assurance lot-blank is 1.5 liters. Pairs of samples analyzed on the same date had been frozen and stored at laboratory before analysis. Samples processed at 20.0 degrees Celsius and 760 millimeters of mercury. Abbreviations: E, estimated or having a higher degree of uncertainty; mm/dd/yyyy, month/day/year; - , compound was not detected at a concentration above laboratory reporting level; LRL, laboratory reporting level]

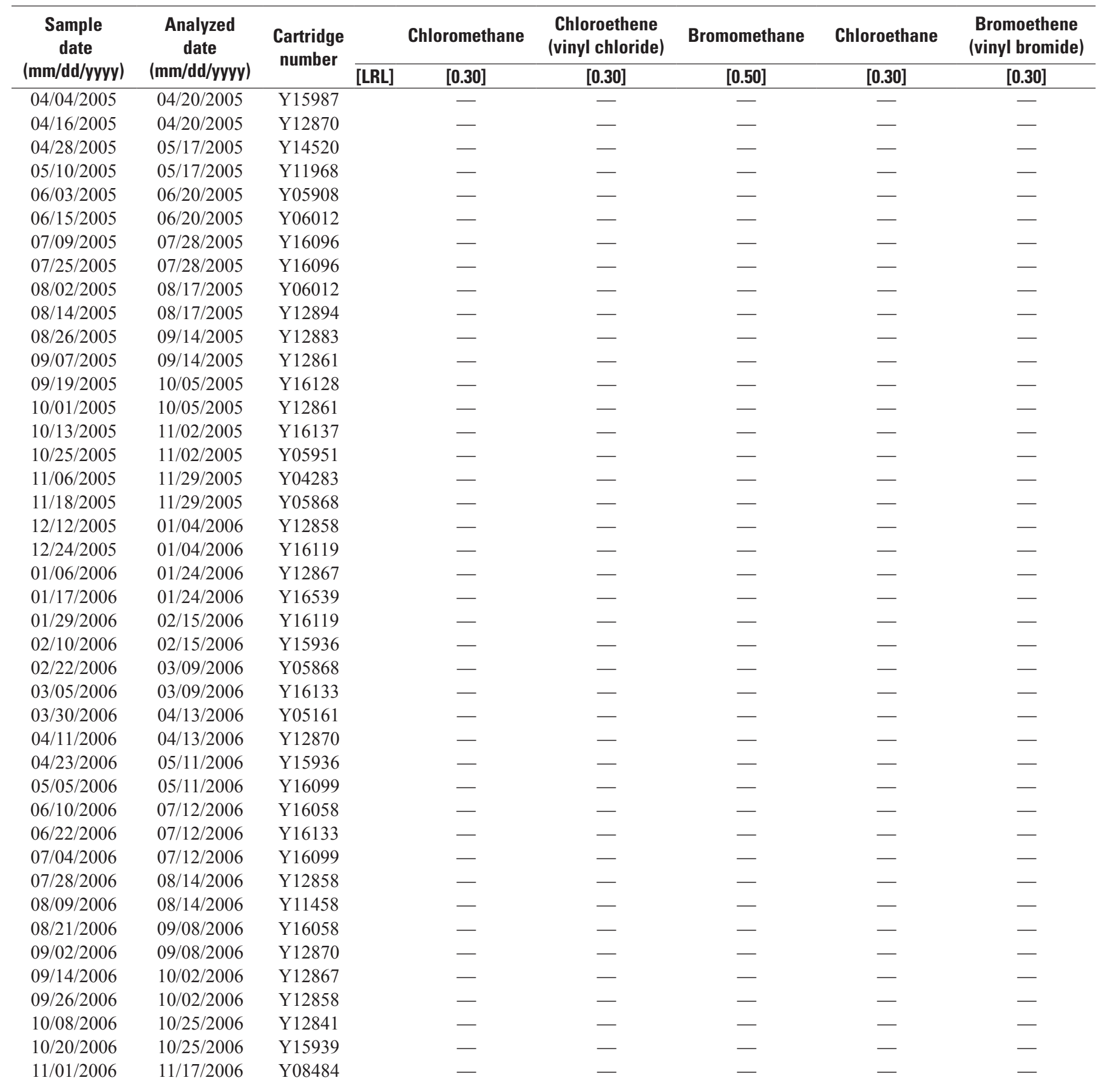


Table B2C. Quality-control analytical lot blank results for high-volatility volatile organic compounds (VOCs) processed at the Oregon Graduate Institute for air samples collected from the Sweetwater Reservoir atmospheric site, San Diego County, California.—Continued

[The site-identification number is 324141117001601 . Concentrations are given in part per billion by volume (ppbv). Sample volume for quality-assurance lot-blank is 1.5 liters. Pairs of samples analyzed on the same date had been frozen and stored at laboratory before analysis. Samples processed at 20.0 degrees Celsius and 760 millimeters of mercury. Abbreviations: E, estimated or having a higher degree of uncertainty; mm/dd/yyyy, month/day/year; —, compound was not detected at a concentration above laboratory reporting level; LRL, laboratory reporting level]

\begin{tabular}{|c|c|c|c|c|c|c|c|}
\hline $\begin{array}{c}\text { Sample } \\
\text { date }\end{array}$ & $\begin{array}{c}\text { Analyzed } \\
\text { date }\end{array}$ & Cartridge & Chloromethane & $\begin{array}{l}\text { Chloroethene } \\
\text { (vinyl chloride) }\end{array}$ & Bromomethane & Chloroethane & $\begin{array}{c}\text { Bromoethene } \\
\text { (vinyl bromide) }\end{array}$ \\
\hline (mm/dd/yyyy) & & number [LRL] & {$[0.30]$} & {$[0.30]$} & [0.50] & {$[0.30]$} & {$[0.30]$} \\
\hline $11 / 13 / 2006$ & $11 / 17 / 2006$ & Y08484 & - & - & - & - & - \\
\hline $11 / 25 / 2006$ & $12 / 15 / 2006$ & Y05161 & - & - & - & - & - \\
\hline $12 / 07 / 2006$ & $12 / 15 / 2006$ & Y12874 & - & - & - & - & - \\
\hline $12 / 31 / 2006$ & $01 / 19 / 2007$ & Y12867 & - & - & - & - & - \\
\hline $01 / 12 / 2007$ & $01 / 19 / 2007$ & Y12870 & - & - & - & - & - \\
\hline $01 / 24 / 2007$ & $02 / 07 / 2007$ & Y05908 & - & - & - & - & - \\
\hline $02 / 05 / 2007$ & $02 / 07 / 2007$ & Y16058 & - & - & - & - & - \\
\hline $02 / 17 / 2007$ & $03 / 08 / 2007$ & Y05951 & - & - & - & - & - \\
\hline 03/01/2007 & 03/08/2007 & Y16099 & - & - & - & - & - \\
\hline 03/13/2007 & $03 / 29 / 2007$ & Y12889 & - & - & - & - & - \\
\hline $03 / 25 / 2007$ & 03/29/2007 & Y05868 & - & - & - & - & - \\
\hline $04 / 06 / 2007$ & $05 / 01 / 2007$ & Y14520 & - & - & - & - & - \\
\hline $04 / 18 / 2007$ & 05/01/2007 & Y15989 & - & - & - & - & - \\
\hline $04 / 30 / 2007$ & $05 / 16 / 2007$ & Y12883 & - & - & - & - & - \\
\hline 05/12/2007 & $05 / 16 / 2007$ & Y12841 & - & - & - & - & - \\
\hline $05 / 24 / 2007$ & $06 / 14 / 2007$ & Y16046 & - & - & - & - & - \\
\hline $06 / 05 / 2007$ & $06 / 14 / 2007$ & Y15939 & - & - & - & - & - \\
\hline 06/17/2007 & $07 / 11 / 2007$ & Y16113 & - & - & - & - & - \\
\hline $06 / 29 / 2007$ & $07 / 11 / 2007$ & Y16134 & - & - & - & - & - \\
\hline $07 / 11 / 2007$ & $07 / 13 / 2007$ & Y12889 & - & - & - & - & - \\
\hline $07 / 23 / 2007$ & 08/09/2007 & Y05908 & - & - & - & - & - \\
\hline $08 / 04 / 2007$ & 08/09/2007 & Y12874 & - & - & - & - & - \\
\hline 08/16/2007 & 09/05/2007 & Y12867 & - & - & - & - & - \\
\hline $08 / 28 / 2007$ & 09/05/2007 & Y16139 & - & - & - & - & - \\
\hline 09/09/2007 & $10 / 02 / 2007$ & Y05951 & - & - & - & - & - \\
\hline $09 / 21 / 2007$ & $10 / 02 / 2007$ & Y16099 & - & - & - & - & - \\
\hline $10 / 03 / 2007$ & $10 / 19 / 2007$ & Y16052 & - & - & - & - & - \\
\hline $10 / 15 / 2007$ & $10 / 19 / 2007$ & Y16052 & - & - & - & - & - \\
\hline $10 / 27 / 2007$ & $11 / 13 / 2007$ & Y12889 & - & - & - & - & - \\
\hline $11 / 08 / 2007$ & $11 / 13 / 2007$ & Y12841 & - & - & - & - & - \\
\hline $11 / 20 / 2007$ & $10 / 02 / 2006$ & Y15939 & - & - & - & - & - \\
\hline $12 / 02 / 2007$ & $12 / 06 / 2007$ & Y05868 & - & - & - & - & - \\
\hline $12 / 14 / 2007$ & $01 / 03 / 2008$ & Y12874 & - & - & - & - & - \\
\hline $12 / 26 / 2007$ & $01 / 03 / 2008$ & Y16139 & - & - & - & - & - \\
\hline $01 / 07 / 2008$ & $01 / 23 / 2008$ & Y05868 & - & - & - & - & - \\
\hline 01/19/2008 & $01 / 23 / 2008$ & Y12870 & - & - & - & - & - \\
\hline $01 / 31 / 2008$ & $02 / 19 / 2008$ & Y15936 & - & - & - & - & - \\
\hline $02 / 12 / 2008$ & $02 / 19 / 2008$ & Y12889 & - & - & - & - & - \\
\hline $02 / 24 / 2008$ & 03/13/2008 & Y12089 & - & - & - & - & - \\
\hline $03 / 07 / 2008$ & $03 / 13 / 2008$ & Y12874 & - & - & - & - & - \\
\hline 03/19/2008 & $04 / 03 / 2008$ & Y16139 & - & - & - & - & - \\
\hline
\end{tabular}


Table B2C. Quality-control analytical lot blank results for high-volatility volatile organic compounds (VOCs) processed at the Oregon Graduate Institute for air samples collected from the Sweetwater Reservoir atmospheric site, San Diego County, California.—Continued

[The site-identification number is 324141117001601 . Concentrations are given in part per billion by volume (ppbv). Sample volume for quality-assurance lot-blank is 1.5 liters. Pairs of samples analyzed on the same date had been frozen and stored at laboratory before analysis. Samples processed at 20.0 degrees Celsius and 760 millimeters of mercury. Abbreviations: E, estimated or having a higher degree of uncertainty; mm/dd/yyyy, month/day/year; - , compound was not detected at a concentration above laboratory reporting level; LRL, laboratory reporting level]

\begin{tabular}{|c|c|c|c|c|c|c|c|c|}
\hline \multirow{2}{*}{$\begin{array}{c}\text { Sample } \\
\text { date } \\
\text { (mm/dd/yyyy) }\end{array}$} & \multirow{2}{*}{$\begin{array}{c}\text { Analyzed } \\
\text { date } \\
\text { (mm/dd/yyyy) }\end{array}$} & \multirow{2}{*}{$\begin{array}{c}\text { Cartridge } \\
\text { number }\end{array}$} & \multicolumn{2}{|r|}{ Chloromethane } & \multirow{2}{*}{$\begin{array}{c}\begin{array}{c}\text { Chloroethene } \\
\text { (vinyl chloride) }\end{array} \\
{[0.30]}\end{array}$} & \multirow{2}{*}{$\begin{array}{c}\text { Bromomethane } \\
{[0.50]}\end{array}$} & \multirow{2}{*}{$\begin{array}{c}\text { Chloroethane } \\
{[0.30]}\end{array}$} & \multirow{2}{*}{$\begin{array}{c}\begin{array}{c}\text { Bromoethene } \\
\text { (vinyl bromide) }\end{array} \\
{[0.30]}\end{array}$} \\
\hline & & & [LRL] & {$[0.30]$} & & & & \\
\hline $03 / 31 / 2008$ & $04 / 03 / 2008$ & Y05016 & & - & - & - & - & - \\
\hline $04 / 12 / 2008$ & $04 / 30 / 2008$ & Y05908 & & - & - & - & - & - \\
\hline $04 / 24 / 2008$ & $04 / 30 / 2008$ & Y16099 & & - & - & - & - & - \\
\hline 05/06/2008 & $05 / 22 / 2008$ & Y15989 & & - & - & - & - & - \\
\hline 06/01/2008 & 06/18/2008 & Y12867 & & - & - & - & - & - \\
\hline $06 / 23 / 2008$ & 07/14/2008 & Y26208 & & - & - & - & - & - \\
\hline $07 / 05 / 2008$ & 07/14/2008 & Y05868 & & - & - & - & - & - \\
\hline 07/17/2008 & 08/14/2008 & Y12874 & & - & - & - & - & - \\
\hline $07 / 29 / 2008$ & 08/14/2008 & Y12874 & & - & - & - & - & - \\
\hline 09/27/2008 & $10 / 16 / 2008$ & Y16096 & & - & - & - & - & - \\
\hline $10 / 09 / 2008$ & $10 / 16 / 2008$ & Y05908 & & - & - & - & - & - \\
\hline $10 / 21 / 2008$ & $11 / 06 / 2008$ & Y12867 & & - & - & - & - & - \\
\hline $11 / 02 / 2008$ & $11 / 06 / 2008$ & Y12870 & & - & - & - & - & - \\
\hline $11 / 14 / 2008$ & $12 / 10 / 2008$ & Y12874 & & - & - & - & - & - \\
\hline $11 / 26 / 2008$ & $12 / 10 / 2008$ & Y12841 & & - & - & - & - & - \\
\hline $12 / 08 / 2008$ & $12 / 31 / 2008$ & Y16134 & & - & - & - & - & - \\
\hline $12 / 20 / 2008$ & $12 / 31 / 2008$ & Y12867 & & - & - & - & - & - \\
\hline 01/01/2009 & 01/21/2009 & Y15989 & & - & - & - & - & - \\
\hline 01/13/2009 & 01/21/2009 & Y12874 & & - & - & - & - & - \\
\hline $01 / 25 / 2009$ & 02/11/2009 & Y05868 & & - & - & - & - & - \\
\hline 05/13/2009 & $05 / 21 / 2009$ & Y16119 & & - & - & - & - & - \\
\hline $05 / 25 / 2009$ & 06/11/2009 & Y15989 & & - & - & - & - & - \\
\hline 06/06/2009 & $06 / 11 / 2009$ & Y15989 & & - & - & - & - & - \\
\hline $06 / 18 / 2009$ & 07/09/2009 & Y05951 & & - & - & - & - & - \\
\hline 06/30/2009 & 07/09/2009 & Y15939 & & - & - & - & - & - \\
\hline $07 / 12 / 2009$ & 07/29/2009 & Y16139 & & - & - & - & - & - \\
\hline $07 / 24 / 2009$ & $07 / 29 / 2009$ & Y12870 & & - & - & - & - & - \\
\hline
\end{tabular}


Table B2D. Quality-control analytical laboratory blank results for high-volatility volatile organic compounds (VOCs) processed at the Oregon Graduate Institute for air samples collected from the Sweetwater Reservoir atmospheric site, San Diego County, California.

[The site-identification number is 324141117001601 . Concentrations are given in part per billion by volume (ppbv). Sample volume for quality-assurance labblank is 1.5 liters. Samples processed at 20.0 degrees Celsius and 760 millimeters of mercury. Pairs of samples analyzed on the same date had been frozen and stored at laboratory before analysis. Abbreviations: E, estimated or having a higher degree of uncertainty; - , not detected at a concentration above laboratory reporting level; LRL, laboratory reporting level]

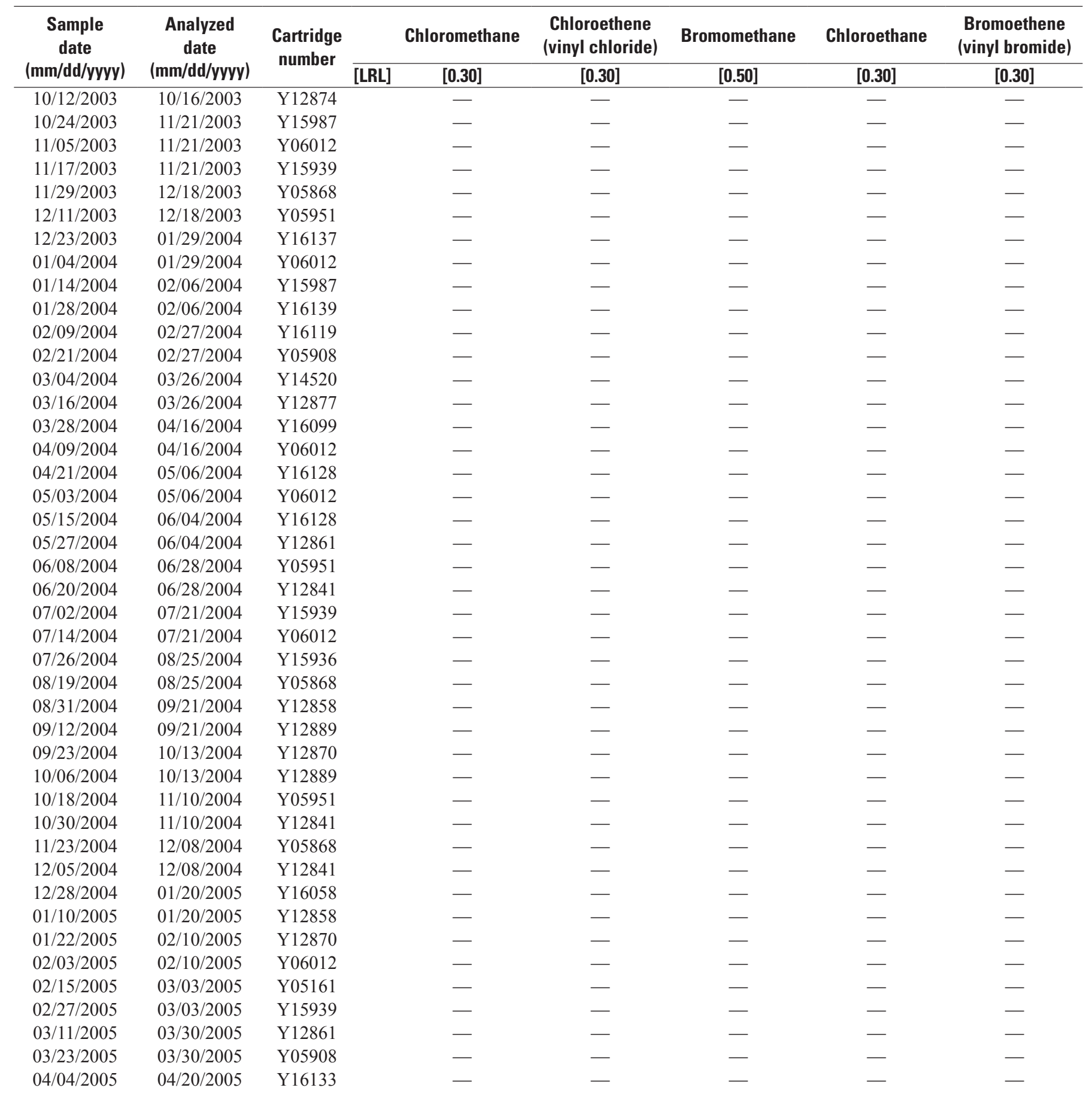


Table B2D. Quality-control analytical laboratory blank results for high-volatility volatile organic compounds (VOCs) processed at the Oregon Graduate Institute for air samples collected from the Sweetwater Reservoir atmospheric site, San Diego County, California. -Continued

[The site-identification number is 324141117001601 . Concentrations are given in part per billion by volume (ppbv). Sample volume for quality-assurance labblank is 1.5 liters. Samples processed at 20.0 degrees Celsius and 760 millimeters of mercury. Pairs of samples analyzed on the same date had been frozen and stored at laboratory before analysis. Abbreviations: E, estimated or having a higher degree of uncertainty; —, not detected at a concentration above laboratory reporting level; LRL, laboratory reporting level]

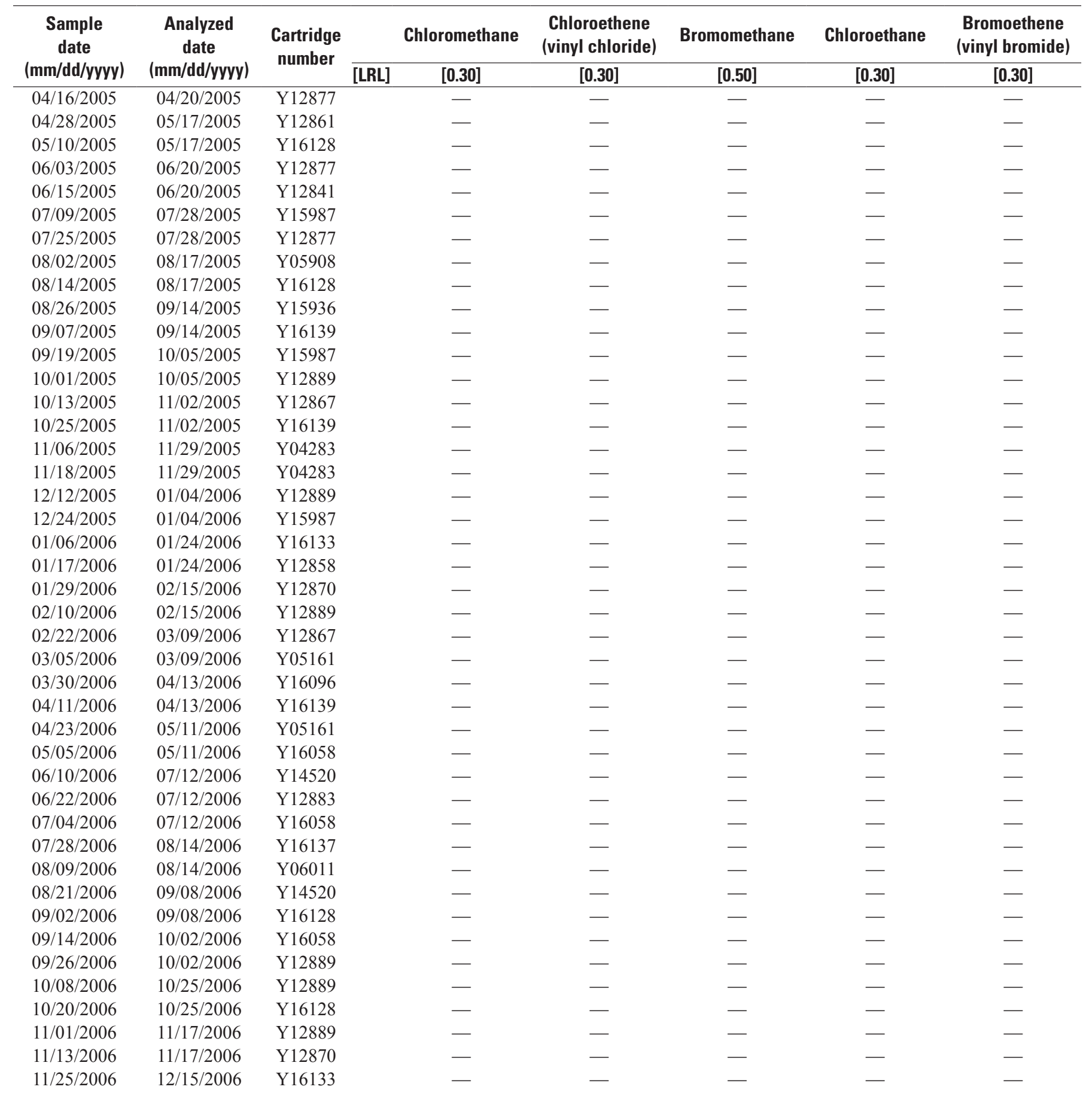


Table B2D. Quality-control analytical laboratory blank results for high-volatility volatile organic compounds (VOCs) processed at the Oregon Graduate Institute for air samples collected from the Sweetwater Reservoir atmospheric site, San Diego County, California.

-Continued

[The site-identification number is 324141117001601 . Concentrations are given in part per billion by volume (ppbv). Sample volume for quality-assurance labblank is 1.5 liters. Samples processed at 20.0 degrees Celsius and 760 millimeters of mercury. Pairs of samples analyzed on the same date had been frozen and stored at laboratory before analysis. Abbreviations: E, estimated or having a higher degree of uncertainty; - , not detected at a concentration above laboratory reporting level; LRL, laboratory reporting level]

\begin{tabular}{|c|c|c|c|c|c|c|c|c|}
\hline \multirow{2}{*}{$\begin{array}{c}\text { Sample } \\
\text { date } \\
\text { (mm/dd/yyyy) }\end{array}$} & \multirow{2}{*}{$\begin{array}{c}\text { Analyzed } \\
\text { date } \\
\text { (mm/dd/yyyy) }\end{array}$} & \multirow{2}{*}{$\begin{array}{l}\text { Cartridge } \\
\text { number }\end{array}$} & \multicolumn{2}{|r|}{ Chloromethane } & \multirow{2}{*}{$\begin{array}{c}\begin{array}{c}\text { Chloroethene } \\
\text { (vinyl chloride) }\end{array} \\
{[0.30]}\end{array}$} & \multirow{2}{*}{$\begin{array}{c}\text { Bromomethane } \\
{[0.50]}\end{array}$} & \multirow{2}{*}{$\begin{array}{c}\text { Chloroethane } \\
{[0.30]}\end{array}$} & \multirow{2}{*}{$\begin{array}{r}\begin{array}{r}\text { Bromoethene } \\
\text { (vinyl bromide }\end{array} \\
{[0.30]} \\
\end{array}$} \\
\hline & & & $\overline{[\text { LRL] }}$ & {$[0.30]$} & & & & \\
\hline $12 / 07 / 2006$ & $12 / 15 / 2006$ & Y16099 & & - & - & - & - & - \\
\hline $12 / 31 / 2006$ & 01/19/2007 & Y16058 & & - & - & - & - & - \\
\hline $01 / 12 / 2007$ & $01 / 19 / 2007$ & Y11217 & & - & - & - & - & - \\
\hline $01 / 24 / 2007$ & $02 / 07 / 2007$ & Y12883 & & - & - & - & - & - \\
\hline $02 / 05 / 2007$ & $02 / 07 / 2007$ & Y16058 & & - & - & - & - & - \\
\hline $02 / 17 / 2007$ & 03/08/2007 & Y15989 & & - & - & - & - & - \\
\hline 03/01/2007 & 03/08/2007 & Y12867 & & - & - & - & - & - \\
\hline 03/13/2007 & 03/29/2007 & Y12841 & & - & - & - & - & - \\
\hline $03 / 25 / 2007$ & 03/29/2007 & Y12870 & & - & - & - & - & - \\
\hline $04 / 06 / 2007$ & 05/01/2007 & Y16099 & & - & - & - & - & - \\
\hline $04 / 18 / 2007$ & 05/01/2007 & Y16046 & & - & - & - & - & - \\
\hline $04 / 30 / 2007$ & 05/16/2007 & Y06011 & & - & - & - & - & - \\
\hline $05 / 12 / 2007$ & $05 / 16 / 2007$ & Y12883 & & - & - & - & - & - \\
\hline $05 / 24 / 2007$ & $06 / 14 / 2007$ & Y12874 & & - & - & - & - & - \\
\hline $06 / 05 / 2007$ & $06 / 14 / 2007$ & Y16134 & & - & - & - & - & - \\
\hline $06 / 17 / 2007$ & $07 / 11 / 2007$ & Y06011 & & - & - & - & - & - \\
\hline $06 / 29 / 2007$ & $07 / 11 / 2007$ & Y14520 & & - & - & - & - & - \\
\hline $07 / 11 / 2007$ & $07 / 13 / 2007$ & Y12841 & & - & - & - & - & - \\
\hline $07 / 23 / 2007$ & 08/09/2007 & Y12883 & & - & - & - & - & - \\
\hline 08/04/2007 & 08/09/2007 & Y16099 & & - & - & - & - & - \\
\hline 08/16/2007 & 09/05/2007 & Y11217 & & - & - & - & - & - \\
\hline 08/28/2007 & 09/05/2007 & Y12841 & & - & - & - & - & - \\
\hline 09/09/2007 & $10 / 02 / 2007$ & Y05868 & & - & - & - & - & - \\
\hline 09/21/2007 & $10 / 02 / 2007$ & Y16058 & & - & - & - & - & - \\
\hline $10 / 03 / 2007$ & $10 / 19 / 2007$ & Y12874 & & - & - & - & - & - \\
\hline $10 / 15 / 2007$ & $10 / 19 / 2007$ & Y12883 & & - & - & - & - & - \\
\hline $10 / 27 / 2007$ & $11 / 13 / 2007$ & Y16058 & & - & - & - & - & - \\
\hline $10 / 27 / 2007$ & $11 / 13 / 2007$ & Y16058 & & - & - & - & - & - \\
\hline $11 / 08 / 2007$ & $11 / 13 / 2007$ & Y12867 & & - & - & - & - & - \\
\hline $11 / 08 / 2007$ & $11 / 13 / 2007$ & Y12867 & & - & - & - & - & - \\
\hline $11 / 20 / 2007$ & $12 / 06 / 2007$ & Y05161 & & - & - & - & - & - \\
\hline $11 / 20 / 2007$ & $12 / 06 / 2007$ & Y05161 & & - & - & - & - & - \\
\hline $12 / 02 / 2007$ & $12 / 06 / 2007$ & Y16119 & & - & - & - & - & - \\
\hline $12 / 02 / 2007$ & $12 / 06 / 2007$ & Y16119 & & - & - & - & - & - \\
\hline $12 / 14 / 2007$ & $01 / 03 / 2008$ & Y05161 & & - & - & - & - & - \\
\hline $12 / 14 / 2007$ & $01 / 03 / 2008$ & Y05161 & & - & - & - & - & - \\
\hline $12 / 26 / 2007$ & $01 / 03 / 2008$ & Y15936 & & - & - & - & - & - \\
\hline $12 / 26 / 2007$ & $01 / 03 / 2008$ & Y15936 & & - & - & - & - & - \\
\hline $01 / 07 / 2008$ & $01 / 23 / 2008$ & Y12889 & & - & - & - & - & - \\
\hline $01 / 19 / 2008$ & $01 / 23 / 2008$ & Y16099 & & - & - & - & - & - \\
\hline $01 / 31 / 2008$ & $02 / 19 / 2008$ & Y16058 & & - & - & - & - & - \\
\hline $02 / 12 / 2008$ & $02 / 19 / 2008$ & Y16052 & & - & - & - & - & - \\
\hline $02 / 24 / 2008$ & 03/13/2008 & Y05016 & & - & - & - & - & - \\
\hline
\end{tabular}


Table B2D. Quality-control analytical laboratory blank results for high-volatility volatile organic compounds (VOCs) processed at the Oregon Graduate Institute for air samples collected from the Sweetwater Reservoir atmospheric site, San Diego County, California. -Continued

[The site-identification number is 324141117001601 . Concentrations are given in part per billion by volume (ppbv). Sample volume for quality-assurance labblank is 1.5 liters. Samples processed at 20.0 degrees Celsius and 760 millimeters of mercury. Pairs of samples analyzed on the same date had been frozen and stored at laboratory before analysis. Abbreviations: E, estimated or having a higher degree of uncertainty; —, not detected at a concentration above laboratory reporting level; LRL, laboratory reporting level]

\begin{tabular}{|c|c|c|c|c|c|c|c|c|}
\hline \multirow{2}{*}{$\begin{array}{c}\text { Sample } \\
\text { date } \\
\text { (mm/dd/yyyy) }\end{array}$} & \multirow{2}{*}{$\begin{array}{c}\text { Analyzed } \\
\text { date } \\
\text { (mm/dd/yyyy) }\end{array}$} & \multirow{2}{*}{$\begin{array}{c}\text { Cartridge } \\
\text { number }\end{array}$} & \multicolumn{2}{|r|}{ Chloromethane } & \multirow{2}{*}{$\begin{array}{c}\begin{array}{c}\text { Chloroethene } \\
\text { (vinyl chloride) }\end{array} \\
{[0.30]}\end{array}$} & \multirow{2}{*}{$\begin{array}{c}\text { Bromomethane } \\
{[0.50]}\end{array}$} & \multirow{2}{*}{$\begin{array}{c}\text { Chloroethane } \\
{[0.30]}\end{array}$} & \multirow{2}{*}{$\begin{array}{r}\begin{array}{r}\text { Bromoethene } \\
\text { (vinyl bromide }\end{array} \\
{[0.30]}\end{array}$} \\
\hline & & & [LRL] & {$[0.30]$} & & & & \\
\hline $\begin{array}{l}03 / 07 / 2008 \\
\end{array}$ & $03 / 13 / 2008$ & Y16099 & & - & - & - & - & - \\
\hline 03/19/2008 & $04 / 03 / 2008$ & Y16096 & & - & - & - & - & - \\
\hline $03 / 31 / 2008$ & $04 / 03 / 2008$ & Y05908 & & - & - & - & - & - \\
\hline $04 / 12 / 2008$ & $04 / 30 / 2008$ & Y14520 & & - & - & - & - & - \\
\hline $04 / 24 / 2008$ & $04 / 30 / 2008$ & Y12874 & & - & - & - & - & - \\
\hline 05/06/2008 & $05 / 22 / 2008$ & Y12867 & & - & - & - & - & - \\
\hline $05 / 18 / 2008$ & $05 / 22 / 2008$ & Y16134 & & - & - & - & - & - \\
\hline 05/30/2008 & $06 / 18 / 2008$ & Y16139 & & - & - & - & - & - \\
\hline 06/01/2008 & $06 / 18 / 2008$ & Y16096 & & - & - & - & - & - \\
\hline $06 / 23 / 2008$ & $07 / 14 / 2008$ & Y11185 & & - & - & - & - & - \\
\hline $07 / 05 / 2008$ & $07 / 14 / 2008$ & Y05951 & & - & - & - & - & - \\
\hline $07 / 17 / 2008$ & 08/14/2008 & Y16658 & & - & - & - & - & - \\
\hline 07/29/2008 & $08 / 14 / 2008$ & Y15889 & & - & - & - & - & - \\
\hline 08/10/2008 & $08 / 28 / 2008$ & Y05016 & & - & - & - & - & - \\
\hline $08 / 22 / 2008$ & 08/28/2008 & Y16139 & & - & - & - & - & - \\
\hline 09/03/2008 & 09/18/2008 & Y16058 & & - & - & - & - & - \\
\hline 09/15/2008 & $09 / 18 / 2008$ & Y14520 & & - & - & - & - & - \\
\hline 09/27/2008 & $10 / 16 / 2008$ & Y16052 & & - & - & - & - & - \\
\hline $10 / 09 / 2008$ & $10 / 16 / 2008$ & Y15989 & & - & - & - & - & - \\
\hline $10 / 21 / 2008$ & $11 / 06 / 2008$ & Y05161 & & - & - & - & - & - \\
\hline $11 / 02 / 2008$ & $11 / 06 / 2008$ & Y05908 & & - & - & - & - & - \\
\hline $11 / 14 / 2008$ & $12 / 10 / 2008$ & Y12883 & & - & - & - & - & - \\
\hline $11 / 26 / 2008$ & $12 / 10 / 2008$ & Y16139 & & - & - & - & - & - \\
\hline $12 / 08 / 2008$ & $12 / 31 / 2008$ & Y16058 & & - & - & - & - & - \\
\hline $12 / 20 / 2008$ & $12 / 31 / 2008$ & Y12883 & & - & - & - & - & - \\
\hline 01/01/2009 & $01 / 21 / 2009$ & Y05951 & & - & - & - & - & - \\
\hline 01/13/2009 & $01 / 21 / 2009$ & Y05161 & & - & - & - & - & - \\
\hline $01 / 25 / 2009$ & $02 / 11 / 2009$ & Y05908 & & - & - & - & - & - \\
\hline 02/06/2009 & $02 / 11 / 2009$ & Y05868 & & - & - & - & - & - \\
\hline 02/18/2009 & 03/04/2009 & Y15939 & & - & - & - & - & - \\
\hline 03/02/2009 & 03/04/2009 & Y16096 & & - & - & - & - & - \\
\hline 03/14/2009 & 04/01/2009 & Y05016 & & - & - & - & - & - \\
\hline 03/26/2009 & 04/01/2009 & Y12867 & & - & - & - & - & - \\
\hline 04/07/2009 & $04 / 23 / 2009$ & Y16119 & & - & - & - & - & - \\
\hline 04/19/2009 & $04 / 23 / 2009$ & Y05951 & & - & - & - & - & - \\
\hline 05/01/2009 & $05 / 21 / 2009$ & Y14520 & & - & - & - & - & - \\
\hline 05/13/2009 & $05 / 21 / 2009$ & Y16052 & & - & - & - & - & - \\
\hline $05 / 25 / 2009$ & 06/11/2009 & Y05016 & & - & - & - & - & - \\
\hline 06/06/2009 & 06/11/2009 & Y16058 & & - & - & - & - & - \\
\hline 06/18/2009 & 07/09/2009 & Y16096 & & - & - & - & - & - \\
\hline 06/30/2009 & 07/09/2009 & Y14520 & & - & - & - & - & - \\
\hline 07/12/2009 & 07/29/2009 & Y16134 & & - & - & - & - & - \\
\hline $07 / 24 / 2009$ & 07/29/2009 & Y12889 & & - & - & - & - & - \\
\hline
\end{tabular}



产

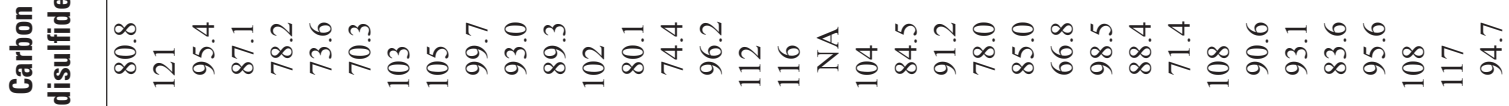

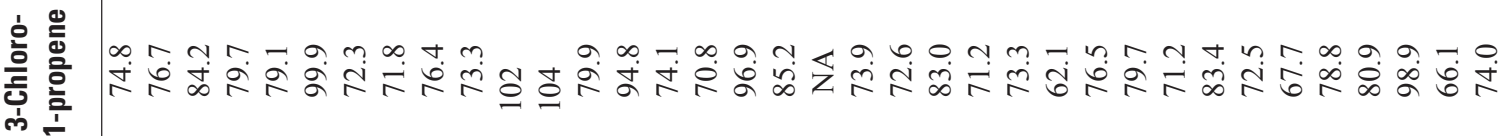

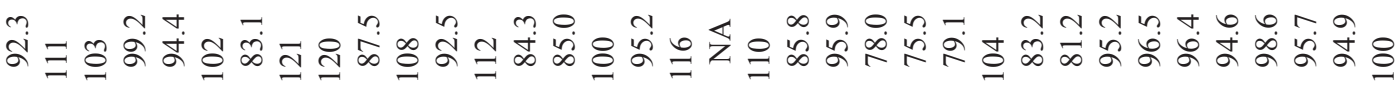

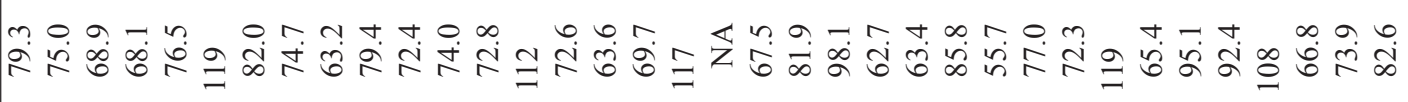
$\stackrel{0}{\frac{\Theta}{\rightleftarrows}}$

小 을

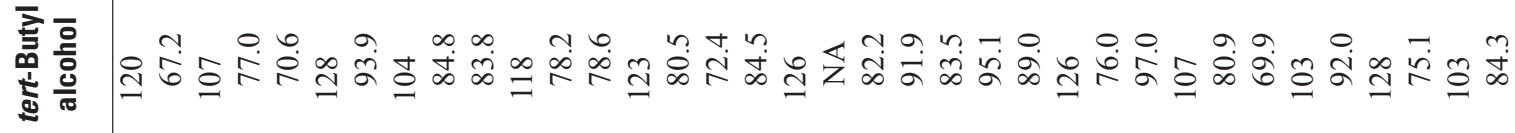

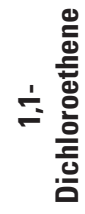

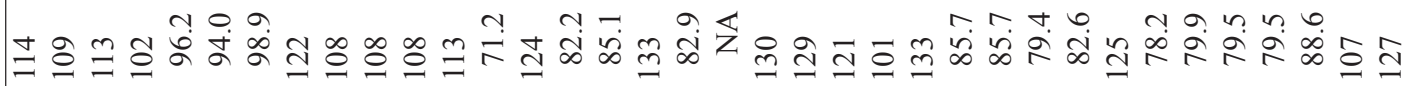

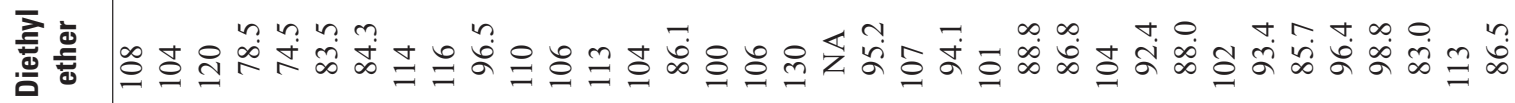

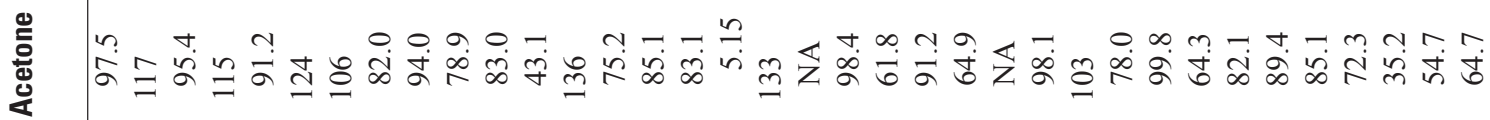

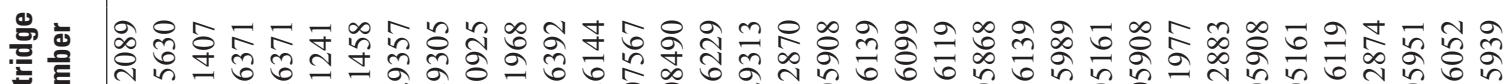
壳 业

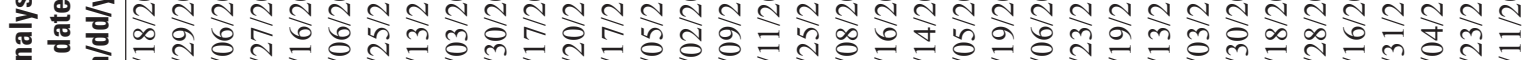

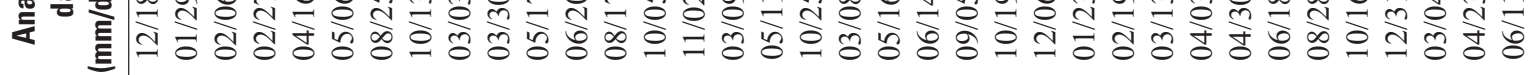

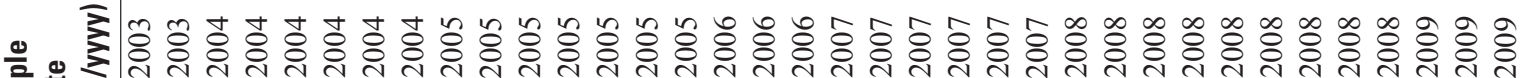

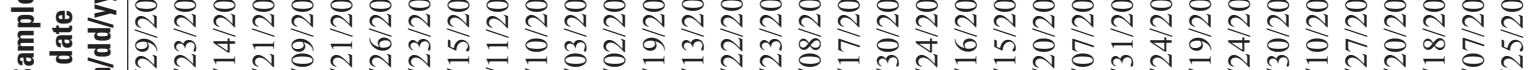

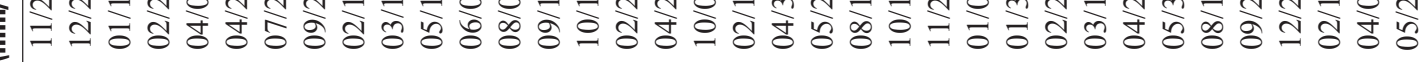




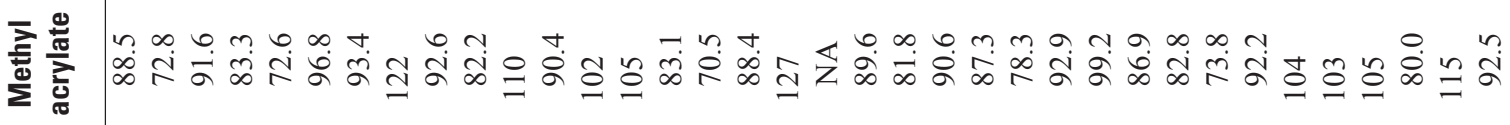

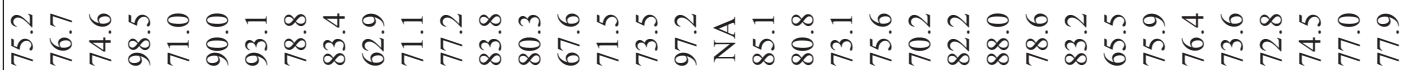

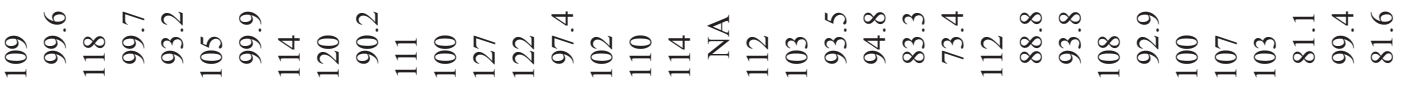

을

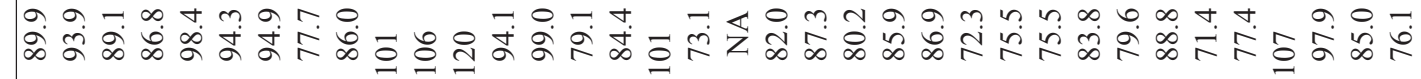

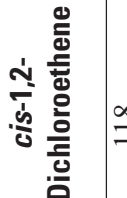

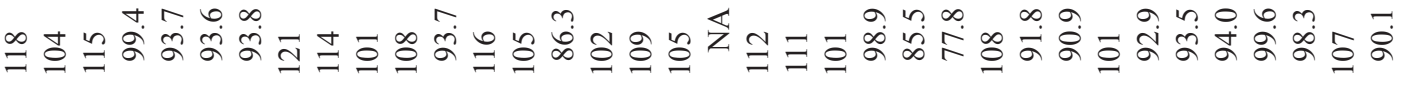

这

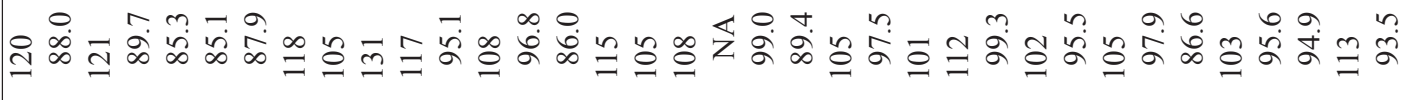

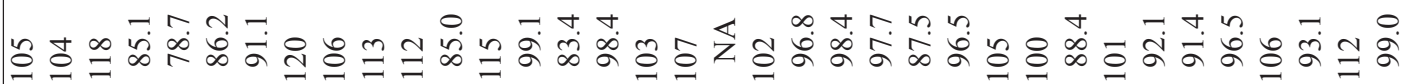
:

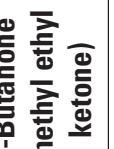

我足

嵌

告

害兽

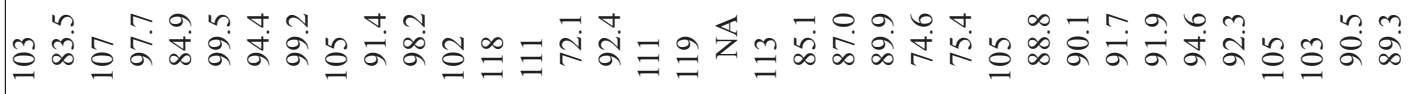

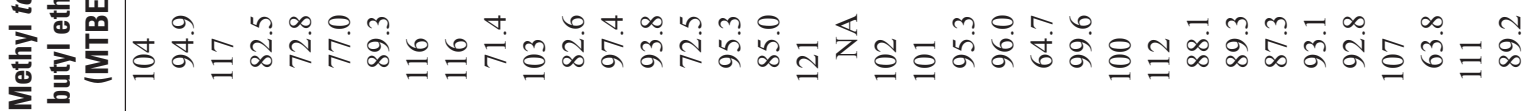

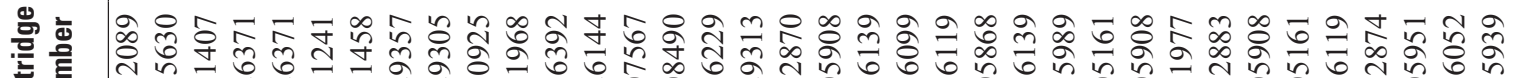

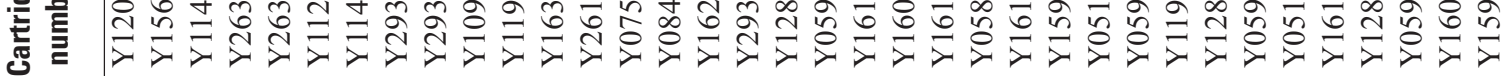

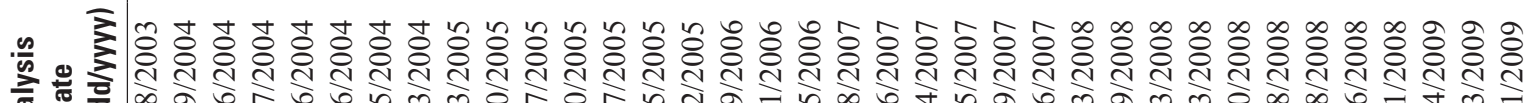

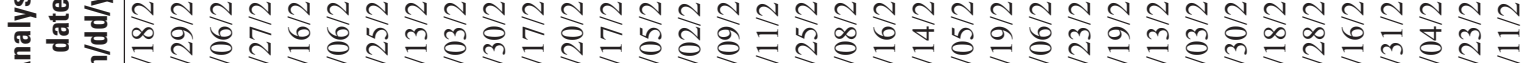

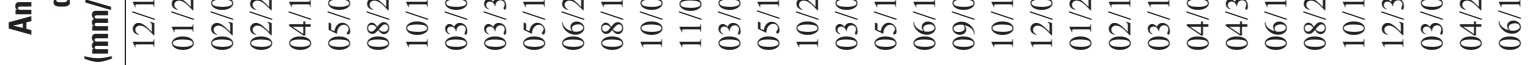

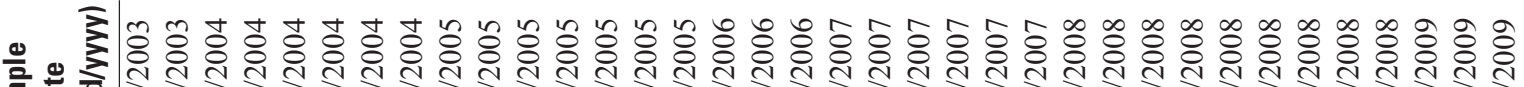

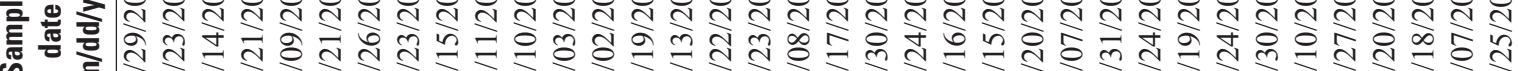

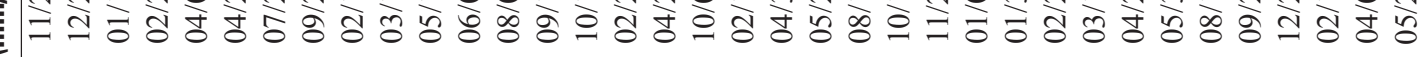




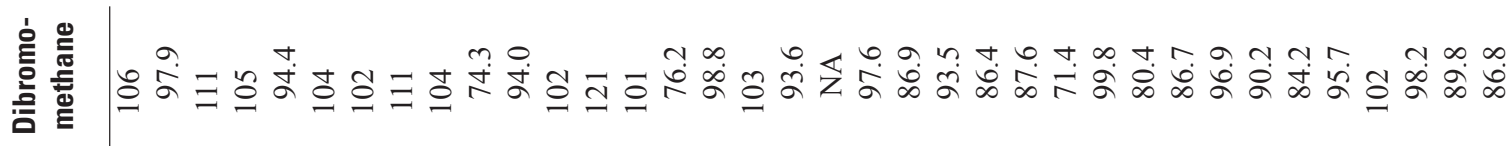

产

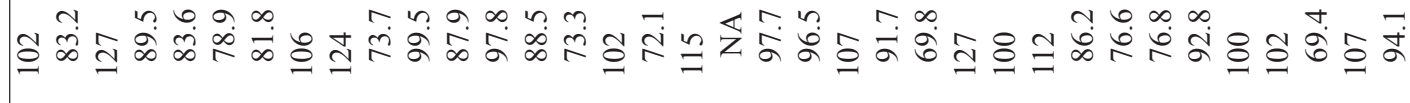

三 ป

客

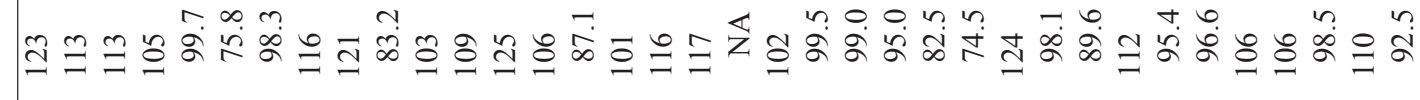

흘

$=$

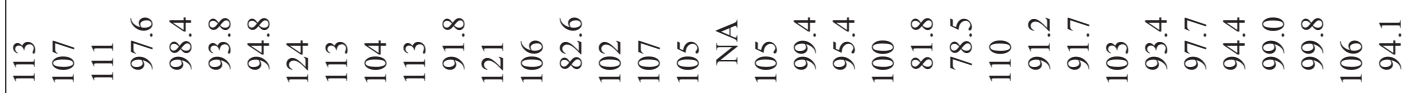

듬

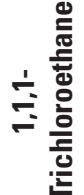

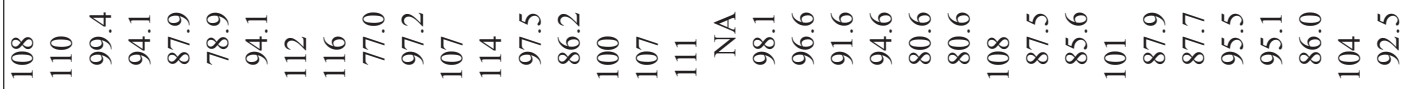

츨

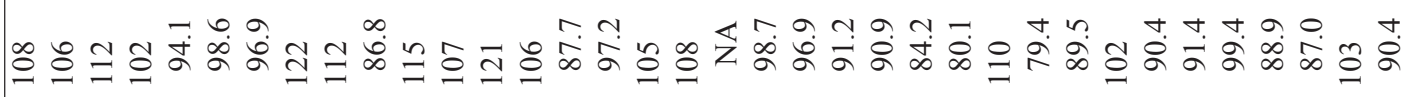

离就

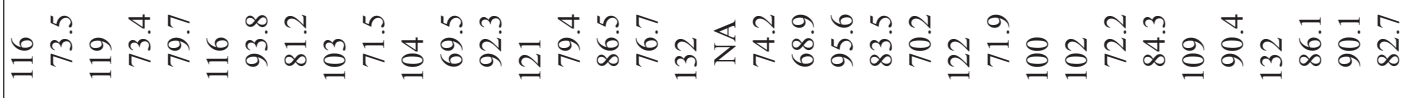

튼

产

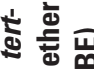

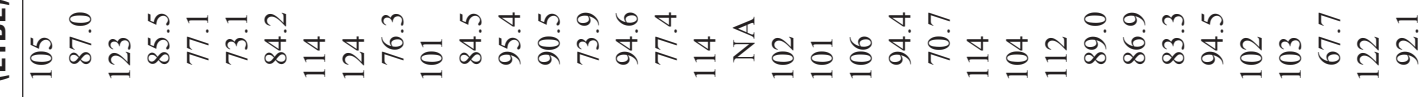

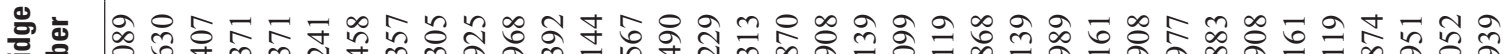

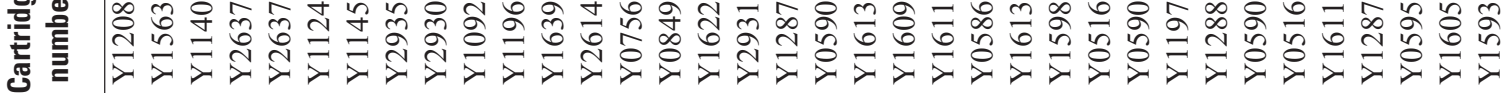
-

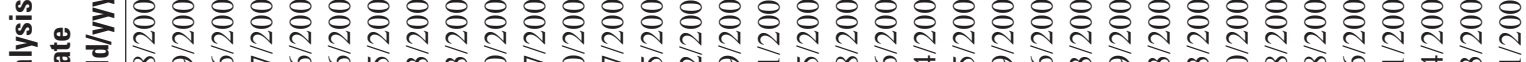
西 뜽

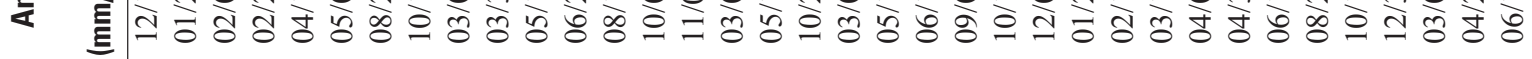

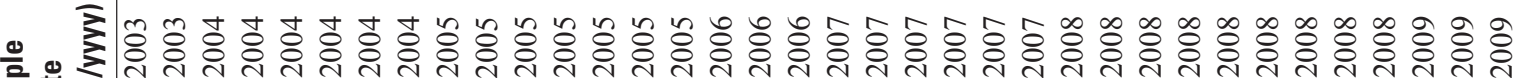
要

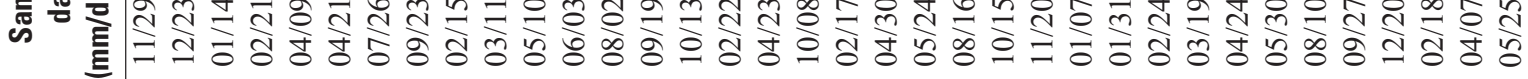




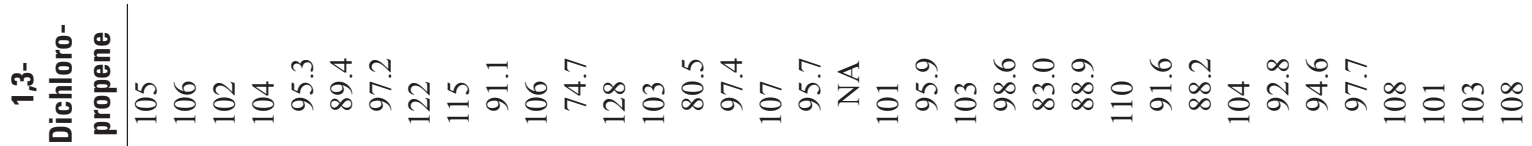
产

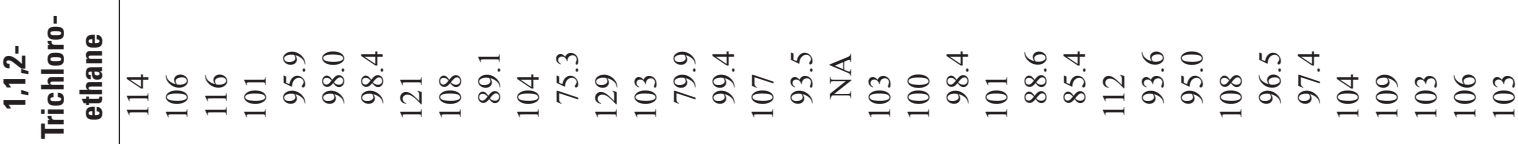

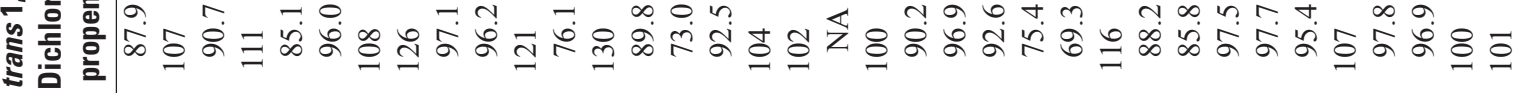

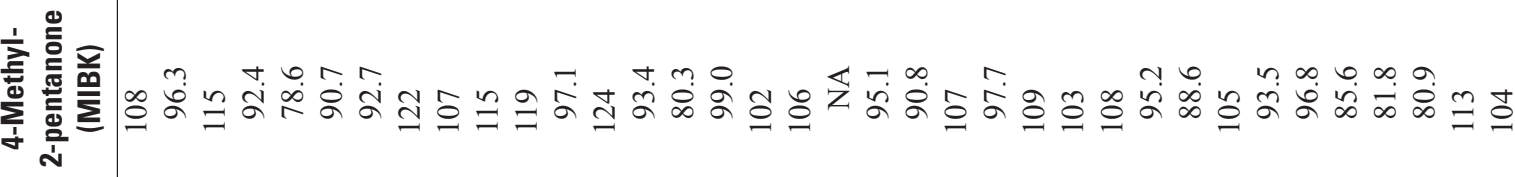

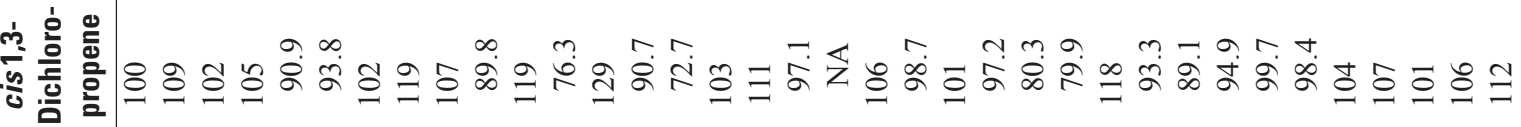

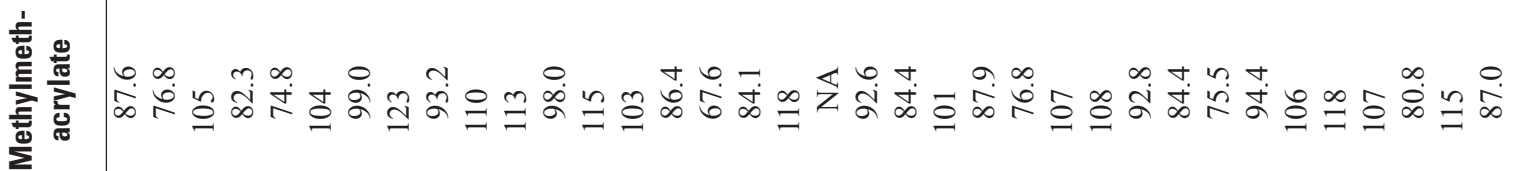
흘

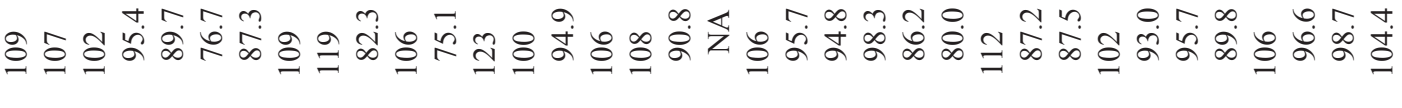
这

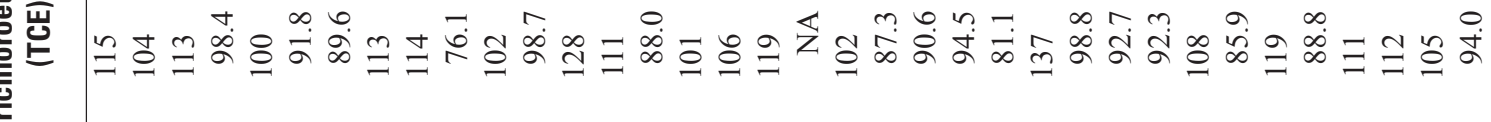

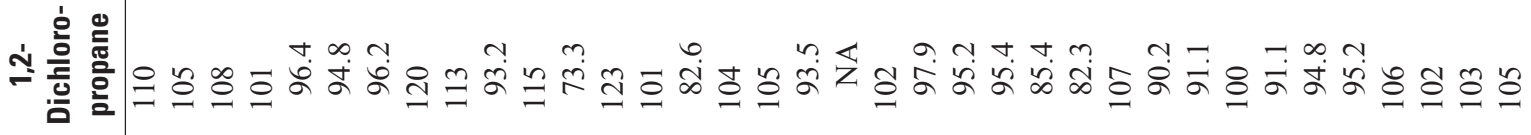

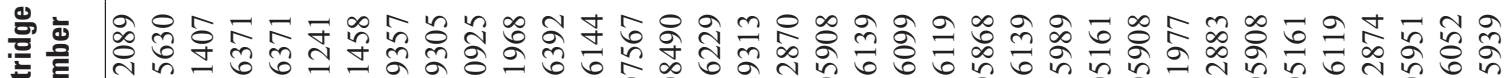

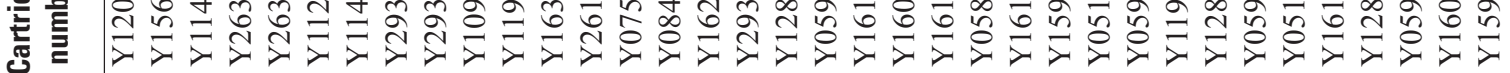

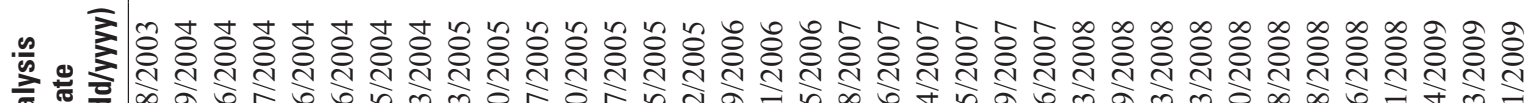

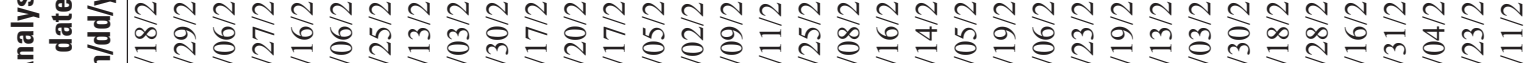

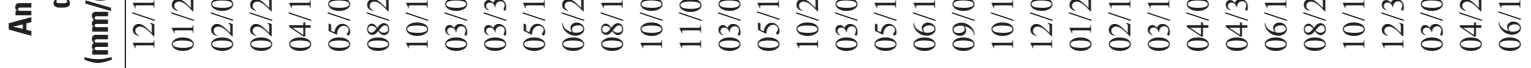

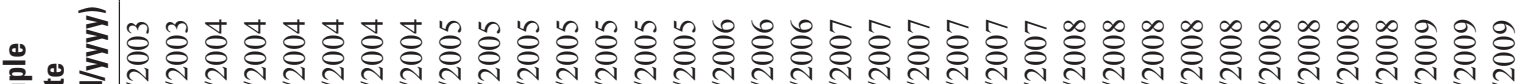
竞

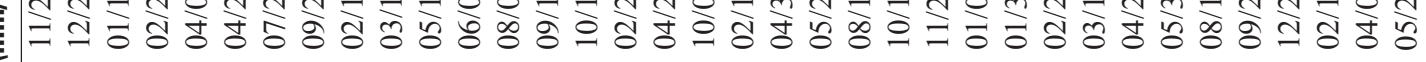




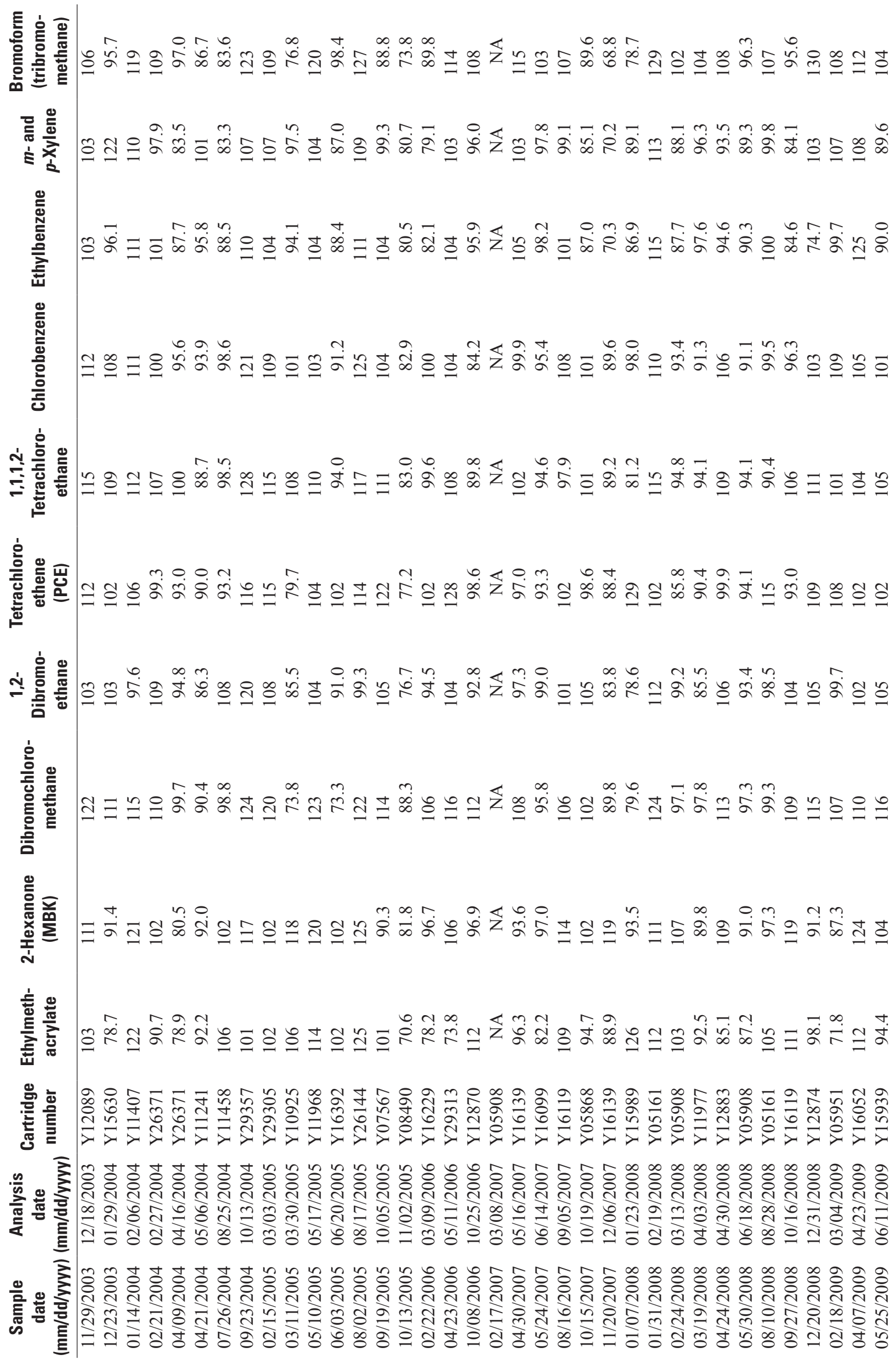




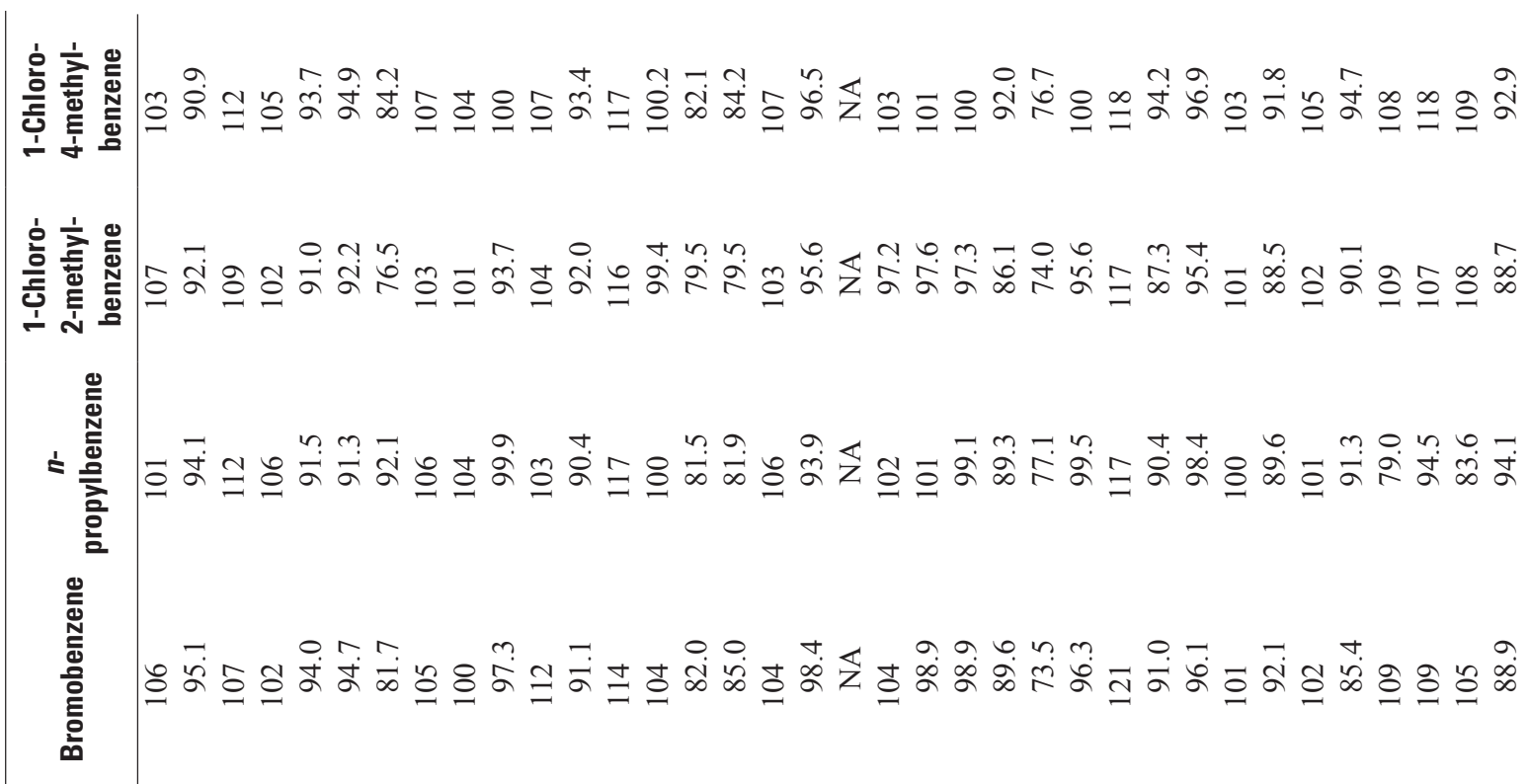

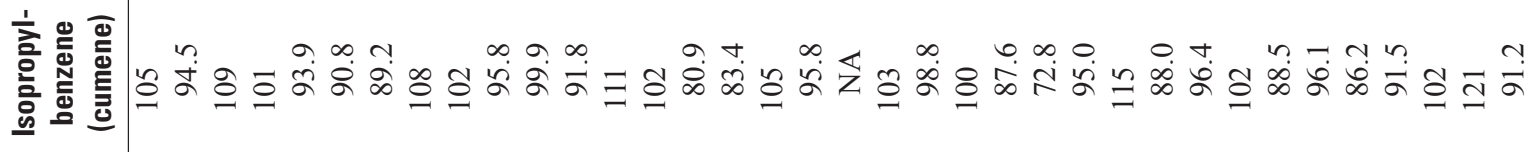

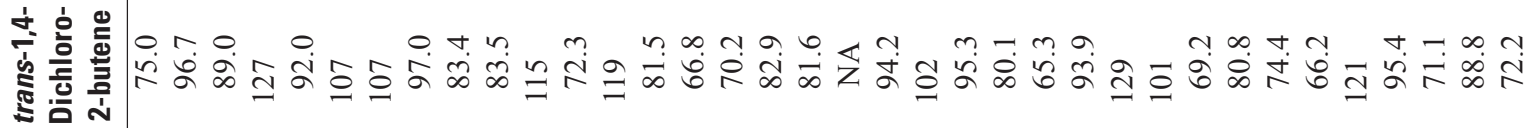

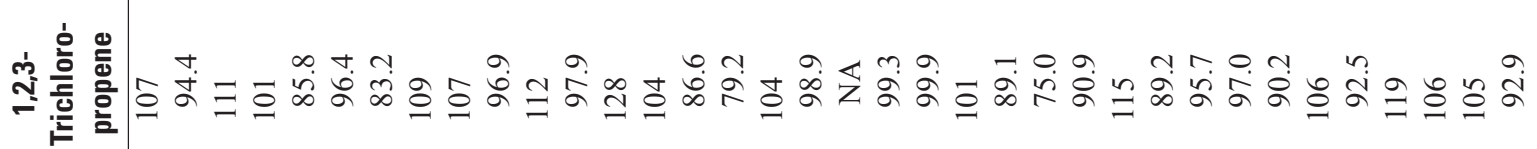

严

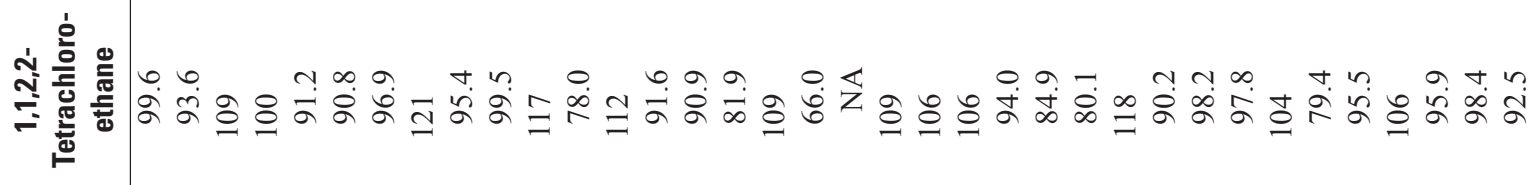

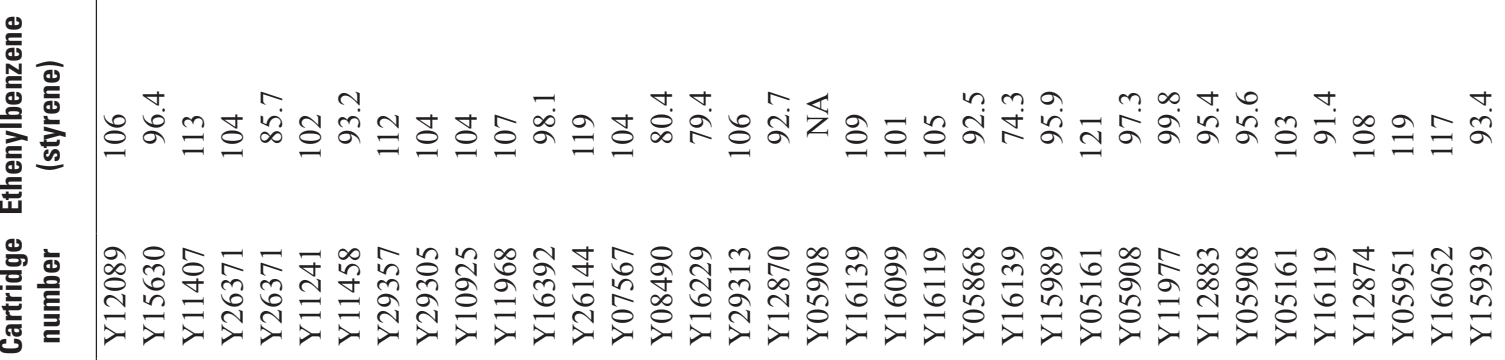

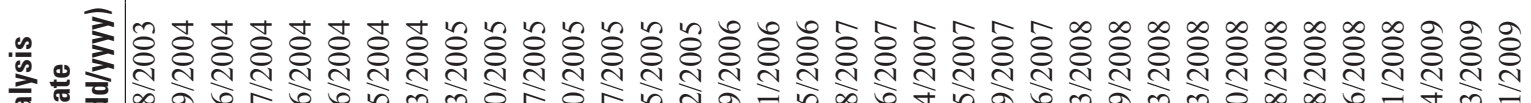

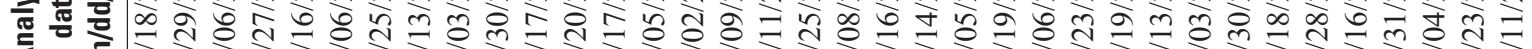

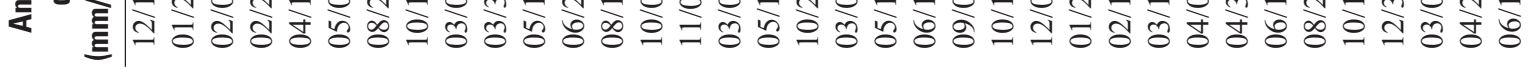

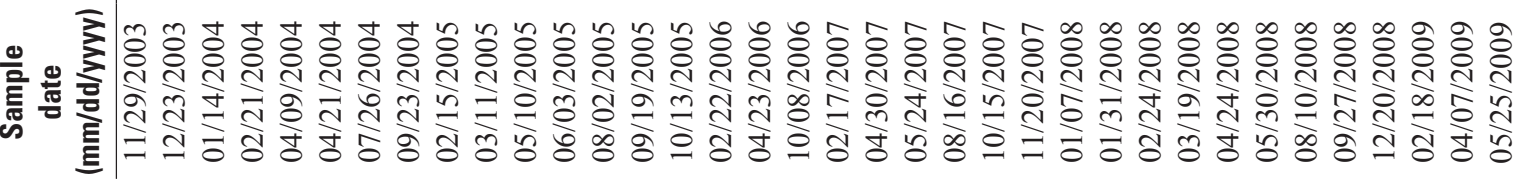


늘 产 至

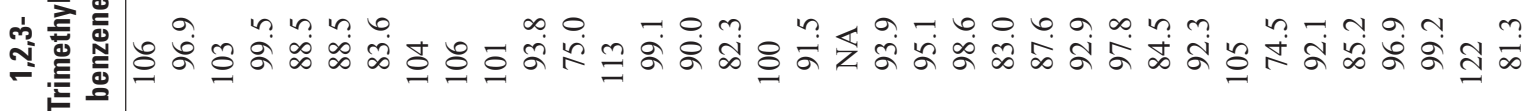

$\frac{1}{1}$

$\stackrel{\underset{\mathbb{N}}{Z}}{\stackrel{Z}{Z}}$

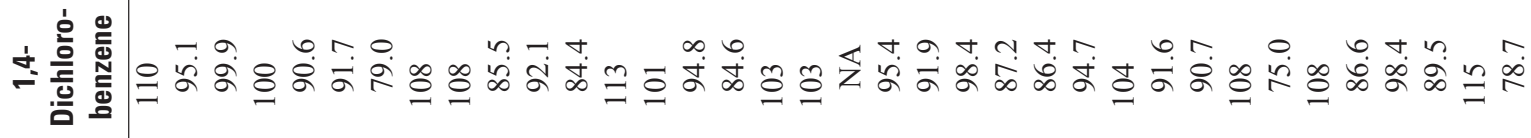

m.

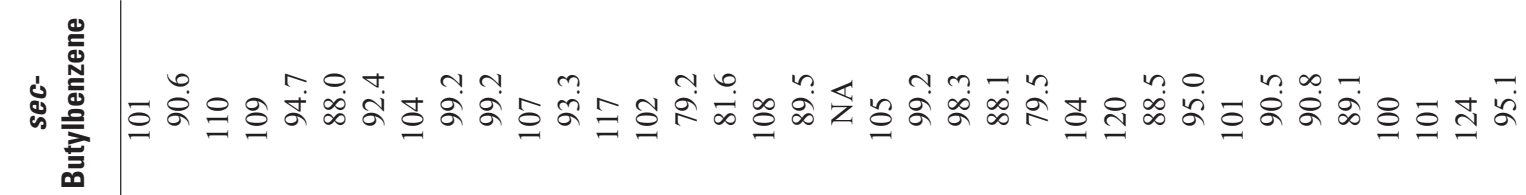

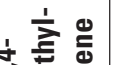

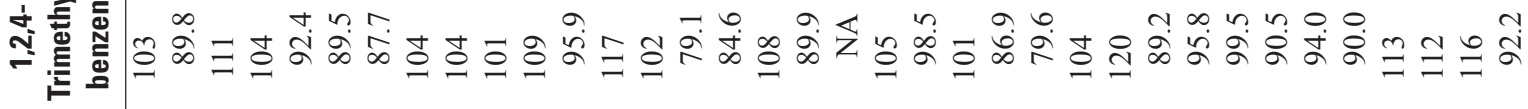

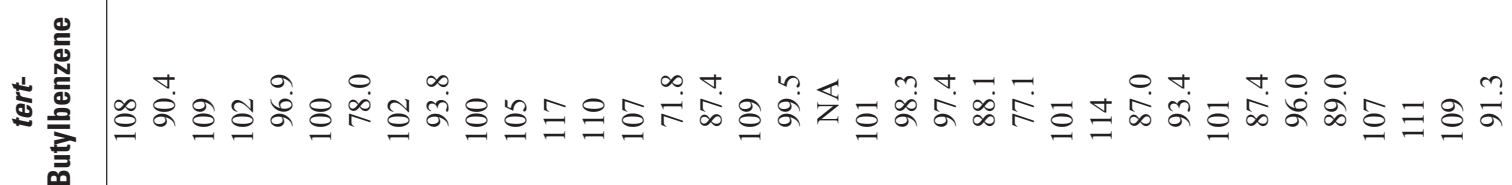

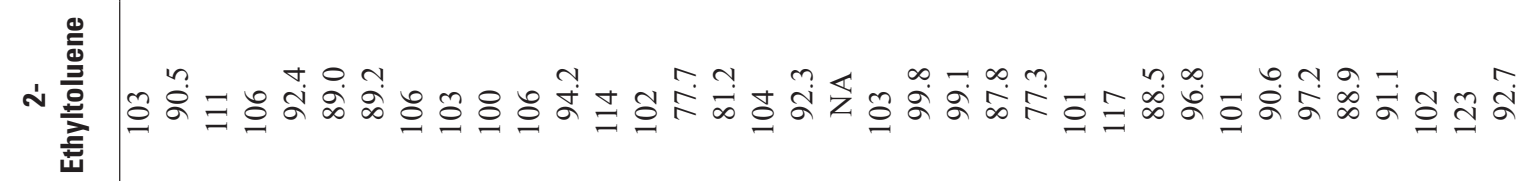

i

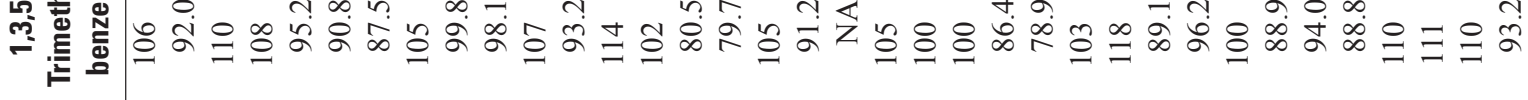

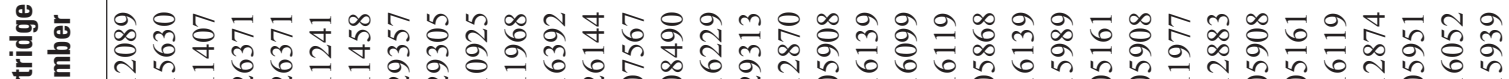

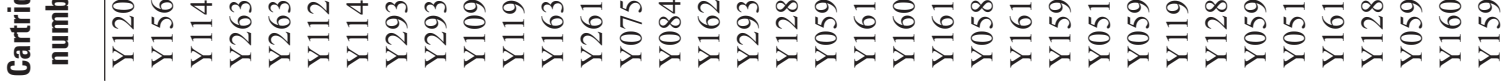

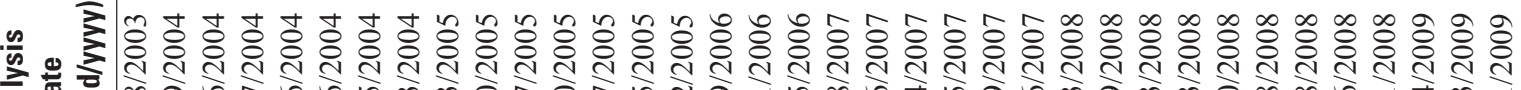

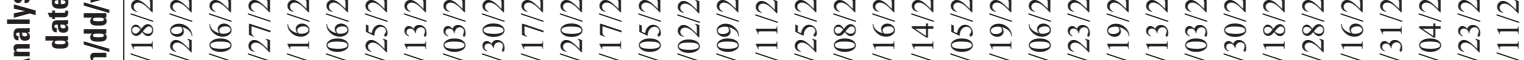

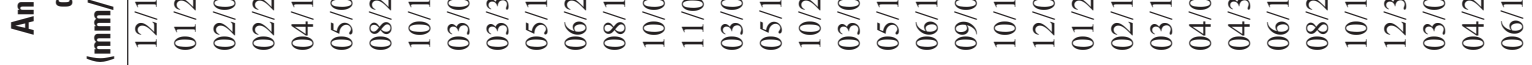

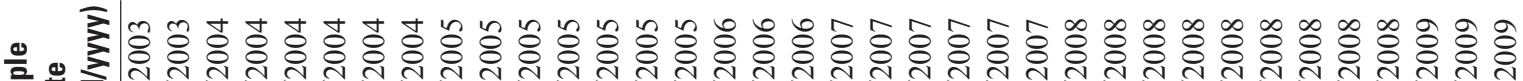

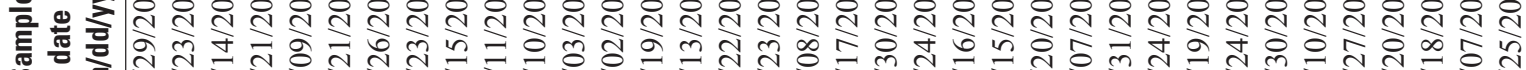

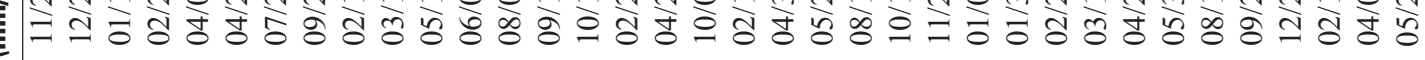




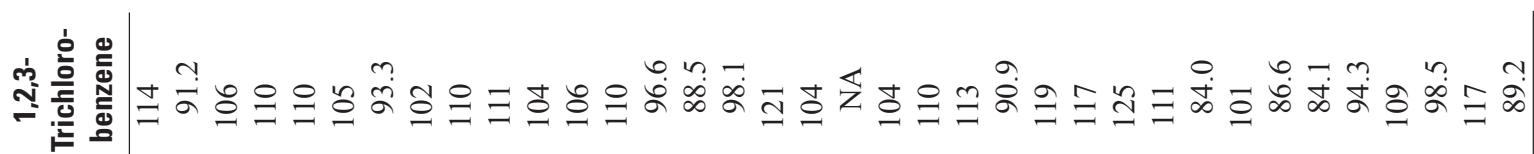

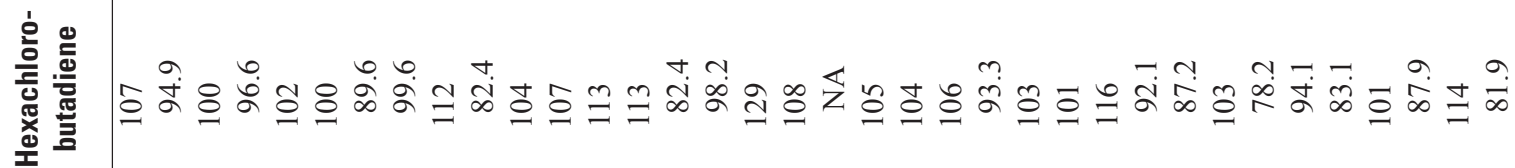

产

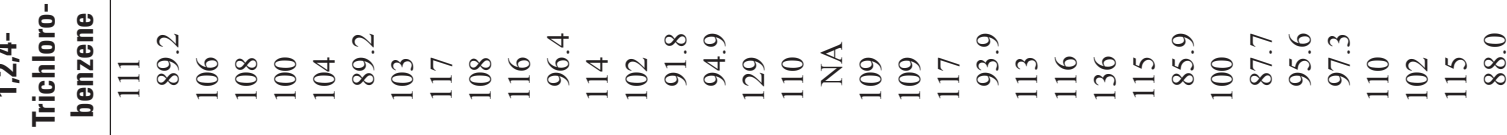

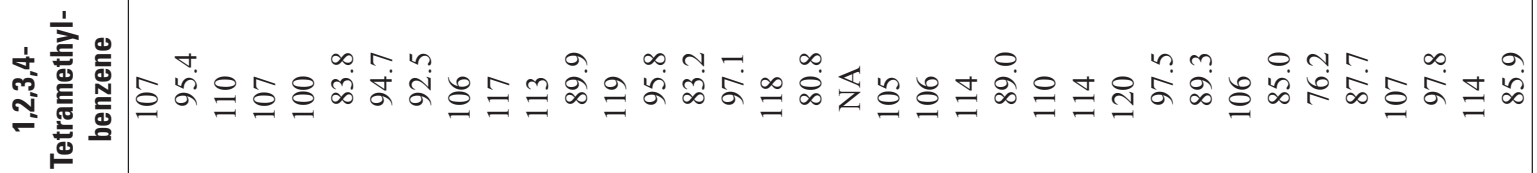

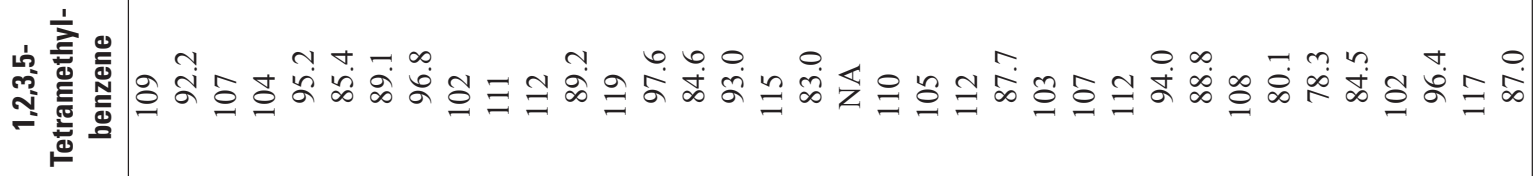

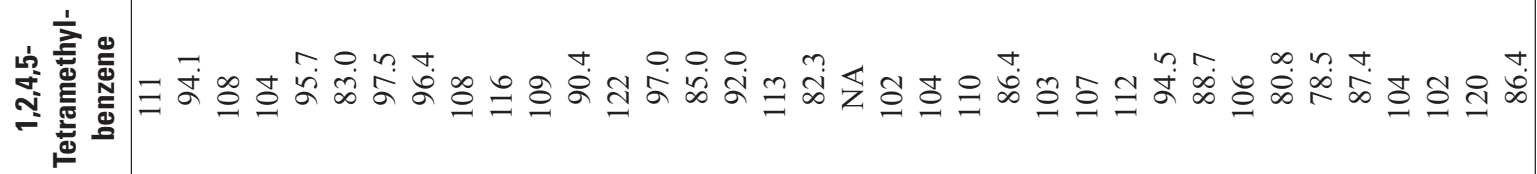

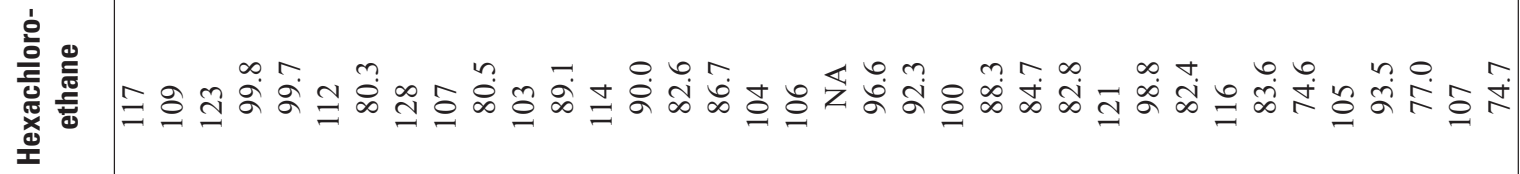
을 흘 흥

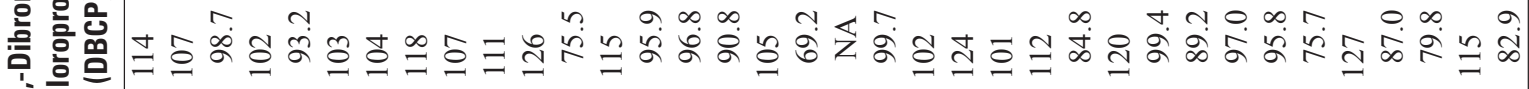
ํํํำ

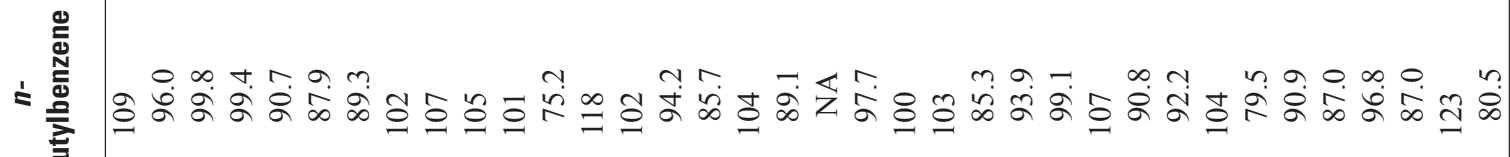

若㐫

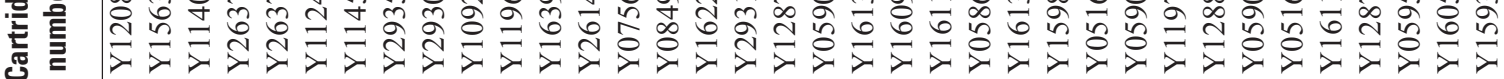
री

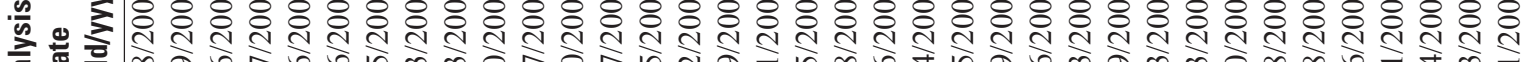

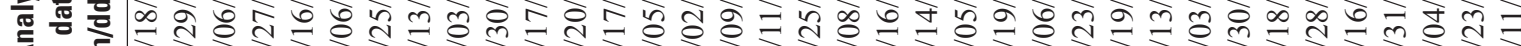

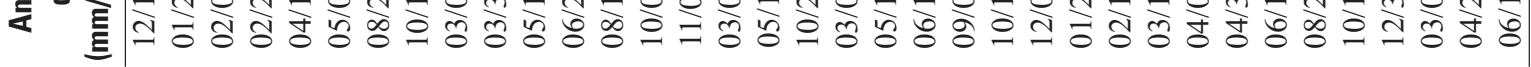
•

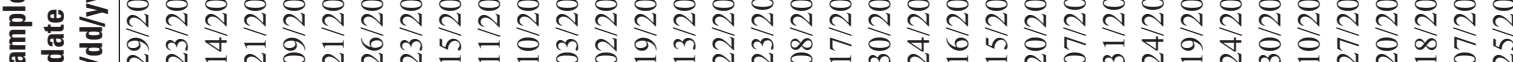

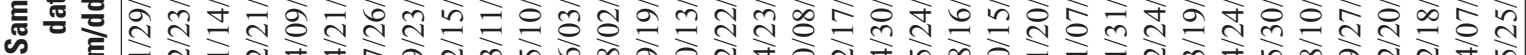




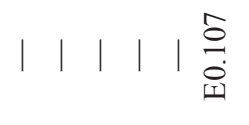

\section{흘}

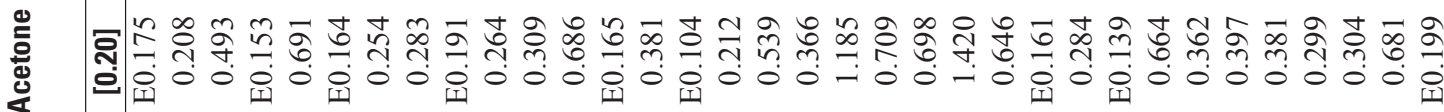

롤

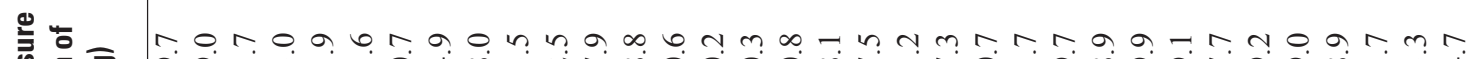
尊互至

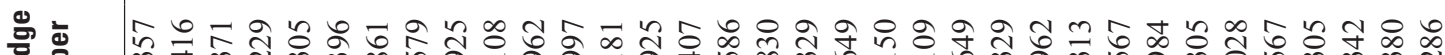

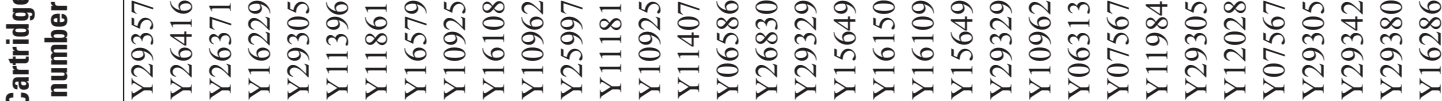
m m

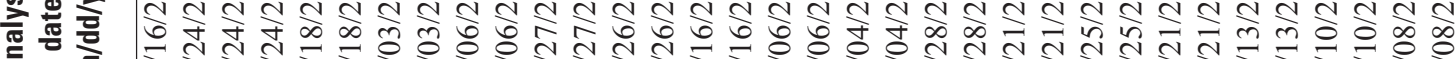

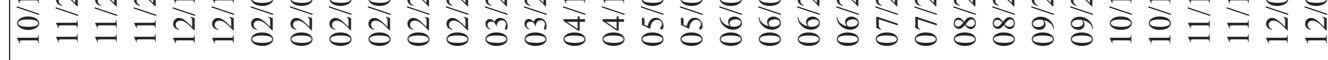




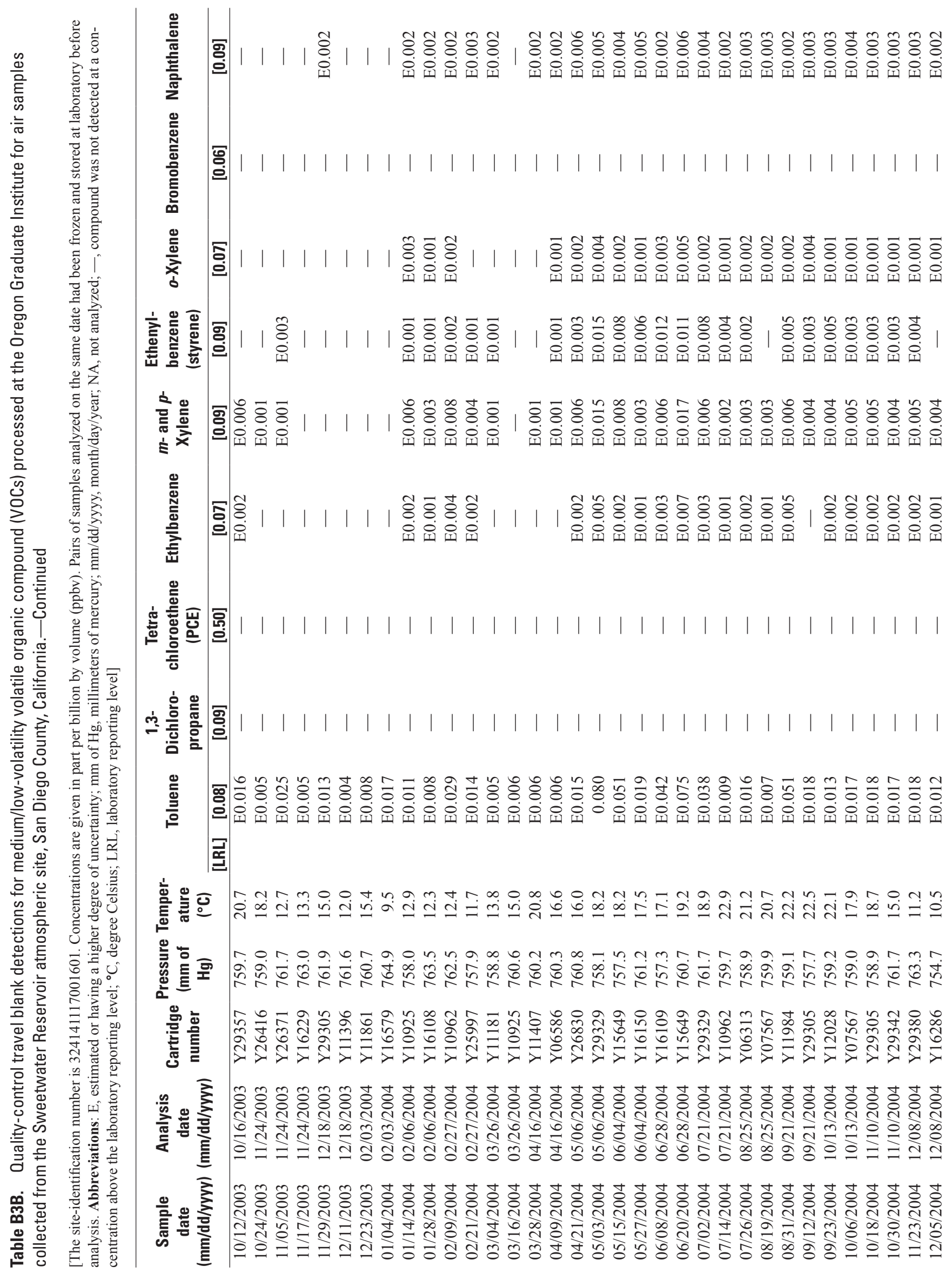



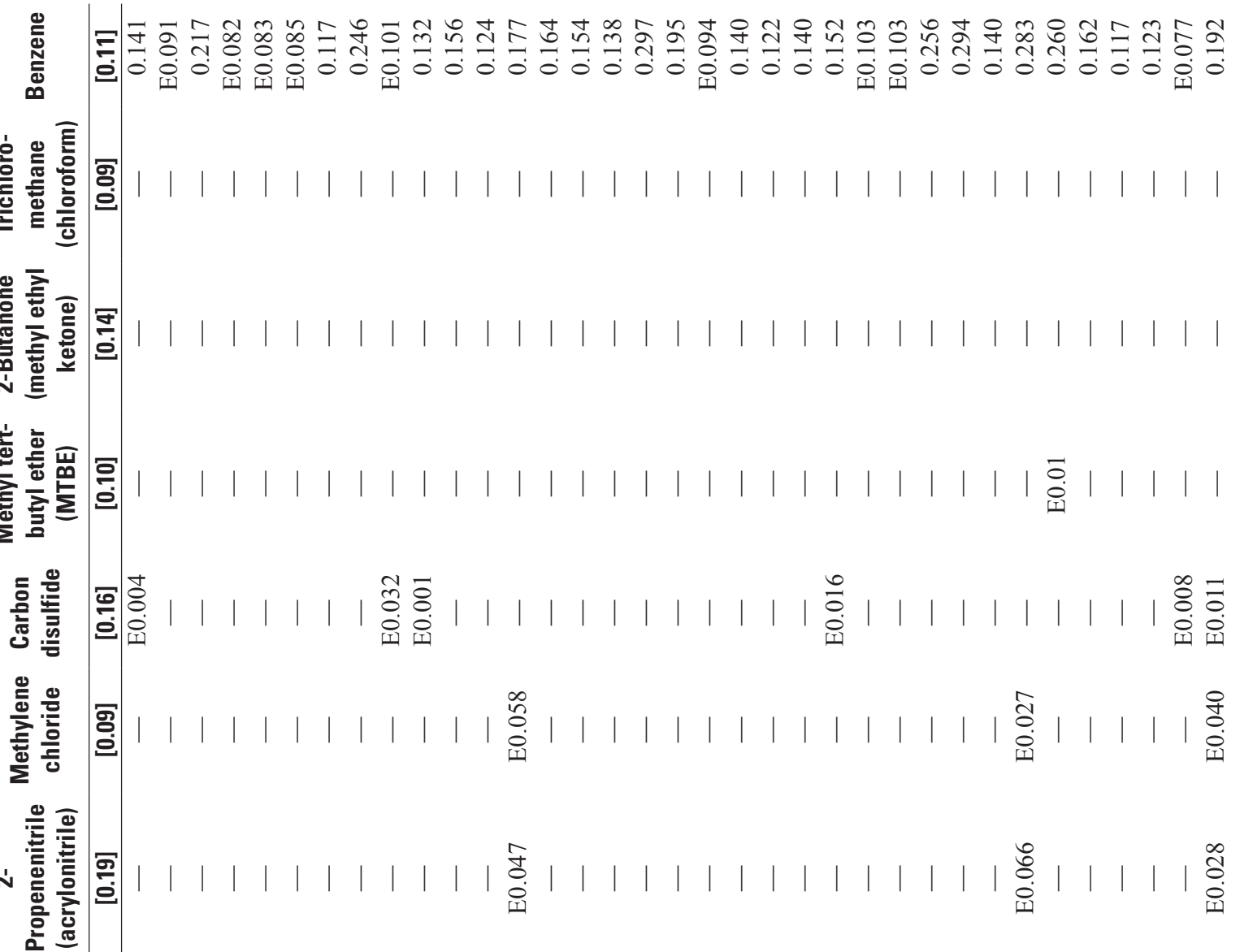

:

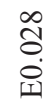

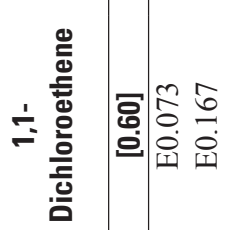

产

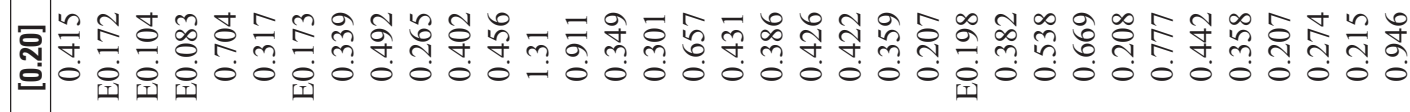

골

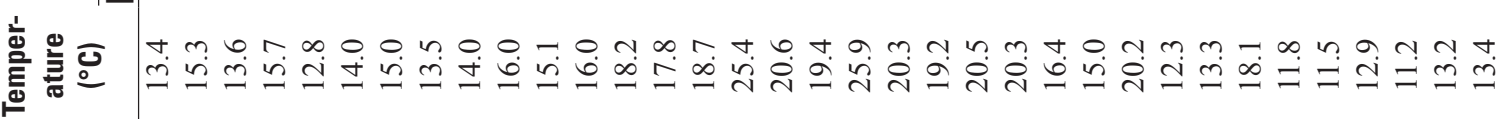

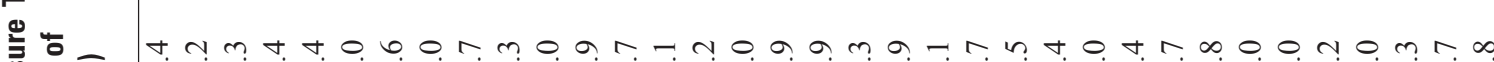

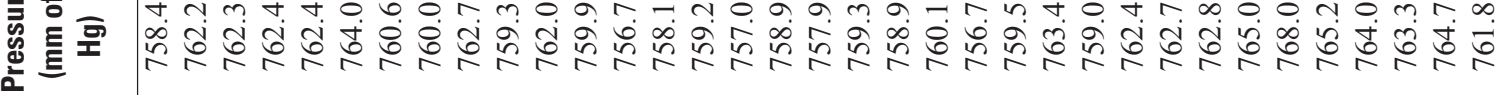

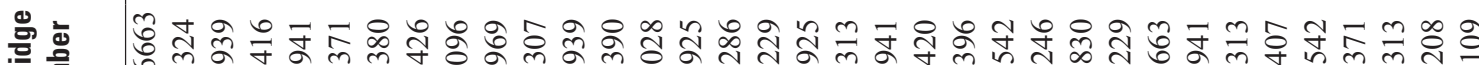

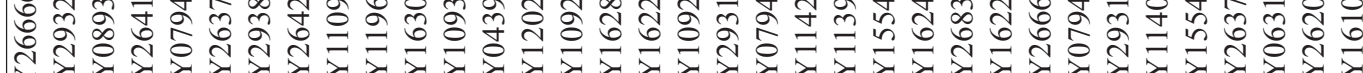

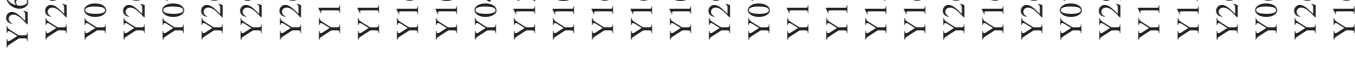

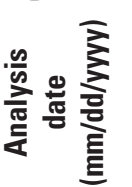

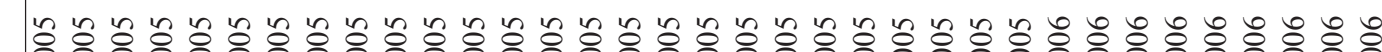

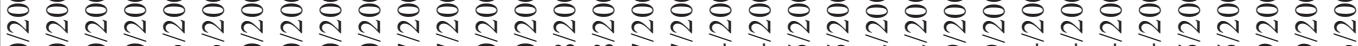
ते

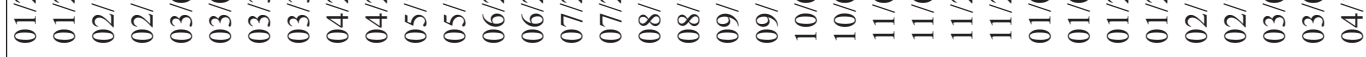

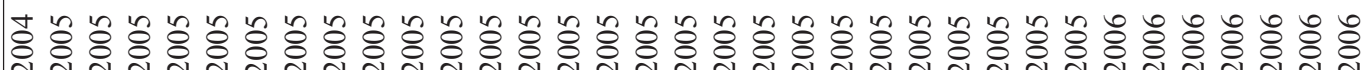

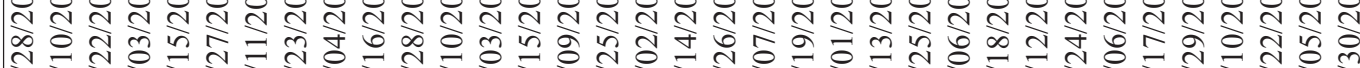
경ㅎㅇㅇㅎㅇㅎㅇ 


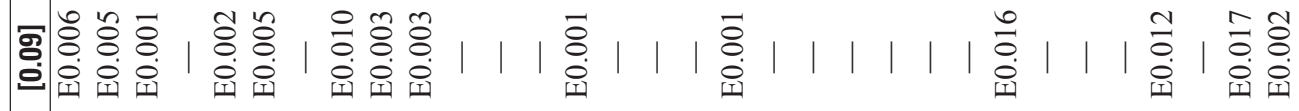

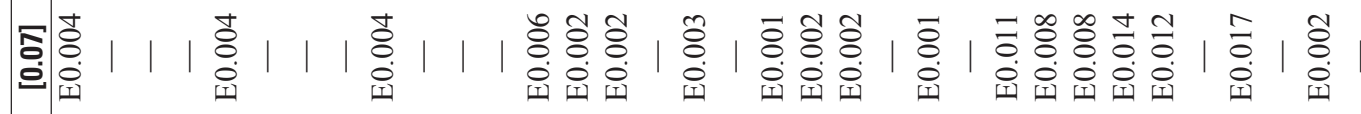

छ.

马ु

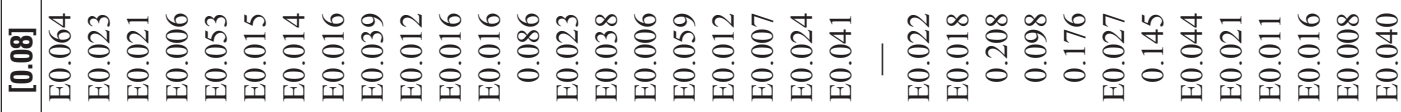

롤

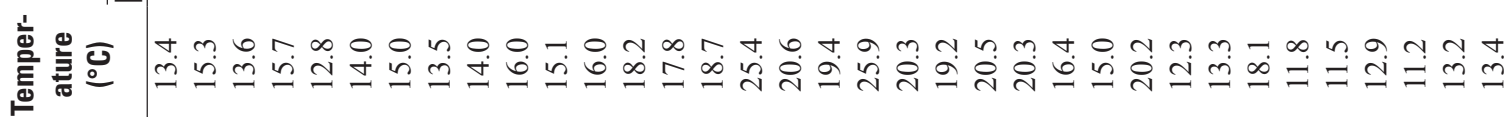

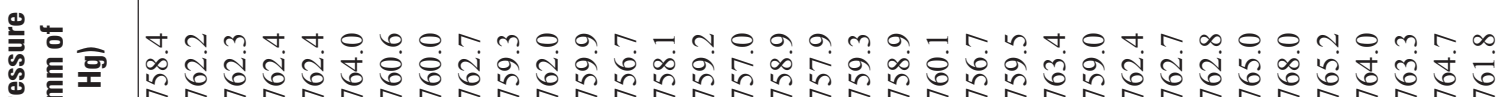
(a)

గ艹

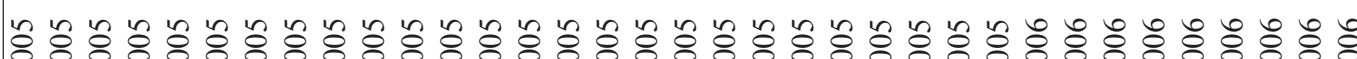

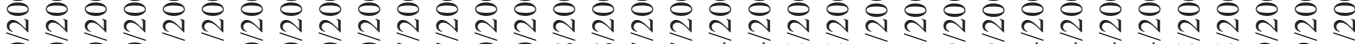

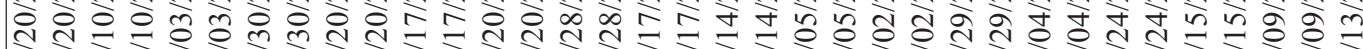

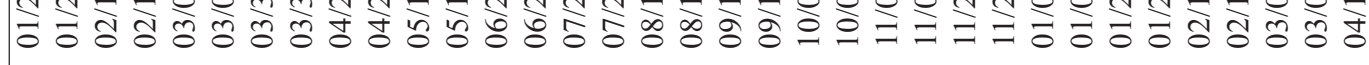




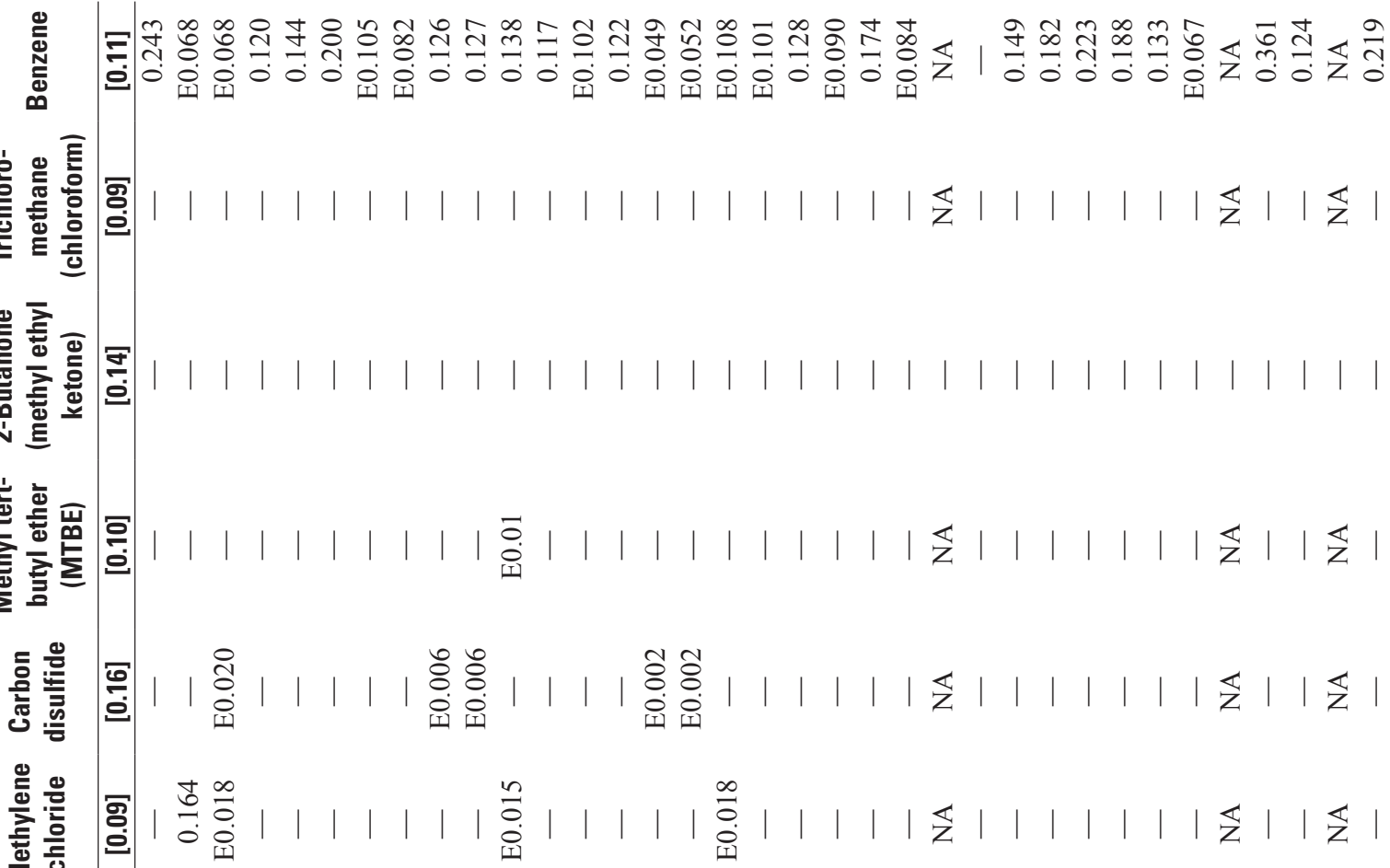

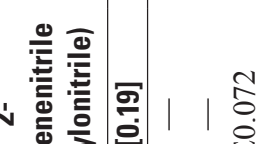

产递

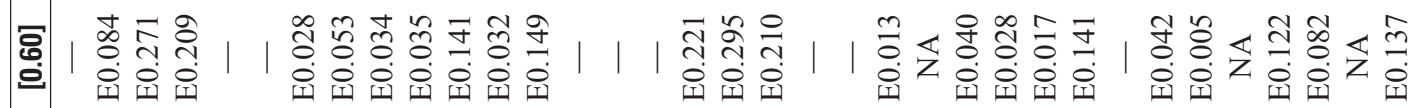

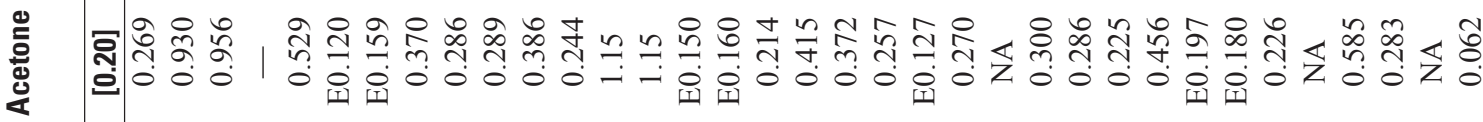

茎

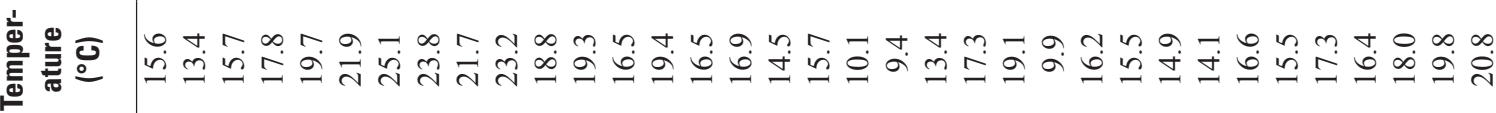

D.

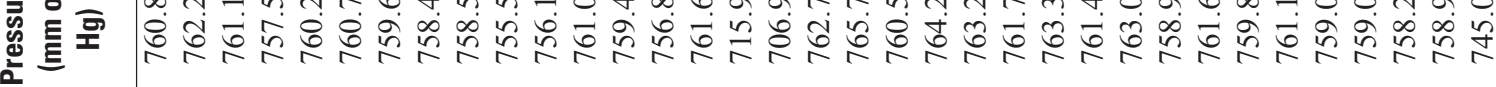

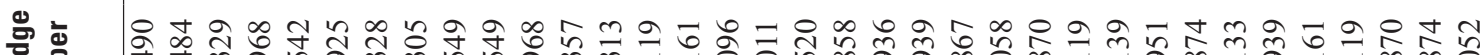

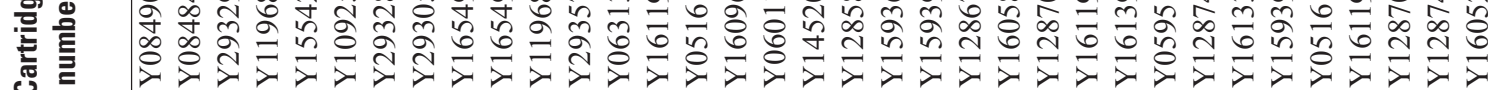
承

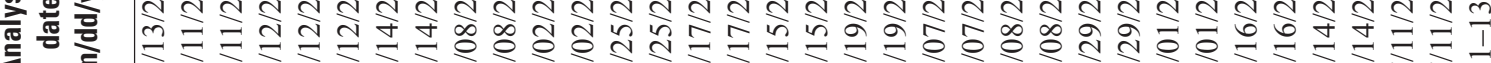

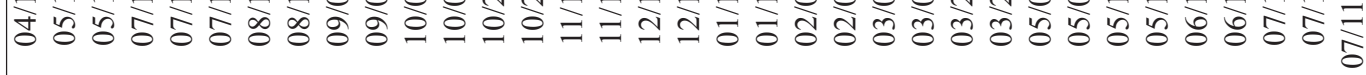

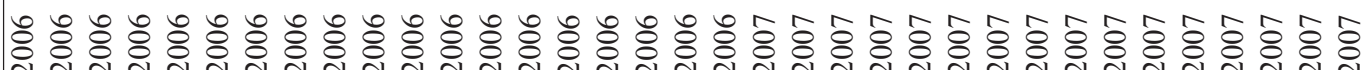
त $\exists$ तn

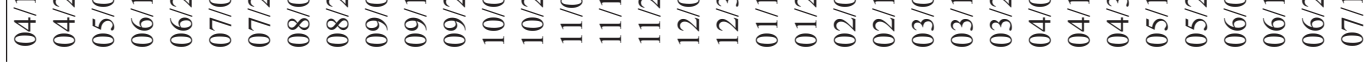




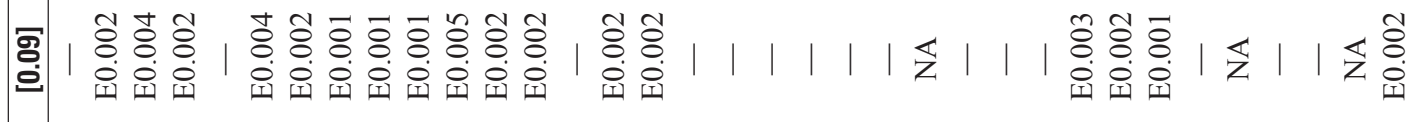




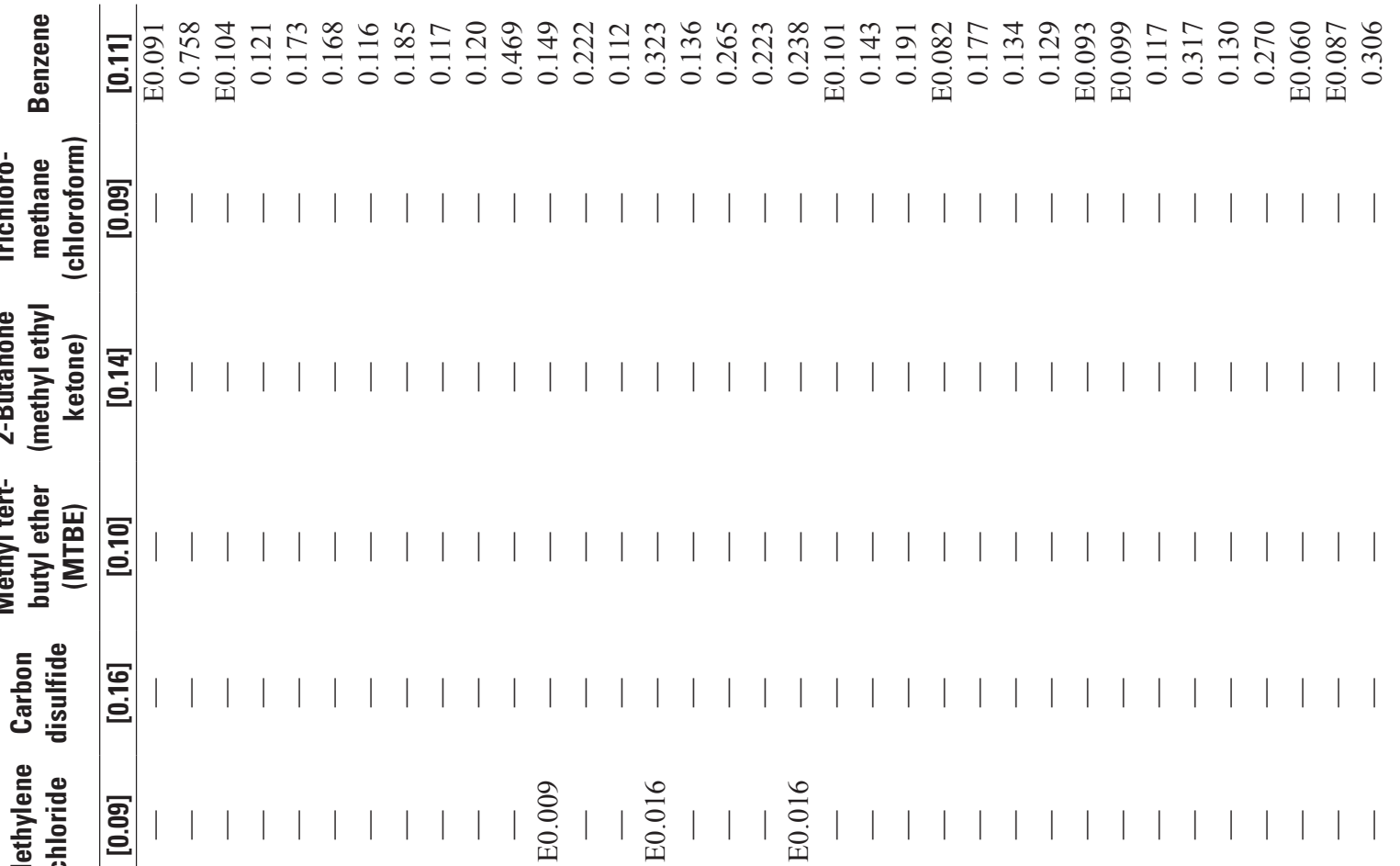

ن

홍

官.

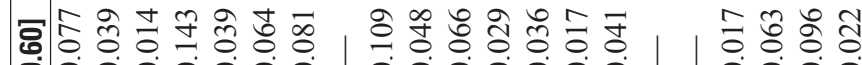

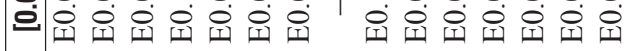

宇宁宫宫

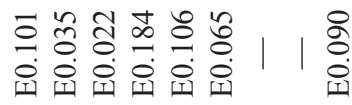

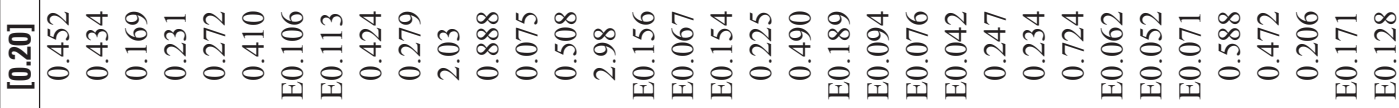

롤

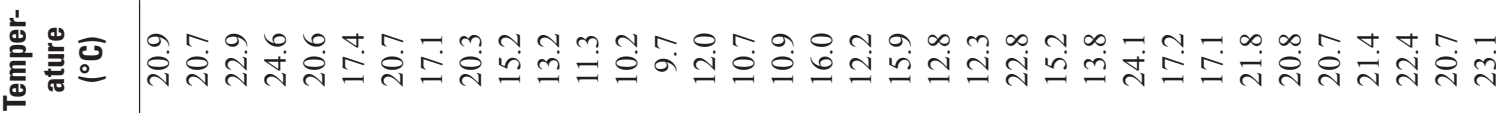

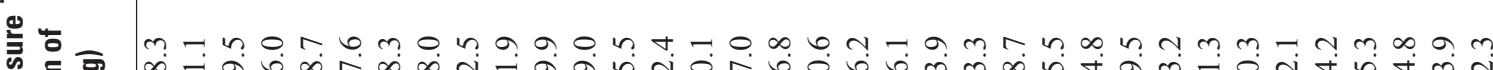

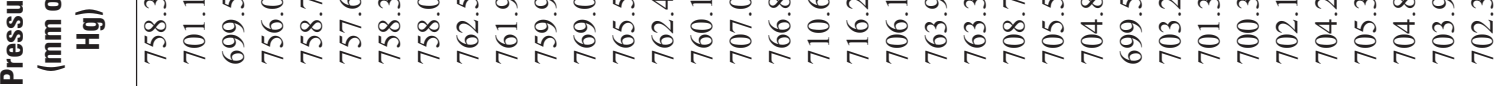

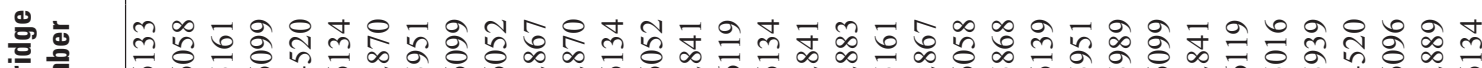

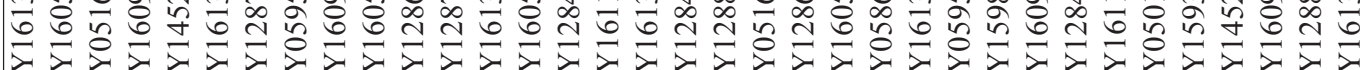

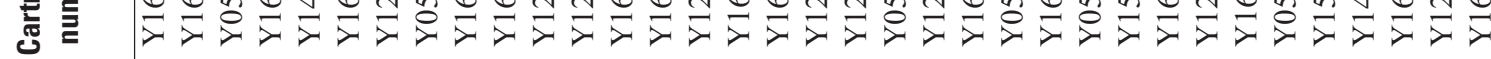
央

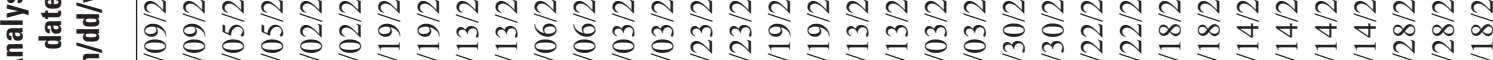

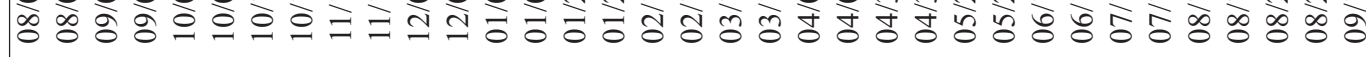

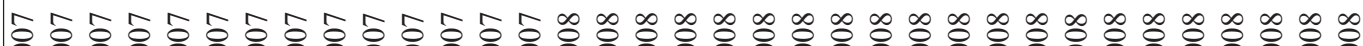

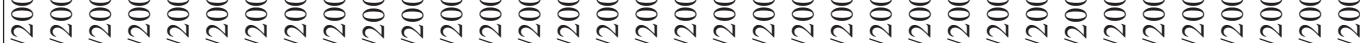
กิ讠寸웡

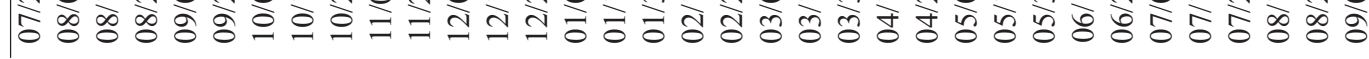




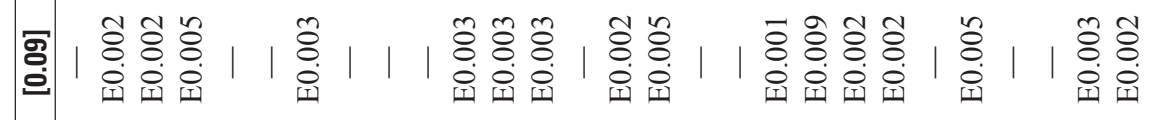

离

票官

총 :

ธี

要

ธ

䂧

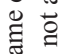

$\stackrel{Ð}{ \pm}$

范

g

:

पु

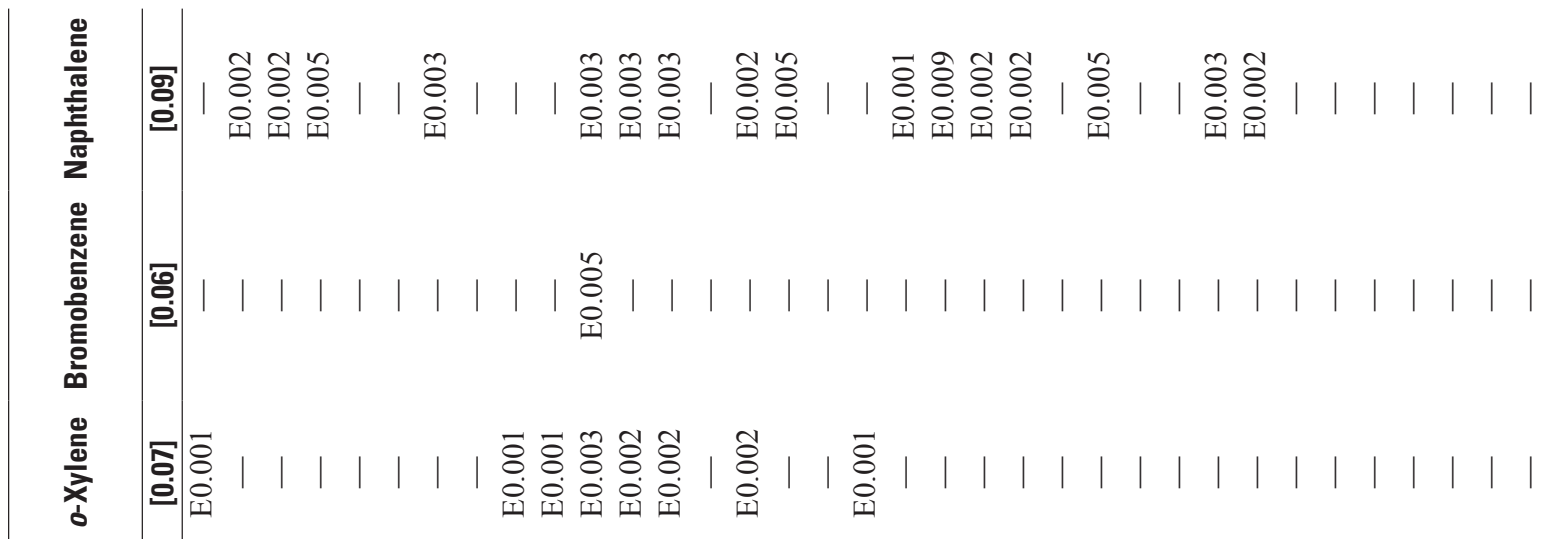

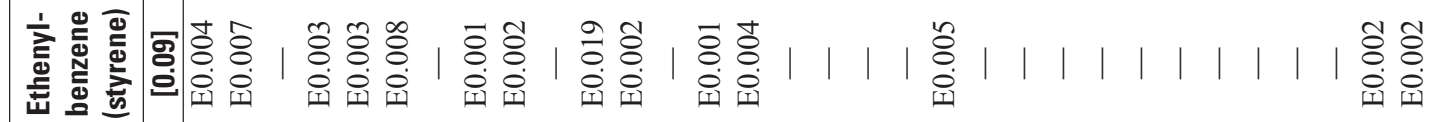

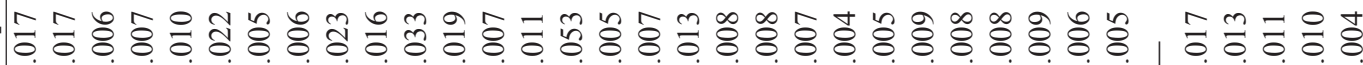

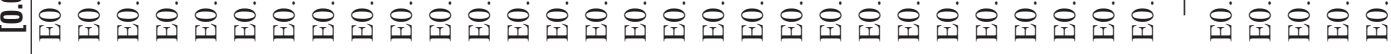

폴

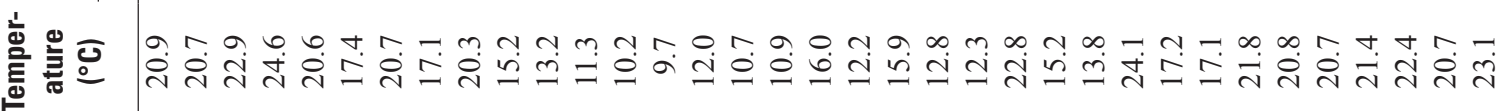

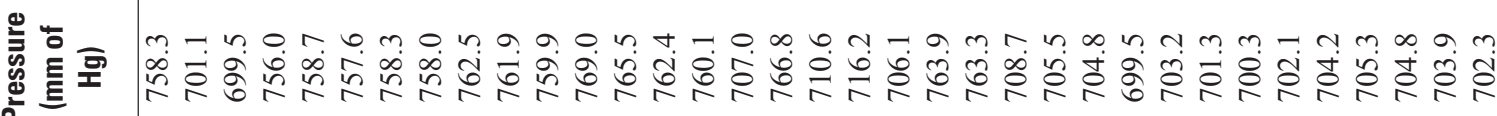

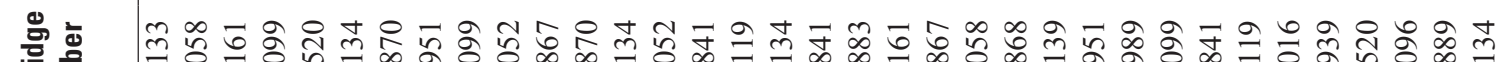

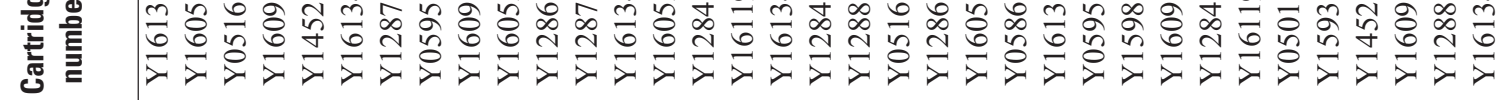




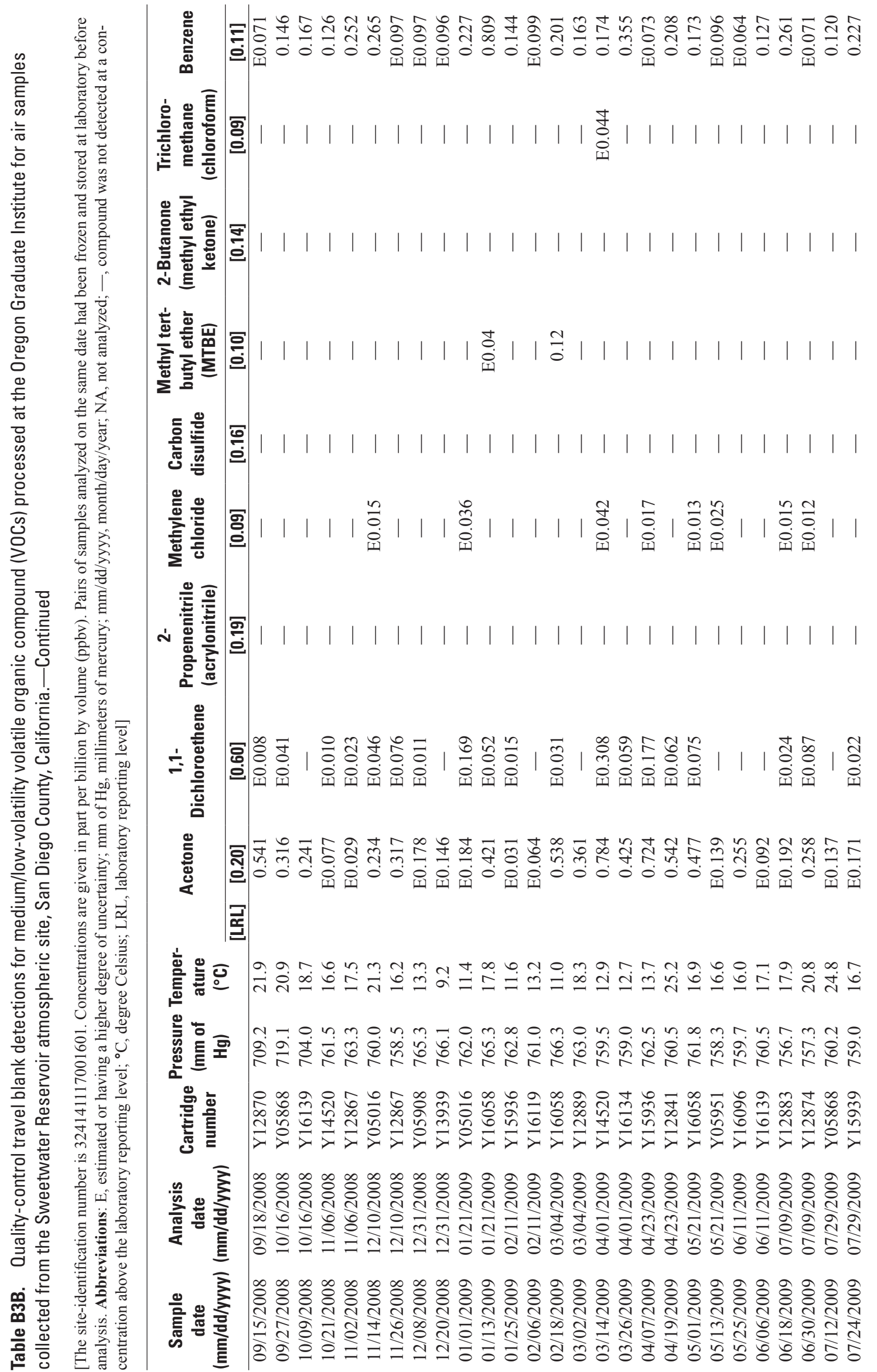




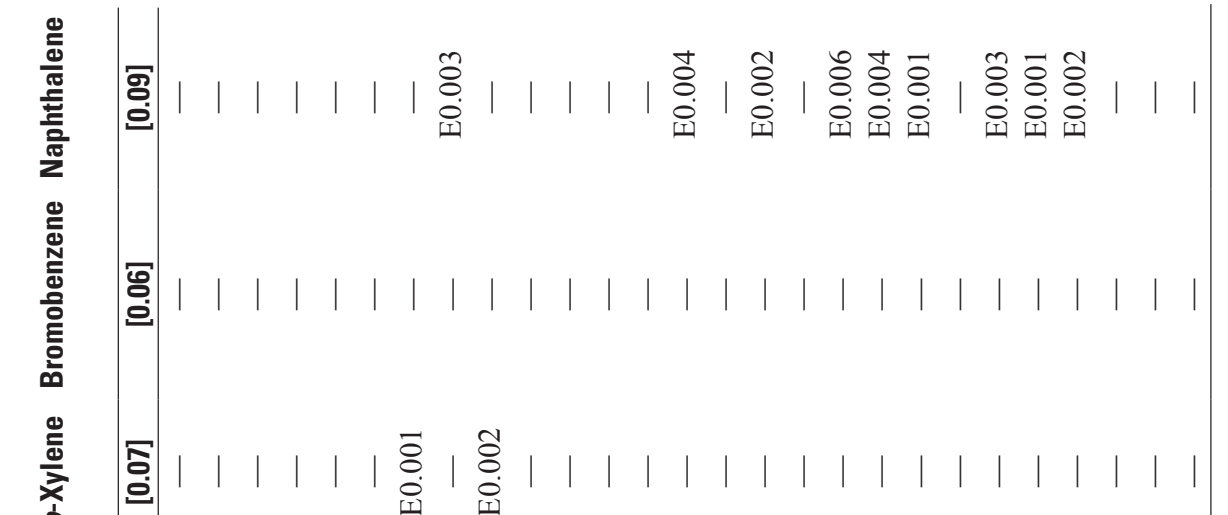

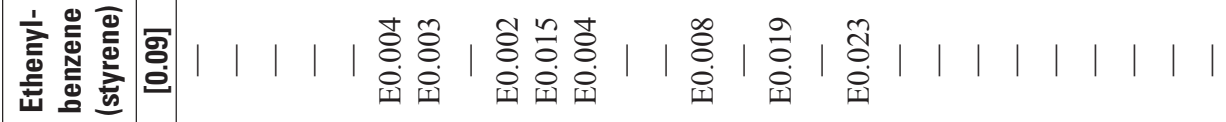

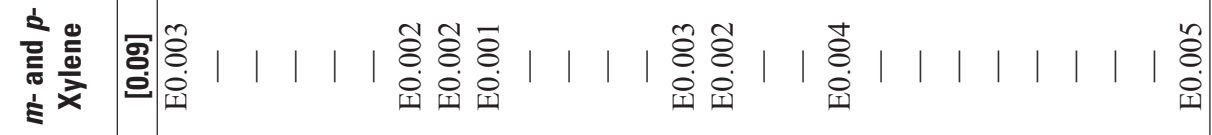

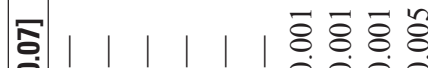

응 i亩宁完
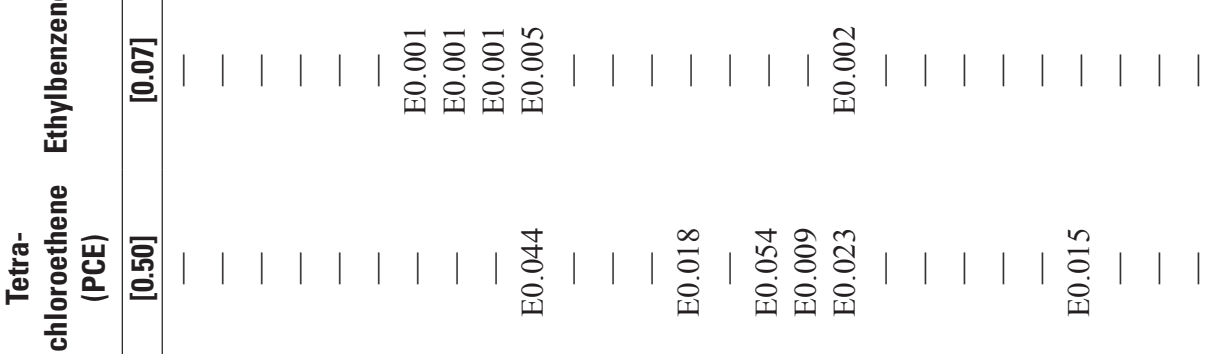

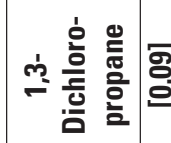

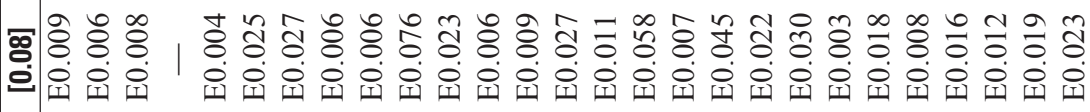
疋 产产恶

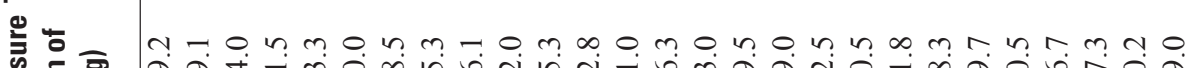

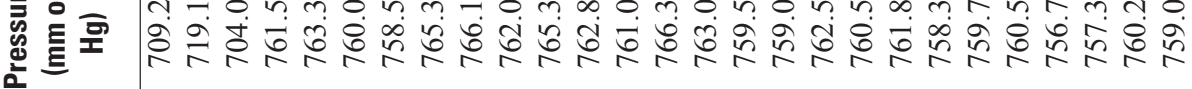
苛市

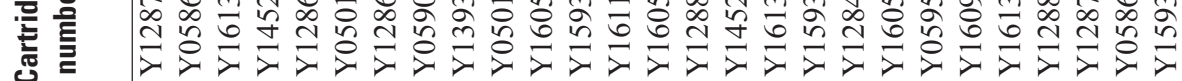

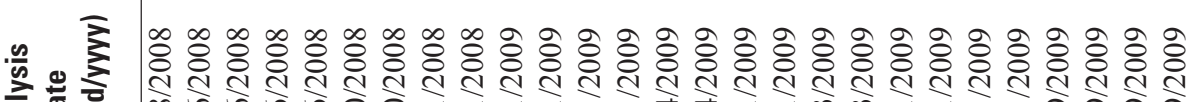
흥 홍

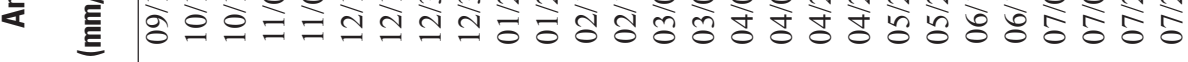

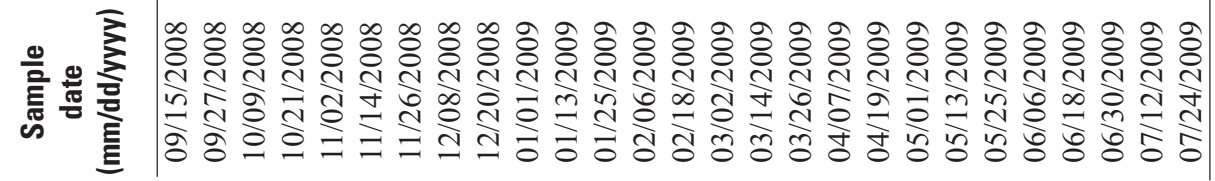




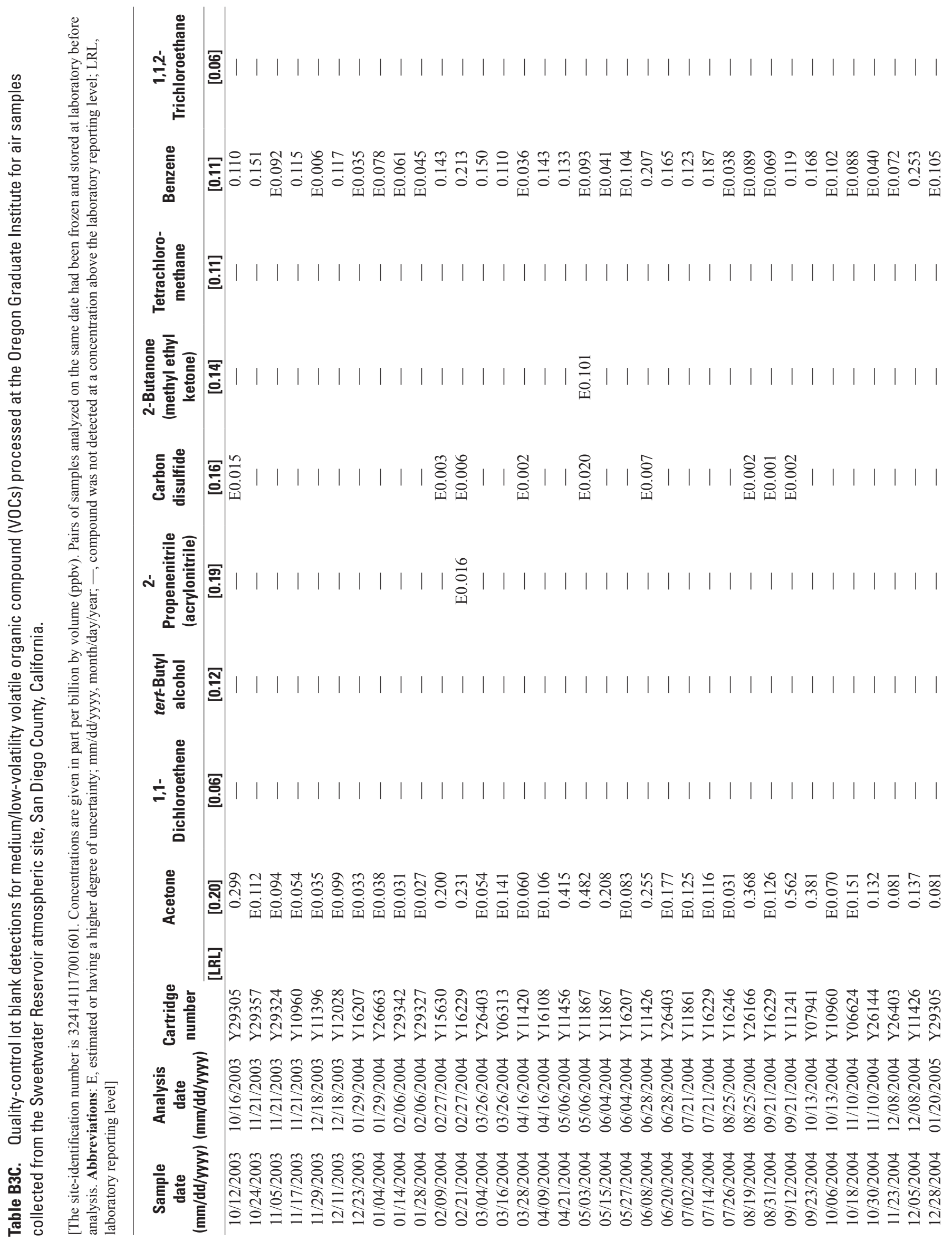




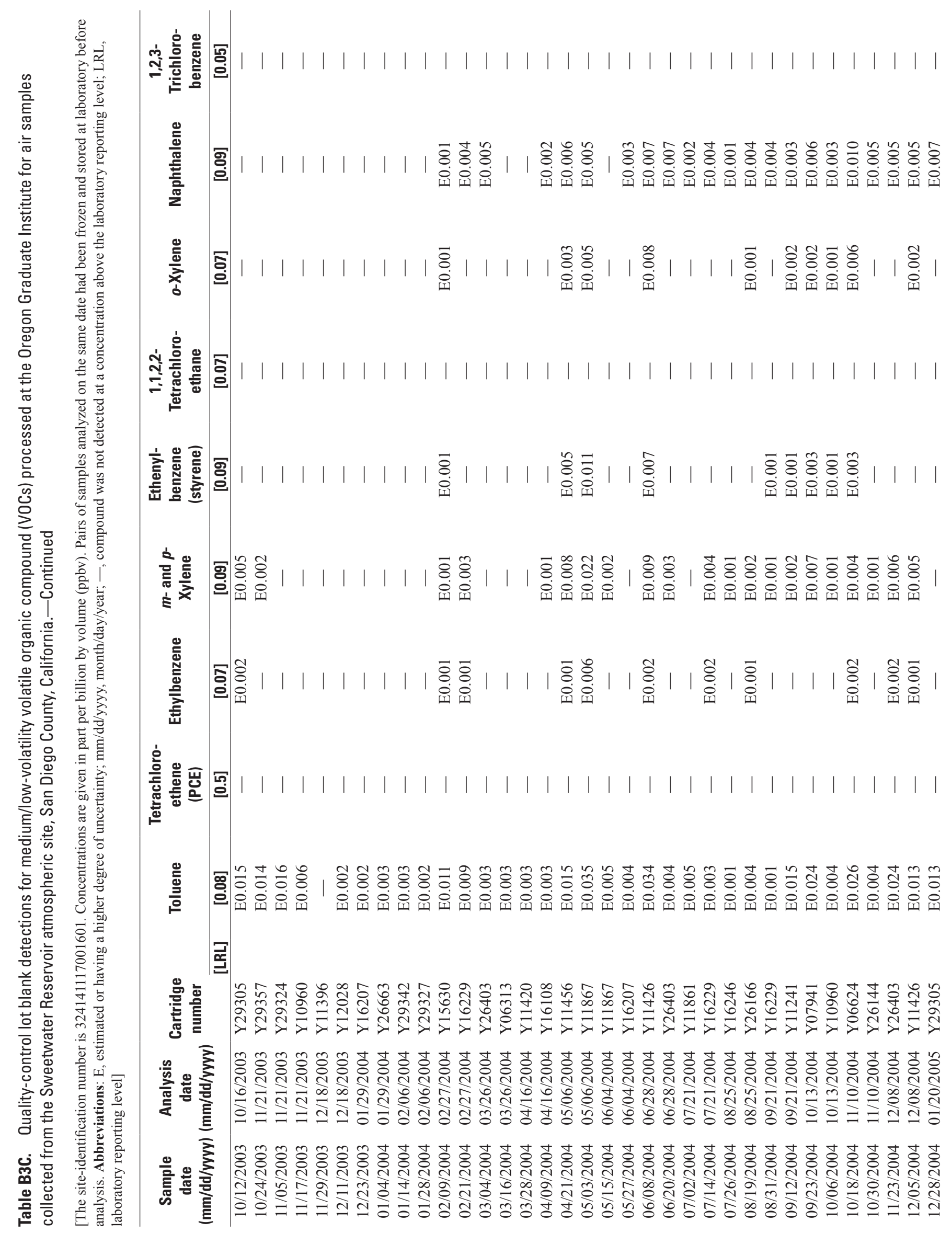




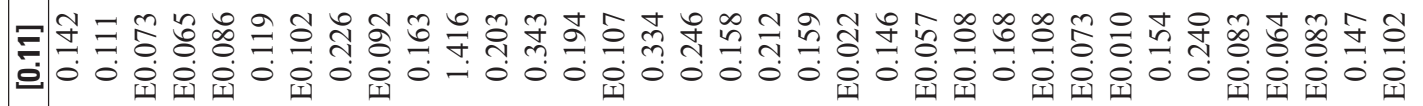

E

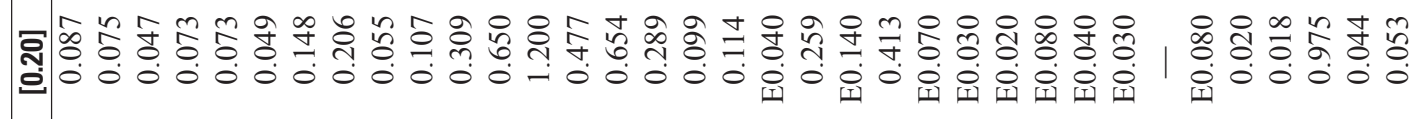

롤

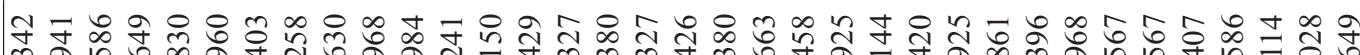

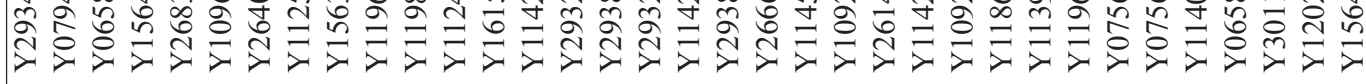

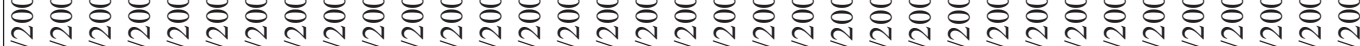
위의윙

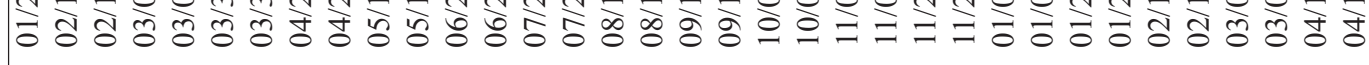




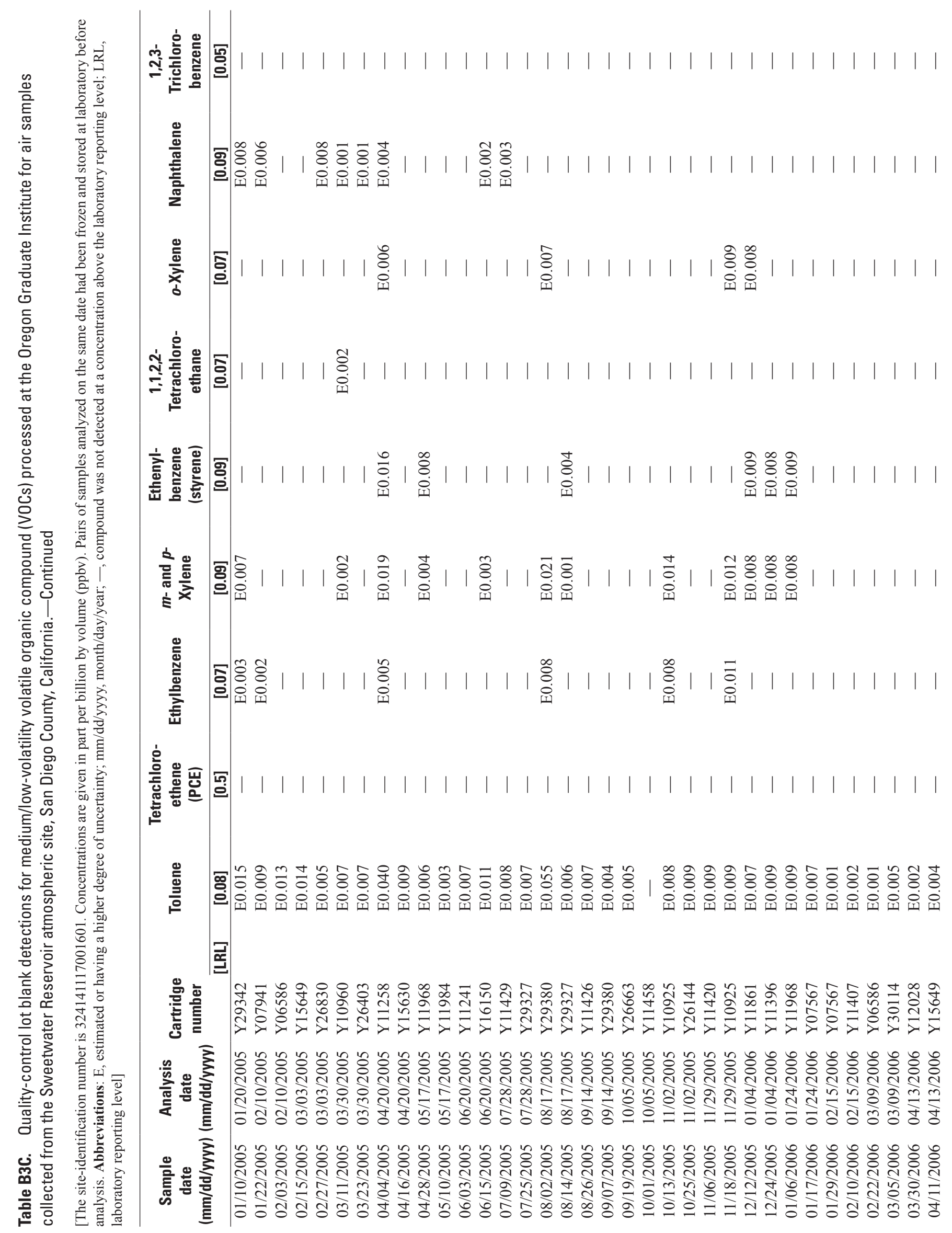




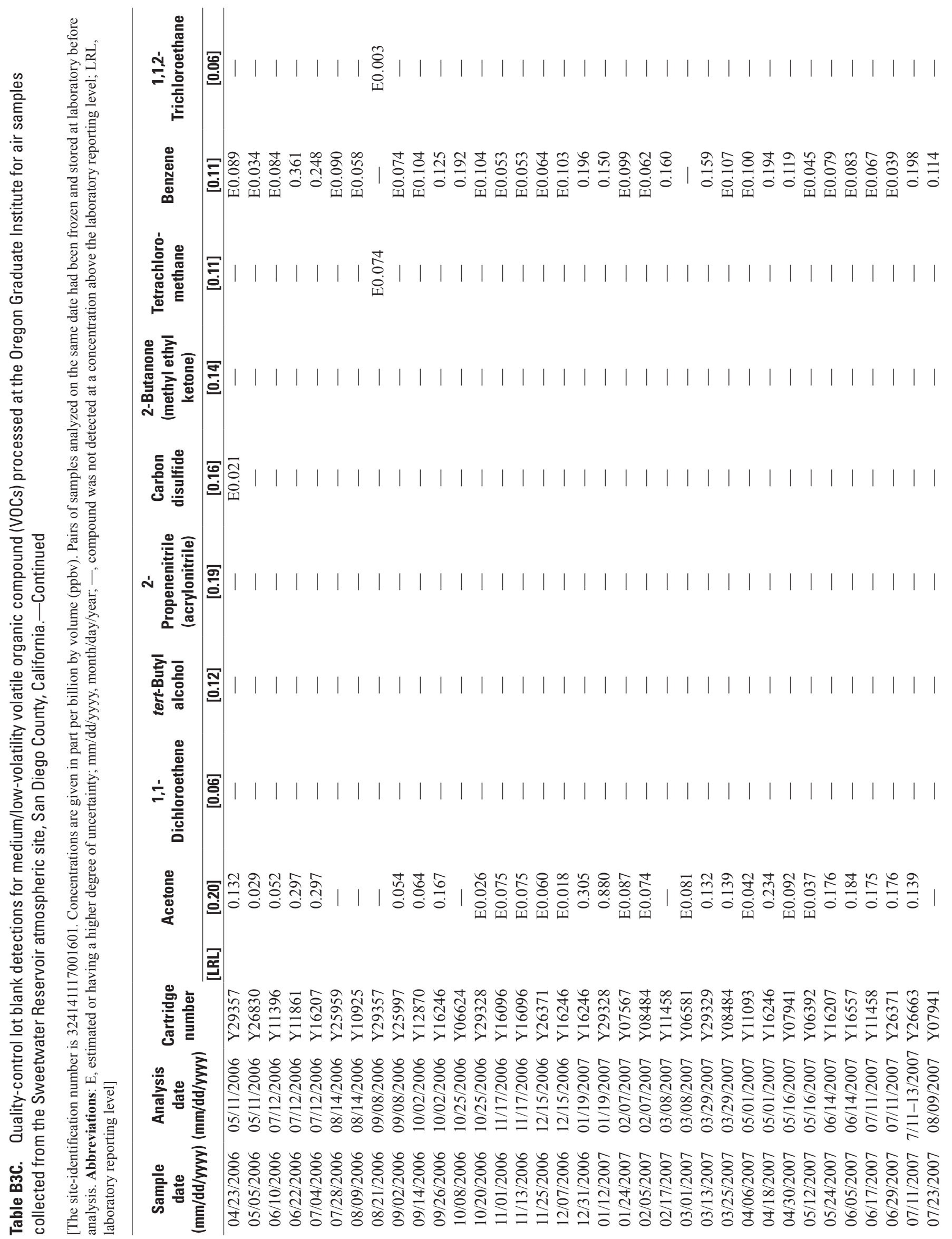


荧

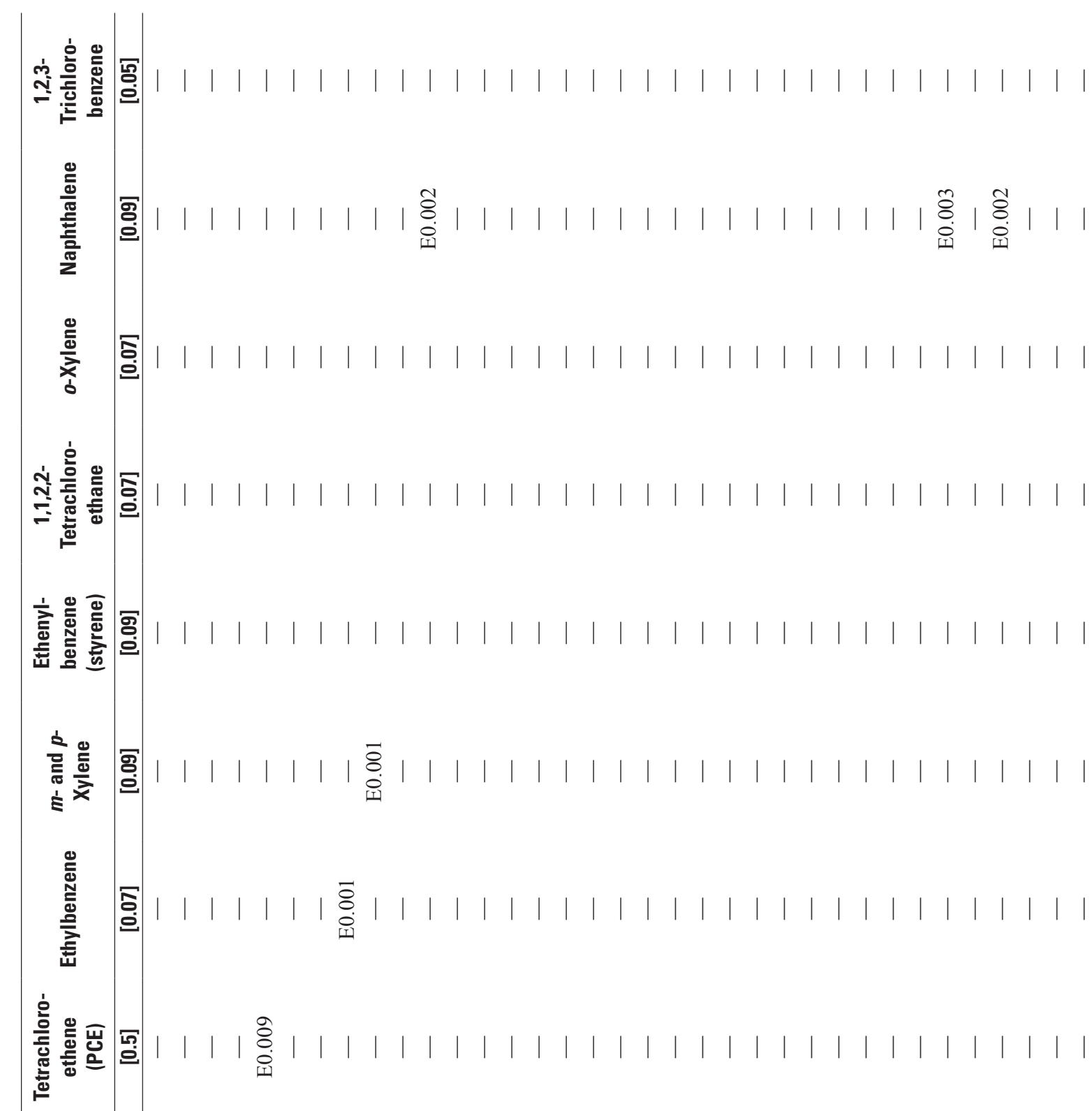

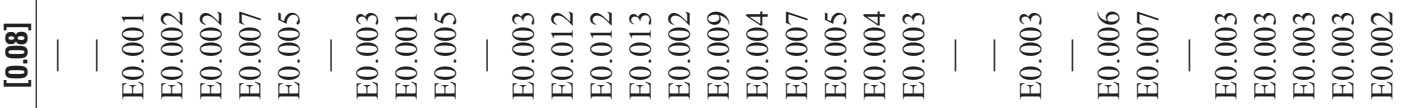

$\underset{\vec{z}}{\underline{2}}$

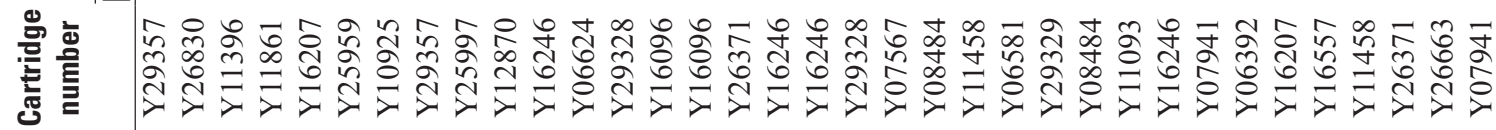

卒

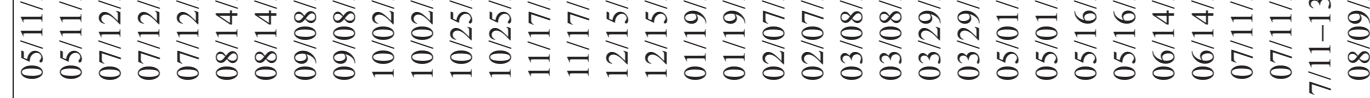




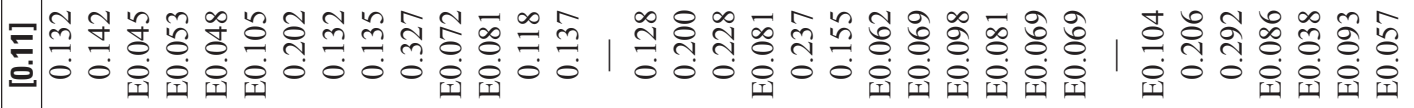

폴

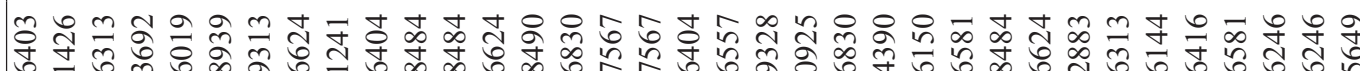

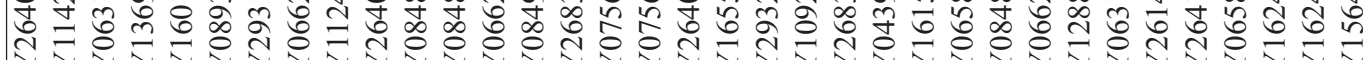
o g.

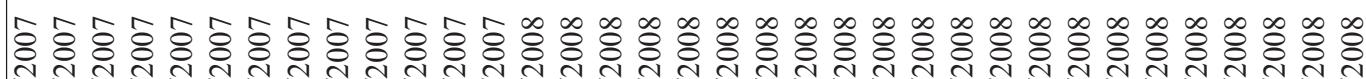

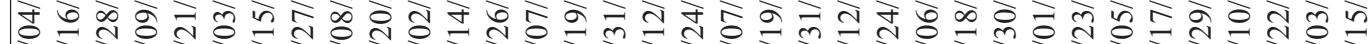

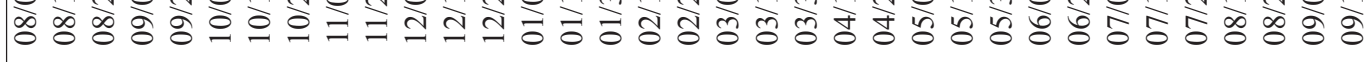




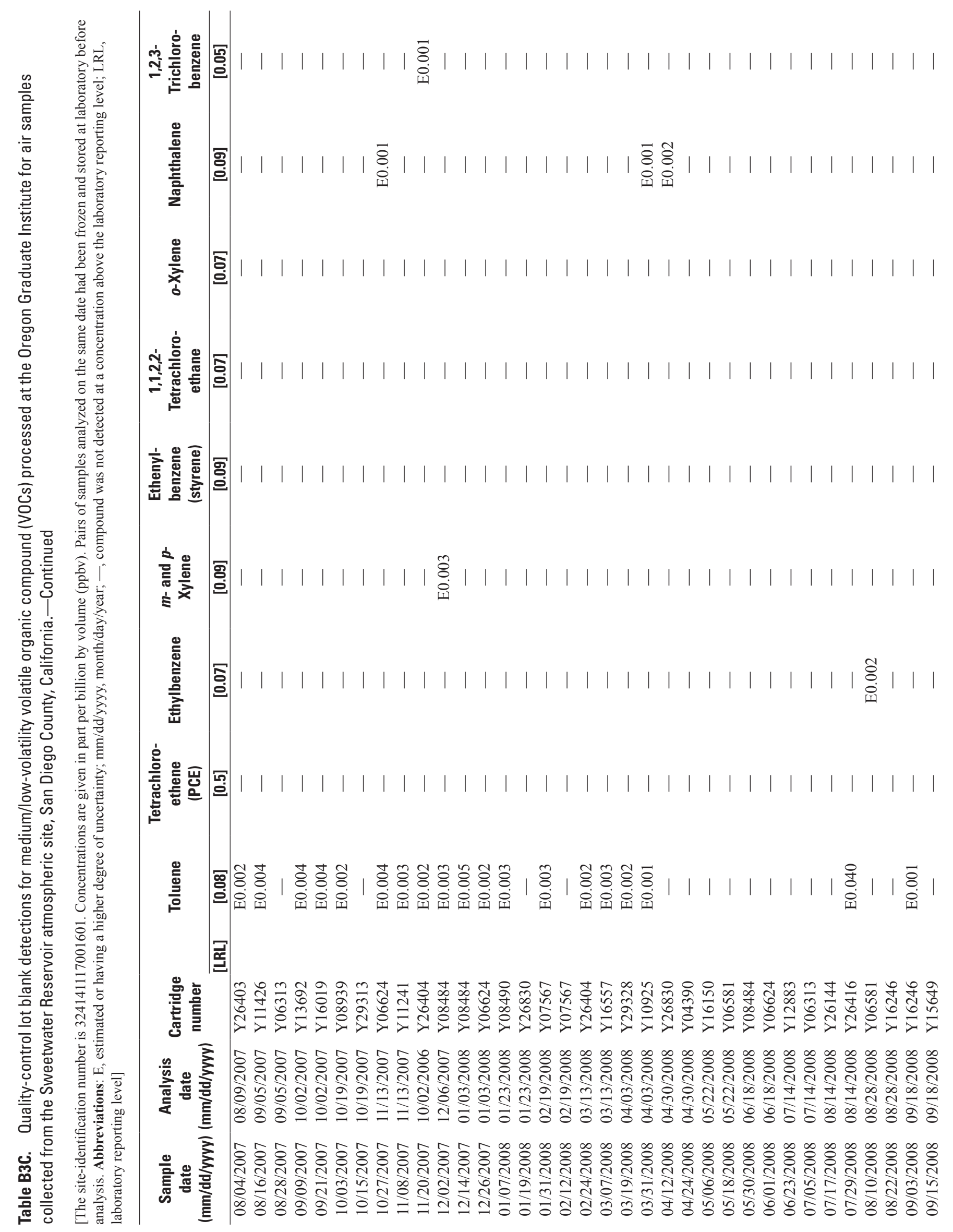




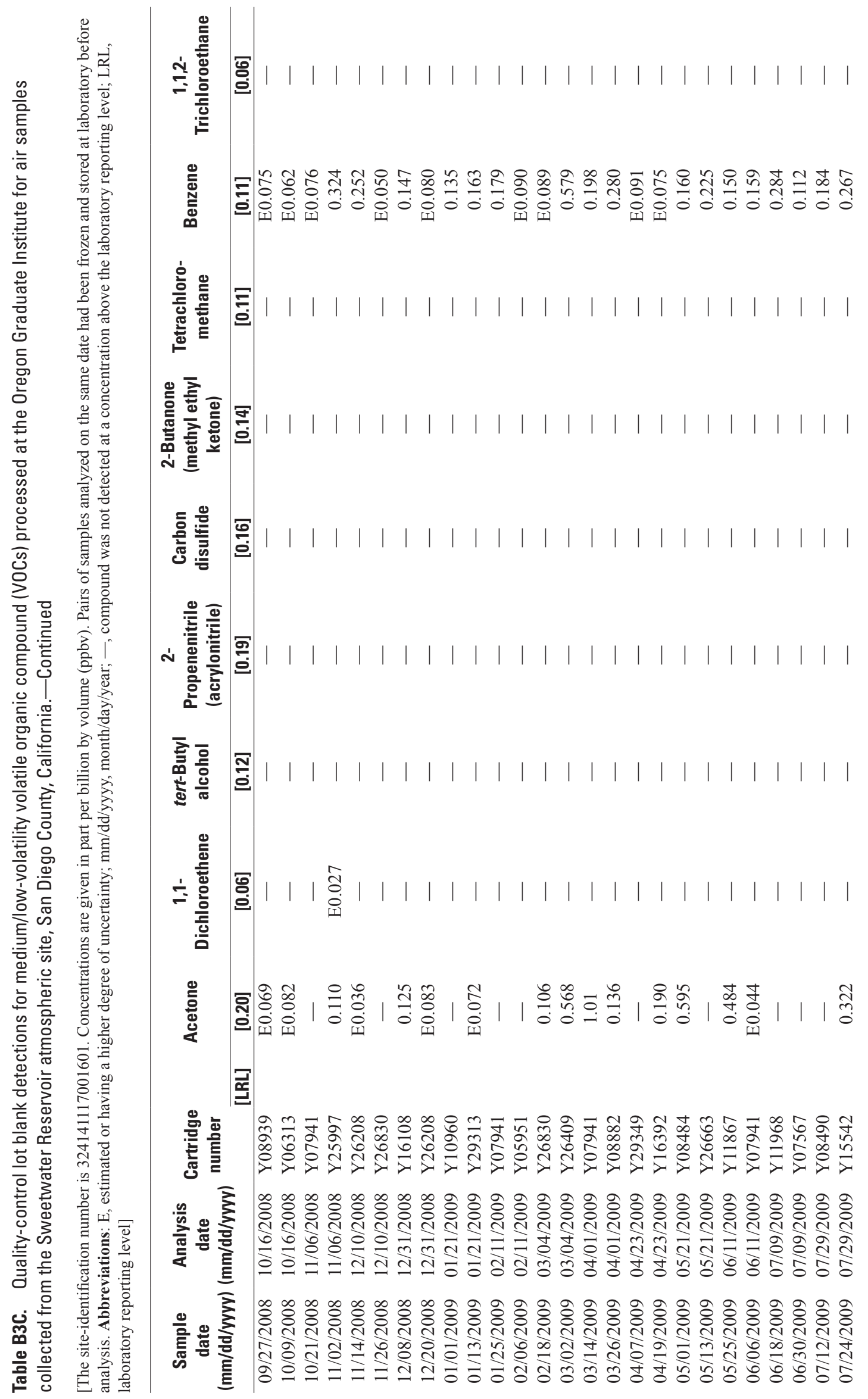




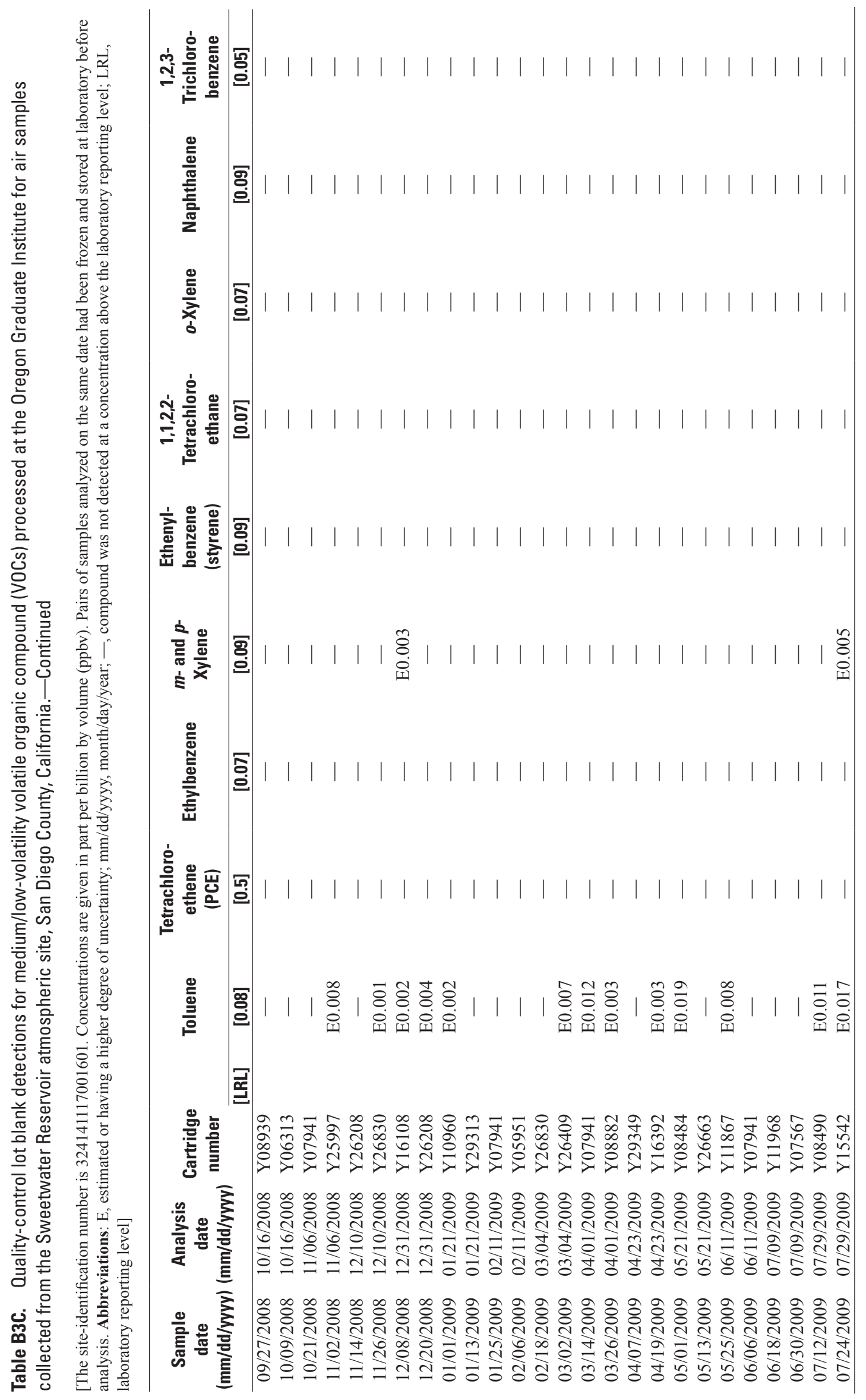




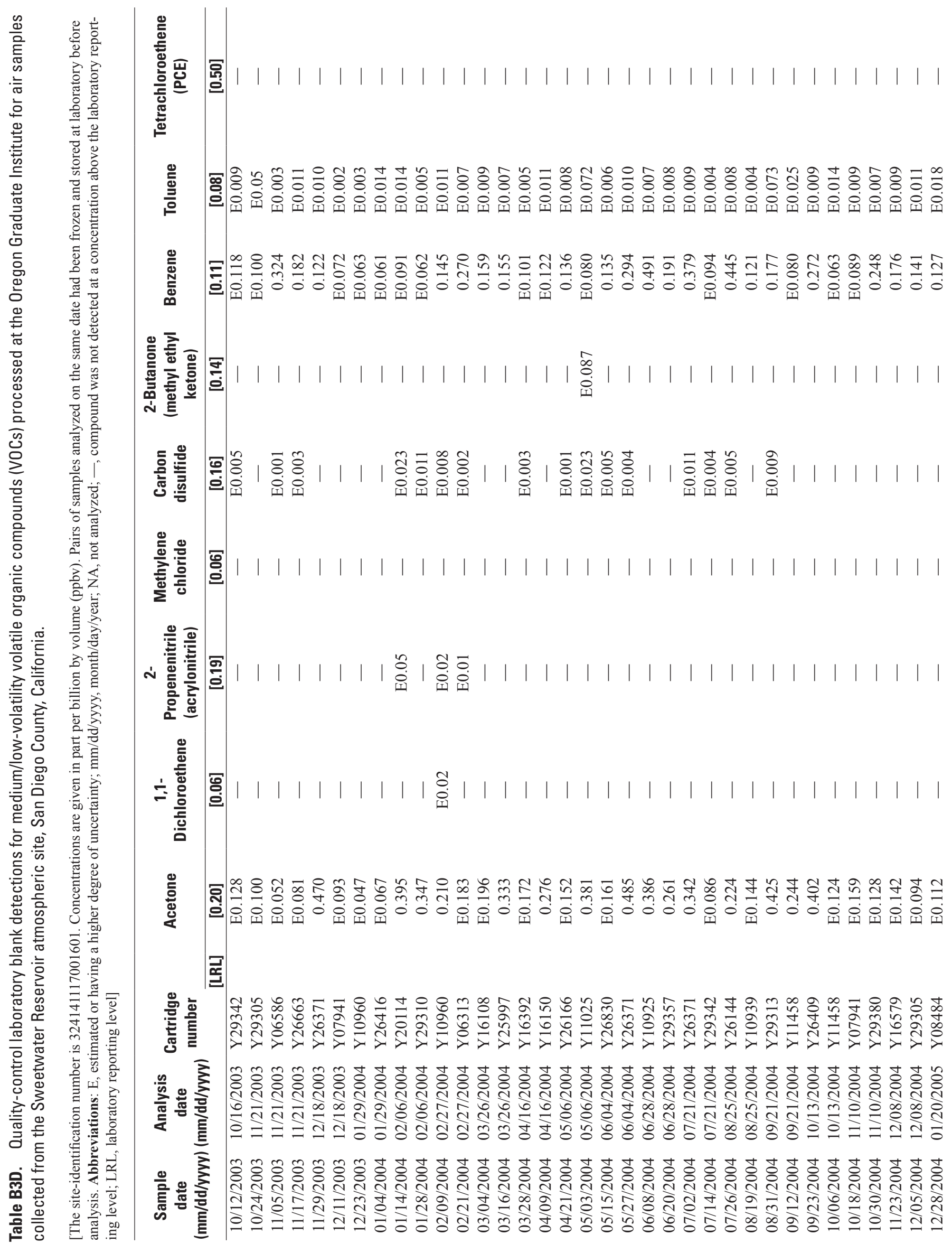




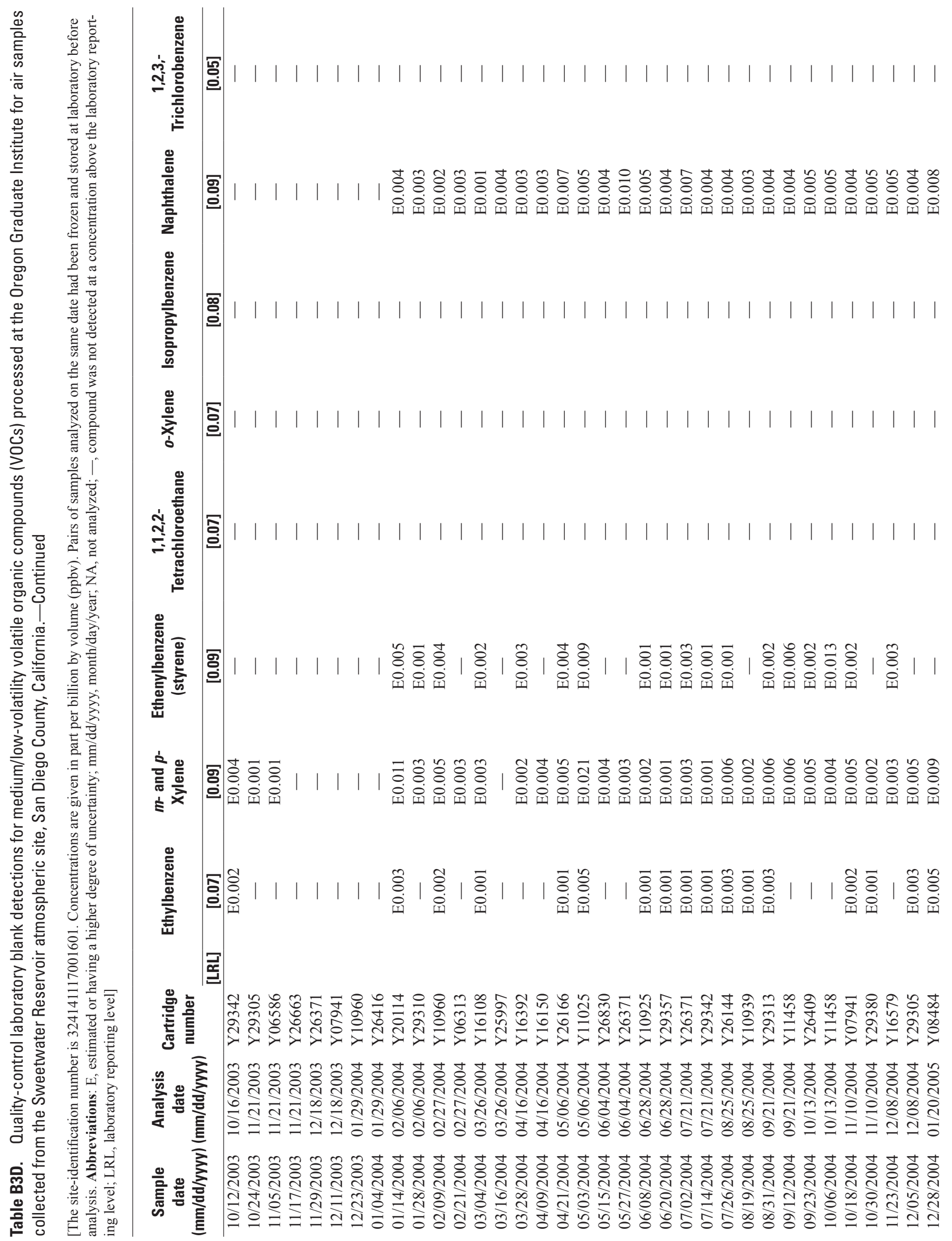




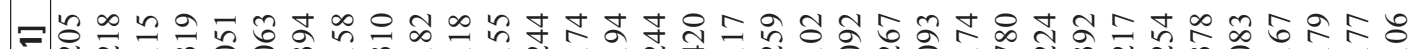
可

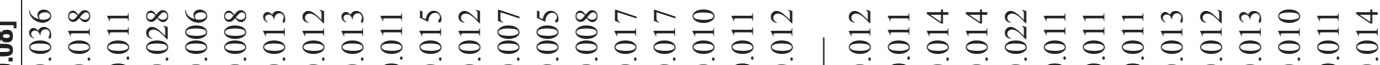

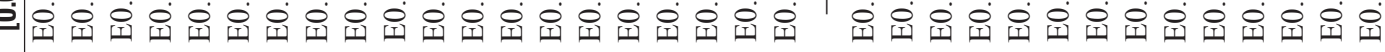

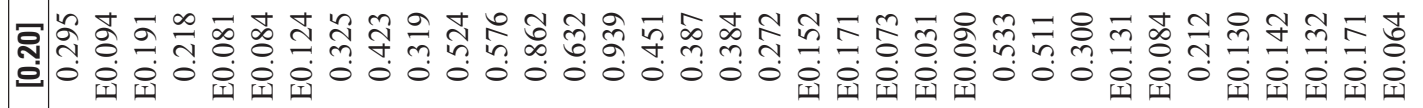

폴

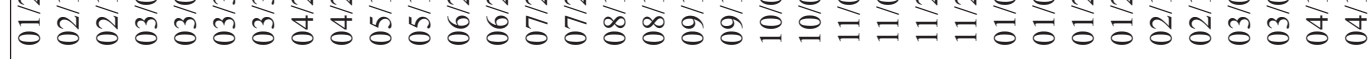




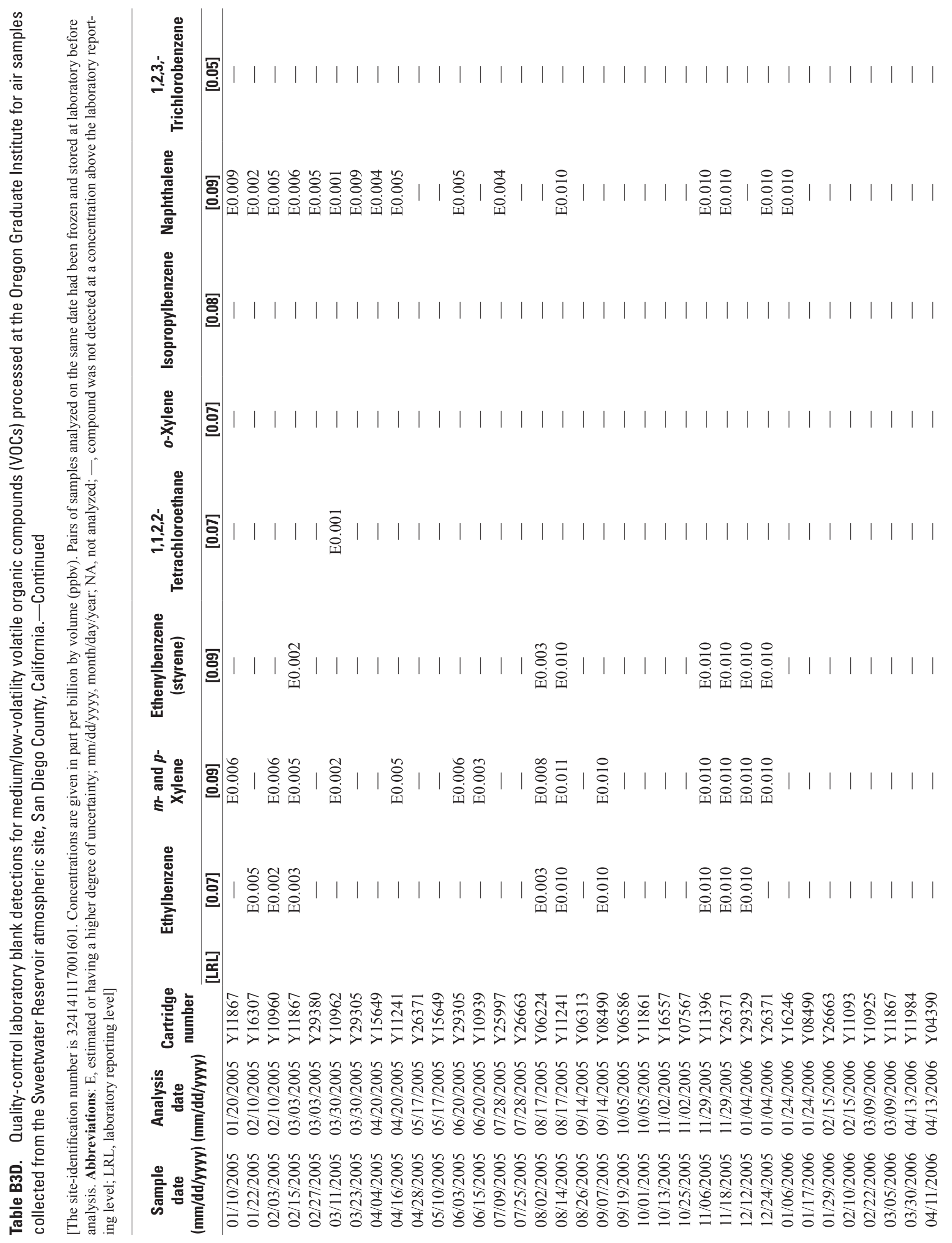




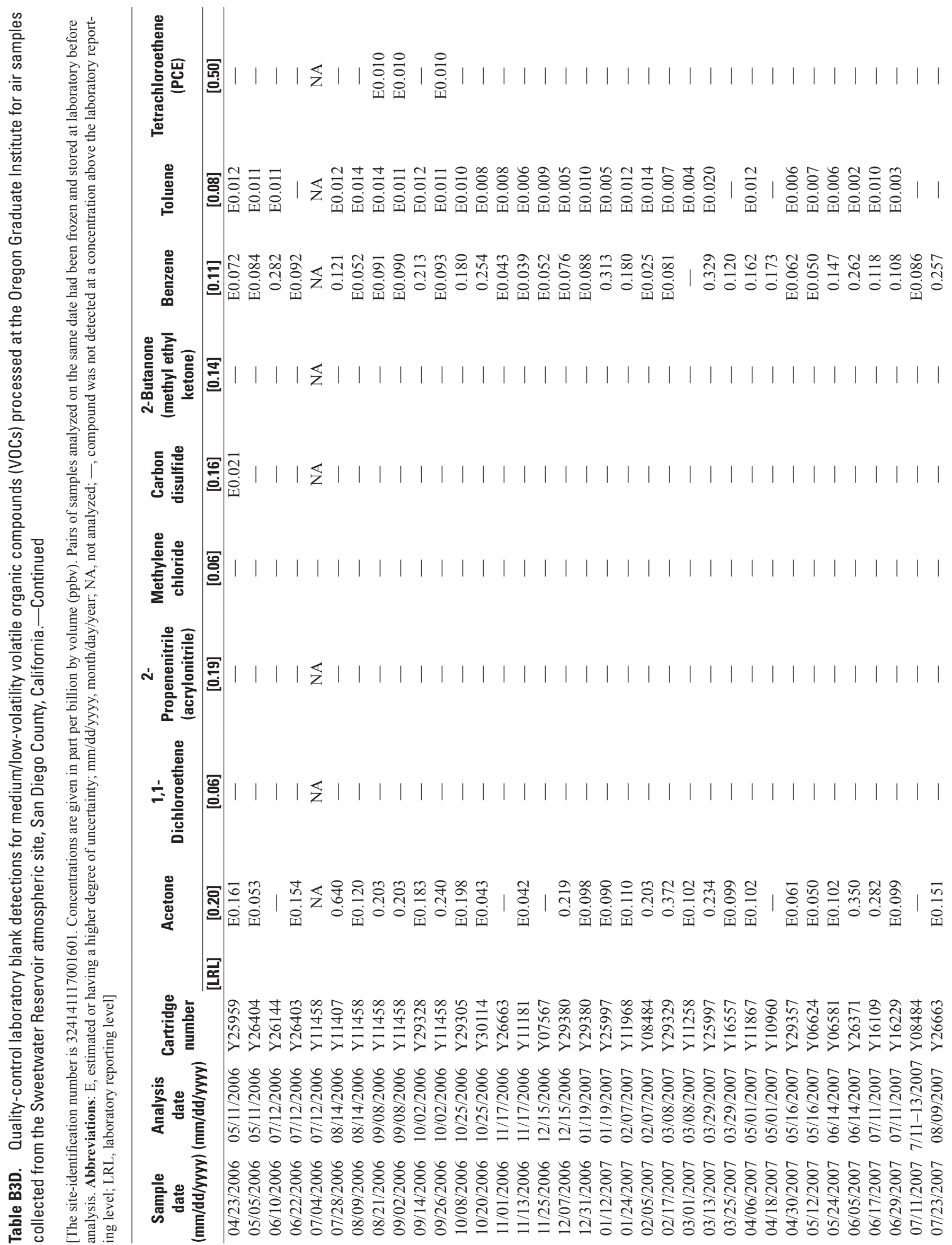




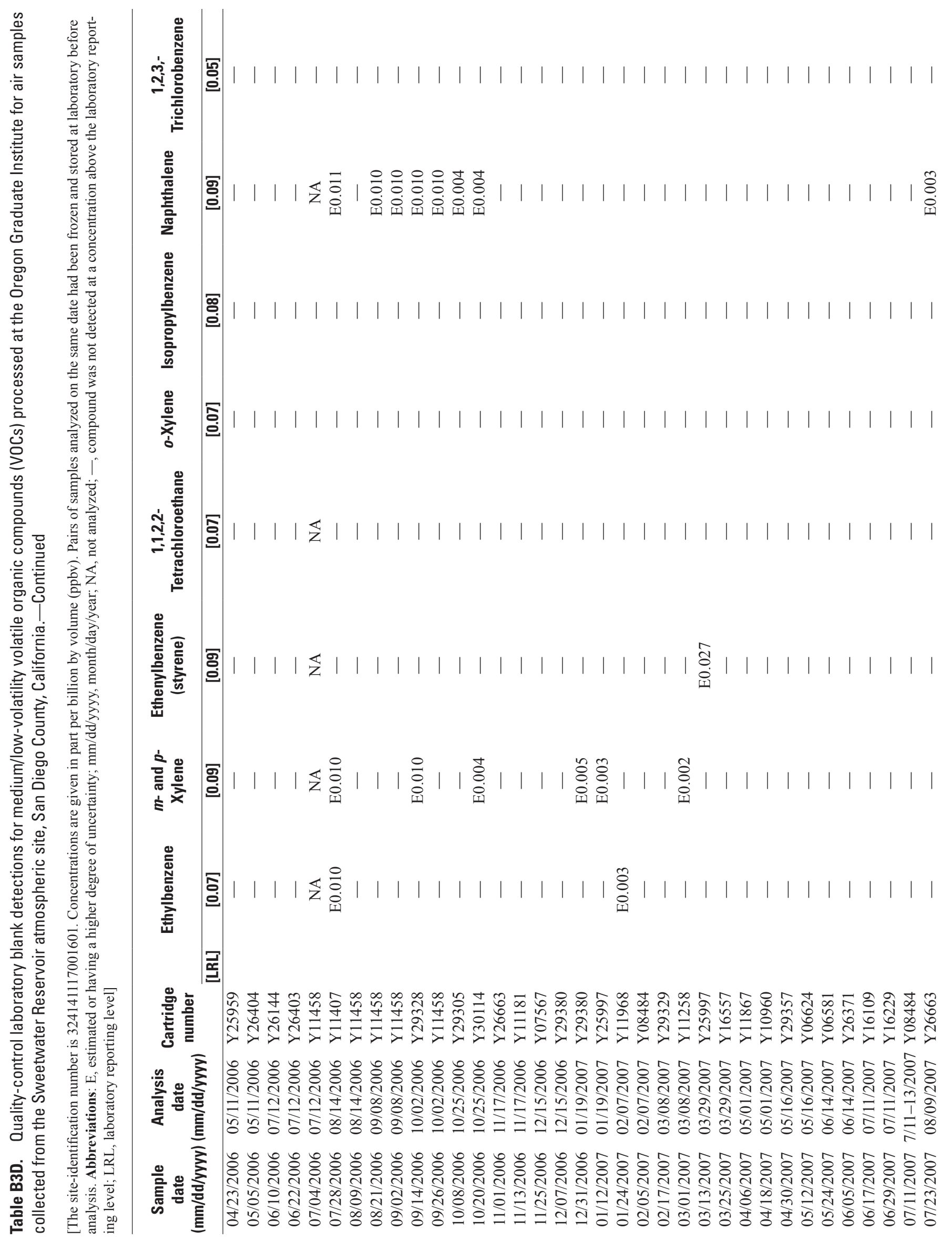


\%

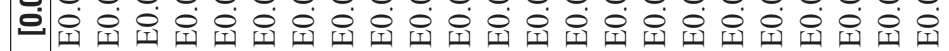

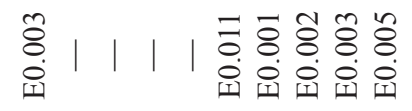

폴

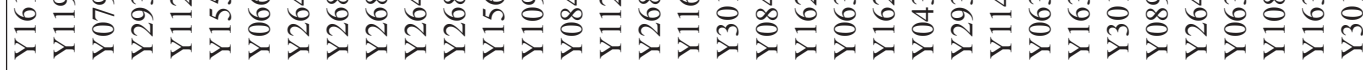

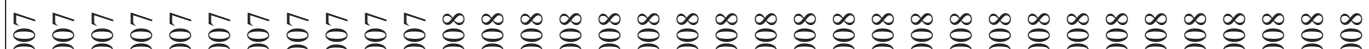
ते के

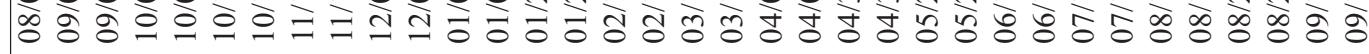




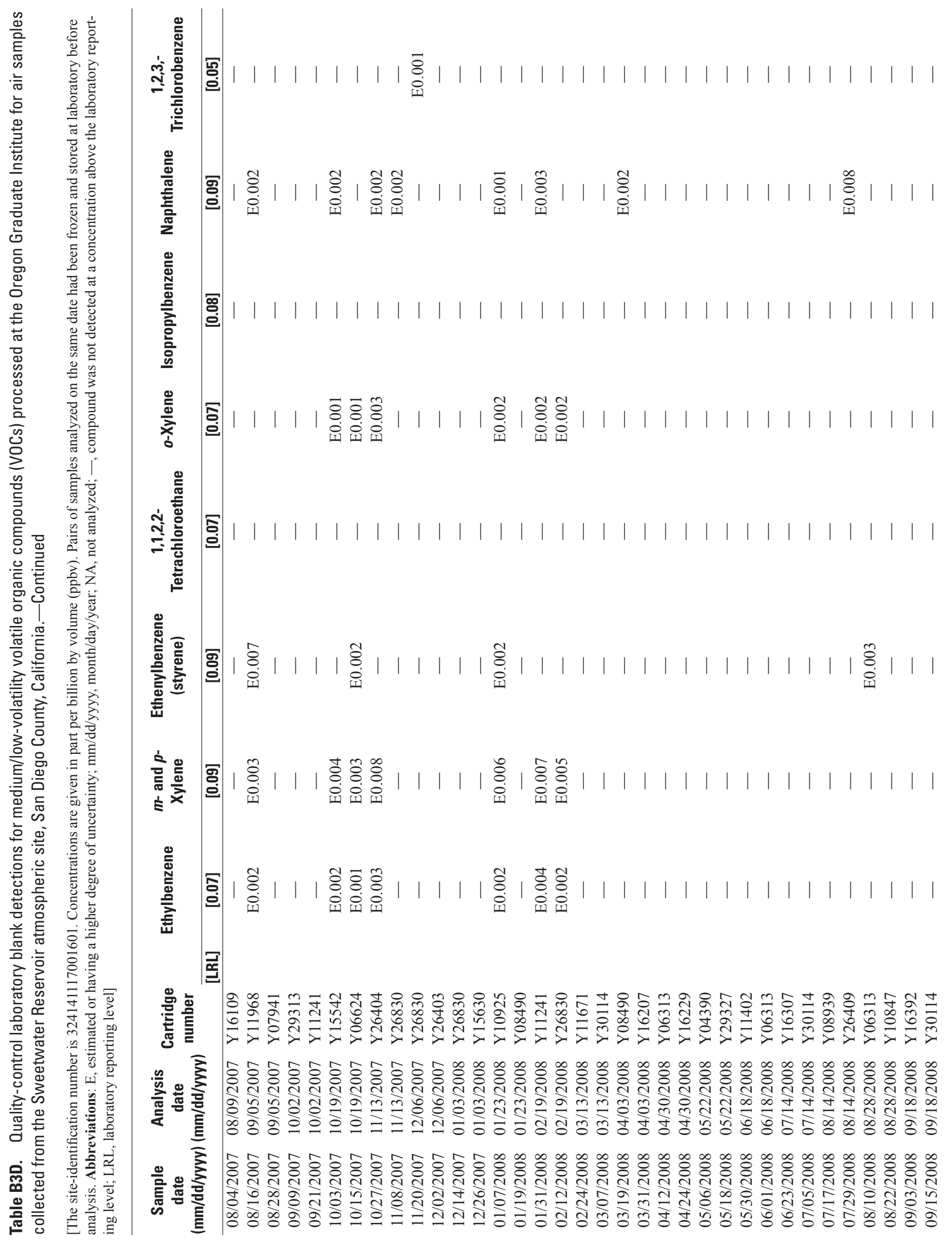




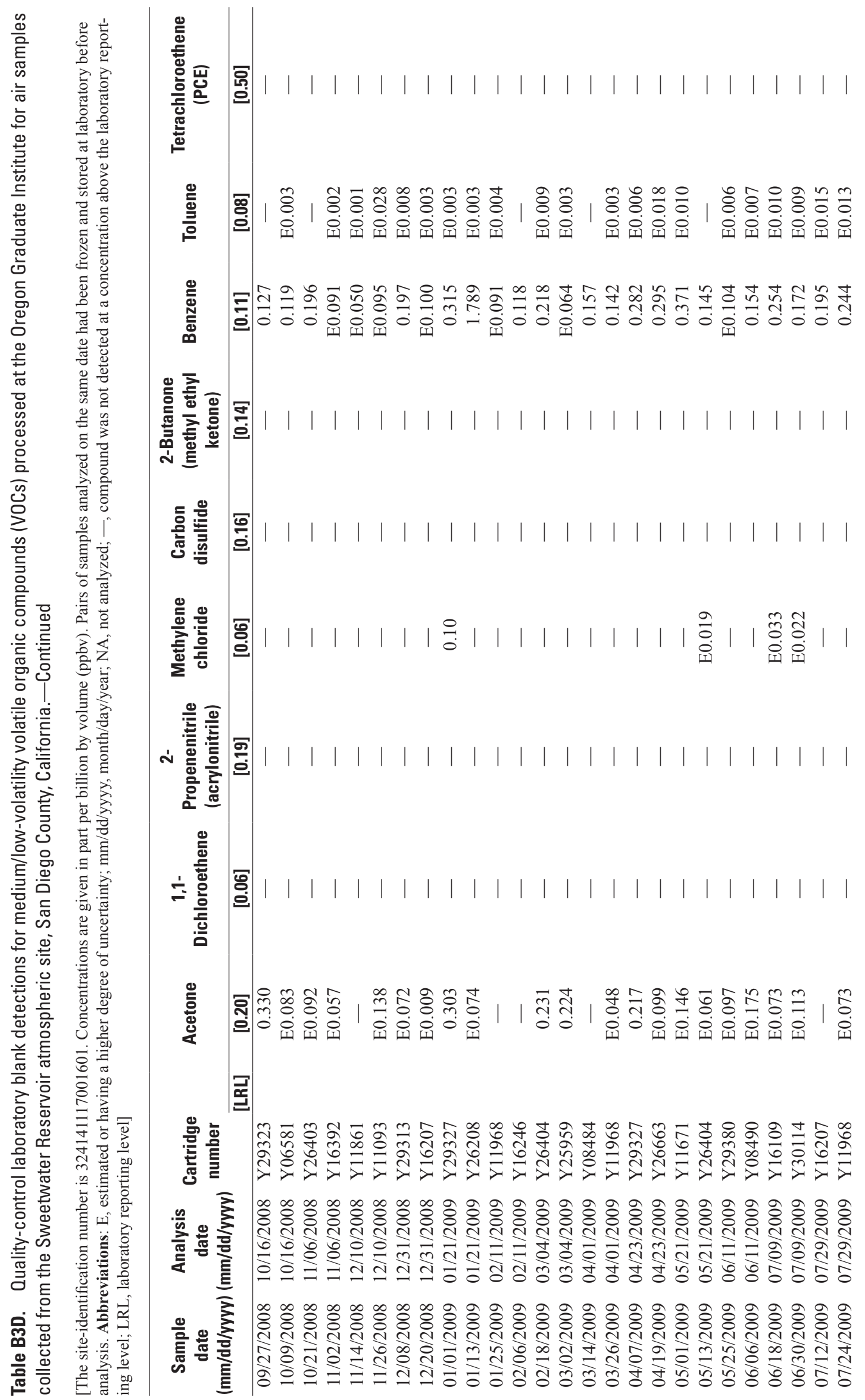




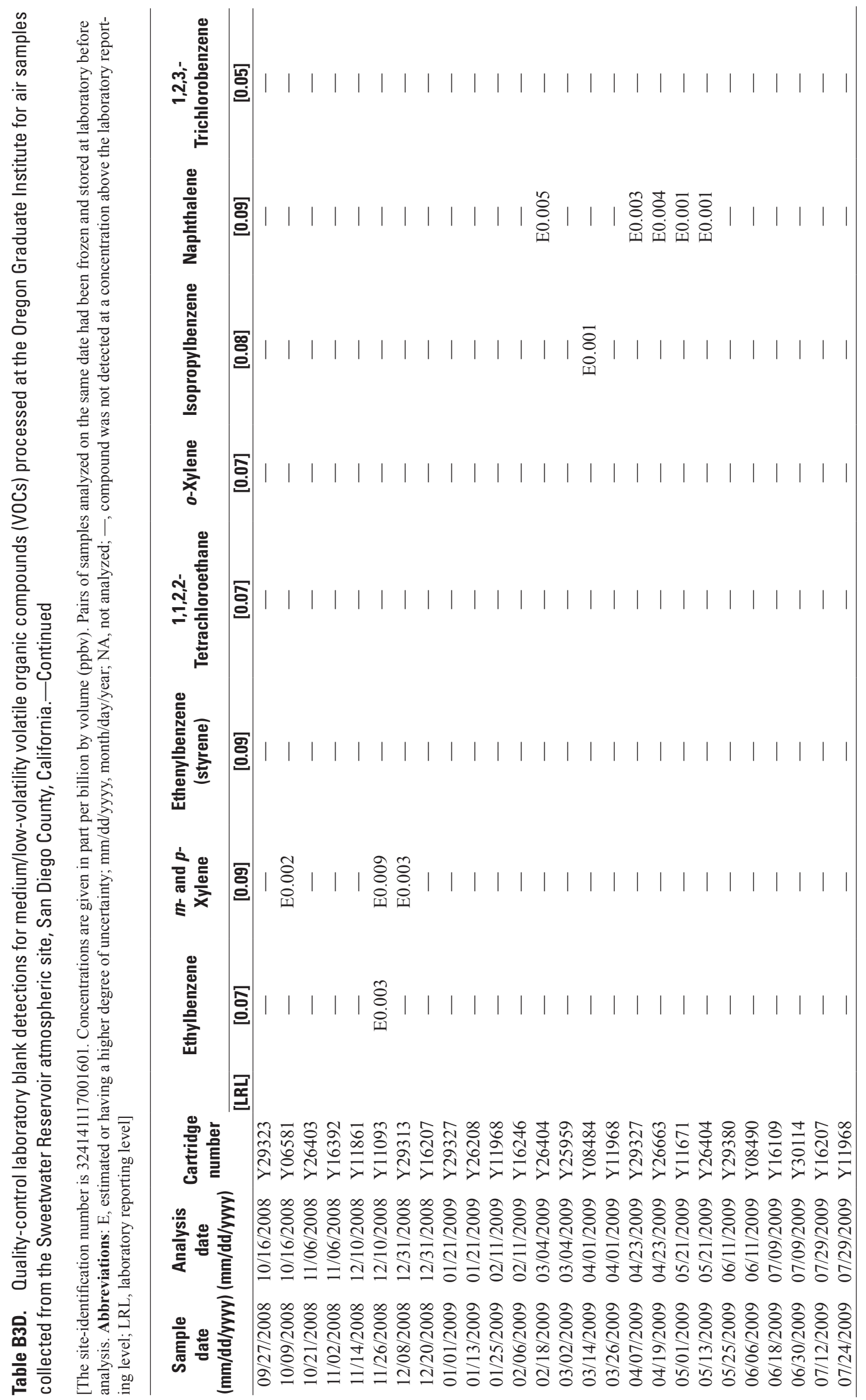


Table B4A. National Water Quality Laboratory quality-control reagent-spike results for polycyclic aromatic hydrocarbon (PAH) compound recoveries for the Sweetwater Reservoir atmospheric site, San Diego County, California.

[Alkylated PAH were not spiked, so they are not listed in this table. Values are given in percentage recovery, unless noted. Abbreviations: GCMS, gas chromatography mass spectrometry; GFF, glass fiber filter; mm/dd/yyyy, month/day/year; NA, not analyzed; PSE, pressurized solvent extraction; PUF, polyurethane foam; Scan, full scan ion monitoring mode; SIM, selected ion monitoring mode; TSP, total suspended particles (calculated for GFF samples only assuming 315 cubic meters air volume); $\mu \mathrm{g} / \mathrm{m}^{3} ;$ micrograms per cubic meter]

\begin{tabular}{|c|c|c|c|c|c|c|c|c|c|c|c|c|}
\hline $\begin{array}{c}\text { Begin } \\
\text { date } \\
\text { (mm/dd/yyyy) }\end{array}$ & $\begin{array}{c}\text { Sample } \\
\text { component } \\
\text { spiked }\end{array}$ & $\begin{array}{c}\text { Sample } \\
\text { t preparation } \\
\text { set number }\end{array}$ & $\begin{array}{c}\text { Extraction } \\
\text { by }\end{array}$ & $\begin{array}{l}\text { Extract } \\
\text { split }\end{array}$ & $\begin{array}{l}\text { GCMS } \\
\text { SIM or } \\
\text { scan }\end{array}$ & $\begin{array}{c}\text { TSP } \\
\left(\mu \mathrm{g} / \mathrm{m}^{3}\right)\end{array}$ & Anth & $\begin{array}{c}\text { Benz[a] } \\
\text { anthracene }\end{array}$ & $\begin{array}{l}\text { Benzo[ghi] } \\
\text { perylene }\end{array}$ & $\begin{array}{l}\text { Benzo[a] } \\
\text { pyrene }\end{array}$ & $\begin{array}{c}\text { Benzo }[b] \\
\text { fluoranthene }\end{array}$ & $\begin{array}{l}\text { Benzo[e] } \\
\text { pyrene }\end{array}$ \\
\hline $\begin{array}{ll}11 / 19 / 2003 \\
\end{array}$ & GFF & 03.32308 & PSE & Yes & SIM & 3.2 & 68.7 & 67.5 & 84.7 & 68.7 & 88.8 & 97.0 \\
\hline $11 / 19 / 2003$ & PUF & 03.32308 & PSE & Yes & SIM & & 85.0 & 85.3 & 91.3 & 85.2 & 91.2 & 95.7 \\
\hline 03/12/2004 & GFF & 04.07205 & PSE & Yes & Scan & 1.3 & 69.7 & 69.3 & 64.7 & 60.5 & 65.8 & 64.7 \\
\hline 03/12/2004 & PUF & 04.07205 & PSE & Yes & Scan & & 72.8 & 78.0 & 70.8 & 64.5 & 69.3 & 70.8 \\
\hline 08/30/2004 & GFF & 04.24305 & PSE & Yes & Scan & 0.0 & 62.9 & 81.1 & 82.6 & 74.9 & 81.9 & NA \\
\hline 08/30/2004 & PUF & 04.24305 & PSE & Yes & Scan & & 77.3 & 85.3 & 98.3 & 87.1 & 103 & NA \\
\hline $09 / 14 / 2005$ & GFF & 05.25707 & Soxhlet & No & Scan & 6.3 & 73.6 & 90.3 & 116 & 92.9 & 118 & 120 \\
\hline $09 / 14 / 2005$ & PUF & 05.25707 & Soxhlet & No & Scan & & 77.6 & 93.2 & 113 & 93.8 & 115 & 117 \\
\hline 01/17/2006 & GFF & 06.01705 & Soxhlet & No & Scan & 3.2 & 84.2 & 97.8 & 118 & 86.6 & 109 & 106 \\
\hline $01 / 17 / 2006$ & PUF & 06.01705 & Soxhlet & No & Scan & & 55.0 & 83.4 & 109 & 52.4 & 89.2 & 97.9 \\
\hline 08/15/2006 & GFF & 06.22308 & Soxhlet & No & Scan & 2.5 & 76.7 & 76.7 & 72.3 & 71.7 & 71.9 & 73.2 \\
\hline 08/15/2006 & PUF & 06.22308 & Soxhlet & No & Scan & & 66.6 & 73.9 & 79.5 & 66.5 & 76.9 & 80.2 \\
\hline $04 / 18 / 2008$ & GFF & 08.10910 & Soxhlet & No & Scan & 3.8 & 48.5 & 51.6 & 51.3 & 50.5 & 53.9 & 54.4 \\
\hline $04 / 18 / 2008$ & PUF & 08.10910 & Soxhlet & No & Scan & & 86.0 & 90.0 & 89.1 & 86.9 & 98.7 & 97.8 \\
\hline
\end{tabular}

Table B4A. National Water Quality Laboratory quality-control reagent-spike results for polycyclic aromatic hydrocarbon (PAH) compound recoveries for the Sweetwater Reservoir atmospheric site, San Diego County, California.-Continued

[Alkylated PAH were not spiked, so they are not listed in this table. Values are given in percentage recovery, unless noted. Abbreviations: GCMS, gas chromatography mass spectrometry; GFF, glass fiber filter; mm/dd/yyyy, month/day/year; NA, not analyzed; PSE, pressurized solvent extraction; PUF, polyurethane foam; Scan, full scan ion monitoring mode; SIM, selected ion monitoring mode; TSP, total suspended particles (calculated for GFF samples only assuming 315 cubic meters air volume); $\mu \mathrm{g} / \mathrm{m}^{3} ;$ micrograms per cubic meter]

\begin{tabular}{|c|c|c|c|c|c|c|c|c|c|c|c|}
\hline $\begin{array}{c}\text { Begin } \\
\text { date } \\
\text { (mm/dd/yyyy) }\end{array}$ & $\begin{array}{c}\text { Sample } \\
\text { component } \\
\text { spiked }\end{array}$ & $\begin{array}{c}\text { Sample } \\
\text { preparation } \\
\text { set number }\end{array}$ & $\begin{array}{l}\text { Extraction } \\
\text { by }\end{array}$ & $\begin{array}{l}\text { Extract } \\
\text { split }\end{array}$ & $\begin{array}{l}\text { GCMS } \\
\text { SIM or } \\
\text { scan }\end{array}$ & $\begin{array}{c}\text { TSP } \\
\left(\mu g / m^{3}\right)\end{array}$ & $\begin{array}{c}\text { Benzo }[k] \\
\text { fluoranthene }\end{array}$ & Chrysene & Coronene & $\begin{array}{l}\text { Dibenzo[a,h] } \\
\text { anthracene }\end{array}$ & Fluoranthene \\
\hline $11 / 19 / 2003$ & GFF & 03.32308 & PSE & Yes & SIM & 3.2 & 91.5 & 81.7 & 86.0 & 73.8 & 78.2 \\
\hline $03 / 12 / 2004$ & GFF & 04.07205 & PSE & Yes & Scan & 1.3 & 70.2 & 72.2 & 60.5 & 61.8 & 72.8 \\
\hline $03 / 12 / 2004$ & PUF & 04.07205 & PSE & Yes & Scan & & 73.7 & 80.5 & 66.8 & 65.7 & 79.8 \\
\hline 09/14/2005 & GFF & 05.25707 & Soxhlet & No & Scan & 6.3 & 114 & 98.0 & 124 & 120 & 92.9 \\
\hline $09 / 14 / 2005$ & PUF & 05.25707 & Soxhlet & No & Scan & & 114 & 93.0 & 117 & 112 & 99.1 \\
\hline $01 / 17 / 2006$ & GFF & 06.01705 & Soxhlet & No & Scan & 3.2 & 102 & 91.8 & 126 & 112 & 104 \\
\hline $01 / 17 / 2006$ & PUF & 06.01705 & Soxhlet & No & Scan & & 86.7 & 113 & 122 & 101 & 103 \\
\hline $08 / 15 / 2006$ & GFF & 06.22308 & Soxhlet & No & Scan & 2.5 & 73.1 & 75.9 & NA & 74.9 & 79.2 \\
\hline $08 / 15 / 2006$ & PUF & 06.22308 & Soxhlet & No & Scan & & 77.1 & 77.6 & NA & 81.8 & 77.6 \\
\hline
\end{tabular}


Table B4A. National Water Quality Laboratory quality-control reagent-spike results for polycyclic aromatic hydrocarbon (PAH) compound recoveries for the Sweetwater Reservoir atmospheric site, San Diego County, California.—Continued

[Alkylated PAH were not spiked, so they are not listed in this table. Values are given in percentage recovery, unless noted. Abbreviations: GCMS, gas chromatography mass spectrometry; GFF, glass fiber filter; mm/dd/yyyy, month/day/year; NA, not analyzed; PSE, pressurized solvent extraction; PUF, polyurethane foam; Scan, full scan ion monitoring mode; SIM, selected ion monitoring mode; TSP, total suspended particles (calculated for GFF samples only assuming 315 cubic meters air volume); $\mu \mathrm{g} / \mathrm{m}^{3} ;$ micrograms per cubic meter]

\begin{tabular}{|c|c|c|c|c|c|c|c|c|c|c|c|}
\hline $\begin{array}{c}\text { Begin } \\
\text { date } \\
\text { (mm/dd/yyyy) }\end{array}$ & $\begin{array}{c}\text { Sample } \\
\text { component } \\
\text { spiked }\end{array}$ & $\begin{array}{c}\text { Sample } \\
\text { preparation } \\
\text { set number }\end{array}$ & $\begin{array}{c}\text { Extraction } \\
\text { by }\end{array}$ & $\begin{array}{l}\text { Extract } \\
\text { split }\end{array}$ & $\begin{array}{l}\text { GCMS } \\
\text { SIM or } \\
\text { scan }\end{array}$ & $\begin{array}{c}\text { TSP } \\
\left(\mu \mathrm{g} / \mathrm{m}^{3}\right)\end{array}$ & $\begin{array}{c}\text { Indeno[1,2,3-cd }] \\
\text { pyrene }\end{array}$ & $\begin{array}{l}\text { 2-Methyl- } \\
\text { anthracene }\end{array}$ & $\begin{array}{c}\text { 4,5- } \\
\text { Methylene- } \\
\text { phen- } \\
\text { anthrene }\end{array}$ & $\begin{array}{c}\text { 1- } \\
\text { Methyl- } \\
\text { phen- } \\
\text { anthrene }\end{array}$ & $\begin{array}{c}\text { 1-Methyl- } \\
\text { pyrene }\end{array}$ \\
\hline $11 / 19 / 2003$ & PUF & 03.32308 & PSE & Yes & SIM & & 80.2 & 85.0 & 92.3 & 91.8 & 96.3 \\
\hline $03 / 12 / 2004$ & GFF & 04.07205 & PSE & Yes & Scan & 1.3 & 59.2 & 66.7 & 71.5 & 70.8 & 69.5 \\
\hline 08/30/2004 & PUF & 04.24305 & PSE & Yes & Scan & & 98.7 & 81.8 & 79.3 & 84.0 & 88.8 \\
\hline 09/14/2005 & GFF & 05.25707 & Soxhlet & No & Scan & 6.3 & 106 & 75.9 & 83.9 & 91.1 & 92.9 \\
\hline $09 / 14 / 2005$ & PUF & 05.25707 & Soxhlet & No & Scan & & 104 & 81.5 & 85.0 & 95.2 & 99.5 \\
\hline $01 / 17 / 2006$ & GFF & 06.01705 & Soxhlet & No & Scan & 3.2 & 109 & 82.5 & 99.1 & 106 & 103 \\
\hline $01 / 17 / 2006$ & PUF & 06.01705 & Soxhlet & No & Scan & & 89.3 & 56.4 & 103 & 110 & 92.9 \\
\hline $08 / 15 / 2006$ & GFF & 06.22308 & Soxhlet & No & Scan & 2.5 & 70.7 & 77.1 & 78.3 & 74.8 & 76.3 \\
\hline
\end{tabular}

Table B4A. National Water Quality Laboratory quality-control reagent-spike results for polycyclic aromatic hydrocarbon (PAH) compound recoveries for the Sweetwater Reservoir atmospheric site, San Diego County, California.-Continued

[Alkylated PAH were not spiked, so they are not listed in this table. Values are given in percentage recovery, unless noted. Abbreviations: GCMS, gas chromatography mass spectrometry; GFF, glass fiber filter; mm/dd/yyyy, month/day/year; NA, not analyzed; PSE, pressurized solvent extraction; PUF, polyurethane foam; Scan, full scan ion monitoring mode; SIM, selected ion monitoring mode; TSP, total suspended particles (calculated for GFF samples only assuming 315 cubic meters air volume); $\mu \mathrm{g} / \mathrm{m}^{3} ;$ micrograms per cubic meter]

\begin{tabular}{|c|c|c|c|c|c|c|c|c|c|c|c|c|}
\hline $\begin{array}{c}\text { Begin } \\
\text { date } \\
\text { (mm/dd/yyyy) }\end{array}$ & $\begin{array}{c}\text { Sample } \\
\text { component } \\
\text { spiked }\end{array}$ & $\begin{array}{l}\text { Sample } \\
\text { preparation } \\
\text { set number }\end{array}$ & $\begin{array}{c}\text { Extraction } \\
\text { by }\end{array}$ & $\begin{array}{c}\text { Extract } \\
\text { split }\end{array}$ & $\begin{array}{l}\text { GCMS } \\
\text { SIM or } \\
\text { scan }\end{array}$ & $\begin{array}{c}\text { TSP } \\
\left(\mu \mathrm{g} / \mathrm{m}^{3}\right)\end{array}$ & Perylene & $\begin{array}{l}\text { Phen- } \\
\text { anthrene }\end{array}$ & Pyrene & $\begin{array}{l}\text { 2-Fluoro- } \\
\text { biphenyl } \\
\text { surrogate }\end{array}$ & $\begin{array}{c}\text { Nitro- } \\
\text { benzene- } d 5 \\
\text { surrogate }\end{array}$ & $\begin{array}{c}\text { Terphenyl- } \\
\text { d14 } \\
\text { surrogate }\end{array}$ \\
\hline $11 / 19 / 2003$ & GFF & 03.32308 & PSE & Yes & SIM & 3.2 & 78.5 & 79.0 & 79.3 & 73.8 & 99.8 & 86.2 \\
\hline $11 / 19 / 2003$ & PUF & 03.32308 & PSE & Yes & SIM & & 84.7 & 93.3 & 96.8 & 97.8 & 108 & 99.6 \\
\hline $03 / 12 / 2004$ & GFF & 04.07205 & PSE & Yes & Scan & 1.3 & 59.7 & 73.7 & 71.7 & 62.4 & 45.6 & 66.2 \\
\hline $03 / 12 / 2004$ & PUF & 04.07205 & PSE & Yes & Scan & & 64.0 & 80.3 & 79.7 & 66.0 & 49.2 & 73.4 \\
\hline 08/30/2004 & GFF & 04.24305 & PSE & Yes & Scan & 0.0 & NA & 72.4 & 81.3 & 42.5 & 44.1 & 96.4 \\
\hline 08/30/2004 & PUF & 04.24305 & PSE & Yes & Scan & & NA & 83.8 & 91.5 & 84.0 & 78.9 & 102 \\
\hline $09 / 14 / 2005$ & GFF & 05.25707 & Soxhlet & No & Scan & 6.3 & 86.8 & 85.7 & 93.3 & 74.2 & 69.8 & 95.0 \\
\hline $09 / 14 / 2005$ & PUF & 05.25707 & Soxhlet & No & Scan & & 90.5 & 89.7 & 98.7 & 74.7 & 68.9 & 99.8 \\
\hline $01 / 17 / 2006$ & GFF & 06.01705 & Soxhlet & No & Scan & 3.2 & 75.9 & 102 & 103 & 65.4 & 33.8 & 106 \\
\hline $01 / 17 / 2006$ & PUF & 06.01705 & Soxhlet & No & Scan & & 61.3 & 105 & 104 & 105 & 85.6 & 122 \\
\hline $08 / 15 / 2006$ & GFF & 06.22308 & Soxhlet & No & Scan & 2.5 & 72.5 & 76.3 & 79.1 & 58.6 & 39.5 & 81.5 \\
\hline 08/15/2006 & PUF & 06.22308 & Soxhlet & No & Scan & & 67.2 & 76.5 & 76.7 & 79.6 & 61.7 & 79.5 \\
\hline $04 / 18 / 2008$ & GFF & 08.10910 & Soxhlet & No & Scan & 3.8 & 51.9 & 49.6 & 51.1 & 54.5 & 53.7 & 68.3 \\
\hline $04 / 18 / 2008$ & PUF & 08.10910 & Soxhlet & No & Scan & & 87.0 & 92.0 & 92.1 & 78.8 & 69.0 & 89.7 \\
\hline
\end{tabular}



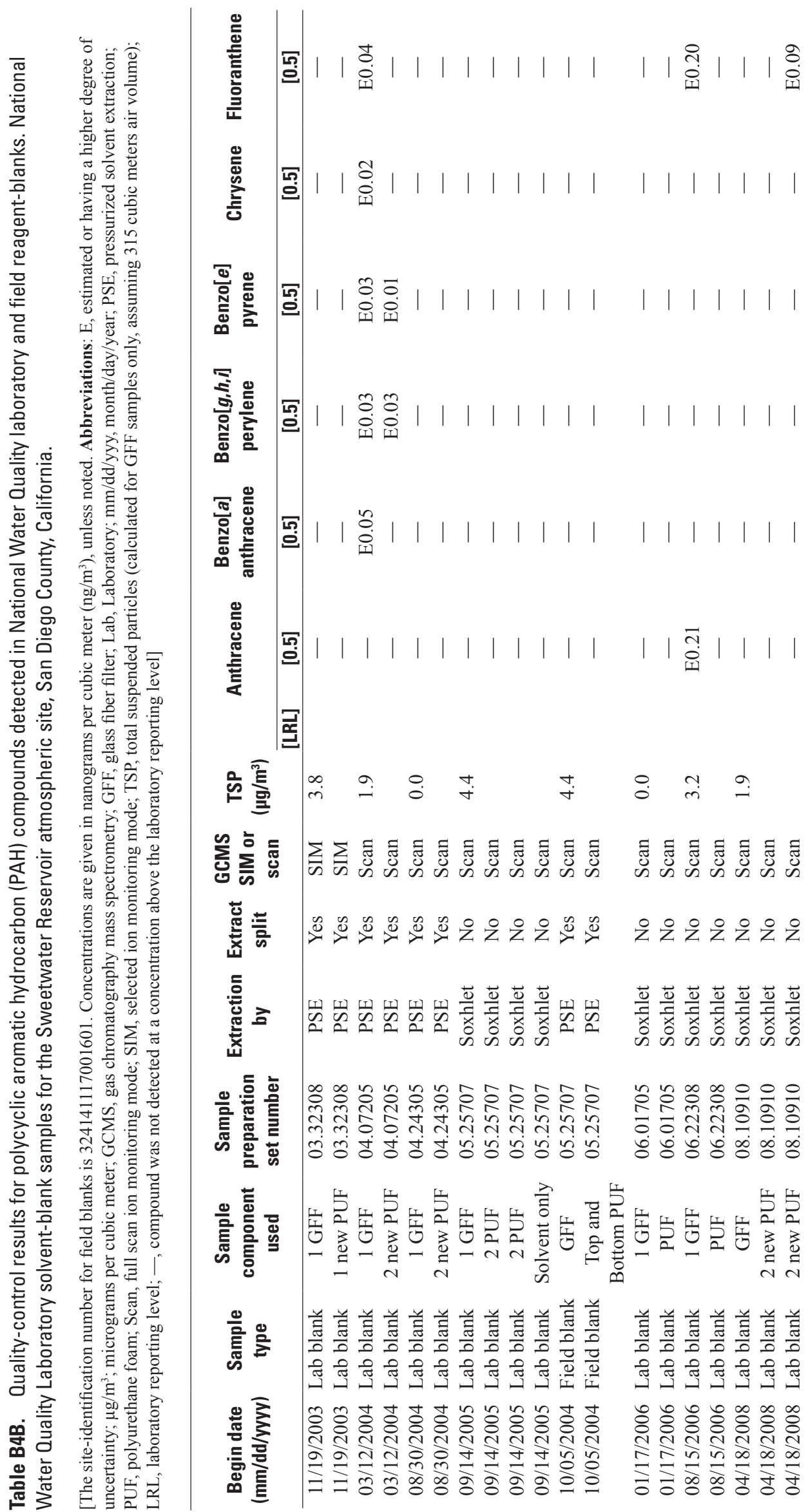

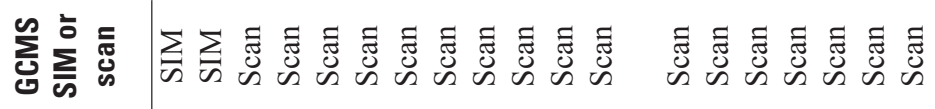

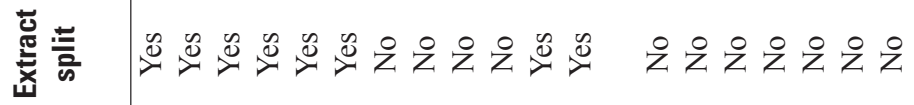

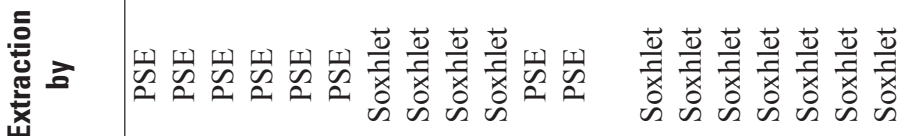

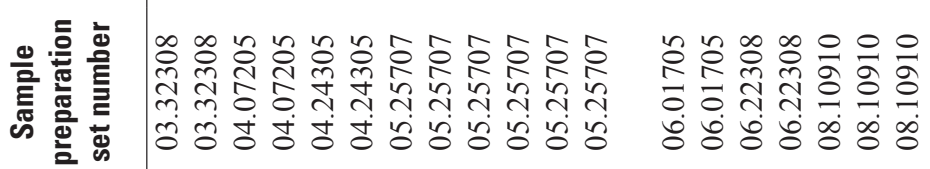

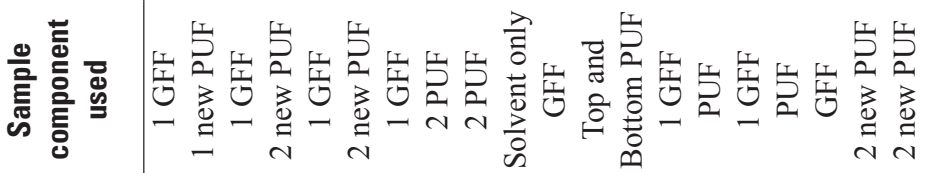

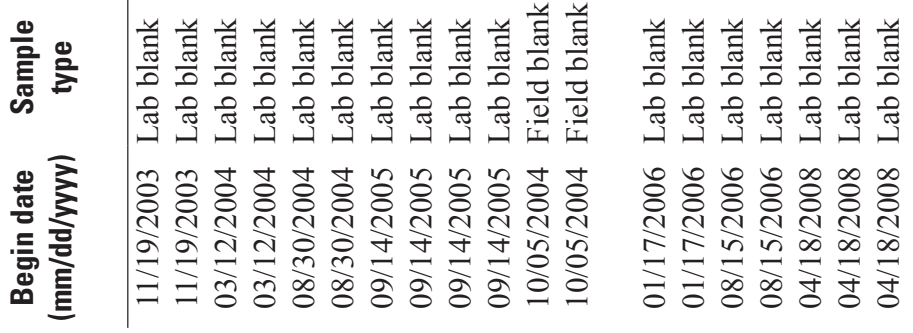




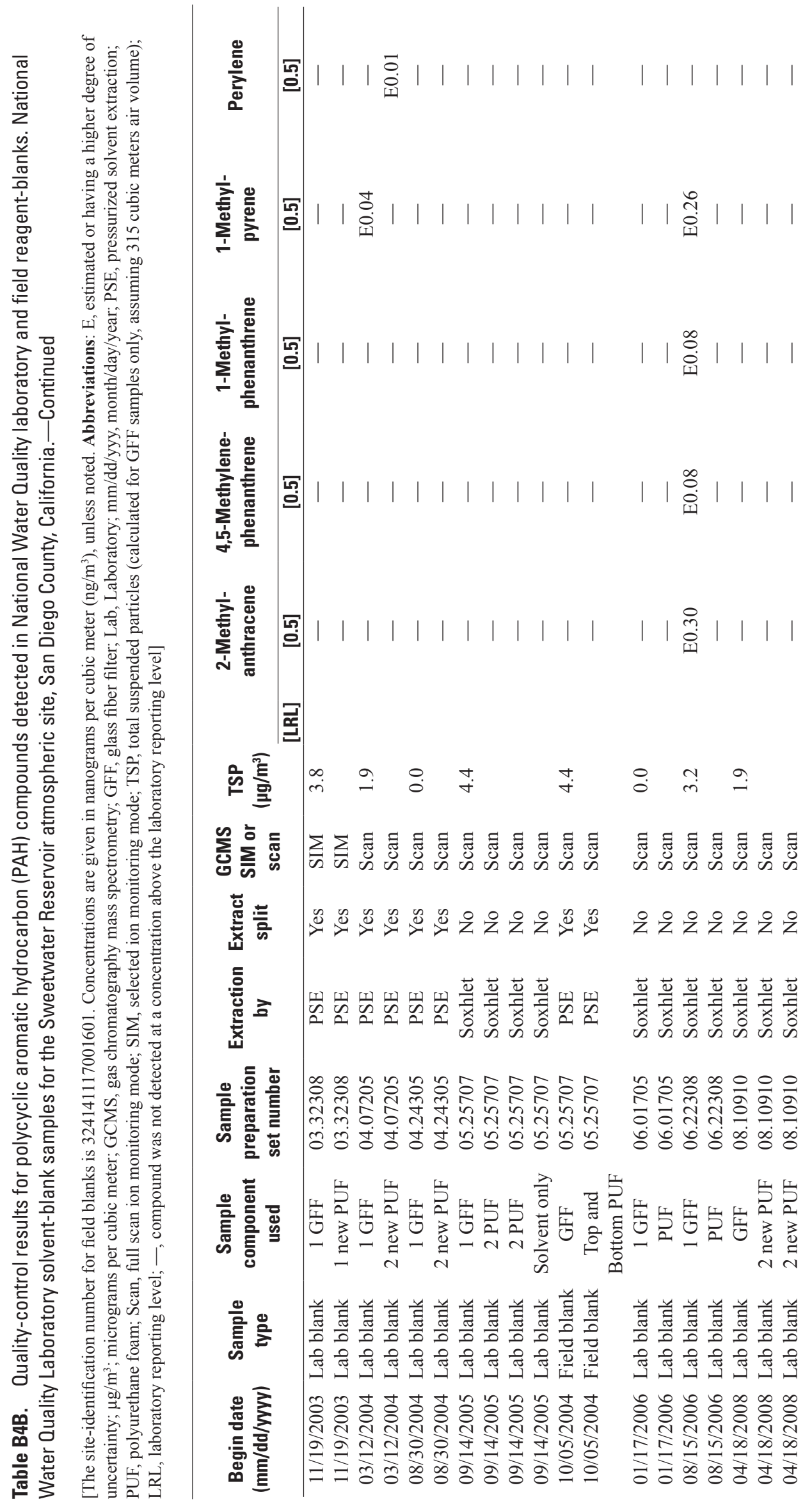



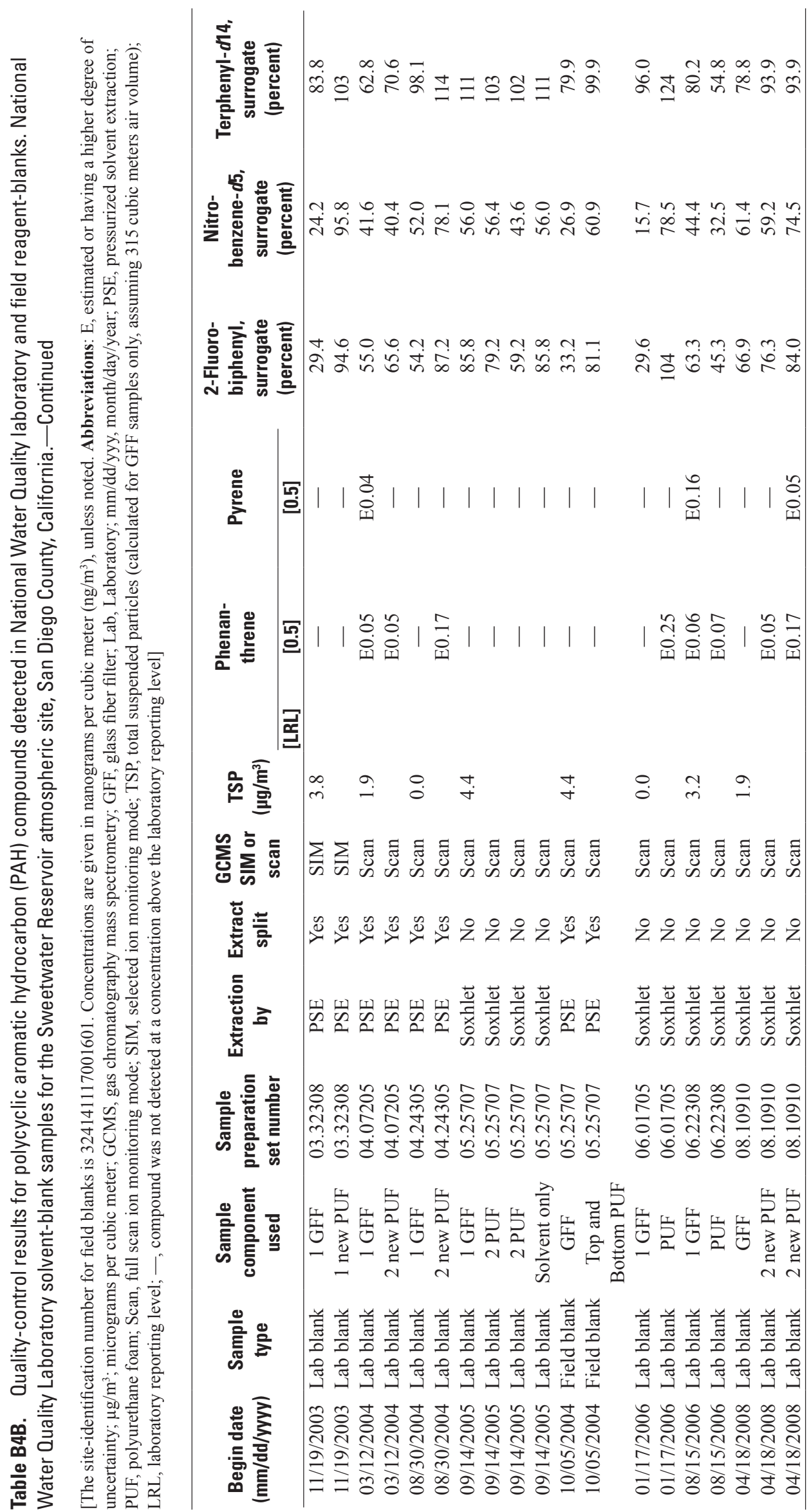


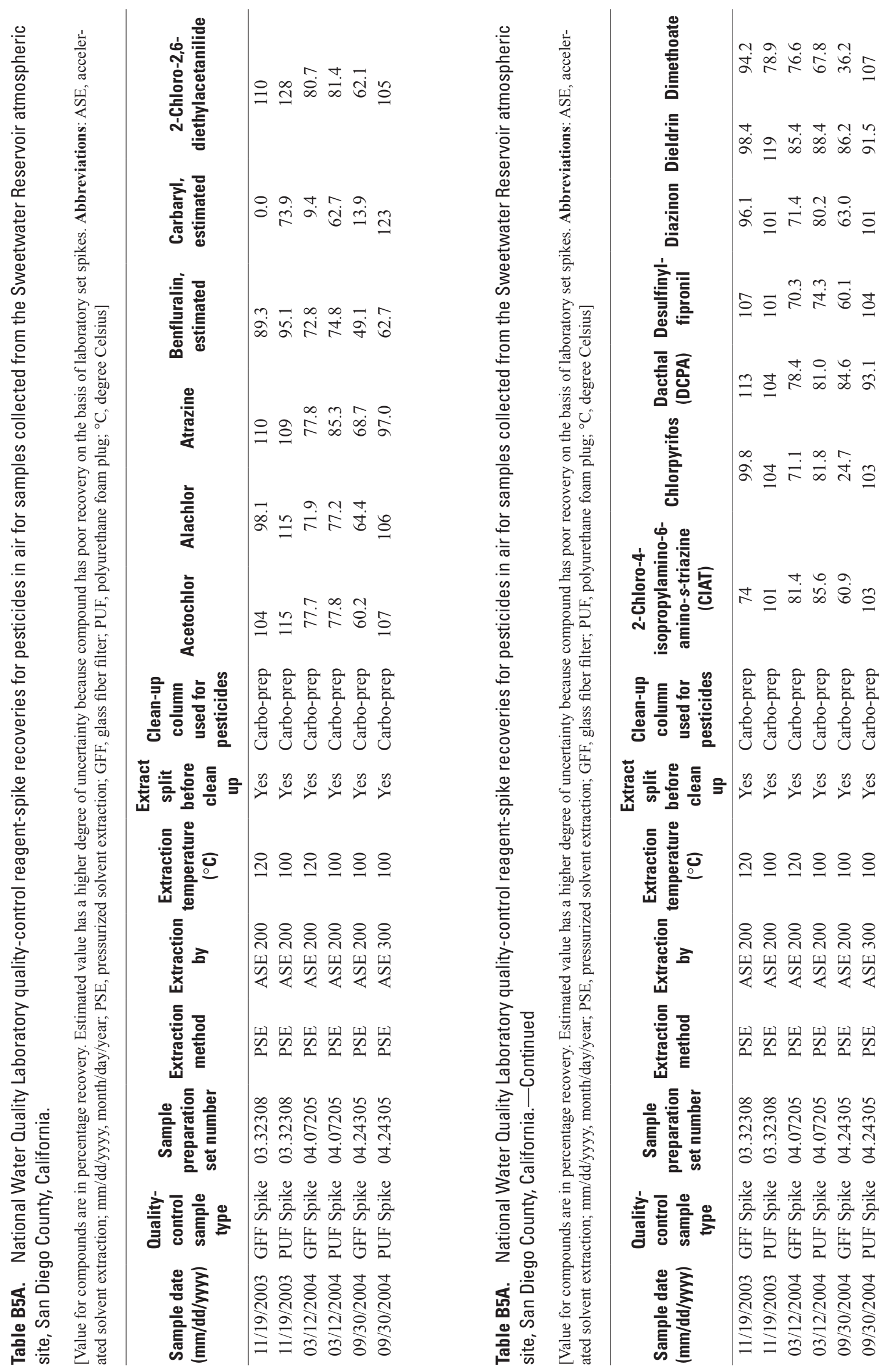



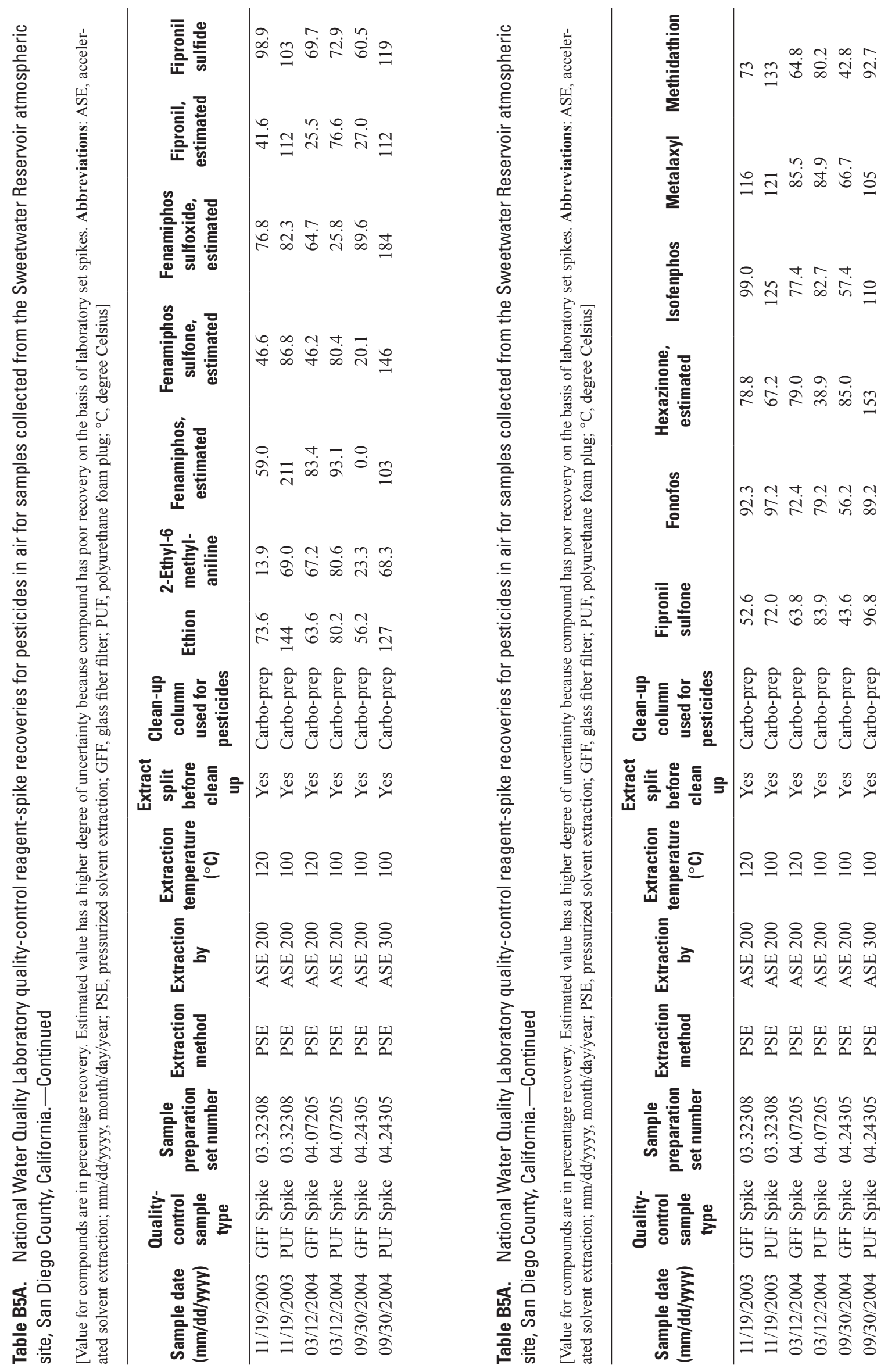


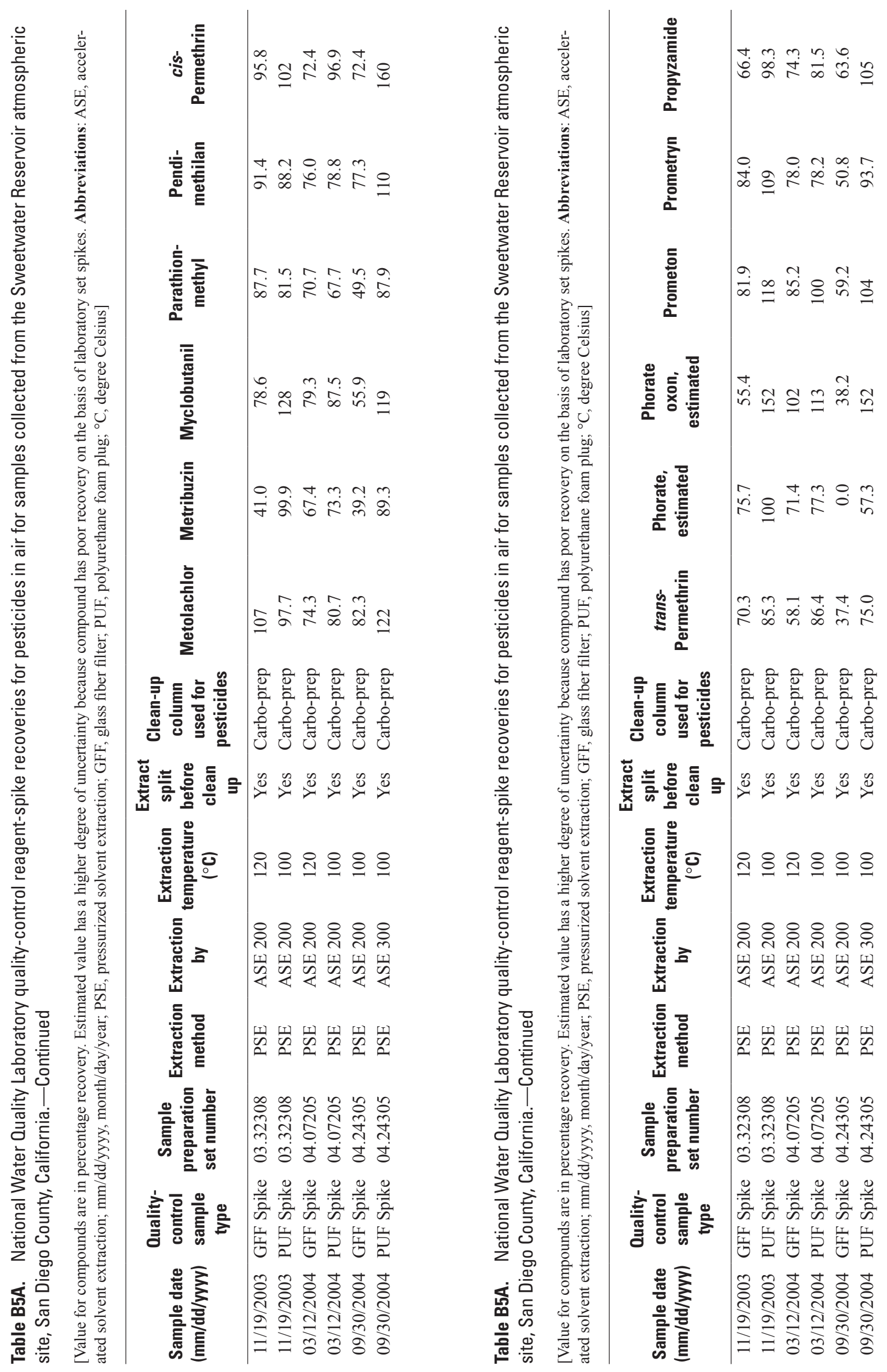




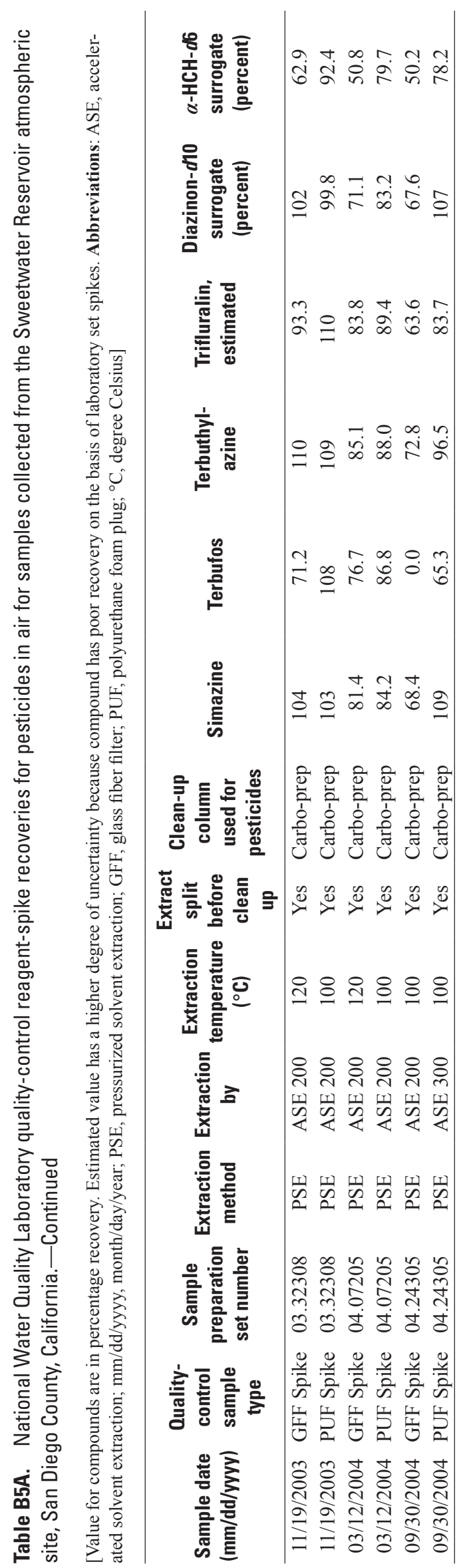



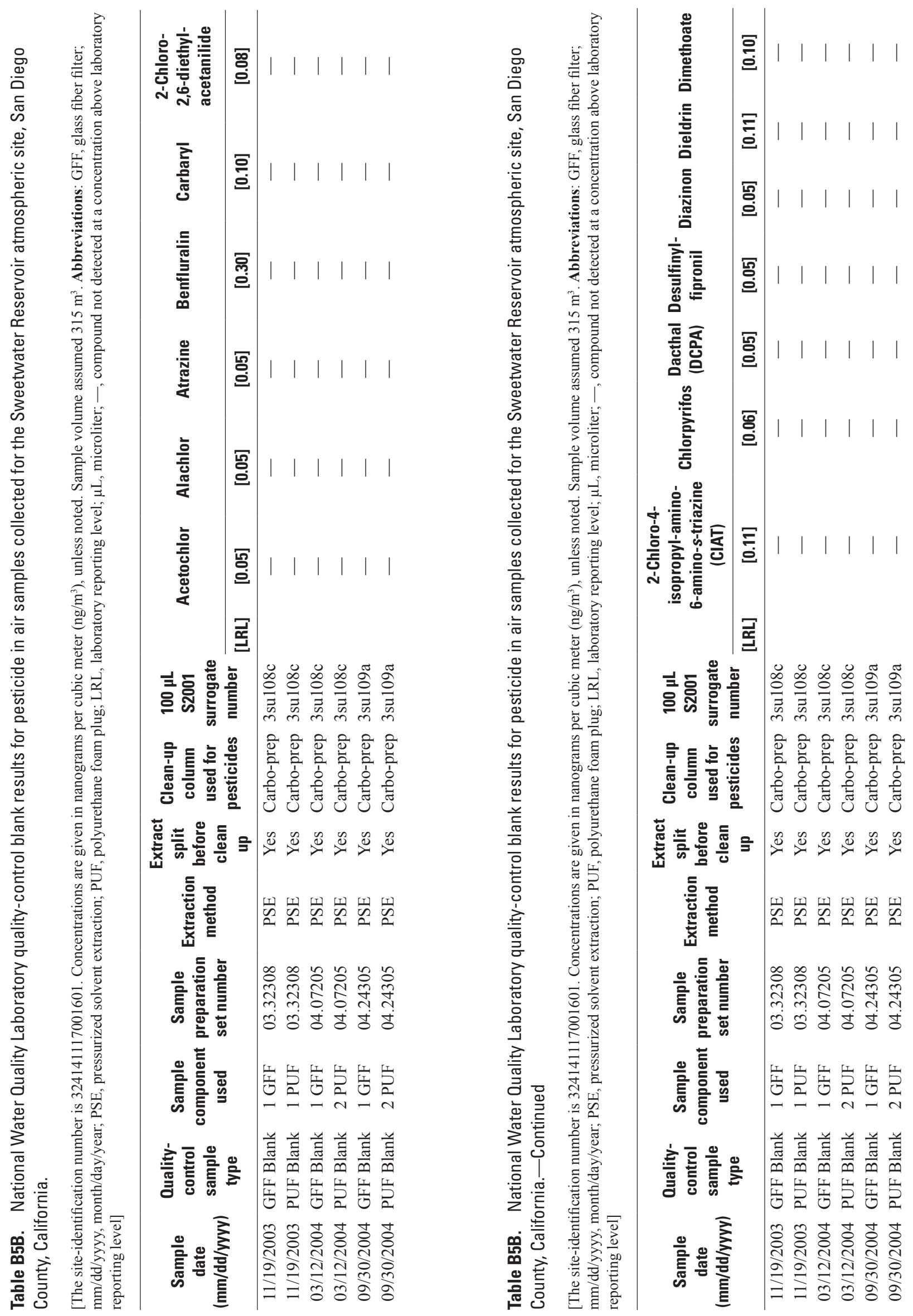

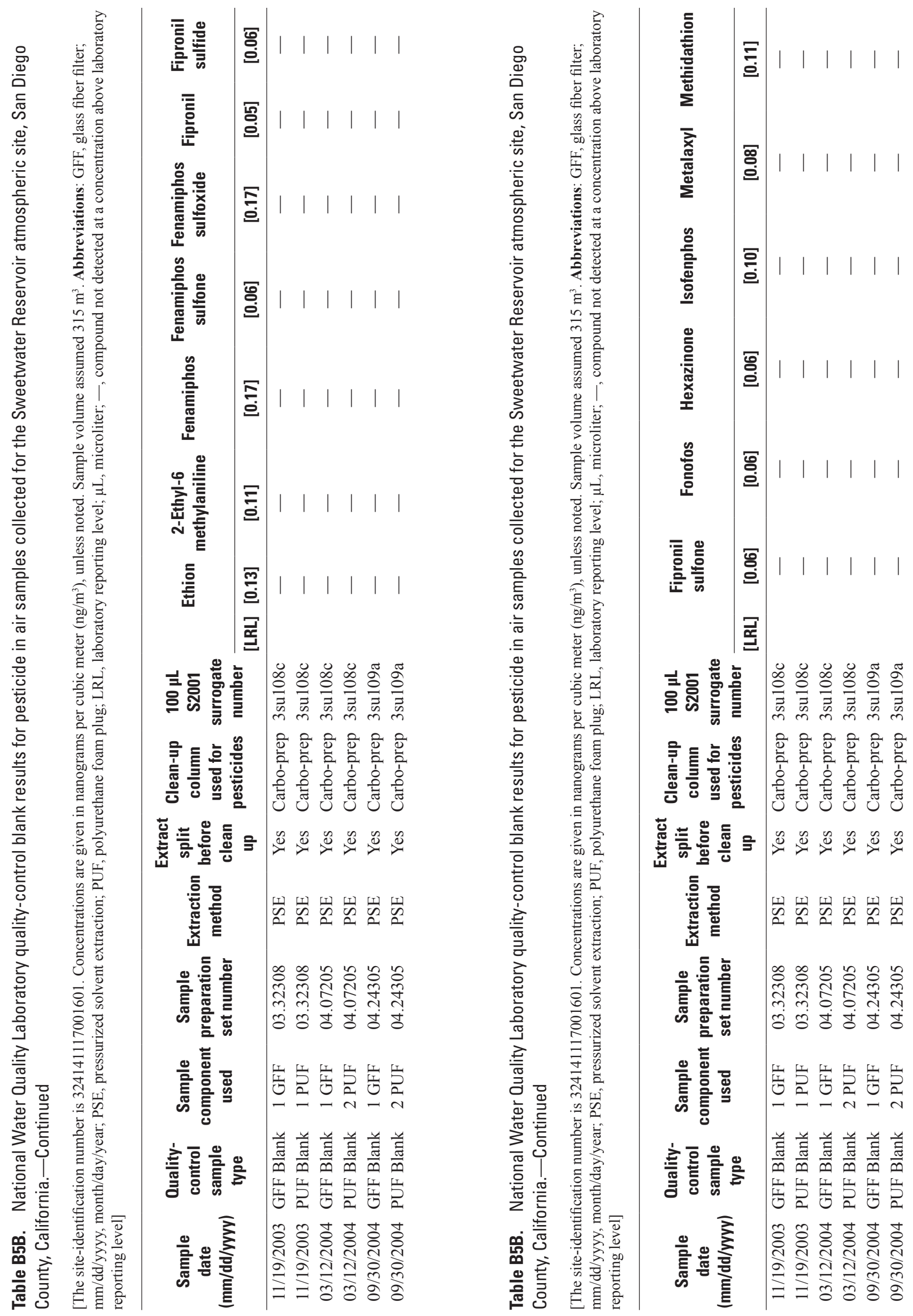

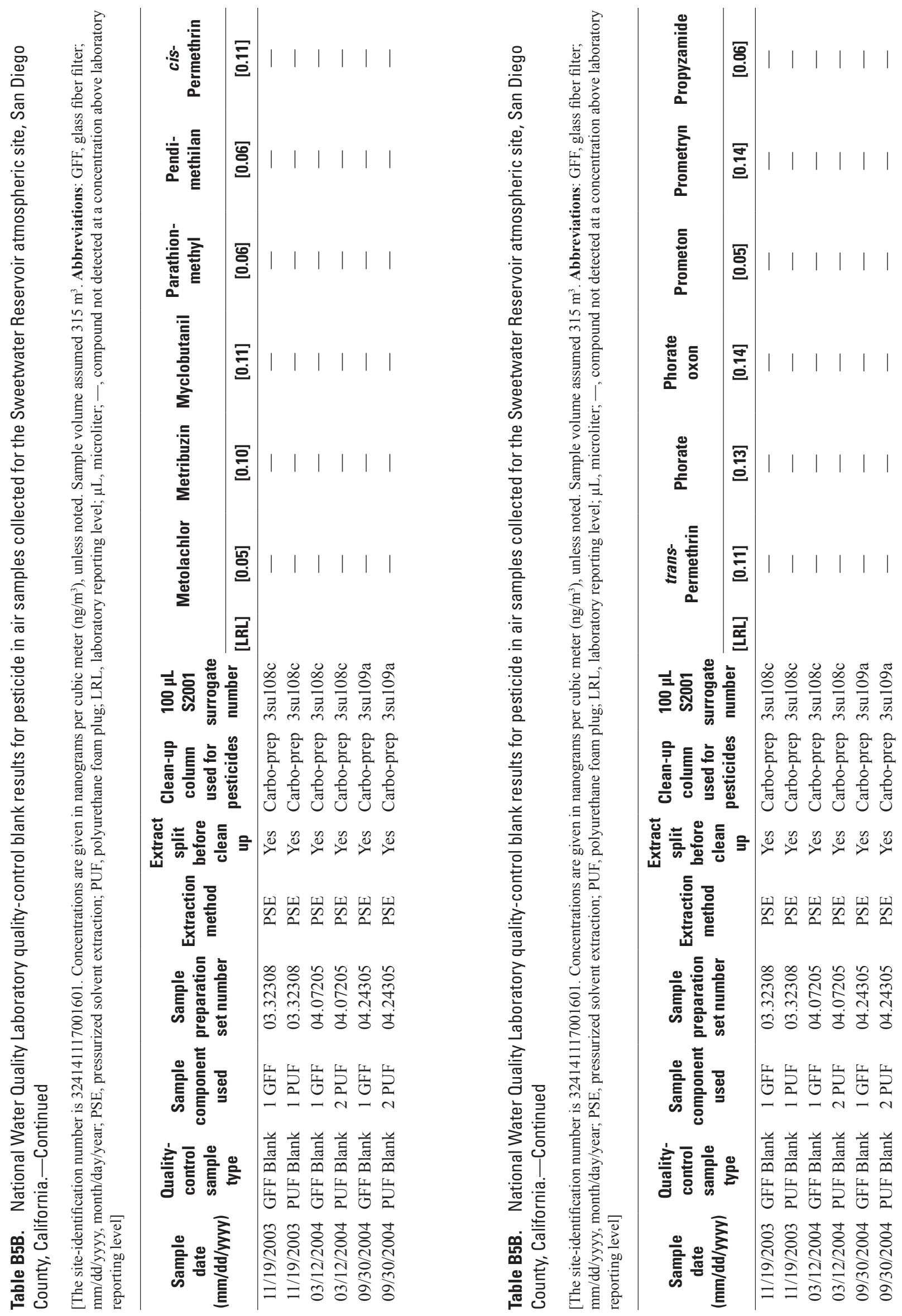


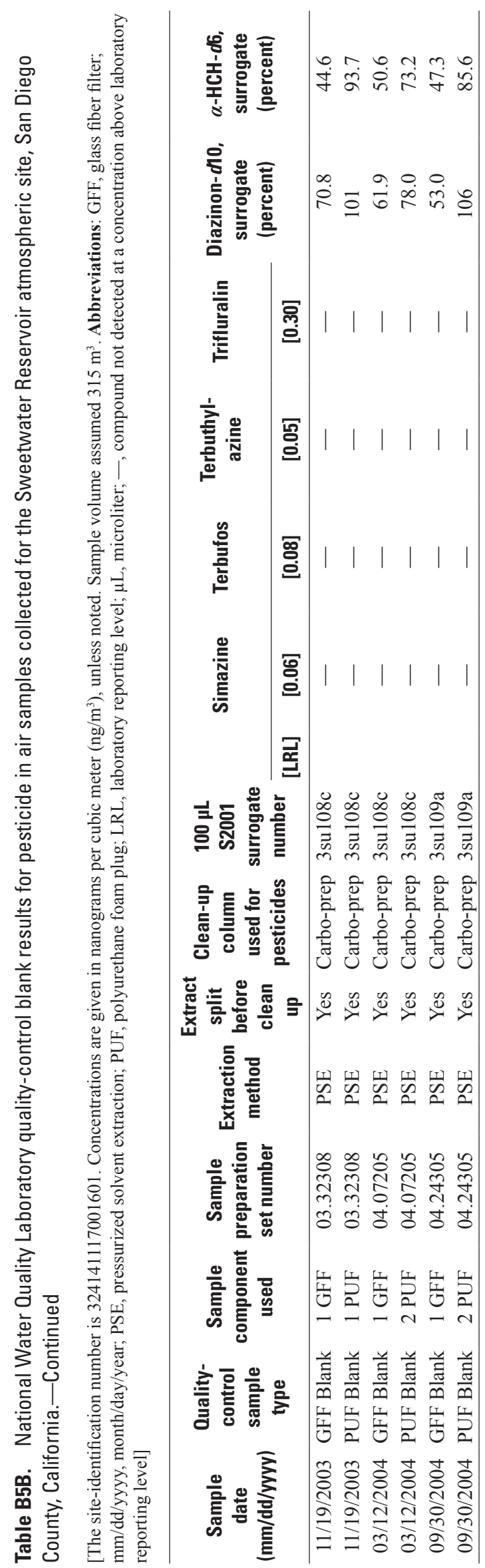




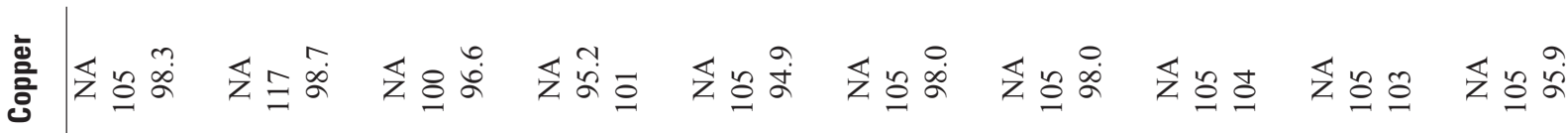

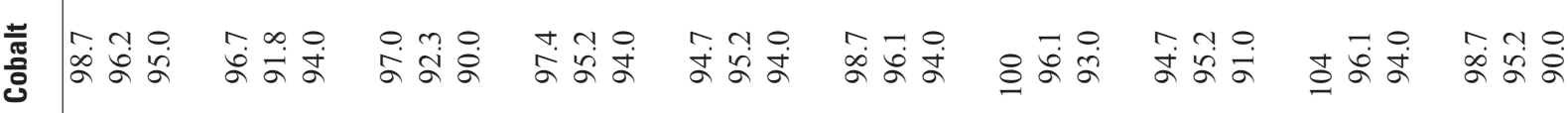
言

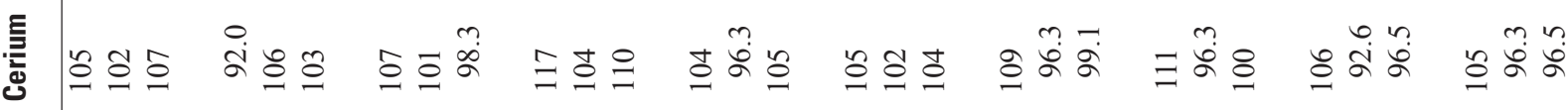

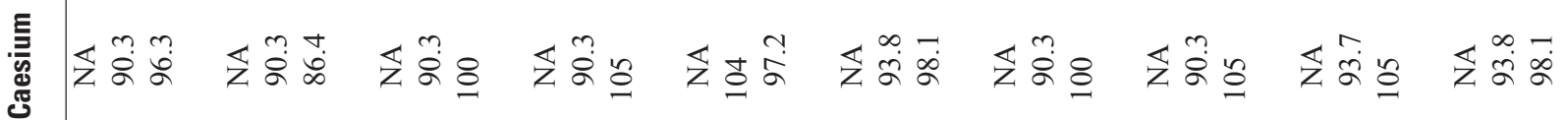
重

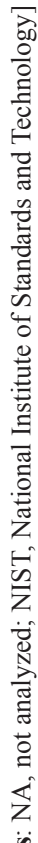

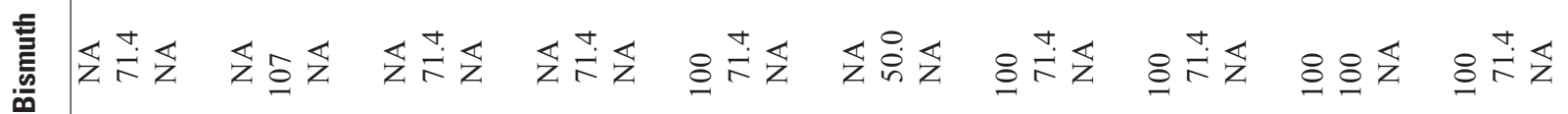

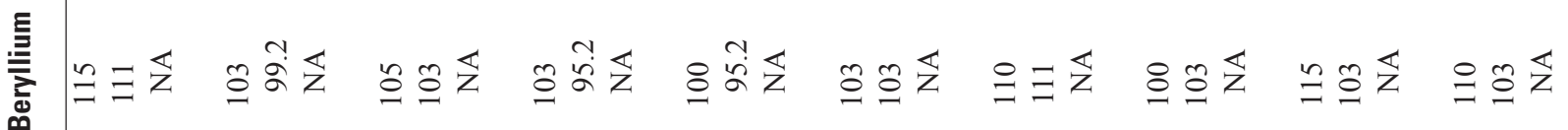

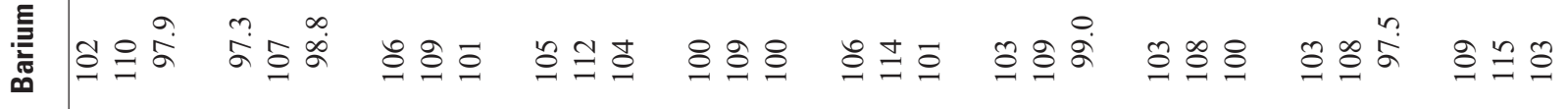

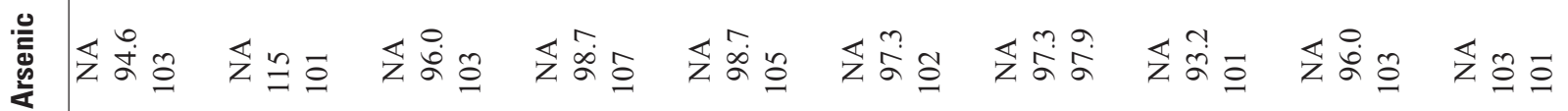

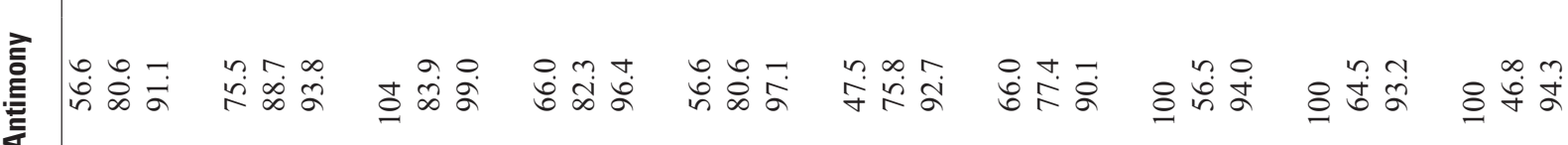

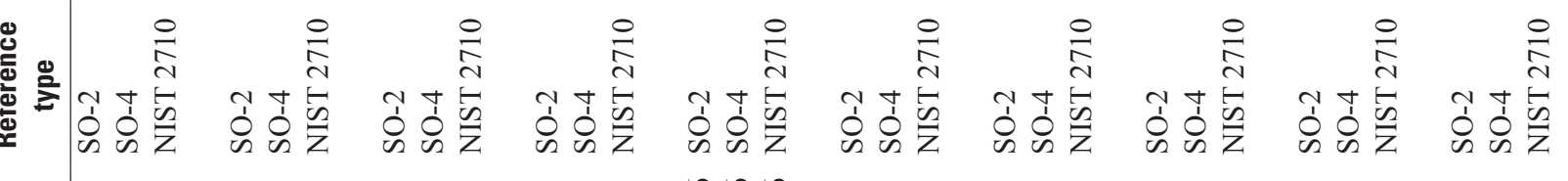

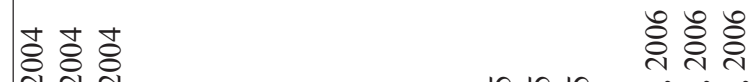

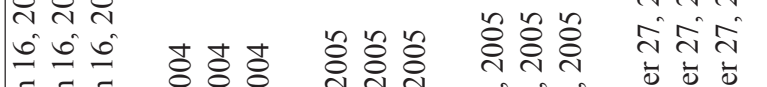

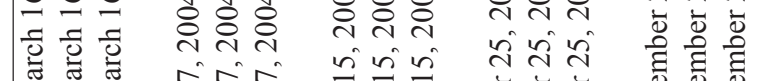

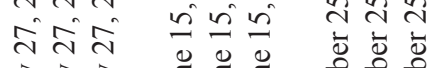

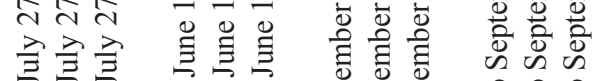
$\therefore$ 웅 ถิ ซิ 의

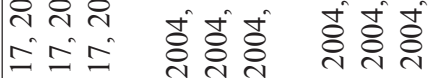
ㅇㅇㅇ 过

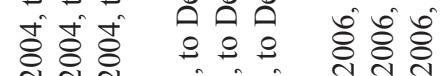
iิ तิ तิ ती กิ่

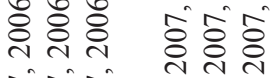

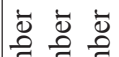

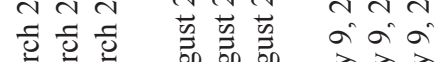
ニニะ

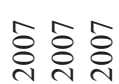
में ते मे 롴를 090 तैंते

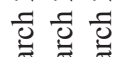

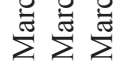

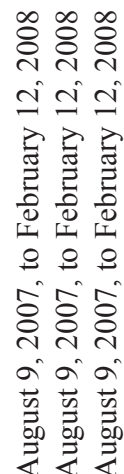

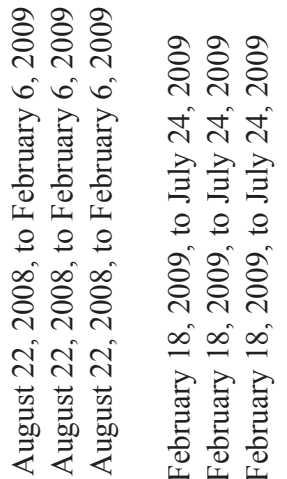

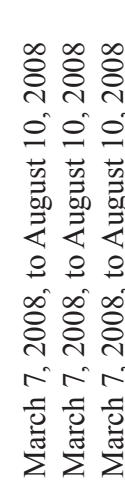




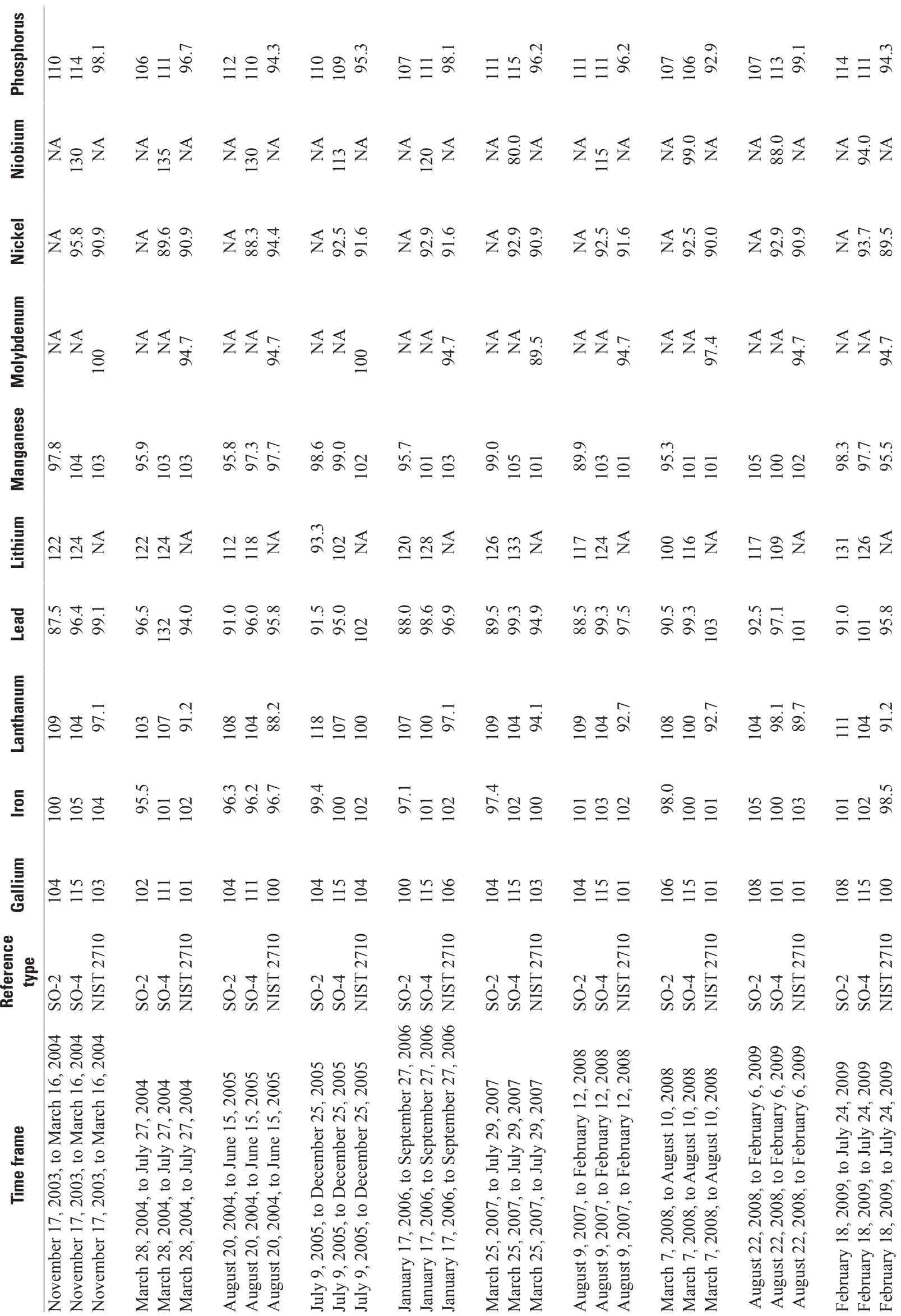




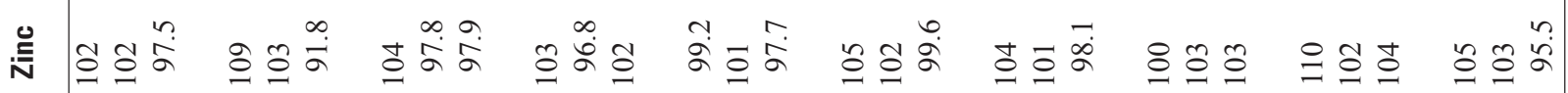

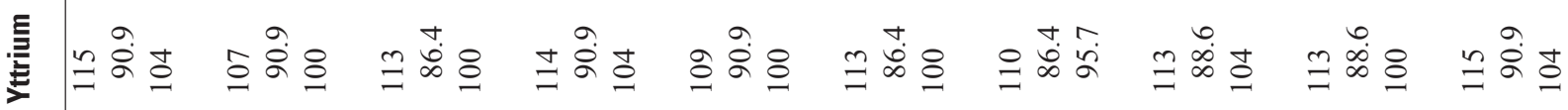
言 言 盇

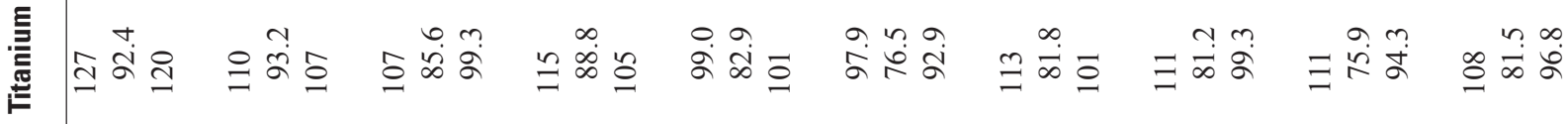

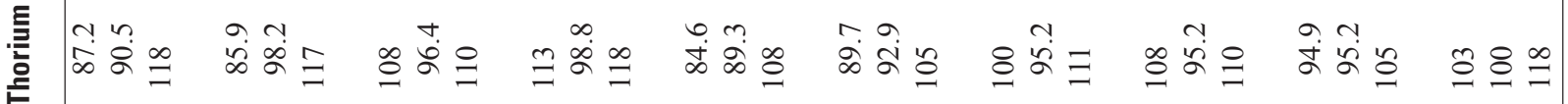
重

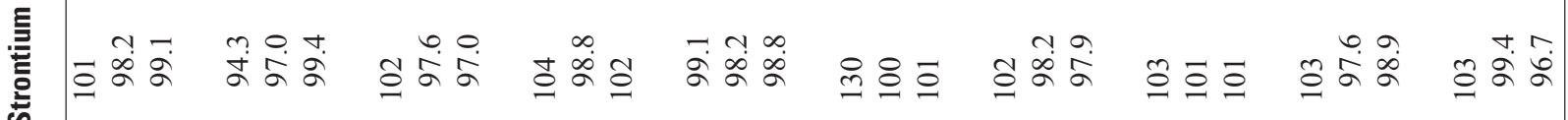

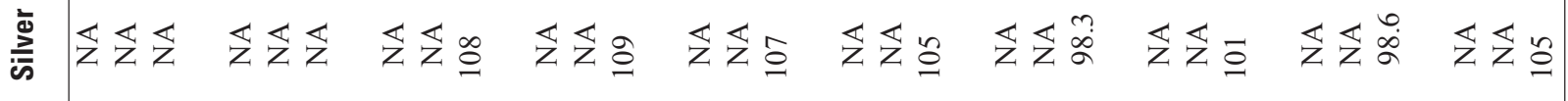
衰密

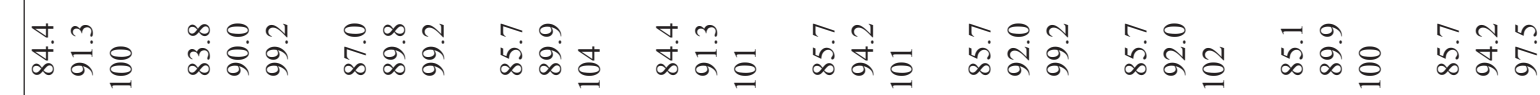

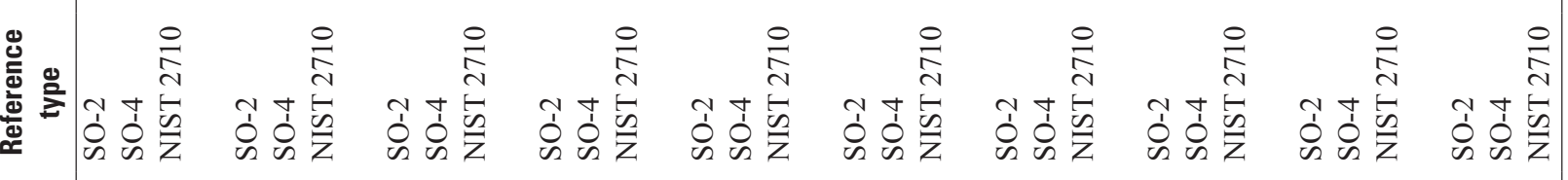
t蓬恶

ํำ

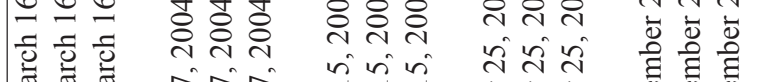

范

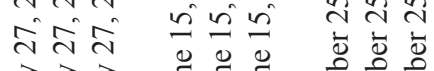

o ?

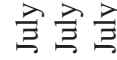

㤩兽兽

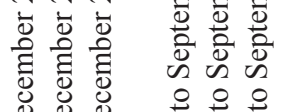

ชิ ซิ ชิ ㅇㅇㅇㅇ 永永 $\therefore$ 요

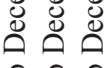

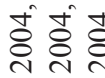
○ \& 8์

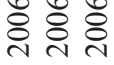

㤹 की तิ ते ते in

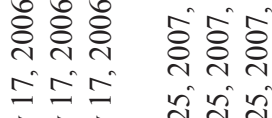

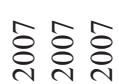
วิจे จे

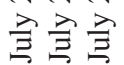
$\circ 20$ ते चे

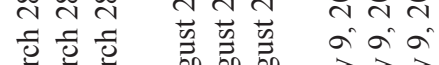
등 छ 究完完

椋

를럴

듄 온

in:

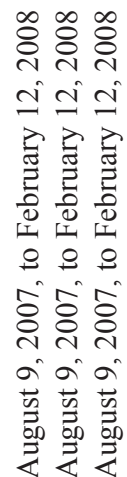

ठิેे ڤે ڤે

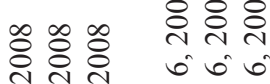
00

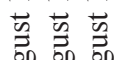
至考去 $\circ \%$ ôे \&ᄋ ㅇํㅇ

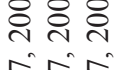

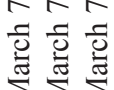

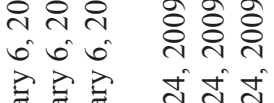

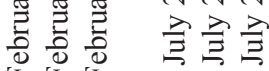
I of of

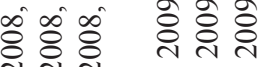
สิ่ี่

$\overrightarrow{\mathrm{g}} \overrightarrow{\mathrm{s}} \overrightarrow{\mathrm{v}}$ 至表专

류률 


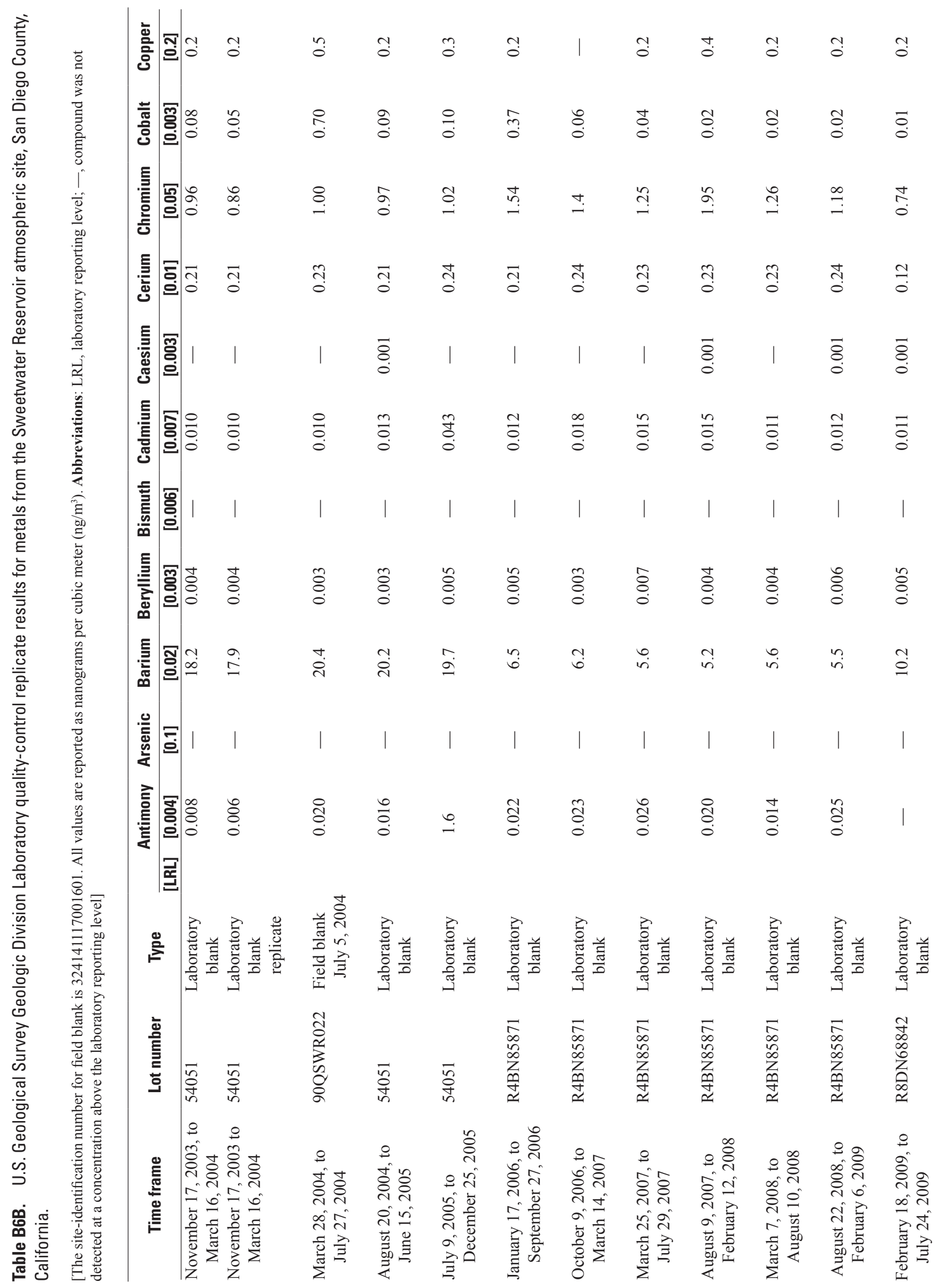




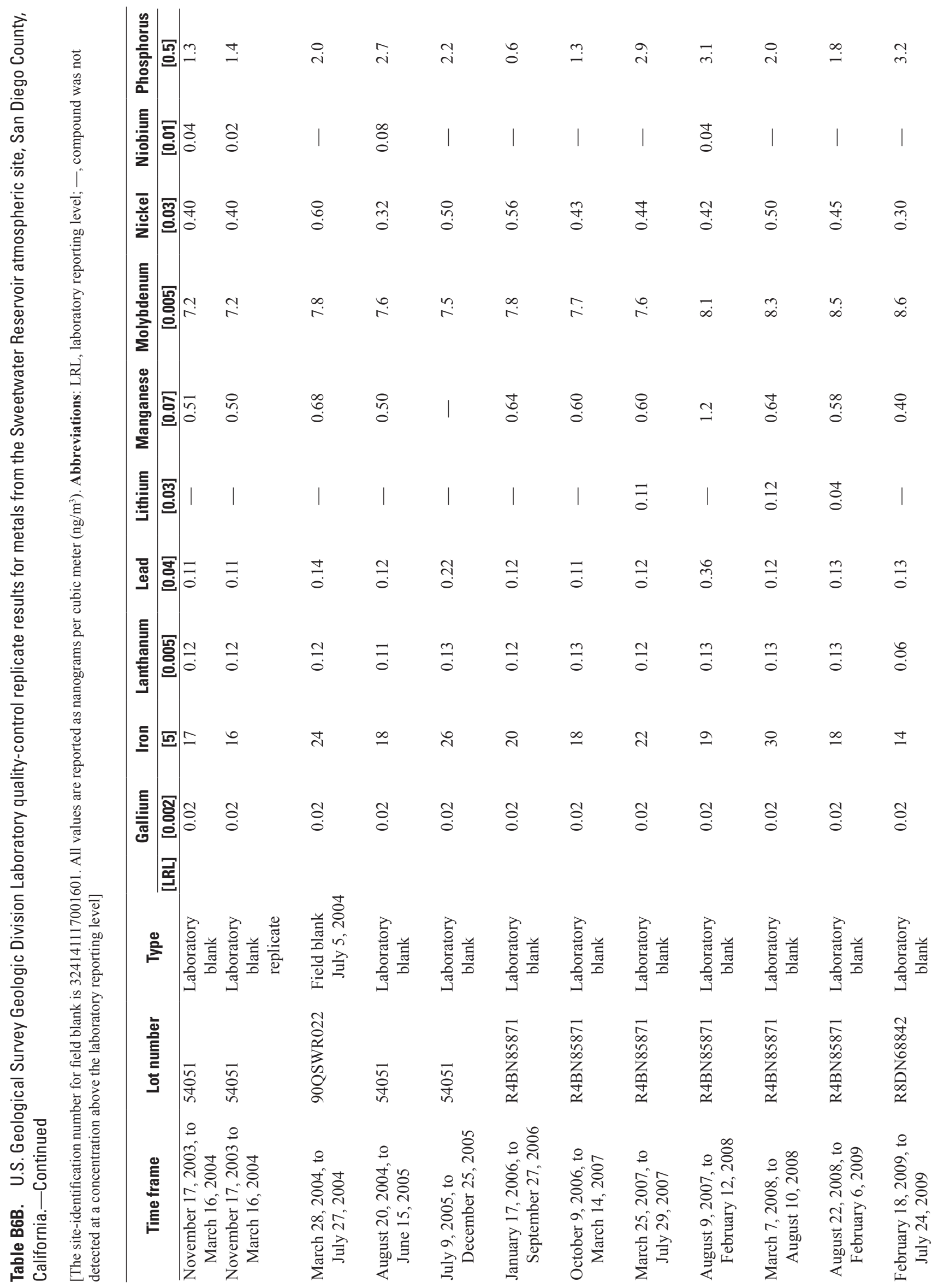




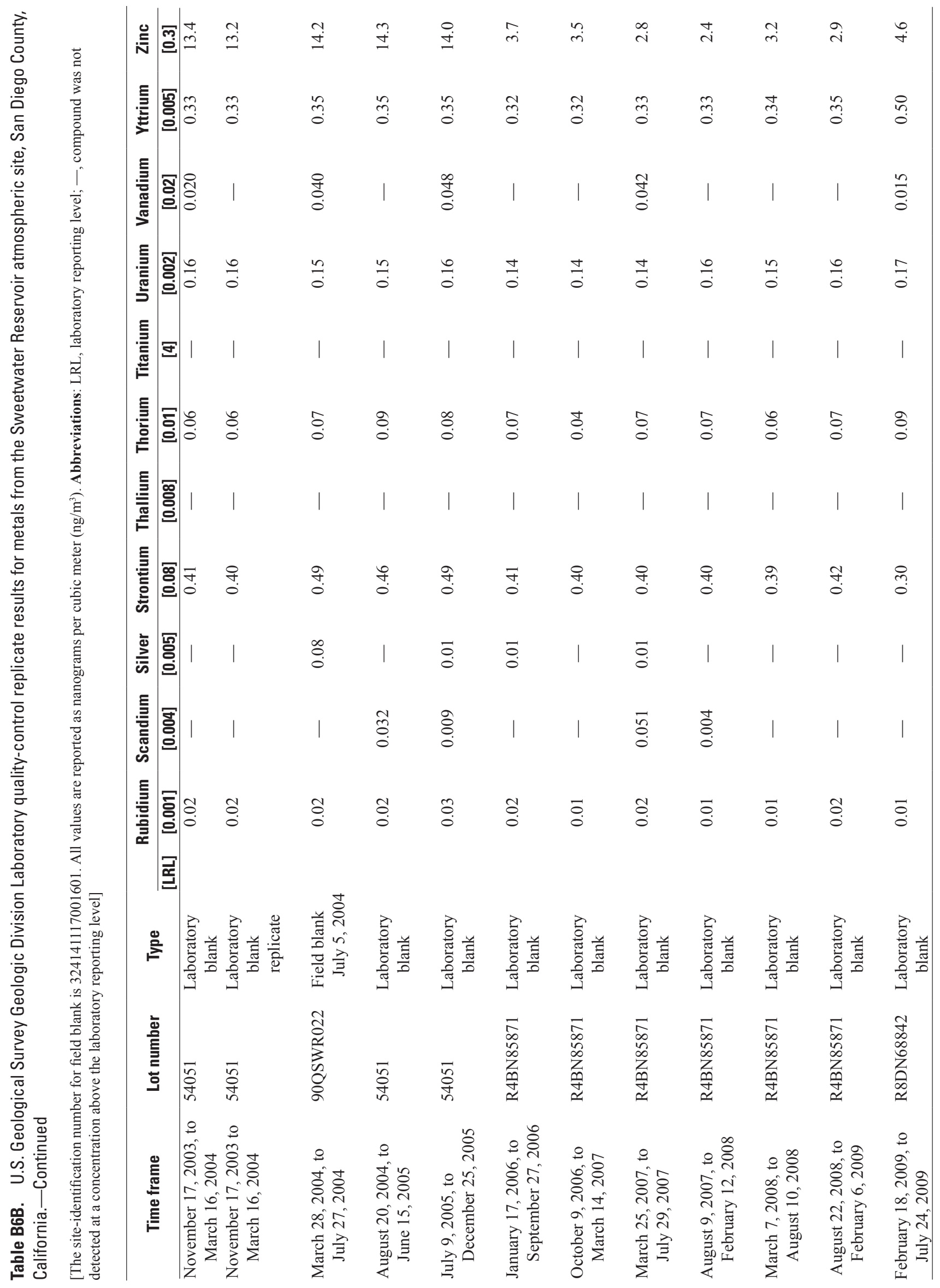




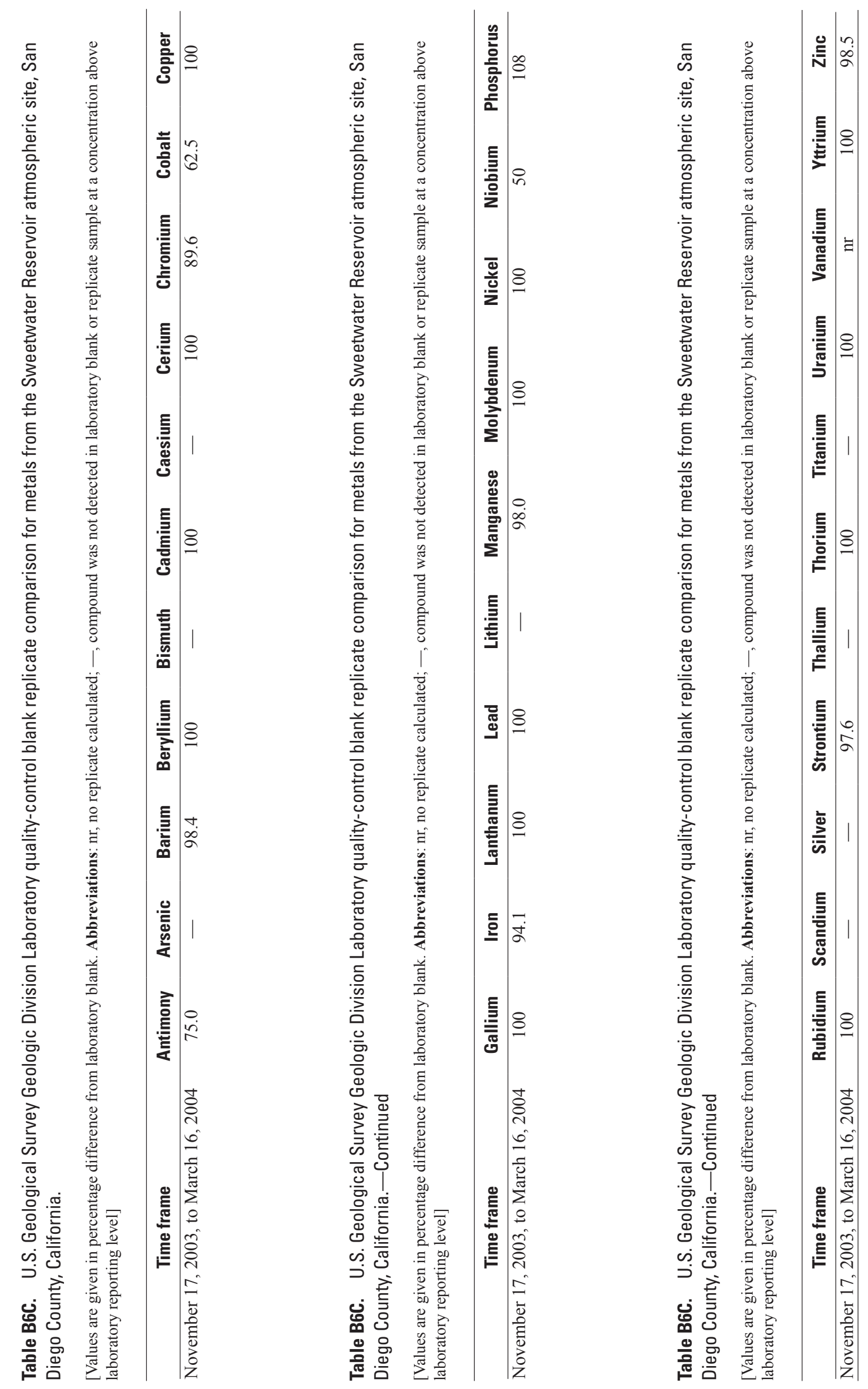


214 Water- and Air-Quality and Surficial Bed-Sediment Monitoring of Sweetwater Reservoir Watershed

Appendix C Tables. 


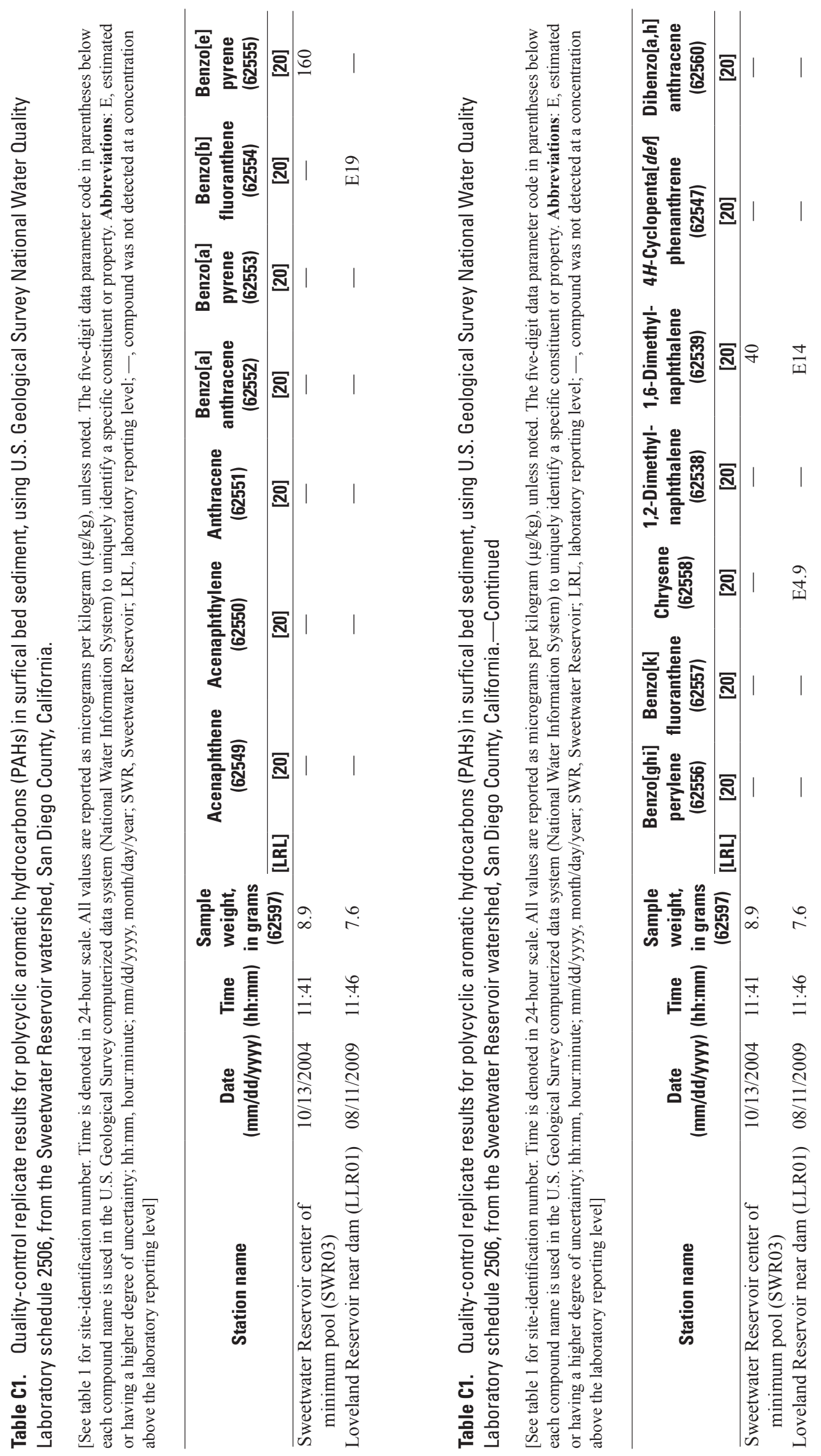




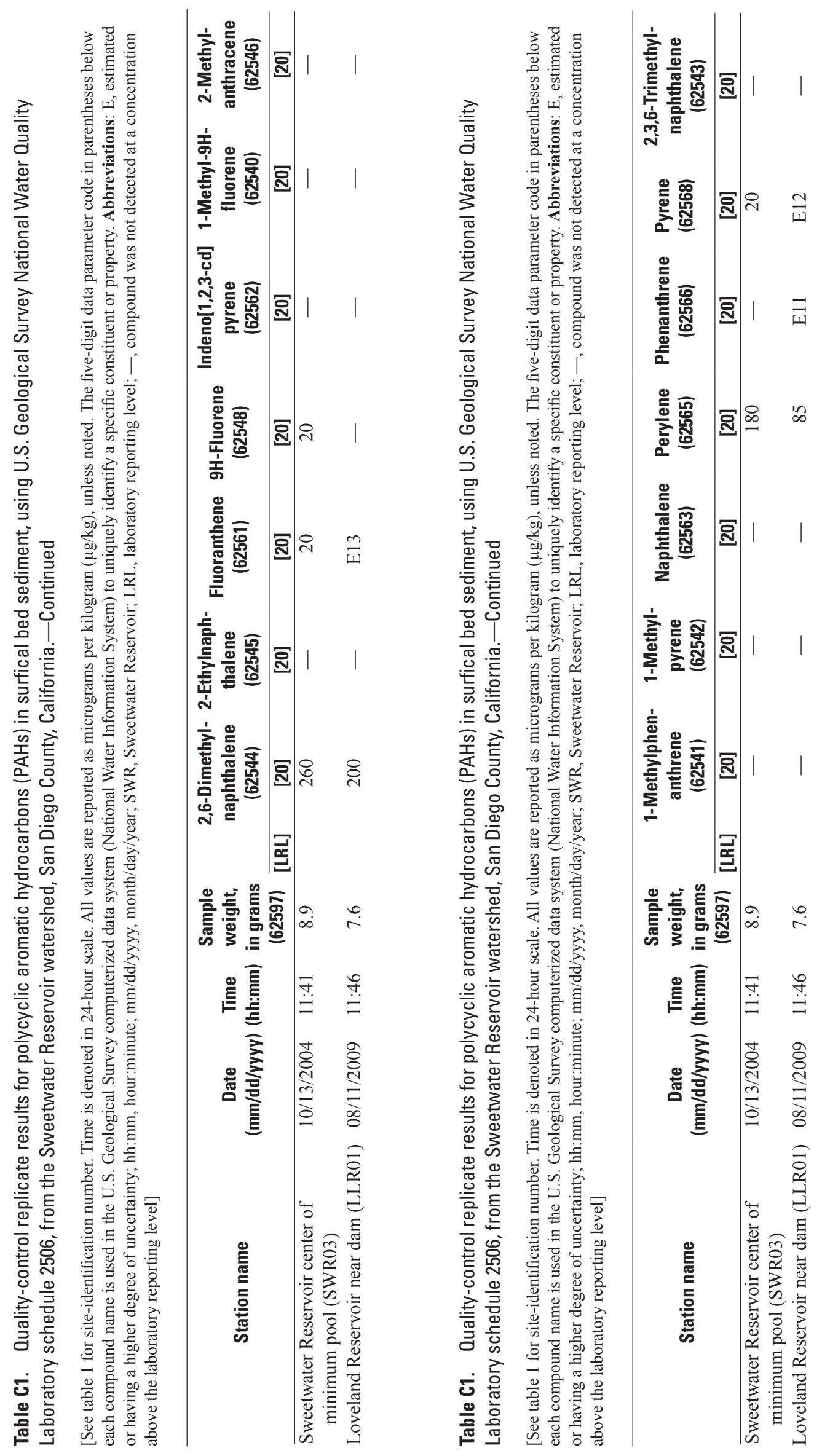




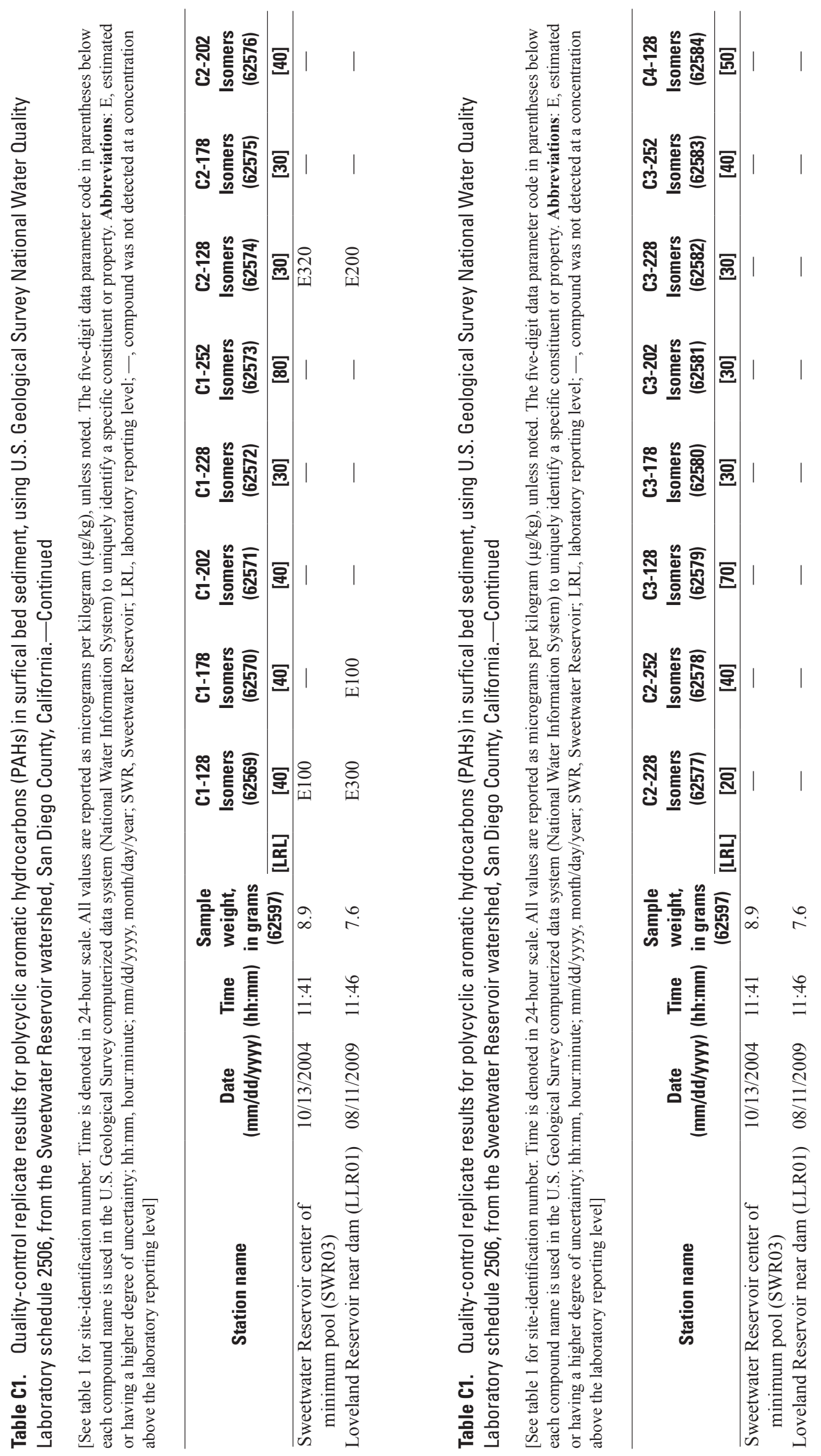




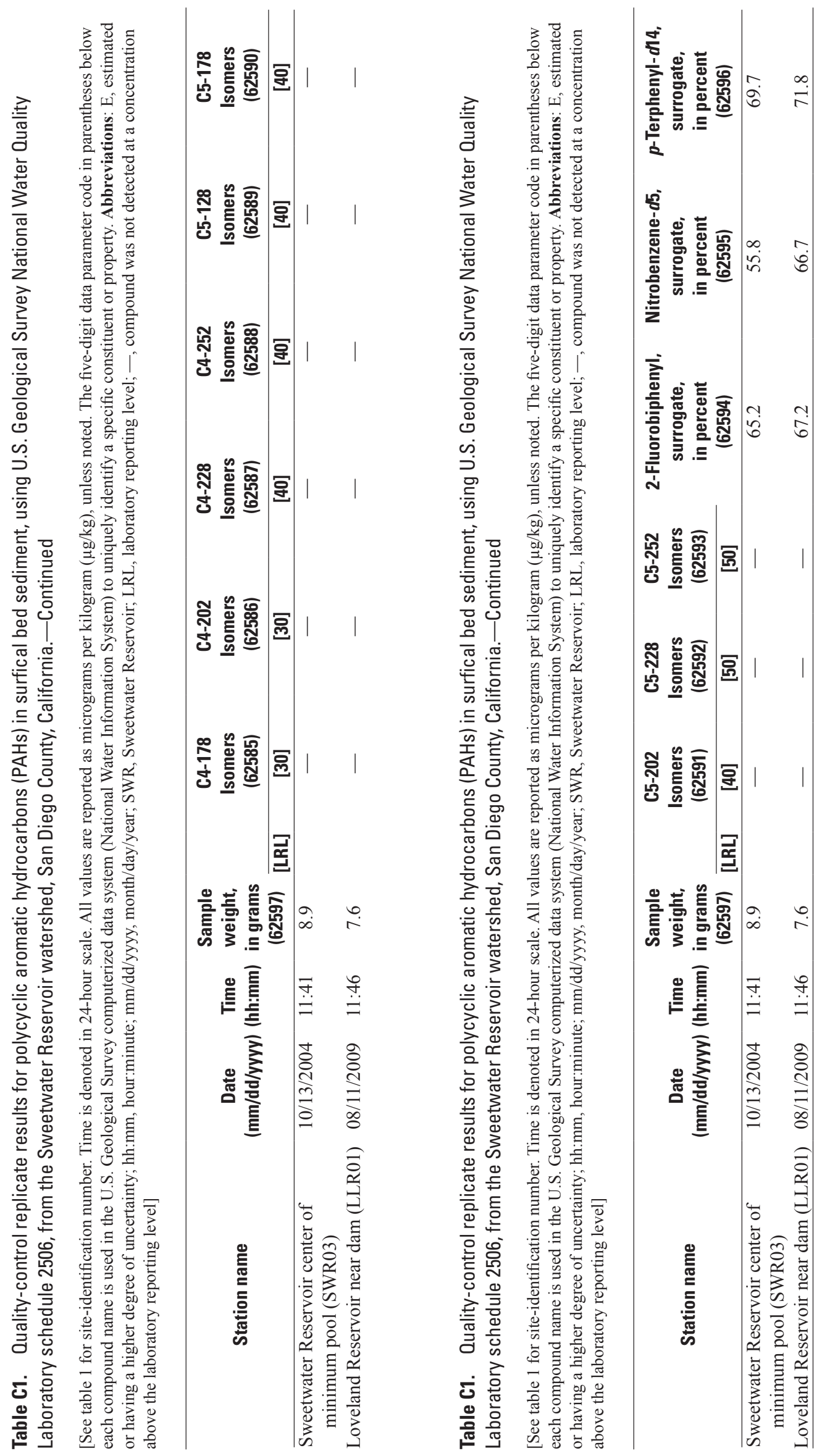



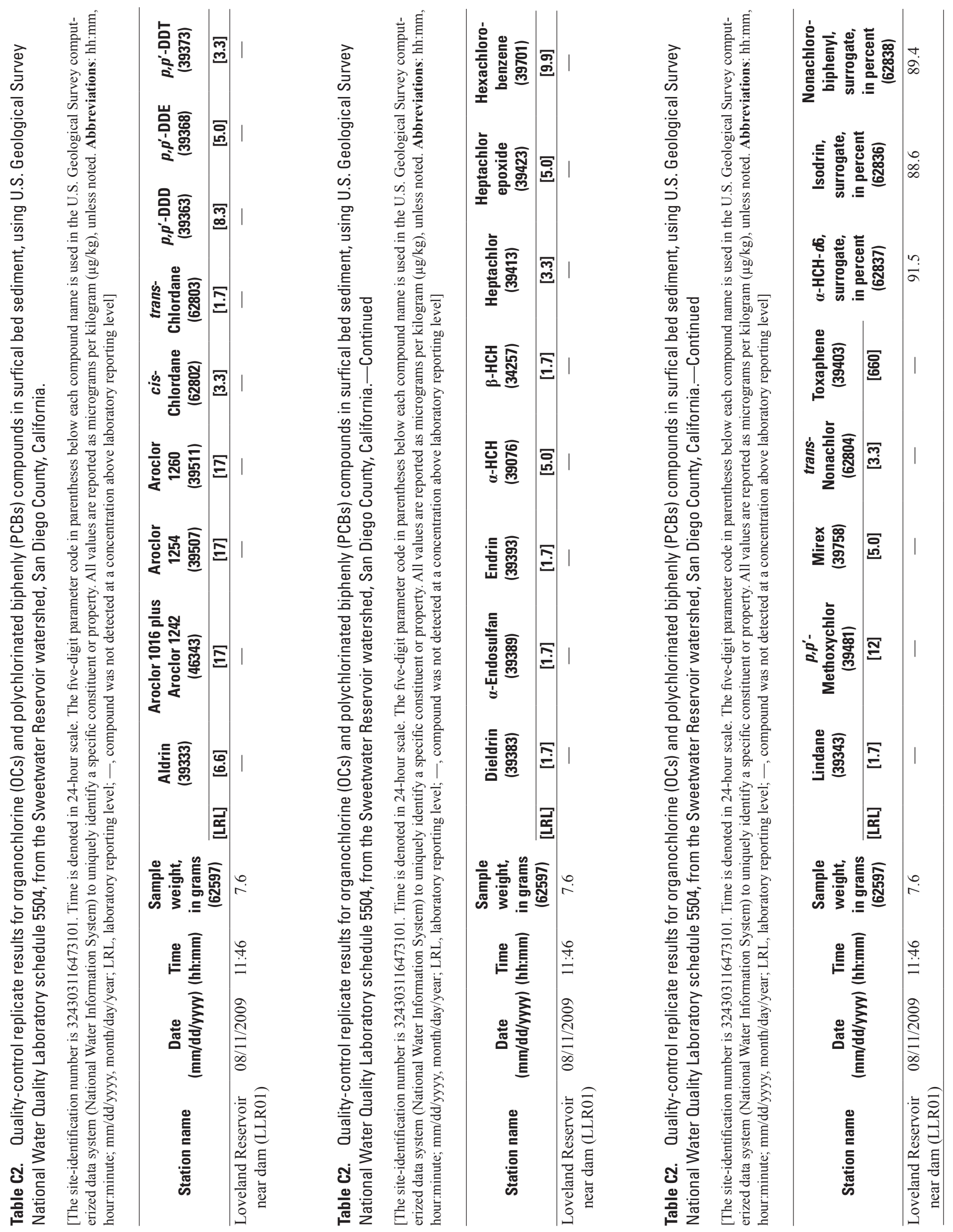


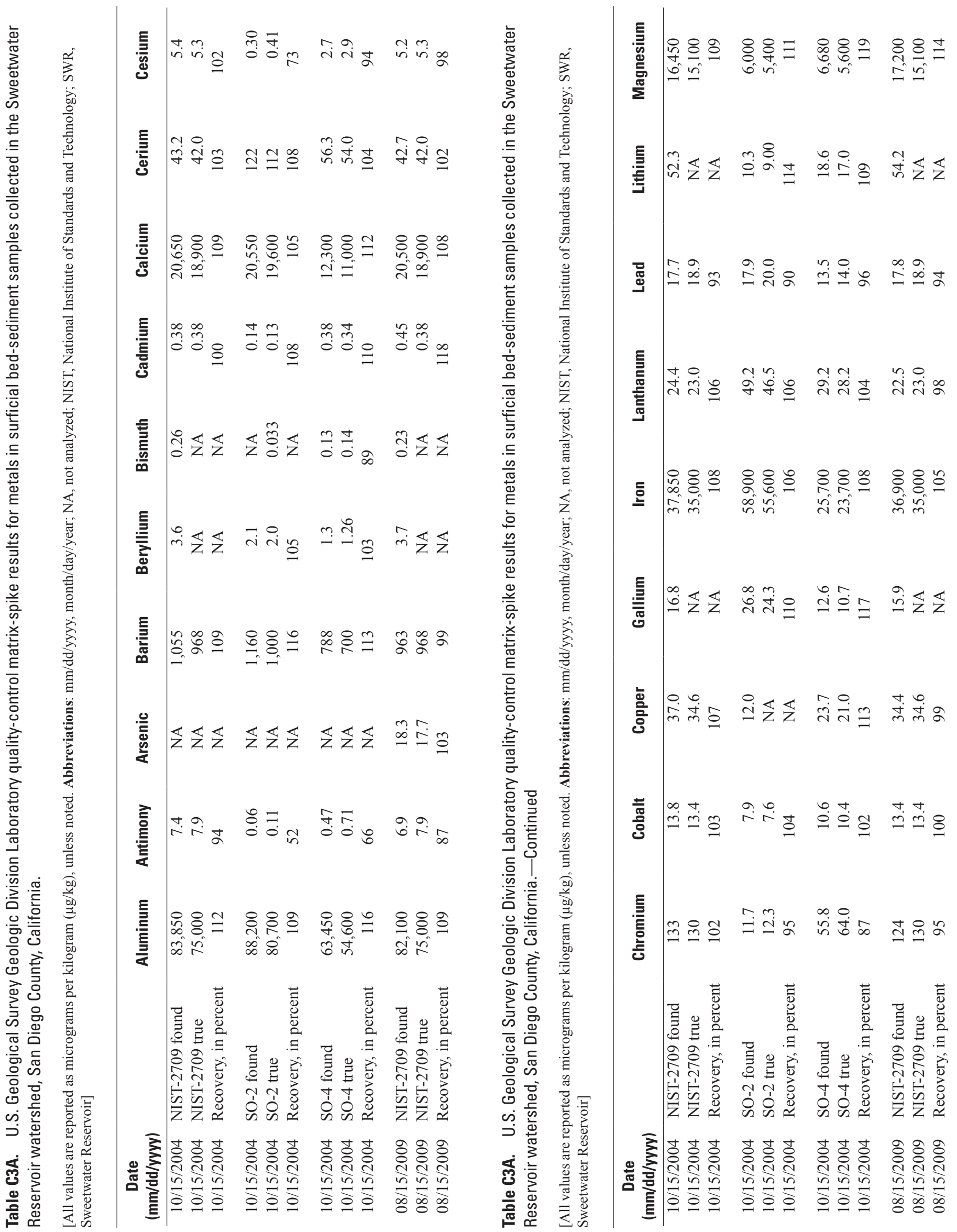



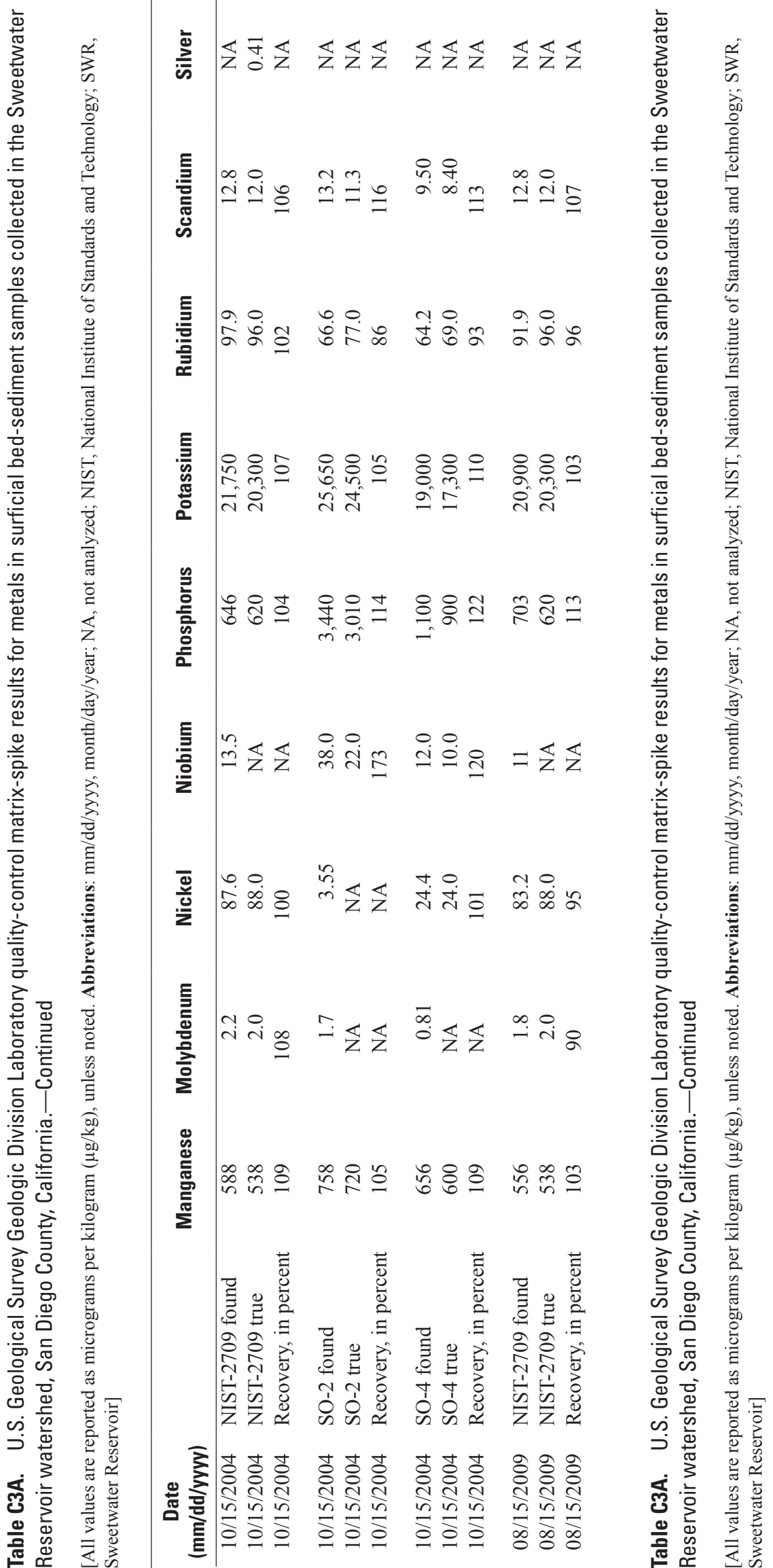

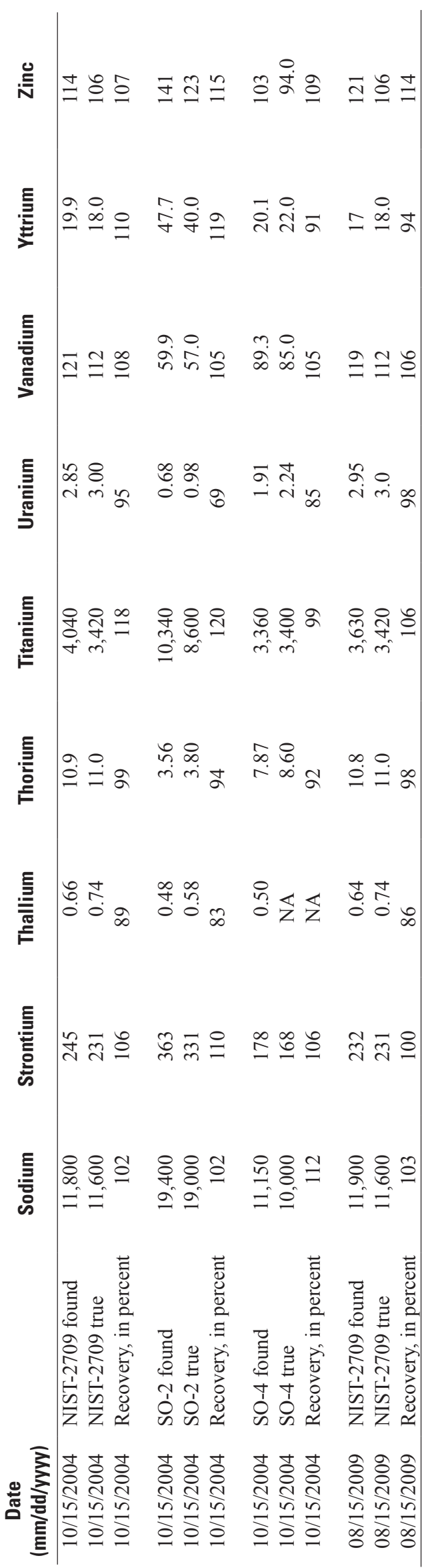




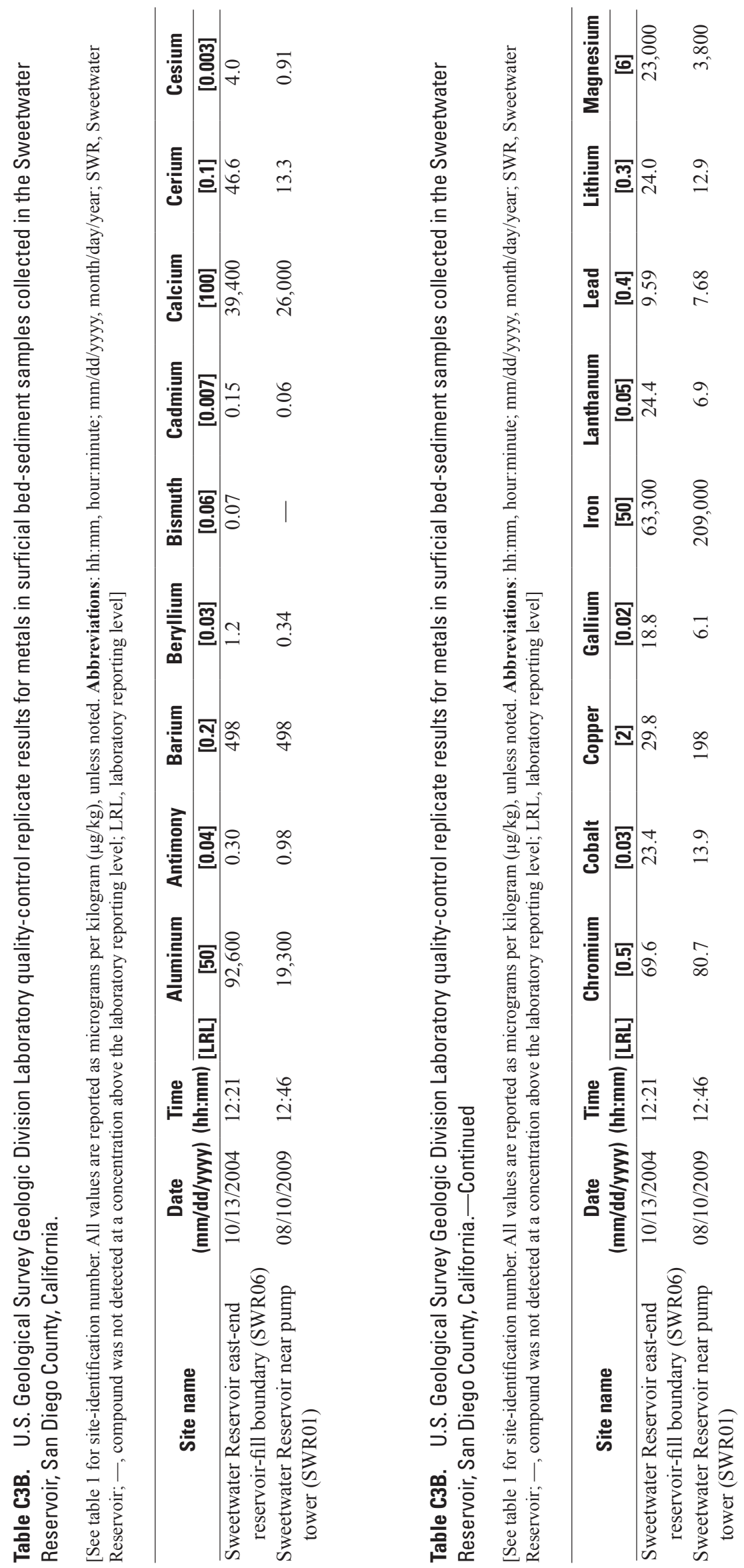




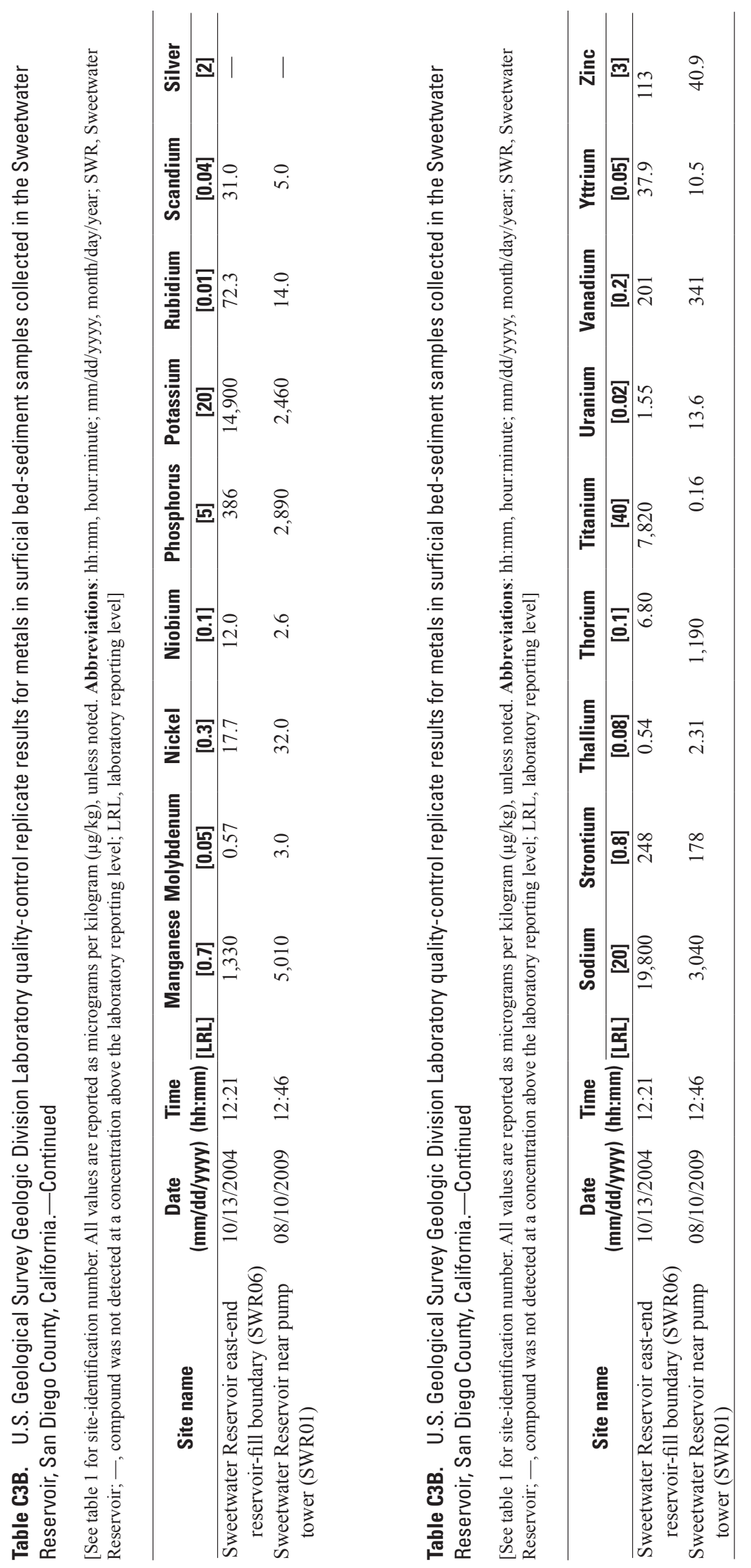




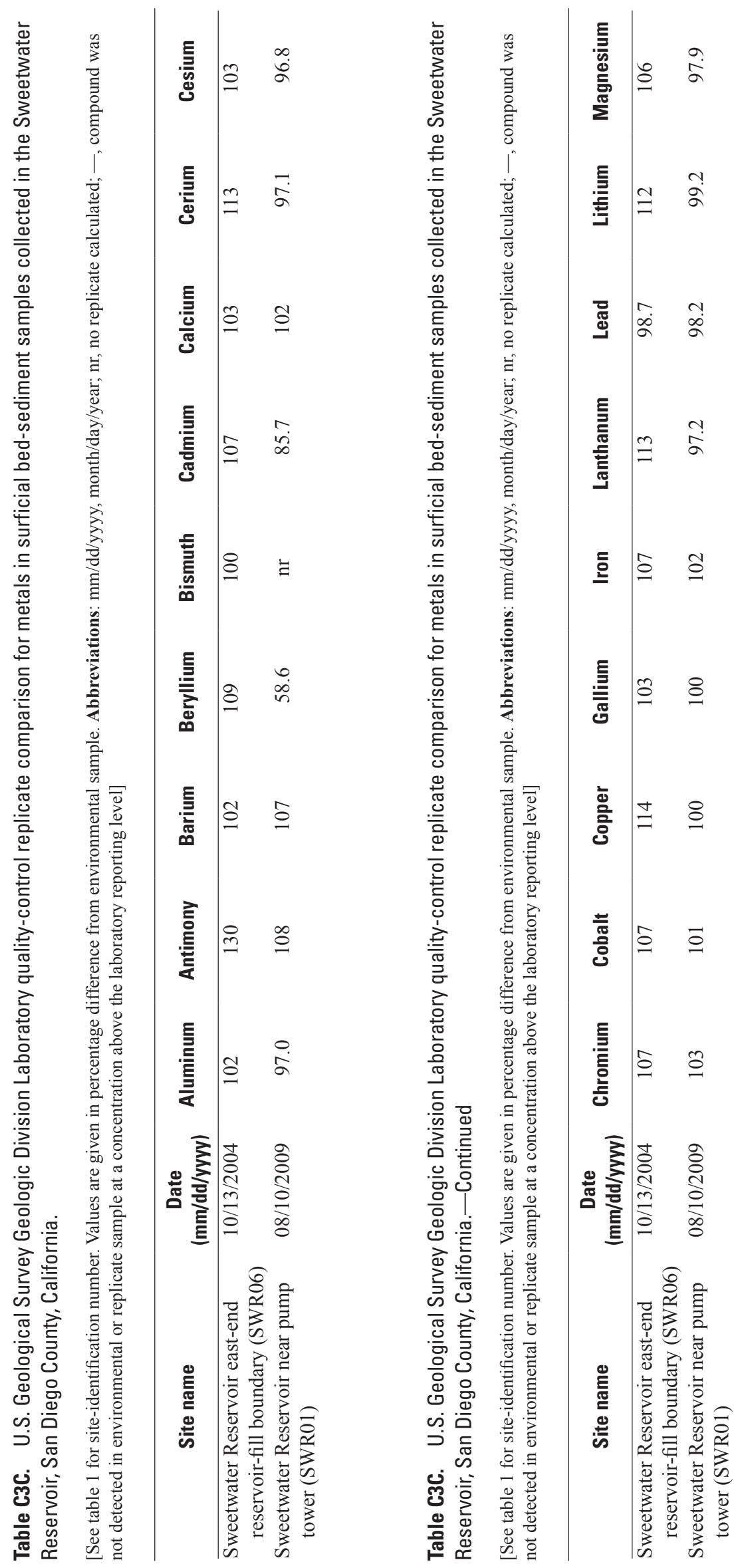




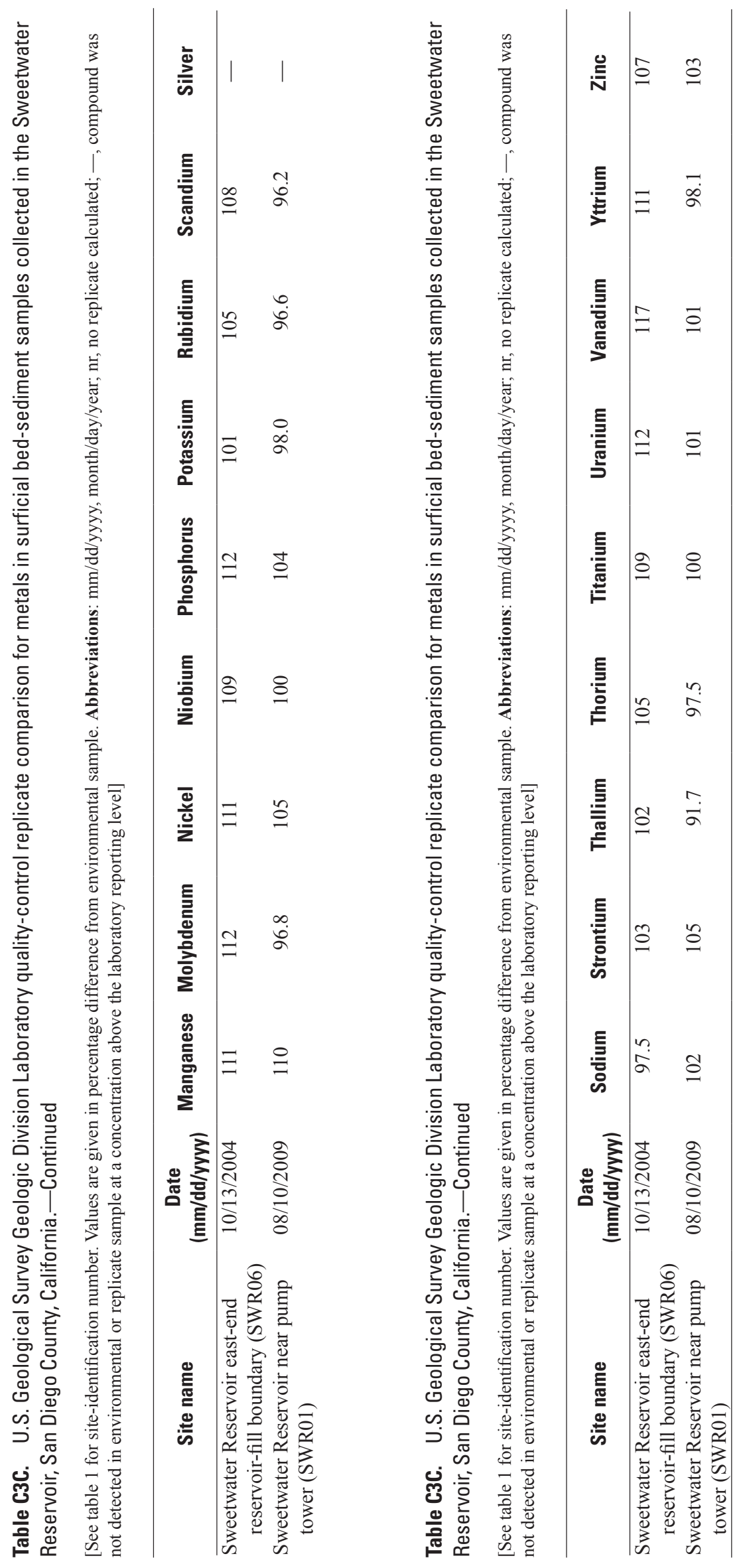


This page intentionally left blank. 
Prepared by the Sacramento Publishing Service Center.

For more information concerning this report, contact:

Director

U.S. Geological Survey

California Water Science Center

6000 J Street, Placer Hall

Sacramento, CA 95829

or visit our Web site at:

http://ca.water.usgs.gov 


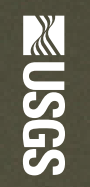

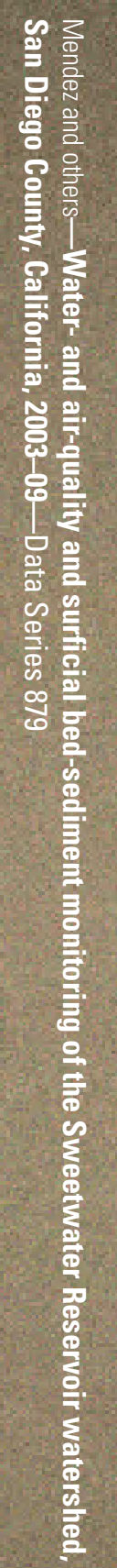

